\title{
Gestaltung der elektronischen Korrelationen in Perowskit-Heterostrukturen auf atomarer Skala
}

\author{
Dissertation \\ zur Erlangung des mathematisch-naturwissenschaftlichen Doktorgrades \\ „Doctor rerum naturalium“ \\ der Georg-August-Universität Göttingen \\ im Promotionsprogramm PROPHYS \\ der Georg-August University School of Science (GAUSS)
}

vorgelegt von

Markus Jungbauer

aus Speyer

Göttingen, 2015 


\section{Betreuungsausschuss}

Prof. Dr. Vasily Moshnyaga,

I. Physikalisches Institut, Georg-August-Universität Göttingen

Prof. Dr. Konrad Samwer,

I. Physikalisches Institut, Georg-August-Universität Göttingen

\section{Mitglieder der Prüfungskommission}

\section{Referent:}

Prof. Dr. Vasily Moshnyaga,

I. Physikalisches Institut, Georg-August-Universität Göttingen

\section{Korreferent:}

Prof. Dr. Christian Jooß,

Institut für Materialphysik, Georg-August-Universität Göttingen

\section{Korreferent:}

Prof. Dr. Guus Rijnders (nur Gutachter),

Faculty of Science \& Technology, University of Twente

\section{Weitere Mitglieder der Prüfungskommission}

Prof. Dr. Hans Hofsäss,

2. Physikalisches Institut, Georg-August-Universität Göttingen

Prof. Dr. Wolfram Kollatschny,

Institut für Astrophysik, Georg-August-Universität Göttingen

Prof. Dr. Hans-Ulrich Krebs,

Institut für Materialphysik, Georg-August-Universität Göttingen

Prof. Dr. Michael Seibt,

IV. Physikalisches Institut, Georg-August-Universität Göttingen

\section{Tag der mündlichen Prüfung: 16. Dezember 2015}




\section{Inhaltsverzeichnis}

\begin{tabular}{ll}
\hline 1. Einleitung & 1
\end{tabular}

\begin{tabular}{lll}
\hline I. & Theoretische Grundlagen & 7
\end{tabular}

2. Manganate 9

2.1. Kristallstruktur . . . . . . . . . . . . . . . . . . . . . . . . . 9 9

2.2. Elektronische Struktur der Mn-Ionen . . . . . . . . . . . . . . . . . . . . 10

2.3. Austauschmechanismen . . . . . . . . . . . . . . . . . 12

2.3.1. Superaustausch und Goodenough-Kanamori-Anderson-Regeln . . . . . 12

2.3 .2$. Doppelaustausch . . . . . . . . . . . . . . 14

2.4. Phasendiagramm von $\mathrm{La}_{1-\mathrm{x}} \mathrm{Sr}_{\mathrm{x}} \mathrm{MnO}_{3} \ldots \ldots \ldots \ldots \ldots$

$\begin{array}{ll}\text { 3. Grenzflächeneffekte } & 19\end{array}$

3.1. Grenzflächenchemie und Valenz der Übergangsmetallionen . . . . . . . . . . . 21

3.2. Orbitale Freiheitsgrade $\ldots \ldots \ldots \ldots \ldots \ldots$

3.3. Magnetismus . . . . . . . . . . . . . . . . . . . . . . . . . 29

3.3.1. Magnetismus und orbitale Polarisation . . . . . . . . . . . . . . . 29

3.3.2. "Tote" Lagen . . . . . . . . . . . . . . . . . . . . . . 31

3.3.3. Kollektive Phänomene . . . . . . . . . . . . . . . . . . . . . . 32

3.3.4. Exchange Bias . . . . . . . . . . . . . . . . . 35

$\begin{array}{ll}\text { II. Experimentelle Methoden } & 41\end{array}$

\begin{tabular}{|l|l|}
\hline 4. Metallorganische Aerosol Deposition (MAD) mit in-situ Ellipsometrie & 43
\end{tabular}

4.1. MAD Grundlagen . . . . . . . . . . . . . . . . . . . 43

4.2. Motivation zur in-situ Ellipsometrie . . . . . . . . . . . . . . . . . 45

4.3. Theorie Ellipsometrie . . . . . . . . . . . . . . . . . . . . . . . . . 47

4.3.1. Fresnel-Gleichungen . . . . . . . . . . . . . . . . . . 48

4.3.2. Reflexion an Multilagen . . . . . . . . . . . . . . . . . . . . 50

4.3.3. Rauigkeiten: Effektiv-Medium-Theorie . . . . . . . . . . . . . . . . . 52

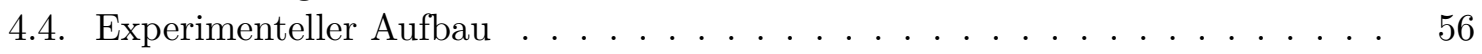

4.4.1. Ellipsometriemessung durch Polarisationsmodulation . . . . . . . . . . 60

4.4.2. Details zur Schichtpräparation . . . . . . . . . . . . . . . 63

4.4.3. Substrattemperatur . . . . . . . . . . . . . . . . . 65 
4.4.4. Brechungsindex von $\mathrm{SrTiO}_{3} \ldots \ldots \ldots \ldots \ldots$

4.4.5. Einfluss des Aerosols auf die Polarisationsmessungen . . . . . . . . . . 69

4.4.6. Auswertung und Modellierung der in-situ Ellipsometrie . . . . . . . . 71

5. Charakterisierung $\quad \mathbf{7 5}$

5.1. Rastersondenmikroskopie (Tunnelmikroskopie, Kraftmikroskopie) . . . . . . . 75

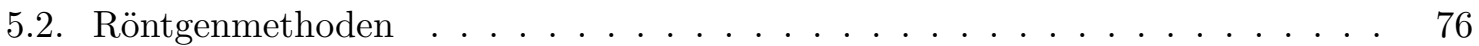

5.2.1. Röntgenbeugung (XRD) . . . . . . . . . . . . . . . . . 77

5.2.2. Röntgenreflektometrie (XRR) _ . . . . . . . . . . . . . . 82

5.3. Transmissionselektronenmikroskopie (TEM) . . . . . . . . . . . . . . . 85

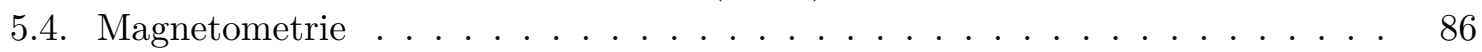

5.5. Elektrischer Widerstand . . . . . . . . . . . . . . . . . . . . 86

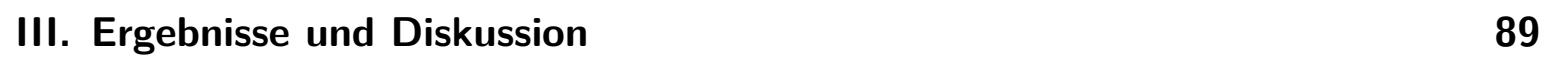

\begin{tabular}{ll}
\hline 6. In-situ Ellipsometrie beim Wachstum dünner Manganatfilme & 91
\end{tabular}

6.1. Stöchiometrie von $\mathrm{La}_{0.7} \mathrm{Sr}_{0.3} \mathrm{MnO}_{3} \ldots \ldots \ldots \ldots$. . . . . . . . . . . . . . . . . 91

6.2. $\quad$ Brechungsindex von $\mathrm{La}_{1-\mathrm{x}} \mathrm{Sr}_{\mathrm{x}} \mathrm{MnO}_{3}$ für $\mathrm{x}=0-1 \ldots \ldots$. . . . . . . . . . . 94

6.3. Die ersten Monolagen:

Durchmischung mit Substrat und Monolagenoszillationen . . . . . . . . . . . 97

\begin{tabular}{ll}
\hline 7. Atomlagenepitaxie & 107
\end{tabular}

7.1. Motivation . . . . . . . . . . . . . . . . . . . . 107

7.2. $\mathrm{Sr}_{\mathrm{n}+1} \mathrm{Ti}_{\mathrm{n}} \mathrm{O}_{3 \mathrm{n}+1}$ Ruddlesden-Popper Phase $\ldots \ldots \ldots \ldots \ldots$

7.2 .1$. Einführung . . . . . . . . . . . . . . . . . . 109

7.2.2. Experimentelle Vorbereitungen . . . . . . . . . . . . . . . . . . . 110

7.2.3. Wachstum und in-situ Ellipsometrie . . . . . . . . . . . . . . . . 112

7.2.4. Strukturelle Charakterisierung . . . . . . . . . . . . . . . . . . 112

7.2.5. Diskussion . . . . . . . . . . . . . . . . . . . . . 117

7.3. $\left(\left(\mathrm{SrMnO}_{3}\right)_{1} /\left(\mathrm{LaMnO}_{3}\right)_{2}\right)_{\mathrm{m}}$ : Rolle der ersten Lagen . . . . . . . . . . . . . . 122

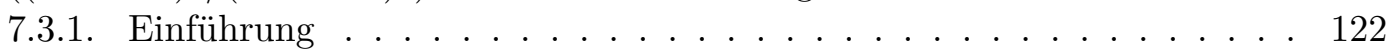

7.3.2. Experimentelle Vorbereitungen . . . . . . . . . . . . . . . . . . . . . 123

7.3.3. In-situ Ellipsometrie . . . . . . . . . . . . . . . . . . . . . . . . . . . 124

7.3.4. Strukturelle Charakterisierung . . . . . . . . . . . . . . . . . . . . 129

7.3.5. Magnetische und elektrische Eigenschaften. . . . . . . . . . . . . . . 131

7.3.6. Diskussion . . . . . . . . . . . . . . . . . . . . . 133

7.4. Zusammenfassung . . . . . . . . . . . . . . . . . . . . . 139

8. Exchange bias in $\mathrm{La}_{0.7} \mathrm{Sr}_{0.3} \mathrm{MnO}_{3} / \mathrm{La}_{1-\mathrm{x}} \mathrm{Sr}_{\mathrm{x}} \mathrm{MnO}_{3}(\mathrm{x}=0.6-1.0)$ Heterostrukturen 141

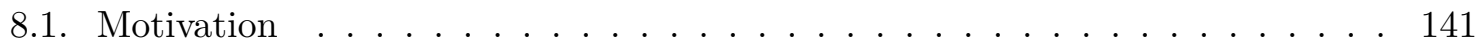

8.2. Probenherstellung und Charakterisierung . . . . . . . . . . . . . . . . 143

8.2.1. In-situ Ellipsometrie . . . . . . . . . . . . . . . . . . . . . . . 143

8.2.2. Oberflächenmorphologie . . . . . . . . . . . . . . . . . . 144

8.2.3. Schärfe der Grenzflächen: Röntgenreflektometrie . . . . . . . . . . . . 146

8.2.4. Epitaktische Verspannung: Röntgendiffraktion. . . . . . . . . . . . . . 149

8.2.5. Simulation der in-situ Ellipsometrie . . . . . . . . . . . . . . . . . 152

8.3. Magnetische Eigenschaften . . . . . . . . . . . . . . . . . . . 155

8.4. Diskussion . . . . . . . . . . . . . . . . . . . . . . . . . . . 160

8.5. Zusammenfassung . . . . . . . . . . . . . . . . . . . . 168 
$\begin{array}{ll}\text { 9. } \mathrm{LaMnO}_{3} / \mathrm{SrMnO}_{3} \text {-Übergitter } & 171\end{array}$

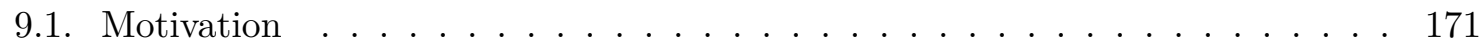

9.2. Vorbereitung: Einfache $\mathrm{La}_{1-\mathrm{x}} \mathrm{Sr}_{\mathrm{x}} \mathrm{MnO}_{3}$-Filme . . . . . . . . . . . . . . . . 172

9.2.1. Die Ausgangsmaterialien $\mathrm{SrMnO}_{3}$ und $\mathrm{LaMnO}_{3}$. . . . . . . . . . . . 172

9.2.2. Magnetismus von $\mathrm{La}_{1-\mathrm{x}} \mathrm{Sr}_{\mathrm{x}} \mathrm{MnO}_{3}$-Filmen auf $\mathrm{SrTiO}_{3}$ (001)- und (111)-

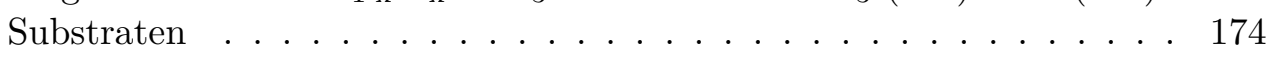

9.3. Wachstum von $\mathrm{LaMnO}_{3} / \mathrm{SrMnO}_{3}$-Übergittern . . . . . . . . . . . . . . . 176

9.3.1. $\mathrm{SrTiO}_{3}$ (111): $\mathrm{Sr} /$ La-Durchmischung . . . . . . . . . . . . . . . . . . 177

9.3.2. $\mathrm{SrTiO}_{3}(001)$ : Beobachtung der Delokalisierung von $\mathrm{e}_{\mathrm{g}}$-Elektronen durch

in-situ Ellipsometrie . . . . . . . . . . . . . . . . . . 181

9.4. Magnetismus der $\mathrm{LaMnO}_{3} / \mathrm{SrMnO}_{3}$-Übergitter . . . . . . . . . . . . . . . . . 191

9.4.1. Vergleich $\mathrm{SrTiO}_{3}$ (111)- und (001)-Substrat . . . . . . . . . . . . . . . 191

9.4.2. Neue ferromagnetische Phase? . . . . . . . . . . . . . . . . . 195

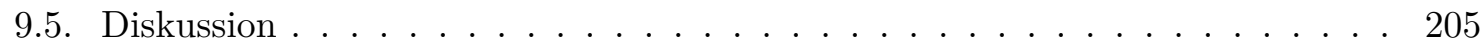

9.6. Zusammenfassung . . . . . . . . . . . . . . . . . . . . . . 214

\begin{tabular}{ll}
\hline 10. Zusammenfassung und Ausblick & 217
\end{tabular}

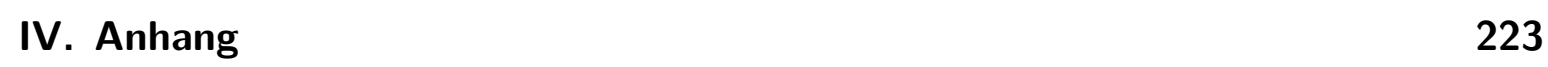

\begin{tabular}{ll}
\hline Abkürzungsverzeichnis & 225
\end{tabular}

\begin{tabular}{ll}
\hline Literatur & 227
\end{tabular}

\begin{tabular}{lr}
\hline Publikationen & 249
\end{tabular}

\begin{tabular}{ll}
\hline Danksagungen & 251
\end{tabular} 



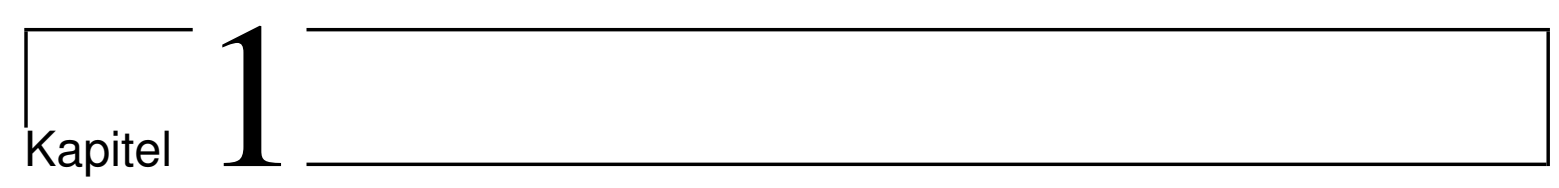

\section{Einleitung}

„The interface is the device." In diesem Ausspruch aus der Nobelpreisrede von Herbert Kroemer [1] wird die Bedeutung der Grenzflächen für die Funktionalitäten von Halbleiterstrukturen zusammengefasst. Diese auf konventionellen Halbleitern aufbauenden Technologien sind eine Grundlage für den zukünftigen Fortschritt unserer heutigen Gesellschaft. Mit zunehmender Verkleinerung der Halbleiterstrukturen stößt man aber an materialbedingte Grenzen, die aus der geringen Ladungsträgerdichte der konventionellen Halbleiter resultieren. Dadurch kommt es unterhalb bestimmter lateraler Abmessungen zu Quanteneffekten, die die angestrebte Funktionalität stören [2, 3]. Deshalb sucht man nach neuen Materialien, die eine weitere Verkleinerung der Strukturen erlauben und nach Möglichkeit zusätzliche Funktionalitäten bereitstellen.

Eine mögliche Materialklasse, die diese Rolle ausfüllen könnte, ist die der komplexen Oxide. Diese stellen eine Vielzahl an funktionalen Eigenschaften wie Ferro-, Antiferromagnetismus, Ferroelektrizität oder Supraleitung bereit. Außerdem besitzen die Materialien in der Unterklasse der Perowskit-Oxide ähnliche Gitterstrukturen, so dass die Herstellung von Heterostrukturen mit qualitativ hochwertigen Grenzflächen ermöglicht wird. Es gibt Beispiele von multiferroischen Heterostrukturen aus einem Ferromagneten und einem Ferroelektrikum, bei denen an der Grenzfläche eine Kopplung zwischen der Magnetisierung und der ferroelektrischen Polarisation hergestellt wird 446 .

Neben diesen Kopplungseffekten beobachtet man auch, dass sich an der Grenzfläche ein völlig neuer Zustand einstellt, der in den beiden zusammengebrachten Materialien nicht auftaucht. In diesem Zusammenhang ist das zweidimensionale Elektronengas an der Grenzfläche der beiden Isolatoren $\mathrm{SrTiO}_{3}$ (STO) und $\mathrm{LaAlO}_{3}$ (LAO) das prominenteste Beispiel [7, das ein großes wissenschaftliches und technologisches Interesse für Grenzflächen von komplexen Oxiden induziert hat. Wie bei einem Feld-Effekt-Transistor (FET) kann man auch bei dem Elektronengas an der STO/LAO-Grenzfläche die Ladungsträgerkonzentration durch eine Gate-Spannung variieren und sogar den Übergang zur Supraleitung erreichen [8, 9].

Durch das komplexe Zusammenspiel zwischen lokaler Chemie und Freiheitsgraden der Ladungen, Orbitale, Spins und des Gitters ist das grundlegende physikalische Verständnis der Grenzflächeneffekte sehr anspruchsvoll. Der experimentelle Zugang wird dadurch erschwert, dass sich die Grenzflächeneffekte typischerweise im Bereich weniger Monolagen abspielen. 
Diese im Vergleich zu konventionellen Halbleitern deutlich kleinere Längenskala ist das Resultat der höheren Ladungsträgerdichte, die eine kleinere Abschirmlänge bedingt [10]. Zur Untersuchung der Grenzflächenphänomene von komplexen Oxiden muss man in der Lage sein, Heterostrukturen mit atomar scharfen Grenzflächen herzustellen und deren Eigenschaften auf der Längenskala einer Atomlage zu analysieren.

Im Rahmen dieser Arbeit wurde eine Anlage zur Abscheidung von komplexen Oxiden mittels metallorganischer Aerosol Deposition (MAD) aufgebaut, um diesen Anforderungen gerecht zu werden. Diese Anlage wurde mit einer Wachstumskontrolle über in-situ Ellipsometrie ausgestattet. Dadurch dass die Herstellung der Dünnfilme mittels MAD bei atmosphärischen Bedingungen $\left(p_{\mathrm{O}_{2}} \approx 0.21\right.$ bar $)$ stattfindet, kann man die MAD klar von den Vakuum-Methoden, der gepulsten Laserdeposition (PLD) und Molekularstrahlepitaxie (MBE), abgrenzen. Durch das Vorhandensein von Übergangsmetallionen besitzen die hier untersuchten Perowskit-Oxide ein kompliziertes $T-p_{\mathrm{O}_{2}}$-Phasendiagramm. Unter Anwendung der verbreiteten VakuumMethoden kommt es so häufig zum Einbau unerwünschter Sauerstoffleerstellen in die Oxidfilme. Dieser Störeinfluss kann bei der MAD ausgeschlossen werden. Des Weiteren beeinflussen die Depositionsbedingungen die Beweglichkeit der Adatome auf der Probenoberfläche und damit die chemische Qualität der Grenzflächen. In dieser Arbeit wird am Beispiel von Übergittern aus $\mathrm{LaMnO}_{3}(\mathrm{LMO})$ und $\mathrm{SrMnO}_{3}$ (SMO) gezeigt, dass sich diese Unterschiede in der Art und Verteilung von Defekten in den magnetischen Eigenschaften niederschlagen.

Die typischerweise zur Wachstumskontrolle verwendete Beugung hochenergetischer Elektronen bei Reflexion (RHEED) ist sensitiv auf die Verteilung der Gesamtelektronen an der Probenoberfläche. Mit der hier benutzten Ellipsometrie erhält man zum Teil komplementäre Informationen, da man empfindlich auf die elektronischen Eigenschaften der deponierten Materialien ist. Aus den Messdaten der Ellipsometrie kann man so neben der Identifikation der Wachstumsmode, z.B. über Monolagenoszillationen bei zweidimensionalem Inselwachstum, auch Schlüsse über die Verteilung der Elektronen auf den Übergangsmetallionen ziehen (siehe Kapitel 6, 7, 8 und 9).

Ein Perowskit $\mathrm{ABO}_{3}$ kann man entlang der [001]-Richtung in alternierende $\mathrm{AO}$ - und $\mathrm{BO}_{2}$ Lagen zerlegen. Zur vollständigen Definition der chemischen Umgebung einer Grenzfläche benötigt man also die Kontrolle über diese halben Perowskitlagen. Dieser Freiheitsgrad hat tatsächlich Einfluss auf die elektronischen Eigenschaften der Strukturen. Für die LAO/STOGrenzfläche beobachtet man nur ein zweidimensionales Elektronengas, wenn das STO-Substrat mit einer $\mathrm{TiO}_{2}$-Lage terminiert ist [7]. Hier werden die ersten Experimente zur MAD-Atomlagenepitaxie (ALE) präsentiert. Im Gegensatz zum konventionellen MAD-Verfahren werden die Perowskite abwechselnd aus $\mathrm{AO}$ - und $\mathrm{BO}_{2}$-Lagen aufgebaut. Am Beispiel von gestapelten Ruddlesden-Popper-Strukturen aus $\mathrm{SrO}_{-} \mathrm{SrTiO}_{3}$ und $\mathrm{SMO} / \mathrm{LMO}-$ Heterostrukturen wird dieses Verfahren vorgestellt.

Neben den zuvor genannten technologischen Weiterentwicklungen der MAD werden in dieser Arbeit auch die magnetischen Kopplungen zwischen verschiedenen Manganaten aus dem $\mathrm{La}_{1-\mathrm{x}} \mathrm{Sr}_{\mathrm{x}} \mathrm{MnO}_{3}-\mathrm{Phasendiagramm}$ studiert. Dieses Phasendiagramm enthält ferromagnetische (FM) und antiferromagnetische (AFM) Phasen mit verschiedenen Typen von AFM Ordnung. Die Kopplung zwischen Ferro- und Antiferromagneten an der gemeinsamen Grenzfläche kann zu Veränderungen der $M(H)$-Kurve des Ferromagneten führen. Eine mögliche Modifikation besteht in der Ausbildung von exchange bias (EB), das eine Verschiebung der $M(H)$-Kurve entlang der Feldachse bezeichnet. Es gibt theoretische und experimentelle Hinweise darauf, dass das EB mit einem Spin-Glas-Zustand der Grenzfläche verknüpft 
ist [11, 12. Die genaue Ausprägung dieses Spin-Glases hängt von der Art und Stärke der magnetischen Wechselwirkungen an der Grenzfläche ab. In Heterostrukturen aus PerowskitManganaten kann man die magnetischen Kopplungen durch Veränderungen der mittleren Mn-Valenz und der Polarisation der 3d-Orbitale beeinflussen. Dies wird hier am Beispiel von $\mathrm{La}_{0.7} \mathrm{Sr}_{0.3} \mathrm{MnO}_{3} / \mathrm{La}_{1-\mathrm{x}} \mathrm{Sr}_{\mathrm{x}} \mathrm{MnO}_{3}$-Doppellagen mit $x=0.6-1.0$ durchgeführt, wo sich $\ddot{\mathrm{A}}$ derungen in der Dotierung und des epitaktischen Verspannungszustandes in dem messbaren exchange bias niederschlagen.

In Übergittern aus LMO und SMO wird der Transfer von Elektronen an der Grenzfläche entscheidend für das resultierende magnetische Verhalten der gesamten Struktur. May et al. führen weiterhin an, dass die morphologischen Eigenschaften der LMO/SMO-Grenzflächen Einfluss auf die magnetischen Kopplungen nehmen können [13, 14. An einer Grenzfläche mit Rauigkeiten auf der Nanoskala wird die FM Austauschwechselwirkung durch eine Unordnung der 3d-Orbitale unterdrückt [13, 14]. Wenn die Grenzflächen chemisch scharf sind, unterstützt die Ordnung der La- und Sr-Ionen auf den A-Plätzen den FM Doppelaustausch in der Ebene. May et al. erreichen durch eine solche Kationenordnung die Erhöhung der Néel-Temperatur $T_{N}$ von $\mathrm{La}_{1 / 3} \mathrm{Sr}_{2 / 3} \mathrm{MnO}_{3}$ um $\sim 70 \mathrm{~K}$ [15]. Bei den hier mit MAD abgeschiedenen LMO/SMOÜbergittern stellt man die Koexistenz zweier FM Phasen fest. Diese in der Literatur nicht beschriebene Phänomenologie wird den besonderen Depositionsbedingungen der MAD zugeschrieben. Dadurch unterscheiden sich die Defektstrukturen der Ausgangsmaterialien, LMO und SMO, und die Qualität der Grenzflächen von den Beispielen der Literatur. Das Auftreten dieser beiden Phasen wird mit einer Kationenordnung an den Grenzflächen und der aus der epitaktischen Verspannung folgenden orbitalen Polarisation in Verbindung gebracht.

$\mathrm{Zu}$ Beginn werden die theoretischen Grundlagen für die Physik der Manganate (Kapitel 2) und der Grenzflächen von komplexen Oxiden (Kapitel 3) besprochen. Bei den Grundlagen der Manganate wird ein besonderer Schwerpunkt darauf gelegt, die Faktoren herauszustellen, die Art und Stärke der magnetischen Wechselwirkungen über Super- und Doppelaustausch bestimmen. Daraus wird dann das magnetische Phasendiagramm von $\mathrm{La}_{1-\mathrm{x}} \mathrm{Sr}_{\mathrm{x}} \mathrm{MnO}_{3}$ für $x=0-1$ deduziert. Im anschließenden Kapitel über Grenzflächen werden die Modifikationen der Chemie, Ladungen und Orbitale besprochen, die sich bei der Kombination verschiedener komplexer Oxide an den Grenzflächen ergeben. Dann wird gezeigt, wie sich diese Veränderungen im Magnetismus niederschlagen.

Die im Rahmen dieser Dissertation aufgebaute MAD-Anlage mit Wachstumsüberwachung durch in-situ Ellipsometrie wird in Kapitel 4 vorgestellt. Nach der Einführung in die Grundlagen der MAD wird die Theorie der Ellipsometrie-Messungen besprochen. Dann wird die experimentelle Realisierung der neuen MAD-Anlage und die Auswertung der Messdaten erläutert. In Kapitel 5 werden die Methoden zur ex-situ Charakterisierung der Proben vorgestellt, wobei ein Schwerpunkt auf die Röntgenreflektometrie (XRR) und Röntgendiffraktion (XRD) gelegt wird. Mit diesen Techniken lässt sich die chemische Qualität der vergrabenen Grenzflächen qualifizieren.

Die Darstellung der Ergebnisse wird mit Kapitel 6 eröffnet, in dem die ersten Ergebnisse der in-situ Ellipsometrie für das Wachstum von einfachen $\mathrm{La}_{1-\mathrm{x}} \mathrm{Sr}_{\mathrm{x}} \mathrm{MnO}_{3}(x=0-1)$-Filmen auf STO-Substraten vorgestellt werden. Es wird dargelegt, dass die optischen Konstanten der $\mathrm{La}_{1-\mathrm{x}} \mathrm{Sr}_{\mathrm{x}} \mathrm{MnO}_{3}$-Filme bei Depositionsbedingungen vor allem von der durchschnittlichen Mn-Valenz abhängen. Die Brechungsindizes für $x=0-1$ werden in den späteren Kapiteln zur Simulation der in-situ Ellipsometrie verwendet. Bei der Beobachtung des Wachstums 
der Manganat-Filme erkennt man in den ersten 3 Monolagen eine Durchmischung mit dem STO-Substrat und Monolagenoszillationen, die mit einem zweidimensionalen Inselwachstum zusammenhängen.

Dann werden in Kapitel 7 die ersten Versuche zur MAD-ALE vorgestellt. Als erster Test der Funktionalität werden Ruddlesden-Popper-Filme, $\mathrm{Sr}_{\mathrm{n}+1} \mathrm{Ti}_{\mathrm{n}} \mathrm{O}_{3 \mathrm{n}+1}$ mit $n=2-4$, abgeschieden. Mit der in-situ Ellipsometrie kann man die korrekte Sr/Ti-Stöchiometrie in der Präkursorlösung einstellen. Des Weiteren kann man Rückschlüsse auf Defekte der SrO-STO-Abfolge ziehen. Auch LMO/SMO-Heterostrukturen wurden mit MAD-ALE abgeschieden. Im Gegensatz zu den SrO-STO-Strukturen liegen in den Manganat-Übergittern zum Teil geladene AOund $\mathrm{BO}_{2}$-Lagen vor. Dadurch können sich Coulomb-Potentiale ergeben, die das Wachstum stören. Es wird gezeigt, dass ein absichtlicher Mn-Überschuss in der ersten $\mathrm{MnO}_{2}$-Lage zu einem stabilen lagenweisen Wachstum führt. Mit der in-situ Ellipsometrie können die Änderungen der Mn-Valenzen in den oberflächlichen $\mathrm{MnO}_{2}$-Lagen bei jedem Wachstumsschritt nachvollzogen werden.

In Kapitel 8 werden die Studien zum exchange bias (EB) von $\mathrm{La}_{0.7} \mathrm{Sr}_{0.3} \mathrm{MnO}_{3} / \mathrm{La}_{1-\mathrm{x}} \mathrm{Sr}_{\mathrm{x}} \mathrm{MnO}_{3}$ $(x=0.6-1.0)$ Doppellagen präsentiert. Durch die Verwendung von variierenden Sr-Dotierungen $x$ kann man verschiedene Typen von AFM Ordnung in der $\mathrm{La}_{1-\mathrm{x}} \mathrm{Sr}_{\mathrm{x}} \mathrm{MnO}_{3}$-Lage einstellen und den resultierenden Einfluss auf das EB in Erfahrung bringen. Bei der Variation der Schichtdicken ergeben sich durch die Relaxation der epitaktischen Verspannungen unterschiedliche tetragonale Verzerrungen. Daraus resultiert eine Variation der orbitalen Polarisation der 3d-Orbitale und damit Veränderungen der magnetischen Kopplungen an der Grenzfläche. Diese Modifikationen der Grenzflächen kann man wiederum im Verhalten der $M(H)$-Kurven ausmachen.

Übergitter aus LMO und SMO auf STO (001)-, (111)- und $\left(\mathrm{La}_{0.3} \mathrm{Sr}_{0.7}\right)\left(\mathrm{Al}_{0.65} \mathrm{Ta}_{0.35}\right) \mathrm{O}_{3}$ (LSAT) (001)-Substraten werden in Kapitel 9 behandelt. Mit der in-situ Ellipsometrie kann man bereits beim Wachstum Aussagen über die strukturellen und elektrischen Eigenschaften der Heterostrukturen treffen. So kann man beim Wachstum auf STO (111) Interdiffusionsprozesse identifizieren und beim Wachstum auf STO (001) die Delokalisierung der Elektronen über die LMO/SMO-Grenzfläche quantifizieren. Dieser Kontrast der chemischen Qualität der Grenzflächen schlägt sich auch im Magnetismus nieder. Bei den Proben auf STO (111) stellt man einen homogenen FM Zustand fest. Dagegen sieht man für STO (001) zwei entkoppelte FM Phasen. Unter Berücksichtigung der experimentellen Ergebnisse bei Variation der LMO/SMO-Bilagendicke und Modifikation der epitaktischen Verspannung durch das Wachstum auf einem LSAT (001)-Substrat entwickelt man ein Modell, um diesen unkonventionellen Zustand zu erklären.

Zum Schluss werden die in der Arbeit gewonnen Ergebnisse zusammengefasst. Dabei wird zum einen auf die in dieser Arbeit erprobten technischen Weiterentwicklungen der MAD eingegangen. Andererseits werden die verschiedenen magnetischen Kopplungsphänomene, die hier in Manganat-Heterostrukturen beobachtet wurden, beleuchtet. Insgesamt ergeben sich so viele Anknüpfungspunkte für weitere Forschungsaktivitäten, die abschließend erwähnt werden sollen.

Diese Arbeit wurde im Rahmen des IFOX (Interfacing Oxides)-Projektes (FP 7) der Europäischen Union durchgeführt. Damit kam es zu einem regen Austausch mit anderen Arbeitsgruppen. Es ist insbesondere die Verbindung zur Gruppe von Johan Verbeeck der Universität Antwerpen, in der Ricardo Egoavil und Haiyan Tan hochaufgelöste Transmissionselektronenmikroskop (TEM)-Aufnahmen der hier vorgestellten Proben anfertigten, zu erwähnen. Au- 
ßerdem wurden der Gruppe um Donald MacLaren von der Universität Glasgow verschiedene Heterostrukturen zur Untersuchung mit einem Lorentz-TEM bereitgestellt. Neben den Partnern im IFOX-Projekt fertigte Vladimir Roddatis vom Institut für Materialphysik der GeorgAugust-Universität Göttingen TEM-Aufnahmen an. Zudem lieferten Bachelor- und Masterstudenten, die im Umfeld dieser Dissertation betreut wurden, experimentelle Beiträge: Danny Schwarzbach, Sven Esser und Marius Keunecke bearbeiteten Teilaspekte der LMO/SMOÜbergitter (Kapitel 9), Felix Massel führte MAD-ALE mit Manganaten und Fryderyk Lyzwa mit Ruddlesden-Popper-Strukturen aus $\mathrm{SrO}-\mathrm{SrTiO}_{3}$ durch (Kapitel 7 ). 



\section{Teil I.}

\section{Theoretische Grundlagen}



Kapitel 2

\section{Manganate}

Manganate sind stark korrelierte Übergangsmetallperowskite, die in Abhängigkeit ihrer chemischen Zusammensetzung verschiedene elektrische und magnetische Eigenschaften zeigen können. Einige Mitglieder dieser Materialklasse sind empfindlich gegen externe Stimuli, z.B. elektrische und magnetische Felder, hydrostatischen Druck, Licht, die strukturelle, magnetische und elektrische Phasenübergänge treiben können [16, 17]. Dabei ist das prominenteste Beispiel der Kolossale Magnetowiderstand (CMR), der eine drastische Reduktion des Widerstandes durch ein äußeres Magnetfeld bezeichnet. Dabei wird ein Übergang vom ladungsgeordneten (antiferromagnetischen) Isolator (COI) zum ferromagnetischen Metall (FMM) induziert 18, 19. Manganate mit entsprechender Zusammensetzung sind ferromagnetische Metalle mit einer Spinpolarisation von $100 \%$ [20], so dass sie ein großes Potential für spintronische Anwendungen besitzen, insbesondere zur Verwendung als Elektrodenmaterial von Strukturen, in denen der Tunnelmagnetowiderstand (TMR) ausgenutzt wird.

In diesem Kapitel werden die Grundlagen der Physik der Manganate besprochen. Zunächst wird die Kristallstruktur erläutert, um dann die damit verbundene elektronische Struktur der d-Elektronenzustände zu diskutieren. Durch die Mn-O-Mn-Bindungen ergeben sich mit dem Superaustausch und dem Doppelaustausch magnetische Kopplungen zwischen den Spins auf den Mn-Ionen. Schließlich liefert die Betrachtung dieser Austauschwechselwirkungen Ansätze zur Konstruktion eines magnetischen Phasendiagramms, das hier anhand des Beispiels von $\mathrm{La}_{1-\mathrm{x}} \mathrm{Sr}_{\mathrm{x}} \mathrm{MnO}_{3}$ illustriert wird.

\subsection{Kristallstruktur}

Die hier untersuchten Manganate mit der allgemeinen Summenformel $\mathrm{ABO}_{3}$ kristallisieren in verschiedenen Varianten der Perowskit-Struktur. Nach allgemeiner Konvention unterscheidet man in dieser Struktur zwischen den Kationen auf den A- und B-Plätzen (siehe Abbildung 2.1. Die Sauerstoff-Ionen bilden um den B-Platz einen $\mathrm{BO}_{6}$-Oktaeder, der bestimmend ist für die Physik der Übergangsmetallperowskite. Bei den Manganaten wird der B-Platz von $\mathrm{Mn}^{3+} / \mathrm{Mn}^{4+}$-Ionen belegt und der A-Platz von Ionen der Lanthanoiden $\left(\mathrm{La}^{3+}, \mathrm{Pr}^{3+}, \ldots\right)$ oder Erdalkali-Elemente $\left(\mathrm{Sr}^{2+}, \mathrm{Ca}^{2+}, \ldots\right)$. Die in Abbildung 2.1 dargestellte kubische Struktur wird nur für wenige chemische Zusammensetzungen realisiert. In Abhängigkeit von den 


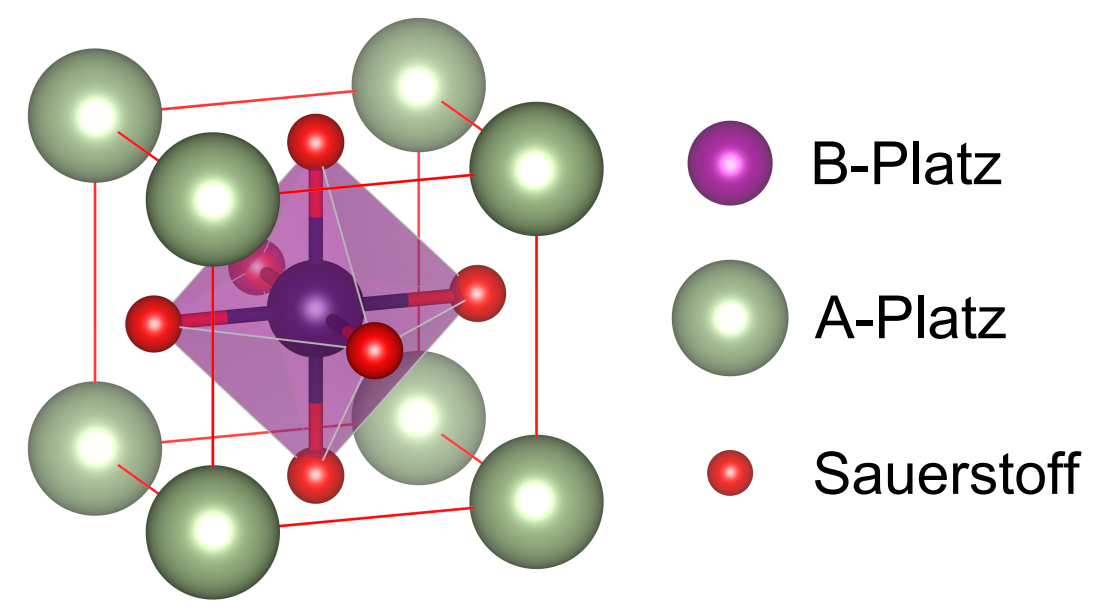

Abbildung 2.1.: Einheitszelle für ein kubisches Perowskit mit Kationen auf den A- und B-Plätzen und den $\mathrm{O}^{2-}$-Anionen (gezeichnet mit VESTA [21]).

Ionenradien der Kationen ergeben sich Abweichungen von dieser Struktur. Entlang der [001]Richtung zerfällt die Perowskit-Struktur in Ebenen aus $\mathrm{AO}$ und $\mathrm{BO}_{2}$. Der Goldschmidtsche Toleranzfaktor

$$
t=\frac{d_{A-O}}{\sqrt{2} d_{B-O}}=\frac{1}{\sqrt{2}} \frac{\left\langle r_{A}\right\rangle+r_{O}}{\left\langle r_{B}\right\rangle+r_{O}}
$$

$\left(d_{A / B-O}\right.$ : durchschnittliche Längen der A/B-O-Bindungen, $\left\langle\mathrm{r}_{\mathrm{A} / \mathrm{B}}\right\rangle$ : durchschnittliche Radien der Kationen auf den A/B-Plätzen, $r_{O}$ : Radius der $\mathrm{O}^{2-}$-Ionen) ist ein Maß für die Fehlpassung der $\mathrm{AO}$ - und $\mathrm{BO}_{2}$-Lagen 22 . Für einen Toleranzfaktor $t=1$ ergibt sich die kubische Struktur, bei der die Bindungswinkel der Mn-O-Mn-Bindungen den Idealwert von $180^{\circ}$ annehmen. Abweichungen von $t=1$ werden durch Verdrehungen und Verkippungen der $\mathrm{BO}_{6}$-Oktaeder

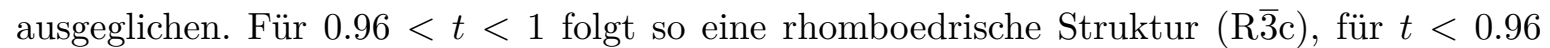
eine orthorhombische Struktur (Pmna) und für $t>1$ eine Struktur mit hexagonaler Symmetrie 22. Diese strukturellen Modifikationen der kubischen Struktur haben durch ihren Zusammenhang mit den Mn-O-Mn-Bindungswinkeln einen zum Teil drastischen Einfluss auf die elektronischen und magnetischen Eigenschaften. Neben den mittleren Bindungswinkeln ist für eine vollständige Beschreibung aber außerdem die Unordnung der Kationenradien zu berücksichtigen, welche über eine Unordnung der Mn-O-Mn-Bindungswinkel auf die beobachteten Eigenschaften rückkoppelt [16. In den folgenden Abschnitten wird man sehen, dass sich die Struktur nicht nur einfach aus dem Vergleich der Kationenradien ergibt, sondern auch elektronische Freiheitsgrade wie der Jahn-Teller-Effekt zu Veränderungen der Kristallstruktur führen können.

\subsection{Elektronische Struktur der Mn-Ionen}

Die $\mathrm{Mn}^{3+} / \mathrm{Mn}^{4+}-$ Ionen liegen in einer Argon-Edelgaskonfiguration mit vier $\left([\mathrm{Ar}] 3 \mathrm{~d}^{4}\right)$ bzw. drei $\left([\mathrm{Ar}] 3 \mathrm{~d}^{3}\right)$ weiteren Elektronen auf der $3 \mathrm{~d}-$ Schale vor. Das Kristallfeld der $\mathrm{O}^{2-}$-Ionen hebt die Entartung der 3d-Orbitale auf: Die $d_{x y}, d_{x z}$ und $d_{y z^{-}}$Orbitale, die man als $t_{2 g^{-}}$ Zustände zusammenfasst, werden gegenüber den $e_{g}$-Zuständen, die aus dem $d_{x^{2}-y^{2}}$ und $d_{3 z^{2}-r^{2}}$-Orbital bestehen, energetisch abgesenkt (siehe Abbildung 2.2). Diese energetische Aufspaltung, die Kristallfeldenergie $\Delta_{C F}$, hat in den hier betrachteten Manganaten die Grö- 


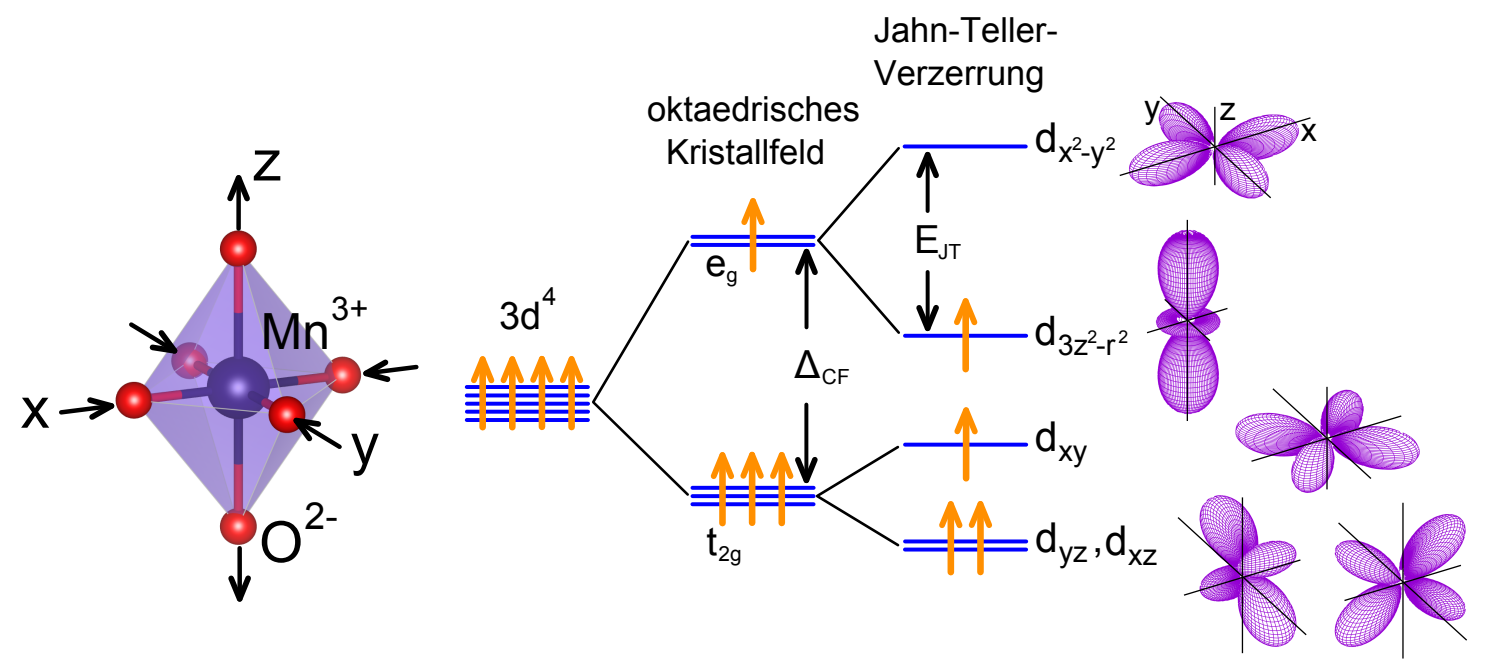

\begin{abstract}
Abbildung 2.2.: Aufhebung der Entartung der 3d-Niveaus eines $\mathrm{Mn}^{3+}$-Ions im oktaedrischen Kristallfeld der $\mathrm{O}^{2-}$-Ionen in der Perowskit-Struktur: Die Kristallfeldenergie $\Delta_{C F}$ separiert die $e_{g^{-}}$und $t_{2 g^{-}}$Niveaus, die durch eine Jahn-Teller-Verzerrung des $\mathrm{MnO}_{6}$-Oktaeders weiter aufspalten. Außerdem sind die winkelabhängigen Wahrscheinlichkeitsverteilungen der 3d-Orbitale, die der Symmetrie des Kristallfeldes entsprechen, dargestellt (nach 22, 23]).
\end{abstract}

ßenordnung $\Delta_{C F} \approx 1 \mathrm{eV}\left[24\right.$. Nach den Hundschen Regeln besetzen die Elektronen die $t_{2 g^{-}}$ Niveaus mit parallelem Spin und bilden einen Rumpfspin mit $S=\frac{3}{2}$. Das vierte Elektron auf den $\mathrm{Mn}^{3+}$-Ionen besetzt ein $e_{g}$-Niveau, da die Energie der Hundschen Kopplung in Manganaten mit $J_{H} \approx 2-3 \mathrm{eV} 22$ deutlich größer ist als die Kristallfeldenergie. Das $\mathrm{Mn}^{3+}$-Ion befindet sich somit im high spin Zustand mit einem Gesamtspin $S=2$. Für die dem Kristallfeld der $\mathrm{MnO}_{6}$-Oktaeder angepassten Wellenfunktionen der 3d-Elektronen verschwinden die Erwartungswerte des Drehimpulsoperators $l_{z}$ und damit das magnetische Bahnmoment [22]. Daher ist das magnetische Moment nur durch die Spins gegeben und es folgt für $\mathrm{La}_{1-\mathrm{x}} \mathrm{Sr}_{\mathrm{x}} \mathrm{MnO}_{3}$ ein Sättigungsmoment von

$$
m_{z}=2\left(2(1-x)+\frac{3}{2} x\right) \mu_{B}=(4-x) \mu_{B}
$$

Die orbitale Entartung der $e_{g}$-Elektronen wird nach dem Jahn-Teller-Theorem durch eine Verzerrung des Gitters aufgehoben [25]. In Abbildung 2.2 wird dies durch die Streckung des $\mathrm{MnO}_{6}$-Oktaeders entlang der z-Achse und gleichzeitiger Stauchung senkrecht dazu erreicht. Die Verzerrung schreitet fort, bis die Erhöhung der elastischen Energie die Energieabsenkung des $d_{3 z^{2}-r^{2}}$-Orbitals kompensiert. Das bei dieser Symmetrieänderung modifizierte CoulombPotential der $\mathrm{O}^{2-}$-Ionen spaltet auch die $t_{2 g}$-Niveaus auf (siehe Abbildung 2.2). Im nächsten Kapitel wird aufgezeigt, dass die Entartung der $e_{g}$-Niveaus auch durch äußere Verzerrungen des Gitters wie epitaktische Verspannungen aufgehoben werden kann. Mit optischer Spektroskopie ergibt sich für die energetische Aufspaltung der $e_{g}$-Niveaus in $\mathrm{LaMnO}_{3}$ (LMO) $E_{J T} \approx 0.7 \mathrm{eV}$ 26, 27. In LMO kommt es zu einer kollektiven Ordnung der Jahn-Tellerverzerrten Oktaeder unterhalb einer Temperatur von $T_{O O} \approx 800 \mathrm{~K}$, woraus eine Gitterstruktur mit orthorhombischer Symmetrie resultiert [28]. Die durch den Jahn-Teller-Effekt vermittelte Elektron-Phonon-Kopplung mündet in dem Quasiteilchen des Jahn-Teller-Polarons, das die Kombination aus einem Elektron auf den $e_{g}$-Niveaus und der daraus resultierenden Verzerrung des $\mathrm{MnO}_{6}$-Oktaeders und seiner Umgebung bezeichnet 22. 


\subsection{Austauschmechanismen}

Da der Überlapp der benachbarten 3d-Orbitale in Manganaten gering ist, findet der magnetische Austausch indirekt über die Hybridisierung mit den p-Orbitalen der $\mathrm{O}^{2-}$-Ionen statt. Der Energiegewinn bei der Delokalisierung von Elektronen zwischen den $\mathrm{O}_{2 \mathrm{p}^{-}}$und $\mathrm{Mn}_{3 \mathrm{~d}^{-}}$ Zuständen wird durch das p-d-Transferintegral $t_{p d}$ charakterisiert. Der $\sigma$-Überlapp zwischen den $\mathrm{O}_{2 \mathrm{p}^{-}}$und $e_{g^{-}}$-Orbitalen ist deutlich stärker als der $\pi$-Überlapp zwischen den $\mathrm{O}_{2 \mathrm{p}^{-}}$und $t_{2 g^{-}}$ Orbitalen: $t_{p d \pi} \sim(1 / 2) t_{d p \sigma}[29$. Bei einer Delokalisierung eines Elektrons über zwei durch eine Sauerstoffbrücke verbundene Mn-Ionen ergibt sich eine Verringerung der kinetischen Energie gemäß 22, 29, 30]

$$
t_{d d}=\frac{t_{p d}^{2}}{\Delta}
$$

mit der Energielücke des $\mathrm{O}_{2 \mathrm{p}} \rightarrow \mathrm{Mn}_{3 \mathrm{~d}}$-Ladungstransfers (siehe Abbildung 2.3 a))

$$
\Delta=E\left(d^{n+1} p^{5}\right)-E\left(d^{n} p^{6}\right) .
$$

Über den Mn-O-Mn-Bindungswinkel $\phi$ ist $t_{d d}$ direkt mit der Struktur des Netzwerkes aus $\mathrm{MnO}_{6}$-Oktaedern verknüpft und verkleinert sich bei Abweichungen von dem Bindungswinkel $\phi_{k u b}=180^{\circ}$ für ideale kubische Strukturen proportional zu $|\cos (\phi)|[31]$.

\subsubsection{Superaustausch und Goodenough-Kanamori-Anderson-Regeln}

Da der Austausch der Spins auf den 3d-Ionen indirekt über die Hybridisierung mit den Sauerstoffionen stattfindet, wird dieser Superaustausch genannt. Dieser wurde von Goodenough, Kanamori und Anderson zuerst theoretisch beschrieben und mit den nach ihnen benannten Goodenough-Kanamori-Anderson (GKA)-Regeln ist es möglich, die Art und Stärke der magnetischen Kopplung in Abhängigkeit der Besetzung der 3d-Orbitale abzuschätzen [22, 30]. Zur Illustration des Superaustausches betrachtet man $\mathrm{LaMnO}_{3}$ (LMO), in dem alle Mn-Ionen im $\mathrm{Mn}^{3+}$-Zustand vorliegen (siehe Abbildung 2.3). Der Transfer eines Elektrons zwischen den Mn-Ionen führt auf einem Ion zu einer doppelten Besetzung des $e_{g}$-Niveaus, welche mit einer Erhöhung der Coulomb-Energie einhergeht. Diese wird durch die sogenannte Mott-HubbardEnergie, $U$, charakterisiert 29]:

$$
U=E\left(d^{n+1} d^{n-1}\right)-E\left(d^{n} d^{n}\right)
$$

Für 3d-Perowskite ergeben sich $U \sim 3-7 \mathrm{eV}$ und $t_{d d} \sim 0.1-0.4 \mathrm{eV}$ [29] (LMO: $U \approx$ $3.1 \mathrm{eV}, t_{d d} \approx 0.4 \mathrm{eV}\left[26\right.$ ). Wegen $U \gg t_{d d}$ ist der Gewinn an kinetischer Energie bei der Delokalisierung über mehrere Mn-Ionen zu klein, um die erhöhte Coulomb-Energie bei einer doppelten Besetzung eines $e_{g}$-Orbitals zu kompensieren. LMO ist im Grundzustand ein MottHubbard-Isolator. Die Elektron-Elektron-Korrelationen lokalisieren die Elektronen in diesem Material, das wegen seiner halb-gefüllten $e_{g}$-Niveaus ohne Korrelationen ein Metall wäre $[22$, 29 .

Mit $U \gg t_{d d}$ sieht man im Hamilton-Operator des Hubbard-Modells den zu $t_{d d}$ proportionalen Hüpfterm als Störung an. Im ungestörten Grundzustand sind also die durch den Jahn-Teller-Effekt energetisch bevorzugten $e_{g}$-Orbitale einfach besetzt. Um Aussagen über die gegenseitige Ordnung der Spins auf den Mn-Ionen zu treffen, betrachtet man virtuelle Hüpfprozesse zwischen den Mn-Ionen (siehe Abbildung 2.3). Bei einer parallelen Ausrichtung 
a)

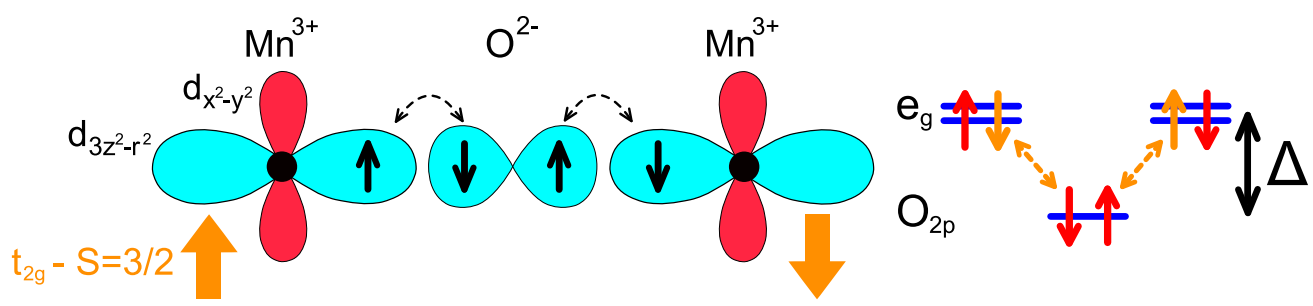

b)
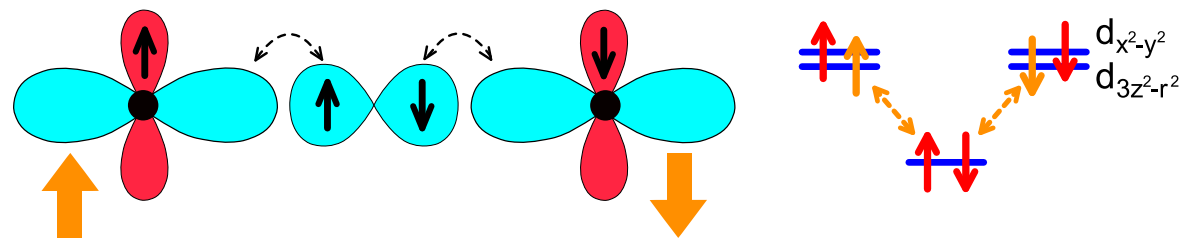

c)
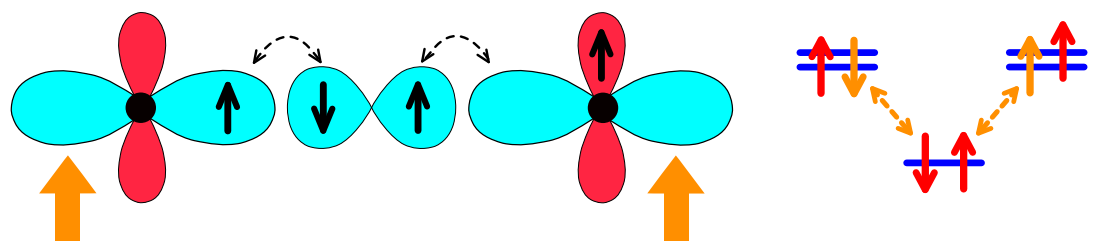

Abbildung 2.3.: Zum Superaustausch und den Goodenough-Kanamori-Anderson (GKA)Regeln am Beispiel einer Bindung zwischen zwei $\mathrm{Mn}^{3+}$-Ionen (nach [22, 30]): Virtuelle Hüpfprozesse zwischen den $e_{g^{-}}$und $\mathrm{O}_{2 \mathrm{p}}$-Niveaus, die durch die Energie des Ladungstransfers $\Delta$ separiert sind, vermitteln Austauschkopplungen. Die $t_{2 g}$-Rumpfspins $(S=3 / 2)$ folgen der Ausrichtung der $e_{g}$-Elektronen aufgrund der Hundschen Kopplung. In den Beispielen sind die $d_{3 z^{2}-r^{2}}$-Orbitale entlang der Mn-O-Mn-Bindungen ausgerichtet und die $d_{x^{2}-y^{2}}$-Orbitale senkrecht dazu. a) Aufgrund des Pauli-Prinzips sind die Hüpfprozesse zu beiden Seiten nur erlaubt, wenn die Spins auf den Mn-Ionen entgegengesetzt orientiert sind. b) Bei unbesetzten $d_{3 z^{2}-r^{2}}$-Orbitalen diktiert die Hundsche Kopplung eine antiparallele Ausrichtung der Spins. c) Wenn auf einem Mn-Ion das $d_{3 z^{2}-r^{2}}$-Orbital und auf dem anderen das zur Bindung orthogonale $d_{x^{2}-y^{2}}$-Orbital besetzt ist, wird eine ferromagnetische Ausrichtung bevorzugt.

der Spins auf den benachbarten und einfach besetzten $e_{g}$-Orbitalen schließt das Pauli-Prinzip Hüpfprozesse aus. Für eine antiparallele Orientierung können die Elektronen virtuell hüpfen und es ergibt sich nach störungstheoretischen Rechnungen zweiter Ordnung eine Reduktion der Energie um 22, 29]

$$
J_{S E}=-\frac{2 t_{d d}^{2}}{U}
$$

Auch zwischen den Elektronen auf den $t_{2 g}$-Orbitalen ergibt sich eine Austauschwechselwirkung nach dem Superaustausch. Durch $t_{p d \pi} \sim(1 / 2) t_{d p \sigma}$ ist die Austauschwechselwirkung der Spins auf den $t_{2 g}$-Zuständen um einen Faktor $\sim 1 / 16$ kleiner als zwischen den $e_{g}$-Orbitalen [29]. Damit nimmt man hier an, dass die $t_{2 g}$-Elektronen nicht aktiv an den Austauschprozessen teilnehmen und einen Rumpfspin $S=\frac{3}{2}$ bilden, der über die Hundsche Kopplung die Ausrichtung der Spins der $e_{g}$-Elektronen annimmt. Nach Zaanen, Sawatzky und Allen differenziert man zwischen dem genannten Mott-Hubbard-Isolator und einem sogenannten Ladungstransfer-Isolator. Man unterscheidet diese beiden Typen, indem man betrachtet, ob das bei den virtuellen Hüpfprozessen entstehende Loch auf dem $O^{2-}$-Anion besteht (Ladungstransfer-Isolator) oder auf dem 3d-Ion (Mott-Hubbard-Isolator) [22, 29]. Im LMO ist $\Delta \approx 5 \mathrm{eV}>U \approx 3.1 \mathrm{eV}[26$, wodurch ein Loch auf einem Mn-Ion bevorzugt wird. Für $\Delta<U$ liegt das Loch auf dem Sauerstoff-Ion. Bei einem Ladungstransfer-Isolator muss man in der Gleichung 2.6 den Faktor $1 / U$ durch $1 / \Delta$ ersetzen $[22,29] 1$.

\footnotetext{
$\overline{{ }^{1} \text { Diese einfache Form der Austauschenergie }} J_{S E}$ gilt streng nur für die Grenzfälle mit $\Delta \gg U$ bzw. $U \gg \Delta$.
} 


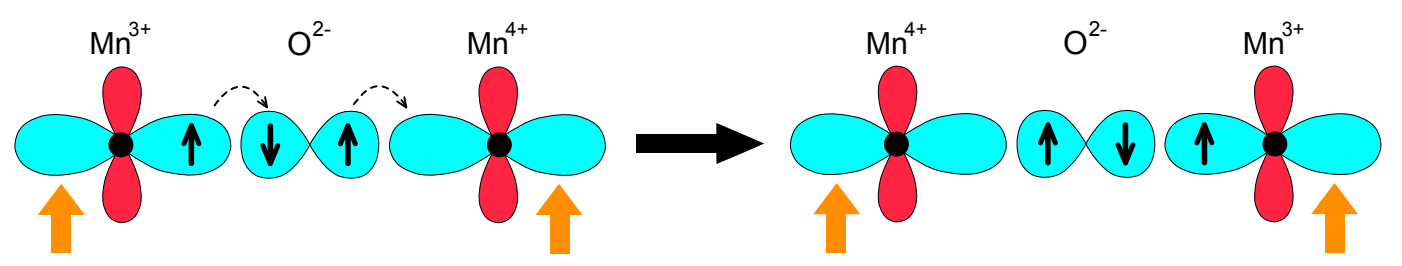

Abbildung 2.4.: Zum Doppelaustausch: Bei paralleler Ausrichtung der $t_{2 g}$-Spins auf den benachbarten Mn-Ionen erlaubt die Hundsche Kopplung ein Springen des $e_{g}$-Elektrons vom $\mathrm{Mn}^{3+}$ - zum $\mathrm{Mn}^{4+}$-Ion, ohne dass sich die Energie dabei verändert (nach [22, 30]).

In der dreidimensionalen Kristallstruktur der Manganate ist nicht nur die in Abbildung 2.3 a) gezeigte Situation denkbar, sondern auch andere Fälle, in denen sich gefüllte und leere $e_{g^{-}}$ Orbitale an einer Sauerstoffbindung gegenüber stehen. Dabei ist zu berücksichtigen, dass sich das $e_{g}$-Elektron auf dem $\mathrm{Mn}^{3+}$-Ion auch auf dem Orbital befinden kann, das senkrecht auf der betrachteten Mn-O-Mn-Bindung steht ${ }^{2}$. Mit den Goodenough-Kanamori-Anderson (GKA)Regeln ist es möglich, in diesen Fällen die Art und Stärke der magnetischen Austauschwechselwirkung abzuschätzen. Dabei ergibt sich die Austauschwechselwirkung analog zu den oben angestellten Überlegungen daraus, dass die $e_{g^{-}}$und $\mathrm{O}_{2 \mathrm{p}}$-Elektronen ihre kinetische Energie durch virtuelle Hüpfprozesse vermindern können. Dadurch dass das Pauli-Prinzip und die Hundsche Kopplung das Hüpfen der Elektronen nur bei einer bestimmten relativen Spinorientierung erlauben, wird das Vorzeichen der Austauschwechselwirkung festgelegt. Durch die starke Hundsche-Kopplung $J_{H}$ folgt der Rumpfspin der $t_{2 g}$-Elektronen der Orientierung der $e_{g}$-Elektronen [22, 30]. In Abbildung 2.3 sind die ersten beiden GKA-Regeln für eine $\mathrm{Mn}^{3+}$ $\mathrm{O}-\mathrm{Mn}^{3+}$-Bindung skizziert, die dritte Regel wird hier nicht gesondert aufgeführt, da sie den $90^{\circ}$-Austausch betrifft, der in Perowskit-Manganaten aus geometrischen Gründen nicht relevant ist. Nach der ersten GKA-Regel ist der $180^{\circ}$-Austausch zwischen gefüllten oder leeren Orbitalen antiferromagnetisch (siehe $2.3 \mathrm{a}$ ) und b) ${ }^{3}$. Teil c) der Abbildung 2.3 entspricht der zweiten GKA-Regel. Beim zweiten $\mathrm{Mn}^{3+}$-Ion ist das $d_{x^{2}-y^{2}}$-Orbital senkrecht zur Mn-O-MnBindung besetzt, so dass eine ferromagnetische Ausrichtung der Spins auf den Mn-Ionen eine Delokalisierung der $\mathrm{O}_{2 \mathrm{p}}$-Elektronen unterstützt. Der ferromagnetische Zustand wird dabei einer antiferromagnetischen Ordnung vorgezogen, da bei einer ferromagnetischen Ordnung die Hundsche Kopplung zwischen den beiden Elektronen auf den orthogonalen $e_{g}$-Orbitalen erfüllt werden kann 22,30 .

\subsubsection{Doppelaustausch}

Der in diesem Abschnitt besprochene Mechanismus des Doppelaustausches erklärt den für einen weiten Dotierungsbereich vorherrschenden ferromagnetisch metallischen (FMM) Grundzustand gemischtvalenter Manganate und liefert eine qualitative Begründung für den CMR. Wie in Abbildung 2.4 dargestellt, betrachtet man ein $\mathrm{Mn}^{3+}$ - und ein $\mathrm{Mn}^{4+}$-Ion, deren $e_{g^{-}}$ Orbitale über eine Sauerstoffbrücke verbunden sind. Im Gegensatz zu der zuvor geschilderten

\footnotetext{
${ }^{2}$ In Abbildung 2.3 werden immer Mn-O-Mn-Bindungen aus zwei $d_{3 z^{2}-r^{2}}$-Orbitalen dargestellt. In der Realität kann es auch gemischte Bindungen mit jeweils einem $d_{3 z^{2}-r^{2}}$ und $d_{x^{2}-y^{2}}$-Orbital geben. Durch die orbitale Ordnung im LMO werden genau diese gemischten Bindungen in den ferromagnetisch ordnenden Ebenen realisiert.

${ }^{3}$ In Abbildung 2.3 a) ist bei einer Besetzung des $d_{3 z^{2}-r^{2}}$ mit zwei Elektronen mit antiparallelem Spin die Korrelationsenergie $U$ zu bezahlen. Für die in Abbildung 2.3 b) gezeigte Situation ist die Energie bei einer doppelten Besetzung der $e_{g}$-Orbitale durch die Verteilung der beiden Elektronen auf verschiedene Orbitale mit parallelem Spin um die Hundsche Kopplungsenergie $J_{H}$ reduziert und beträgt $U-J_{H}$.
} 
Situation des Superaustausches ist nun ein reelles Hüpfen des $e_{g}$-Elektrons zwischen den Mn-Ionen möglich, ohne dass eine zusätzliche Coulomb-Energie $U$ aufgebracht werden muss. Stehen die beiden $t_{2 g}$-Rumpfspins parallel (Abbildung 2.4), so ist der Zustand nach dem Hüpfen energetisch gleichwertig mit dem Ausgangszustand, die $e_{g}$-Elektronen sind itinerant. Bei einer antiparallelen Orientierung muss beim Hüpfen die Hundsche Kopplungsenergie bezahlt werden. Mit den Hüpfprozessen ist eine Reduktion der kinetischen Energie der $e_{g}$-Elektronen verbunden, woraus eine ferromagnetische Austauschwechselwirkung folgt. Die Beweglichkeit der Ladungsträger und die ferromagnetische Ordnung bedingen einander also 22 .

Mit Blick auf die Rolle der Hundschen Kopplung führen Anderson und Hasegawa ein effektives Transfermatrixelement ein 31]:

$$
t_{d d}^{e f f}=t_{d d} \cos \left(\frac{\theta_{i j}}{2}\right)
$$

mit dem Winkel $\theta_{i j}$ zwischen den $t_{2 g}$-Spins auf benachbarten Gitterplätzen i und j. In der Nähe zur Curie-Temperatur verringert sich die ferromagnetische Ordnung und es gibt Abweichungen vom idealen Wert $\theta_{i j}=0^{\circ}$. Dadurch nimmt die durch $t_{d d}^{e f f}$ bestimmte Bandbreite ab, was gleichbedeutend ist mit einer Erhöhung der effektiven Masse und somit des elektrischen Widerstandes. Ein externes Magnetfeld ordnet die $t_{2 g}$-Spin teilweise und bewirkt damit eine Reduktion des Widerstandes. In der Nähe der Curie-Temperatur ist die magnetische Suszeptibilität am größten, so dass der Effekt des Feldes auf die relative Orientierung der Spins dort maximal wird. Damit erwartet man auch ein Maximum des CMR in der Nähe von $T_{C}$ und nur einen kleinen Effekt bei höheren und tieferen Temperaturen. Dies wird in der Tat experimentell für viele Manganate wie z.B. $\mathrm{La}_{0.7} \mathrm{Sr}_{0.3} \mathrm{MnO}_{3}$ beobachtet. Es gibt jedoch auch Materialien, die bei tiefen Temperaturen weit unterhalb von $T_{C}$ einen starken CMR aufweisen [16, 17. Auch kann der Doppelaustausch nicht die Amplitude des CMR und deren Variation zwischen verschiedenen Manganaten erklären [16, 17. Der absolute Wert des CMR, den man mit theoretischen Berechnungen im Modell des Doppelaustausches erhält, ist deutlich kleiner als die im Experiment gemessenen Werte 17.

Der Doppelaustausch greift also zu kurz, es müssen weitere Freiheitsgrade berücksichtigt werden. Diese sind in der Elektron-Phonon-Kopplung zu suchen, die durch den bereits oben eingeführten Jahn-Teller-Effekt vermittelt wird und sich im Quasiteilchen des Jahn-TellerPolarons manifestiert. Bei der Lokalisierung eines Elektrons in einem Jahn-Teller-verzerrten Oktaeder gewinnt man die Energie $E_{\text {latt }}$. Dies steht in Konkurrenz zur Delokalisierung der $e_{g}$-Elektronen über den Doppelaustausch, der über einen FMM-Zustand zu einer Verringerung der kinetischen Energie führt. Zum Vergleich dieser beiden widerstrebenden Prozesse führt Millis den Quotienten $\lambda=E_{\text {latt }} / t_{d d}^{\text {eff }}$ ein [32], welcher ein Maß für die Korrelationen zwischen Elektronen und Gitter darstellt [17, 32, 33]. Da $t_{d d}$ von dem Mn-O-Mn-Bindungswinkel abhängt, lässt sich somit die Stärke der Elektron-Phonon-Kopplung durch Variation der Kationen auf den A-Plätzen verändern. Mathur und Littlewood konstruieren mit diesem Parameter ein generisches Phasendiagramm der Manganate [33]. Für $\mathrm{La}_{0.7} \mathrm{Sr}_{0.3} \mathrm{MnO}_{3}$ ist $\lambda$ sehr klein, so dass dieses Material den Vorhersagen des Doppelaustausches gehorcht [24]. In $\mathrm{Pr}_{0.7} \mathrm{Ca}_{0.3} \mathrm{MnO}_{3}$ ist der Mn-O-Mn-Bindungswinkel deutlich kleiner, woraus eine stärkere Elektron-Phonon-Kopplung folgt, die zu einem isolierenden Grundzustand führt [16]. Zhao et al. beobachten beim Austausch der ${ }_{8}^{16} \mathrm{O}^{2-}$-Sauerstoffionen durch das schwerere Isotop ${ }_{8}^{18} \mathrm{O}^{2-}$ in $\mathrm{La}_{0.8} \mathrm{Ca}_{0.2} \mathrm{MnO}_{3}$ eine Verringerung der Curie-Temperatur um $\Delta T \approx 20 \mathrm{~K}$ [34]. Der Austausch der Sauerstoffisotope beeinflusst die Schwingungsfrequenz der Phononen, hat aber keine Auswirkungen auf $E_{\text {latt }}$ und $t_{d d}^{e f f}$. Der eingeführte Parameter $\lambda$ kann also die verschiedenen Manganate nicht vollständig klassifizieren. Sudheendra et al. führen den Quotienten $\lambda_{v i b}=\frac{E_{\text {latt }}}{\hbar \omega_{L}}\left(\omega_{L}\right.$ bezeichnet die Schwingungsfrequenz des longitudinalen optischen Phonons) 
ein [35, 36. $\lambda_{\text {vib }}$ ist wie $\lambda$ ein Maß der Elektron-Phonon-Kopplung [35]. Wenn Zhao et al. den Sauerstoff durch ein schwereres Isotop ersetzen, verringert sich die Schwingungsfrequenz der Gitterschwingungen, bei denen die Sauerstoffionen beteiligt sind. Damit steigt $\lambda_{v i b}$, was eine erhöhte Kopplung der Elektronen an das Gitter signalisiert. Daraus resultiert dann die beobachtete Reduktion der Curie-Temperatur.

Korrelationseffekte zwischen Polaronen sind ein entscheidender Baustein für die Erklärung des CMR. Im Zusammenhang mit dem CMR beobachtet man korrelierte Polaronen, deren orbitale Ordnung eine ähnliche Struktur aufweist wie in $\mathrm{La}_{0.5} \mathrm{Ca}_{0.5} \mathrm{MnO}_{3}$. Diese sorgen für eine Lokalisierung der Ladungsträger in einer ladungsgeordneten Struktur des CE-Typs und die Unterdrückung des Ferromagnetismus. Durch äußere Felder (z.B. Magnetfeld) werden die Korrelationen aufgebrochen und es kommt zum Metall-Isolator-Übergang (MIT) [16, 17, 37. Theoretische Überlegungen und experimentelle Befunde zeigen eine Koexistenz der FMMund COI-Phase. Diese ist bei Manganaten mit mittleren Werten von $\lambda$ nur in der Nähe des MIT zu finden, für größere $\lambda$ liegt diese Phasenseparation auch bei tiefen Temperaturen vor 16, 17, 37. In diesem Zustand lassen sich die großen Werte des CMR mit einem feldgetriebenen Wachstum der FMM-Phase, die perkolative Pfade durch die COI-Matrix bildet, erklären 16, 17.

\subsection{Phasendiagramm von $\mathrm{La}_{1-\mathrm{x}} \mathrm{Sr}_{\mathrm{x}} \mathrm{MnO}_{3}$}

Mithilfe des Zusammenspiels zwischen Superaustausch und Doppelaustausch lassen sich weite Teile des in Abbildung 2.5 gezeigten magnetischen Phasendiagramms von $\mathrm{La}_{1-\mathrm{x}} \mathrm{Sr}_{\mathrm{x}} \mathrm{MnO}_{3}$ $(\operatorname{LSMO}(x))$ verstehen. Für $\mathrm{LaMnO}_{3}(\mathrm{LMO})$ führt die bei höheren Temperaturen, $T_{O O} \approx$ $800 \mathrm{~K}$, stattfindende orbitale Ordnung dazu, dass die besetzten $e_{g}$-Orbitale senkrecht zur cAchse der orthorhombischen Struktur stehen. In den Ebenen orthogonal zur c-Achse ordnen die Orbitale dann so, dass sich jeweils ein besetztes $d_{3 z^{2}-r^{2}}$ Orbital und ein unbesetztes $d_{x^{2}-y^{2}}$ an einer Mn-O-Mn-Bindung gegenüber stehen. Nach den GKA-Regeln ist die Kopplung in diesen Ebenen dann ferromagnetisch. Entlang der c-Achse ergibt sich durch die beiderseitig unbesetzten $e_{g}$-Orbitale dann eine antiferromagnetische Wechselwirkung. Insgesamt resultiert so eine antiferromagnetische Ordnung des A-Typs, die im Experiment eine Néel-Temperatur $T_{N} \approx 150 \mathrm{~K}$ zeigt 38,39 . Die Coulomb-Abstoßung führt zu einer Lokalisierung der $e_{g^{-}}$ Elektronen, so dass LMO ein Mott-Hubbard-Isolator ist. Für $\mathrm{SrMnO}_{3}$ (SMO) am anderen Ende des Phasendiagramms sind alle $e_{g}$-Orbitale unbesetzt, so dass sich nach der ersten GKA-Regel für jedes Mn-Ion eine antiferromagnetische Kopplung zu allen Nachbarn ergibt. Damit ist SMO ein Antiferromagnet des G-Typs mit einer Néel-Temperatur $T_{N} \approx 260 \mathrm{~K}[40$. Durch das Fehlen von Ladungsträgern auf den $e_{g}$-Orbitalen ist SMO ein Bandisolator. Wie es der Doppelaustausch vorhersagt, liegt im Bereich von $x \approx 0.17-0.5$ eine ferromagnetisch metallische Phase vor, die in der Region um $x=0.3-0.4$ seine maximale Curie-Temperatur, $T_{C} \approx 370 \mathrm{~K}$, zeigt.

Für die Regionen zwischen den zuvor genannten Phasen lassen sich keine einfachen Erklärungen des magnetischen Zustandes angeben. Diese Übergangsregionen werden von der Konkurrenz des Super- und Doppelaustausches bestimmt und sind oft von einer Koexistenz ferromagnetischer und antiferromagnetischer Phasen geprägt. Wenn man von LMO ausgehend Sr-Ionen zudotiert, verringert sich durch die reduzierte Anzahl der Jahn-Teller-verzerrten Oktaeder die treibende Kraft zur orbitalen Ordnung. Zusätzlich wird an einigen Stellen der Doppelaustausch ermöglicht, wodurch die antiferromagnetische Ordnung des A-Typs schon 


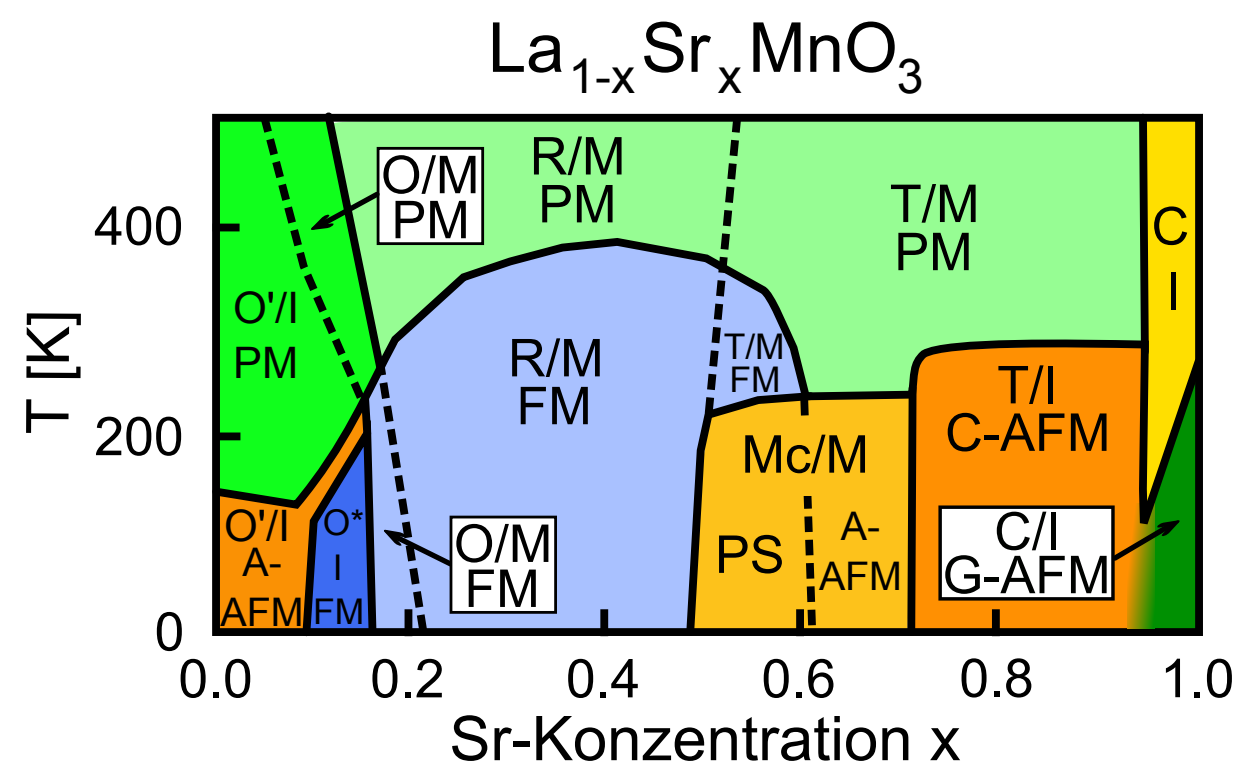

\begin{abstract}
Abbildung 2.5.: Strukturelles, elektronisches und magnetisches Phasendiagramm von $\mathrm{La}_{1-\mathrm{x}} \mathrm{Sr}_{\mathrm{x}} \mathrm{MnO}_{3}$ in seiner bulk-Form nach 38, 39] (Kristallstrukturen: JahnTeller verzerrt orthorhombisch-O', orthorhombisch-O, orbital-geordnet orthorhombisch$\mathrm{O}^{*}$, rhomboedrisch-R, tetragonal-T, monoklin-Mc, kubisch-C, elektronische Eigenschaften: isolierend-I, metallisch-M, magnetische Ordnung: ferromagnetisch-FM, paramagnetischPM, phasensepariert-PS, antiferromagnetisch A-Typ-A-AFM, antiferromagnetisch C-TypC-AFM, antiferromagnetisch G-Typ-G-AFM.).
\end{abstract}

bei einer Dotierung $x=0.09$ verloren geht und eine ferromagnetische Ordnung erscheint Übersteigt die Sr-Dotierung einen Wert von $x=0.175$, so verschwindet die langreichweitige Ordnung der Jahn-Teller-verzerrten Oktaeder und der FMM-Zustand setzt ein [22, 38 . Allerdings zeigen Messungen der Paar Dichtefunktion (pair density function (PDF)) mittels gepulster Neutronenstreuung das Vorhandensein von lokalen Jahn-Teller-Verzerrungen auch im FMM-Grundzustand für Proben mit $x \geq 0.175$ [41]. Diese Verzerrungen besitzen keine langreichweitige Ordnung, so dass sie keinen Einfluss auf die bulk-Gitterstruktur haben [41]. Erst bei größeren Dotierungen, $x \gtrsim 0.35$ verschwinden die Signaturen für die Verzerrung der $\mathrm{MnO}_{6}$-Oktaeder aus den Messungen der PDF [41].

Der sich der FMM-Phase anschließende Bereich für $x>0.5$ zeigt ein komplexes Verhalten mit unscharfen Phasengrenzen. Ein Grund für diese Asymmetrie des Phasendiagramms um $x=0.5$ ist in den strukturellen Veränderungen zu suchen, die sich mit steigender Sr-Dotierung ergeben. Der Toleranzfaktor steigt von $t=0.89$ für $x=0$ auf $t=1.01$ für $x=1$. Mit steigendem $x$ nähert sich der Mn-O-Mn-Bindungswinkel also immer mehr dem Idealwert von $\phi=180^{\circ}$ an. Dadurch dass die anisotropen Verzerrungen des Gitters bei größerem SrGehalt schwach ausgeprägt sind, wird die Separierung von den Energieniveaus der $d_{x^{2}-y^{2-}}$ und $d_{3 z^{2}-r^{2}}$-Orbitale deutlich kleiner als in LMO. Die beiden $e_{g}$-Orbitale sind fast entartet [38. Theoretische Betrachtungen des Doppelaustausches zwischen entarteten $e_{g}$-Orbitalen resultieren in der experimentell beobachteten Abfolge von magnetischen Phasen für $0.5<$ $x<0.9$, bei der auf die ferromagnetische Phase zunächst ein Antiferromagnet des A-Typs und dann des C-Typs folgt [42]. Der Übergang zwischen dem Antiferromagneten des A- und des C-Typs bei $x \approx 0.7$ hängt damit zusammen, dass sich die kleine tetragonale Verzerrung

\footnotetext{
${ }^{4}$ Auch im Bereich für $x<0.09$ zeigt sich ferromagnetisches Verhalten, das entweder auf Phasenseparation oder einen gekanteten antiferromagnetischen Zustand zurückgeführt wird 38.
} 
des Gitters $((c-a) / a \approx \pm 0.4 \%)$ von $c / a<1$ für $x \lesssim 0.7$ auf $c / a>1$ für $x \gtrsim 0.7$ ändert [38. Auch die teilweise Unterdrückung des Ferromagnetismus oberhalb von $x=0.5$ fällt mit einem Übergang von einer rhomboedrischen zu einer tetragonalen Struktur zusammen [38. Im nächsten Kapitel wird dargelegt, wie diese Änderung der Gitterstruktur die Besetzung der $e_{g}$-Orbitale und damit den magnetischen Ordnungsparameter beeinflusst. Die letzte und bisher am wenigsten untersuchte Grenze im Phasendiagramm befindet sich bei $x \approx 0.95$. Im Bereich von $x=0.9-0.95$ beobachtet man eine starke Reduktion der Néel-Temperatur im Vergleich zu den benachbarten Phasen im Diagramm. Man stellt für Proben mit dieser Dotierung eine Koexistenz von Phasen mit antiferromagnetischer Ordnung des C- und G-Typs fest [39]. Weitere theoretische und experimentelle Befunde zeigen, dass es für diese hohen Dotierungen auch zur Bildung von ferromagnetischen Clustern in der antiferromagnetischen Matrix kommen kann [43, 44]. 


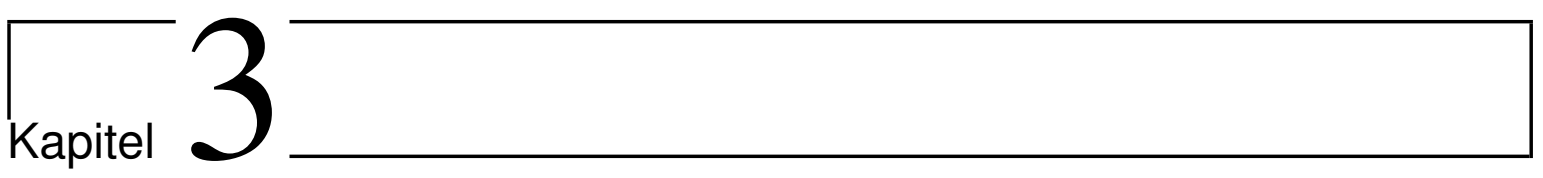

\section{Grenzflächeneffekte}

Materialien, die an einer Grenzfläche zusammentreffen, wechselwirken über die Freiheitsgrade des Gitters, der Ladungen, der Orbitale und der Spins. Durch die Manipulation der Ladungsträgerkonzentration an der Grenzfläche zwischen konventionellen Metallen und Halbleitern ergeben sich eine Vielzahl an Funktionalitäten, die eine außerordentliche technische Bedeutung besitzen. Gegenüber diesen bereits gut verstandenen Effekten ergeben sich bei der Kombination von Übergangsmetallperowskiten bisher unbekannte Phänomene, die das Potential haben, in der Anwendung neuartige Funktionalitäten bereitzustellen. Bei der Oxidation der Übergangsmetalle werden die s-Elektronen an die Sauerstoffionen abgegeben, so dass nur noch 3d-Elektronen verbleiben. Wie oben für die Manganate beschrieben wurde, bestimmen diese stark korrelierten d-Elektronen die Physik dieser Materialklasse. Es ergibt sich auf jedem Ion eine lokale Verflechtung von Ladung, Spin und den Orbitalen [45].

Die im Vergleich zu konventionellen Halbleitern deutlich größere Ladungsträgerdichte führt dazu, dass die Abschirmlängen deutlich kleiner sind und somit Bandverbiegungen an Grenzflächen in deutlich schmäleren Bereichen stattfinden 10. Typischerweise ergibt sich eine Breite von wenigen Monolagen, in denen es zu Veränderungen der Elektronendichte kommt. Aufgrund der starken Korrelationen verändert diese lokale Modifikation der Elektronendichte die Bandstruktur und kann so Änderungen des Typs der magnetischen Ordnung oder der elektrischen Eigenschaften treiben 10,45 . Des Weiteren spielt bei der Kombination zweier Perowskite $\mathrm{ABO}_{3}$ und $\mathrm{A}^{\prime} \mathrm{B}^{\prime} \mathrm{O}_{3}$ die lokale Chemie eine große Rolle. Neben einem einfachen diffusiven Austausch der Ionen A, A' auf den A-Plätzen und B, B' auf den B-Plätzen sind auch Redoxreaktionen der Übergangsmetallionen zu erwarten. Diese können einerseits unter bestimmten Herstellungsbedingungen zum Einbau von Sauerstoffleerstellen in eines der beiden Materialien [46] und andererseits zu einem spezifischen Elektronentransfer zwischen den B- und B'-Ionen führen 29.

Die magnetische Austauschwechselwirkung wird von den Eigenheiten der jeweiligen B-OB'-Bindung bestimmt, wobei nach den GKA-Regeln zunächst die Besetzung der 3d-Orbitale ausschlaggebend ist. Gegenüber den bulk-Materialien kann es an Grenzflächen jedoch zu Umbesetzungen der Orbitale kommen, die genau wie veränderte B-O-B'-Bindungswinkel einen beachtlichen Einfluss auf die magnetischen Kopplungen ausüben [45]. Die Verkippungswinkel der $\mathrm{BO}_{6}$-Oktaeder und damit die B-O-B'-Bindungswinkel ergeben sich durch den strukturellen Übergang zwischen den Materialien, der sich durch die Wahl der Kationen auf den A- 
Plätzen beeinflussen lässt. Abseits von der Betrachtung der Phänomene, die an einer Grenzfläche auftreten, kann man in Übergittern aus zwei oder mehr Materialien kollektive Phänomene bemerken, die sich z.B. durch den Bruch der Inversionssymmetrie bei Dreifachlagen oder die erzwungene Ordnung der Ionen auf den A-Plätzen ergeben [29, 45. Somit lassen sich bei der Kombination verschiedener komplexer Oxide elektronische Zustände einstellen, die weder in den einzelnen Komponenten auftreten, noch durch eine komplette chemische Mischung aller Bestandteile zugänglich sind $[29,45,47$.

Auch aus praktischen Erwägungen bieten sich die oxidischen Übergangsmetallperowskite für systematische Studien an. In dieser Stoffgruppe treten verschiedenste Typen von Ordnungsübergängen wie Ferro- und Antiferromagnetismus, Ferroelektrizität oder Supraleitung auf. Trotz dieser stark unterschiedlichen elektronischen Eigenschaften weisen diese Materialien ähnliche Strukturen auf und lassen sich somit leicht in Heterostrukturen mit qualitativ hochwertigen Grenzflächen integrieren [47]. Fortschritte bei der Erforschung von Phänomenen an Grenzflächen waren immer wieder getrieben von Verbesserungen der Methoden zur Herstellung und Untersuchung oxidischer Heterostrukturen. Da die räumliche Ausdehnung der Bereiche, in denen sich die Grenzflächeneffekte abspielen, deutlich kleiner sind als bei konventionellen Halbleitern und im Bereich weniger Monolagen liegen können, muss man in der Lage sein Heterostrukturen mit entsprechender Genauigkeit und chemischer Schärfe der Grenzflächen herzustellen. Für diese Anforderungen ist eine Beobachtung des Wachstums mit in-situ Methoden unerlässlich. Ebenso müssen die Methoden zur Charakterisierung, wo zuallererst die Transmissionselektronenmikroskopie (TEM) zu nennen ist, in der Lage sein die lokale Chemie mit einer Ortsauflösung im Bereich einer Atomlage aufzulösen.

Da man zur Untersuchung von Grenzflächeneffekten zumeist Heterostrukturen aus dünnen Filmen heranzieht, muss man neben den Effekten, die sich direkt an der Grenzfläche abspielen, zusätzliche Dünnfilmeffekte betrachten. Relevant sind dabei die Einschränkung in einer Dimension, die epitaktische Verspannung und mögliche Veränderungen der Chemie, die sich durch den Kontakt mit dem Substrat und der freien Oberfläche ergeben [46, 47. Auch bei Metallen und Halbleitern kommen diese Dünnfilmeffekte zum Tragen. Eine reine Einschränkung der Dimensionalität, wie sie bei dünnen Schichten auftritt, führt bei konventionellen Metallen und Halbleitern zu Quanteneffekten [2, 3. Durch die geometrische Beschränkung wird der Gewinn an kinetischer Energie bei Delokalisierung der Elektronen kleiner als für bulk-Materialien, wodurch Korrelationseffekte ein stärkeres Gewicht erhalten. Für die metallischen Stoner-Ferromagneten kann die stärkere Lokalisierung der d-Elektronen und die damit verbundene Vergrößerung der Zustandsdichte $D\left(E_{F}\right)$ an der Fermi-Kante eine Erhöhung der Austauschenergie und der Sättigungsmagnetisierung nach sich ziehen. Die CurieTemperatur beschreibt die kollektive magnetische Ordnung und wird somit sowohl durch die Austauschwechselwirkung als auch durch das kollektive thermodynamische Verhalten des Materials bestimmt. Nach dem Mermin-Wagner-Theorem [48] verschwindet so die kollektive ferromagnetische Ordnung in einem zweidimensionalem Materia 5 Im Verlauf dieses Kapitels wird ersichtlich, dass bei komplexen Oxiden zumeist die Effekte an Grenz- bzw. Oberflächen gegenüber einer reinen Größeneinschränkung dominieren. Dadurch gibt es nur wenige Beispiele, anhand derer man bei komplexen Oxiden den direkten Einfluss der dimensionalen Einschränkung feststellen kann [49]. Aufgrund der Magnetostriktion beeinflussen epitaktische Verspannungen bei konventionellen Metallen genauso wie bei komplexen Oxiden die

\footnotetext{
${ }^{5}$ Im Mermin-Wagner-Theorem wird vom Heisenberg-Modell ausgegangen. Wenn man eine zusätzliche Anisotropie einführt, die es bei dünnen Filmen oft in Form einer in-plane-Anisotropie gibt, ergibt sich ein Übergang zum Ising-Modell, in dem eine kollektive Ordnung auch in zwei Dimensionen möglich ist.
} 
magnetische Anisotropie. Durch die starke Kopplung an das Gitter ergeben sich für Oxide aber zum Teil deutlich drastischere Effekte, so dass biaxiale Verspannungen zu einem Wechsel des Typs magnetischer Ordnung oder einem Metall-Isolator-Übergang führen können.

In diesem Kapitel werden die Veränderungen besprochen, die sich in komplexen Oxiden durch Grenzflächen- und Dünnfilmeffekte im Vergleich zu bulk-Materialien ergeben. Diese Modifikationen sollen im Hinblick ihres Einflusses auf die magnetischen Eigenschaften der Heterostruktur untersucht werden. Deshalb werden zunächst die treibenden Kräfte diskutiert, die zur Veränderung der Chemie und der Valenz der Übergangsmetallionen führen, um dann die Modifikationen der Besetzung der 3d-Orbitale zu diskutieren. Wenn diese Parameter festgelegt sind, kann man die magnetischen Kopplungen konstruieren, die dann in kollektiven magnetischen Phänomenen wie exchange bias münden können. Teilweise lassen sich die unten diskutierten Modifikationen an der Grenzfläche aufgrund deutlich unterschiedlicher Energieskalen getrennt betrachten. So sind die treibenden Kräfte für eine Umordnung der Kationen mit $\Delta E \sim 1-10 \mathrm{eV}$ (siehe Abschnitt 3.1) sehr groß und lassen sich somit gut von anderen Effekten separieren. Andere Bereiche wie die Freiheitsgrade der Ladungen $(\Delta E \sim 0.1-10 \mathrm{eV}$, siehe Abschnitt 3.1), der Orbitale $\left(\Delta E \sim E_{J T} \sim 0.5 \mathrm{eV}\right.$, siehe Abschnitt 3.2 und 2.2) und der Spins $\left(\Delta E \sim k_{B} T_{C / N} / 6 \sim 1-10 \mathrm{meV}\right.$, siehe Abschnitt 3.3 und 2.4) lassen sich nicht immer direkt separieren und man muss im Einzelfall betrachten, ob eine hierarchische Herangehensweise möglich ist. Im Verlauf dieses Kapitels soll außerdem verdeutlicht werden, dass man bei der Erklärung der Phänomene an Grenzflächen neben den Prozessen, die direkt an der Grenzfläche stattfinden, immer auch Dünnfilmeffekte wie z.B. den Verspannungszustand der Heterostruktur berücksichtigen muss.

\subsection{Grenzflächenchemie und Valenz der Übergangsmetallionen}

Komplexe oxidische Perowskite sind durch das Vorhandensein von unterschiedlichen Kationen auf den A- und B-Plätzen, die in verschiedenen Mischungsverhältnissen vorliegen können, sehr empfindlich auf Änderungen der Stöchiometrie. Wie man es im Phasendiagramm der Manganate gesehen hat (siehe Abschnitt 2.4), sind die Eigenschaften von mischvalenten Manganaten in der bulk-Phase abhängig vom Verhältnis zwischen der Anzahl an $\mathrm{Mn}^{3+}$ - und $\mathrm{Mn}^{4+}$-Ionen. Dies wird beeinflusst durch die Ionenladung der Kationen auf den A-Plätzen und das Vorhandensein von Sauerstoffleerstellen. Die Auswirkungen verschiedener Ionenradien auf die Struktur, die die Bindungswinkel der B-O-B'-Bindungen und damit das Austauschintegral bestimmt, sollen hier zunächst nicht weiter berücksichtigt werden. Die in die Heterostrukturen eingebrachten oxidischen Perowskite weisen eine ähnliche Kristallstruktur auf und es gibt zumeist keine Mischungslücke für den Austausch der Kationen auf den A- und B-Plätzen. Diffusion führt so zu einer unscharfen Grenzfläche, da die Depositionstemperatur deutlich unterhalb der Schmelzpunkte der beteiligten Materialien liegt und ein mischbares Phasendiagramm besteht.

Bei dünnen Filmen kann die Ausgangsstöchiometrie $\mathrm{ABO}_{3}$ modifiziert werden. Dabei sind zunächst die Anreicherung oder Verarmung bestimmter Kationen bzw. Anionen an der Oberfläche zu nennen. Bei Manganaten beobachtet man unter bestimmten Depositionsbedingungen oft eine Verarmung von Sauerstoffionen an der Oberfläche. Für Depositionsmethoden, die im Vakuum arbeiten, wie gepulster Laser-Deposition (PLD) und Molekularstrahlepitaxie (MBE) kann es bei zu niedrigem Sauerstoffpartialdruck zur Begünstigung von Sauerstoffleerstellen kommen. Diese machen sich einerseits in der Veränderung des Verhältnisses von $\mathrm{Mn}^{3+}$ zu 
$\mathrm{Mn}^{4+}$-Ionen bemerkbar [50], andererseits beobachtet man außerdem das Vorhandensein von $\mathrm{Mn}^{2+}$-Ionen an der Oberfläche mittels oberflächensensitiver Röntgenabsorptionsspektroskopie (XAS) 51. Bei geeigneten Depositionsbedingungen, die zum Beispiel bei der Metallorganischen Aerosol Deposition (MAD) vorliegen, lässt sich das Vorhandensein dieser Oberflächenmodifikation unterdrücken [51]. Veränderungen des Gehalts an Sauerstoffionen können auch getrieben werden durch Redoxreaktionen zwischen verschiedenen Schichten in einer Heterostruktur, wobei auch dies nur bei entsprechenden Depositionsbedingungen zu beobachten ist. So wird bei der Deposition von $\mathrm{LaAlO}_{3}$ auf $\mathrm{SrTiO}_{3}$ der Einbau von Sauerstoffleerstellen im Titanat beobachtet, der zu einer $\mathrm{Ti}^{3+}-\mathrm{Ti}^{4+}{ }_{-}$Mischvalenz führt, was der reduzierenden Wirkung der La- und Al-Atome zugeschrieben wird [52. Auch epitaktische Verspannungen, die sich in Heterostrukturen ergeben, können die Sauerstoffstöchiometrie beeinflussen, da der Einbau von Sauerstoffleerstellen zu einer vergrößerten Gitterkonstanten führt und damit die Bildungsenergie für Sauerstoffleerstellen unter Zugspannungen herabgesetzt wird [53]. Im Experiment beobachtet man für Übergitter aus $\mathrm{LaMnO}_{3}$ (LMO) und $\mathrm{SrMnO}_{3}$ (SMO), die durch PLD auf $\mathrm{SrTiO}_{3}$-Substraten gewachsen wurden, mittels TEM-Elektronen-EnergieVerlustspektroskopie (EELS) eine Verarmung von Sauerstoff in den SMO-Lagen, die auf den Übergang vom kubischen $\mathrm{SrMnO}_{3}$ zum orthorhombischen $\mathrm{SrMnO}_{2.5}$ hinweist [54]. Dass diese Modifikation von SMO, die zu einer gleichmäßigen Verteilung von $\mathrm{Mn}^{3+}$-Ionen im Übergitter führt, in anderen Studien nicht beobachtet werden konnte [55], zeigt den großen Einfluss der Depositionsbedingungen auf die Bildung von Sauerstoffleerstellen. Da die Sauerstoffanionen gegenüber den Kationen eine deutlich größere Beweglichkeit aufweisen [53, kann man eine Modifikation der Verteilung der Sauerstoffionen deutlich häufiger observieren. Aufgrund der kleineren Diffusionskonstanten für die Kationen müssen die treibenden Kräfte, die zu einer feststellbaren Umverteilung dieser führen, deutlich größer sein als für die Sauerstoffionen. Für $\mathrm{La}_{0.7} \mathrm{Sr}_{0.3} \mathrm{MnO}_{3}$ beobachtet man eine Anreicherung von $\mathrm{Sr}^{2+}$-Ionen an der Oberfläche [56, 57]. Diese Segregation wird anscheinend durch das Vorhandensein von Sauerstoffleerstellen begünstigt, da sie bei niedrigem Sauerstoffpartialdruck vermehrt auftritt. Sauerstoffleerstellen sind allerdings nicht die einzige treibende Kraft, da die Anreicherung von $\mathrm{Sr}^{2+}$ auch bei oxidierenden Bedingungen stattfindet [57]. In Übergittern aus $\mathrm{La}_{2 / 3} \mathrm{Ca}_{1 / 3} \mathrm{MnO}_{3}$ (LCMO) und $\mathrm{BaTiO}_{3}$ (BTO) schließen die LCMO-Lagen bevorzugt mit einer CaO-Lage statt einer $\mathrm{La}_{2 / 3} \mathrm{Ca}_{1 / 3} \mathrm{O}$-Lage, die sich im stöchiometrischen Fall ergeben würde, an der Grenzfläche zum BTO ab [58]. Als treibende Kraft für die Umverteilung von Kationen wird wiederholt die Vermeidung der polaren Katastrophe diskutiert, welche im Folgenden dargelegt werden soll.

Ionische Kristalle lassen sich in Abhängigkeit der Orientierung ihrer Oberfläche in alternierende Lagen zerlegen, welche unterschiedliche formale Ladungen pro Formeleinheit aufweisen. Für dünne Filme ist die elektrostatische Betrachtung einer Abfolge solcher Lagen stark vereinfacht, da man nach dem Gaußschen Gesetz [59] annehmen kann, dass das von jeder Lage ausgehende Feld unabhängig vom Abstand zu dieser Lage ist ${ }^{6}$ In Ionenkristallen mit einer $\mathrm{NaCl}$-Struktur ergibt sich bei einem Schnitt entlang der (001)-Ebene unabhängig von den beteiligten Ionen eine Abfolge neutraler Lagen. Schneidet man einen Kristall dieser Struktur, z.B. $\mathrm{Ni}^{2+} \mathrm{O}^{2-}$, allerdings entlang der (111)-Ebene, befinden sich die Kationen und Anionen getrennt in unterschiedlichen Lagen parallel zur (111)-Fläche. Für oxidische Perowskite ergibt sich entlang der (001)-Richtung ein alternierender Stapel von AO- und $\mathrm{BO}_{2}$-Lagen. Für das hier als Substrat verwendete $\mathrm{SrTiO}_{3}$ ergeben sich so neutrale Lagen $\left(\mathrm{Sr}^{2+} \mathrm{O}^{2-}\right)^{0}$ und

\footnotetext{
${ }^{6} \mathrm{Da}$ man das Feld nur mikroskopisch untersucht, ist diese Näherung gerechtfertigt. Bei makroskopischen Abständen, die in die Größenordnung der lateralen Dimension des Filmes kommen, bricht diese Näherung zusammen.
} 


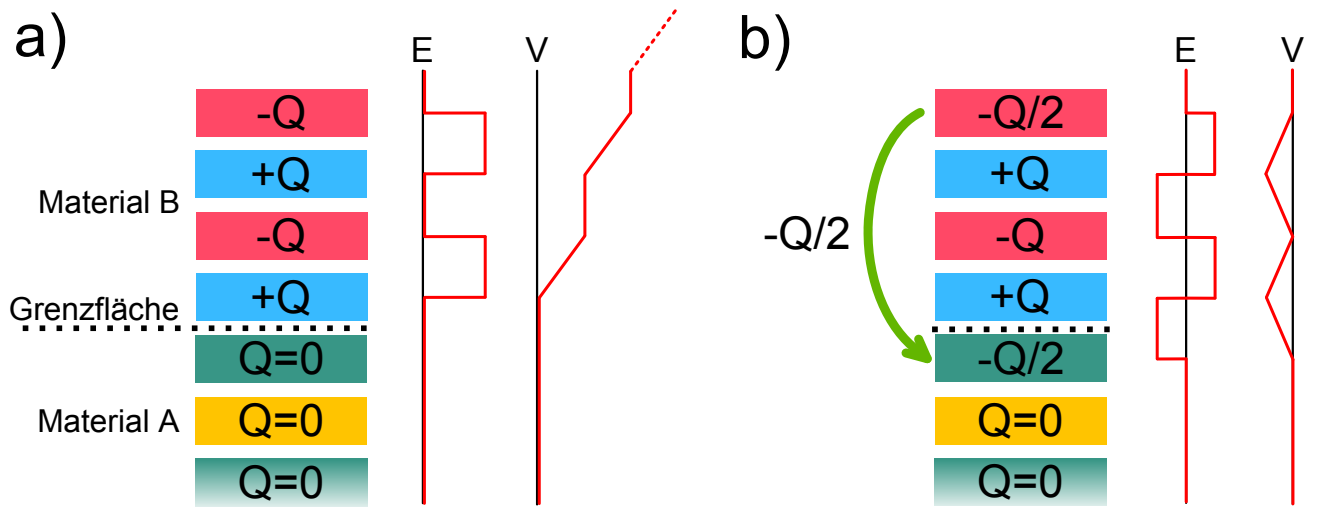

Abbildung 3.1.: Illustration der polaren Katastrophe an der Grenzfläche zweier Ionenkristalle: a) Ohne Rekonstruktion ergibt sich durch den Symmetriebruch an der Grenzfläche der gezeigte Verlauf des elektrischen Feldes $E$ und des elektrostatischen Potentials $V$, das mit der Schichtdicke von Material B divergiert. b) Wenn eine Ladung $-Q / 2$ von der Oberfläche an die Grenzfläche transferiert wird, oszilliert das elektrische Feld um null und das elektrostatische Potential um einen endlichen Wert (angelehnt an Zeichnung aus 61]).

$\left(\mathrm{Ti}^{4+} \mathrm{O}_{2}^{2-}\right)^{0}$. Wenn die Oberfläche des Substrates entlang einer anderen Ebene geschnitten wird, ist der Kristall aus Ebenen aufgebaut, die elektrisch geladen sind. So ergibt sich für die (110)-Ebene eine Sequenz aus Ebenen, die formal aus $\left(\mathrm{O}_{2}^{2-}\right)^{4-}$ bzw. $\left(\mathrm{Sr}^{2+} \mathrm{Ti}^{4+} \mathrm{O}^{2-}\right)^{4+}$ aufgebaut sind. Wie in Abbildung 3.1 a) zu sehen ist, ergibt sich durch eine Anordnung von abwechselnd mit $\pm Q$ geladenen Lagen ein elektrostatisches Potential $V$, das linear mit der Zahl der Einheitszellen ansteigt. Da sich die Potentialänderung $\Delta V$, die sich mit dem Abschluss einer Einheitszelle ergibt, in der Größenordnung $\Delta V \sim 1 \mathrm{~V}$ bewegt, ergeben sich schon bei einer kleinen Zahl von Ebenen große treibende Kräfte, die deutlich größer sind als die Aktivierungsenergien für Diffusion, z. B. $\Delta E \sim 4 \mathrm{eV}$ für Sr-Diffusion in $\mathrm{SrTiO}_{3}$ [60. Diese Divergenz des elektrostatischen Potentials in Ionenkristallen bezeichnet man auch als polare Katastrophe 61.

Theoretische Überlegungen 62 64 und experimentelle Beobachtungen 61, 65,68 weisen nun auf verschiedene Möglichkeiten hin, die Divergenz des elektrostatischen Potentials zu vermeiden. Diese basieren jedoch alle auf einem ähnlichen Grundprinzip, das abhängig von den beteiligten Materialien und den Herstellungsbedingungen unterschiedlich umgesetzt wird. In Abbildung 3.1 b) ist dies für das Beispiel eines Filmes (Material B), der mit abwechselnd $\pm Q$ geladenen Lagen auf einem Substrat (Material A) aus komplett neutralen Lagen aufwächst, dargestellt. Der kontinuierliche Anstieg des Potentials $V$ mit der Filmdicke lässt sich vermeiden, wenn die Ladung $-Q / 2$ von der Oberfläche zur Grenzfläche transferiert wird. Nach diesem Ladungstransfer oszilliert das elektrische Feld $E$ um null und das Potential um einen endlichen Wert unabhängig von der Filmdicke. Eine einfache Durchmischung an der Grenzfläche von Material A und B verhindert den kontinuierlichen Anstieg des elektrostatischen Potentials nicht. Experimentell wurden verschiedene Varianten zur Bewerkstelligung des Ladungstransfers festgestellt: Für NiO mit der NaCl-Struktur beobachtet man eine oktopolare Rekonstruktion der (111)-Oberfläche, bei der $\frac{1}{4}$ der äußeren $\mathrm{Ni}^{2+}$-Ionen und $\frac{3}{4}$ der $\mathrm{O}^{2-}$-Ionen entfernt werden, so dass eine Oberflächenlage mit einer Ladung +1 entsteht 62 , 64, 65. Auch ionische Adsorbate an der Oberfläche können eine Lage mit einer Ladungsdichte bilden, die zur Vermeidung der Polaren Katastrophe notwendig ist. Man beobachtet so keine oktopolare Rekonstruktion der (111)-Oberfläche von NiO, wenn sich eine Adsorbat-Lage 
aus $\mathrm{OH}^{-}$-Ionen auf die Oberfläche legt [65]. Auch $\mathrm{SrTiO}_{3}$, das entlang der (110)-Ebene geschnitten wurde, zeigt eine Rekonstruktion der Oberfläche. Es bilden sich Nanofacetten mit $\{001\}$-Orientierungen 68 .

In Heterostrukturen aus komplexen Oxiden kann die Vermeidung der polaren Katastrophe eine Änderung der Valenz der Übergangsmetallionen treiben. Das prominenteste Beispiel für diesen Vorgang sind Heterostrukturen aus $\mathrm{LaAlO}_{3}$ (LAO) und $\mathrm{SrTiO}_{3}$ (STO), die entlang der (001)-Richtung aufgewachsen wurden. Im LAO wechseln sich geladene Lagen $(\mathrm{LaO})^{+}$ und $\left(\mathrm{AlO}_{2}\right)^{-} \mathrm{ab}$, so dass für das Wachstum von LAO auf einem STO-Substrat eine Situation vorliegt, die mit Abbildung 3.1 a) vergleichbar ist. Untersuchungen mit TEM-EELS zeigen, dass für eine $\mathrm{TiO}_{2}$-Terminierung des STO-Substrates Ti-Ionen mit einer Valenz von 3+ statt $4+$ in der Nähe der Grenzfläche lokalisiert sind [61]. Die Elektronen werden also von der Oberfläche auf die Ti-Ionen transferiert. Die Elektronen auf den Ti-Ionen delokalisieren über einen Bereich mit einer Breite von etwa $5 \mathrm{~nm}$. Der dadurch entstehende elektrische Dipol treibt die Durchmischung der La- und Sr-Ionen, die deutlich ausgeprägter ist, als der Austausch der Al- und Ti-Ionen [61, 69]. Für eine SrO-Terminierung des STO-Substrates kann die polare Katastrophe durch Elektronentransfer von der Grenzfläche zur Oberfläche vermieden werden. Dieser wird durch den Einbau von Sauerstoffleerstellen an der Grenzfläche hergestellt 61.

Wenn man LAO auf einem $\mathrm{TiO}_{2}$-terminiertem STO aufwächst, bilden die Elektronen auf den $\mathrm{Ti}^{3 / 4+}$-Ionen ein zweidimensionales Elektronengas, das neben einem metallischen Verhalten des Widerstandes [7] auch weitere Effekte wie stark anisotropen Magnetowiderstand [70], Ferromagnetismus und Supraleitung [8] zeigt. Experimentelle Indizien weisen darauf hin, dass die polare Katastrophe tatsächlich die treibende Kraft für das zweidimensionale Elektronengas an der STO/LAO-Grenzfläche darstellt. Man beobachtet kein metallisches Verhalten, wenn man LAO auf einem mit SrO terminierten STO-Substrat deponiert [7], und es gibt eine minimale LAO-Schichtdicke, die zur Entstehung des Elektronengases notwendig ist [9]. Die Vermeidung der polaren Katastrophe als treibende Kraft für die Entstehung des zweidimensionalen Elektronengases an der STO/LAO-Grenzfläche bleibt aber umstritten, da STO seine elektrischen Eigenschaften durch das Einbringen von chemischen Defekten stark verändern kann 47 . Es gibt verschiedene Autoren, die die das zweidimensionale Elektronengas an der STO/LAO-Grenzfläche mit einer einfachen Dotierung des Titanats aufgrund von La/Sr-Durchmischung oder Sauerstoffleerstellen erklären. Sauerstoffleerstellen können einerseits durch die Eigenheiten der Depositionstechnik, z.B. den reduzierten Sauerstoffpartialdruck in der Depositionskammer und das Auftreffen von hochenergetischen Ionen bei gepulster Laserdeposition (PLD), eingebracht werden 52 . Andererseits können die deponierten Laund Al-Atome die Ti-Ionen an der Oberfläche des STO von $\mathrm{Ti}^{4+}$ nach $\mathrm{Ti}^{3+}$ reduzieren [71]. In letzter Zeit vorgenommene Experimente weisen darauf hin, dass auch das La/Al-Verhältnis in der LAO-Schicht eine Rolle spielt. Bei einem reduzierten Al-Anteil beobachtet man kein zweidimensionales Elektronengas an der Grenzfläche, da dann die polare Katastrophe durch eine Akkumulation von Leerstellen auf dem B-Platz an der Grenzfläche zum STO verhindert wird. Bei einem stöchiometrischen La/Al-Verhältnis oder einem Al-Überschuss ist dies nicht mehr möglich und es bildet sich ein zweidimensionales Elektronengas im Titanat nach dem oben dargelegten Ansatz [72. Man sieht an diesem vielfach studierten Beispiel, dass die Verteilung der Atome in der Grenzregion sehr genau betrachtet werden muss bei der Erforschung von Effekten, die sich an der Grenzfläche abspielen. Experimentelle Artefakte lassen sich am besten durch eine systematische Variation der Zusammensetzung von den beteiligten Komponenten ausschließen. 
Wie man anhand des Phasendiagrammes von $\mathrm{La}_{1-\mathrm{x}} \mathrm{Sr}_{\mathrm{x}} \mathrm{MnO}_{3}$ gesehen hat, ist die durchschnittliche Ladung der Mn-Ionen von großer Bedeutung für die magnetischen und elektrischen Eigenschaften. In Heterostrukturen aus Manganaten mit unterschiedlichem La/SrVerhältnis, z.B. Übergittern aus $\mathrm{LaMnO}_{3}$ (LMO) und $\mathrm{SrMnO}_{3}$ (SMO), ist die polare Katastrophe eine treibende Kraft, die die Verteilung der Elektronen auf den Mn-Ionen bestimmt [73. An der Grenzfläche zwischen zwei Manganaten mit unterschiedlicher Zahl von Elektronen in den $e_{g}$-Orbitalen kommt es auch bei einem chemisch abrupten Verlauf der Grenzfläche zu einer Delokalisierung über die Grenzfläche. Die Länge, die die $e_{g}$-Elektronen über die Grenzfläche zurücklegen, ergibt sich aus dem Wechselspiel zwischen dem Gewinn der kinetischen Energie bei Delokalisierung und der Coulomb-Energie, die man aufgrund der Trennung der Elektronen von den Ionen auf den A-Plätzen bezahlen muss. Der Gewinn der kinetischen Energie wird durch das Hopping-Integral $t_{d d}$ zwischen den d-Orbitalen charakterisiert. Die Coulomb-Energie für die Ladungsseparation um eine Gitterkonstante $a$ ergibt sich für eine Permittivität $\varepsilon$ des umgebenden Materials zu $\frac{e^{2}}{\varepsilon a}$, wobei $e$ die Elementarladung bezeichnet [74. Analog zur Thomas-Fermi-Abschirmlänge ergibt sich dann die Längenskala, über die die $e_{g}$-Elektronen an der Grenzfläche delokalisieren 29,74 :

$$
L_{T F} \sim \frac{t_{d d} \varepsilon a^{2}}{e^{2}}
$$

Diese liegt bei typischen Werten für Perowskit-Oxide $(t \sim 0.2 \mathrm{eV}, \varepsilon \sim 15)$ im Bereich von etwa 1-3 Monolagen [74]. Dabei ist anzumerken, dass das Hopping-Integral $t_{d d}$ von der magnetischen Ordnung beeinflusst wird. Außerdem wird man im folgenden Abschnitt sehen, dass die Besetzung der beiden $e_{g}$-Orbitale durch epitaktische Verspannungen und Grenzflächeneffekte verändert wird, wodurch auch $t_{d d}$ richtungsabhängig modifiziert wird [75]. Diese Delokalisierung der d-Elektronen führt zu einem elektrostatischen Potential, das einen Ausgleich des chemischen Potentials der in Kontakt stehenden Materialien bewirkt. Bei reellen Systemen verkompliziert sich die einfache Herangehensweise von oben, da das chemische Potential aufgrund der starken Korrelationen von der Zahl der $e_{g}$-Elektronen abhängig wird [29. Wenn Materialien mit unterschiedlichen Elementen auf den B-Plätzen in Kontakt gebracht werden, muss man außerdem den Sprung der Energieniveaus zwischen den verschiedenen Ionen berücksichtigen. Des Weiteren kann es in Analogie einer Redox-Reaktion zu einer Trennung der Ladungen kommen. In $\mathrm{LaCoMnO}_{6}$ kommt es beispielsweise zur Reaktion $\mathrm{Co}^{3+}+\mathrm{Mn}^{3+} \rightarrow \mathrm{Co}^{2+}+\mathrm{Mn}^{4+}[29]$. Man beobachtet diese Ladungstrennung auch in Heterostrukturen von $\mathrm{LaNiO}_{3}$ und $\mathrm{LaMnO}_{3}$, wo in den Monolagen an der Grenzfläche die Reaktion $\mathrm{Ni}^{3+}+\mathrm{Mn}^{3+} \rightarrow \mathrm{Ni}^{2+}+\mathrm{Mn}^{4+}$ stattfindet 76 .

\subsection{Orbitale Freiheitsgrade}

Wie man im Kapitel über die Manganate gesehen hat, sind die Ausrichtung und Besetzung der 3d-Orbitale von überragender Bedeutung für die magnetischen Eigenschaften. In diesem Abschnitt soll beschrieben werden, wie sich die Besetzung und Ausrichtung dieser Orbitale in Heterostrukturen verändern können. Für die hier hauptsächlich untersuchten Manganate wird betrachtet, wie in Heterostrukturen die Entartung der beiden $e_{g}$-Orbitale, des $d_{x^{2}-y^{2-}}$ und des $d_{3 z^{2}-r^{2}}$-Orbitals, aufgehoben wird. Dabei sind einerseits epitaktische Verspannungen wirksam und andererseits der Symmetriebruch an Grenzflächen. Ebenso werden die MnO-Mn-Bindungswinkel verändert, was aufgrund des winkelabhängigen Transferintegrals $t_{d d}$ 


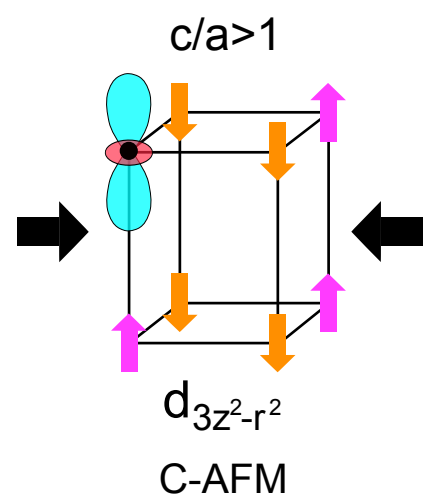

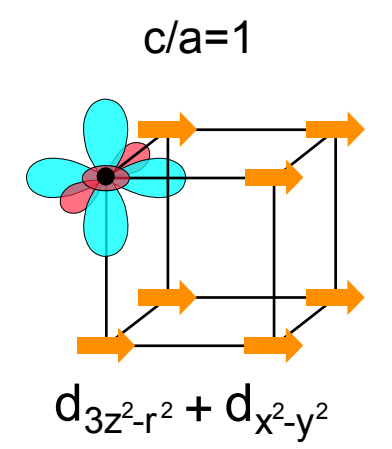

FM

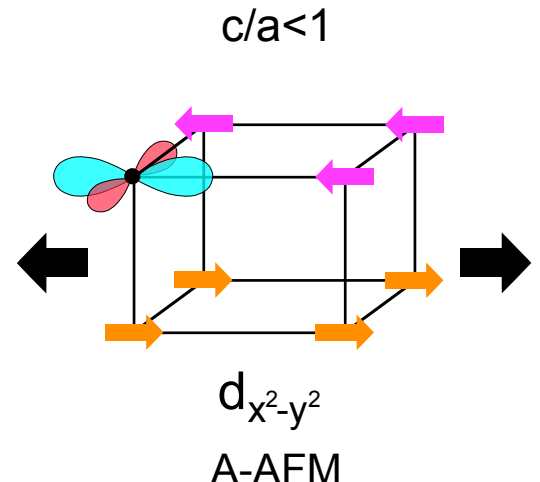

A-AFM

\begin{abstract}
Abbildung 3.2.: Aufhebung der Entartung der $e_{g}$-Orbitale $\left(d_{x^{2}-y^{2}}, d_{3 z^{2}-r^{2}}\right)$ durch epitaktische Verspannungen und der daraus resultierenden Modifikationen der magnetischen Ordnung (FM: Ferromagnet, C-AFM: C-Typ Antiferromagnet, A-AFM: A-Typ Antiferromagnet). Das Verhältnis $c / a$ zwischen der Gitterkonstanten in der Filmebene, $a$, und senkrecht dazu, $c$, beschreibt die Stärke und Richtung der tetragonalen Verzerrung (nach $77,78$,$) .$
\end{abstract}

einen großen Einfluss auf die Eigenschaften ausübt. Es lassen sich so in Heterostrukturen Zustände der 3d-Orbitale herstellen, die in bulk-Materialien nicht auftreten und zu unerwarteten Eigenschaften führen können.

Die biaxialen Verspannungen in einem verspannten Film verringern die Symmetrie des Kristallgitters. So wird aus einer kubischen Struktur eine tetragonale. Diese Symmetrieänderung hebt die Entartung der $t_{2 g^{-}}$und $e_{g}$-Niveaus auf, vergleichbar mit dem Jahn-Teller-Effekt (siehe Abbildung 2.2. . Bei Manganaten wirkt sich die Aufhebung der Entartung der $t_{2 g}$-Niveaus nicht auf die messbaren Eigenschaften aus, da die $t_{2 g}$-Elektronen den lokalisierten Rumpfspin bilden. Bei einer ursprünglich energetischen Gleichwertigkeit der $d_{x^{2}-y^{2}}$ und $d_{3 z^{2}-r^{2}}$ Orbitale führt eine Kompression durch das Substrat zu einer bevorzugten Besetzung der $d_{3 z^{2}-r^{2-}}$ Orbitale und eine Dehnung zu einer Bevorzugung der $d_{x^{2}-y^{2}}$-Orbitale [78, 79] (siehe Abbildung 3.2). Diese orbitale Polarisation wurde im Experiment eingehend an $\mathrm{La}_{0.7} \mathrm{Sr}_{0.3} \mathrm{MnO}_{3}$ (LSMO) untersucht, das man auf Substraten deponiert, die verschiedene Gitterparameter ober- und unterhalb der pseudokubischen Gitterkonstanten von LSMO aufweisen. Theoretische Berechnungen von Fang et al. 80] und Experimente von Konishi et al. 81] lieferten erste Hinweise auf die aufgehobene Entartung der $e_{g}$-Orbitale in verspannten Manganatfilmen. Eine direkte experimentelle Bestätigung dieser Tatsache wurde jedoch erst später geliefert: Da die $d_{x^{2}-y^{2}}$ und $d_{3 z^{2}-r^{2}}$-Orbitale jeweils eine Orientierung senkrecht und parallel zur Oberflächennormalen der Schicht aufweisen, kann man die Unterschiede der Besetzung dieser beiden Orbitale mittels linearem Röntgendichroismus (XLD) analysieren. Dabei vergleicht man die Absorption von Strahlung, die parallel und senkrecht zur Filmebene polarisiert ist. Indem man Anregungen im Energiebereich der Mn-L-Kante betrachtet, ist man sensitiv auf die elektronische Struktur der Mn-Ionen, insbesondere auf die unbesetzten 3d-Zustände, welche durch die Bevorzugung einzelner Orbitale zu einer polarisationsabhängigen Anregungswahrscheinlichkeit führen [79. Die von Aruta et al. durchgeführten XLD-Messungen an vollständig verspannten LSMO-Filmen zeigten tatsächlich das erwartete Ergebnis. Wenn man LSMO auf $\mathrm{SrTiO}_{3}$ (STO) (001) aufwächst, das eine größere Gitterkonstante besitzt, steht der Film unter Zugspannung und die $d_{x^{2}-y^{2}}$-Orbitale werden bevorzugt besetzt. Beim Wachstum auf $\mathrm{LaAlO}_{3}$ (LAO) (001) wird der Film in der Ebene komprimiert und es wird eine bevorzugte Besetzung der $d_{3 z^{2}-r^{2}}$-Orbitale festgestellt 79 . 
Nicht nur die veränderte Symmetrie des Gitters bei verspannten Filmen kann eine bevorzugte Besetzung der einzelnen $e_{g}$-Orbitale treiben, sondern auch der Symmetriebruch an Grenz- und Oberflächen. Tebano et al. erfassen bei sehr dünnen LSMO-Filmen auf STO $(d \sim 6 \mathrm{ML})$ neben der durch die Verspannung induzierten Bevorzugung der $d_{x^{2}-y^{2}}$-Orbitale zusätzlich auch eine Besetzung der $d_{3 z^{2}-r^{2}}$-Orbitale. Da diese Signatur nur bei diesen sehr dünnen Filmen im XLD-Spektrum zu erfassen ist und bei dickeren Filmen verschwindet, wird diese Besetzung der $d_{3 z^{2}-r^{2}}$-Orbitale den Mn-Ionen an Grenz- und Oberfläche zugeschrieben [77]. Ebenso zeigen Cui et al., dass bei dünnen LSMO-Filmen $(d \sim 5 \mathrm{ML})$ unabhängig von der durch das Substrat aufgeprägten Verspannung bevorzugt die $d_{3 z^{2}-r^{2}}$-Orbitale besetzt werden 82 . Lepetit et al. begründen diese Phänomenologie für LSMO-Filme auf STO folgendermaßen [83]: Die Elektronen auf den teilweise besetzten Orbitalen der Mn-Ionen delokalisieren an der Grenzfläche in die vollständig unbesetzten d-Orbitale der Ti-Ionen, was nur über die $d_{3 z^{2}-r^{2}}$-Orbitale effektiv geschehen kann. Diese orbitale Umbesetzung führt zu einer Jahn-Teller-Verzerrung senkrecht zur Filmebene, welche entgegen den Auswirkungen der epitaktischen Verspannung gerichtet ist. Die damit einhergehende Erhöhung der elastischen Energie wird aber durch den Gewinn der kinetischen Energie überkompensiert. Nach 1-3 Monolagen ist der Austausch mit den Ti-Ionen nicht mehr relevant und es stellt sich der durch die elastischen Verzerrungen bestimmte Zustand mit einer präferentiellen Besetzung der $d_{x^{2}-y^{2}}$-Orbitale ein. Auch für die Mn-Ionen an der freien Oberfläche von LSMO, das mit einer $\mathrm{MnO}_{2}$-Lage terminiert ist, deuten theoretische Berechnungen von Calderon daraufhin, dass in der obersten Lage von Mn-Ionen nur die $d_{3 z^{2}-r^{2}}$-Orbitale besetzt sind [84]. Huijben et al. deponierten LSMO-Filme auf STO, wobei sie ein besonderes Augenmerk auf die Vermeidung von Sauerstoffleerstellen im Film legten. In diesen Filmen konnte mit XLD auch für sehr kleine Schichtdicken $(d \sim 3 \mathrm{ML})$ keinerlei Besetzung der $d_{3 z^{2}-r^{2}}$-Orbitale detektiert werden, es waren gemäß der epitaktischen Verspannung nur die $d_{x^{2}-y^{2}}$-Orbitale besetzt [85]. Die weiter oben beschriebene Besetzung der $d_{3 z^{2}-r^{2}}$-Orbitale an den Grenzflächen wird also möglicherweise von Defekten in der Chemie, z.B. Sauerstoffleerstellen, an Ober- und Grenzfläche getrieben. Spätere Studien von Pesquera et al. signalisieren die Abhängigkeit der orbitalen Polarisation an der Oberfläche von LSMO-Filmen von der lokalen Umgebung der Mn-Ionen, indem sie eine Relation zwischen der orbitalen Polarisation und der Terminierung der Oberfläche herstellen [86]. Endet der LSMO-Film mit einer $\mathrm{MnO}_{2}$-Lage, so findet man eine Präferenz der $d_{3 z^{2}-r^{2}}$-Orbitale an der Oberfläche. Bei einem Abschluss mit einer $\mathrm{La}_{2 / 3} \mathrm{Sr}_{1 / 3} \mathrm{O}$-Lage findet man eine Bevorzugung der $d_{x^{2}-y^{2}}$-Orbitale entsprechend dem Einfluss des STO-Substrates [86]. Die XLD-Messungen liefern keine direkten Aussagen über die Längenskala, auf der sich die durch die Grenzfläche induzierte Veränderung der orbitalen Besetzung fortsetzt. Mit orbitaler Röntgenreflektometrie an der L-Kante von Ni konnte ermittelt werden, wie weit die orbitale Polarisation an der Grenzfläche in das Material eindringt 87]: In $\mathrm{LaNiO}_{3} / \mathrm{LaAlO}_{3}$-Übergittern wurde beobachtet, dass die $e_{g}$-Elektronen auf den Ni-Ionen bevorzugt die $d_{x^{2}-y^{2}}$-Orbitale besetzen. Diese Präferenz ist auf den Monolagen an der Grenzfläche zum LAO etwa doppelt so stark ausgeprägt wie in den folgenden Lagen im Zentrum der $\mathrm{LaNiO}_{3}$-Schicht. Da die Al-O-Ni-Bindung zwischen dem metallischen Nickelat und LAO, einem Isolator mit großer Bandlücke, sehr schwach ist, werden die senkrecht zur Filmebene ausgerichteten $d_{3 z^{2}-r^{2}}$-Orbitale gegenüber den $d_{x^{2}-y^{2}}$-Orbitalen energetisch angehoben 45 .

Neben einer einfachen Änderung der Bindungsabstände, sprich einer tetragonalen Verzerrung der $\mathrm{MnO}_{6}$-Oktaeder, kann die epitaktische Dehnung des Films auch durch Verkippungen der Oktaeder im Film realisiert werden. Wie eingangs beschrieben, hat dies über das Transfermatrixelement $t_{d d}$ einen starken Einfluss auf die magnetischen Kopplungskonstanten. In der Realität ist es schwer vorherzusagen, ob die epitaktischen Dehnungen eher durch eine tetragonale Verzerrung oder Verkippung der Oktaeder realisiert werden. Zumeist spielen beide Va- 
rianten eine Rolle [88. Dies ist auch stark abhängig von der bulk-Struktur des Filmmaterials und der kristallinen Orientierung der Substratoberfläche [88]. Das System von Verkippungen der Oktaeder im Film kann durch die epitaktischen Verspannungen modifiziert werden. Für das in der bulk-Phase rhomboedrische $\mathrm{LaAlO}_{3}$ (LAO) werden durch Druckspannungen die Rotationen entlang der [110]-Richtung und für Zugspannung entlang der [001]-Richtung [89] unterdrückt. Bei einer orthorhombischen Ausgangsstruktur ergibt sich ein Unterschied, ob die c-Achse des orthorhombischen Gitters in der Ebene des Substrates oder senkrecht dazu steht. Die Messung der Verkippungen und Elongationen der $\mathrm{MO}_{6}$-Oktaeder ( $\mathrm{M}$ bezeichnet Metallion auf B-Platz) ist technisch anspruchsvoll, da die gebräuchlichen Methoden wie Röntgendiffraktion und TEM vor allen Dingen die Positionen der schweren Kationen auf den Aund B-Plätzen auflösen und die Sauerstoffionen fast unsichtbar sind. Für makroskopische Einoder Polykristalle können aufgrund des großen Streuvolumens dennoch die genauen Gitterstrukturen, inklusive der Verkippungen der Oktaeder aufgeklärt werden. In Heterostrukturen wird der experimentelle Zugang neben der Reduktion des Streuvolumens dadurch erschwert, dass die Struktur der Verkippungen nicht homogen ist, sondern über die Filmdicke variiert. Deshalb sind detaillierte Informationen über die Ausrichtung der $\mathrm{MO}_{6}$-Oktaeder erst seit etwa fünf Jahren verfügbar, da diese nur durch eine Verbesserung der Auflösung bei der TEM und Methoden der Synchrotron-Röntgenbeugung zugänglich sind 90].

Tatsächlich werden die Bindungswinkel nicht allein durch die epitaktischen Verspannungen bestimmt, sondern auch durch die Struktur des Materials, das an der Grenzfläche liegt. Dies folgt zunächst einfach geometrisch aus der Tatsache, dass sich zwei an einer Grenzfläche benachbarte Oktaeder ein Sauerstoff-Ion teilen. Damit setzt sich das Schema von den Verkippungen der Oktaeder des Substrates über eine gewisse Längenskala in den Film fort und geht dann in den Zustand über, der durch den Verspannungszustand des Filmes diktiert wird. Diese Längenskala wird einerseits durch die Strukturen der beiden Materialien bestimmt. Wenn sich die Oktaeder nur entlang der z-Achse senkrecht zur Filmebene verdrehen, wird die Kopplung wesentlich kleiner ausfallen als für Drehungen, die die Sauerstoff-Ionen in der Grenzebene verschieben. Außerdem besitzen die Netzwerke aus $\mathrm{MO}_{6}$-Oktaedern materialabhängige Steifigkeiten. Eine Verdrehung der Oktaeder aus der Gleichgewichtslage der bulk-Phase führt also zu einer materialabhängigen Energieerhöhung. Die Breite der Übergangsregion steigt dann mit der Steifigkeit des Netzwerkes der Oktaeder an [91]. Damit zeigen konkrete theoretische Berechnungen und Experimente auch je nach Materialkombination Längenskalen von $d_{\text {relax }} \sim 2-9 \mathrm{ML}$, auf der das System an Verkippungen des Substrates in den Film hinein abklingt. Bei reinen Drehungen um die z-Achse ist die Länge sehr klein. Theoretische Berechnungen zeigen für ein in der tetragonalen Tieftemperaturphase befindliches STO-Substrat mit einem kubischen Film aus $\mathrm{SrFeO}_{3}$ eine Relaxationslänge von etwa 2 Monolagen [92. Gibt es zusätzlich Drehungen in der Filmebene, so wird die Längenskala deutlich größer. Dies zeigt sich beispielsweise in Heterostrukturen aus LSMO und $\mathrm{BiFeO}_{3}(\mathrm{BFO})$, wo die Rotationen auf einer Länge von 4-5 Monolagen relaxieren [93. Eine ähnliche Längenskala ergibt sich auch theoretisch für einen $\mathrm{La}_{0.75} \mathrm{Sr}_{0.25} \mathrm{MnO}_{3}$-Film auf STO 91. Für $\mathrm{La}_{0.67} \mathrm{Sr}_{0.33} \mathrm{MnO}_{3}$-Filme auf STO zeigt sich nach einer präzisen Analyse von Synchrotron-Beugungsspektren eine Übergangsregion, die mit zunehmender Filmdicke von $d_{\text {relax }} \sim 5 \mathrm{ML}$ (für $d_{L S M O} \approx 10 \mathrm{~nm}$ ) auf $d_{\text {relax }} \sim 24 \mathrm{ML}$ (für $d_{L S M O} \approx 60 \mathrm{~nm}$ ) ansteigt $[94$ In Übergittern aus Materialien mit verschiedenen Rotationssymmetrien stellen sich die Verdrehungen der Oktaeder abhängig von den jeweiligen Schichtdicken ein. Bei Übergittern aus kubischem $\mathrm{SrMnO}_{3}$ (SMO) und rhomboedrischem $\mathrm{LaNiO}_{3}$ (LNO) werden je nach Wahl der relativen Schichtdicken verschiedene Muster der Oktaederrotationen beobachtet. Ist das LNO dünner als das SMO, so werden die Rotationen im LNO komplett unterdrückt, für dickere LNO-Schichten werden in den SMOSchichten Drehungen induziert 95. Gehrke et al. erreichen eine Veränderung des Systems 
von Verkippungen der Oktaeder durch die Modifikation von Grenzflächen 96: In Übergittern aus $\mathrm{La}_{2 / 3} \mathrm{Ca}_{1 / 3} \mathrm{MnO}_{3}(\mathrm{LCMO}, d \approx 15 \mathrm{~nm})$ und $\mathrm{BaTiO}_{3}(\mathrm{BTO}, d \approx 8 \mathrm{~nm}$ ) besitzen die LCMO-Lagen eine orthorhombische (Pnma) Struktur. Durch Einführen von zwei Monolagen $\mathrm{LaMnO}_{3}$ (LMO) an den LCMO/BTO-Grenzflächen kommt es zu einem strukturellen Übergang der LCMO-Lagen zu einer rhomboedrischen (R $\overline{3} \mathrm{c})$ Struktur. Mit den LMO-Lagen werden in der Grenzregion zusätzliche Elektronen eingebracht, die zur Stabilisierung der rhomboedrischen Phase führen, mit der ein FMM-Grundzustand einhergeht [96].

\subsection{Magnetismus}

In diesem Abschnitt werden zunächst die Unterschiede im Magnetismus eines einfachen Filmes auf einem Substrat im Vergleich zum bulk-Material besprochen. Dabei wird zum einen auf den Zusammenhang zwischen dem Magnetismus und der aus der epitaktischen Verspannung folgenden Veränderung der orbitalen Freiheitsgrade eingegangen und zum anderen auf das Phänomen der magnetisch „toten“ Lagen an der Grenzfläche zum Substrat und der freien Oberfläche. Dann wird das kollektive magnetische Verhalten in Heterostrukturen aus verschiedenen Materialien besprochen. In diesem Zusammenhang werden allgemeine Konzepte des exchange bias als Kopplungsphänomen zwischen ferro- und antiferromagnetischen Schichten beleuchtet.

Die zuvor beschriebenen Modifikationen der Perowskite hinsichtlich ihrer Chemie, Valenz des Übergangsmetall-Ions und der orbitalen Parameter legen die magnetischen Wechselwirkungen fest. Die durch die epitaktischen Verspannungen verursachten Veränderungen der orbitalen Besetzungen und Bindungswinkel setzen sich in den Film fort, solange der Film epitaktisch verzerrt aufwächst. Für $\mathrm{La}_{0.7} \mathrm{Sr}_{0.3} \mathrm{MnO}_{3}$-Filme auf STO (001) entspricht dies einer Schichtdicke von über $100 \mathrm{~nm}$ [83, 97. Bei sehr dünnen ferromagnetischen oxidischen Filmen, wie z.B. $\mathrm{La}_{0.7} \mathrm{Sr}_{0.3} \mathrm{MnO}_{3}$ oder $\mathrm{SrRuO}_{3}$ spricht man von magnetisch „toten“ Lagen mit denen man die Monolagen bezeichnet, die auch im Grundzustand keinen Ferromagnetismus mehr zeigen. Die Zahl dieser Lagen wird durch kurzreichweitige Effekte wie den Ladungstransfer, Veränderung der Stöchiometrie (Interdiffusion, Sauerstoffleerstellen) und Modifikationen der 3d-Orbitale bestimmt. Tauscht man bei $\mathrm{La}_{1-\mathrm{x}} \mathrm{Sr}_{\mathrm{x}} \mathrm{MnO}_{3}$ die Kationen auf dem A-Platz aus, ändert sich das $\mathrm{Mn}^{3+} / \mathrm{Mn}^{4+}$-Verhältnis, woraus eine Verschiebung der Eigenschaften gemäß des Phasendiagramms folgt. Ebenso wirken sich Sauerstoffleerstellen aus: Bei einem Anteil der Sauer-

stoffleerstellen von $\delta$ ergibt sich eine effektive Dotierung $\mathrm{La}_{1-\mathrm{x}} \mathrm{Sr}_{\mathrm{x}} \mathrm{Mn}_{1-\mathrm{x}+2 \delta}^{3+} \mathrm{Mn}_{\mathrm{x}-2 \delta}^{4+} \mathrm{O}_{3-\delta}$. An der Position der Sauerstoffleerstelle werden zudem die über den Sauerstoff vermittelten Austauschwechselwirkungen unterbrochen. Durch den Austausch von Kationen auf dem B-Platz kommen außerdem konkurrierende Wechselwirkungen hinzu abhängig von den getauschten Kationen. Die dadurch induzierte räumliche Unordnung in den Austauschkonstanten hat wie bei einem Spin-Glas eine drastische Reduktion der Ordnungstemperatur zur Folge [50, 98.

\subsubsection{Magnetismus und orbitale Polarisation}

Epitaktische Verzerrungen bewirken eine Umbesetzung der Mn- $e_{g}$-Orbitale im LSMO. Wie es bei den Ausführungen zu den GKA-Regeln angeklungen ist, sind die Art und Stärke der MnO-Mn-Austauschwechselwirkungen stark von der gegenseitigen Ausrichtung der $e_{g}$-Orbitale abhängig. So wird experimentell und theoretisch von Konishi und Fang eine Verschiebung der Grenzen des magnetischen Phasendiagramms von $\mathrm{La}_{1-\mathrm{x}} \mathrm{Sr}_{\mathrm{x}} \mathrm{MnO}_{3}(\mathrm{LSMO}(\mathrm{x})$ ) als Funk- 


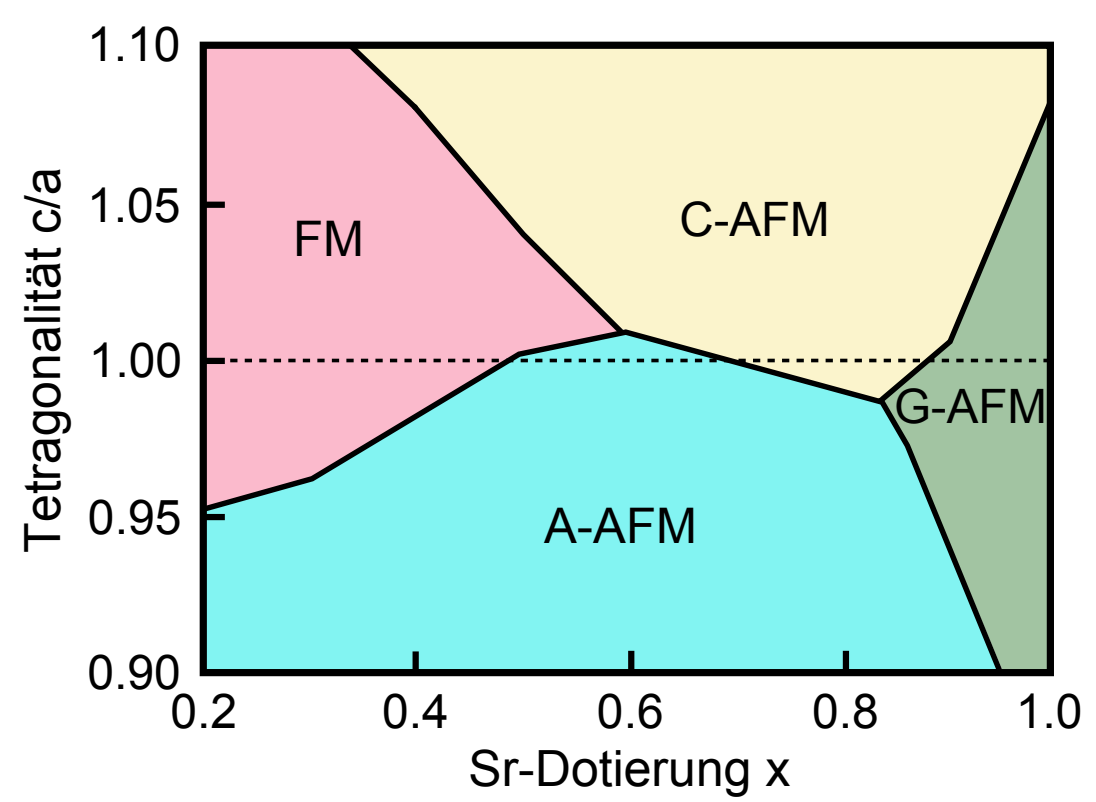

\begin{abstract}
Abbildung 3.3.: Magnetisches Phasendiagramm von in der (001)-Ebene biaxial verspannten $\mathrm{La}_{1-\mathrm{x}} \mathrm{Sr}_{\mathrm{x}} \mathrm{MnO}_{3}$-Filmen gemäß den theoretischen Berechnungen von Fang et al. [80] (Die Tetragonalität $c / a$ bezeichnet das Verhältnis zwischen den Gitterparametern in der Ebene, $a$, und senkrecht dazu, $c$. Es treten verschiedene Typen magnetischer Ordnung im Grundzustand auf: Ferromagnetismus (FM) und antiferromagnetische Ordnung verschiedenen Typs, C-Typ (C-AFM), A-Typ (A-AFM) und G-Typ (G-AFM)).
\end{abstract}

tion der durch epitaktische Verspannungen bewirkten tetragonalen Verzerrung beobachtet 80, 81. Man charakterisiert die tetragonale Verzerrung durch das Verhältnis $c / a$ zwischen den Atomabständen in der Filmebene, $a$, und senkrecht dazu, $c$. Durch das Wachstum auf Substraten (alle entlang der (001)-Ebene geschnitten) mit verschiedenen Gitterparametern wurden in $\operatorname{LSMO}(\mathrm{x}=0.5)$ verschiedene tetragonale Verzerrungen mit $c / a=0.97, c / a=0.99$ und $c / a=1.04$ verursacht. Für die kleinste Verzerrung $(c / a=0.99)$ bleibt der Film wie in der bulk-Phase ferromagnetisch-metallisch. Bei Zugspannung $(c / a=0.97)$ wird der Film isolierend und zeigt eine antiferromagnetische Ordnung des A-Typs. Befindet sich der Film unter Druckspannung $(c / a=1.04)$, ist dieser ein antiferromagnetischer Isolator des C-Typs 81 .

Die durch die Zugspannungen begünstigten $d_{x^{2}-y^{2}}$-Orbitale vermitteln in den (001)-Ebenen ferromagnetische Kopplungen durch den Doppelaustausch. Die Kopplung senkrecht zu diesen Ebenen ist nach den GKA-Regeln dann antiferromagnetisch, da die entlang der [001]-Richtung orientierten $d_{3 z^{2}-r^{2}}$-Orbitale weniger stark besetzt sind. Druckspannungen sorgen für einen Vorzug der $d_{3 z^{2}-r^{2}}$-Orbitale, so dass entlang der [001]-Richtung der Doppelaustausch zu einer ferromagnetischen Ordnung führt. Diese ferromagnetischen Linien koppeln in der Ebene antiferromagnetisch, so dass insgesamt eine antiferromagnetische Ordnung des C-Typs folgt 77 , 80, 81 (siehe Abbildung 3.2 . Bei einer kleinen tetragonalen Verzerrung sind die $e_{g}$-Orbitale gleichmäßig besetzt und es ergibt sich ein ferromagnetischer Doppelaustausch in alle Richtungen. Die Verspannungen verursachen außerdem eine Änderung des Mn-O-Bindungsabstandes $d_{M n-O}$. Das bereits zuvor eingeführte Transfermatrixelement $t_{d d}$, das den Gewinn kinetischer Energie bei der Delokalisierung beschreibt, fällt entsprechend $t_{d d} \sim d_{M n-O}^{-7}$ mit dem Abstand der Ionen ab 99,100 . Wie aus den zuvor geschilderten Beobachtungen folgt, überwiegt der Effekt der orbitalen Umbesetzung gegen diese Abstandsabhängigkeit, da ja gerade die fer- 
romagnetischen Wechselwirkungen entlang der gestreckten Richtungen überwiegen. Durch first-principle DFT-Rechnungen erhalten Fang et al. dann ein LSMO(x)-Phasendiagramm, wo sich die Phasengrenzen als Funktion der tetragonalen Verzerrung verschieben [80] (siehe Abbildung 3.3). Dieses theoretische Phasendiagramm bestätigt qualitativ die Tendenzen der zuvor geschilderten experimentellen Untersuchungen an $\operatorname{LSMO}(\mathrm{x}=0.5)$-Filmen 80, 81. Über den gesamten Sr-Dotierungsbereich setzt sich die beschriebene Tendenz fort, wobei oberhalb einer Dotierung von etwa $x=0.6$ mittels epitaktischer Verspannung direkt zwischen einem antiferromagnetischen Grundzustand des C- und A-Typs gewechselt werden kann.

\subsection{2. "Tote“ Lagen}

Die räumliche Ausdehnung und die Ursachen der „toten“ Lagen in dünnen Filmen von LSMO mit der für die ferromagnetisch-metallische (FMM) Phase optimalen Dotierung von $\mathrm{x}=0.3-0.4$ sind immer noch Gegenstand intensiver Forschungsaktivitäten [101. Dabei werden zumeist LSMO-Filme auf STO (001)-Substraten untersucht. Neben den magnetisch „toten“ Lagen werden auch die elektrisch „toten“ Lagen festgestellt, die auch bei tiefen Temperaturen kein metallisches Verhalten aufweisen. Typischerweise ist die Zahl der magnetisch „toten“ Lagen kleiner als die der elektrischen, was auf perkolative Effekte zurückgeführt wird [85]. In der Literatur liegen die kleinsten Filmdicken, bei denen noch ferromagnetisches Verhalten sichtbar ist, bei etwa 4-5 Monolagen 85, 102 104. Bei einfachen LSMO-Schichten auf STO liegt die untere Grenze für metallisches Verhalten bei 7-8 Monolagen 101, 104, 105.

Elektrostatische Überlegungen können eine Änderung der Mn-Valenz an der Grenzfläche zum Substrat oder der Oberfläche erklären. Für das Wachstum auf einem $\mathrm{TiO}_{2}$-terminierten STO (001)-Substrat erwartet man so eine Valenz von $\mathrm{Mn}^{3+}$ an der Grenzfläche [105, 106]. In der Tat beobachtet man experimentell mittels Röntgenabsorptionsspektroskopie, dass bei dünnen LSMO-Filmen mit einer Dicke im Bereich $d \sim 6-8 \mathrm{ML}$ die Valenz von dem Wert der bulk-Phase zum $\mathrm{Mn}^{3+}$-Zustand wechselt [102, 103, 107]. Allerdings erwartet man aufgrund reiner elektrostatischer Wechselwirkungen die Valenzänderung nur auf einer Monolage, da die Thomas-Fermi-Abschirmlänge für LSMO etwa $0.3 \mathrm{~nm}$ beträgt 106. Oft wird diese Diskrepanz mit Sauerstoffleerstellen erklärt, die sich leicht über größere Bereiche verteilen können [101 103. Dabei kann es speziell in der Nähe zum STO-Substrat zu einer Akkumulation von $\mathrm{O}^{2-}$-Leerstellen kommen, da das Substrat bei UHV-Depositionsverfahren mit Leerstellen angereichert wurde und dann als getter die Mn-Ionen in der wachsenden Schicht reduziert 77 , 108.

Neben diesen Modifikationen werden auch die im vorherigen Abschnitt erwähnten Änderungen der orbitalen Polarisation an den Grenzflächen als Ursachen für die „toten“ Lagen diskutiert. Die beschriebene Bevorzugung der $d_{3 z^{2}-r^{2}}$-Orbitale an der Ober- und Grenzfläche führt zu einer Unterdrückung des ferromagnetischen Doppelaustausches in der Filmebene und einem antiferromagnetischen Austausch des C-Typs [77. Die Konkurrenz dieser Wechselwirkungen in der Nähe der Grenzflächen und der ferromagnetischen Wechselwirkungen im Inneren des Filmes verursacht dann eine Reduktion der Ordnungstemperaturen [98]. Durch die geringe Hybridisierung der $d_{3 z^{2}-r^{2}}$-Orbitale in der (001)-Ebene wird zudem auch der typischerweise in der Filmebene gemessene elektrische Transport unterdrückt 77,83 .

Die Veränderung der Verdrehungen der $\mathrm{MnO}_{6}$-Oktaeder an Grenzflächen zeigt sich auch bei dünnen LSMO-Filmen und wirkt sich auf die magnetischen und elektrischen Eigenschaften aus: Moon et al. wachsen dünne LSMO-Filme mit einer Dicke von $d \sim 11 \mathrm{ML}$ auf einem kubi- 
schen Substrat, $\left(\mathrm{LaAlO}_{3}\right)_{0.3}\left(\mathrm{Sr}_{2} \mathrm{AlTaO}_{6}\right)_{0.7}$ (LSAT), und einem orthorhombischen Substrat, $\mathrm{NdGaO}_{3}$ (NGO). Obwohl beide Substrate etwa die gleiche pseudokubische Gitterkonstante aufweisen 7 zeigt sich dennoch für den Film auf LSAT eine um $140 \mathrm{~K}$ höhere Metall-IsolatorÜbergangstemperatur. Durch die sich an der Grenzfläche zwischen Substrat und Film fortsetzende Verkippung der Oktaeder sind die Bindungswinkel für den Film auf NGO deutlich kleiner als $180^{\circ}$, was die elektronische Bandbreite und damit die Übergangstemperatur reduziert 109 .

Zur Vermeidung oder Reduzierung der „toten“ Lagen werden verschiedene Pufferlagen an der Grenzfläche zum Substrat oder auf der Oberfläche eingeführt. Dabei wird meist die Beeinflussung eines der verschiedenen Phänomene an der Grenzfläche, die zu den „toten“ Lagen führen, Modifikationen der Chemie, Veränderung der Mn-Valenz oder orbitale Polarisation, angestrebt. Welche dieser drei Ursachen für die „toten“ Lagen man letztendlich mit der Grenzflächenmodifikation unterbindet, ist nur schwer auszumachen, da ein gleichzeitiger Einfluss auf alle drei Aspekte anzunehmen ist. In verschiedenen Experimenten wird als Pufferlage $\mathrm{La}_{1-\mathrm{x}} \mathrm{Sr}_{\mathrm{x}} \mathrm{MnO}_{3}$ mit einer anderen Sr-Dotierung als der Hauptfilm mit $x=0.3-0.4$ verwendet. Dabei werden von den verschiedenen Arbeitsgruppen im Experiment widersprüchliche Auswirkungen gleichartiger Modifikationen festgestellt. So beobachten Yamada et al. eine Verstärkung des ferromagnetischen Momentes in dünnen LSMO-Filmen, wenn an der Grenze zum STO-Substrat zwei Monolagen LMO eingeführt werden [110. Wenn Peng et al. bei dünnen LSMO-Filmen $(x=0.33)$ die Grenzfläche zum STO $(001)$ mit einer Monolage LSMO(x) mit variabler Sr-Dotierung $x$ versehen, stellen sie fest, dass die Curie-Temperatur gerade für $x=0$ am niedrigsten ist und für $x=1$ am höchsten. Durch das Einführen der $\mathrm{SrMnO}_{3}$-Lage auf einem mit einer $\mathrm{TiO}_{2}$-Lage terminierten Substrat wird der Sprung des elektrostatischen Potentials an der Grenzfläche verhindert, welcher chemische und elektronische Umverteilungen innerhalb des Filmes treiben kann 101, 106. Diese Art der Modifikation wirkt allerdings auch einer durch Sauerstoffleerstellen verursachten Fehldotierung entgegen. Ein ähnlicher Ansatz wird von Boschker et al. verfolgt, wo die Divergenz des elektrostatischen Potentials und sein Sprung an der Grenzfläche direkt durch die Deposition von einer Lage $\mathrm{La}_{0.33} \mathrm{Sr}_{0.67} \mathrm{MnO}_{3}$ vermieden wird [105]. Dadurch wird die elektrostatisch getriebene Diffusion mit dem Substrat vermindert, was die elektrischen und magnetischen Eigenschaften verbessert. Shapoval et al. beseitigen den störenden Einfluss des STO-Substrates auf die 3d-Orbitale des aufgewachsenen Manganat-Filmes durch Einführen einer Pufferlage aus $\mathrm{SrMn}_{\mathrm{x}} \mathrm{Ti}_{1-\mathrm{x}} \mathrm{O}_{3}$ [104]. Zur Erzeugung dieser Lage werden auf ein Ti-terminiertes STO-Substrat zwei Monolagen von $\mathrm{SrMnO}_{3}$ (SMO) aufgebracht, wobei sich die Ti- und Mn-Ionen in der ersten Monolage stark durchmischen. Auf diesen Puffer werden drei Monolagen von $\mathrm{LaMnO}_{3}(\mathrm{LMO})$ und zwei Monolagen von SMO deponiert, worauf zum Abschluss eine $\mathrm{LaO} / \mathrm{TiO}_{2}$-Doppellage folgt. In die LMO-Lagen werden durch den Austausch von Sr- und La-Ionen bzw. durch Elektronentransfer mit den SMO-Lagen Löcher eingebracht und es resultiert ein FMM-Grundzustand 104.

\subsubsection{Kollektive Phänomene}

Die zuvor erwähnten Veränderungen der magnetischen Kopplungen für die einfachste Heterostruktur, ein dünner Film auf einem Substrat, kommen auch bei komplizierteren Strukturen und Übergittern zum Tragen. Ein Beispiel dafür sind Strukturen aus $\mathrm{LaMnO}_{3}$ (LMO) und $\mathrm{SrMnO}_{3}$ (SMO). Wie man dem Phasendiagramm von $\mathrm{La}_{1-\mathrm{x}} \mathrm{Sr}_{\mathrm{x}} \mathrm{MnO}_{3}$ entnehmen kann, ist LMO in der stöchiometrischen bulk-Phase ein Antiferromagnet des A-Typs und SMO einer

${ }^{7} a_{L S A T}=0.3862 \mathrm{~nm}, a_{N G O}=0.3868 \mathrm{~nm}[109]$. 
des G-Typs (siehe Abbildung 2.5). Im Experiment zeigt LMO als dünner Film zumeist ein ferromagnetisches Verhalten [11 113]. Dies wird einer nicht perfekten Stöchiometrie in den Filmen zugesprochen. Nach dem Phasendiagramm benötigt man nur eine kleine Dotierung von $\mathrm{Mn}^{4+}$-Ionen im Bereich von $x \approx 0.09 \mathrm{im} \mathrm{LMO}$, um einen Übergang vom Antiferromagneten zum ferromagnetischen Isolator zu induzieren. Wenn man Polykristalle und dünne Filme aus LMO in Atmosphären mit hohen Sauerstoffpartialdrücken, $p_{O_{2}} \approx 0.2-1$ bar, auslagert, beobachtet man eine Änderung der Stöchiometrie, es werden Mn- und La-Leerstellen eingebaut, einen strukturellen Übergang von der orthorhombischen Struktur zu einer rhomboedrischen und das Erscheinen einer ferromagnetischen Ordnung [114 116]. Die unstöchiometrische LMO-Phase wird also bei hohen Sauerstoffpartialdrücken thermodynamisch bevorzugt. Zudem unterdrücken die epitaktischen Verzerrungen durch ein STO (001)-Substrat die orbitale Ordnung, die für eine antiferromagnetische Ordnung des A-Typs notwendig ist [117, 118].

Bei Heterostrukturen aus diesen beiden Materialien haben insbesondere Übergitter der Form $\left(\mathrm{LaMnO}_{3}\right)_{2 \mathrm{n}} /\left(\mathrm{SrMnO}_{3}\right)_{\mathrm{n}}$ auf STO (001)-Substraten, wobei $n$ die Anzahl der Monolagen bezeichnet, große wissenschaftliche Aufmerksamkeit auf sich gezogen. Bei einer vollständigen Durchmischung der Kationen dieses Übergitters erhält man nach dem Phasendiagramm von $\operatorname{LSMO}(\mathrm{x})$ ein ferromagnetisches Metall. Für eine chemisch abrupte Trennung der Kationen auf den A-Plätzen wird der magnetische Zustand zunächst durch die Verteilung der Ladungen auf den Mn-Ionen bestimmt [117], welche sich unter Anwendung der zuvor geschilderten Prinzipien ergibt. Da im LMO mit $(\mathrm{LaO})^{+}$und $\left(\mathrm{Mn}^{3+} \mathrm{O}_{2}\right)^{-}$geladene Halblagen vorliegen und im SMO mit $(\mathrm{SrO})^{0}$ und $\left(\mathrm{Mn}^{4+} \mathrm{O}_{2}\right)^{0}$ ungeladene, kommt das Konzept der polaren Katastrophe 73 genauso zur Anwendung wie eine durch Minimierung der kinetischen Energie getriebene Delokalisierung der Elektronen auf den $e_{g}$-Orbitalen [74, 119]. Theoretische Berechnungen im Rahmen der Dichtefunktionaltheorie (DFT) und experimentelle Untersuchungen zeigen für $n=1$ eine homogene Verteilung der Elektronen auf den $e_{g}$-Orbitalen der Mn-Ionen [73, 113 , 117, 119]. Dies hat einen homogenen ferromagnetisch metallischen (FMM) Grundzustand zur Folge. In den Experimenten von Bhattacharya et al. zeigt sich dabei eine um etwa $10 \mathrm{~K}$ reduzierte Curie-Temperatur gegenüber Filmen mit zufällig verteilten La/Sr-Ionen [119]. Für eine Periode von $n=2$ wird von Dong et al. unter Anwendung von Monte-Carlo-Simulationen eine Lage von Mn-Ionen mit einer antiferromagnetischen Ordnung des G-Typs zwischen den beiden SrO-Lagen vorhergesagt [117]. Im Experiment sieht man jedoch erst ab einer Periode von $n=3$ eine deutliche Reduktion der Sättigungsmagnetisierung bei tiefen Temperaturen und somit das Vorhandensein von antiferromagnetisch geordneten Bereichen [119]. Diese Diskrepanz ist womöglich durch eine leichte Durchmischung an den Grenzflächen zu erklären, die in der Theorie unberücksichtigt bleibt.

Tatsächlich zeigt sich, dass die Eigenschaften der LMO/SMO-Grenzflächen auf atomarer Skala einen großen Einfluss auf die lokalen magnetischen Eigenschaften ausüben: May et al. beobachten bei $\left(\mathrm{LaMnO}_{3}\right)_{2 \mathrm{n}} /\left(\mathrm{SrMnO}_{3}\right)_{\mathrm{n}}$ - Übergittern mit $n \approx 6$ eine asymmetrische Rauigkeit. Die Grenzfläche, bei der in Wachstumsrichtung LMO auf SMO folgt (SMO/LMO), ist schärfer als jene, bei der SMO auf LMO folgt (LMO/SMO). Bei letzterer werden in der LMO-Schicht Täler mit einer Breite von etwa $10 \mathrm{~nm}$ und einer Tiefe von zwei Monolagen beobachtet 13, 14. Mit Blick auf die Durchmischung der Kationen auf den A-Plätzen weist die LMO/SMO-Grenzfläche eine stärkere Delokalisierung der Ladungsträger auf, womit man für diese rauere Grenzfläche eine erhöhte Magnetisierung erwarten würde. Im Gegensatz zu dieser intuitiven Vermutung zeigt das mittels Neutronenreflektometrie bestimmte Profil der Magnetisierung aber deutliche Maxima an den strukturell scharfen Grenzflächen und eine Reduktion an den diffusen LMO/SMO-Grenzen. Shah et al. argumentieren, dass dieses Verhalten durch lokale Änderungen der orbitalen Polarisation verursacht wird, welche durch die chemische Inhomogenität an der rauen Grenzfläche getrieben wird [13, 14]. 
Durch epitaktische Verspannungen induzierte orbitale Polarisationen erweisen sich auch bei diesen Heterostrukturen als starker Einflussfaktor: Die zuvor beschriebene Delokalisierung senkrecht zur Wachstumsrichtung wird durch eine vermehrte Besetzung der $d_{3 z^{2}-r^{2} \text {-Orbitale }}$ begünstigt, welche durch biaxiale Druckspannungen hervorgerufen werden kann. Ab initio Rechnungen der LMO/SMO-Grenzfläche zeigen, dass unter Verwendung der typischerweise für oxidische Heterostrukturen gebrauchten Substrate eine deutliche orbitale Polarisation erreicht werden kann [78]. Beim Wachstum auf LAO ${ }^{8}$ liegt eine starke Druckspannung vor, wodurch fast alle $e_{g}$-Elektronen die $d_{3 z^{2}-r^{2}}$-Orbitale besetzen. Trotz der dadurch verstärkten Delokalisierung der $e_{g}$-Elektronen [73] ergibt sich im Experiment für $\left(\mathrm{LaMnO}_{3}\right)_{3 \mathrm{n}} /\left(\mathrm{SrMnO}_{3}\right)_{2 \mathrm{n}^{-}}$ Übergitter, die auf LAO (001)-Substraten gewachsen wurden, kein ferromagnetischer Zustand, da die starke orbitale Ordnung eine antiferromagnetische Ordnung des C-Typs favorisiert 120. In $\operatorname{LSMO}(\mathrm{x})$ wird die Verkleinerung des Gitterparameters mit steigender SrDotierung $x$ durch das Verhältnis von $\mathrm{Mn}^{3+}$ - und $\mathrm{Mn}^{4+}$-Ionen getrieben. Damit hängt der lokale Verspannungszustand in LMO/SMO-Heterostrukturen auch von der lokalen Besetzung der $e_{g}$-Orbitale ab. Dies wird für Wachstum auf STO-Substraten bedeutend, da die Gitterkonstante von STO mit $a_{S T O}=0.3905 \mathrm{~nm}$ zwischen den Gitterparametern von LSMO $(\mathrm{x}=0.33)\left(a_{L S M O}=0.387 \mathrm{~nm}[121)\right)$ und LMO $\left(a_{L M O}=0.394 \mathrm{~nm}[122]\right)$ liegt. In der Nähe der LMO/SMO-Grenzfläche findet man eine über wenige Monolagen delokalisierte Ladungsverteilung der $e_{g}$-Elektronen, womit sich der Verspannungszustand und damit auch die orbitale Polarisation in dieser Region kontinuierlich ändert. Diese Tatsache manifestiert sich auch bei der Untersuchung der orbitalen Freiheitsgrade in $\left(\mathrm{LaMnO}_{3}\right)_{2 \mathrm{n}} /\left(\mathrm{SrMnO}_{3}\right)_{\mathrm{n}}$-Übergittern mittels XLD. Für $n=1$ liegt eine homogene Ladungsverteilung vor, so dass eine bevorzugte Besetzung der $d_{x^{2}-y^{2}}$-Orbitale folgt. Für $n \geq 5$ zeigt sich der dominante Beitrag der $d_{3 z^{2}-r^{2}}$ Orbitale auf den Mn-Ionen im Inneren der LMO-Lagen [113].

Auch die in einem Übergitter veränderte Verkippung der $\mathrm{MnO}_{6}$-Oktaeder kann einen Einfluss auf die magnetischen Eigenschaften haben. So untersuchten May et al. Proben mit einer Dotierung $x=2 / 3$ im LSMO(x)-Phasendiagramm. Für bulk-Kristalle liegt $x=2 / 3$ in der Nähe der Phasengrenze zwischen antiferromagnetischer Ordnung des A- und C-Typs [15]. Die epitaktische Zugspannung auf den verwendeten STO (001)-Substraten stabilisiert aber die Ordnung des A-Typs. Neben einem Film mit gemischten Kationen, $\mathrm{La}_{1 / 3} \mathrm{Sr}_{2 / 3} \mathrm{MnO}_{3}$, stellten May et al. auch $\left(\mathrm{LaMnO}_{3}\right)_{\mathrm{n}} /\left(\mathrm{SrMnO}_{3}\right)_{2 \mathrm{n}}$-Übergitter her, die das gleiche $\mathrm{Mn}^{3+} / \mathrm{Mn}^{4+}$-Verhältnis aufweisen. Unter Anwendung von Neutronendiffraktion konnte dann festgestellt werden, dass die Néel-Temperatur $T_{N}$ für das Übergitter um $70 \mathrm{~K}$ höher liegt als für die ungeordnete Probe. In der ungeordneten Probe gibt es Verkippungen der $\mathrm{MnO}_{6}$-Oktaeder in den (001)-Ebenen, was den Doppelaustausch zwischen den $d_{x^{2}-y^{2}}$-Orbitalen schwächt. Aufgrund der kubischen Struktur des SMO wird dies in den Übergittern nicht verzeichnet, so dass das Transfermatrixelement zwischen den $d_{x^{2}-y^{2}}$-Orbitalen größer wird und die Ordnungstemperatur steigt 15.

\footnotetext{
${ }^{8} a_{L A O}=0.3792 \mathrm{~nm} 120$.
} 


\subsubsection{Exchange Bias}

Gesondert soll in diesem Abschnitt auf das Phänomen des exchange bias eingegangen werden, das bei der Kopplung zwischen Ferromagnet und Antiferromagnet auftritt und sich in einer Verschiebung der magnetischen Hystereseschleife bemerkbar macht. Dazu sollen hier zunächst allgemeine Modelle zu diesem Effekt vorgestellt werden, die unabhängig von dem betrachteten Materialsystem gültig sind. Die speziellen Gegebenheiten, die bei oxidischen Heterostrukturen auftreten, sollen in der späteren Diskussion der in dieser Arbeit behandelten Materialkombinationen aufgeführt werden.

Exchange bias (EB) als Kopplungsphänomen zwischen Ferromagnet (FM) und Antiferromagnet (AFM) wurde schon 1956 von Meiklejohn und Bean in Co/CoO core-shell Nanopartikeln beobachtet als Verschiebung der $M(H)$-Hysteresekurve nach Kühlen der Probe durch $T_{N}$ mit angelegtem Feld 123. Zudem wurde eine Erhöhung des Koerzitivfeldes registriert. Konventionsgemäß wird für die verschobene Hysteresekurve dann das Koerzitivfeld durch

$$
H_{C}=\frac{H_{C}^{+}-H_{C}^{-}}{2}
$$

und die Amplitude des exchange bias durch

$$
H_{E}=\frac{H_{C}^{+}+H_{C}^{-}}{2}
$$

definiert 124, 125 $\left(H_{C}^{+}\right.$und $H_{C}^{-}$bezeichnen die Feldwerte, an denen die $M(H)$-Kurve die Feldachse schneidet mit $H_{C}^{+}>H_{C}^{-}$.). Das einfache Bild zur Erklärung dieser unidirektionalen Anisotropie, das bereits von Meiklejohn und Bean beschrieben wurde, ist folgendes: Der Antiferromagnet hat in der Kontaktebene zum Ferromagneten eine unkompensierte Spinkonfiguration, wobei die Grenzfläche keinerlei Rauigkeiten aufweisen soll und die Kopplung entweder vollständig ferromagnetisch oder antiferromagnetisch sein soll (siehe Abbildung 3.4). Die Curie-Temperatur $T_{C}$ des Ferromagneten ist größer als die Néel-Temperatur $T_{N}$ des Antiferromagneten. Wenn man diesen Schichtstapel nun mit angelegtem Feld von $T_{C} \geq T>T_{N}$ einkühlt, legt der durch das Feld ausgerichtete Ferromagnet mittels der Austauschkopplung an der Grenzfläche die Orientierung des Antiferromagneten fest. Nimmt man nun an, dass die Ausrichtung des Antiferromagneten starr ist, vermittelt die Austauschwechselwirkung an der FM/AFM-Grenze mit einer Energiedichte $J_{I F}$ der Kopplungsenergie pro Fläche die unidirektionale Anisotropie [123].

Bei einer genaueren Betrachtung im Stoner-Wolfarth-Modell berücksichtigt man die Anisotropie des Antiferromagneten. Man nimmt vereinfachend eine uniaxiale Anisotropieenergiedichte $K_{A F M}$ für den Antiferromagneten an. Mit dieser beschreibt man die Energie, die zur kollektiven Drehung der Magnetisierungen aller in sich ferromagnetisch ausgerichteten Ebenen im AFM aufgewendet werden muss. Außerdem setzt man im FM und AFM einen eindomänigen Zustand voraus. Wie in Abbildung 3.5 dargestellt, werden die Winkel zwischen der uniaxialen Anisotropieachse des Antiferromagneten, dem äußeren Feld $H\left(\theta_{K H}\right)$, der Magnetisierungsrichtung der ferromagnetisch geordneten Ebenen im Antiferromagneten $M_{A F M}\left(\theta_{K A F M}\right)$ und der Magnetisierung des Ferromagneten $M_{F M}\left(\theta_{K F M}\right)$ eingeführt. Im erweiterten Modell von Meiklejohn und Bean für eine ferromagnetische Schicht der Dicke $t_{F M}$ und Magnetisierung $M_{F M}$, die an die antiferromagnetische Schicht der Dicke $t_{A F M}$ koppelt, ergibt sich dann unter 


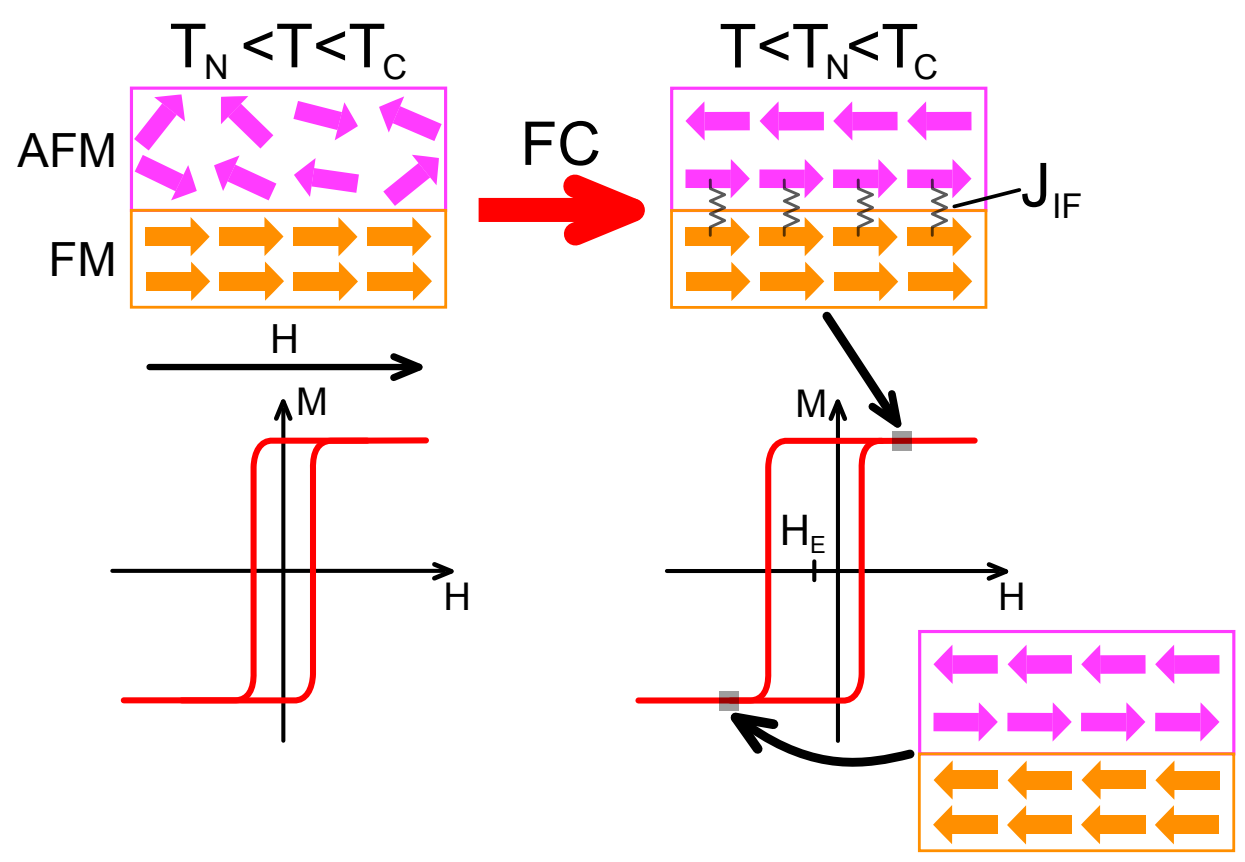

Abbildung 3.4.: Erklärung von exchange bias nach Meiklejohn und Bean 123: Ein Ferromagnet (FM) und ein Antiferromagnet (AFM) mit einer unkompensierten Spinkonfiguration haben eine gemeinsame Grenzfläche und es gilt $T_{N}<T_{C}$. Bei einer Temperatur $T_{N}<T<T_{C}$ beginnt man damit die Probe in einem äußeren Feld $H$, das den Ferromagneten ausrichtet, auf eine Temperatur $T<T_{N}<T_{C}$ einzukühlen (FC). Durch die (hier) ferromagnetische Kopplung $J_{I F}$ zwischen Ferromagnet und Antiferromagnet wird beim Einkühlen die Ausrichtung des Antiferromagneten festgelegt. Diese bleibt beim Durchlaufen der $M(H)$-Hysterese unverändert und führt vermittels der Austauschkopplung an der Grenzfläche $J_{I F}$ zu einer Verschiebung $H_{E}$ der $M(H)$-Kurve entlang der Feldachse (nach [11, 124 $125]$ ).

Vernachlässigung der Anisotropie des Ferromagneten folgende Flächendichte der Energie9

$$
\begin{aligned}
E= & -\mu_{0} H M_{F M} t_{F M} \cos \left(\theta_{K F M}-\theta_{K H}\right) \\
& +K_{A F M} t_{A F M} \sin ^{2}\left(\theta_{K A F M}\right) \\
& -J_{I F} \cos \left(\theta_{K A F M}-\theta_{K F M}\right)
\end{aligned}
$$

Bei einer sehr großen Anisotropie des Antiferromagneten bleibt die Ausrichtung der Spins in diesem fest und es ergibt sich die EB-Amplitude $H_{E}$, um die Kopplung an der Grenzfläche zu überwinden $\left(\theta_{K A F M}=0^{\circ}\right.$, das Kühlfeld war also positiv und entlang der Anisotropieachse des Antiferromagneten ausgerichtet):

$$
-\mu_{0} H_{E} M_{F M} t_{F M}=J_{I F} \Leftrightarrow H_{E}=-\frac{J_{I F}}{\mu_{0} M_{F M} t_{F M}}
$$

Im Falle einer kleinen Anisotropie des Antiferromagneten $\left(J_{I F} \geq K_{A F M} t_{A F M}\right)$ wird die Richtung der Spins in den ferromagnetisch geordneten Lagen des Antiferromagneten bei der Ummagnetisierung des Ferromagneten mitgedreht. Dadurch verschwindet das exchange bias, nur das Koerzitivfeld wird erhöht. Es gibt somit eine kritische Schichtdicke des Antiferromagneten, unterhalb der kein exchange bias auftritt:

$$
t_{A F M}^{c r i t}=\frac{J_{I F}}{K_{A F M}}
$$

\footnotetext{
${ }^{9} J_{I F}>0$, hier wird also eine ferromagnetische Kopplung zwischen FM und AFM an der Grenzfläche angenommen.
} 


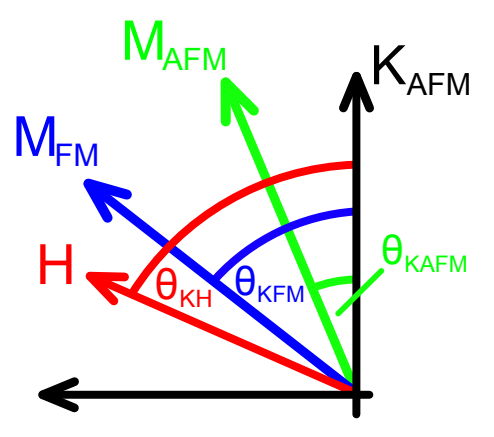

Abbildung 3.5.: Zur Definition der Winkel, die die Richtung der Magnetisierung $M_{F M}$ des Ferromagneten $\left(\theta_{K F M}\right)$, die Richtung der Magnetisierung $M_{A F M}$ der ferromagnetisch geordneten Lagen im Antiferromagneten $\left(\theta_{K A F M}\right)$ und das äußere Feld $H\left(\theta_{K H}\right)$ mit der Anisotropieachse des Antiferromagneten einschließen.

Für

$$
R=\frac{K_{A F M} t_{A F M}}{J_{I F}}>1
$$

steigt $H_{E}$ dann monoton in Richtung seines asymptotischen Grenzwertes von

$$
H_{E}^{\infty}=-\frac{J_{I F}}{\mu_{0} M_{F M} t_{F M}}
$$

an [11]:

$$
H_{E}\left(t_{A F M}\right)=H_{E}^{\infty} \sqrt{1-\frac{1}{4 R^{2}}}
$$

Das oben skizzierte Modell zum exchange bias ermöglicht die Erklärung des exchange bias und die Vorhersage einiger qualitativer Eigenschaften. Jedoch liefert es Werte für $H_{E}$, die zwei bis drei Größenordnungen zu hoch sind [11, 124]. Zudem kann es keine Erklärung für das Auftreten von EB bei einer vollständig kompensierten Spinkonfiguration des Antiferromagneten an der Grenzfläche liefern. Es gibt nun eine Fülle unterschiedlicher Ansätze, um diese Widersprüche aufzulösen, wobei anzumerken ist, dass es bisher kein Modell gibt, das das experimentell beobachtete Verhalten vollständig erklären kann.

Néel führte ein Modell ein, bei dem analog zu einer Domänenwand in einem Ferromagneten eine kontinuierliche Drehung der Magnetisierungsvektoren in Ferromagnet und Antiferromagnet parallel zur Grenzfläche stattfindet [125]. Dadurch wird die Energiedifferenz bei der Drehung der Magnetisierung des Ferromagneten stark herabgesetzt, was zu einer Verringerung von $H_{E}$ führt. Dabei muss allerdings die Anisotropie in Ferromagnet und Antiferromagnet klein sein, so dass sich eine breite Domänenwand ausbilden kann. Bei sehr großen Anisotropien geht das Modell von Néel in das von Meiklejohn und Bean über. Die Notwendigkeit breiter Domänenwände erzwingt auch sehr große Schichtdicken $\left(t_{F M / A F M}>100 \mathrm{~nm}\right)$, um die nötige Herabsetzung der Austauschkopplung $J_{I F}$ zu erreichen [11].

Malozemoff berücksichtigt atomare Rauigkeiten an der Grenzfläche [126], um das Vorhandensein von exchange bias bei einer vollständig kompensierten Magnetisierung in der Grenzebene zum Ferromagneten zu erklären. Bei einer monoatomaren Rauigkeit des Antiferromagneten an der Grenzfläche ergeben sich unkompensierte Austauschkopplungen, die eine bevorzugte Ausrichtung des Ferromagneten induzieren. Da die Rauigkeiten an zufälligen Stellen auftreten können, ergeben sich Austauschkopplungen mit unterschiedlichen Vorzeichen, die sich 
insgesamt weitestgehend aufheben [23. Nach Malozemoff werden nun aber in den Antiferromagneten beim Einkühlen im Feld Domänengrenzen senkrecht zur Grenzfläche eingebaut, so dass die Kopplungsenergie mit den in den Ferromagneten ragenden Atomen des Antiferromagneten minimiert wird. So addieren sich die lokalen Beiträge der Rauigkeiten. Die Größe der Domänen und damit die Dichte der für das exchange bias verantwortlichen Kopplungen hängt von den Materialparametern des Antiferromagneten, der Austauschsteifigkeit $A_{A F M}$ und der Anisotropie $K_{A F M}$, ab. Mit diesem Modell können zum Teil quantitativ richtige Werte für $H_{E}$, beispielsweise für das $\mathrm{Co} / \mathrm{CoO}-$ System, abgeschätzt werden [11.

Ein weiteres Modell, das domain-state-Modell [127], basiert ähnlich wie das Modell von Malozemoff darauf, dass beim Einkühlen im Feld eine Domänenstruktur im Antiferromagneten festgelegt wird, die zum exchange bias führt. Im domain-state-Modell werden aber nicht die Defekte an der Grenzfläche herangezogen, sondern Defekte im Inneren des Antiferromagneten, wie Korngrenzen und Fremdatome, die die magnetischen Wechselwirkungen lokal modifizieren. Durch Rechnungen im Ising-Modell zeigt sich dann, dass beim Einkühlen durch den Einfluss des äußeren Feldes und der Kopplung mit dem Ferromagneten an der Grenzfläche ein Domänenzustand im Antiferromagneten induziert wird, der bei tiefen Temperaturen eine irreversible Netto-Magnetisierung enthält, welche das EB verursacht. Dieses Spin-Glas-Verhalten resultiert aus dem pinning der Domänenwände an den Defekten. Mit diesem Modell kann somit auch exchange bias für Antiferromagneten mit einer kompensierten Spinkonfiguration an der Grenzfläche erklärt werden. Auch der training-Effekt, der die Verringerung von $H_{E}$ nach mehrmaligem Durchlaufen der $M(H)$-Kurve bezeichnet, kann damit verstanden werden: Bei der Ummagnetisierung des Ferromagneten kommt es zu Veränderungen der Domänenstruktur im AFM, die einer Verringerung der Netto-Magnetisierung entsprechen, so dass $H_{E}$ abnimmt 11 .

Um dem Symmetriebruch der magnetischen Wechselwirkungen an der FM/AFM-Grenzfläche Rechnung zu tragen, wurde von Radu et al. ein Modell vorgestellt, bei dem die Grenzfläche als Spin-Glas betrachtet wird [11. An der Grenzfläche gibt es so einen Bereich, in dem die Anisotropie des Antiferromagneten kontinuierlich abnimmt (siehe Abbildung 3.6). Spins in der Nähe der Grenzfläche haben dabei eine so schwache Anisotropie, dass diese mit dem Ferromagneten rotieren. Der Einfluss dieser kann als uniaxiale Anisotropie mit der Energiedichte $K_{S G}^{\text {eff }}$ beschrieben werden. Dadurch wird das Koerzitivfeld erhöht, aber kein exchange bias verursacht. Die Spins in der Nähe zum Antiferromagneten besitzen eine größere Anisotropie, so dass diese sich nicht mit dem Ferromagneten drehen und zu EB führen. Diese als fest angesehenen Spins koppeln über die reduzierte Kopplungsenergiedichte $J_{I F}^{\text {eff }}$ an die ferromagnetische Schicht. Mit diesen Überlegungen modifiziert man Gleichung $\left.3.4\right|^{\mid 0}$

$$
\begin{aligned}
E= & -\mu_{0} H M_{F M} t_{F M} \cos \left(\theta_{K F M}-\theta_{K H}\right) \\
& +K_{A F M} t_{A F M} \sin ^{2}\left(\theta_{K A F M}\right) \\
& -J_{I F}^{e f f} \cos \left(\theta_{K A F M}-\theta_{K F M}\right) \\
& +K_{S G}^{e f f} \sin ^{2}\left(\theta_{K S G}-\theta_{K F M}\right)
\end{aligned}
$$

Dabei bezeichnet $\theta_{K S G}$ den Winkel zwischen der uniaxialen Anisotropieachse des Antiferromagneten und der Anisotropieachse der drehbaren Momente des Spin-Glases an der Grenzfläche. Zur Charakterisierung der Unordnung an der Grenzfläche führen Radu et al. den

\footnotetext{
${ }^{10}$ Man vernachlässigt die Zeeman-Energie der rotierbaren Spins an der Grenzfläche, da diese in den meisten Fällen deutlich kleiner ist als die Zeeman-Energie des Ferromagneten 11.
} 


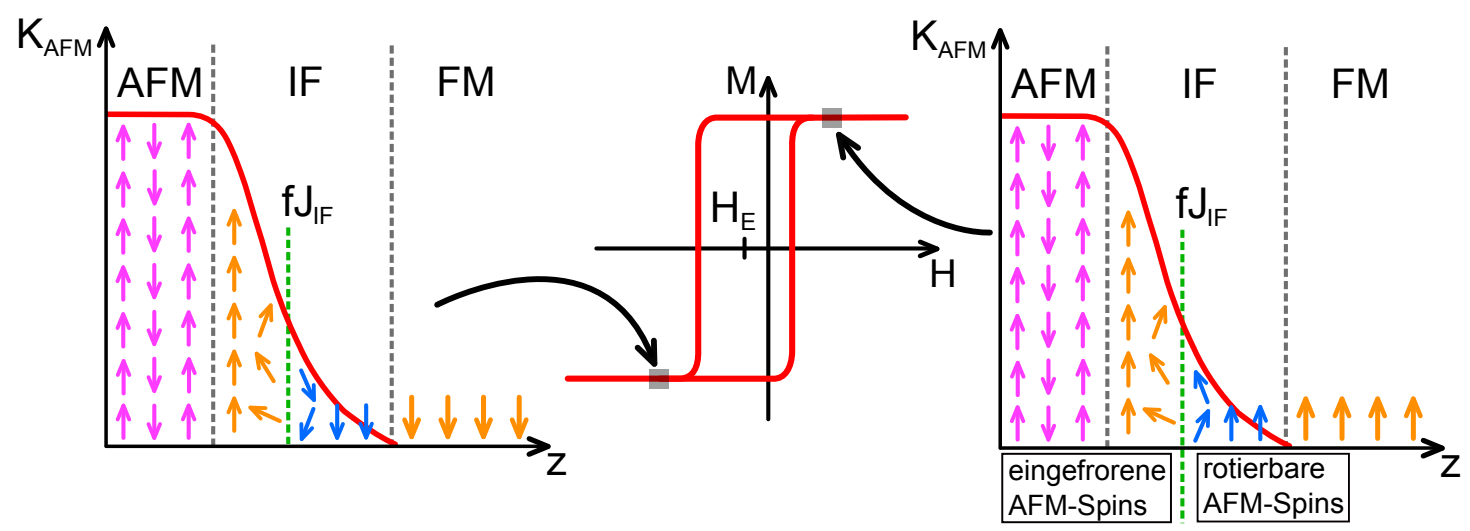

\begin{abstract}
Abbildung 3.6.: Zum Spin-Glas-Modell der Grenzfläche (IF) zwischen Ferromagnet (FM) und Antiferromagnet (AFM) nach Radu et al. [11]: An der Grenzfläche nimmt die Anisotropie des Antiferromagneteten $K_{A F M}$ kontinuierlich ab, so dass es in der Nähe zum Ferromagneten Spins des Antiferromagneten gibt, die sich mit der Magnetisierung des FM drehen. Wenn man sich weiter in Richtung des Antiferromagneten bewegt, sind die Spins eingefroren und vermitteln über die Kopplungsenergiedichte $f J_{I F}$ das exchange bias (nach [11).
\end{abstract}

Parameter $f=0-1$ ein, $f=1$ bedeutet eine vollständige Ordnung, $f=0$ totale Unordnung 11. Dieser Parameter verknüpft $J_{I F}$ mit $J_{I F}^{e f f}$ und $K_{S G}^{\text {eff }}$ :

$$
\begin{aligned}
K_{S G}^{e f f} & =(1-f) J_{I F} \\
J_{I F}^{e f f} & =f J_{I F}
\end{aligned}
$$

Numerische Rechnungen in diesem Modell zeigen, dass das $H_{E}$ monoton mit $f$ ansteigt, wogegen das Koerzitivfeld einen umgekehrten Trend zeigt [11. Analog zum Modell von Meiklejohn und Bean gibt es eine kritische Schichtdicke des Antiferromagneten

$$
t_{A F M}^{c r i t}=\frac{f J_{I F}}{K_{A F M}}
$$

unterhalb derer kein exchange bias zu beobachten ist, da die Spins im AFM der Magnetisierung der ferromagnetischen Schicht folgen. Als Funktion der Schichtdicke $t_{A F M}$ folgt je nach Grad von Unordnung entweder ein monotoner Anstieg von $H_{E}$ mit $t_{A F M}$ oder ein Verlauf mit einem Maximum in der Nähe von $t_{A F M}^{c r i t}[11]$. Im Experiment ist die blocking-Temperatur, die höchste Temperatur, bei der man noch exchange bias messen kann, gewöhnlich deutlich kleiner als die Néel-Temperatur des Antiferromagneten [124]. Das Koerzitivfeld zeigt aber sofort beim Kühlen unterhalb von $T_{N}$ einen Anstieg. Dies ist eine Folge der Temperaturabhängigkeit von $K_{A F M}$. Für das Erscheinen einer endlichen EB-Amplitude muss die Ungleichung

$$
K_{A F M}>\frac{f J_{I F}}{t_{A F M}}
$$

erfüllt sein. Damit gibt es einen kritischen Wert der Anisotropie des Antiferromagneten, unterhalb derer kein EB auftritt. Da $K_{A F M}$ monoton mit steigender Temperatur fällt und bei $T_{N}$ verschwindet, folgt daraus die blocking-Temperatur $T_{B}<T_{N}[11$.

Mit dem freien Parameter $f$ lassen sich die experimentell beobachteten $M(H)$-Kurven gut rekonstruieren. Es gibt jedoch auch direkte experimentelle Bestätigungen des Spin-Glas-Modells für exchange bias. Für Ferromagneten im Kontakt zu Materialien, die ein Spin-Glas-Verhalten 
zeigen, beobachtet man exchange bias [128]. Zudem ist es mittels elementselektiver Methoden wie zirkularem Röntgendichroismus (XMCD) und resonanter magnetischer Röntgenstreuung (XRMS) möglich, fixierte und rotierbare Momente an vergrabenen Grenzflächen zu erfassen. Die rotierbaren Momente zeigen dabei eine Hysterese wie der Ferromagnet, während sich die eingefrorenen Momente als Verschiebung entlang der Magnetisierungsachse manifestieren. Brück et al. bestimmten unter Anwendung von XRMS die Konzentration und Position der rotierbaren und fest stehenden Momente an der Grenzfläche zwischen dem Ferromagneten Fe und dem Antiferromagneten $\mathrm{MnPd}$, der in der Grenzebene eine kompensierte Spinkonfiguration aufweist. Die drehbaren Spins der Mn-Atome befinden sich in einem Bereich mit einer Breite von zwei Atomlagen innerhalb des MnPd-Filmes. Dies ist vergleichbar mit der chemischen Rauigkeit der Grenzfläche. Der Bereich, in dem sich die statischen Momente befinden, besitzt mit etwa sechs Atomlagen eine wesentlich größere Breite. Zudem ist die Konzentration dieser Momente um einen Faktor fünf kleiner als die der drehbaren [12. 


\section{Teil II.}

\section{Experimentelle Methoden}



$\Gamma_{\text {Kapitel }} 4$

\section{Metallorganische Aerosol Deposition (MAD) mit in-situ Ellipsometrie}

In diesem Kapitel soll die im Rahmen dieser Arbeit realisierte Anlage zur metallorganischen Aerosol Deposition (MAD) mit in-situ Wachstumsüberwachung durch Ellipsometrie beschrieben werden. Sämtliche hier betrachteten Heterostrukturen wurden mit dieser Anlage deponiert. Dazu wird zunächst das grundsätzliche Prinzip der MAD vorgestellt und Möglichkeiten der in-situ Wachstumskontrolle mit Blick auf die technischen Begebenheiten der MAD diskutiert. Dann werden die theoretischen Grundlagen für die Ellipsometriemessungen und deren Interpretation gelegt, um dann schließlich den realisierten experimentellen Aufbau einzuführen.

\subsection{MAD Grundlagen}

Die metallorganische Aerosol Deposition (MAD) ist ein unkonventionelles Verfahren zur Herstellung qualitativ hochwertiger dünner Filme aus Metalloxiden 129, 130. Mit dieser Methode konnten schon verschiedene komplexe Oxide wie Manganate, Cobaltate, Ruthenate, Cuprate, Titanate und Doppelperowskite wie $\mathrm{La}_{2} \mathrm{CoMnO}_{6}$ deponiert werden. Auch einfache Oxide wie z.B. $\mathrm{ZnO}, \mathrm{Al}_{2} \mathrm{O}_{3}, \mathrm{SrO}$ und $\mathrm{MgO}$ wurden mit dieser Methode bereits abgeschieden.

Metallorganische Präkursoren werden im gewünschten molaren Verhältnis in Dimethylformamid, einem organischen polaren Lösungsmittel, gelöst. In dieser Arbeit kommen dabei für Mn, La und Sr Acetylacetonat-Komplexe zum Einsatz, für Ti wird ein TetramethylheptadionatKomplex verwendet. Das Substrat wird mit einer Messing-Klammer auf den Heizer gedrückt und auf die Depositionstemperatur aufgeheizt, die, je nach deponierter Materialkombination, bei $T_{D e p}=600-930^{\circ} \mathrm{C}$ liegt. Bei den in dieser Arbeit hergestellten Filmen war die Kammer der MAD-Anlage immer mit Umgebungsluft gefüllt. Während des gesamten Depositionsprozesses inklusive Aufheiz- und Abkühlvorgang wird durch die Düse Druckluft in Richtung des Substrates geblasen. Nach Erreichen der Depositionstemperatur wird die Präkursorlösung in die pneumatische Düse [131] dosiert, die einen auf das Substrat gerichteten Aerosolkegel erzeugt (siehe Abbildung 4.1). In diesem Aerosol liegt der mittlere Tröpfchendurchmesser bei etwa $20 \mu \mathrm{m}$ 129. In einer Region unmittelbar über dem beheizten Substrat kommt es zur 


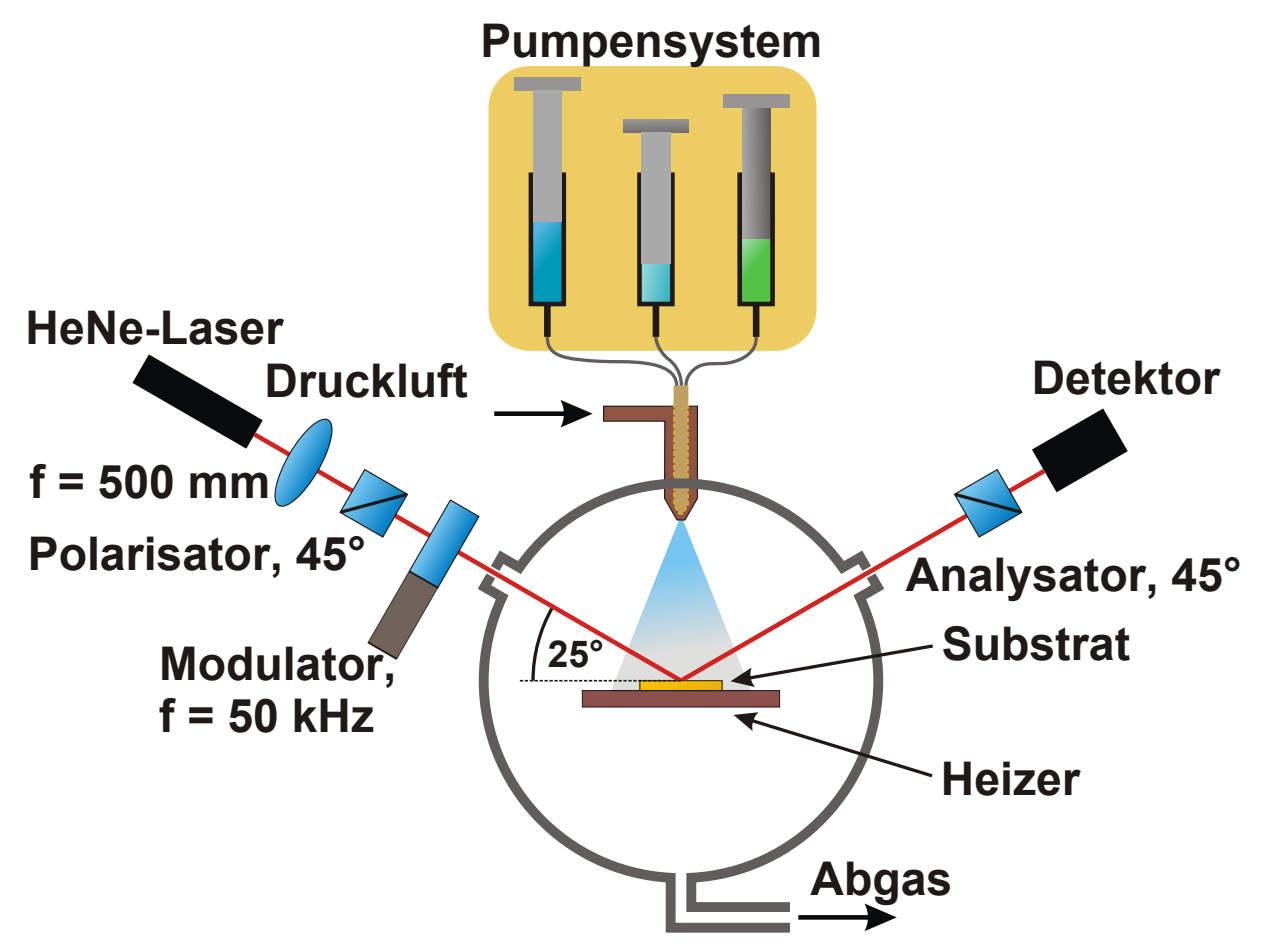

Abbildung 4.1.: Prinzipskizze von neu aufgebauter Anlage zur metallorganischen Aerosol Deposition (MAD) mit in-situ Wachstumskontrolle durch Ellipsometrie (Zeichnung wurde angefertigt von Camillo Ballani).

Verbrennung der organischen Bestandteile der Präkursoren und des Lösungsmittels. So ergibt sich beispielsweise folgende Reaktion für den Mn-Präkursor:

$$
2 \mathrm{Mn}\left(\mathrm{C}_{5} \mathrm{H}_{7} \mathrm{O}_{2}\right)_{3}+36 \mathrm{O}_{2} \rightarrow \mathrm{Mn}_{2} \mathrm{O}_{3}+30 \mathrm{CO}_{2}+21 \mathrm{H}_{2} \mathrm{O}
$$

Die Metalloxide scheiden sich dann auf dem Substrat ab und bilden den dünnen Film.

Da die Zersetzungsreaktionen der verschiedenen Präkursoren unterschiedlich ablaufen, erfolgt der Übertrag der Metall-Kationen vom Aerosol auf die wachsende Schicht nicht im ursprünglichen molaren Verhältnis, das in der Lösung vorliegt. Eine mögliche Erklärung dieser Tatsache ist folgendermaßen: Kurz oberhalb des Substrates kommt es zur Zersetzung der Präkursoren, so dass sich über dem Substrat für jedes Metallion ein spezifischer Dampfdruck einstellt. Dieser Druck und damit der resultierende Fluss von Metallionen auf das Substrat hängt von den thermodynamischen Eigenschaften des jeweiligen Präkursors ab. Damit unterscheidet sich der relative Übertrag von der Präkursorlösung in die gewachsene Schicht für die verschiedenen Präkursoren. Um diese verschiedenen Effizienzen der Präkursoren auszugleichen, werden Korrekturfaktoren eingeführt. Für das Wachstum von Filmen aus dem $\mathrm{La}_{1-\mathrm{x}} \mathrm{Sr}_{\mathrm{x}} \mathrm{MnO}_{3}$-System betrachtet man so zum Beispiel die gewünschten molaren Verhältnisse zwischen $\mathrm{Mn}$ und $\mathrm{Sr}$ bzw. La im deponierten Film. Man berechnet nun zunächst die Mengen der Präkursoren, die man bei stöchiometrischem Übertrag benötigt, und multipliziert die Mengen der Präkursoren von Sr bzw. La dann mit den empirischen Faktoren $C_{S r / M n}$ bzw. $C_{L a / M n}$. Die Faktoren ergeben sich durch Optimierung der Filme hinsichtlich ihrer strukturellen, morphologischen, elektrischen und magnetischen Eigenschaften. Beim $\mathrm{La}_{1-\mathrm{x}} \mathrm{Sr}_{\mathrm{x}} \mathrm{MnO}_{3}$-System werden insbesondere die Materialien an den Enden des Phasendiagramms, $\mathrm{LaMnO}_{3}$ und $\mathrm{SrMnO}_{3}$, betrachtet. Die Korrekturfaktoren sind dann für alle Sr-Dotierungen verwendbar, was sich experimentell durch die gute Qualität der so hergestellten $\mathrm{La}_{1-\mathrm{x}} \mathrm{Sr}_{\mathrm{x}} \mathrm{MnO}_{3}$-Filme zeigt (siehe z.B. Kapitel 
8). Somit ist es sehr einfach möglich die Dotierung zu ändern und verschiedene Materialien aus einem Phasendiagramm zu kombinieren, was z.B. für das $\left(\mathrm{La}_{1-\mathrm{y}} \mathrm{Pr}_{\mathrm{y}}\right)_{0.67} \mathrm{Ca}_{0.33} \mathrm{MnO}_{3^{-}}$ Phasendiagramm gezeigt wurde [132. Die Wachstumsrate lässt sich einfach durch eine Änderung der Dosiergeschwindigkeit und über die Molarität der Präkursorlösung beeinflussen. Man kann die Geschwindigkeit der Deposition so über einen weiten Bereich von $v=1-100 \mathrm{~nm} / \mathrm{min}$ regulieren.

Durch die Funktion bei atmosphärischen Bedingungen und die Verwendung von metallorganischen Präkursoren als Ausgangsmaterialien grenzt sich dieses Verfahren von den üblichen Methoden der Molekularstrahlepitaxie (MBE), der gepulsten Laserdeposition (PLD) und der Sputter-Deposition ab, die alle prinzipbedingt unter Vakuumbedingungen arbeiten. Insbesondere bei dünnen Manganatfilmen ergeben sich bei diesen Methoden Probleme mit Sauerstoffleerstellen, die zu einer Verschlechterung der magnetischen und elektrischen Eigenschaften führen, was bei der MAD aufgrund des hohen Sauerstoffpartialdrucks unproblematisch ist. Bei PLD und Sputter-Deposition ist es außerdem aufwendig die Zusammensetzung anzupassen, da die Filme aus polykristallinen Targets hergestellt werden. Die relativ hohen Substrattemperaturen unterbinden bei der MAD die Kontamination der Oberflächen mit Adsorbaten. Bei den in dieser Arbeit gezeigten Heterostrukturen war die Depositionstemperatur immer oberhalb von $800^{\circ} \mathrm{C}$, wo typische Adsorbat-Moleküle wie $\mathrm{H}_{2} \mathrm{O}$ oder $\mathrm{CO}_{2}$ nicht mehr am $\mathrm{SrTiO}_{3}$-Substrat haften 133,134 .

In den letzten Jahren vorgenommene Weiterentwicklungen der MAD erlauben die Herstellung weiterer Klassen von Metalloxiden durch die Verwendung anderer Umgebungsgase als Luft während der Deposition. So konnten die verschiedenen Phasen des Fe-O-Diagramms, Hematit, Maghemit, Magnetit und Wüstit 135 sowie $\mathrm{V}_{\mathrm{x}} \mathrm{O}_{\mathrm{y}}$ hergestellt werden.

\subsection{Motivation zur in-situ Ellipsometrie}

Bei der Erforschung von Effekten an Grenzflächen zwischen komplexen Oxiden werden an die Depositionsmethode höchste Anforderungen gestellt. Man muss in der Lage sein, Heterostrukturen mit einer Präzision im Bereich einer einzelnen Atomlage und einer hohen Reproduzierbarkeit herstellen zu können. Um diese Anforderungen zu sichern, ist eine in-situ Überwachung des Wachstums von großer Bedeutung. Die zumeist verwendeten Techniken zur Herstellung dünner Filme aus komplexen Oxiden, gepulste Laser Deposition (PLD) und Molekularstrahl Epitaxie (MBE) arbeiten im Vakuum. Damit wird typischerweise die Beugung hochenergetischer Elektronen bei Reflexion (RHEED) zur Wachstumskontrolle herangezogen. Durch den kleinen Einfallswinkel $\theta<5^{\circ}$ werden die Elektronen nur von der Oberfläche gestreut. Das entstehende Beugungsbild liefert dann Informationen über die Struktur der ersten Lage. So können Rekonstruktionen der Oberfläche und Änderungen der Wachstumsmode beobachtet werden. Wenn der dünne Film in zweidimensionalen Inseln aufwächst, ergeben sich aufgrund der diffusen Streuung am unvollständig geschlossenen Film Oszillationen der Intensität im Beugungsmuster, deren Periode einer Monolage entspricht 136. Diese Oszillationen treten bei der step-flow-Wachstumsmode nicht auf, da die diffuse Streuung dann beim fortschreitenden Wachstum der Schicht unverändert bleibt. Durch eine differentiell abgepumpte Elektronenkanone lässt sich der maximale Druck über dem Substrat, bei dem noch ein Beugungsbild mittels RHEED aufgenommen werden kann, auf etwa 1 mbar steigern [137. Somit ist diese Technik nicht geeignet für die atmosphärischen Bedingungen bei der MAD. 
Die bekannten ex-situ Methoden, um dünne Filme mittels Röntgen-Reflektometrie zu charakterisieren, lassen sich theoretisch auch in-situ nutzen. Bei Verwendung von harter Röntgenstrahlung sind auch die atmosphärischen Bedingungen der MAD kein Hindernis für diese Technik. Da die zeitliche Auflösung bei der Beobachtung der Wachstumsprozesse im Bereich von $0.1 \mathrm{~s}$ liegen soll, ist es aufgrund der Zählstatistik mit Labor-Röntgenquellen nicht möglich zu jedem Zeitpunkt der Deposition ein winkelabhängiges Spektrum der Reflektivität aufzunehmen. Bei Messung unter fester Geometrie erwartet man dann bei einem 2D-Inselwachstum Oszillationen der Intensität durch die periodischen Änderungen der Oberflächenrauigkeit, bei einer step-flow-Wachstumsmode sind die Änderungen der Intensität deutlich kleiner und ergeben sich rein durch die Änderung der durchschnittlichen Dicke [138]. Die Verwendung von brillanterer Synchrotronstrahlung ermöglicht die rapide Aufnahme ganzer Spektren, womit z.B. unter Anwendung von nicht-spekularer Röntgen-Reflektometrie Informationen über die zeitliche Entwicklung der Oberflächenmorphologie extrahiert werden können [139].

Eine Wachstumsüberwachung mittels optischer Techniken wie Ellipsometrie und Reflectance Difference Spectroscopy (RDS) findet in der Halbleitertechnologie aufgrund seiner einfachen und flexiblen Instrumentierung vielfache Anwendungen 140 143. Die RDS bezeichnet die Messung des Unterschiedes der Reflektivität für zwei zueinander senkrecht ausgerichtete Polarisationen bei senkrechtem Einfall [144]. Da bei dieser Geometrie kein Unterschied der Reflektivitäten aufgrund der Fresnel-Gleichungen induziert wird, ist man sensitiv auf die anisotropen dielektrischen Eigenschaften der Probe. Insbesondere für die Epitaxie von III-VSystemen wie GaAs wird RDS häufig für in-situ Beobachtungen des Wachstums eingesetzt, da es je nach Verhältnis zwischen Ga und As an der Oberfläche zu verschiedenen anisotropen Rekonstruktionen der Oberfläche kommt 144]. So werden Änderungen der Ga/As Stöchiometrie an der Oberfläche 144 und Monolagenoszillationen 143 beobachtet. Für das hier hauptsächlich untersuchte Wachstum auf kubischen $\mathrm{SrTiO}_{3}$ (001) Substraten erwartet man zunächst keine Anisotropie in der (001)-Ebene. Allenfalls kann die aufgrund des Fehlschnitts der Substrate verursachte Terrassenstruktur eine Anisotropie hervorrufen. Zudem wäre die Instrumentierung bei der MAD schwierig, da das Lichtbündel komplett innerhalb des Aerosolkegels verlaufen müsste, um einen senkrechten Einfall sicherzustellen (siehe Abbildung 4.1.

Bei der Ellipsometrie wird die Änderung des Polarisationszustandes bei Reflexion betrachtet. Wie später in den theoretischen Betrachtungen zur Ellipsometrie erläutert wird, ist die Sensitivität dieser Technik am größten, wenn das Licht unter dem Brewster-Winkel auf das Substrat trifft. Damit ist der Einfallswinkel bei dieser Technik typischerweise im Bereich $\theta=60^{\circ}-70^{\circ}$, wodurch der Lichtstrahl den Aerosolkegel nur in einem kleinen Bereich streift (siehe Bild 4.1). Mit dieser Technik ist es neben der Ermittlung der durchschnittlichen Schichtdicke möglich morphologische Veränderungen (Inselwachstum an der Oberfläche [141]) oder chemische Modifikationen (Durchmischung [142], Oxidationsprozesse, Veränderungen der Stöchiometrie) zu beobachten [140, 145]. Diese Informationen sind in den meisten Fällen aber nur indirekt durch Anpassen von Modellen an die gemessenen Daten zu gewinnen 140 , 145. Bei der Homo- und Heteroepitaxie von III-V-Halbleitern wie GaAs mittels metallorganischer Gasphasenepitaxie (MOVPE) konnten Monolagenoszillationen bei 2D-Inselwachstum beobachtet werden [143, 146]. Das Auftreten dieser wird mit dem Vorhandensein von Ga-CBindungen, welche vom metallorganischen Präkursor stammen, an den Kanten der zweidimensionalen Inseln begründet [146]. Bei der Epitaxie komplexer Oxide gibt es Beispiele für die beiden hier diskutierten optischen Methoden, jedoch waren die bisherigen Experimente auf Homoepitaxie von $\mathrm{SrTiO}_{3}\left[147-149\right.$ oder einfache Heterostrukturen von $\mathrm{La}_{0.67} \mathrm{Ba}_{0.33} \mathrm{MnO}_{3-\delta}$ [150, 151] bzw. $\mathrm{La}_{0.67} \mathrm{Sr}_{0.33} \mathrm{MnO}_{3}$ [152] auf $\mathrm{SrTiO}_{3}$, beschränkt, wobei die Deposition in al- 


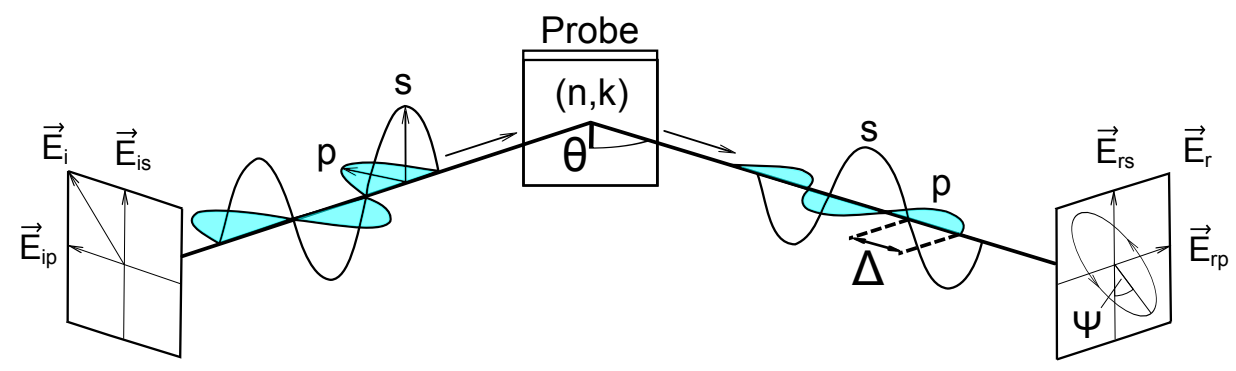

\begin{abstract}
Abbildung 4.2.: Veranschaulichung des Messprinzips der Ellipsometrie: Ein eingehender Strahl, dessen lineare Polarisation einen Winkel von $45^{\circ}$ mit der Einfallsebene, die aus dem Normalenvektor auf die Probenoberfläche und der Propagationsrichtung des Lichtstrahls aufgespannt wird, einschließt, wird an der zu untersuchenden Probe reflektiert. Für den reflektierten Strahl schließt die Polarisation einen Winkel von $\Psi$ mit der Einfallsebene ein und es gibt eine Phasenverschiebung $\Delta$ zwischen p- und s- Komponente (nach [140]).
\end{abstract}

len genannten Beispielen mit PLD ausgeführt wurde. Zhu et al. untersuchten die Kinetik der Oxidation von dünnen Filmen von $\mathrm{SrTiO}_{3}$ und $\mathrm{La}_{0.67} \mathrm{Ba}_{0.33} \mathrm{MnO}_{3-\delta}$ auf $\mathrm{SrTiO}_{3}$, die bei den Bedingungen ihrer Experimente nach der Deposition noch Sauerstoffleerstellen aufweisen [147, 149, 151. Zudem werden bei der Homoepitaxie von $\mathrm{SrTiO}_{3}$ die Wachstumsmoden klassifiziert und die Zeitskalen von Diffusionsprozessen der Atome auf der Oberfläche ermittelt [147, 150]. Beim Vorliegen von zweidimensionalem Inselwachstum werden Oszillationen mit der Periode einer Perowskit-Monolage für das Wachstum von Nb-dotierten $\mathrm{SrTiO}_{3}$ Filmen auf $\mathrm{SrTiO}_{3}$ (001) beobachtet. Bei den reduzierenden Bedingungen von PLD hängt die Rate, mit der die Sauerstoffleerstellen im wachsenden Film durch Diffusion aus dem Substrat aufgefüllt werden, von der Dichte der Kanten der Inseln ab. Da die Depositionsrate zu schnell ist für eine vollständige Relaxation der Sauerstoffkonzentration im Film, hängt dessen dielektrische Funktion von der Dichte der Kanten an den Inseln ab. Die Zahl der Kanten oszilliert mit der relativen Bedeckung der Monolage, wodurch sich die periodischen Schwankungen der Messgrößen ergeben [148].

\title{
4.3. Theorie Ellipsometrie
}

Bei der Ellipsometrie beobachtet man die Änderungen der Polarisation von Licht nach der Reflexion an der zu untersuchenden Probe. Wie es in Abbildung 4.2 illustriert ist, zerlegt man den elektrischen Feldvektor der Polarisation in eine Komponente parallel zur Einfallsebene (p) und senkrecht dazu (s). Beim Einstrahlen von Licht, dessen Polarisationsvektor einen Winkel von $45^{\circ}$ mit der Einfallsebene einschließt, ergibt sich aufgrund der unterschiedlichen komplexen Reflexionskoeffizienten $r_{p / s}$ für den parallel/senkrecht zur Einfallsebene polarisierten Anteil der elektromagnetischen Welle eine elliptische Polarisation mit einer gegenüber dem ursprünglichen Winkel verdrehten Hauptachse. Die hier und in der Literatur bei der Beschreibung von Ellipsometrie-Messungen verwendeten Größen $(\Delta, \Psi)$ werden über die Reflexionskoeffizienten für p- und s-polarisierte Strahlung (siehe Abbildung 4.2)

$$
r_{p}=\frac{E_{r p}}{E_{i p}}, r_{s}=\frac{E_{r s}}{E_{i s}}
$$

definiert [140]:

$$
\rho=\tan (\Psi) \exp (i \Delta)=\frac{r_{p}}{r_{s}}
$$


Nach dieser Definition bezeichnet $\Psi$ den Winkel zwischen der Einfallsebene und der Hauptachse des Polarisationsvektors und $\Delta$ die Phasenverschiebung zwischen der p- und der sKomponente nach der Reflexion von Licht, das vor der Reflexion mit $45^{\circ}$ linear polarisiert war.

\subsubsection{Fresnel-Gleichungen}

Um die Reflexionskoeffizienten $r_{p / s}$ zu erhalten, betrachtet man zunächst die Ausbreitung elektromagnetischer Wellen in Materie: Im Allgemeinen sind in Materie die dielektrische Verschiebungsdichte $\vec{D}$ und das elektrische Feld über den frequenzabhängigen Dielektrizitätstensor $\varepsilon_{P}(\omega)$ sowie die magnetische Kraftflussdichte $\vec{B}$ mit dem Magnetfeld $\vec{H}$ über den Permeabilitätstensor verknüpft $\mu(\omega)$ :

$$
\vec{D}=\varepsilon_{P} \vec{E}, \vec{B}=\mu \vec{H}
$$

In ferromagnetischen Materialien kann $\mu$ bei kleinen Frequenzen sehr große Werte annehmen $\left(\mu \propto 10^{5}\right)$, bei den hohen Frequenzen für sichtbares Licht ist $\mu$ jedoch mit der Permeabilität des Vakuums gleichzusetzen: $\mu=\mu_{0}$ [153]. Die hier betrachteten Materialien weisen nur kleine optische Anisotropien auf, da ihre Gitterstrukturen fast kubisch sind (z.B. kubisches $\mathrm{SrTiO}_{3}$ oder $\mathrm{SrMnO}_{3}$ ) und die durch epitaktische Verspannungen erzeugten Anisotropien im Bereich weniger Prozent liegen. Damit besitzt der $\varepsilon$-Tensor näherungsweise nur Diagonaleinträge, die alle identisch sind, und geht somit als Skalar in die Gleichungen ein. Man schreibt dann für die Beziehung zwischen elektrischem Feld und Verschiebungsdichte $\vec{D}=\varepsilon_{0} \varepsilon \vec{E}$ mit der Permittivität des Vakuums $\varepsilon_{0}$ und der dielektrischen Konstanten $\varepsilon$.

Damit erhält man für die Maxwellgleichungen in quellen- und senkenfreien Medien [59]:

$$
\begin{aligned}
\vec{\nabla} \cdot \vec{B} & =0 \\
\vec{\nabla} \cdot \vec{D} & =0 \\
\vec{\nabla} \times \vec{E}+\frac{\partial \vec{B}}{\partial t} & =0 \\
\vec{\nabla} \times \vec{H}-\frac{\partial \vec{D}}{\partial t} & =0
\end{aligned}
$$

Diese lassen sich durch ebene Transversalwellen lösen:

$$
\vec{E}(\vec{r}, t)=\vec{E}_{0} e^{i(\omega t-\vec{r} \cdot \vec{k})}, \vec{H}(\vec{r}, t)=\vec{H}_{0} e^{i(\omega t-\vec{r} \cdot \vec{k})}
$$

mit der Phasengeschwindigkeit

$$
c=\frac{1}{\sqrt{\mu_{0} \varepsilon \varepsilon_{0}}}
$$

und dem Wellenzahlvektor

$$
\vec{k}=\frac{2 \pi N}{\lambda_{0}} \overrightarrow{e_{k}}
$$

der die Ausbreitungsrichtung beschreibt. Dabei ist $\lambda_{0}$ die Wellenlänge im Vakuum und $N=$ $n-i k$ der komplexe Brechungsindex, der über folgende Relationen mit $\varepsilon$ und der optischen Leitfähigkeit $\sigma$ verknüpft ist [48]:

$$
N=\sqrt{\varepsilon}=\sqrt{1+\frac{4 \pi i \sigma}{\omega}}
$$




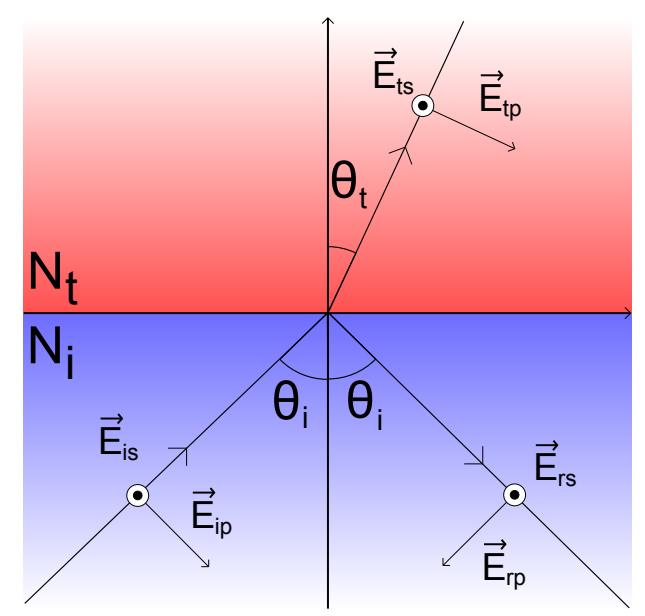

Abbildung 4.3.: Illustration der Geometrie und relevanten Komponenten des elektrischen Feldes für die Fresnel-Koeffizienten.

Die mit einem Photodetektor messbare Intensität erhält man dann durch Bildung des komplexen Betragsquadrates $I \propto|E|^{2}$.

Wenn der Lichtstrahl von einem Medium mit Brechungsindex $N_{i}$ in ein anderes Material mit Brechungsindex $N_{t}$ übertritt, kommt es zu einer Aufspaltung in einen reflektierten und transmittierten Strahl (siehe Abbildung 4.3). An der Grenzfläche sind die Komponenten von $\vec{E}$ und $\vec{B}$ parallel zur Grenzfläche stetig $[\overline{140}$. Diese Bedingungen legen dann die Koeffizienten fest, die die Feldamplituden von transmittierter und reflektierter Welle mit der Amplitude der eingehenden Welle in Beziehung setzen. Aufgeteilt in Koeffizienten für die Feldkomponenten parallel (p) und senkrecht (s) zur Einfallsebene erhält man so [140:

$$
r_{p}=\frac{E_{r p}}{E_{i p}}=\frac{N_{t} \cos \theta_{i}-N_{i} \cos \theta_{t}}{N_{t} \cos \theta_{i}+N_{i} \cos \theta_{t}}, r_{s}=\frac{E_{r s}}{E_{i s}}=\frac{N_{i} \cos \theta_{i}-N_{t} \cos \theta_{t}}{N_{i} \cos \theta_{i}+N_{t} \cos \theta_{t}}
$$

für den reflektierten Strahl und

$$
t_{p}=\frac{E_{t p}}{E_{i p}}=\frac{2 N_{i} \cos \theta_{i}}{N_{t} \cos \theta_{i}+N_{i} \cos \theta_{t}}, t_{s}=\frac{E_{t s}}{E_{i s}}=\frac{2 N_{i} \cos \theta_{i}}{N_{i} \cos \theta_{i}+N_{t} \cos \theta_{t}}
$$

für den transmittierten Anteil. Der Winkel $\theta_{t}$ wird durch das Snelliussche Brechungsgesetz bestimmt:

$$
N_{i} \sin \theta_{i}=N_{t} \sin \theta_{t}
$$

Die Reflexionskoeffizienten für die Grenzfläche zwischen Luft $N=1.0$ und $\mathrm{SrTiO}_{3}$ mit $N=$ $2.388(\lambda=632.8 \mathrm{~nm}) 154$ sind in Abbildung 4.4 dargestellt. Beim Brewster-Winkel $\theta_{B}$, der für Medien mit verschwindendem Imaginärteil von $N$ durch $\tan \theta_{B}=N_{t} / N_{i}$ gegeben ist, verschwindet die Reflektivität für die p-Komponente $\left(r_{p}=0\right)$. Im Beispiel liegt dieser bei $\theta_{B} \approx 67.3^{\circ}$. Für praktische Messungen wählt man einen Einfallswinkel in der Nähe von $\theta_{B}$. Bei diesem Einfallswinkel kommt es beim Aufbringen weiterer Schichten auf das Substrat zu rapiden Änderungen der Phase $\Delta$, was durch den Phasensprung um $180^{\circ}$ beim Überschreiten von $\theta_{B}$ illustriert wird. Außerdem ist $\Psi=0^{\circ}$, womit bei dem in dieser Arbeit verwendeten Modulationsaufbau die Sensitivität maximiert wird (siehe Abschnitt 4.4.1). 


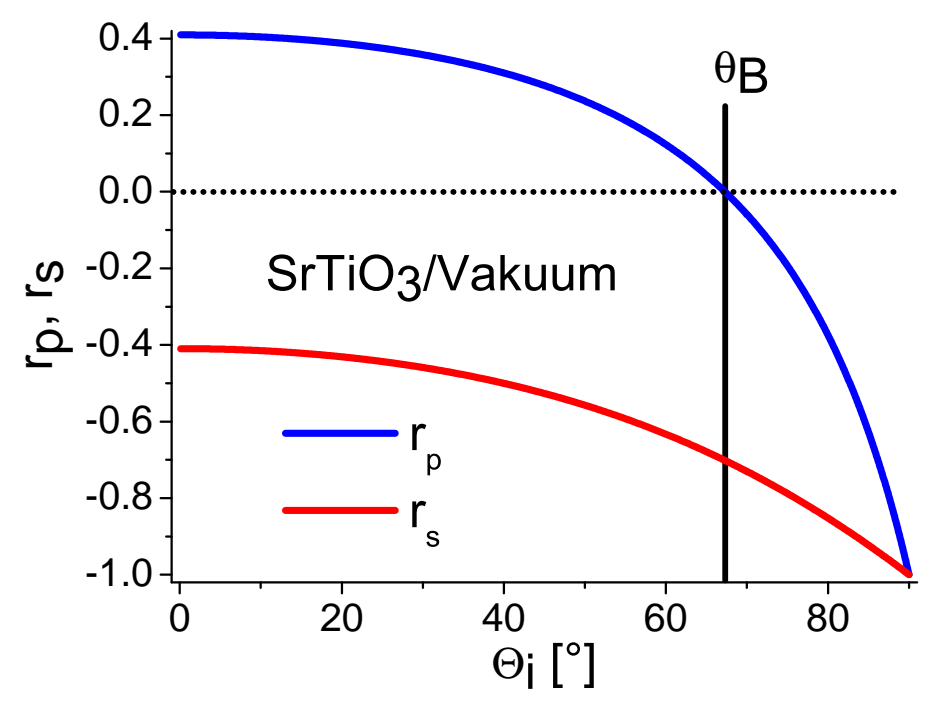

Abbildung 4.4.: Reflexionsamplituden für die Komponenten des elektrischen Feldes an der Grenzfläche zwischen Luft $(N=1.0)$ und $\mathrm{SrTiO}_{3}(N=2.388$ für $\lambda=632.8 \mathrm{~nm})$, eingezeichnet ist der Brewster-Winkel $\theta_{B}$ als Nullstelle von $r_{p}$.

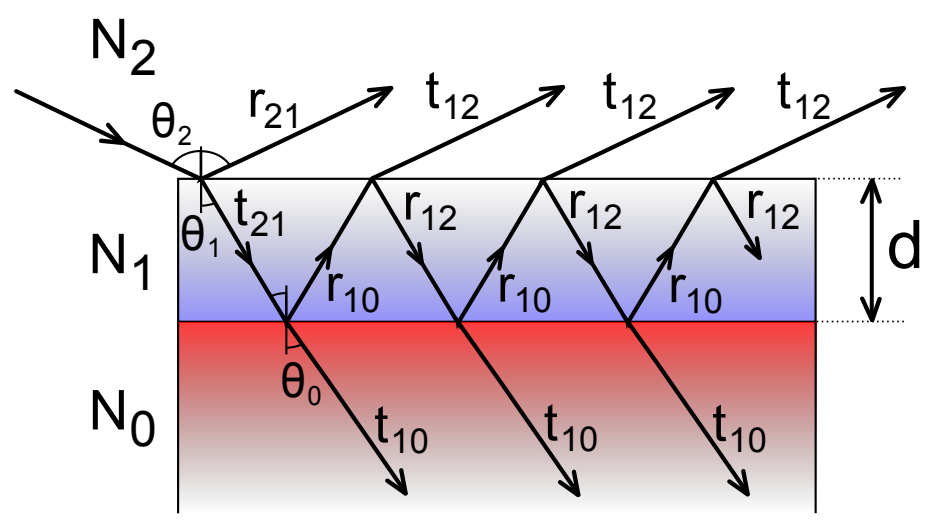

Abbildung 4.5.: Herleitung der totalen Reflexions- und Transmissionskoeffizienten für eine Schicht der Dicke $d$ auf einem Substrat. Den Pfeilen folgend ergeben sich die verschiedenen Pfade, die das Licht bis zur endgültigen Reflexion bzw. Transmission zurücklegt. Die Amplituden-Koeffizienten an den Pfeilen ergeben sich aus den Fresnel-Gleichungen.

\subsubsection{Reflexion an Multilagen}

Wenn man die Reflexion an einer dünnen Schicht auf einem Substrat untersucht, muss man die Überlagerung der vielfachen Reflexionen an den beiden vorhandenen Grenzflächen berücksichtigen. In Abbildung 4.5 sieht man einen Film der Dicke $d$ und dem Brechungsindex $N_{1}$ auf einem Substrat mit dem Brechungsindex $N_{0}$. An der Oberseite grenzt der Film an ein Medium mit dem Brechungsindex $N_{2}$, für das wie beim Substrat eine unendliche Ausdehnung in eine Richtung angenommen wird. Die Reflexions- und Transmissionskoeffizienten $r_{j k, p / s}$, $t_{j k, p / s}$ beim Auftreffen eines Strahls aus Medium j auf die Grenzfläche zum Medium k erhält man aus den Fresnelkoeffizienten oben. Die Einfallswinkel für die verschiedenen Medien ergeben sich wieder über das Snelliussche Brechungsgesetz:

$$
N_{0} \sin \theta_{0}=N_{1} \sin \theta_{1}=N_{2} \sin \theta_{2}
$$




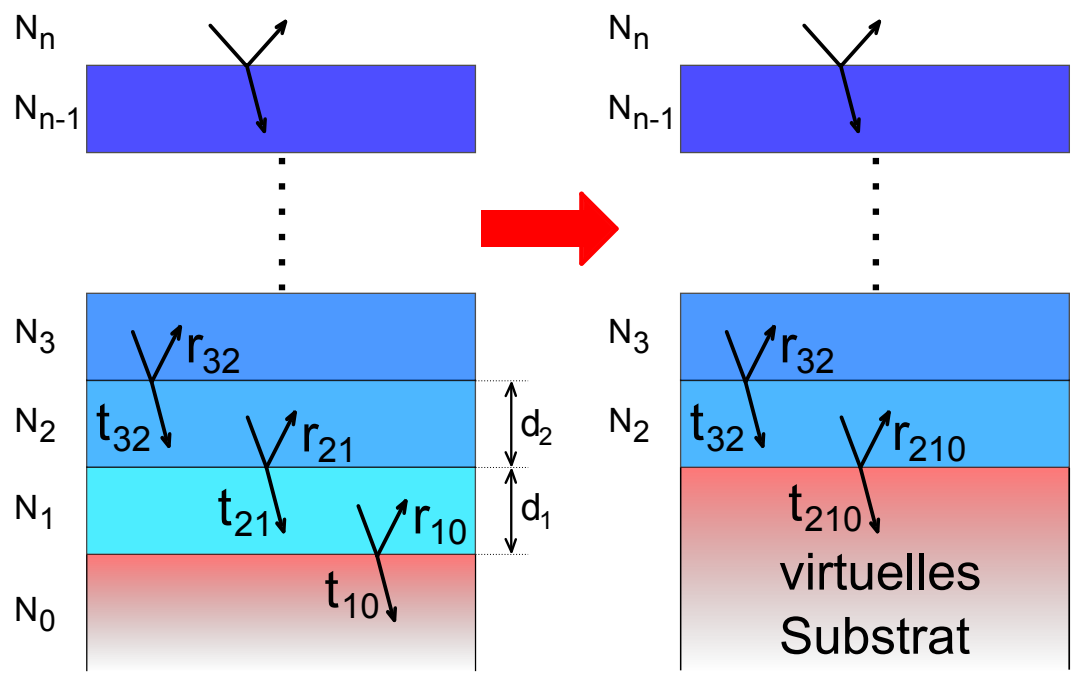

\begin{abstract}
Abbildung 4.6.: Zur Berechnung der Reflexionskoeffizienten komplizierter Heterostrukturen mit $n-1$ Lagen auf einem Substrat: Von der ersten Lage auf dem Substrat ausgehend, werden die totalen Reflexions- und Transmissionskoeffizienten $r_{210}$ und $t_{210}$ berechnet. Lage 1 wird dann zusammen mit dem Substrat als virtuelles Substrat aufgefasst und die Koeffizienten $r_{210}$ bzw. $t_{210}$ für Reflexion bzw. Transmission an der Grenzfläche zwischen Lage 2 und Lage 1 eingesetzt.
\end{abstract}

Bei der einfachen Propagation durch den Film ändert sich die Phase der Welle um exp $(-i \beta)$ mit 140

$$
\beta=\frac{2 \pi d}{\lambda} N_{1} \cos \theta_{1}
$$

Zur Berechnung der totalen Reflexions- und Transmissionskoeffizienten an der Grenzfläche zwischen dem Medium mit Brechungsindex $N_{2}$ und dem Film muss man die Beiträge aller mehrfach reflektierten Strahlen phasenrichtig aufaddieren (siehe Bild 4.5). Für die insgesamt von der Heterostruktur reflektierten Strahlen ergibt sich so:

$$
r_{210}=r_{21}+t_{21} t_{12} r_{10} \exp (-i 2 \beta)+\ldots+t_{21} t_{12} r_{10}^{n} r_{12}^{n-1} \exp (-i 2 n \beta)+\ldots
$$

und für die transmittierten Strahlen:

$$
t_{210}=t_{21} t_{10} \exp (-i \beta)+t_{21} t_{10} r_{10} r_{12} \exp (-i 3 \beta)+\ldots+t_{21} t_{10} r_{10}^{n} r_{12}^{n} \exp (-i(2 n+1) \beta)+\ldots
$$

Die dabei entstehende geometrische Reihe lässt sich dann zu folgenden totalen Koeffizienten zusammenfassen [140]:

$$
r_{210, p / s}=\frac{r_{21, p / s}+r_{10, p / s} \exp (-i 2 \beta)}{1+r_{21, p / s} r_{10, p / s} \exp (-i 2 \beta)}
$$

für die reflektierten Strahlen und

$$
t_{210, p / s}=\frac{t_{21, p / s} t_{10, p / s} \exp (-i \beta)}{1+r_{21, p / s} r_{10, p / s} \exp (-i 2 \beta)}
$$

für die transmittierten.

Wenn man kompliziertere Heterostrukturen untersucht, die eine Vielzahl von Lagen enthalten, kann man die Reflexionskoeffizienten iterativ bestimmen: Bei einem Schichtstapel mit $n-1$ Lagen auf einem Substrat geht man von Lage 1 auf dem Substrat aus und bestimmt wie oben die Koeffizienten $r_{210}$ und $t_{210}$ für die Heterostruktur aus Substrat und Lage 1, die in Kontakt 
zu einem äußeren Medium mit Brechungsindex $N_{2}$ steht (siehe Abbildung 4.6). Damit kann man dann Lage 1 zusammen mit dem Substrat als virtuelles Substrat auffassen. Bei der Berechnung der totalen Koeffizienten für die Grenzfläche zwischen Lage 3 und 2 setzt man dann $r_{210}$ und $t_{210}$ ein, um die Reflexion und Transmission an der Grenzfläche zwischen Lage 2 und dem virtuellen Substrat zu beschreiben. Der Winkel $\theta_{i}$, den die Ausbreitungsrichtung der Strahlen in der Schicht $i$ mit der Normalen auf die Grenzfläche einschließt, lässt sich aufgrund des Snelliusschen Brechungsgesetzes direkt aus dem Winkel $\theta_{n}$ für die Ausbreitung im Vakuum ableiten:

$$
N_{0} \sin \theta_{0}=N_{1} \sin \theta_{1}=\ldots=N_{n} \sin \theta_{n}
$$

Nach diesem Prinzip kann man nach und nach die totalen Koeffizienten an der Grenzfläche zwischen der i-ten und (i+1)-ten Lage berechnen und die resultierenden Reflexions- und Transmissionskoeffizienten verwenden, um den Stapel vom Substrat bis einschließlich zur i-ten Lage als virtuelles Substrat aufzufassen. Damit kann man wiederum die totalen Koeffizienten für die Grenzfläche zwischen der (i+1)-ten und (i+2)-ten Lage ermitteln. Wie beim Algorithmus von Parratt zur Berechnung von Röntgenreflektometriespektren [155] führt man dies dann für $i=1 \ldots n-1$ durch und erhält so die totalen Koeffizienten für die ganze Struktur.

\subsubsection{Rauigkeiten: Effektiv-Medium-Theorie}

Wie oben gezeigt, lässt sich die lagenweise Kombination von verschiedenen Materialien in Heterostrukturen mathematisch exakt behandeln. Reale Übergitter weisen jedoch keine perfekt scharfen Grenzflächen auf, sondern besitzen immer einen gewissen Grad von Grenz- bzw. Oberflächenrauigkeit. Für die in-situ Beobachtung von Wachstumsprozessen ist dabei insbesondere die Oberflächenrauigkeit interessant, die bei zweidimensionalem bzw. dreidimensionalem Inselwachstum auftritt. Es ist also insbesondere die Frage zu klären, wie sich die optischen Eigenschaften einer Mischung von dem Material, aus dem der Film aufgebaut ist, und Vakuum darstellen (siehe Abbildung 4.7). Die hier in Frage kommenden Rauigkeiten sind mikroskopisch, das heißt sowohl die lateralen als auch die horizontalen Größenskalen des Höhenprofils sind deutlich kleiner als die zur Untersuchung gebrauchte Wellenlänge $\lambda$. Damit tritt keine makroskopische Streuung des Lichts auf und es kommt auch nicht zu Depolarisierungseffekten aufgrund von vielfachen Ablenkungen der Strahlen [156, 157]. Numerisch lässt sich für mikroskopische Mischungen verschiedener Materialien mittels Wellenoptik die Verteilung der Feldamplituden im Fernfeld berechnen. Diese Lösung liefert allerdings zumeist zu detaillierte Informationen, die bei einer realen Messung aufgrund der durch endliche Strahlquerschnitte erfolgenden Mittelung nicht erfasst werden können [145]. Deshalb geht man dazu über einen Film mit mikroskopischer Rauigkeit durch zwei Schichten zu nähern (siehe Abbildung 4.7): Die erste Schicht der Dicke $d_{B}$ besitzt die optischen Eigenschaften des Ausgangsmaterials $N_{1}$ und die zweite Schicht, die zur Beschreibung der Rauigkeiten dient, die Dicke $d_{S}$ und den Brechungsindex $N_{E M A}$ 140, 145. Dabei ist der Brechungsindex der Mischlage $N_{E M A}$ eine Funktion der Brechungsindizes der beiden beteiligten Phasen (in Abbildung 4.7 also $N_{1}$ für das Material des Films und $N_{2}$ für das Vakuum) und deren Mischungsverhältnis $f$. Im Folgenden wird die Berechnung von $N_{E M A}$ motiviert und die Grenzen für die Anwendbarkeit der Näherung durch ein effektives Medium diskutiert. 
a)

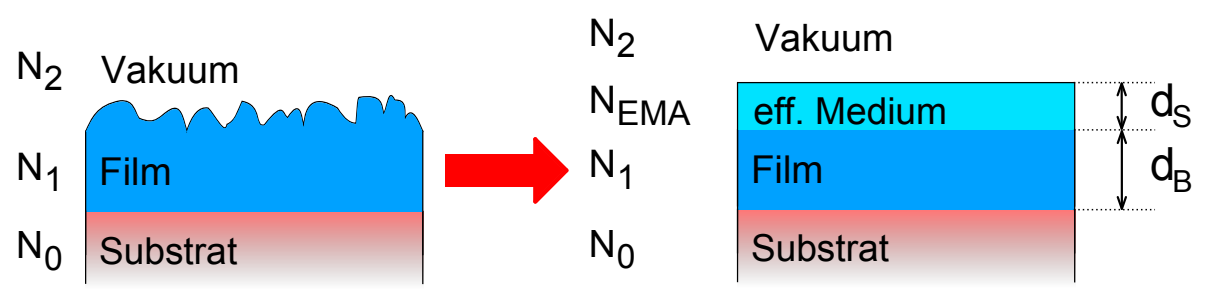

b)

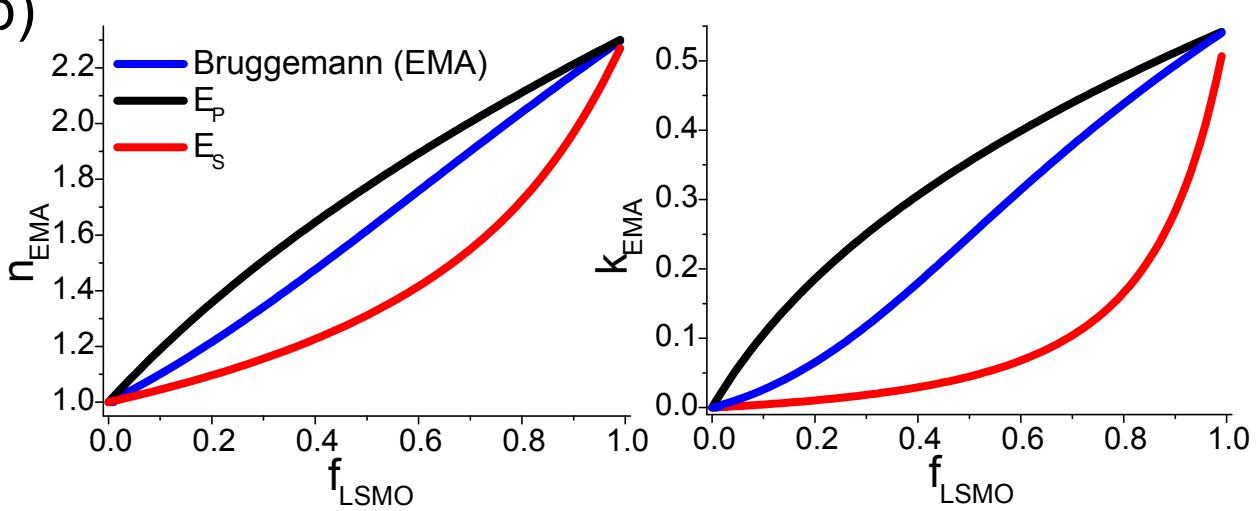

\begin{abstract}
Abbildung 4.7.: a) Näherung der Rauigkeit eines dünnen Filmes durch eine zusätzliche Lage der Dicke $d_{S}$ mit dem effektiven Brechungsindex $N_{E M A}$, der sich aus den Brechungsindizes des Filmmaterials, $N_{1}$, und des Vakuums, $N_{2}$, ergibt. b) effektive Brechungsindizes $N_{E M A}=n_{E M A}-i k_{E M A}$ für eine Mischung aus $\mathrm{La}_{0.7} \mathrm{Sr}_{0.3} \mathrm{MnO}_{3}$ (LSMO) $\left(N_{L S M O}=2.3-i \cdot 0.55\right)$ und Vakuum $\left(N_{V a k}=1.0\right)$, die sich durch das Modell von Bruggeman (Gleichung 4.31) sowie die Grenzfälle für eine Lagenstruktur, bei der das elektrische Feld parallel ( $E_{p}$, Gleichung 4.32) oder senkrecht $\left(E_{s}\right.$, Gleichung 4.3.3 auf den Lagen steht, ergeben. $f_{L S M O}$ bezeichnet dabei den relativen Volumenanteil von LSMO.
\end{abstract}

Für jedes Atom in einem dielektrischen Festkörper wird durch ein äußeres Feld $\vec{E}_{l o k}$ ein über die Polarisierbarkeit $\alpha(\omega)$ verknüpftes atomares elektrisches Dipolmoment

$$
\vec{p}_{e l}=\varepsilon_{0} \alpha \vec{E}_{l o k}
$$

induziert. $\vec{E}_{l o k}$ ist das Feld an der Position des Atoms und entspricht im Allgemeinen nicht dem von außen angelegten Feld $\vec{E}_{\text {ext }}$. Daraus ergibt sich bei einer atomaren Dichte von $n_{V}=$ $N / V$ die makroskopische elektrische Polarisation

$$
\vec{P}=\varepsilon_{0} n_{V} \alpha \vec{E}_{l o k}
$$

Diese ist durch

$$
\vec{D}=\varepsilon_{0} \varepsilon \vec{E}=\varepsilon_{0} \vec{E}+\vec{P}
$$

mit der dielektrischen Verschiebungsdichte verknüpft [48, 140, 158]. Um die makroskopischen Felder, für die die Variationen auf atomarer Skala herausgemittelt werden, mit der mikroskopischen Polarisierbarkeit zu verknüpfen, muss man das lokale Feld $\vec{E}_{l o k}$ an einem Gitterplatz ermitteln. Dieses wird neben dem äußeren Feld $\vec{E}_{\text {ext }}$ auch durch das von der Polarisation induzierte Feld $\vec{E}_{\text {ind }}$ verursacht. Dazu betrachtet man einen kugelförmigen Bereich innerhalb eines dielektrischen Materials. Der Durchmesser der Kugel ist so gewählt, dass er viele Einheitszellen des Festkörpers enthält und die Polarisation homogen ist. Für sichtbares Licht lässt sich diese Anforderung erfüllen, da die Wellenlänge sehr viel größer ist als der Gitterparameter. Die Summation der elektrischen Felder aller atomaren Dipole innerhalb dieser Kugel führt zu einem verschwindenden Feld 158. Das die Kugel umgebende Material, das 
die makroskopische Polarisation $\vec{P}$ aufweist, induziert ein Feld innerhalb der Kugel. Dieses ergibt sich, wenn man sich vorstellt, dass sich im Material ein kugelförmiger Hohlraum befindet. Das Feld in diesem Hohlraum kann man durch induzierte Ladungen auf der Oberfläche beschreiben. Wegen

$$
\vec{\nabla} \cdot \vec{D}=0=\vec{\nabla} \cdot\left(\varepsilon_{0} \vec{E}+\vec{P}\right) \Leftrightarrow \vec{\nabla} \cdot \vec{E}=-\frac{1}{\varepsilon_{0}} \vec{\nabla} \cdot \vec{P}
$$

entspricht eine lokal nichtverschwindende Divergenz von $\vec{P}$ analog zur elektrischen Ladungsdichte $\rho$ der Quelle eines elektrischen Feldes. Mit dem Gaußschen Satz ergibt sich insbesondere an Grenzflächen zwischen Materialien verschiedener Polarisation eine nichtverschwindende Divergenz und damit eine Oberflächenladungsdichte, die sich durch Projektion der Polarisationsdifferenz $\Delta \vec{P}$ auf die Oberflächennormale $\vec{n}$ zu $\sigma_{P}=\vec{n} \cdot \Delta \vec{P}$ ergibt. Wenn man das durch diese Ladungen verursachte Feld aufintegriert, erhält man das Lorentz-Feld $\vec{E}_{\text {ind }}=\frac{1}{3 \varepsilon_{0}} \vec{P}$ und damit für das lokale Feld im Inneren dieses Hohlraumes [158:

$$
\vec{E}_{l o k}=\vec{E}_{e x t}+\frac{1}{3 \varepsilon_{0}} \vec{P}
$$

Damit lässt sich ein Zusammenhang zur mikroskopischen Definition der Polarisation herstellen:

$$
\vec{P}=\varepsilon_{0} n_{V} \alpha \vec{E}_{l o k}=\varepsilon_{0} n_{V} \alpha\left(\vec{E}_{e x t}+\frac{1}{3 \varepsilon_{0}} \vec{P}\right)
$$

Wenn man diese Gleichung nach $\vec{P}$ auföst und die makroskopische Definition für die Dielektrizitätskonstante $\vec{P}=\varepsilon_{0}(\varepsilon-1) \vec{E}_{\text {ext }}$ einsetzt erhält man die Clausius-Mossotti Relation 158:

$$
\frac{\varepsilon-1}{\varepsilon+2}=\frac{1}{3} n_{V} \alpha
$$

Besteht das Material aus verschiedenen Atomsorten mit Polarisierbarkeiten $\alpha_{a, b}$ und Dichten $n_{V}^{a, b}$, ergibt sich 159

$$
\frac{\varepsilon-1}{\varepsilon+2}=\frac{1}{3}\left(n_{V}^{a} \alpha_{a}+n_{V}^{b} \alpha_{b}\right)
$$

Dies kann man mit den Dielektrizitätskonstanten $\varepsilon_{a, b}$ für Materialien mit Polarisierbarkeiten $\alpha_{a, b}$ und den entsprechenden Clausius-Mossotti Relationen zur Lorentz-Lorenz Beziehung für ein effektives Medium umschreiben:

$$
\frac{\varepsilon-1}{\varepsilon+2}=f_{a} \frac{\varepsilon_{a}-1}{\varepsilon_{a}+2}+f_{b} \frac{\varepsilon_{b}-1}{\varepsilon_{b}+2}
$$

mit den Volumen-Anteilen $f_{a, b}$ der beiden Phasen a und b.

Sind die Materialien a, b ausgedehnt und in ein Medium mit der Dielektrizitätskonstanten $\varepsilon_{h}$ eingebettet, wird Gleichung $4.28 \mathrm{zu} 159$

$$
\frac{\varepsilon-\varepsilon_{h}}{\varepsilon+2 \varepsilon_{h}}=f_{a} \frac{\varepsilon_{a}-\varepsilon_{h}}{\varepsilon_{a}+2 \varepsilon_{h}}+f_{b} \frac{\varepsilon_{b}-\varepsilon_{h}}{\varepsilon_{b}+2 \varepsilon_{h}}
$$

Im Maxwell Garnett Modell für das effektive Medium wird angenommen, dass Phase b fein verteilt in Form von sphärischen Partikeln in einer Matrix aus Phase a vorliegt, und damit $\varepsilon_{h}=\varepsilon_{a}$ gilt:

$$
\frac{\varepsilon-\varepsilon_{a}}{\varepsilon+2 \varepsilon_{a}}=f_{b} \frac{\varepsilon_{b}-\varepsilon_{a}}{\varepsilon_{b}+2 \varepsilon_{a}}
$$


In der Näherung von Bruggeman (EMA) wird $\varepsilon=\varepsilon_{h}$ angenommen, so dass sich die Bestimmungsgleichung für $\varepsilon$ ergibt [160]:

$$
f_{a} \frac{\varepsilon_{a}-\varepsilon}{\varepsilon_{a}+2 \varepsilon}+f_{b} \frac{\varepsilon_{b}-\varepsilon}{\varepsilon_{b}+2 \varepsilon}=0
$$

Während mit der Näherung von Maxwell und Garnett eher eine Mikrostruktur von beschichteten Kugeln (core-shell-Partikel) beschrieben wird, ist die Näherung von Bruggeman mit einer zufälligen Mischung der Phasen a und b verbunden, wo beide Phasen im effektiven Medium eingebettet sind.

Bisher wurden nur kugelförmige Mikrostrukturen angenommen, die keinerlei Anisotropie aufweisen. Die extremalen Fälle für anisotrope dielektrische Eigenschaften ergeben sich für eine aus Lagen aufgebaute Probe, wenn man die Situationen, wo das Feld parallel bzw. senkrecht zur Ebene der Lagen angelegt wird, vergleicht. Verläuft $\vec{E}$ parallel zu den Grenzflächen, ist das Feld aufgrund der Stetigkeitsbedingungen in der ganzen Struktur homogen. Damit ist die dielektrische Verschiebung $\vec{D}$ in jeder dieser Lagen entweder proportional zu $\varepsilon_{a}$ oder $\varepsilon_{b}$, je nachdem, ob man sich in Phase a oder b befindet. Mittelung ergibt dann

$$
\varepsilon=f_{a} \varepsilon_{a}+f_{b} \varepsilon_{b}
$$

Wenn $\vec{E}$ senkrecht auf den Grenzflächen der Lagen steht, ist $\vec{D}$ homogen und man erhält durch Mittelung:

$$
\frac{1}{\varepsilon}=\frac{f_{a}}{\varepsilon_{a}}+\frac{f_{b}}{\varepsilon_{b}}
$$

Die beiden obigen Gleichungen stellen die Grenzen für die dielektrischen Eigenschaften von zusammengesetzten Materialien dar, wenn man beliebige Mikrostrukturen zulässt [159]. Aus der dielektrischen Konstanten $\varepsilon$ für das effektive Medium ergibt sich über $N_{E M A}=$ $\sqrt{\varepsilon}$ der Brechungsindex. In Abbildung 4.7 ist der effektive Brechungsindex für eine raue $\mathrm{La}_{0.7} \mathrm{Sr}_{0.3} \mathrm{MnO}_{3}$-Schicht gezeigt, wobei die Berechnung nach dem Modell von Bruggeman erfolgte und die sich über Gleichungen 4.32 und 4.3 .3 ergebenden Grenzfälle angegeben sind.

Wie schon eingangs erwähnt, müssen die typischen Längenskalen der vermischten Materialien deutlich kleiner sein als die zur Untersuchung verwendete Wellenlänge $\lambda$, damit die Näherung durch ein effektives Medium gültig ist. Bei Lagenstrukturen kann man die reflektierten und transmittierten Feldamplituden exakt berechnen und mit den Ergebnissen vergleichen, die sich bei der Näherung durch ein effektives Medium ergeben würden. Damit ergibt sich, dass für laterale Separationen $d<\lambda / 10$ der Einfluss der Wellenlänge auf die messbaren optischen Eigenschaften zu vernachlässigen sind. Die Eigenschaften, die sich aus der exakten Rechnung und der Näherung mit einem effektiven Medium ergeben, sind identisch 159, 161. Wenn $d / \lambda>>0.1$, gibt es deutliche Abweichungen von diesen Näherungen, da sich die Strahlung durch einen Wellenleiter-Effekt für $\varepsilon_{b}>\varepsilon_{a}$ (es werden zur Vereinfachung reelle $\varepsilon_{a, b}$ angenommen) in den Bereichen von Phase b konzentriert [161. Bei einem anderen Ansatz wird die Gültigkeit der Approximation durch ein effektives Medium dadurch definiert, dass die Wellenfront unbeeinflusst durch den gemischten Bereich propagiert und keine Lichtstreuung stattfindet [156]. Bei Vergrößerung der Teilchendurchmesser lassen sich die Abweichungen von diesem Verhalten mittels der Mie-Theorie für Lichtstreuung beschreiben. Die Näherung mit einem effektiven Medium nach Bruggeman ist dann gültig für $d<\lambda / 30$ [156].

Werden die Längenskalen der Phasen a und b sehr klein und erreichen atomare Dimensionen, bricht die Näherung durch ein effektives Medium zusammen, da Quanteneffekte eine Rolle spielen 140. Bei einem so gemischten Material verschiebt sich so z.B. die Position der Absorptionsmaxima, bei einer einfachen Mischung wäre eine Verbreiterung zu beobachten. Auch 
bei den in dieser Arbeit untersuchten Manganaten zeigen sich deutliche Abweichungen, wenn man den Brechungsindex von $\mathrm{La}_{1-\mathrm{x}} \mathrm{Sr}_{\mathrm{x}} \mathrm{MnO}_{3}$ aus der Mischung vn $\mathrm{LaMnO}_{3}$ und $\mathrm{SrMnO}_{3}$ berechnet (siehe Abbildung 6.5). Diese untere Grenze lässt sich nicht allgemein festlegen, sondern hängt von den wechselseitigen Beeinflussungen der beiden Materialien, z.B. von der elektrischen Abschirmlänge, ab [156].

Experimentell zeigt sich, dass man mit der Näherung von Bruggeman Rauigkeiten von dünnen Filmen gut modellieren kann, wenn man ellipsometrische Messungen simulieren möchte. Mittels spektroskopischer Ellipsometrie wurden auf Silizium basierende Heterostrukturen mit verschiedenen Rauigkeiten extensiv untersucht. Dabei erfolgte ein systematischer Vergleich der Berechnung der optischen Konstanten von rauen Lagen an der Oberfläche mit den Modellen von Bruggeman, Maxwell-Garnett und Lorentz-Lorenz. Die gemessenen spektralen Abhängigkeiten von $(\Delta, \Psi)$ lassen sich am besten durch das Modell von Bruggeman anpassen 157. Wie in Abbildung 4.7 wird die Rauigkeit mit einer zusätzlichen Lage der Dicke $d_{S}$, die aus einer Mischung von dem Material des Films und Vakuum besteht, beschrieben. Eine zusätzlich zur Ellipsometrie erfolgende Charakterisierung der mikroskopischen Rauigkeit mit Hilfe von Rasterkraftmikroskopie zeigt einen linearen Zusammenhang zwischen $d_{S}$ und der mit dem Rasterkraftmikroskop bestimmten RMS-Rauigkeit $d_{R M S}\left[162,163, d_{S}\right.$ liegt dabei zwischen dem Wert für die durchschnittliche Peak-zu-Peak-Rauigkeit und $d_{R M S}$. Für die zuletzt genannten Studien wurden amorphe Silizium-Kohlenstoff Filme $\left(\mathrm{a}-\mathrm{Si}_{1-\mathrm{x}} \mathrm{C}_{\mathrm{x}}: \mathrm{H}\right)$ untersucht, deren Morphologie durch Cluster mit einer Höhe von ca. $h \approx 1-4 \mathrm{~nm}$ und lateralen Abmessungen im Bereich von $d \approx 40 \mathrm{~nm}$ bestimmt wird. Aufgrund dieser experimentellen und der zuvor beschriebenen theoretischen Befunde werden in dieser Arbeit Rauigkeiten an der Oberfläche der dünnen Filme mit dem Modell von Bruggeman beschrieben.

\subsection{Experimenteller Aufbau}

Um den in der Motivation genannten Anforderungen an eine Depositionstechnik von Heterostrukturen aus komplexen Oxiden gerecht zu werden, wurde in dieser Arbeit eine neue Anlage zur metallorganischen Aerosol Deposition (MAD) aufgebaut. Zuerst wird hier der grundlegende Aufbau dieser Anlage erklärt. Dann wird genau darauf eingegangen, wie die in-situ Messung des Polarisationszustandes abläuft und die ellipsometrischen Größen $(\Delta, \Psi)$ mathematisch mit den gemessenen Größen verknüpft sind. Die detaillierten Abläufe bei der Präparation von Heterostrukturen sollen erläutert werden, wobei insbesondere auf die verwendeten Substrate und deren Vorbehandlung eingegangen wird und eine Betrachtung der möglichen Fehlerquellen erfolgt. Im darauf folgenden Abschnitt wird die Bestimmung der Abhängigkeit der Substrattemperatur vom Strom durch den Heizer beschrieben. Der für die Auswertung der Daten zur in-situ Ellipsometrie wichtige Brechungsindex von $\mathrm{SrTiO}_{3}$ bei Depositionstemperatur wird besprochen und der Einfluss des Aerosols auf die Messung des Polarisationszustandes diskutiert. Schließlich wird die Auswertung und Modellierung der bei der in-situ Ellipsometrie gewonnen Daten beschrieben.

Der in Abbildung 4.1 gezeigte Aufbau der neuen MAD-Anlage folgt vom Grundprinzip der ursprünglichen Idee dieses Depositionsprinzipes 129, 130. Zum einfacheren Aufbau der optischen Komponenten strahlt die Düse hier entgegen dem ursprünglichen Konzept, wo die Düse entlang des Vektors der Gravitationskraft gerichtet ist, waagerecht. Aufgrund der kleinen Teilchendurchmesser im Aerosol spielt die Gravitationskraft gegenüber den Reibungskräften im Luftstrom nur eine kleine Rolle. Bei sehr großen Tropfen führt die Schwerebeschleunigung 

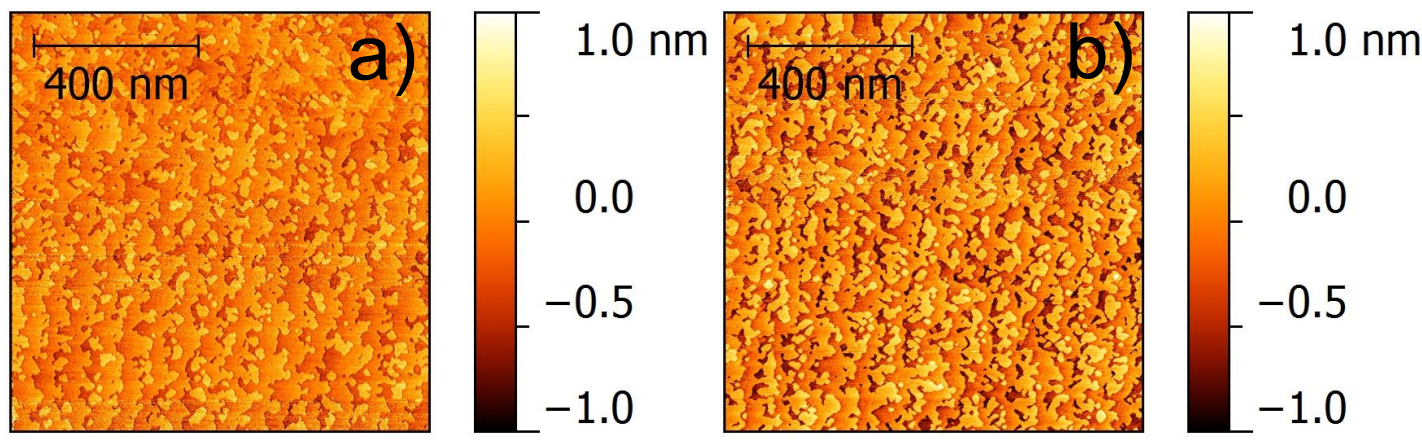

\begin{abstract}
Abbildung
4.8.:

Oberflächenmorphologien

für

eine $\mathrm{La}_{0.7} \mathrm{Sr}_{0.3} \mathrm{MnO}_{3} / \mathrm{SrMnO}_{3} / \mathrm{La}_{0.7} \mathrm{Sr}_{0.3} \mathrm{MnO}_{3}$ Dreifachlage, gemessen mit Rastertunnelmikroskopie (STM); a) aufgenommen in der Mitte des Substrates, b) aufgenommen ca. $0.5 \mathrm{~mm}$ entfernt vom durch das Abdecksubstrat verursachten diffusen Rand.
\end{abstract}

zu einer Ablenkung auf dem Weg zum Heizer, so dass diese nicht mehr auf das Substrat auftreffen. Sehr große Tropfen werden nicht komplett oberhalb des Substrates zersetzt, sondern treffen noch flüssig auf das Substrat und stören dabei das Wachstum aufgrund einer inhomogenen Verteilung der Lösungsbestandteile nach dem Verdampfen der Flüssigkeit. Die Luftströmung innerhalb des Aerosolkegels, der in der Ebene des Substrates einen Durchmesser von $D \sim 2 \mathrm{~cm}$ besitzt, wird von der Strömung der Düse bestimmt. Damit erfolgt die Pyrolysereaktion in der gleichen Weise oberhalb der Substratoberfläche, unabhängig davon, ob die Düse waagerecht oder entlang der Erdbeschleunigung orientiert ist (In Bereichen der Kammer, die in großer Entfernung vom Strahlkegel der Düse liegen, wird der Einfluss der Konvektion größer. Dies ist aber nicht von Belang für die Pyrolysereaktion, die direkt über dem Substrat stattfindet). Das gleichartige Verhalten der Pyrolyse für diese beiden Konfigurationen wurde verifiziert, indem dünne Filme von $\mathrm{La}_{0.7} \mathrm{Sr}_{0.3} \mathrm{MnO}_{3}$ auf $\mathrm{SrTiO}_{3}$ (001) in der hier vorgestellten Anlage und einer schon bestehenden Anlage, bei der die Düse entlang der Erdbeschleunigung strahlt, deponiert wurden. Dabei wurden identische molare Verhältnisse für die Präkursoren und ähnliche Depositionsbedingungen (Heizertemperatur, Depositionsrate) gewählt. Die beiden dabei hergestellten Filme waren dann hinsichtlich ihrer morphologischen, strukturellen, elektrischen und magnetischen Eigenschaften vergleichbar. Zur Optimierung der Deposition wird der Abstand zwischen Düse und Substrat variiert und die Größe des Ringspaltes, durch den die Luft aus der Düse austritt [131]. Zudem wird der Heizer so positioniert, dass der Strahlkegel des Aerosols zentral auf das Substrat gerichtet ist.

Durch die waagerechte Stellung der Düse und der damit verbundenen vertikalen Lage des Heizers muss das Substrat mit einer entsprechenden Klemmung auf den Heizer gedrückt werden. Dies geschieht folgendermaßen (siehe Abbildung 4.9): Das zu beschichtende Substrat liegt mittig auf dem Heizer aus SiC. Dann legt man zwei benutzte STO-Substrate auf die Kanten des frischen Substrates, so dass Streifen mit einer Breite von etwa $w=0.5-1.0 \mathrm{~mm}$ bedeckt werden. Diese werden dann mit einer Messingklammer fest auf den Heizer gedrückt. Die Messingklammer ist flexibel genug, so dass sich das Substrat beim Aufheizen ausdehnen kann und dabei die beiden Abdecksubstrate zur Seite schiebt. Die Abdecksubstrate an den Rändern schatten das Aerosol ab und sorgen dabei für einen diffusen Rand der Schichten mit einer Breite von etwa $0.1 \mathrm{~mm}$ an beiden Seiten des Substrates. Abgesehen von diesem Rand ist die Schicht homogen und es gibt keine Anzeichen einer starken Veränderung der Depositionsparameter (Temperatur, Wachstumsrate) hin zu den Rändern: Untersuchungen mit dem STM zeigen, dass die Morphologie an Stellen, die sich am Rand befinden $(0.5 \mathrm{~mm}$ 


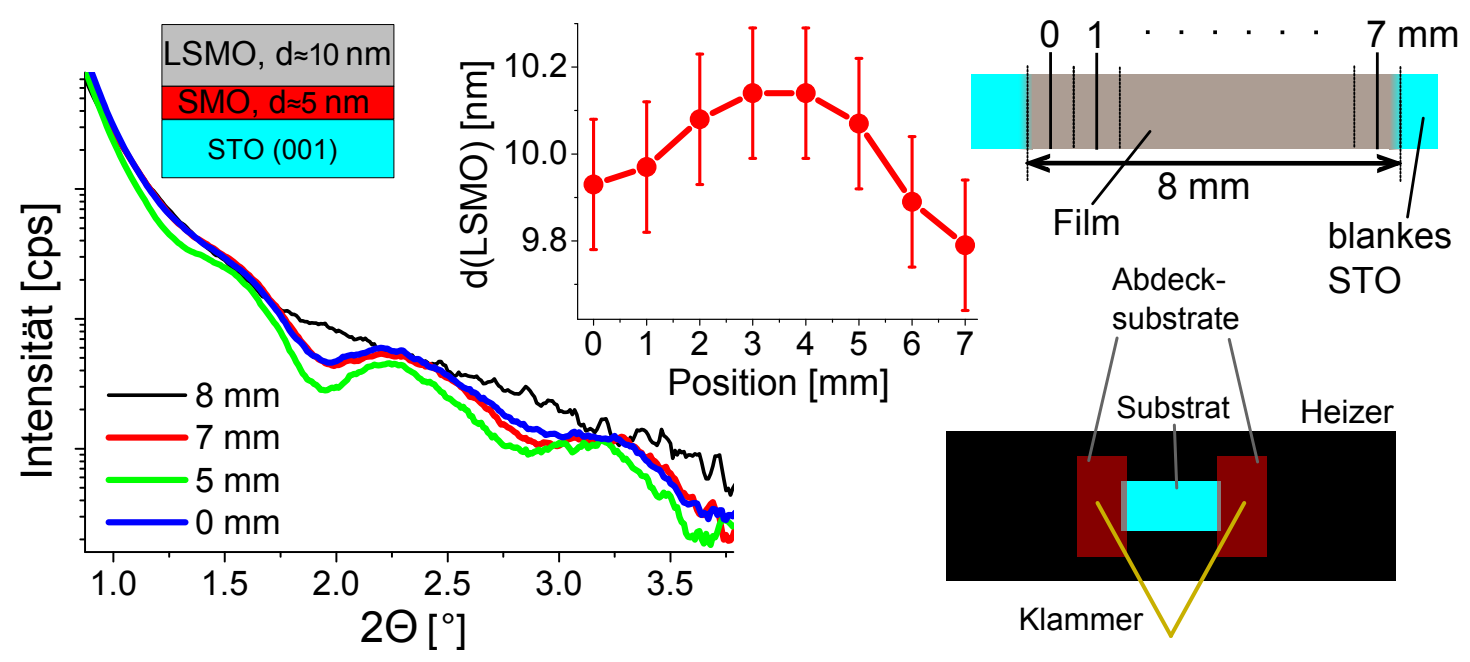

\begin{abstract}
Abbildung 4.9.: Die Klemmung des Substrates auf den Heizer mit bereits benutzten $\mathrm{SrTiO}_{3}$-Substraten und Messingklammer (rechts) erzeugt abgeschatteten Bereich am Rand der Schicht mit einer diffusen Grenze. Die Homogenität der Schichtdicke wird mit Röntgenreflektometrie an verschiedenen Positionen der Schicht überprüft: Für eine Doppellage $\mathrm{La}_{0.7} \mathrm{Sr}_{0.3} \mathrm{MnO}_{3} / \mathrm{SrMnO}_{3}$ (LMO/SMO) auf $\mathrm{SrTiO}_{3} \quad(\mathrm{STO})$ (001) sind Röntgenreflektometrie-Spektren an verschiedenen Positionen gezeigt (bei $8 \mathrm{~mm}$ befindet man sich auf dem abgedeckten Teil des Substrates, es wird nur das blanke STO gemessen). Das Inset zeigt dann die Variation der Schichtdicke der LSMO-Schicht d(LSMO) über die Länge der Probe.
\end{abstract}

entfernt von der diffusen Grenze der Schicht), so aussieht wie in der Mitte (siehe Abbildung 4.8). Mittels Röntgenreflektometrie kann man die Homogenität der Schichtdicke quantifizieren (siehe Abschnitt 5.2.2): Bei kleinen Einfallswinkeln $\theta$ (bei Röntgenmessungen wird der Einfallswinkel $\theta$ nach Konvention anders als bei den optischen Reflexionsmessungen nicht vom Lot auf die Oberfläche, sondern direkt von der Oberfläche gemessen, vergleiche dazu Abschnitt 5.2 wird die Breite des Bereichs, der von der Röntgenstrahlung erfasst wird, durch die Schneidblende oberhalb der Probe begrenzt und nicht durch die Spaltblende im Strahl. Bei einer Spaltbreite $w$ ergibt sich eine Breite $b_{\text {Spalt }}=\frac{w}{\sin \theta}$ auf der Probenoberfläche. Die Schneidblende mit einem Abstand $h$ von der Probenoberfläche schränkt den für die Messung verwendeten Querschnitt der Probe auf eine Breite von $b_{\text {Schneid }}=\frac{2 h}{\tan \theta}$ ein. Bei den typischen hier verwendeten Werten für Kleinwinkelbeugung, $\theta=2^{\circ}, h=0.02 \mathrm{~mm}, w=0.2 \mathrm{~mm}$ [164], ergibt sich so $b_{\text {Spalt }} \approx 5.7 \mathrm{~mm}$ und $b_{\text {Schneid }} \approx 1.1 \mathrm{~mm}$. Aufgrund zusätzlicher Spaltblenden vor dem Detektor wird der durch den Röntgenstrahl abgetastete Streifen weiter verkleinert. Man kann somit die Schichtdicke lokal entlang der Probe ermitteln, indem man die Probe unterhalb der Schneidblende so verschiebt, dass die zu untersuchende Stelle an der Kante der Schneidblende liegt. Um nun einen Eindruck der Homogenität der Schichtdicke zu gewinnen, verschiebt man die Probe in Schritten von $1 \mathrm{~mm}$ und nimmt Kleinwinkelspektren auf (siehe Abbildung 4.9). Die Verschiebung von Position 7 zu Position 8 erzeugt ein abruptes Verschwinden der Oszillationen der Schicht, man sieht nur noch das blanke Substrat. Damit ist bei den für die Messung in Abbildung 4.9 verwendeten Einstellungen der Bereich, der von dem Röntgenbündel erfasst wird, schmäler als $1 \mathrm{~mm}$. Die an den verschiedenen Stellen der Schicht (Position 0-7) gemessenen Kleinwinkelspektren weisen einen sehr ähnlichen Verlauf auf, insbesondere die Stellen der Minima und Maxima sind fast deckungsgleich. Wenn man die Schichtdicken der verwendeten $\mathrm{SrMnO}_{3} / \mathrm{La}_{0.7} \mathrm{Sr}_{0.3} \mathrm{MnO}_{3}$ (SMO/LSMO) Doppellage mit ReMagX 165 anpasst, erhält man den in Abbildung 4.9 gezeigten Verlauf für die Schichtdi- 
cke von LSMO. Die Schichtdicke fällt zu beiden Rändern der Probe leicht ab, die maximale Änderung der Schichtdicke über die Breite von $8 \mathrm{~mm}$ (der gemessene Bereich erstreckt sich etwa $0.5 \mathrm{~mm}$ rechts und links von der Position der Schneidblende) beträgt etwa $3 \%$.

Die MAD-Kammer wird fest auf einem optischen Tisch verschraubt. Die optischen Komponenten befinden sich auf zwei separaten Schienen, die auf demselben Tisch angebracht werden. Durch die verwindungssteife Ausführung der Halterung des Heizers verursacht die aus der Düse tretende Luft keine Vibrationen der Probe und damit kein zusätzliches Rauschen der Intensität auf dem Detektor. Der resistive Heizer, der aus einem Stück dotiertem $\mathrm{SiC}$ besteht, weist im mittleren Bereich, wo das Substrat angebracht ist, einen kleineren Querschnitt auf. Dadurch wird der größte Anteil der eingebrachten elektrischen Heizleistung in diesem Bereich konzentriert. Die beiden Enden des SiC-Heizers, an denen die Kontakte zur Einleitung des Heizstromes angeklemmt sind, werden kontinuierlich mit Wasser gekühlt. Damit kommt es beim Aufheizen des Substrates bis zur Depositionstemperatur nur in einem kleinen Bereich des Heizers zu einer starken Temperaturänderung. Aufgrund des zusätzlich geringen Wärmeausdehnungskoeffizienten von $\mathrm{SiC}[166$

$$
\alpha_{S i C}(T=1300 \mathrm{~K}) \approx 5 \cdot 10^{-6} \frac{1}{\mathrm{~K}}
$$

ist die resultierende Veränderung der Position des Substrates im optischen Strahlengang beim Aufheizen von Raumtemperatur zur typischen Depositionstemperatur von $T_{D e p} \approx 930^{\circ} \mathrm{C}$ minimal. Experimentell kann man die Änderung der Geometrie beim Aufheizen des Substrates quantifizieren, indem man die Bewegung des vom Substrat reflektierten Laserstrahls auf einem vor dem Detektor angebrachten Schirm verfolgt. Man erhält so für die typische hier verwendete Situation, bei der man ein STO-Substrat von Raumtemperatur auf $T_{D e p} \approx 930^{\circ} \mathrm{C}$ aufheizt eine Änderung des Einfallswinkels von $\Delta \theta \approx 0.02(1)^{\circ}$. Wie man später bei Bestimmung des Brechungsindexes für STO bei der Depositionstemperatur sehen wird, führt diese Änderung nur zu sehr kleinen Fehlern bei den optischen Konstanten. Zur sehr präzisen Messung der temperaturabhängigen Konstanten kann man die Veränderung der Geometrie gleichzeitig mit den ellipsometrischen Größen $(\Delta, \Psi)$ messen und so die Winkeländerung in die Berechnung einbeziehen. Es ist damit möglich, die optischen Konstanten der oxidischen Filme für Temperaturen oberhalb von Raumtemperatur zu beobachten und temperaturabhängige Veränderungen zu registrieren.

Wie es in den Grundlagen zur metallorganischen Aerosol Deposition dargelegt wurde, ist die Schichtdicke der deponierten Schicht proportional zum in die Düse dosierten Volumen der Präkursorlösung. Zur hochpräzisen und reproduzierbaren Dosierung der Lösungen werden hier deshalb Spritzendosierer des Typs SyrDos von der HiTec Zang GmbH verwendet. Bei einem Volumen von $V_{S y r}=500 \mu \mathrm{l}$ der hier verwendeten Spritzen ergibt sich dann eine maximale Auflösung von $\Delta V \approx 0.01 \mu \mathrm{l}$ und Förderraten von $P_{\min } \approx 0.6 \mu \mathrm{l} / \mathrm{s}$ bis $P_{\max } \approx 60 \mu \mathrm{l} / \mathrm{s}$ [167]. Dies entspricht bei den typischen Depositionsparametern für die Herstellung dünner Manganatfilme einer maximalen Aufösung im Bereich von 1/500 einer Monolage (ML) und einer Bandbreite hinsichtlich der Wachstumsrate von $P_{\min } \approx 0.1 \mathrm{ML} / \mathrm{s}$ bis $P_{\min } \approx 10 \mathrm{ML} / \mathrm{s}$. Zum Wachstum von Heterostrukturen aus verschiedenen Materialien wurden 4 separate Teflonleitungen von den einzelnen Spritzendosierern bis zur Spitze der Düse verlegt, durch die Präkursorlösungen injiziert werden. Durch ein Computerprogramm, das in Visual C\# implementiert wurde, werden die Spritzendosierer vollautomatisch gesteuert, so dass beliebige Abfolgen verschiedener Materialien deponiert werden können. Es ergibt sich nun die Problematik, dass diese Depositionsleitungen vor der Deposition exakt bis zu ihrem Ende gefüllt werden müssen. Ein Fehler dabei führt zu Abweichungen der Schichtdicke der ersten deponierten Schicht für jedes in der Heterostruktur vorkommende Material vom ursprünglich 
gewünschten Wert. Für die präzise Füllung der Depositionsleitungen wurden verschiedene Verfahren angewendet 11 . Zunächst kann man die Länge der Leitungen ermitteln, indem man pulsweise DMF in die Leitung dosiert, bis es aus der Düse austritt. Der Fehler dieses Verfahrens liegt im Bereich von $\Delta V \approx 0.5 \mu \mathrm{l}$. Zudem kann sich die Länge der Leitung durch Verformungen im System aus Teflonleitungen verändern, so dass man diese Eichung der Leitungslängen regelmäßig durchführen muss. Eine andere Möglichkeit besteht darin, die in-situ Ellipsometrie zu verwenden. Dabei wird bei der Deposition der ersten Schicht jedes Materials pulsweise Präkursorlösung dosiert, bis eine Änderung der ellipsometrischen Größen registriert wird. Hier ergibt sich ebenso ein Fehler von $\Delta V \approx 0.5 \mu \mathrm{l}$, es müssen aber keine zusätzlichen Eichmessungen durchgeführt werden. Durch den zusätzlichen Einbau eines „aktiven“ Shutters in die MAD-Anlage lässt sich der Fehler fast komplett vermeiden. Dazu wird direkt vor der Düse eine Metallplatte positioniert, die das Aerosol zum Substrat hin abschattet. An der dem Substrat zugewandten Seite der Metallplatte befindet sich eine weitere Düse, aus der Luft strömt, um das Substrat auf eine der Depositionstemperatur ähnliche Temperatur zu kühlen. Dieser Shutter ist beweglich angebracht und kann außerhalb der MAD-Kammer betätigt werden. Zur groben Kontrolle der richtigen Luftmenge kann dabei der Widerstand des SiC-Heizers herangezogen werden (siehe Abbildung 4.12). Insbesondere wenn man auf einer bereits bestehenden Heterostruktur eine weitere Deposition durchführen möchte, ist es zu vermeiden, dass die Substrattemperatur die Depositionstemperatur übersteigt. Direkt vor der Deposition werden dann alle Depositionsleitungen mit einem Volumen gefüllt, das die zur Füllung nötige Menge sicher übersteigt. Die austretenden Präkursorlösungen werden nicht deponiert, da sie von der Metallplatte abgeschattet werden. Dies wird zusätzlich durch Beobachtung der in-situ Ellipsometrie verifiziert. In allen verwendeten Leitungen reicht die eingefüllte Lösung nun jeweils genau zum Leitungsende. Der Shutter kann nun aus dem Kegel der Hauptdüse geklappt werden. Nachdem die Luftzufuhr des Shutters abgeschaltet worden ist und die Substrattemperatur sich nach einer Wartezeit von etwa 1 min stabilisiert hat, kann die eigentliche Deposition begonnen werden. Der Fehler mit dem Shutter kann durch mögliche Kapillareffekte an den Enden der Leitungen mit einem Innendurchmesser von ca. $0.2 \mathrm{~mm}$ auf $\Delta V \approx 10^{-2} \mu \mathrm{l}$ abgeschätzt werden.

\subsubsection{Ellipsometriemessung durch Polarisationsmodulation}

Die Messung der ellipsometrischen Größen $(\Delta, \Psi)$ erfolgt hier mit einem Aufbau, der auf dem Prinzip der Polarisationsmodulation durch einen photoelastischen Modulator beruht. Dieses Konzept wurde zuerst von Jasperson und Schnatterly eingeführt [168. Analog zum Aufbau dieser Autoren ist die Polarisationsmessung auch hier gestaltet (siehe Abbildung 4.1): Licht aus einem linear polarisierten Helium-Neon-Laser $(\lambda=632.8 \mathrm{~nm})$ wird durch einen Polarisator, ein Glan-Thompson-Prisma der Firma CVI Melles-Griot [169, geschickt, dessen Durchlassrichtung um $45^{\circ}$ gegen die Einfallsebene verkippt ist, um die Richtung der Polarisation exakt festzulegen und den bereits vorhandenen Grad der Polarisation des Lasers zu verbessern. Darauf passiert der Strahl den photoelastischen Modulator (PEM), der zwischen der p- und s-Komponente des Polarisationsvektors eine mit der Frequenz $f=50 \mathrm{kHz}$ oszillierende Phasenverschiebung, $\delta(t)=\delta_{0} \sin (\omega t)$, einführt [170].

Der PEM besteht aus einem Quarzglas-Quader, der an einen piezoelektrischen Quarzkristall geklebt ist. Durch eine angelegte Wechselspannung wird der Kristall zu elastischen Schwingungen mit einer Frequenz $f=50 \mathrm{kHz}$ angeregt, die im Glaskörper elastische Wellen verursa-

\footnotetext{
${ }^{11}$ Bei den in dieser Arbeit vorgestellten Heterostrukturen wurden verschiedene Methoden zur Eichung der Leitungslängen verwendet. Wenn es relevant ist, wird die im Einzelfall angewendete Methode aufgeführt.
} 
chen. Diese werden an der Rückseite des Glasquaders reflektiert und es bildet sich aufgrund einer geeigneten Wahl der Abmessungen eine stehende elastische Welle aus. Die elastischen Schwingungen sorgen für Variationen der lokalen Dichte und damit des Brechungsindexes (vgl. Lorentz-Modell zu dielektrischen Eigenschaften 140]). Aufgrund der Querkontraktion des Materials besitzt die Dichteänderung in der Richtung senkrecht zur angeregten Schwingungsebene ein anderes Vorzeichen. Dadurch wird zwischen den Komponenten der elektromagnetischen Welle mit Polarisation parallel und senkrecht zur angeregten Schwingungsebene eine Phasenverschiebung induziert. Die Amplitude der Phasenverschiebung $\delta_{0}$ lässt sich durch Variation der angelegten Spannung an den piezoelektrischen Kristall und damit der Schwingungsamplitude variieren $[170]$.

Dann wird der Lichtstrahl durch eine Lochöffnung in die MAD-Kammer gelassen, von der Probe reflektiert und dann durch eine weitere Öffnung aus der Kammer geleitet. Der Durchmesser des Laserstrahlenbündels beim Substrat beträgt etwa $0.5 \mathrm{~mm}$ (1/e-Radius des Gaußschen Strahlprofils). Auf dem Substrat ergibt sich durch den Einfallswinkel von $\theta \approx 65^{\circ}$ ein elliptischer Querschnitt mit einer Breite von etwa $1.2 \mathrm{~mm}$. Dadurch dass in der MADAnlage atmosphärische Bedingungen herrschen und durch den Luftfluss in der Kammer ein leichter Unterdruck herrscht, muss man zur Einkopplung des Lichtstrahls keine Fenster verwenden. Diese modifizieren aufgrund der Fresnel-Koeffizienten in Transmission den Polarisationszustand des Lichts, was zu einer Verfälschung der gemessenen Werte von $(\Delta, \Psi)$ führt. Da die inhomogene Erwärmung der Kammer zudem Dichtegradienten innerhalb des Fensterglases erzeugt, kommt es zu einer zusätzlichen Phasenverschiebung zwischen der p- und s-Komponente. Die Depositionskammer heizt sich auch nach dem Erreichen der endgültigen Depositionstemperatur weiter auf, wodurch diese Phasenverschiebung auch während der Deposition driften und den Verlauf von $\Delta(t)$ stören würde.

Der nach der Reflexion an der Probe aus der Kammer tretende Strahl passiert einen weiteren Polarisator [169], der ebenfalls einen Winkel von $45^{\circ}$ mit der Einfallsebene einschließt, und trifft schließlich auf einen Si-Detektor, der die Intensität über das Prinzip der Fotoleitfähigkeit erfasst. Der Vorverstärker dieses Photodetektors der Firma Hinds Instruments besitzt eine ausreichende Bandbreite, um die modulierten Signale auf den Frequenzen $f=50 \mathrm{kHz}$ und $2 f=100 \mathrm{kHz}$ zu erfassen 171 .

Mathematisch wird die Veränderung des Polarisationszustandes beim Durchlaufen der verschiedenen Elemente im Strahlengang hier durch den Jones-Formalismus beschrieben [140]. Dieser setzt vollständig polarisierte Strahlung voraus, was aufgrund der vernachlässigbaren Depolarisation nach der Reflexion an den hier betrachteten Proben gegeben ist (siehe Diskussion in 4.4.6). Für den hier verwendeten Aufbau ergibt sich so als Polarisationsvektor $\vec{E}_{t o t}$ nach Durchlaufen aller polarisationsbeeinflussenden Elemente, die mit den zugehörigen Jones-Matrizen berücksichtigt werden:

$$
\vec{E}_{\text {tot }}=\underbrace{\frac{1}{2}\left(\begin{array}{ll}
1 & 1 \\
1 & 1
\end{array}\right)}_{\text {Analysator } 45^{\circ}} \underbrace{\left(\begin{array}{cc}
r_{p} & 0 \\
0 & r_{s}
\end{array}\right)}_{\begin{array}{c}
\text { Reflexion } \\
\text { an Probe }
\end{array}} \underbrace{\left(\begin{array}{cc}
1 & 0 \\
0 & e^{i \delta_{0} \sin (\omega t)}
\end{array}\right)}_{\text {Modulator }} \underbrace{\frac{1}{\sqrt{2}\left(\begin{array}{l}
1 \\
1
\end{array}\right)}}_{\begin{array}{c}
\text { Zustand } \\
\text { nach Polarisator }
\end{array}}=\frac{1}{2 \sqrt{2}}\left(\begin{array}{l}
r_{p}+r_{s} e^{i \delta(t)} \\
r_{p}+r_{s} e^{i \delta(t)}
\end{array}\right)
$$

In der Matrix, die die Reflexion an der Probe beschreibt, stehen die totalen Reflexionskoeffizienten $r_{p / s}$. Um die gemessene Intensität auf dem Detektor zu bestimmen, berechnet man das komplexe Betragsquadrat von $\vec{E}_{t o t}$, wobei numerische Vorfaktoren vernachlässigt werden können [140]: 


$$
\begin{aligned}
I(t)=\left|\vec{E}_{t o t}\right|^{2}=I_{0}( & +J_{0}\left(\delta_{0}\right) \sin 2 \Psi \cos \Delta \\
& +2 J_{1}\left(\delta_{0}\right) \sin 2 \Psi \sin \Delta \sin (\omega t) \\
& \left.+2 J_{2}\left(\delta_{0}\right) \sin 2 \Psi \cos \Delta \cos (2 \omega t)\right)
\end{aligned}
$$

Der Vorfaktor $I_{0}$ ist dabei bestimmt durch die durchschnittliche Reflektivität für die beiden Polarisationsrichtungen:

$$
I_{0}=\frac{\left|r_{p}\right|^{2}+\left|r_{s}\right|^{2}}{2}
$$

Bei der Berechnung der Intensität wurde die Funktion $e^{i \delta_{0} \sin (\omega t)}$ über die ersten Glieder der Entwicklung nach sin und cos angenähert:

$$
\begin{aligned}
e^{i \delta_{0} \sin (\omega t)} & =J_{0}\left(\delta_{0}\right)+\sum_{n=1}^{\infty} J_{n}\left(\delta_{0}\right)\left(e^{i n \omega t}+(-1)^{n} e^{-i n \omega t}\right) \\
& \approx J_{0}\left(\delta_{0}\right)+2 i J_{1}\left(\delta_{0}\right) \sin (\omega t)+2 J_{2}\left(\delta_{0}\right) \cos (2 \omega t)
\end{aligned}
$$

mit der Bessel-Funktion $J_{i}\left(\delta_{0}\right)$ der i-ten Ordnung. Damit besteht das zeitabhängige Signal der Intensität aus Beiträgen auf drei verschiedenen Frequenzen: Dem DC-Anteil

$$
I_{D C}=I_{0}\left(1+J_{0}\left(\delta_{0}\right) \sin 2 \Psi \cos \Delta\right),
$$

dem Anteil auf der Modulationsfrequenz

$$
I_{\omega}=2 I_{0} J_{1}\left(\delta_{0}\right) \sin 2 \Psi \sin \Delta,
$$

und dem Anteil auf dem zweifachen der Modulationsfrequenz

$$
I_{2 \omega}=2 I_{0} J_{2}\left(\delta_{0}\right) \sin 2 \Psi \cos \Delta
$$

Durch Anpassung der Amplitude von der an den Quarzkristall angelegten Wechselspannung kann man $\delta_{0}=139^{\circ}$ einstellen. Dann ist $J_{0}\left(\delta_{0}\right)=0.011 \approx 0$ und $J_{1}\left(\delta_{0}\right)$ bzw. $J_{2}\left(\delta_{0}\right)$ sind nur etwa $10 \%$ von ihren Maximalwerten entfernt. Damit kann man die Berechnung von $(\Delta, \Psi)$ aus den Messwerten von $I_{D C}, I_{\omega}$ und $I_{2 \omega}$ deutlich vereinfachen, da man die Identitäten nicht zusätzlich nach $I_{0}$ auflösen muss. Außerdem wird das Rauschen für $(\Delta, \Psi)$ reduziert, denn für die modulierten Signale kann man wegen der festen Phasenbeziehung zum Referenzsignal des Modulators den Anteil des Rauschens mittels Lock-in-Verstärkung stark reduzieren. Numerisch erhält man für den hier gewählten Wert $\delta_{0}=139^{\circ} J_{1}\left(\delta_{0}\right)=0.515$ und $J_{2}\left(\delta_{0}\right)=0.435$. Bei der Berechnung von $(\Delta, \Psi)$ kürzt sich die Intensität $I_{0}$ heraus, wodurch man bei der Messung unempfindlich gegen äußere Beeinflussungen der Intensität ist (z.B. Schwankungen der Laserintensität oder teilweise Abschattung des Strahls). Dies ist ein großer Vorteil der Ellipsometrie gegenüber Messungen der einfachen Reflektivität, bei der immer eine Normierung der Intensität nötig ist. Dieser selbstnormierende Charakter von Ellipsometrie sorgt in der Praxis auch für ein besseres Signal-zu Rausch-Verhältnis, da auch Schwankungen von $I_{0}$ herausdividiert werden.

Zur Justage der Drehwinkel der beiden Polarisatoren verschiebt man die beiden Schienen mit dem Laser und dem Detektor so, dass der Laserstrahl ohne Reflexion an einer Probe auf den Detektor trifft. In dieser Transmissionsgeometrie setzt man $r_{p}=r_{s}=1$ und berechnet dann die zu erwartenden Amplituden $I_{D C}, I_{\omega}, I_{2 \omega}$. Die Polarisatoren werden dann solange verdreht, bis die theoretisch kalkulierten Werte erreicht sind. 
Im Experiment wird das Spannungssignal der Photodiode, das proportional zur Intensität ist, mit einem Multimeter (Keithley 2000 [172] ) und zwei Lock-in-Verstärkern (EG\&G 5210 [173]) aufgenommen. Das Multimeter nimmt die Intensität $I_{D C}$ auf und die Lock-in-Verstärker nehmen unter Benutzung des Referenzsignals vom Steuergerät des photoelastischen Modulators $I_{\omega}$ bzw. $I_{2 \omega}$ auf. Durch Anlegen von Referenzsignalen mit den hier betrachteten Frequenzen $(f=50 \mathrm{kHz}, f=100 \mathrm{kHz}$ und $f=0 \mathrm{~Hz})$ an die Messgeräte wurde verifiziert, dass die Messgeräte selektiv die gewünschten Teile des Signals aufnehmen und nicht durch Signale auf den anderen Frequenzen beeinflusst werden. Da innerhalb der Lock-in-Verstärker Filter zur Signalaufbereitung verwendet werden, wird zwischen den gemessenen AC-Signalen und dem Referenzsignal eine zusätzliche Phasenverschiebung eingeführt. Diese Verschiebung ist fest bei gleichbleibenden Filtereinstellungen der Verstärker. Somit sind die Messdaten von $I_{\omega}$ und $I_{2 \omega}$ unbestimmt hinsichtlich eines konstanten Vorfaktors von \pm 1 . Man kann diesen aber leicht ermitteln, indem man aus den gemessenen Werten von $(\Delta, \Psi)$ den Brechungsindex einer deponierten Schicht berechnet. Bei einer falschen Wahl des Vorzeichens ergeben sich unphysikalische Werte für den Brechungsindex $N$ (z.B. mit einem positiven Imaginärteil).

Die Messdatenaufnahme erfolgt mit demselben Computerprogramm, das auch die Dosierung der Präkursorlösungen mit den Spritzendosierern steuert. Aufgrund einer endlichen Antwortzeit der Messgeräte werden die Datenpunkte mit einem zeitlichen Abstand von etwa 0.1 s aufgenommen, was den Zeitkonstanten des DC-Filters am Ausgang der beiden Lock-in-Verstärker entspricht. Die Sensitivität der Lock-in-Verstärker wird vom Messprogramm immer auf den kleinstmöglichen Wert eingestellt, so dass es im Verlauf der Deposition zu mehreren Messbereichsumschaltungen kommen kann. Nach der Umschaltung des Messbereichs benötigen die Verstärker eine Zeitspanne von etwa zwei Sekunden, bis sich die Spannung am Ausgang stabilisiert hat und wieder Messdaten an das Programm weitergegeben werden können. Dadurch gibt es in einigen der später gezeigten zeitlichen Verläufe von $\Delta$ und $\Psi$ Lücken mit einer zeitlichen Dauer von etwa $3 \mathrm{~s}$.

\subsubsection{Details zur Schichtpräparation}

In dieser Arbeit wurden $\mathrm{SrTiO}_{3}$ (STO)-Substrate in (001) und (111)-Orientierung sowie $\left(\mathrm{La}_{0.3} \mathrm{Sr}_{0.7}\right)\left(\mathrm{Al}_{0.65} \mathrm{Ta}_{0.35}\right) \mathrm{O}_{3}$ (LSAT)-Substrate in (001)-Orientierung benutzt. Die Substrate wurden von den jeweiligen Herstellern (CrysTec GmbH, Crystal GmbH, SurfaceNet GmbH) mit einer polierten Oberfläche geliefert. Sie besitzen alle eine Dicke von $0.5 \mathrm{~mm}$ und haben eine Oberfläche von $5 \mathrm{~mm}$ x $10 \mathrm{~mm}$. STO und LSAT weisen beide bei Raumtemperatur und den hier betrachteten Depositionstemperaturen eine kubische Gitterstruktur auf. Bei STO ändert sich die Struktur unterhalb von $T_{t e t}=105-110 \mathrm{~K}$ von der kubischen in eine tetragonale mit einer Tetragonalität $\frac{a}{c}=1.0015[174-176$. Der Gitterparameter von STO beträgt $a_{S T O}=0.3905 \mathrm{~nm}[177,178]$ und der von LSAT $a_{L S A T}=0.3869 \mathrm{~nm}[179$. Die von den verschiedenen Herstellern gelieferten Substrate sind nicht exakt entlang der spezifizierten Kristallrichtungen geschnitten, sondern weisen einen sogenannten misscut von bis zu $\alpha_{\text {cut }} \approx 0.5^{\circ}$ auf. Zum Ausgleich dieses Fehlschnitts bilden sich an der Oberfläche des Substrats Terrassen mit der Höhe einer Gitterzelle $a$. Die Breite dieser Terrassen ergibt sich dann geometrisch direkt aus dem jeweiligen Winkel des Fehlschnittes zu $w_{\text {Terr }}=\frac{a}{\tan \alpha_{c u t}}$. Abbildung 4.10 a) zeigt die mittels Rasterkraftmikroskopie gemessene Morphologie eines STO-Substrates, auf dem man diese durch den misscut induzierten Terrassen sieht. In Teil b) von Bild 4.10 sieht man einen größeren Ausschnitt eines STO-Substrates, wo man die Einteilung der Oberfläche in rechteckige Bereiche mit Kantenlängen im Bereich von 5-10 $\mu \mathrm{m}$ erkennt. Dies wird 

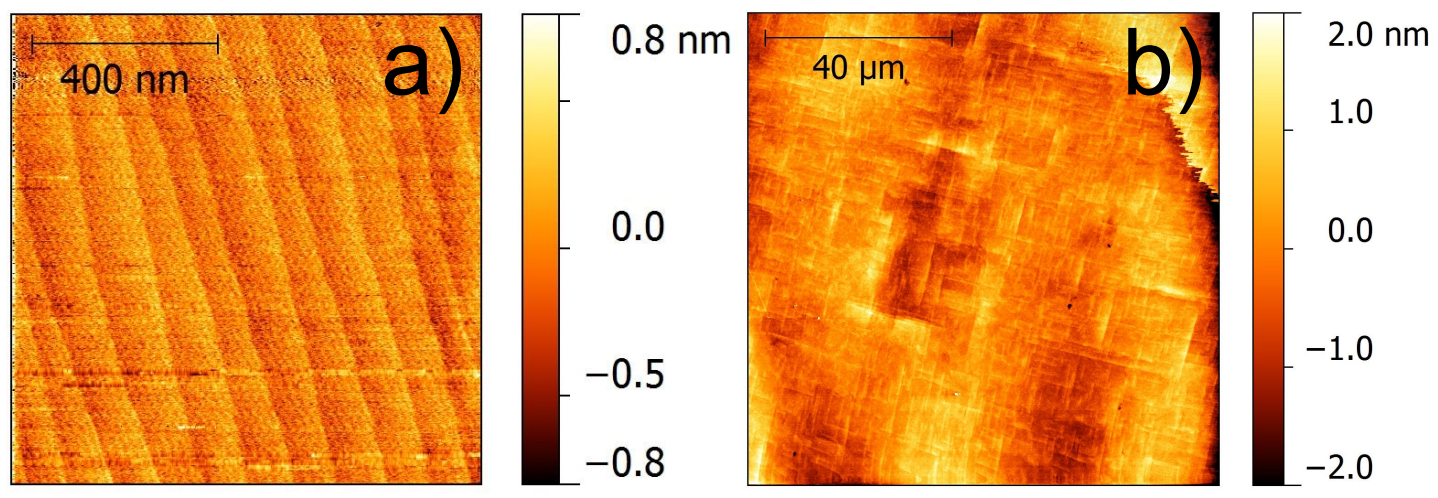

Abbildung 4.10.: Oberflächenmorphologie von verwendeten $\mathrm{SrTiO}_{3}$ (STO) (001)Substraten, gemessen mittels Rasterkraftmikroskopie; a) $1 \mu \mathrm{m}$ x $1 \mu \mathrm{m}$, b) $100 \mu \mathrm{m}$ x $100 \mu \mathrm{m}$.

dadurch verursacht, dass die STO-Substrate aus Mikrokristalliten bestehen, die gegeneinander um Winkel der Größenordnung $0.1^{\circ}$ verkippt sind. Auch die Verbreiterung der mit Röntgendiffraktometrie gemessenen Rocking-Kurven zeigt diese Fehlorientierung zwischen den Kristalliten.

Die durch den Fehlschnitt verursachten Stufen auf der Substratoberfläche sind von großer Bedeutung für das Wachstum der deponierten Filme. Im Zusammenhang mit diesen Stufen ergeben sich durch die Wachstumskinetik und Minimierung der elastischen Energie drei verschiedene Wachstumsmoden [180: Beim step-flow-Wachstum diffundieren die auf dem Substrat ankommenden Adatome komplett an die Stufenkanten, so dass sich die Kanten auf den Terrassen kontinuierlich voranbewegen. Das step-bunching-Wachstum bezeichnet das Zusammendrängen von mehreren Stufen, so dass sich sehr breite Terrassen bilden, die durch Stufen mit einer Höhe, die einem Vielfachen der Gitterkonstanten a entspricht, getrennt sind. Zuletzt kann es zum Inselwachstum kommen, wobei die Dimensionalität der Inseln (zweioder dreidimensional) von den Grenzflächenenergien zwischen Substrat, Film und Vakuum abhängen [181]. Bei den hier betrachteten Materialkombinationen kommt es typischerweise zu einer Konkurrenz zwischen dem zweidimensionalen Inselwachstum und der step-flowWachstumsmode. Welche dieser beiden Moden dominiert, wird durch ein Wechselspiel von der Beweglichkeit der Adatome auf den Terrassen $D$, der Stufenbreite $L$ und der Depositionsrate $F$ bestimmt [180] : Die mittlere von einem Adatom in der Zeit $t$ zurückgelegte Strecke senkrecht zur Richtung der Stufenkanten ist durch $l=\sqrt{2 D t}$ gegeben. Damit ist die mittlere Zeit, nach der sich ein Adatom an die Stufenkante angelagert hat, $\tau_{\text {Kante }}=L^{2} / 2 D$. In dieser Zeit überstreicht das Atom im Mittel eine Fläche von $L^{2}$. Mit der Depositionsrate $F$ in Monolagen (ML) pro Sekunde ergibt sich die mittlere Zeit zwischen dem Aufkommen zweier Adatome auf einer Fläche von $L^{2}$ zu $\tau_{D e p}=(a / L)^{2} / F$, wobei $a$ die Gitterkonstante an der Oberfläche ist. Für $\tau_{\text {Kante }}>\tau_{D e p}$ steigt die Zahl an Adatomen auf der Oberfläche der Terrassen und es kommt zur Inselbildung. Damit wird die Bildung von Inseln verhindert, wenn $\tau_{\text {Kante }}<\tau_{D e p}$ und damit

$$
F<\frac{2 D a^{2}}{L^{4}}
$$

gilt. Bei gleichbleibender Depositionsrate und Temperatur ergibt sich so bei größerem $\alpha_{c u t}$ eher Inselwachstum als bei kleineren Werten für den Fehlschnitt, da die Adatome auf den breiten Terrassen nicht genug Zeit haben, um zur Stufenkante zu diffundieren, bevor sie mit anderen Adatomen zu einer Insel agglomerieren. 
Entlang der (001)-Richtung besteht der $\mathrm{SrTiO}_{3}$-Kristall aus Lagen von $\mathrm{SrO}$ und $\mathrm{TiO}_{2}$. Bei der Herstellung der Substrate ergibt sich durch das Schneiden und Schleifen der Oberfläche eine zufällige Terminierung mit diesen beiden Varianten. Aufgrund der unterschiedlichen chemischen Eigenschaften von $\mathrm{SrO}$, das in Atmosphäre schnell zu $\mathrm{Sr}(\mathrm{OH})_{2}$ oder $\mathrm{SrCO}_{3}$ reagiert, und $\mathrm{TiO}_{2}$ kann man durch selektives chemisches Ätzen das SrO ablösen und eine Terminierung mit der $\mathrm{TiO}_{2}$-Halblage erreichen. Auf Kawasaki et al. geht dafür das Verfahren zurück, bei dem man STO mit gepufferter Flusssäure (BHF), einer Mischung aus $\mathrm{NH}_{4} \mathrm{~F}$ und $\mathrm{HF}$ behandelt 182, 183. Diese Methode wurde von Koster et al. weiter verfeinert, so dass das Substrat vor dem eigentlichen Ätzschritt mit BHF zusätzlich demineralisiertem Wasser ausgesetzt wird 184]. Dadurch bildet sich an den mit $\mathrm{SrO}$ terminierten Stellen $\mathrm{Sr}(\mathrm{OH})_{2}$, das sich dann in BHF wesentlich leichter und reproduzierbarer löst als SrO. Angelehnt an die Methode von Koster et al. wird hier folgender Ablauf zur Terminierung der $\mathrm{SrTiO}_{3}$-Substrate mit (001)-Orientierung angewendet:

- 10 min demineralisiertes Wasser bei $20^{\circ} \mathrm{C}$ im Ultraschallbad.

- $45 \mathrm{~s}$ in gepufferter Flusssäure $\left(\mathrm{NH}_{4} \mathrm{~F} \cdot \mathrm{HF}\right.$, CAS Nummer: 70456-74-5).

- Spülen mit demineralisiertem Wasser.

- Auslagern bei $T_{\text {Anneal }}=1000{ }^{\circ} \mathrm{C}$ für $t_{\text {Anneal }}=1 \mathrm{~h}$ in Luft.

Bei der weiter unten erfolgenden Besprechung der Ergebnisse wird gesondert erwähnt, ob die jeweilige Probe auf einem nach diesem Verfahren terminierten STO-Substrat deponiert oder ein unbehandeltes STO-Substrat verwendet wurde. Für die LSAT-Substrate gibt es keine etablierte Vorgehensweise zur Terminierung, diese Substrate wurden nach Lieferung bei $T_{\text {Anneal }}=1000^{\circ} \mathrm{C}$ für $t_{\text {Anneal }}=1 \mathrm{~h}$ in Luft ausgelagert.

Die Genauigkeit und Reproduzierbarkeit der Schichtdicken hängt neben der reinen Dosiergenauigkeit der Volumina der Präkursorlösungen natürlich auch von den Parametern der Lösungen selbst ab. Dabei beeinflusst die Molarität der Präkursorlösung die Genauigkeit der deponierten Schichtdicke. Mit einem typischen Fehler für das Abwiegen der metallorganischen Präkursoren von $0.3 \%$ und einem Fehler von $0.5 \%$ für das Dosieren von Dimethylformamid ergibt sich insgesamt ein Fehler von $0.6 \%$ für die Molarität. Da die resultierende Schichtdicke proportional zur Molarität ist, ergibt sich der gleiche relative Fehler auch für die Schichtdicke.

\subsubsection{Substrattemperatur}

Für die Deposition dünner Filme ist die Temperatur des Substrates von entscheidender Bedeutung. Durch Variation der Temperatur können verschiedene Phasen thermodynamisch begünstigt werden (vgl. Herstellung verschiedener Eisenoxide in [135]). Außerdem werden Geschwindigkeiten von erwünschten und unerwünschten Diffusionsprozessen beeinflusst. Zur reproduzierbaren Herstellung von Heterostrukturen ist die Überprüfung der Depositionstemperatur also von großer Wichtigkeit. Dazu kann man den hier realisierten Messaufbau für die in-situ Ellipsometrie ausnutzen. In Abbildung 4.11 ist eine in-situ Messung der reflektierten Intensität für ein gespaltenes $\mathrm{Al}_{2} \mathrm{O}_{3}$ (0001)-Substrat gezeigt, während dieses von Raumtemperatur zur typischen Depositionstemperatur bei $I_{S i C}=36 \mathrm{~A}$ aufgeheizt und wieder auf Raumtemperatur abgekühlt wird. In der vergrößerten Darstellung sind regelmäßige Oszillationen zu erkennen. Diese ergeben sich durch die Interferenz zwischen den an der Oberfläche und Rückseite reflektierten Teilstrahlen (siehe Abbildung 4.11). Durch die thermische Ausdehnung des Substrates ändert sich die Phasenbeziehung zwischen den beiden Teilstrahlen und es ergeben sich Oszillationen, da die Gesamtdicke des Substrates beim Aufheizen um ein 


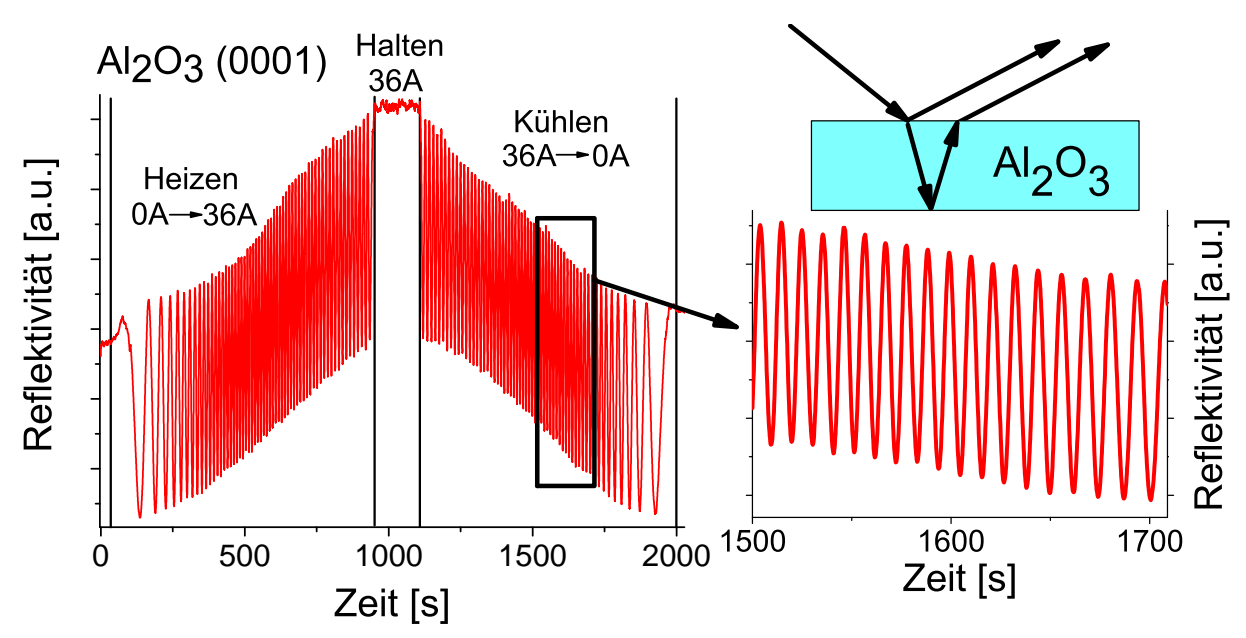

\begin{abstract}
Abbildung 4.11.: Änderung der optischen Reflektivität $\lambda=632.8 \mathrm{~nm}$ eines $\mathrm{Al}_{2} \mathrm{O}_{3}$ (0001)Substrates beim Heizen und Kühlen von Raumtemperatur zur Depositionstemperatur, die bei $I_{S i C}=36 \mathrm{~A}$ erreicht wird. Der vergrößerte Bereich zeigt oszillierendes Verhalten, das sich durch die Interferenz zwischen den an der Ober- und Rückseite reflektierten Teilstrahlen ergibt (siehe Skizze).
\end{abstract}

Vielfaches der verwendeten Wellenlänge zunimmt. Die Gesamtzahl der Oszillationen, die von Raumtemperatur bis zur Depositionstemperatur erscheinen, sind somit ein Maß für die über die Dicke des Substrates $d \approx 0.5 \mathrm{~mm}$ gemittelte Temperatur am Ende des Aufheizvorganges. Über die Zählung der Oszillationen kann man damit die Substrattemperatur reproduzierbar einstellen und auf Änderungen der Bedingungen wie dem Luftstrom durch die Düse oder die thermische Ankopplung an den Heizer reagieren. Im gezeigten Beispiel mit $\mathrm{Al}_{2} \mathrm{O}_{3}$-Substrat $\left(N_{\mathrm{Al}_{2} \mathrm{O}_{3}}=1.766[154]\right)$ zeigt sich dieses Verfahren auch relativ robust gegen die für diesen Aufbau typischen Variationen des Einfallswinkels. Der Einfallswinkel liegt in einem Bereich von $62.5 \pm 2^{\circ}$. Damit ändert sich die Strecke, die das Licht im Substrat zurücklegt um etwa $\pm 2 \%$. Auch die Zahl der Oszillationen und damit der Fehler der Reproduzierbarkeit der Temperatur liegen dann in diesem Bereich, wenn man die Winkeländerung nicht gesondert berücksichtigt.

Zusätzlich wurde die Temperatur mit einem Zweikanal-Pyrometer, welches die Intensitäten der Wärmestrahlung bei zwei Wellenlängen mit dem Gesetz für Schwarzkörperstrahlung abgleicht, ermittelt. Dabei wurde die Temperatur für ein beschichtetes STO-Substrat bestimmt. Der aufgebrachte Film (LSMO, $d \approx 120 \mathrm{~nm}$ ) ist dabei intransparent für die Wärmestrahlung 12 , so dass mit dem Pyrometer die Temperatur an der Oberfläche des Substrates gemessen wird und nicht die Wärmestrahlung des Heizers. Für den Heizerstrom bei der Deposition $I_{S i C}=36 \mathrm{~A}$ ergibt sich damit eine Temperatur von $T_{D e p}=930(5)^{\circ} \mathrm{C}$ für die Oberfläche des Substrates. Bei einem unbeschichteten Substrat misst man mit dem Pyrometer nur die Temperatur des Heizers und erhält mit $T=980^{\circ} \mathrm{C}$ einen um etwa $50^{\circ} \mathrm{C}$ höheren Wert für die Temperatur. Im Folgenden soll der mit dem Pyrometer ermittelte Wert als Temperatur des Substrates herangezogen werden.

Bei den hier hauptsächlich verwendeten STO-Substraten sind die Interferenzoszillationen auch bei beidseitig polierten Substraten nur schwach ausgeprägt und werden bei höheren Temperaturen zumeist durch die allgemeinen Temperaturabhängigkeiten von $(\Delta, \Psi)$ und $I_{0}$

\footnotetext{
${ }^{12}$ Für den bei der Pyrometermessung relevanten Wellenlängenbereich $(\lambda=900-1000 \mathrm{~nm})$ kann man abschätzen, dass etwa $10 \%$ der Wärmestrahlung des Heizers durch den LSMO-Film transmittieren 185.
} 
a)

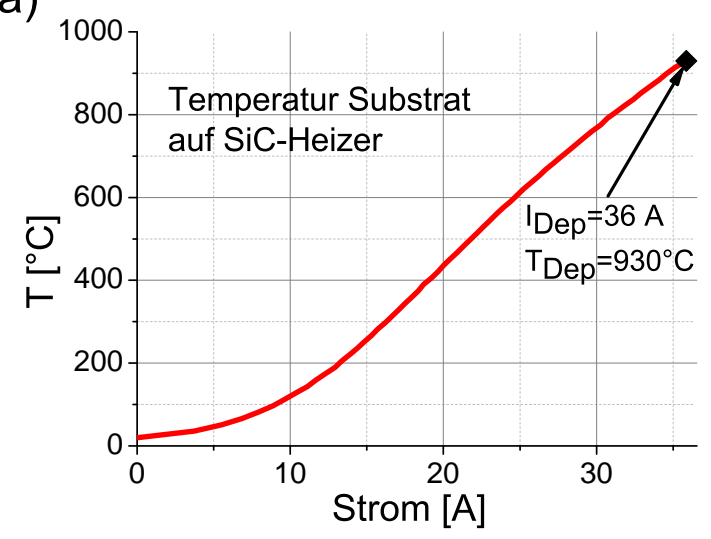

b)

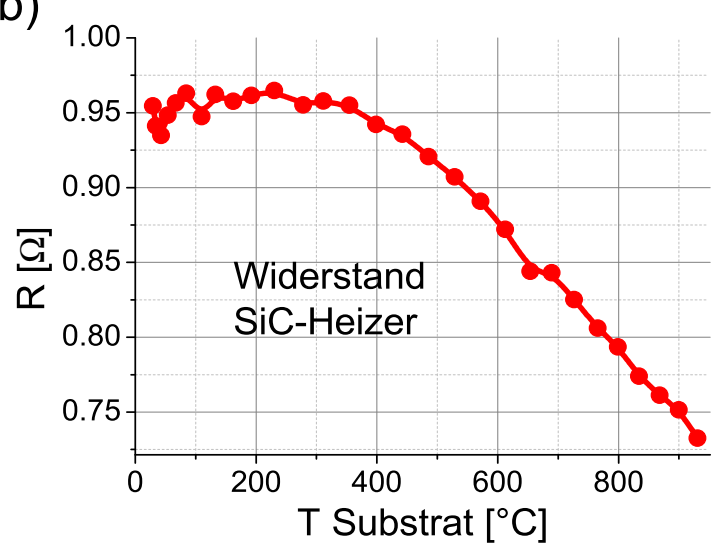

Abbildung 4.12.: Charakteristika des in der hier realisierten MAD-Anlage verwendeten SiC-Heizers: a) Aus den Interferenzoszillationen in Abbildung 4.11 rekonstruierte Abhängigkeit der durchschnittlichen Substrattemperatur vom Strom durch den Heizer, b) Darstellung des Widerstandes vom SiC-Heizer als Funktion der Temperatur.

überdeckt. Dies ist auf die mangelnde Planparallelität dieser Substrate zurückzuführen. STO lässt sich aufgrund seiner Perowskit-Struktur im Vergleich zu $\mathrm{Al}_{2} \mathrm{O}_{3}$ mit seiner hexagonalen und damit stark anisotropen Struktur nur schlecht entlang bestimmter Ebenen spalten. Die von den Herstellern gelieferten, geschliffenen STO-Substrate weisen hinsichtlich ihrer Planparallelität eine Toleranz im Bereich von $5^{\circ}$ auf. Für die Strahlen im Lichtfleck des Lasers mit einer Breite von ca. $1 \mathrm{~mm}$ variiert die Phasenbeziehung zwischen den an der Ober- und Rückseite reflektierten Teilstrahlen somit stark in Abhängigkeit von der Stelle auf der Rückseite, von der ein Strahl reflektiert wird. Dadurch kann es nie zu einer komplett konstruktiven oder destruktiven Interferenz zwischen den an der Ober- und Unterseite reflektierten Strahlen kommen.

Wenn man die aus der Pyrometermessung ermittelte Temperatur $T_{D e p}=930{ }^{\circ} \mathrm{C}$ bei $I_{S i C}=$ $36 \mathrm{~A}$ zugrunde legt und den thermischen Ausdehnungskoeffizienten $\alpha_{\mathrm{Al}_{2} \mathrm{O}_{3}}$ für $\mathrm{Al}_{2} \mathrm{O}_{3}$ kennt, kann man aus den in Abbildung 4.11 gezeigten Oszillationen die Abhängigkeit der Substrattemperatur vom Strom durch den Heizer konstruieren. Von hohen Temperaturen ausgehend zeigt $\alpha_{\mathrm{Al}_{2} \mathrm{O}_{3}}$ bis hinunter zu etwa $180^{\circ} \mathrm{C}$ ein fast temperaturunabhängiges Verhalten $186{ }^{13}$ Damit kann man näherungsweise annehmen, dass die Interferenzmaxima äquidistant im Abstand von $\Delta T=15.4^{\circ} \mathrm{C}$ auftreten. Mit den zu den Daten aus Abbildung 4.11 gehörenden Werten für den Strom durch den Heizer $I_{S i C}$ ergibt sich die in Abbildung 4.12 a) gezeigte Abhängigkeit für die Substrattemperatur. Aus den Daten von Abbildung 4.12 a) kann man den Widerstand des SiC-Heizers $R_{S i C}(T)$ als Funktion der Substrattemperatur ableiten, welche in Teil b) der Abbildung gezeigt ist. $R_{S i C}(T)$ ist zu verwenden, wenn man den Luftstrom durch den Shutter einstellt, so dass die Depositionstemperatur nicht überschritten wird, wenn der Shutter den Strahlkegel der Hauptdüse abdeckt.

Die Änderung der Temperatur des Substrates beim Dosieren der Präkursorlösung kann durch eine in-situ Widerstandsmessung eines STO-Substrates abgeschätzt werden. Dabei verwendet man eine thermisch aktivierte Abhängigkeit der Ladungsträgerdichte $n(T) \propto e^{-\frac{E_{g}}{2 k_{B} T}}$ und den bekannten Wert für die Bandlücke von STO $E_{g}=3.25 \mathrm{eV}$ [187]. Für die typische Förderrate der Präkursorlösung $p \approx 2.1 \mu \mathrm{l} / \mathrm{s}$ ergibt sich eine Abkühlung des Substrates um $\Delta T \approx 15 \mathrm{~K}$.

\footnotetext{
${ }^{13}$ Für $T<180^{\circ} \mathrm{C}$ ist der Ausdehnungskoeffizient kleiner als bei höheren Temperaturen. In diesem Niedertemperaturbereich unterschätzt man somit die wahre Heizertemperatur durch die Annahme eines konstanten Ausdehnungskoeffizienten.
} 

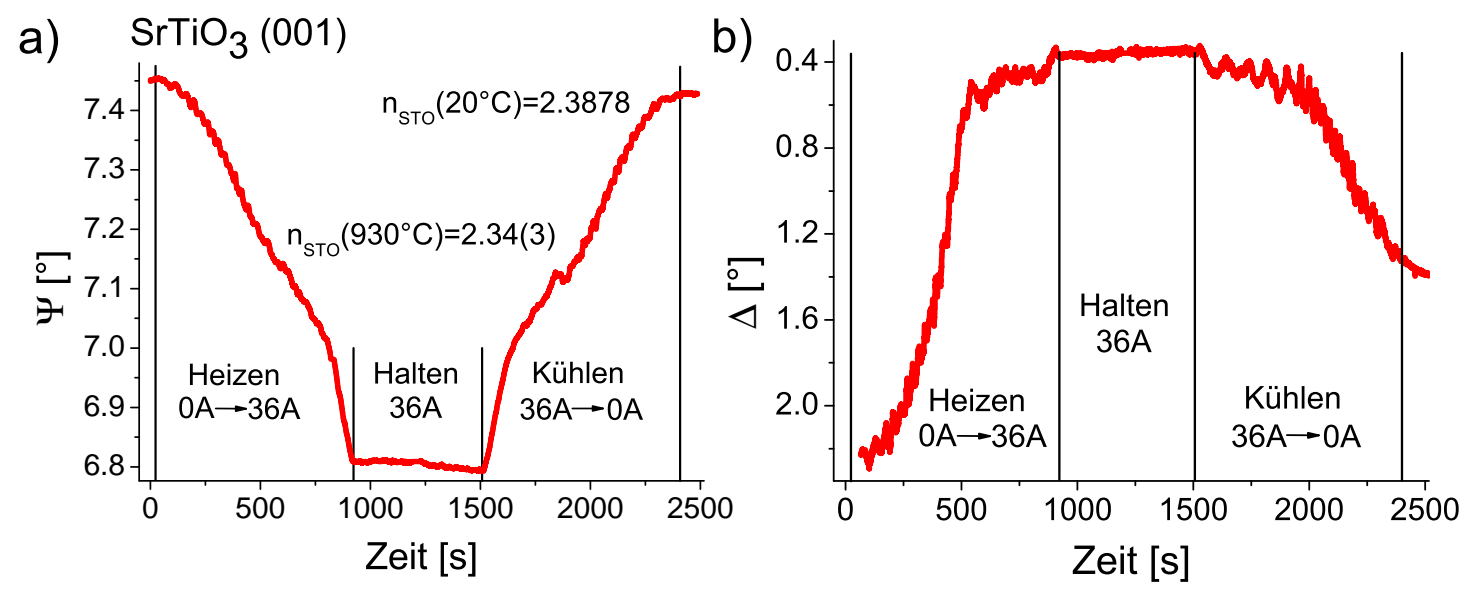

\begin{abstract}
Abbildung 4.13.: In situ-Messung von a) $\Psi$ und b) $\Delta$ für ein frisches $\mathrm{SrTiO}_{3}$ (STO) (001)-Substrat beim Heizen von Raumtemperatur zur Depositionstemperatur, die bei einem Strom $I_{S i C}=36 \mathrm{~A}$ durch den SiC-Heizer erreicht wird, und anschließendem Abkühlen. Der Brechungsindex für STO $N_{S T O}=n_{S T O}+i * 0.0$ bei Raumtemperatur $(\lambda=632.8 \mathrm{~nm})[154$ und der anhand der Messdaten von $\Psi$ berechnete Wert bei den Depositionsbedingungen sind angegeben.
\end{abstract}

\title{
4.4.4. Brechungsindex von $\mathrm{SrTiO}_{3}$
}

Zur späteren Auswertung der in-situ Messungen der Ellipsometrie ist es notwendig, den Brechungsindex des STO-Substrates bei Depositionstemperatur $T_{D e p}$ zu kennen. Dazu misst man $(\Delta, \Psi)$ beim Aufheizen und Abkühlen eines unbehandelten STO (001)-Substrates von Raumtemperatur zur Depositionstemperatur $T_{D e p}=930{ }^{\circ} \mathrm{C}$, die bei $I_{S i C}=36 \mathrm{~A}$ erreicht wird (siehe Abbildung 4.13). Aufheizen und Abkühlen erfolgen dabei mit einer linearen Abhängigkeit des Stromes von der Zeit und in beiden Fällen in einer Zeit von 15 min. $\Psi$ kehrt nach dieser Wärmebehandlung fast auf den gleichen Wert bei Raumtemperatur zurück. Für $\Delta$ ist die Amplitude und die gesamte Änderung beim Aufheizen sehr klein. Jedoch ist eine Irreversibilität zu erkennen, $\Delta$ ist zu Beginn bei Raumtemperatur deutlich größer als nach der Wärmebehandlung. Da der Brechungsindex von STO für die hier verwendete Wellenlänge $\lambda=632.8 \mathrm{~nm}$ einen verschwindenden Imaginärteil hat, erwartet man theoretisch auch ein Verschwinden von $\Delta$. Die hier beobachteten Werte sind einerseits auf Imperfektionen der optischen Instrumente im Strahlengang zurückzuführen und Adsorbatlagen an der Oberfläche des Substrates. Wie weiter oben schon diskutiert wurde, ist insbesondere die SrO-Oberfläche empfindlich auf die Reaktion mit $\mathrm{H}_{2} \mathrm{O}$ und $\mathrm{CO}_{2}$ aus der Luft. Eine Oberflächenlage mit einem anderen Brechungsindex als STO würde dann zu einem nichtverschwindenden Wert von $\Delta$ führen. Man kann auch sehen, dass $\Delta$ beim Aufheizen bis zu einem bestimmten Zeitpunkt, sprich einer bestimmten Temperatur, eine rapide Änderung zeigt, worauf trotz steigender Temperatur ein Plateau folgt. Auf dem Hochtemperatur-Plateau besitzt $\Delta$ einen deutlich kleineren Wert als bei Raumtemperatur sehr nahe zu $\Delta=0^{\circ}$. Dies ist konsistent mit dem Vorhandensein von Adsorbat-Lagen bei niedrigeren Temperaturen, die in einem bestimmten Temperaturbereich $\left(T \sim 400-700^{\circ} \mathrm{C}\right)$ von der Oberfläche desorbieren und ein blankes Substrat hinterlassen 133, 134, 183. Beim Abkühlen des Substrates zeigt $\Delta$ zunächst wieder einen fast waagerechten Verlauf, um dann beim Unterschreiten einer bestimmten Temperatur rapide anzusteigen. Dass $\Delta$ zum Ende hin nicht zu seinem ursprünglichen Wert bei Raumtemperatur zurückkehrt, liegt daran, dass sich die im Ausgangszustand vorhandenen Adsorbate auf dem Substrat über längere Zeit gebildet haben. Nach der kompletten Desorption bei ho- 
hen Temperaturen müsste man das Substrat noch einige Zeit atmosphärischen Bedingungen bei Raumtemperatur aussetzen, um wieder zum Ausgangszustand zurückzukehren. Man kann auch am Ende des Plots von $\Delta(t)$ sehen, wie sich $\Delta$ trotz konstanter Bedingungen ändert, was auf fortschreitende Veränderungen der Oberfläche hindeutet. Bei genauerer Inspektion bemerkt man, dass beim Verlauf von $\Delta(t)$ auch eine gewisse Asymmetrie entlang der Temperaturachse besteht. Daraus folgt, dass die beim Aufheizen beobachtete Desorption bei höheren Strömen (und damit Temperaturen) auftritt als die Wiederkehr der Adsorbate beim Abkühlen. Dies ist konsistent mit der typischen Hysterese, die man bei Phasenübergängen erster Ordnung wie z.B. Kondensation beobachtet.

$\Psi$ ist wesentlich unempfindlicher auf das Vorhandensein einer Adsorbatlage. Man sieht nur eine sehr schwache Irreversibilität und nur Änderungen bei Temperaturänderungen. Es ist anders als bei $\Delta$ kein Drift von $\Psi$ nach Abschluss der Temperaturbehandlung zu sehen. Im Speziellen ist zu den Zeitpunkten, an denen $\Delta(t)$ plötzlich eine Änderung der Steigung aufweist, keine Auffälligkeit im Verlauf von $\Psi(t)$ zu erkennen. Die Adsorption von Molekülen aus der Luft kann bei der Betrachtung von $\Psi$ also vernachlässigt werden. Man kann somit die gemessenen Werte von $\Psi$ verwenden, um den Brechungsindex von STO bei $T_{D e p}$ zu ermitteln. Dabei kann man ein einfaches optisches Modell wählen, wo man nur die Grenzfläche zwischen dem reinen Substrat und Vakuum betrachtet. Die Reflexion von Licht an dieser wird vollständig durch die Fresnel-Koeffizienten beschrieben (siehe Abschnitt 4.3.1). Neben den Brechungsindizes $N_{V a c}$ und $N_{S T O}$ wird der sich bei der Reflexion einstellende Drehwinkel $\Psi$ auch vom Einfallswinkel $\theta$ bestimmt. Der genaue Wert von $\theta$ wird aus dem gemessenen Wert von $\Psi$ bei Raumtemperatur und dem aus der Literatur bekannten Wert $N_{S T O}\left(20^{\circ} \mathrm{C}\right)=2.3878$ $(\lambda=632.8 \mathrm{~nm})$ 154 bestimmt (siehe Abbildung 4.13). Für das hier gezeigte Experiment ergibt sich so $\theta=62.51(1)^{\circ}$. Mit diesem Winkel und dem Wert von $\Psi$ bei den Depositionsbedingungen ergibt sich der Brechungsindex von STO zu $N_{S T O}\left(930^{\circ} \mathrm{C}\right)=2.34(3)$. Der Fehler wird wesentlich durch den möglichen Drift des Einfallswinkels bei der Temperaturänderung bestimmt, welcher mit $\Delta \theta=0.02(1)^{\circ}$ abgeschätzt wurde. Die Reduktion von $N_{S T O}$ bei höheren Temperaturen ist konsistent mit der thermischen Ausdehnung und damit abnehmenden Dichte von polarisierbaren Ladungen ${ }^{14}$.

\subsubsection{Einfluss des Aerosols auf die Polarisationsmessungen}

Da es bei der Herstellung von Heterostrukturen zu einer wechselnden Dosierung von Präkursorlösungen kommt, ist es notwendig den Einfluss des Aerosols in der Depositionskammer auf die in-situ Messung von $(\Delta, \Psi)$ zu untersuchen. Durch Reflexionen an den Lösungsmitteltröpfchen wird ein Teil des Lichts aus dem Strahlengang gestreut und es kommt so zu einer Reduktion der Intensität. Die Wahrscheinlichkeit, mit der ein Strahl an dem Tröpfchen reflektiert wird und nicht transmittiert, kann dabei nach den Fresnel-Gleichungen von der Polarisation abhängen, so dass sich ein möglicher Einfluss auf die Polarisationsmessungen ergibt. Wenn man eine Leerdeposition durchführt, bei der man nur Dimethylformamid ohne Präkursoren in die Düse dosiert und unter den typischen Depositionsbedingungen auf das Substrat sprüht, beobachtet man nur Änderungen der Intensität $I_{0}$ und keinerlei Veränderungen der berechneten Werte für $(\Delta, \Psi)$ beim Aktivieren der Dosierung.

\footnotetext{
${ }^{14} \mathrm{Im}$ Lorentz-Modell gilt für die dielektrische Funktion $\varepsilon=\varepsilon_{1}+i \varepsilon_{2}$ (mit reellem $\left.\varepsilon_{1}, \varepsilon_{2}\right) \varepsilon_{1}-1 \propto N_{e}, \varepsilon_{2} \propto N_{e}$ mit der Volumendichte der polarisierbaren Elektronen $N_{e}$. Dadurch verringert sich auch $n=\sqrt{\varepsilon}$ bei sinkender Elektronendichte $N_{e}$.
} 

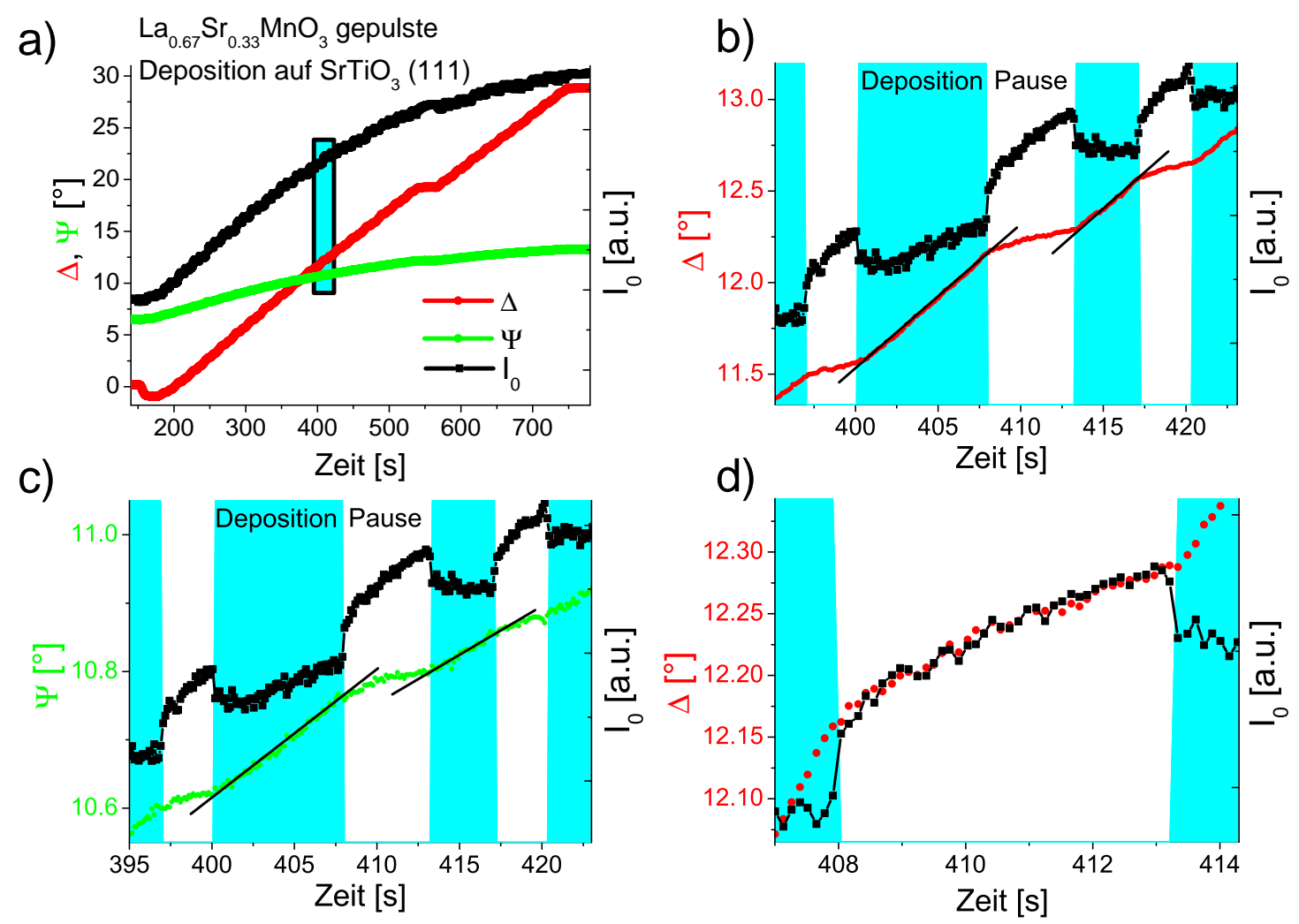

\begin{abstract}
Abbildung 4.14.: Einfluss des Aerosols auf die Messungen von $(\Delta, \Psi)$ wird anhand der gepulsten Deposition eines $\mathrm{La}_{0.67} \mathrm{Sr}_{0.33} \mathrm{MnO}_{3}$ auf einem $\mathrm{SrTiO}_{3}$ (STO) (111)-Substrat überprüft. a) Übersicht der in-situ Aufnahme von $\Delta, \Psi$ und $I_{0}$. Für den in a) markierten Bereich werden in b) und c) die zeitlichen Verläufe von $\Delta$ und $\Psi$ mit den Veränderungen von $I_{0}$ korreliert. Innerhalb der farblich unterlegten Zeitbereiche findet eine Dosierung der Präkursorlösungen statt, in den Bereichen dazwischen ist die Deposition pausiert. In d) sieht man, dass die Relaxation von $I_{0}$ nach Beendigung der Dosierung mit der gleichen Zeitskala abläuft wie das durch $\Delta$ beobachtete Wachstum des Films.
\end{abstract}

Da die Eigenschaften des Aerosols durch einen gelösten Präkursor verändert werden können (Modifikation der Oberflächenspannung, Änderung der optischen Eigenschaften), wurde außerdem ein Versuch durchgeführt, bei dem Material deponiert wird. Man deponiert $\mathrm{La}_{0.67} \mathrm{Sr}_{0.33} \mathrm{MnO}_{3}$ auf einem $\mathrm{SrTiO}_{3}$ (STO) (111)-Substrat mit der in dieser Arbeit zumeist verwendeten Dosiergeschwindigkeit von $p \approx 2.1 \mu \mathrm{l} / \mathrm{s}$ (siehe Abbildung 4.14). Um ähnliche Aerosoleffekte wie bei dem Wachstum einer Heterostruktur zu beobachten, erfolgt die Deposition nicht zusammenhängend. Die Dosierung wird pausiert, wenn Mengen deponiert wurden, die der Dicke von einer bzw. zwei Monolagen entsprechen. Durch die Verwendung von nur einer Komponente wird ausgeschlossen, dass es zur Reaktion von den verschiedenen Materialien in der Heterostruktur kommt, die zu zeitlich veränderlichen Signalen in den Pausen führen könnten. Zudem kann man so beobachten, wie der Depositionsvorgang zeitlich mit der Dosierung der Präkursoren korreliert ist. Die Intensität $I_{0}$ zeigt direkt nach dem Beginn einer Depositionsphase einen sofortigen Einbruch, an den sich ein Bereich anschließt wo $I_{0}$ zunächst abnimmt und danach langsam ansteigt, da sich die Erniedrigung von $I_{0}$ durch die Verdichtung des Aerosols und die Erhöhung der Reflektivität bei Schichtdickenzunahme überlagern (siehe Abbildung 4.14 b), c)). Diese reine Änderung der Intensität wird bei der Berechnung von $\Delta$ 
und $\Psi$ herausnormiert (siehe Gleichungen 4.39 bis $4.41{ }^{15}$. Es gibt keine weiteren Effekte des Aerosols auf den Polarisationszustand des Lichts, da man die sprunghaften Änderungen in der Intensität nicht in $\Delta$ und $\Psi$ wiederfindet. Hätte das Aerosol einen Einfluss auf den Zustand der Polarisation, wäre dieser mit der Dichte des Aerosols in der Kammer und damit mit der Intensitätsänderung korreliert. Während die Lösung in die Düse fließt, zeigen aber $\Delta(t)$ und $\Psi(t)$ einen linearen Verlauf (siehe Abbildungen $4.14 \mathrm{~b}$ ) und c)), man sieht keine Sprünge zu Beginn und Ende der Dosierung wie in $I_{0}$. In diesem Beispiel wird die Intensität durch das Aerosol in der Kammer um $\Delta I_{0} / I_{0} \approx 0.6 \%$ reduziert, die Einflüsse auf die ellipsometrischen Parameter kann man bei dem vorliegenden Rauschen auf $<0.03^{\circ}$ für $\Delta$ und $<0.02^{\circ}$ für $\Psi$ abschätzen.

In den Depositionsphasen ist der Verlauf von $\Delta(t)$ und $\Psi(t)$ perfekt linear, es zeigen sich keine Änderungen der Steigung zu Beginn. Im Rahmen der hier vorhandenen Zeitauflösung ist die Depositionsrate also konstant, das Wachstum der Schicht beginnt instantan mit der Dosierung der Präkursorlösung. In den Pausen zeigt sich ein relaxierendes Verhalten für $I_{0}$, $\Delta$ und $\Psi$ (siehe Abbildung $4.14 \mathrm{~d}$ )), welches sich durch ein langsames Absaugen des Aerosols aus der Kammer ergibt. Gemäß den Ausführungen in Abschnitt 4.4.3 nimmt die Temperatur des Substrates während der Unterbrechungen der Deposition zu, da der kühlende Einfluss des Aerosols dann verschwindet. Aus dieser kleinen Temperaturänderung, $\Delta T \approx 15^{\circ} \mathrm{C}$, resultiert nur eine minimale Änderung des Brechungsindexes, $\Delta n(S T O) \approx 7 \cdot 10^{-4}$, die keinen signifikanten Einfluss auf die hier durchgeführten Messungen besitzt. Die Änderungen in $\Delta$ und $\Psi$ sind mit einem weiteren Wachstum der Schicht zu erklären, welches vom noch in der Kammer vorhandenen Aerosol gespeist wird. Dieser Zusammenhang zeigt sich im gleichen Zeitverhalten der Relaxation von $\Delta(t)$ und $I_{0}(t)$, das in Abbildung 4.14 d) dargestellt wird. Die Pausen zwischen den Depositionsphasen unterschiedlicher Lagen sind also so zu wählen, dass die vom verbleibenden Aerosol gespeiste Deposition abgeklungen ist, um eine künstliche Durchmischung zu vermeiden. Insgesamt kann man so konstatieren, dass die Beobachtungen der in-situ Ellipsometrie während Dosierung der Präkursorlösungen mit Effekten beim Wachstum der Heterostruktur verbunden sind und nicht auf Artefakte wie einer Änderung von $(\Delta, \Psi)$ durch das Aerosol oder einer zeitlich variierenden Depositionsrate zurückzuführen sind.

\subsubsection{Auswertung und Modellierung der in-situ Ellipsometrie}

In Abschnitt 4.3.2 wurde beschrieben, wie die Reflexionskoeffizienten einer Heterostruktur zu berechnen sind. Zur Simulation der in-situ Ellipsometriemessungen muss man also zu jedem Zeitpunkt der Deposition für den aktuellen Zustand der Heterostruktur die Reflexionskoeffizienten $r_{p / s}$ berechnen und dann die gemessen Werte $(\Delta, \Psi)$ über Gleichung 4.3 und $I_{0}$ über Gleichung 4.37 ermitteln. Die genaue Charakterisierung der während des Wachstums eingenommenen Zustände des Übergitters erfordert Informationen über die Wachstumsmode. Bei den hier betrachteten Wachstumsprozessen ergibt sich meist eine Wachstumsmode, die eine Mischung aus einem zweidimensionalen Inselwachstum und step-flow-Wachstum ist. Die lateralen Dimensionen der Inseln sind dabei im Bereich von mehreren $100 \mathrm{~nm}$, wodurch eine Näherung mit einem effektiven Medium nicht in Frage kommt. Auf einer Heterostruktur, die auf den Terrassen eines fehlgeschnittenen Substrates gewachsen ist und deren oberste Lage

\footnotetext{
${ }^{15}$ Bei sehr rapiden Änderungen, die bei großen Depositionsgeschwindigkeiten auftreten, ergibt sich durch die nicht vollständig simultane Messung von $I_{D C}, I_{\omega}$ und $I_{2 \omega}$ ein Fehler bei dieser Normierung, so dass sich die Intensitätsänderungen auch auf $\Delta$ und $\Psi$ auswirken.
} 


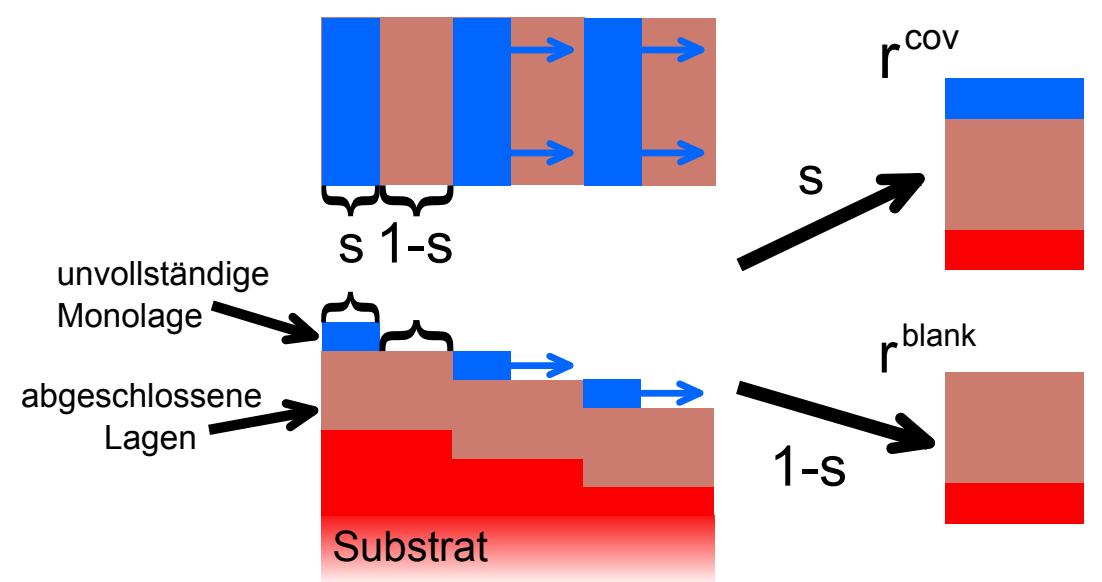

Abbildung 4.15.: Zur Berechnung der Reflexionskoeffizienten bei step-flowWachstumsmode: Bei einer teilweisen relativen Bedeckung $s$ der Terrassen ergeben sich die Reflektivitäten durch die Mittelung über die Heterostruktur mit $\left(r^{c o v}\right)$ und ohne $\left(r^{\text {blank }}\right)$ die teilweise geschlossene Schicht.

komplett abgeschlossen ist, wächst eine weitere Monolage. Diese Monolage bedeckt einen relativen Anteil $s$ der Terrassen (siehe Abbildung 4.15. Die Reflexionskoeffizienten $r_{p / s}^{b l a n k}$ für die Heterostruktur ohne diese teilweise gewachsene Monolage und für eine Heterostruktur, bei der diese zusätzliche Monolage die Terrassen vollständig bedeckt, $r_{p / s}^{c o v}$ lassen sich wie in Abschnitt 4.3.2 berechnen. Nach Muller und Farmer erhält man dann die Reflexionskoeffizienten für die teilweise bedeckte Struktur durch Mittelung [188]:

$$
r_{p / s}^{t o t}=s r_{p / s}^{c o v}+(1-s) r_{p / s}^{b l a n k}
$$

Wenn man dies für jede gewachsene Monolage iterativ ausführt, kann man $r_{p / s}(t)$ während des Wachstums der gesamten Heterostruktur modellieren.

Depolarisationseffekte werden hier nicht berücksichtigt. Es wird angenommen, dass die Strahlung auch nach Reflexion an der Probe eine feste Polarisation aufweist und keine Anteile zufälliger Polarisation enthält. Im Folgenden wird diese Vereinfachung gerechtfertigt, indem die typischen Ursachen für Depolarisationseffekte [140] im Hinblick auf die hier hergestellten Proben diskutiert werden. Zufällige Polarisationen können bei der Wechselwirkung mit der Probe induziert werden, wenn verschiedene Teilstrahlen des auf die Probe treffenden Lichtbündels unterschiedliche Modifikationen der Polarisation erfahren. Die hier betrachteten Proben zeigen nur mikroskopische Rauigkeiten, deren laterale Dimensionen klein sind gegen die Wellenlänge, so dass man diese durch effektive Medien behandeln kann. Durch die leichte Divergenz des Laserstrahls $(\alpha \approx 1 \mathrm{mrad})$ ergibt sich eine Abweichung vom mittleren Einfallswinkel $\Delta \theta \approx \pm 0.06^{\circ}$. Bei diesen kleinen Änderungen verhalten sich $(\Delta, \Psi)$ linear bei einer Änderung des Einfallswinkels und der Effekt auf die Messung mittelt sich heraus.

Eine weitere Quelle der Depolarisierung ergibt sich durch eine zusätzliche Reflexion des Lichts an der Rückseite des Substrates. Die an der Rückseite des Substrates reflektierten Teilstrahlen erhalten beim Durchlaufen der Dicke des Substrates einen zusätzlichen Phasenfaktor. Durch die makroskopische Dicke kommt es schon durch kleine relative Inhomogenitäten der Dicke zu großen Änderungen der Phasenfaktoren und damit Depolarisation. Bei den hier hauptsächlich verwendeten einseitig polierten Substraten kann man die Reflexion an der Rückseite vernachlässigen. Dies kann man überprüfen, wenn man die reflektierte Intensität für ein einseitig poliertes Substrat mit der eines doppelseitig polierten vergleicht. Zur Berechnung dieser 


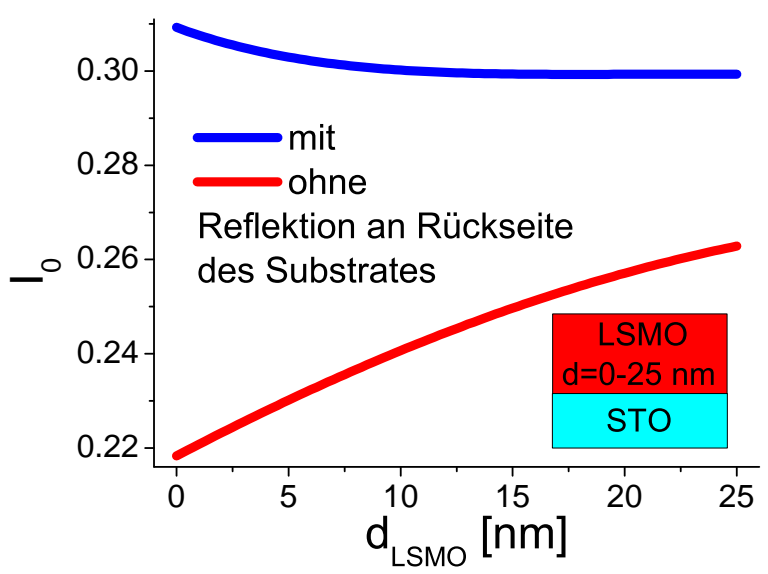

\begin{abstract}
Abbildung 4.16.: Zur Diskussion der Reflexion an den Rückseiten der Substrate: $I_{0}$ (vgl. Gleichung 4.37) wird berechnet als Funktion der Dicke $d_{L S M O}=0-25 \mathrm{~nm}$ einer $\mathrm{La}_{0.7} \mathrm{Sr}_{0.3} \mathrm{MnO}_{3}$ (LSMO) Schicht auf $\mathrm{SrTiO}_{3}$ (STO), wobei in einem Fall Reflexionen an der Rückseite des Substrates zugelassen werden, im anderen Fall werden diese vernachlässigt und die ins Substrat transmittierten Strahlen werden nicht weiter betrachtet $\left(N_{L S M O}=2.31-i \cdot 0.55, \theta=62.5^{\circ}\right)$. Aufgrund der makroskopischen Dicke des Substrates wird zwischen den an der Rückseite reflektierten Strahlen und den an den beiden oberen Grenzflächen reflektierten Strahlen keine feste Phase angenommen, sondern über alle Phasen von $0^{\circ}$ bis $360^{\circ}$ gemittelt.
\end{abstract}

Intensitäten geht man grundsätzlich vor, wie es in Abschnitt 4.3 .2 beschrieben wurde. Allerdings mittelt man bei der Betrachtung der Reflexionen an der Rückseite des Substrates über verschiedene Dicken des Substrates. Der Dickenbereich, über den man mittelt, wird so gewählt, dass die Interferenz zwischen den an der Rückseite reflektierten Strahlen mit den anderen Teilstrahlen am Anfang und Ende des Bereichs der Mittelung destruktiv ist. In Abbildung 4.16 sind die so berechneten Intensitäten $I_{0}$ für die beiden Fälle bei einem mit einer $\mathrm{La}_{0.7} \mathrm{Sr}_{0.3} \mathrm{MnO}_{3}$ (LSMO) Schicht versehenen $\mathrm{SrTiO}_{3}$ (STO) Substrat dargestellt. Bei Einbeziehung der rückseitigen Reflexion sinkt die Reflektivität mit steigender Dicke des LSMO, da der an der Rückseite reflektierte Strahl im Film absorbiert wird. Bei Vernachlässigung dieser Reflexion ist ein monotoner Anstieg von $I_{0}$ zu beobachten, der sich auch im Experiment zeigt (siehe Abbildung 4.17). Wenn man das Verhältnis zwischen der insgesamt reflektierten Intensität unter Einbeziehung der rückseitigen Reflexion, $I_{0}^{\text {Back }}$, und unter Vernachlässigung dieser, $I_{0}^{\text {Surf }}$, berechnet, ergibt sich $I_{0}^{\text {Back }} / I_{0}^{\text {Surf }}=1.41$. Dies stimmt mit dem experimentell ermittelten Wert von $I_{0}^{\text {Back }} / I_{0}^{\text {Surf }}=1.42(2)$ überein, den man durch die in-situ Messungen eines einseitig und doppelseitig polierten Substrates erhält. Die rückseitigen Reflexionen an den hier verwendeten Substraten sind also vernachlässigbar für die hier vorgestellten Ellipsometriemessungen.

Die gemessenen Daten $I_{D C}, I_{\omega}$ und $I_{2 \omega}$ werden mit Gleichungen 4.39 bis 4.41 in $\Delta$ und $\Psi$ umgerechnet. Beim transparenten STO-Substrat ergibt sich theoretisch ein verschwindender Wert von $\Delta$. Der bei der Messung beobachtete Wert zu Beginn der Deposition $\Delta_{0}$ wird durch Imperfektionen der optischen Komponenten verursacht. Diese rein apparative Verschiebung von $\Delta(t)$ subtrahiert man von den gemessenen Daten. Den genauen Einfallswinkel $\theta$ kann man mit den Fresnel-Gleichungen aus dem Startwert von $\Psi$ und dem in Abschnitt 4.4 .4 ermittelten Wert für den Brechungsindex $N_{S T O}$ für das STO-Substrat bei Depositionstemperatur berechnen. Für eine einfache Schicht auf einem Substrat mit bekanntem Brechungsindex kann man durch numerische Inversion aus den Messwerten von $(\Delta, \Psi)$ den durchschnittlichen Brechungsindex bestimmen. Dafür verwendet man die alglib-Bibliothek für Numerik unter Visual $\mathrm{C} \#$ [189]. 


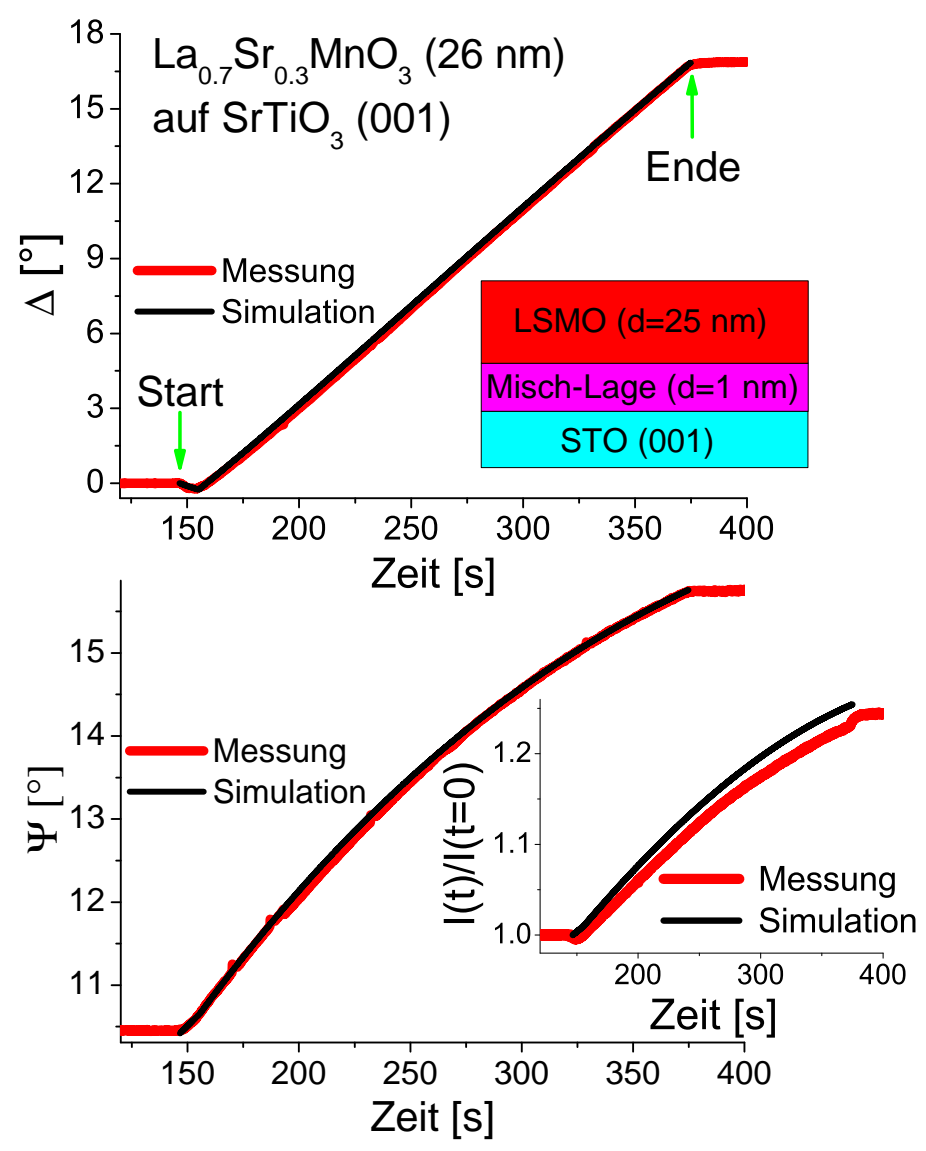

\begin{abstract}
Abbildung 4.17.: Simulation der in-situ Ellipsometrie für das Wachstum einer Schicht aus $\mathrm{La}_{0.7} \mathrm{Sr}_{0.3} \mathrm{MnO}_{3}$ (LSMO) auf $\mathrm{SrTiO}_{3}$ (STO) (001): Die gemessenen Daten für $(\Delta, \Psi)$ und der reflektierten DC-Intensität $I=I_{D C}=I_{0}$ wurden durch das Wachstum von zwei Schichten, einer gemischten Lage der Dicke $d_{m i x}=1 \mathrm{~nm}$ und dem Brechungsindex $N_{m i x}=$ $2.47(4)-i \cdot 0.35(2)$ und einer reinen Manganat-Lage $N_{L S M O}=2.29(1)-i \cdot 0.57(2)$, für einen Einfallswinkel $\theta=60^{\circ}$ beschrieben.
\end{abstract}

In Abbildung 4.17 ist ein Beispiel für die in-situ gemessene Ellipsometrie während der Deposition einer $\mathrm{La}_{0.7} \mathrm{Sr}_{0.3} \mathrm{MnO}_{3}$ (LSMO)-Schicht auf einem $\mathrm{SrTiO}_{3}$ (STO) (001)-Substrat zusammen mit numerisch simulierten Kurven für $\Delta(t), \Psi(t)$ und $I_{0}(t)$ gezeigt. Neben einer Manganat-Schicht der Dicke $d_{L S M O}=25 \mathrm{~nm}$ wird eine zusätzliche Mischlage der Dicke $d_{\text {mix }}=1 \mathrm{~nm}$ eingeführt, die zu Beginn auf dem STO-Substrat wächst. In Abschnitt 6.3 wird genauer auf die Mischlage zwischen Manganat-Filmen und STO sowie deren Einfluss auf die in-situ Ellipsometrie eingegangen. Die simulierten Kurven von $\Delta$ und $\Psi$ passen sehr gut zu den experimentellen Kurven, was auf die Gültigkeit der angenommenen Wachstumsmode zwischen step-flow-Wachstum und zweidimensionalem Inselwachstum über die gesamte Messzeit hindeutet. Die Abweichungen, die sich für den Verlauf von $I_{0}(t)$ ergeben, werden durch das Aerosol verursacht, das sich während der Deposition langsam in der Kammer verteilt und damit $I_{0}$ modifiziert (siehe Abbildung 4.17). Man sieht aber, dass der Endwert von $I_{0}$, der nach dem Absaugen des Aerosols erreicht wird, gut mit den berechneten Werten übereinstimmt. Für $I_{0}$ ist der Fehler allerdings größer, da diese Größe keiner Selbstnormierung unterworfen ist wie $(\Delta, \Psi)$ und somit Fehlerquellen wie Drift der Laserintensität oder kleine Bewegungen der Elemente im Strahlengang eine Rolle spielen. 


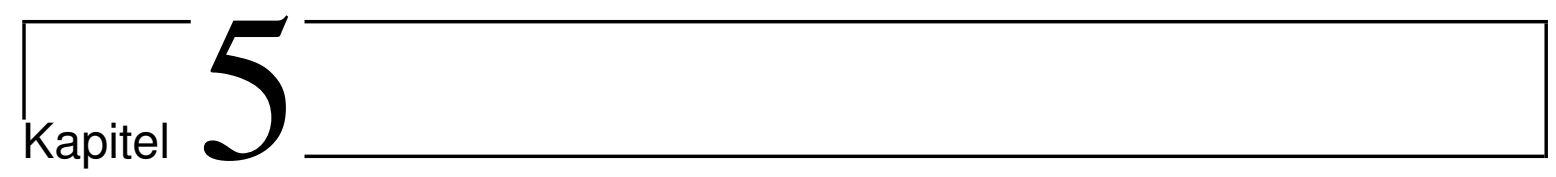

\section{Charakterisierung}

Neben den Charakterisierungen mittels Ellipsometrie während des Wachstums werden die hier vorgestellten Heterostrukturen nach der Deposition mit weiteren Methoden untersucht. Mit Methoden der Rastersondenmikroskopie werden die Oberflächen untersucht, wodurch auf die vorherrschende Wachstumsmode geschlossen werden kann. Die anschließenden strukturellen Untersuchungen durch Röntgenbeugung und Transmissionselektronenmikroskopie erlauben eine Analyse der Struktur in der gesamten Heterostruktur. Für die Untersuchung von Grenzflächeneffekten sind dabei besonders die Verteilungen der Kationen an den Grenzflächen interessant, welche sich durch numerische Simulationen der Röntgenbeugungsspektren ergeben. Die funktionalen Eigenschaften werden durch feld- und temperaturabhängige Messungen der Magnetisierung und des elektrischen Widerstandes bestimmt.

\subsection{Rastersondenmikroskopie (Tunnelmikroskopie, Kraftmikroskopie)}

Mit den hier verwendeten Rastersondenmethoden, der Rastertunnelmikroskopie (STM) und der Rasterkraftmikroskopie, wurden Höhenprofile der hier hergestellten Proben ausgemessen. Bei der Rastertunnelmikroskopie nutzt man aus, dass der Tunnelstrom zwischen einer metallischen Spitze und der Probe exponentiell mit steigendem Abstand absinkt. Wenn man nun die Spitze über die Oberfläche der Probe bewegt und die Höhe der Spitze so anpasst, dass der Tunnelstrom konstant bleibt (constant current-Modus), kann man aus den Höhenanpassungen eine topographische Karte der Oberfläche rekonstruieren. Die Tunnelmikroskopie ist nur auf elektrisch leitende Proben anwendbar, bei isolierenden Proben wie $\mathrm{SrTiO}_{3}$-Substraten wird die Kraftmikroskopie herangezogen. Bei dieser wird eine Si-Spitze über die Probe bewegt, die sich am Ende eines Biegebalkens (cantilever) befindet. Die Kraft auf die Spitze wird durch die Verbiegung des cantilevers detektiert (contact mode) und ist abhängig vom Abstand zwischen Probe und Spitze. Damit kann man analog zur Tunnelmikroskopie eine Aufnahme der Topographie gewinnen, indem man den Abstand zwischen Spitze und Probe regelt, so dass die Kraft konstant bleibt. Durch die exponentielle Abstandsabhängigkeit des Tunnelstroms ist nur ein kleiner Teil der metallischen Spitze relevant für die Abtastung der Oberfläche (nur wenige Atome). Dadurch kann die laterale Auflösung auch atomare Dimensionen erreichen [190]. Bei der Kraftmikroskopie bewirkt die komplexe Kontaktmechanik, 


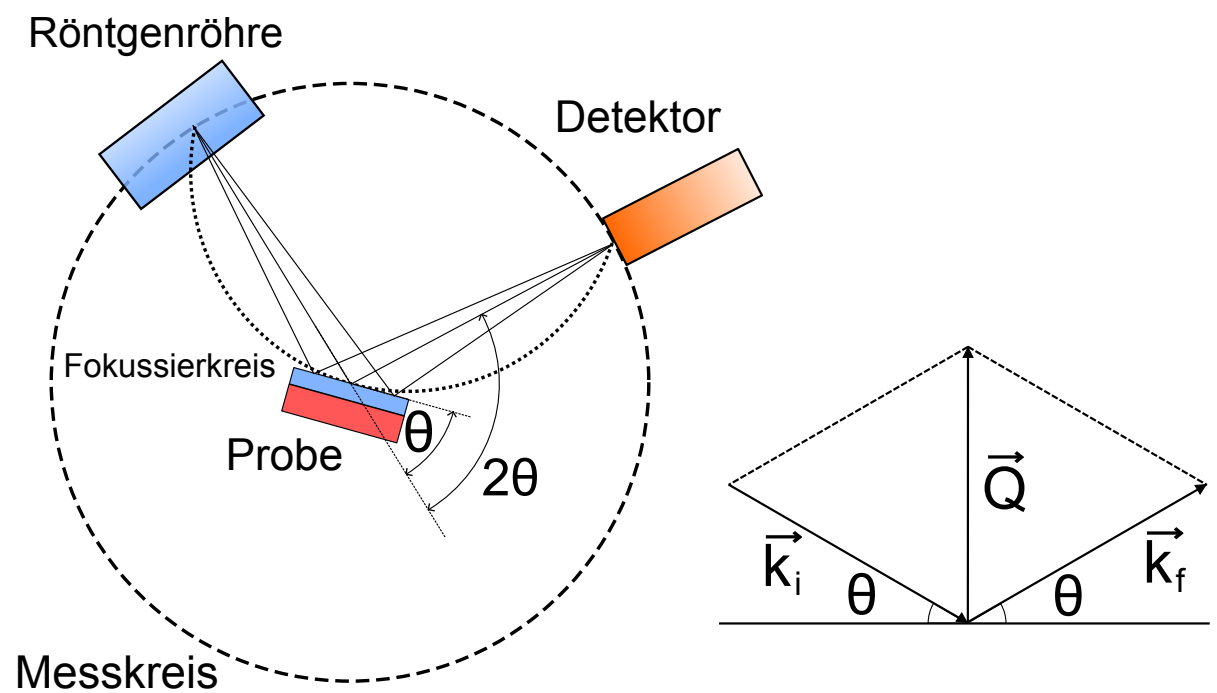

Abbildung 5.1.: Bei der Röntgenbeugung verwendete Bragg-Brentano-Geometrie und Definition des Streuvektors $\vec{Q}$ mit den Vektoren für die unter dem Winkel $\theta$ auf die Probenoberfläche treffende Welle $\vec{k}_{i}$ und die spekular gestreute Welle $\vec{k}_{f}$.

dass ein effektiver Spitzenradius von $d \approx 20-50 \mathrm{~nm}$ mit der Probe wechselwirkt [191, 192 . Somit sind die hier gezeigten STM-Aufnahmen deutlich feiner aufgelöst als die RasterkraftAufnahmen, bei denen deutliche laterale Mittelungen zu erkennen sind. Die meisten STMund Rasterkraft-Bilder wurden mit einem kommerziellen Gerät des Typs Nanoscope IV der Firma Veeco Instruments 191 erstellt. Da dieses maximal Bilder mit einer Kantenlänge von $5 \mu \mathrm{m}$ aufnehmen kann, wurde für größere Aufnahmen mit Kantenlängen bis zu $100 \mu \mathrm{m}$ außerdem ein Rasterkraftmikroskop von Bruker verwendet [192].

\subsection{Röntgenmethoden}

Die Röntgenmessungen wurden mit dem kommerziellen Diffraktometer D8 Advance der Firma Bruker AXS durchgeführt 164. Aus einer Röntgenröhre mit Cu-Anode kommend trifft die Strahlung unter einem Winkel $\theta$ auf die Probe. Die spekular gestreute Strahlung wird dann in einem Aufbau, der der Geometrie von Bragg und Brentano folgt, im Detektor gesammelt (siehe Abbildung 5.1). Das Spektrum der verwendeten Strahlung wird von den $\mathrm{Cu}-\mathrm{K}_{\alpha}$-Linien dominiert. Es liegt jedoch kein monochromatisches Spektrum vor, es gibt weitere charakteristische Linien wie $\mathrm{Cu}-\mathrm{K}_{\beta}$ und $\mathrm{W}-\mathrm{L}_{\alpha / \beta}$, die durch Verunreinigungen der Anode verursacht werden, sowie einen Untergrund aus Bremsstrahlung. Da die Verunreinigungen des Spektrums eine deutlich kleinere Intensität aufweisen als der von der $\mathrm{Cu}-\mathrm{K}_{\alpha}$-stammende Anteil, ergeben sich durch diese nur bei der Messung von sehr dicken Schichten bzw. Substraten zusätzliche Linien im gemessenen Spektrum. In Spektren von dünnen Schichten kann man die vom Substrat stammenden parasitären Linien durch die Messung eines unbeschichteten Substrates identifizieren. Später wird ohne weitere Erwähnung ein solches Spektrum mit den Spektren der Heterostrukturen gezeigt bzw. das Spektrum des Substrates subtrahiert, um die Teile des Spektrums zu erkennen, die von den deponierten Lagen stammen und die Intensitäten der Schichtpeaks exakt zu ermitteln. Die $\mathrm{Cu}-\mathrm{K}_{\alpha}$-Strahlung spaltet aufgrund der Spin-Bahn-Kopplung in $\mathrm{Cu}-\mathrm{K}_{\alpha 1}$ der Wellenlänge $\lambda_{K_{\alpha 1}}=0.1540593 \mathrm{~nm}$ und $\mathrm{Cu}-\mathrm{K}_{\alpha 2}$ der 
Wellenlänge $\lambda_{K_{\alpha 2}}=0.1544427 \mathrm{~nm}$ auf. Bei der Beugung an dünnen Schichten kann man diese Wellenlängen aufgrund der Linienverbreiterung nicht voneinander trennen und man rechnet mit dem gewichteten Mittelwert $\lambda_{K_{\alpha}}=0.1541871 \mathrm{~nm}$ [164].

In diesem Abschnitt werden separat die Röntgenbeugung (XRD), mit der man durch Messung der gestreuten Strahlung im Weitwinkelbereich $\left(2 \theta \gtrsim 10^{\circ}\right)$ Informationen über die Gitterstruktur erhält, und die Röntgenreflektometrie (XRR), wo man durch Messungen im Kleinwinkelbereich $\left(2 \theta \lesssim 10^{\circ}\right)$ die chemische Modulation in Heterostrukturen erfassen kann, behandelt. Bei allen Messungen schließen der einlaufende und gestreute Strahl mit der Probenoberfläche den gleichen Winkel $\theta$ ein, so dass der Streuvektor $\vec{Q}$ senkrecht auf der Probenoberfläche steht. Es werden also ausschließlich Strukturen entlang der c-Achse abgetastet. Der Röntgenstrahl wird durch Spaltblenden vor und nach Wechselwirkung mit der Probe eingeschränkt 164. Hier werden bei allen Messungen Spaltbreiten von $w=0.2 \mathrm{~mm}$ verwendet. Dies führt zu einer deutlichen Verkleinerung des Bereichs, der vom Strahlbündel getroffen wird, auf eine winkelabhängige Breite $b_{\text {Spalt }}=\frac{w}{\sin \theta}$. Bei den Kleinwinkelmessungen wird zudem eine Schneidblende mit einem Abstand von $h=10-30 \mu \mathrm{m}$ vor der Probe positioniert, um den Anteil der Probe, der bei streifendem Einfall berücksichtigt wird, auf eine Breite $b_{\text {Schneid }}=\frac{2 h}{\tan \theta}$ einzuschränken. Für Einfallswinkel $\theta_{s} \lesssim 1.4^{\circ}$ ist die Breite des Strahlbündels auf der Probe bei den gewählten Blendeneinstellungen größer als die Breite des beschichteten Bereichs von ca. $8 \mathrm{~mm}$, so dass die gemessene Intensität für $\theta<\theta_{s}$ kleiner wird als in den Simulationen. Die Kombination von Schneid- und Spaltblende bewirkt nur eine schwache Modifikation von der winkelabhängigen Intensitätsverteilung $I(\theta)$, da $b_{\text {Schneid }} / b_{\text {Spalt }} \propto \cos \theta \approx 1$ für kleine $\theta$.

\subsubsection{Röntgenbeugung (XRD)}

In der kinematischen Theorie der Beugung werden nur einfache Wechselwirkungen der Strahlung mit Materie berücksichtigt ${ }^{16}$. Der eintreffende Röntgenstrahl regt die Elektronen im Material an, so dass von der vorhandenen Elektronenverteilung, die durch eine Elektronendichte $n(\vec{r})$ beschrieben wird, Kugelwellen ausgehen. Bei einem Streuvektor $\vec{Q}$ (siehe Abbildung 5.1 ) ist die Phasenverschiebung zwischen einer im Ursprung und an der Stelle $\vec{r}$ gestreuten Welle im Fernfeld $\Delta \phi=\vec{Q} \cdot \vec{r}$. Die Interferenz dieser Wellen resultiert in folgender Feldamplitude:

$$
A(\vec{Q}) \propto \int_{V} n(\vec{r}) e^{-i \vec{Q} \cdot \vec{r}} \mathrm{~d} V
$$

Die gemessene Intensität erhält man durch Bildung des komplexen Betragsquadrates

$$
I(\vec{Q})=|A(\vec{Q})|^{2}
$$

Kristalline Strukturen kann man durch ein Bravais-Gitter der Form

$$
\vec{R}\left(n_{1}, n_{2}, n_{3}\right)=n_{1} \vec{a}_{1}+n_{2} \vec{a}_{2}+n_{3} \vec{a}_{3}
$$

\footnotetext{
${ }^{16}$ Bei den dünnen Filmen, die man hier betrachtet, ist diese Näherung für die Geometrie bei der Röntgenbeugung gerechtfertigt. Wenn dickere Filme $d>200 \mathrm{~nm}$ untersucht werden, führt die Absorption der gestreuten Strahlung im Film zu einer Verbreiterung der Bragg-Peaks im Vergleich zur Simulation im Rahmen der kinematischen Näherung. Der Fall des streifenden Einfalls wird im Abschnitt über Reflektometrie behandelt, wo eine rein kinematische Betrachtung ebenso zu ungenauen Ergebnissen führt 193.
} 
mit ganzzahligen $n_{i}$ und den primitiven Gittervektoren $\vec{a}_{i}$ sowie einer Basis mit $d$ Atomen, die sich an den Positionen $\vec{r}_{j}$ befinden, beschreiben. Wenn man daraus die Ladungsdichte $n(\vec{r})$ konstruiert, kann man die im Fernfeld messbare Feldamplitude in zwei Faktoren aufspalten:

$$
A(\vec{Q}) \propto\left(\sum_{n_{1}, n_{2}, n_{3}} e^{-i \vec{R} \cdot \vec{Q}}\right) \cdot\left(\sum_{j} f_{j} e^{-i \vec{r}_{j} \cdot \vec{Q}}\right)
$$

Mit $f_{j}$ werden die atomaren Streufaktoren bezeichnet, welche durch

$$
f_{j}=\int n_{j}(\vec{r}) e^{-i \vec{r} \cdot \vec{Q}} \mathrm{~d} V
$$

definiert sind. Dabei ist $n_{j}(\vec{r})$ die Elektronendichteverteilung der Atomsorte, die sich an Position $j$ der Basis befindet. Der erste Faktor in Gleichung 5.4 wird durch die Gitterstruktur des Materials bestimmt. Mit der Konstruktion des reziproken Gitters kann man aus diesem die $\vec{Q}$-Vektoren ableiten, für die Beugungsmaxima erscheinen [48. Für eine kubische Gitterstruktur mit Gitterparameter $a$ ergibt sich daraus dann die Bragg-Bedingung für die Position der Maxima in den winkelabhängigen Röntgenspektren

$$
2 d_{h k l} \sin \theta=n \lambda \text { mit } d_{h k l}=\frac{a}{\sqrt{h^{2}+k^{2}+l^{2}}}
$$

mit der Ordnung $n=1,2, \ldots$ und den Millerschen Indizes $(\mathrm{h}, \mathrm{k}, \mathrm{l})$.

Der zweite Faktor in Gleichung 5.4. der Strukturfaktor, hängt vom genauen Aufbau der Basis ab. Dieser beeinflusst die Intensitätsverhältnisse der Reflexe, die sich durch die BraggBedingung ergeben. Eine quantitative Auswertung der Intensitätsverhältnisse erlaubt Rückschlüsse auf die chemische Zusammensetzung oder die Quantifizierung einer etwaigen Ordnung der Atome in der Basis. Für die genaue Berechnung der Intensitäten muss man aber zusätzliche Faktoren berücksichtigen: Zunächst sind die atomaren Streufaktoren keine Konstanten, sondern zeigen eine Abhängigkeit vom Streuvektor [194 196]. Man kann diese Abhängigkeit numerisch mit hoher Genauigkeit berechnen und mit einem empirischen Gesetz der Form

$$
f(\sin \theta / \lambda)=\sum_{i=1}^{4} a_{i} \exp \left(-b_{i} \sin ^{2} \theta / \lambda^{2}\right)+c
$$

anpassen [196. Die Parameter $a_{i}, b_{i}$ und $\mathrm{c}$ sind in [196 tabelliert und hängen im hier betrachteten Fall der nichtresonanten Anregung mit harter Röntgenstrahlung nicht von der Umgebung des jeweiligen Ions ab, sondern nur von dem betrachteten Element und dessen Ionisationszustand ab. Eine weitere Winkelabhängigkeit der Intensität folgt aus der endlichen Breite der Ewaldkugeloberfläche und der Punkte im reziproken Raum, welche sich durch experimentelle Verbreiterungen der Beugungslinien ergeben. Dadurch wird die Schnittfläche zwischen der Kugelfäche der Ewaldkugel und den Punkten im reziproken Raum und damit die Intensität des betreffenden Reflexes abhängig vom Einfallswinkel, was durch den LorentzFaktor

$$
I \propto \frac{1}{\sin ^{2} \theta \cos \theta}
$$

berücksichtigt wird 194, 195. Die hier verwendete Röntgenstrahlung der Cu-Röhre ist unpolarisiert, was durch einen Faktor nach Thompson für die Streuung unpolarisierter Strahlung einbezogen wird 194 196:

$$
I \propto 1+\cos ^{2} 2 \theta
$$

In der Literatur werden noch weitere Faktoren für eine winkelabhängige Korrektur der Intensitäten aufgeführt. Diese werden hier aber nicht weiter beachtet [194, 195], da sie entweder 


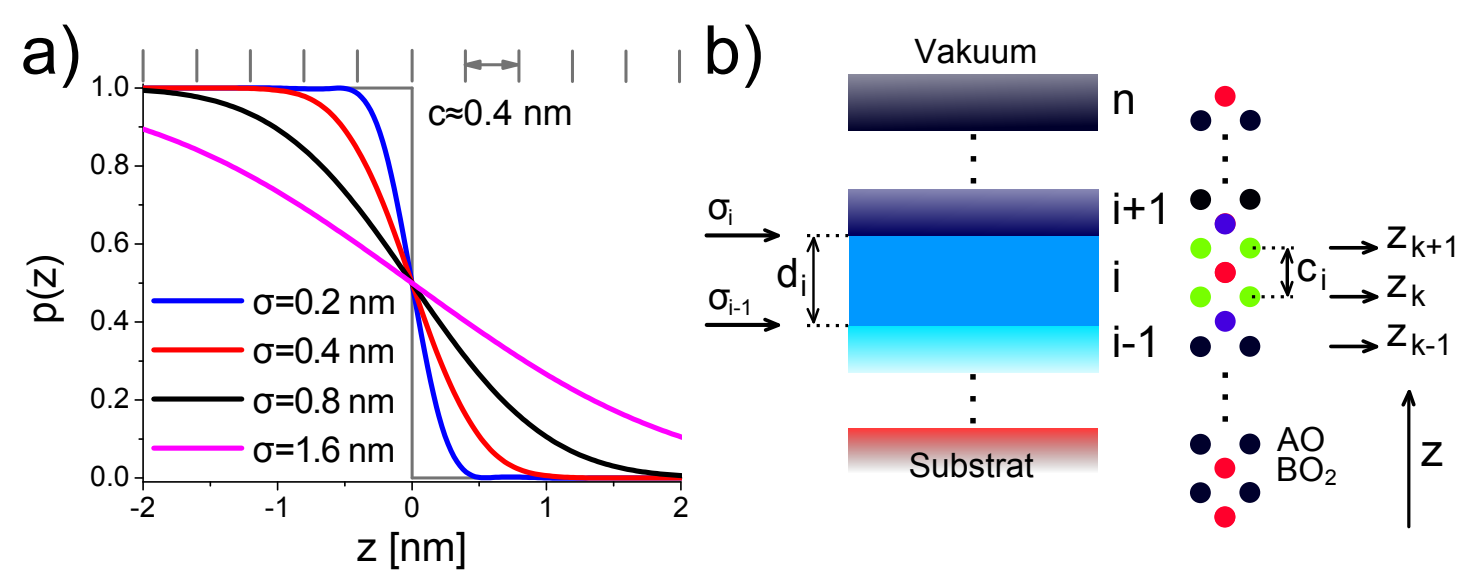

\begin{abstract}
Abbildung 5.2.: Zur Simulation der Röntgenbeugungsspektren: a) Illustration der Durchmischungsprofile an einer rauen Grenzfläche bei $z=0$ für verschiedene Rauigkeiten $\sigma$. Zur Orientierung sind vertikale Linien gezeichnet, deren Abstand im Bereich einer Gitterkonstanten $c \approx 0.4 \mathrm{~nm}$ liegen. b) Darstellung der Größen, die zur atomaren Konstruktion der entlang der (001)-Richtung gewachsenen Heterostrukturen aus Perowskit-Oxiden betrachtet werden: Die Materiallagen mit $i=1-n$ weisen eine Dicke $d_{i}$ auf. Entlang der (001)-Richtung besteht das Perowskit aus $\mathrm{AO}$ - und $\mathrm{BO}_{2}$-Lagen, für die die chemische Zusammensetzung in Lage $i$ durch $A_{i}$ und $B_{i}$ bestimmt wird. Der Gitterparameter in Lage $i$ ist durch $c_{i}$ gegeben.
\end{abstract}

zur Beschreibung der Beugung an Polykristallen vorgesehen sind oder zu vernachlässigbaren Veränderungen der Intensitätsverhältnisse für die hier betrachteten Spektren führen (z.B. Debye-Waller-Faktor).

Bei der Röntgenbeugung von Übergittern, die aus einer periodischen Anordnung von Lagen verschiedener Materialien bestehen, erscheinen neben den Hauptpeaks, die zum durchschnittlichen Gitterebenenabstand $\bar{d}$ gehören, weitere Satellitenpeaks. Aus den Positionen dieser Peaks lässt sich die Periode, $\Lambda$, der Heterostruktur berechnen. Diese entspricht der Dicke des sich in der Heterostruktur periodisch wiederholenden Elements. Nach [197] sind die Positionen der Beugungsmaxima über

$$
2 \frac{\sin \theta}{\lambda}=\frac{1}{\bar{d}}+\frac{n}{\Lambda}
$$

mit $\Lambda$ verbunden, wobei $n$, eine ganze Zahl, die Ordnung der jeweiligen Peaks bezeichnet.

Eine genauere Betrachtung der Intensitäten der Satellitenpeaks erlaubt zusätzlich Aussagen über die Gitterparameter und die genauen Schichtdicken der einzelnen Lagen innerhalb des sich periodisch wiederholenden Elementes. Außerdem lassen sich die chemischen Rauigkeiten abschätzen. Dafür wurde im Rahmen dieser Arbeit ein Computerprogramm zur numerischen Simulation der Beugungsspektren von Heterostrukturen aus Perowskit-Oxiden in Visual C\# implementiert. Für das Wachstum entlang der (001)-Richtung nimmt man tetragonale Einheitszellen an, wo die Modulation der Gitterkonstanten allein durch eine Veränderung der Bindungslängen bewirkt wird. Eine Verkippung der Oktaeder wird komplett vernachlässigt. Da die $\mathrm{O}^{2-}$-Ionen eine deutlich kleinere Gesamtelektronenanzahl aufweisen als die Kationen, führt die Verkippung der Oktaeder nur zu kleinen Modifikationen der Spektren, was bei den hier durchgeführten Beugungsexperimenten nicht aufzulösen ist. Eine Heterostruktur wird dann durch $n$ Lagen aufgebaut, für die folgende Parameter definiert werden $(i=1-n)$ : 
- Dicke der Lage $d_{i}$.

- Chemische Zusammensetzung der Kationen auf dem A-Platz $A_{i}$.

- Chemische Zusammensetzung der Kationen auf dem B-Platz $B_{i}$.

- Rauigkeit zur in Wachstumsrichtung nächsten Lage $\sigma_{i}$.

- Gitterparameter in c-Richtung $c_{i}$.

Der Index $i$ wird in Wachstumsrichtung hochgezählt (siehe Abbildung 5.2). Die Rauigkeiten an den Grenzflächen der Lage $i$ führen zu einer Beimischung von Ionen aus den benachbarten Lagen $i-1$ und $i+1^{17}$. Zur Behandlung der Unschärfe der Grenzflächen nimmt man eine Gaußverteilung der Gesamtschichtdicke $z$ am Ende der Lage $i$ mit der Standardabweichung $\sigma_{i}$ um ihren Mittelwert $d_{i}^{t o t}$ an:

$$
w_{i}(z)=\frac{1}{\sqrt{2 \pi \sigma_{i}^{2}}} e^{-\frac{\left(z-d_{i}^{t o t}\right)^{2}}{2 \sigma_{i}^{2}}}
$$

Durch Integration erhält man dann die Wahrscheinlichkeit

$$
p_{i}(z)=1-\frac{1}{2}\left(1+\operatorname{erf}\left(\frac{z-d_{i}^{t o t}}{\sqrt{2 \sigma_{i}^{2}}}\right)\right),
$$

mit der man an Position $z$ in der Heterostruktur (hier werden wieder nur die Lagen mit den Indizes $i-1, i$ und $i+1$ berücksichtigt) Atome aus der Lage $i$ findet. Mit den Wahrscheinlichkeiten $p_{i}(z)$ werden dann die mittleren chemischen Zusammensetzungen $A(z), B(z)$ und die Gitterparameter $c(z)$ für die entsprechenden Atomlagen, die sich an der Position $z$ befinden, berechnet (siehe Abbildung 5.2. Innerhalb der Materiallage mit dem Index $i$ ergibt sich so die folgende Rechenvorschrift für den Gitterparameter $c(z)$ :

$$
c(z)=c_{i-1} p_{i-1}(z)+c_{i}\left(p_{i}(z)-p_{i-1}(z)\right)+c_{i+1}\left(1-p_{i}(z)\right)
$$

Diese wird analog für die Zusammensetzung auf dem A- und B-Platz angewendet, um die mittlere chemische Zusammensetzung der jeweiligen Atomlage an der diskreten Position $z_{k}$ (siehe Abbildung 5.2 festzulegen.

Für eine nach der obigen Beschreibung konstruierte Struktur kann man dann unter Anwendung von Gleichung 5.4 und den winkelabhängigen Korrekturen der Intensität gemäß den Gleichungen 5.7 5.9 die Beugungsspektren numerisch berechnen. Anhand der gemessenen Daten eines $\mathrm{LaMnO}_{3} / \mathrm{SrMnO}_{3}$-Übergitters soll diskutiert werden, welche Informationen über die Heterostrukturen aus der Anpassung der Beugungsspektren im Weitwinkelbereich extrahiert werden können und die Sensitivität auf mögliche Variationen der angepassten Größen aufgezeigt werden. Das Übergitter wurde auf einem $\mathrm{SrTiO}_{3}$ (STO) (001)-Substrat mit elf Wiederholungen des Elements, das aus einer Lage $\mathrm{LaMnO}_{3}$ der Dicke $d_{L M O}$ und einer Lage $\mathrm{SrMnO}_{3}$ mit der Dicke $d_{S M O}$ besteht, gewachsen. In Abbildung 5.3 a) sieht man das Beugungsspektrum der Heterostruktur in der Umgebung des STO (001)-Peaks (es wurde das Spektrum eines unbeschichteten STO-Substrates subtrahiert). Die Anpassung eines simulierten Spektrums liefert dann die strukturellen Informationen über das Übergitter: die individuellen Schichtdicken, $d_{L M O}, d_{S M O}$, die Gitterkonstanten in c-Richtung, $c_{L M O}, c_{S M O}$ und die Rauigkeit zwischen den Lagen $\sigma_{L M O / S M O}$ (siehe Abbildung 5.3 a)). Man betrachtet hier

\footnotetext{
${ }^{17}$ Durchmischungen mit anderen Lagen als den direkten Nachbarn werden vernachlässigt, da bei Heterostrukturen mit solch einer starken chemischen Durchmischung die genaue Analyse der Beugungsspektren aufgrund des Fehlens von Übergitterreflexen nicht sinnvoll ist.
} 
a)

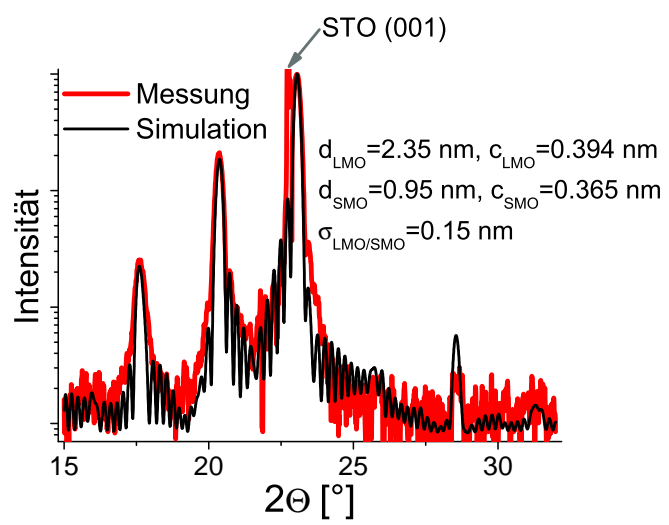

c)

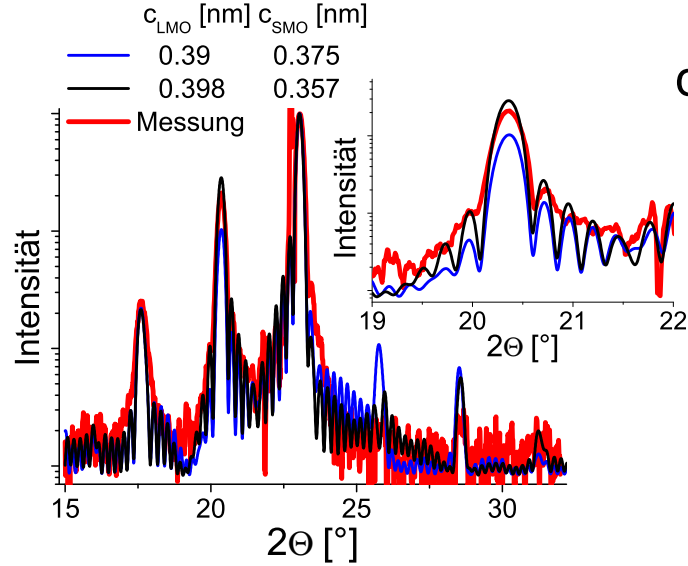

b)

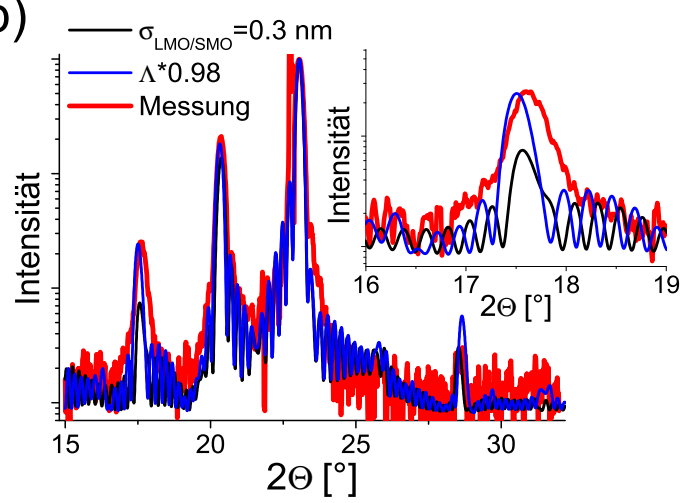

- Messung $\mathrm{d}_{\text {LMO }}[\mathrm{nm}] \mathrm{c}_{\text {LMO }}[\mathrm{nm}] \mathrm{d}_{\text {SMO }}[\mathrm{nm}] \mathrm{c}_{\text {SMO }}[\mathrm{nm}]$

d) $\longrightarrow \begin{array}{llll}2.6 & 0.392 & 0.7 & 0.363 \\ 2.1 & 0.394 & 1.2 & 0.372\end{array}$

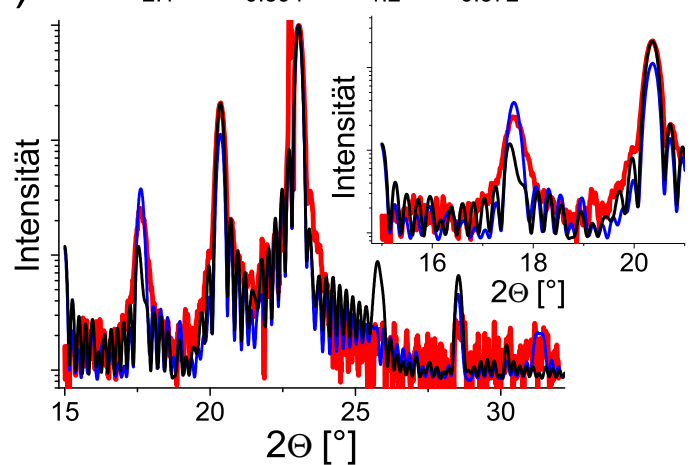

Abbildung 5.3.: Simulation der Weitwinkelbeugung eines $\mathrm{LaMnO}_{3} / \mathrm{SrMnO}_{3}$-Übergitters mit elf Wiederholungen der Doppellage, das auf einem $\mathrm{SrTiO}_{3}$ (001)-Substrat gewachsen wurde: a) Experimentelle Messung mit der numerisch simulierten Anpassung mit den in der Abbildung aufgeführten Parametern. Veränderungen der simulierten Spektren bei Variation einzelner struktureller Parameter unter Fixierung der anderen: b) Grenzflächenrauigkeit $\sigma_{L M O / S M O}$ und Bilagendicke $\left.\Lambda, \mathrm{c}\right)$ individuelle Gitterparameter $c_{S M O}, c_{L M O}$ bei fester durchschnittlicher Gitterkonstanten $\bar{c}$, d) $d_{L M O}, d_{S M O}$ bei festem $\bar{c}$ und $\Lambda$. Die veränderten Parameter sind in der jeweiligen Abbildung notiert und die Insets zeigen vergrößerte Bereiche zur Illustration der sich ergebenden Abweichungen von der Messung bei Modifikation der strukturellen Parameter des Übergitters.

nur den Bereich um den (001)-Peak, da die Intensität der Satelliten-Peaks beim (002)-Peak deutlich kleiner ist. Zudem minimiert man durch die Fokussierung auf einen kleinen Winkelbereich den störenden Einfluss von weiteren möglichen Winkelabhängigkeiten der Intensität, die sich z.B. aus einer Wölbung der Substratoberfläche oder kleinen Fehlern bei der Ausrichtung der Probe im Strahlengang ergeben können. Im gezeigten Beispiel weist die Anpassung des Spektrum auf scharfe Grenzflächen und eine starke Modulation der Gitterkonstanten zwischen den verschiedenen Materialien hin. Um die Signifikanz der angepassten Parameter zu prüfen, werden die strukturellen Parameter einzeln variiert und die sich damit ergebenden simulierten Spektren mit der Messung verglichen (Abbildung 5.3 b)-d)). Eine Veränderung der Periode $\Lambda=d_{L M O}+d_{S M O}$ führt zu einer Verschiebung der Übergitter-Peaks, womit $\Lambda$ sehr präzise bestimmt werden kann $(\Delta \Lambda / \Lambda \approx 1 \%)$. Die Höhe der Satelliten-Peaks sinkt stark bei einer Vergrößerung der Grenzflächenrauigkeit $\sigma_{L M O} / S M O$. Wenn man $c_{S M O}, c_{L M O}$ verändert und dabei die durchschnittliche Gitterkonstante $\bar{c}=\left(d_{L M O} c_{L M O}+d_{S M O} c_{S M O}\right) / \Lambda$ festhält, verschieben sich die Intensitäten der Peaks rechts und links vom mittleren Hauptpeak (Abbildung $5.3 \mathrm{c}$ )). Die starke Modulation zwischen $c_{S M O}, c_{L M O}$ verursacht eine stark 
asymmetrische Verteilung der Intensitäten für die Peaks rechts und links des Hauptpeaks. Neben der gesamten Periode $\Lambda$ kann man auch die individuellen Schichtdicken $d_{L M O}, d_{S M O}$ ermitteln. Eine Veränderung dieser Parameter für konstante $\bar{c}$ und $\Lambda$ ruft komplizierte Modifikationen des Spektrums hervor (siehe Abbildung $5.3 \mathrm{~d}$ )). Dadurch ist der Fehler von $d_{L M O}$, $d_{S M O}$ mit $\Delta d_{L M O / S M O} / d_{L M O / S M O} \approx 5-10 \%$ deutlich größer als für $\Lambda$.

Wie schon zuvor angedeutet, kann man mit der numerischen Simulation der Beugungsspektren auch Rückschlüsse auf die chemische Zusammensetzung innerhalb der Basis schließen. Für dünne Filme aus $\mathrm{La}_{1-\mathrm{x}} \mathrm{Sr}_{\mathrm{x}} \mathrm{MnO}_{3}$, die auf STO (001) gewachsen wurden, vergleicht man die Intensität des (001)- und (002)-Reflexes. Die numerische Berechnung zeigt, dass das Intensitätsverhältnis $I_{(001)} / I_{(002)}$ monoton mit der Dotierung $x$ abfällt. Bei dünnen Filmen wird dieses Verhältnis auch durch die Durchmischung mit dem Substrat beeinflusst, die durch die Rauigkeit $\sigma_{L S M O / S T O}$ charakterisiert wird. Für die im Folgenden betrachteten Filme mit einer Dicke von $d \approx 40 \mathrm{~nm}$ ist dies die größte Fehlerquelle. Die Unsicherheit, die sich durch die unbekannten Gitterparameter im Bereich der Grenzfläche zum Substrat ergibt, hängt außerdem von der Dotierung $x$ ab. Wenn man $I_{(001)} / I_{(002)}$ für verschiedene Konfigurationen der Grenzfläche zum Substrat simuliert, kann man Fehlerintervalle für das Intensitätsverhältnis abschätzen. Für die aus den Messungen abgelesenen Werte $R_{\exp }=I_{(001)}^{\exp } / I_{(002)}^{\exp }$ wird der Fehler durch den Untergrund der Reflexe des STO-Substrates bestimmt. Die experimentell ermittelten Werte $R_{\text {exp }}$ liegen nah an den Werten $R_{\text {sim }}=I_{(001)}^{\text {sim }} / I_{(002)}^{\text {sim }}$, die sich aus der Simulation ergeben: Für $x=0.33$ ist $R_{\text {sim }}=0.37(1)$ und $R_{\exp }=0.38(1)$, für $x=0.5$ ist $R_{\text {sim }}=0.30(2)$ und $R_{\text {exp }}=0.32(1)$, für $x=1.0$ ist $R_{\text {sim }}=0.09(2)$ und $R_{\text {exp }}=0.09(3)$ Da sich $R_{\text {sim }}$ mit einer Dotierungsveränderung von $\Delta x=0.1 \mathrm{um} \Delta R_{\text {sim }}=0.04$ verändert, kann man unter Berücksichtigung der Fehlergrenzen die Dotierung x der LSMO-Filme mit einer Genauigkeit von $\Delta x=0.025-0.05$ verifizieren. Wenn man deutlich dickere Filme $d \gtrsim 500 \mathrm{~nm}$ untersucht, kann man die Modifikationen des Spektrums durch Dünnfilmeffekte ausschließen und damit die Fehlerintervalle für $R_{\text {exp }}$ und $R_{\text {sim }}$ deutlich reduzieren. Für dicke $\mathrm{La}_{1-\mathrm{x}} \mathrm{Ca}_{\mathrm{x}} \mathrm{MnO}_{3}$-Filme, die mit der MAD hergestellt wurden, konnte so die Dotierung mit einer Genauigkeit von $\Delta x=0.02$ verifiziert werden 198 . Das bei der MAD angewendete Verfahren zur Einstellung verschiedener Dotierungen $x$, bei dem man die Präkursormengen der reinen Materialien (hier $\mathrm{LaMnO}_{3}$ und $\mathrm{SrMnO}_{3}$ bzw. $\mathrm{CaMnO}_{3}$ ) entsprechend der Dotierung mischt, führt also zu grundsätzlich korrekten Verhältnissen der Kationen im Film.

\subsubsection{Röntgenreflektometrie (XRR)}

Für streifenden Einfall $2 \theta \lesssim 10^{\circ}$ wird der Betrag von $\vec{Q}$ klein gegenüber den Beträgen der reziproken Gittervektoren, so dass die Streuung in diesem Winkelbereich nicht mehr empfindlich auf die atomaren Abstände ist. Man untersucht dadurch die durchschnittlichen chemischen Eigenschaften der Schichten, aus denen die Heterostruktur besteht. Man geht von der Diffraktometrie zur Reflektometrie über. In diesem Winkelbereich ergibt sich das Beugungsspektrum dann analog zur Behandlung der Reflexion von sichtbarem Licht an Vielfachlagen durch die phasenrichtige Addition der innerhalb der Heterostruktur mehrfach reflektierten Teilstrahlen [193, 199]. Die Ausbreitung der Röntgenstrahlen in einem Medium wird durch den Brechungsindex

$$
n=1-\delta-i \beta
$$

\footnotetext{
${ }^{18}$ Für $\mathrm{LaMnO}_{3}$ kann $R_{\text {exp }}$ nicht ermittelt werden, da die Peaks von den Reflexen des Substrates überdeckt werden.
} 
beschrieben. Für die hier betrachtete Röntgenstreuung mit harter Röntgenstrahlung sind $\delta \sim 10^{-5}, \beta \sim 10^{-6}$ positiv. Damit ergibt sich eine Totalreflexion bis zum maximalen Winkel

$$
\theta_{c} \approx \sin \theta_{c}=\sqrt{2 \delta}
$$

Über das optische Theorem ist die Absorption $\beta$ mit dem Imaginärteil des atomaren Streufaktors verknüpft [200], so dass man $\delta, \beta$ aus diesen berechnen kann 201]:

$$
n=1-\delta-i \beta=1-\frac{r_{e}}{2 \pi} \lambda^{2} \sum_{i} n_{i} f_{i}(0)
$$

Dabei bezeichnet $r_{e}$ den klassischen Elektronenradius und $n_{i}$ die Teilchendichte der Atomsorte $i$. Für den Kleinwinkelbereich kann man die Winkelabhängigkeit der Streufaktoren $f_{i}$ vernachlässigen und die Werte für Vorwärtsstreuung verwenden, die man aus den Tabellen von Chantler et al. entnimmt 202. Die Realteile der $f_{i}(0)$ sind dann im Wesentlichen durch die Gesamtelektronenzahl $Z$ des jeweiligen Elements gegeben. Zur Modellierung der Röntgenreflektometrie (XRR) einer Heterostruktur kann man sich nun für jedes vorkommende Material unter Kenntnis der chemischen Zusammensetzung und der Teilchendichte nach Gleichung 5.16 die Brechungsindizes für die Röntgenstrahlung verschaffen. Analog zur Ellipsometrie (siehe Abschnitt 4.3 wird die Probe dann durch die Angabe der aufgewachsenen Lagen mit ihrer Dicke $d_{i}$ und deren Brechungsindex $n_{i}$ definiert. Rauigkeiten $\sigma_{i}$ zwischen den Lagen werden wie im vorherigen Abschnitt über Röntgenbeugung behandelt. Nach Gleichung 5.16 ist der Brechungsindex einer Mischung zweier Materialien einfach durch das mit den jeweiligen Volumenanteilen gewichtete Mittel der Brechungsindizes beider Materialien gegeben. Analog zu Gleichung 5.13 für die Variation der Gitterkonstanten an einer Grenzfläche kann man dann auch ein Tiefenprofil $n(z)$ für den Brechungsindex aufstellen. Die Breite der Übergangszone zwischen den Brechungsindizes $n_{1}, n_{2}$ zweier benachbarter Lagen ist dann ein Maß für die Rauigkeit. Dabei ist die mathematische Definition identisch zu der im vorigen Abschnitt über Diffraktion (vergleiche Abbildung $5.2 \mathrm{~b}$ )). Schließlich diskretisiert man das Profil $n(z)$ mit $n$ virtuellen Lagen der Dicke $\Delta d=0.2 \mathrm{~nm}$, in denen der Brechungsindex durch $n(i \Delta d)$ für $i=0-n$ gegeben ist. Diese so konstruierte virtuelle Heterostruktur repräsentiert dann die reale Probe mit rauen Grenzflächen zwischen den Materiallagen. Die Reflektivität einer Mehrfachlagenstruktur kann man dann wie in Abschnitt 4.3 durch Vielstrahlinterferenz bestimmen, wobei man den Algorithmus von Parratt verwendet [155].

In dieser Arbeit werden die XRR-Kurven mit dem Programm ReMagX, das von Macke et al. am MPI Stuttgart entwickelt wurde, angepasst [165. Dies führt die oben genannten Schritte zur Erstellung einer virtuellen Lagenstruktur zur Einbeziehung der chemischen Rauigkeiten und die Berechnung der XRR-Spektren für diese Strukturen unter Anwendung des Algorithmus von Parratt aus. Die Anpassung der Messdaten an die Simulation erfolgt mit dem Simplex-Verfahren. Um die Signifikanz der daraus resultierenden Größen, die die chemische Struktur der Probe beschreiben, zu prüfen und Fehlerintervalle für diese abzuschätzen, variiert man jeweils einzelne Größen unter Fixierung aller übrigen und untersucht die daraus folgenden Abweichungen der Simulation von der gemessenen Kurve. Hier soll das am einfachen Beispiel einer $\mathrm{La}_{0.7} \mathrm{Sr}_{0.3} \mathrm{MnO}_{3}$ (LSMO)-Schicht $\left(d=26.1 \mathrm{~nm}\right.$ ) auf $\mathrm{SrTiO}_{3}$ (STO) demonstriert werden (siehe Abbildung 5.4): Der in Teil a) der Abbildung 5.4 gezeigte Fit reproduziert die Messdaten mit den in der Grafik vermerkten Parametern sehr exakt, nur im Bereich der Totalreflexion bei kleinen Winkeln kommt es wegen der Messgeometrie (bei kleinen Einfallswinkeln überstrahlt der Röntgenstrahl die Probe) zu Abweichungen. Die Schichtdicke ist aus den XRR-Messungen sehr präzise bestimmbar, Simulationen mit leicht veränderter Schichtdicke führen zu einer deutlich anderen Periode der Interferenzoszillationen (Abbildung 5.4 

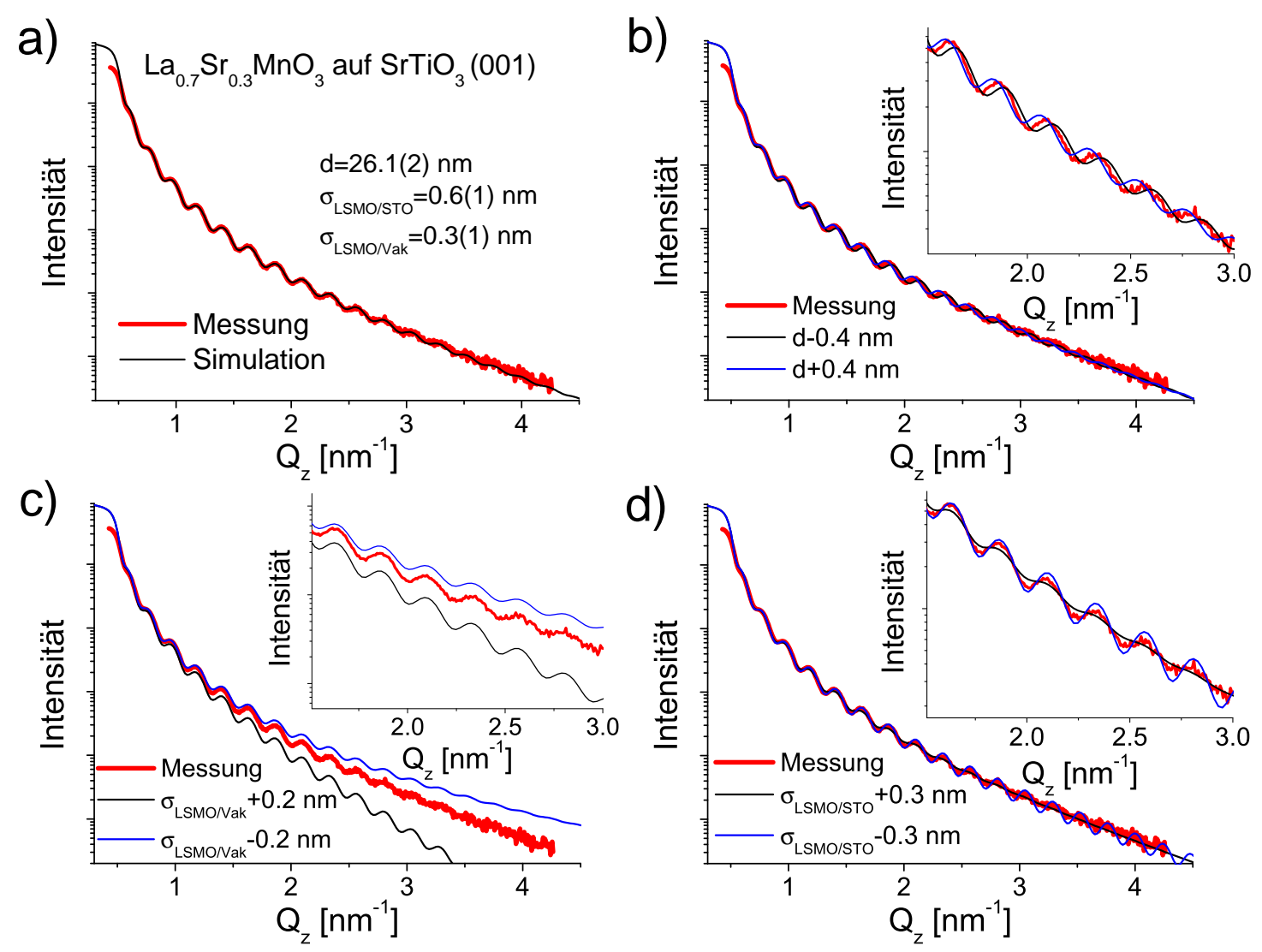

\begin{abstract}
Abbildung 5.4.: Zur Prüfung der Signifikanz der durch die Anpassung der XRR-Spektren erhaltenen Informationen wird die Messung an einer dünnen $\mathrm{La}_{0.7} \mathrm{Sr}_{0.3} \mathrm{MnO}_{3}$ (LSMO)Schicht $(d=26.1 \mathrm{~nm})$ auf $\mathrm{SrTiO}_{3}$ (STO) betrachtet: a) Messung und Fit mit den angegebenen Parametern, b) Vergleich der Messung mit Simulationen, bei der die Schichtdicke $d$ um $\Delta d= \pm 0.4 \mathrm{~nm}$ verändert wurde, c) Vergleich der Messung mit Simulationen, bei der die Rauigkeit zwischen Vakuum und LSMO-Film um $\Delta \sigma_{L S M O / V a k}= \pm 0.2 \mathrm{~nm}$ verändert wurde, d) Vergleich der Messung mit Simulationen, bei der die Rauigkeit zwischen STO und LSMO um $\Delta \sigma_{L S M O / S T O}= \pm 0.3 \mathrm{~nm}$ verändert wurde. Die Insets in den Teilen b)-d) zeigen eine vergrößerte Darstellung der jeweiligen Kurven. (Die simulierten XRR-Kurven wurden mit ReMagX berechnet 165].)
\end{abstract}

b)). Eine raue Oberfläche wird durch einen stetigen Übergang vom Brechungsindex des Vakuums zum Brechungsindex der ersten Lage dargestellt. Bei dieser kontinuierlichen Variation des Brechungsindexes ist die Reflektivität deutlich kleiner als bei einer scharfen Grenzfläche. Dies kann man dadurch verstehen, dass die diffuse Grenzfläche aus vielen Lagen mit leicht unterschiedlichem Brechungsindex aufgebaut ist. An den Grenzflächen dieser Lagen ist der Reflexionskoeffizient sehr klein, da die Brechungsindizes sehr ähnlich sind. Für die scharfe Grenzfläche gibt es eine plötzliche Änderung von $n(z)$, wodurch die Reflektivität groß wird. Dadurch verändert die Rauigkeit der Oberfläche den winkelabhängigen Abfall der Intensität nach dem Peak der Totalreflexion (Abbildung 5.4 c)), bei einer rauen Oberfläche fällt die reflektierte Intensität deutlich schneller ab als bei einer glatten. Wie bei der Oberfläche ist die Reflektivität an der Grenzfläche zwischen LSMO und STO abhängig von ihrer Abruptheit. Da sich die Oszillationen in der XRR-Kurve durch Interferenz der Strahlen, die an der LSMO/STO- und LSMO/Vakuum-Grenzfläche reflektiert werden, ergeben, hängt ihre Ausprägung von der Reflektivität an der LSMO/STO-Grenze ab. Bei einer stark durchmischten Grenzfläche wird diese Reflektivität klein und die Interferenzoszillationen haben eine deut- 
lich kleinere Amplitude als bei einer scharfen Grenzfläche (Abbilung 5.4 d)). Aufgrund der Tatsache, dass die Reflektivitäten innerhalb der Heterostrukturen direkt mit der chemischen Schärfe der Grenzflächen verbunden sind, ist die Röntgenreflektometrie eine wertvolle Methode zur Charakterisierung von Grenzflächen.

\subsection{Transmissionselektronenmikroskopie (TEM)}

Transmissionselektronenmikroskopie (TEM) ermöglicht die Abbildung der lokalen Struktur und Chemie, wobei die räumliche Auflösung Bruchteilen einer Gitterkonstanten entspricht. Die in dieser Arbeit gezeigten TEM-Aufnahmen wurden von Ricardo Egoavil und Haiyan Tan aus der Arbeitsgruppe von Jo Verbeeck an der Universität Antwerpen sowie Vladimir Roddatis vom Institut für Materialphysik der Universität Göttingen erstellt. An der Universität Antwerpen wurden die Messungen mit einem FEI Titan G3 Mikroskop aufgenommen, das bei einer Beschleunigungsspannung von $300 \mathrm{kV}$ betrieben wurde, im Institut für Materialphysik wurde das Titan ETEM G2 mit der gleichen Beschleunigungsspannung verwendet. Experimentelle Details dazu können z.B. in der Arbeit von Ricardo Egoavil nachgelesen werden 203. Die Abbildung erfolgt durch einen Elektronenstrahl, mit dem man bei einer Beschleunigungsspannung von $300 \mathrm{kV}$ eine Auflösung von $0.05 \mathrm{~nm}$ erreichen kann. Für TEM-Untersuchungen muss aus der Probe zunächst eine Lamelle hergestellt werden, die einen Querschnitt durch die Heterostruktur darstellt. Damit der Elektronenstrahl nicht vollständig in der Lamelle absorbiert wird, muss die Dicke der Lamelle kleiner als $100 \mathrm{~nm}$ sein. Typischerweise werden die TEM-Lamellen mit einem fokussierten Ionenstrahl (FIB) aus der Probe geschnitten. Durch den Beschuss mit hochenergetischen Ionen im Vakuum können aber besonders bei komplexen Oxiden Defekte eingebracht werden, die die Ergebnisse verfälschen. So wurde beobachtet, dass es bei $\left(\mathrm{SrTiO}_{3}\right)_{n} \mathrm{SrO}-\mathrm{Mehrfachlagen} \mathrm{zu} \mathrm{einer} \mathrm{Bewegung} \mathrm{der}$ SrO-Lagen kommt. Deshalb kann man die Lamellen auch alternativ mit einer Kombination aus mechanischem Schleifen und Ionenätzen präparieren, wobei die verwendeten Ar-Ionen das Material unter streifendem Einfall abtragen und so keine Defekte in tieferen Schichten induzieren. Durch diese Lamelle kann dann der Elektronenstrahl transmittieren. Die Elektronen des Strahls wechselwirken mit den Elektronen in der Probe, der Elektronenstrahl wird elastisch gestreut (Rutherford-Streuung) und verliert bei Anregungen von Elektronen in der Probe Energie (Anregung von charakteristischer Röntgenstrahlung). Die Wahrscheinlichkeit, mit der die Elektronen aus dem Strahl gestreut werden, ist abhängig von der lokalen Elektronendichte in der Probe. Wenn man nun einen fokussierten Elektronenstrahl über die Lamelle bewegt (scanning TEM - STEM), kann man durch Messung der Intensität des gestreuten Elektronenstrahls eine Karte der Elektronendichte aufnehmen. Bei dieser Art der Abbildung, die high angle annular dark field (HAADF) genannt wird, erscheinen die Atome dann umso heller, je größer ihre totale Elektronendichte $Z$ ist. Für eine Aufnahme im annular dark field $(\mathrm{ADF})$ werden die gestreuten Elektronen bei kleineren Ablenkungswinkeln gesammelt als für HAADF, wodurch sich ein invertierter Kontrast ergibt. Wenn man die Energieverteilung der inelastisch gestreuten Elektronen analysiert (electron energy loss spectroscopy (EELS)), kann man aus der Identifikation der auftretenden Linien auf die lokale chemische Zusammensetzung der Probe schließen. Bei Übergangsmetallionen kann man zudem durch eine genaue Betrachtung der Gestalt der Linien auf die Valenz und die chemische Umgebung schließen. Für die hier vorkommenden chemischen Elemente wurden folgende charakteristische Kanten zur Identifikation herangezogen: $\mathrm{O}-\mathrm{K}, \mathrm{Ti}-\mathrm{L}_{2 ; 3}, \mathrm{Mn}-\mathrm{L}_{2 ; 3}, \mathrm{Sr}-\mathrm{M}_{4 ; 5}, \mathrm{La}-\mathrm{M}_{4 ; 5}$. 


\subsection{Magnetometrie}

Die Messung der Magnetisierung wurde mit einem kommerziellen SQUID (superconducting quantum interference device)-Magnetometer des Typs MPMS (magnetic property measurement system) XL der Firma Quantum Design ausgeführt [204]. Mit diesem Gerät kann das magnetische Moment $m$ der Proben als Funktion des Feldes $\left(B_{\max }=5 \mathrm{~T}\right)$ und der Temperatur $(T=5-400 \mathrm{~K})$ erfasst werden. Die in einer Kunststoffkapsel befindliche Probe wird in einen Plastikstrohhalm geklemmt, der zur Messung des magnetischen Moments in einer Induktionsspule oszilliert. Wenn es nicht anders angegeben wird, liegt das magnetische Feld in der Filmebene. Außerdem war das Feld im Bezug auf die kristallinen Richtungen der Substrate entlang der [100]-Richtung bei einem Schnitt des Substrates in der (001)-Ebene und entlang der [1̄12]-Richtung bei einem Schnitt in der (111)-Ebene ausgerichtet. Der Fehler der Ausrichtung des Feldes liegt durch Ungenauigkeiten des Einbaus in den Strohhalm bei $\Delta \alpha \pm 10^{\circ}$. Die Projektion des Feldes in die Filmebene wird dadurch jedoch um weniger als $2 \%$ verkleinert, so dass dieser Fehler in der Ausrichtung des magnetischen Feldes bei einer inplane Anisotropie nicht ins Gewicht fällt. Aus dem gemessenen totalen magnetischen Moment wird das magnetische Moment pro Übergangsmetallion $M$ berechnet. Bei einer ferromagnetischen Schicht betrachtet man dazu das totale Moment in einem äußeren Feld, bei dem sich der Ferromagnet in Sättigung befindet. Neben der Schicht tragen dann noch das Substrat und die Kunststoffkapsel zum Moment bei. Diese sind wegen ihrer abgeschlossenen Elektronenschalen diamagnetisch und weisen somit eine temperaturunabhängige Suszeptibilität auf. Diese Suszeptibilität kann man so oberhalb der Curie-Temperatur des Ferromagneten bestimmen und dann von den Messungen bei den anderen Temperaturen abziehen, um den Beitrag der isolierten Schicht zu gewinnen ${ }^{19}$, Mit der bekannten Schichtdicke des Films und der von diesem bedeckten Fläche auf dem Substrat kann man dann das magnetische Moment pro Übergangsmetallion berechnen. Der Fehler der Berechnung des Volumens resultiert aus Unsicherheiten bei der mittleren Schichtdicke $d$, die man mit $\frac{\sigma_{d}}{d}=2 \%$ ansetzen kann, und der bedeckten Fläche, die bei $\frac{\sigma_{A}}{A}=2.5 \%$ liegt (vergleiche Abbildung 4.9 ). Außerdem kommt es bei einer nicht perfekt zentrierten Ausrichtung der Probe in der Mitte der Induktionsspule zu einer Verfälschung der Messung des magnetischen Moments. Die Feldlinien, die sich innerhalb der Spule schließen, tragen nicht zum magnetischen Fluss bei. Damit kommt es bei einer dezentralen Position der Probe zu einer Vergrößerung des magnetischen Flusses 205. Unter Berücksichtigung der Geometrie der Probenkammer des SQUID-Magnetometers erhält man bei der typischen Probengröße von $5 \mathrm{~mm}$ x $10 \mathrm{~mm}$ einen relativen Fehler von $\frac{\sigma_{m}}{m}=2.5 \%$, wobei man die Berechnungen aus 205] verwendet ${ }^{20}$. Insgesamt ist der Fehler bei der Berechnung des magnetischen Moments pro Übergangsmetallion also mit $\frac{\sigma_{M}}{M}=5 \%$ anzusetzen.

\subsection{Elektrischer Widerstand}

Der elektrische Widerstand als Funktion des magnetischen Feldes $\left(B_{\max }=9 \mathrm{~T}\right)$ und der Temperatur ( $T=5-400 \mathrm{~K}$ ) wurde im PPMS (physical property measurement system) der Firma Quantum Design gemessen 206. Dazu wurden vier elektrische Kontakte mit Leitsilber

\footnotetext{
${ }^{19}$ Der Fehler dieser Prozedur hängt vom absoluten Moment des Films und dem Sättigungsfeld ab, wodurch dieser hier nicht pauschal angegeben werden kann.

${ }^{20}$ Diese Abschätzung ergibt sich für die Messung kompletter Substrate mit Kantenlängen 5 mm x 10 mm. Wenn man kleinere Probenstücke vermisst, vergrößert sich $\frac{\sigma_{m}}{m}$ deutlich, da es dann zu größeren Änderungen der räumlichen Verteilung des magnetischen Flusses in den Induktionsspulen kommen kann.
} 
und Cu-Drähten auf die Probe aufgebracht, so dass durch die Messung in Vierpunktkonfiguration die Widerstände der Kontakte keine Rolle spielen. Der spezifische Widerstand $\rho$ wird aus der Kontaktgeometrie mit Kontakten eines Durchmessers $d_{A g} \approx 2 \mathrm{~mm}$ und Abstand $d_{\text {Ag-dist }} \approx 2 \mathrm{~mm}$ sowie der Dicke des dünnen Films $d_{\text {Film }}$ zu $\rho \approx R_{\text {meas }} d_{\text {Film }}$ abgeschätzt, wobei $R_{\text {meas }}$ den gemessenen Widerstand bezeichnet. Durch die manuelle Erstellung der Leitsilberkontakte ist der relative Fehler dabei sehr groß und liegt aufgrund von Variationen in $d_{A g}$ und $d_{A g-d i s t}$ im Bereich von $50 \%$. Bei den Manganaten kommt es im Zusammenhang mit dem Phasenübergang vom Paramagneten zum Ferromagneten zu einer Änderung der Steigung im Verlauf von $R(T)$. Um diese zu quantifizieren, betrachtet man die Funktion

$$
T C R(T)=\frac{1}{R(T)} \frac{d R}{d T}(T),
$$

die man aus dem gemessenen Verlauf von $R(T)$ berechnet. Die Position des Maximums von $T C R(T)$ definiert dann die Metall-Isolator-Übergangstemperatur $T_{M I}$ (Dieses Kriterium kann auch herangezogen werden, wenn die Probe einen Metall-Metall-Übergang aufweist, um die Position des Überganges zu bestimmen). Der Wert beim Maximum ist ein Maß für die Schärfe des Überganges. 



\section{Teil III.}

\section{Ergebnisse und Diskussion}





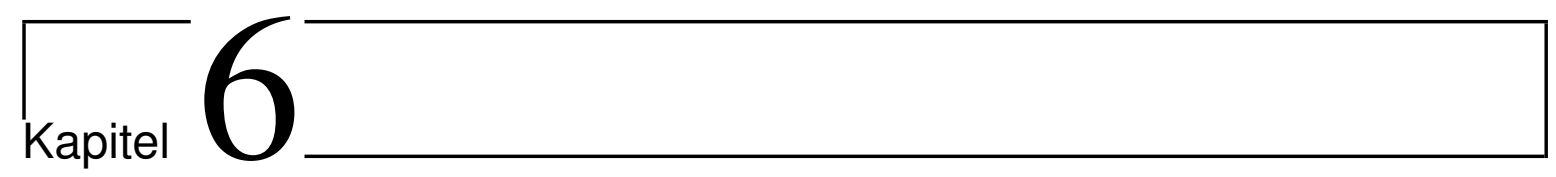

\section{In-situ Ellipsometrie beim Wachstum dünner Manganatfilme}

Bevor in den kommenden Kapiteln das Wachstum von Heterostrukturen aus verschieden dotierten Manganatfilmen besprochen wird, sollen in diesem Kapitel Vorarbeiten dafür gezeigt werden, die sich mit dem Wachstum einzelner $\mathrm{La}_{1-\mathrm{x}} \mathrm{Sr}_{\mathrm{x}} \mathrm{MnO}_{3}$-Filme auf $\mathrm{SrTiO}_{3}$ (001) und (111)-Substraten befassen. Es wird zunächst gezeigt, dass sich Fehler bei der Stöchiometrie der metallorganischen Präkursoren in den Messungen der in-situ Ellipsometrie abzeichnen. Dann erfolgt die Bestimmung der Brechungsindizes von $\mathrm{La}_{1-\mathrm{x}} \mathrm{Sr}_{\mathrm{x}} \mathrm{MnO}_{3}(x=0-1)$ für die hier verwendete Depositionstemperatur von $T_{D e p}=930{ }^{\circ} \mathrm{C}$. Diese werden bei der späteren Auswertung des Wachstums von Übergittern aus verschieden dotierten Manganaten benötigt. Schließlich wird ein Fokus auf das Wachstum der ersten 4-6 Monolagen (ML) des Manganatfilmes auf STO (001) gelegt, wo man mit Hilfe der in-situ Ellipsometrie Interdiffusionsprozesse und Änderungen der Wachstumsmode beobachten kann.

\subsection{Stöchiometrie von $\mathrm{La}_{0.7} \mathrm{Sr}_{0.3} \mathrm{MnO}_{3}$}

Bei der Herstellung dünner Filme komplexer Oxide mittels MAD ist es notwendig, die korrekten molaren Verhältnisse der Präkursoren für die gewünschte Zusammensetzung im Film empirisch zu bestimmen. Dabei können schon die in-situ gemessenen Daten der Ellipsometrie Aufschlüsse über die Korrektheit der verwendeten Präkursor-Zusammensetzung liefern. Dies wird hier anhand des Wachstums von $\mathrm{La}_{0.7} \mathrm{Sr}_{0.3} \mathrm{MnO}_{3}$-Filmen mit einer Dicke von etwa $26 \mathrm{~nm}$ auf $\mathrm{SrTiO}_{3}$ (001) aufgezeigt. Man lässt die Mengen der Präkursoren für Sr und Mn konstant und variiert die Menge des La-Präkursors in Relation zu dem Präkursor von Mn. Das hier angegebene La/Mn-Verhältnis bezeichnet das Gewichtsverhältnis der zur Herstellung der gezeigten Proben verwendeten Präkursoren, welches proportional zum molaren Verhältnis ist. In Abbildung 6.1 sind Messungen der in-situ Ellipsometrie für drei verschiedene La/Mn-Verhältnisse gezeichnet. Bei den Kurven in Teil a) der Abbildung von $\Delta(t)$ während der Deposition der Filme erkennt man für La/Mn=1.67 und La/Mn=1.69 einen qualitativ sehr ähnlichen Verlauf. Bei La/Mn=1.74 zeigt sich nach etwa $40 \mathrm{~s}$, was einer Dicke von etwa $5 \mathrm{~nm}$ entspricht, eine plötzliche Änderung der Steigung. Dies ist möglicherweise auf eine Änderung der Wachstumsmode von einem zweidimensionalen Inselwachstum zu einer 

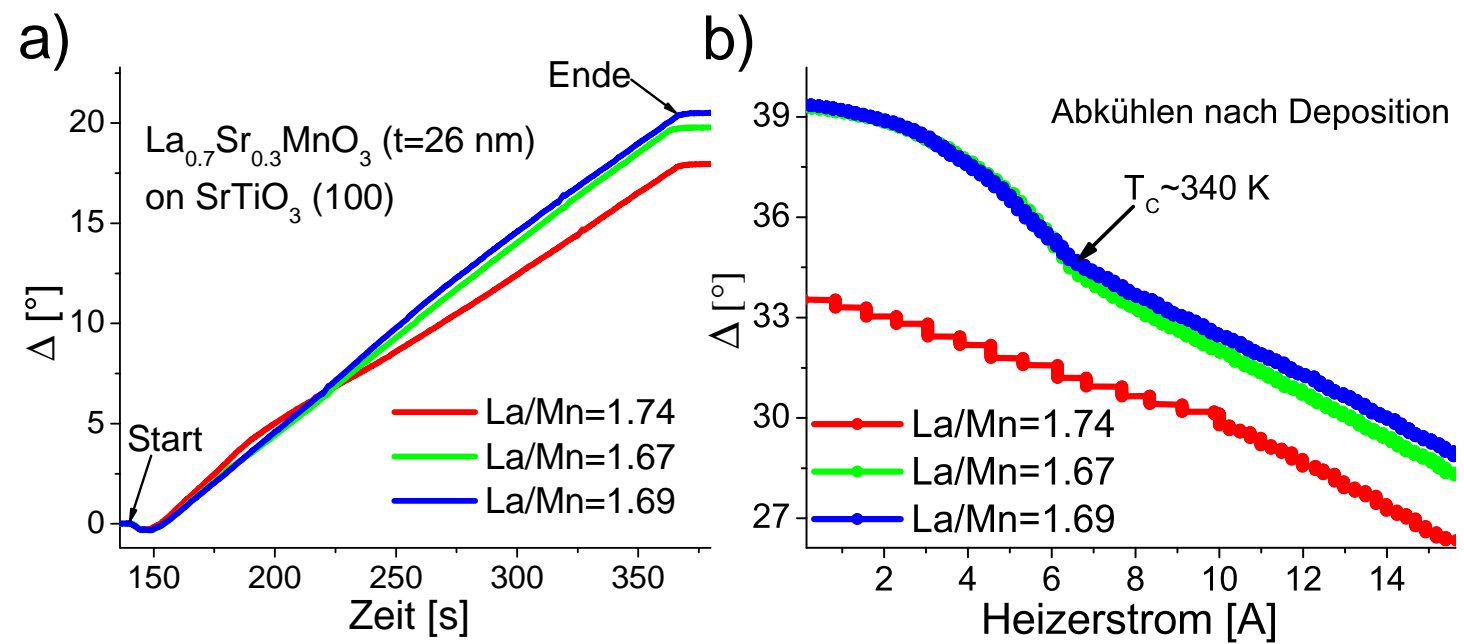

Abbildung 6.1.: In-situ Ellipsometrie bei der Deposition von LSMO-Filmen auf STO (001) mit verschiedenen Verhältnissen von La-und Mn-Präkursoren in der Präkursorlösung: a) $\Delta(t)$ während des Wachstums, b) $\Delta$ als Funktion des Heizerstroms beim Abkühlen der Schichten nach der Deposition, die Temperatur des Überganges $T_{C} \approx 340 \mathrm{~K}$ wurde mit den Daten aus Abschnitt 4.4.3 bestimmt.
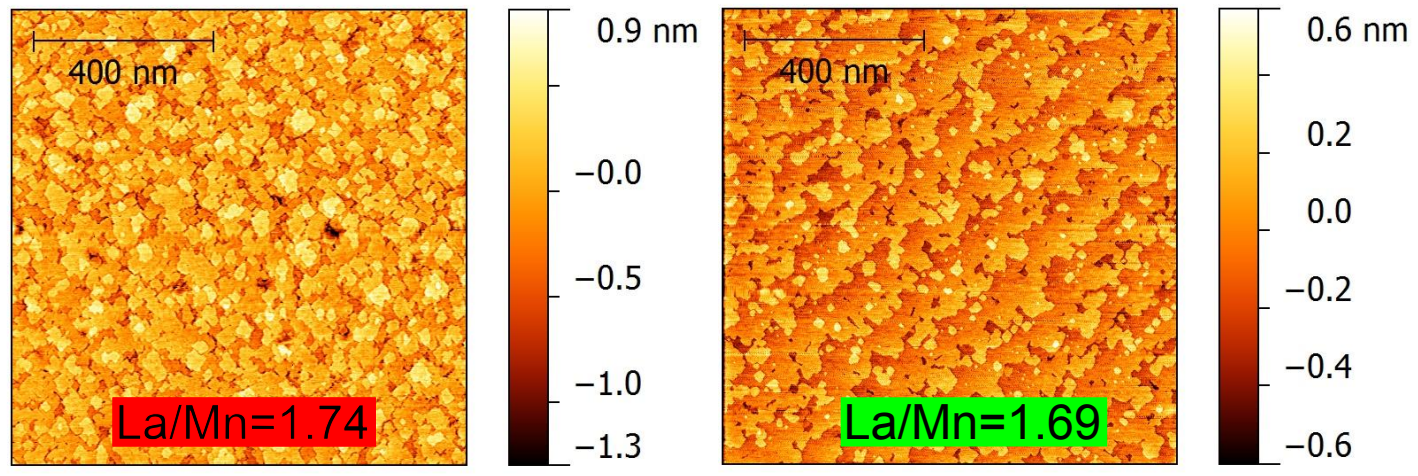

Abbildung 6.2.: Oberflächenmorphologien der Filme mit La/Mn=1.69 und La/Mn=1.74, gemessen mit Rastertunnelmikroskopie (STM).

dreidimensionalen Mode zu erklären. Die bei einem Überschuss von La entstehenden Ausscheidungen behindern die Diffusion der Ionen auf den Terrassen und unterstützen so das Inselwachstum. Die raue Oberfläche hat im Modell des effektiven Mediums einen anderen Brechungsindex als der geschlossene Film, wodurch sich andere Reflexionskoeffizienten $r_{p / s}$ ergeben. Nach dem Ende der Deposition kann man die Brechungsindizes der Schichten berechnen (siehe Tabelle 6.1). Der Imaginärteil $k$ fällt monoton mit dem La/Mn-Verhältnis, wogegen sich der Realteil kaum für $\mathrm{La} / \mathrm{Mn}=1.67$ und $\mathrm{La} / \mathrm{Mn}=1.69$ unterscheidet. Dies ist konsistent mit dem Verhalten von $N$ für $\mathrm{La}_{1-\mathrm{x}} \mathrm{Sr}_{\mathrm{x}} \mathrm{MnO}_{3}$ als Funktion von $x$ (siehe Abbildung 6.4). Ein größeres La/Mn-Verhältnis entspricht einem kleineren $x$. In der Nähe von $x=0.3$ steigt $k$ monoton mit $x$ und $n$ zeigt ein Minimum.

Nach Abschluss der Deposition wurden die Proben abgekühlt und dabei in-situ die Temperaturabhängigkeit von $(\Delta, \Psi)$ gemessen. Für die beiden niedrigeren La-Mengen zeigt sich eine Steigungsänderung in den Messdaten von $\Delta$ als Funktion des Stroms durch den Heizer, für $\mathrm{La} / \mathrm{Mn}=1.74$ steigt $\Delta$ mit sinkendem Heizerstrom monoton an. Die beobachtete Änderung 
der optischen Eigenschaften bei $I_{S i C} \approx 6.5 \mathrm{~A}$ hängt mit der Änderung der optischen Eigenschaften der Manganatfilme beim Einsetzen der ferromagnetischen Ordnung unterhalb von $T_{C}$ zusammen [27, 207 210. Mit dem Zusammenhang zwischen der Substrattemperatur und dem Strom durch den Heizer $I_{S i C}$ aus Abschnitt 4.4.3 kann man die Position $I_{S i C} \approx 6.5 \mathrm{~A}$ einer Temperatur von $T_{C}^{\text {ellip }} \approx 340 \mathrm{~K}$ zuordnen. Genauere Betrachtung zeigt, dass dieser Wert mit $T_{C}^{\text {ellip }} \approx 338 \mathrm{~K}$ für $\mathrm{La} / \mathrm{Mn}=1.69$ etwas kleiner ist als für $\mathrm{La} / \mathrm{Mn}=1.67$, wo der Übergang bei $T_{C}^{\text {ellip }} \approx 343 \mathrm{~K}$ auftritt. Dieser Wert ist kleiner als die Curie-Temperatur $T_{C} \approx 350 \mathrm{~K}$, die sich durch SQUID-Magnetometrie ergibt, was sich durch den systematischen Fehler der Bestimmung kleiner Substrattemperaturen auf dem SiC-Heizer ergibt (siehe Abschnitt 4.4.3).

Die Ex-situ Charakterisierung ist im Einklang mit den Informationen, die sich bei den Ellipsometriemessungen ergeben haben. Die Oberfläche des Films für La/Mn=1.69 zeigt ein step-flow-Wachstum mit klar ausgeprägten Terrassen, für $\mathrm{La} / \mathrm{Mn}=1.74$ sind die durch den Fehlschnitt des Substrates verursachten Terrassen nur schwer zu erahnen, da die Schicht aus vielen kleinen Inseln aufgebaut ist (siehe Abbildung 6.2). In der Röntgenreflektometrie (XRR) kann man die drei in diesem Abschnitt behandelten Proben nicht unterscheiden. Die Rauigkeiten an der Oberfläche und der Grenzfläche zum STO sind innerhalb des Fehlerintervalls identisch. Dagegen schlagen sich die Veränderungen der La/Mn Dotierung in der Gitterkonstanten und damit den Röntgenbeugungsspektren nieder (Abbildung 6.3 a)). Der Wert der c-Achse zeigt aber keinen klaren Trend mit dem La/Mn-Verhältnis (siehe Tabelle 6.1). Die Verkleinerung von $c$ bei $\mathrm{La} / \mathrm{Mn}=1.74$ ist nicht konsistent mit einer Vergrößerung des La/Sr-Verhältnisses und der damit verbundenen Vergrößerung des Verhältnisses zwischen den $\mathrm{Mn}^{3+}$ - und $\mathrm{Mn}^{4+}$-Ionen, was zu einer Vergrößerung des Gitterparameters führen müsste. Dies wäre auch der Fall, wenn die Filme für die beiden niedrigeren La/Mn-Verhältnisse LaLeerstellen enthalten würden, welche dann bei $\mathrm{La} / \mathrm{Mn}=1.74$ aufgefüllt worden würden [211]. Die Größe der c-Achse hängt daneben auch vom Verspannungszustand der Schicht ab und der genauen Gitterstruktur des Materials, aus der der Film besteht, wodurch es schwierig ist an dem Gitterparameter Änderungen der Dotierung zu identifizieren. Im DC-Widerstand zeigen die beiden Proben mit den niedrigeren La/Mn-Verhältnissen einen Metall-Metall-Übergang, wogegen die Probe mit $\mathrm{La} / \mathrm{Mn}=1.74$ einen isolierenden Verlauf im Hochtemperaturbereich aufweist (siehe Abbildung 6.3 b)). Die für den Übergang im Widerstand charakteristische Temperatur $T_{M I}$ folgt dem Trend, der sich schon bei $T_{C}^{\text {ellip }}$ gezeigt hat, wo bei La/Mn=1.67 die Übergangstemperatur am höchsten ist und bei $\mathrm{La} / \mathrm{Mn}=1.74$ am niedrigsten (vergleiche Tabelle 6.1).

Insgesamt sind also die mit der in-situ Ellipsometrie gemessenen Größen, der qualitative Verlauf von $\Delta(t)$, der Brechungsindex $N$ und die Übergangstemperatur in der Abkühlkurve $T_{C}^{\text {ellip }}$, mit den ex-situ gemessenen Eigenschaften der LSMO-Filme verknüpft. Somit liefern die Ellipsometriemessungen Hinweise auf die korrekte Stöchiometrie und die Richtung, in der die Anpassung für eine optimale Zusammensetzung erfolgen muss. In dem hier gezeigten Beispiel werden für $\mathrm{La} / \mathrm{Mn}=1.67$ die elektrischen und magnetischen Eigenschaften optimiert. Über den Brechungsindex $N$ kann man auch sehr kleine Änderungen der Zusammensetzung erfassen, was durch die auflösbaren Unterschiede zwischen den Proben mit $\mathrm{La} / \mathrm{Mn}=1.67$ und 1.69 demonstriert wird. Der Brechungsindex $N$ ist dabei direkt mit dem Verhältnis zwischen den $\mathrm{Mn}^{3+}$ - und $\mathrm{Mn}^{4+}$-Ionen verknüpft und hängt nicht kompliziert vom Grad der Verspannung und der Gitterstruktur ab wie der Gitterebenenabstand entlang der c-Achse.

Hier wurde aufgezeigt, wie man das korrekte molare Verhältnis der La- und Mn-Ionen in der Präkursorlösung über in-situ Ellipsometrie ermitteln kann. Ähnliche Betrachtungen würden auch zum korrekten molaren Verhältnis der Sr- und Mn-Ionen, $\mathrm{Sr} / \mathrm{Mn}$, führen, da dies ebenso 

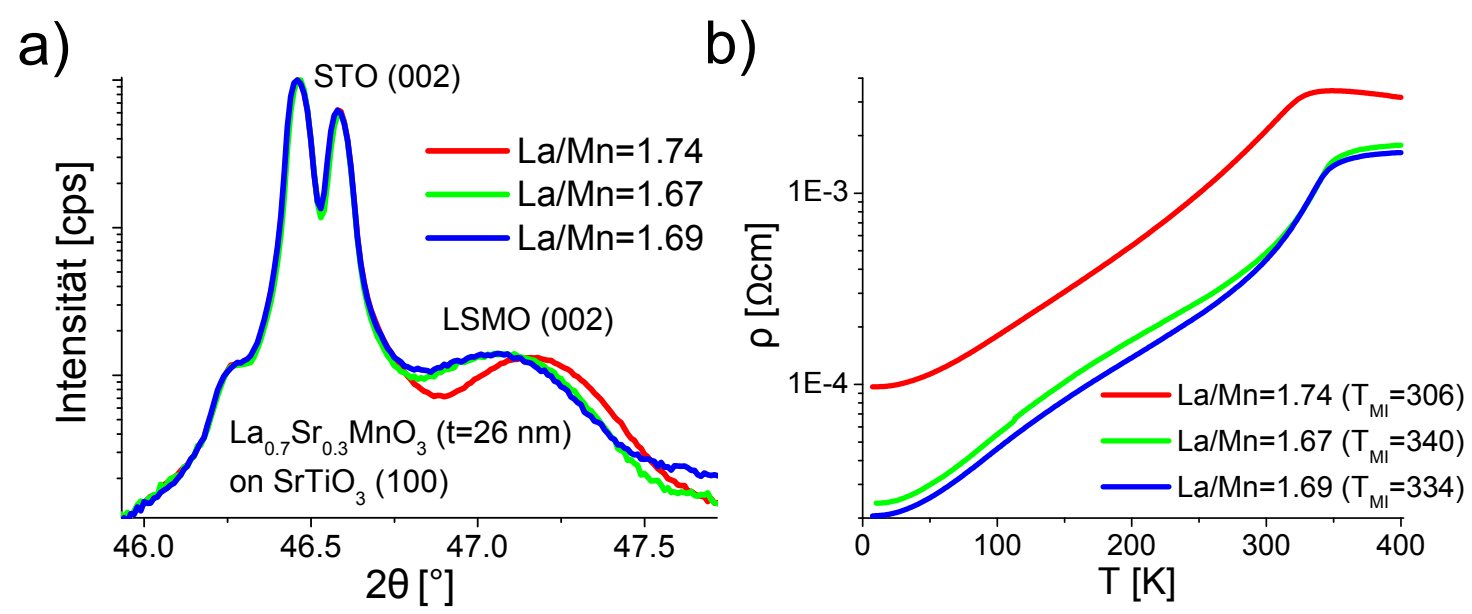

Abbildung 6.3.: Ex-situ Charakterisierung der LSMO-Filme auf STO (001) mit verschiedener La/Mn-Stöchiometrie: a) Röntgenbeugungsspektrum um den (002)-Peak des STOSubstrates, b) Temperaturabhängigkeit des elektrischen Widerstandes mit den aus den Messdaten ermittelten Werten für $T_{M I}$, die den Metall-Isolator-Übergang charakterisieren.

\begin{tabular}{cccccc}
\hline \multirow{2}{*}{ La/Mn-Verhältnis } & \multirow{2}{*}{$k$} & \multirow{2}{*}{$T_{C}^{\text {ellip }}[\mathrm{K}]$} & $T_{M I}[\mathrm{~K}]$ & \multirow{2}{*}[\mathrm{nm}]{} \\
\hline 1.74 & $2.380(4)$ & $0.470(5)$ & - & $306(1)$ & $0.3853(2)$ \\
\hline 1.69 & $2.300(4)$ & $0.522(5)$ & $338(2)$ & $334(1)$ & $0.3862(2)$ \\
\hline 1.67 & $2.310(4)$ & $0.531(5)$ & $343(2)$ & $340(1)$ & $0.3858(3)$ \\
\hline
\end{tabular}

Tabelle 6.1.: Zusammenfassung der Eigenschaften der LSMO-Filme auf STO (001) mit verschiedener La/Mn-Stöchiometrie: Brechungsindex $N=n-i k$ aus den in-situ Messungen der Ellipsometrie, der Fehler wird durch das Rauschen von $(\Delta, \Psi)$ bestimmt, Übergangstemperatur $T_{C}^{\text {ellip }}$ in den Abkühlkurven von $\Delta$, charakteristische Temperatur $T_{M I}$ für den Metall-Isolator-Übergang, Gitterkonstante senkrecht zur Filmebene.

Einfluss auf die Anteile der $\mathrm{Mn}^{3+}$ - und $\mathrm{Mn}^{4+}$-Ionen im Film besitzt. Auch Abweichungen vom stöchiometrischen Verhältnis der Ionen auf den A-Plätzen insgesamt und den Mn-Ionen, $(\mathrm{La}+\mathrm{Sr}) / \mathrm{Mn}$, schlagen sich in Veränderungen der durchschnittlichen Mn-Valenz wieder bzw. verursachen eine Veränderung des Wachstums.

\subsection{Brechungsindex von $\mathrm{La}_{1-x} \mathrm{Sr}_{x} \mathrm{MnO}_{3}$ für $\mathrm{x}=0-1$}

Bei der späteren Betrachtung der in-situ Ellipsometrie des Wachstums von Heterostrukturen ist es notwendig, den Brechungsindex der Manganat-Filme in Abhängigkeit des Verhältnisses der $\mathrm{Mn}^{3+}$ - und $\mathrm{Mn}^{4+}$-Ionen bei den Depositionsbedingungen und für die benutzte Wellenlänge $(\lambda=632.8 \mathrm{~nm})$ zu kennen. Als Prototyp wird dafür der von der Sr-Dotierung $x$ abhängige Brechungsindex von dünnen $\mathrm{La}_{1-\mathrm{x}} \mathrm{Sr}_{\mathrm{x}} \mathrm{MnO}_{3}(x=0.0-1.0)$-Filmen herangezogen. Die Filme wurden auf $\mathrm{SrTiO}_{3}$ (001) bzw. (111)-Substraten gewachsen, wobei eine Schichtdicke von $d \approx 40 \mathrm{~nm}$ gewählt wurde. Durch die relativ große Schichtdicke wird der durch die Durchmischung an der Grenzfläche zum Substrat und die Rauigkeit der Oberfläche verursachte Fehler minimiert. Durch orbitale Ordnung ergibt sich für $\mathrm{LaMnO}_{3}$ in der bulk-Phase eine starke Anisotropie der optischen Konstanten, welche aber oberhalb der Ordnungstemperatur $T_{O O}=780 \mathrm{~K}$ verschwindet $\left[28\right.$. Bei Depositionstemperatur $T_{D e p} \approx 1208 \mathrm{~K}$ ist also nicht 

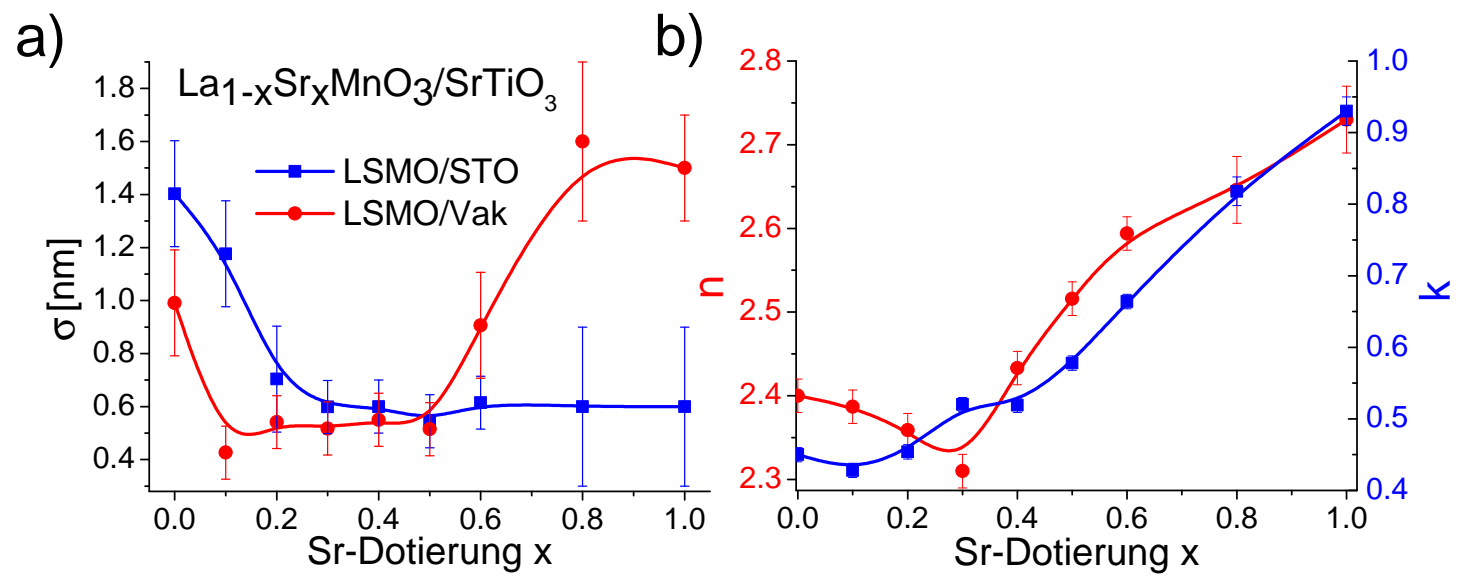

\begin{abstract}
Abbildung 6.4.: Zur Bestimmung des Brechungsindexes $N=n-i k(\lambda=632.8 \mathrm{~nm})$ von $\mathrm{La}_{1-\mathrm{x}} \mathrm{Sr}_{\mathrm{x}} \mathrm{MnO}_{3}$-Filmen $(d \approx 40 \mathrm{~nm})$ auf $\mathrm{SrTiO}_{3}$-Substraten mit (111)- und (001)Orientierung bei Depositionstemperatur $T_{\text {Dep }} \approx 1208 \mathrm{~K}$ : a) Rauigkeiten zwischen STOSubstrat und Manganatfilm, $\sigma_{L S M O / S T O}$, sowie zwischen Film und Luft, $\sigma_{L S M O / V a k}$, als Funktion der Sr-Dotierung $x, \mathrm{~b}$ ) Realteil $n$ und Imaginärteil $k$ des Brechungsindexes $N$ als Funktion der Sr-Dotierung $x$.
\end{abstract}

damit zu rechnen, dass sich optische Anisotropien durch eine orbitale Ordnung ergeben. Zur Berechnung der optischen Konstanten der $\mathrm{La}_{1-\mathrm{x}} \mathrm{Sr}_{\mathrm{x}} \mathrm{MnO}_{3}$-Filme verwendet man die Werte von $(\Delta, \Psi)$ am Ende der Deposition, bevor der Abkühlvorgang eingeleitet wurde.

Mittels Röntgenreflektometrie wurden die genauen Schichtdicken $d$ und Rauigkeiten zwischen Substrat und Film, $\sigma_{L S M O / S T O}$, sowie zwischen Substrat und Luft, $\sigma_{L S M O / V a k}$, bestimmt (siehe Abbildung 6.4). Man sieht, dass die Durchmischung zum Substrat in weiten Teilen des Phasendiagramms konstant bei einem Wert von $\sigma_{L S M O / S T O} \approx 0.6 \mathrm{~nm}$ liegt und dann bei $x \leq 0.2$ stark ansteigt. Der Fehler von $\sigma_{L S M O / S T O}$ vergrößert sich bei großen Sr-Dotierungen, da sich der Kontrast zum STO-Substrat bei der Röntgenreflektometrie verschlechtert. Die starke Durchmischung am linken Ende des $\mathrm{La}_{1-\mathrm{x}} \mathrm{Sr}_{\mathrm{x}} \mathrm{MnO}_{3}$-Phasendiagrammes ergibt sich durch die polare Katastrophe analog zur Situation an der Grenzfläche von $\mathrm{LaAlO}_{3} / \mathrm{SrTiO}_{3}$ (siehe Abschnitt 3.1). Beim Zufügen von Sr ermöglicht die Mischvalenz der Mn-Ionen eine Umverteilung der Ladungen an der Grenzfläche zur Abschirmung des elektrischen Dipols, so dass dies nicht mehr durch Umlagerung der Ionen auf den A-Plätzen erfolgen muss. Für große $x$ rauen die Oberflächen der Filme stark auf, was aus der großen Fehlpassung zwischen den Gitterkonstanten von Substrat und Film folgt $\left(\mathrm{SrTiO}_{3}\right.$ hat einen Gitterparameter von $a_{S T O}=0.3905 \mathrm{~nm}$ und $\mathrm{SrMnO}_{3}(\mathrm{SMO})$ von $\left.a_{S M O}=0.3805 \mathrm{~nm} 212\right)$. Wie in Kapitel 8 beschrieben wird, bilden sich in SMO-Filmen auf STO (001) ab einer Dicke von etwa $5 \mathrm{~nm}$ Spannungsrisse, die zu einem Inselwachstum führen.

Mit den Werten von $d$ und $(\Delta, \Psi)$ am Ende der Deposition kann man die Brechungsindizes der Filme numerisch erhalten, indem man die Gleichungen aus Abschnitt 4.3 invertiert (siehe Abbildung 6.4 b)). Der Fehler wird dabei hauptsächlich durch die Vernachlässigung der rauen Bereiche an den Grenzflächen verursacht. Eine Abschätzung des Fehlers von $n$ und $k$ erfolgt durch die Betrachtung von Heterostrukturen, bei denen die durchmischte Grenzfläche und die raue Oberfläche durch zusätzliche Schichten modelliert werden, deren Dicke mit den Rauigkeiten $\sigma_{L S M O / S T O}, \sigma_{L S M O / V a k}$ genähert wird und deren Brechungsindizes sich aus der Mischung der entsprechenden Brechungsindizes von Luft, STO und $\mathrm{La}_{1-\mathrm{x}} \mathrm{Sr}_{\mathrm{x}} \mathrm{MnO}_{3}$ nach der Gleichung von Bruggeman ergeben. Die Dicke der Manganatschicht in diesen Trilagen behält 

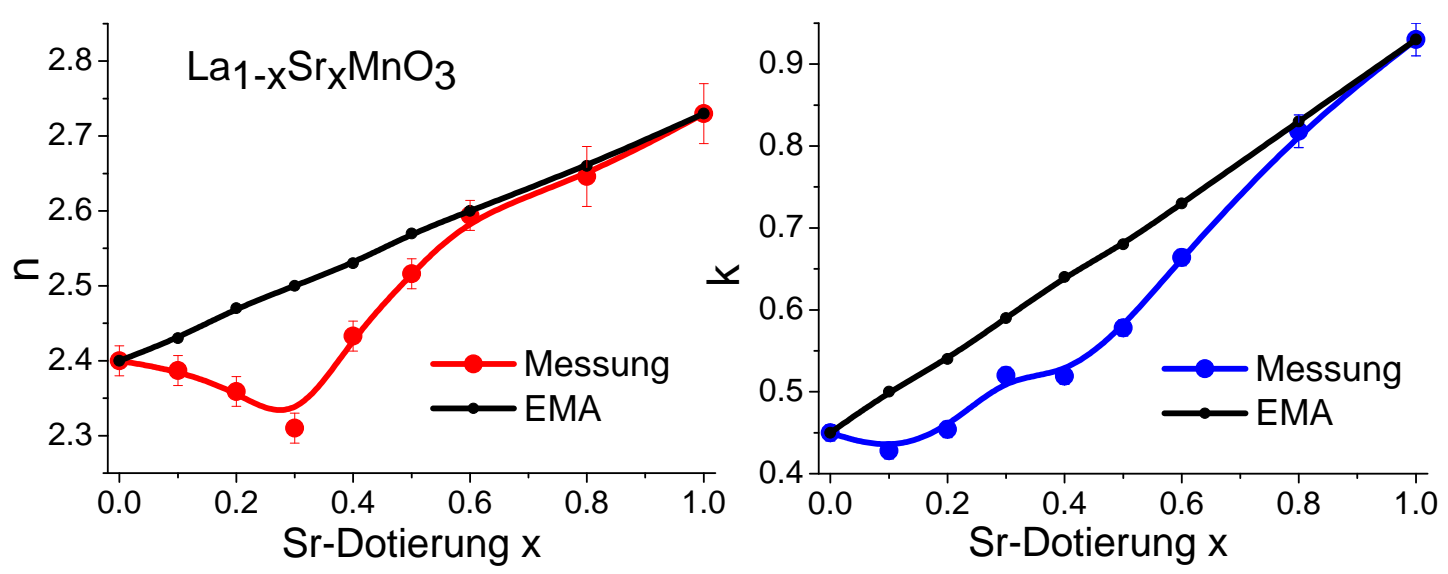

Abbildung 6.5.: Vergleich des Brechungsindexes $N=n-i k$ von $\mathrm{La}_{1-\mathrm{x}} \mathrm{Sr}_{\mathrm{x}} \mathrm{MnO}_{3}$-Filmen $(d \approx 40 \mathrm{~nm})$ auf $\mathrm{SrTiO}_{3}$-Substraten mit (111)- und (001)-Orientierung bei Depositionstemperatur $T_{D e p} \approx 1208 \mathrm{~K}$ mit den korrespondierenden Werten, die sich aus dem Modell des effektiven Mediums durch die Mischung von $\mathrm{LaMnO}_{3}$ und $\mathrm{SrMnO}_{3}$ nach der Gleichung von Bruggeman ergeben.

den Wert, der sich aus der Röntgenreflektometrie ergeben hat. Für diese Multilagen berechnet man nun numerisch die Werte von $(\Delta, \Psi)$. Aus diesen wird wiederum der Brechungsindex unter Annahme eines Einschichtmodells durch numerische Inversion berechnet. Der Unterschied zwischen den direkt aus den Messdaten berechneten Werten von $n, k$ und den Werten, die sich aus den konstruierten Trilagen ergeben, wird dann als Fehler angenommen. Man sieht in Abbildung 6.4 b), dass der Fehler sich bei den Filmen mit großer Sr-Dotierung stark vergrößert, da Modifikationen der Oberfläche wegen der Absorption der Lichtstrahlen im Film stärker ins Gewicht fallen als Rauigkeiten an der Grenzfläche zum Substrat.

Dass die durch Verspannungen verursachten optischen Anisotropien bei der hier erreichbaren experimentellen Auflösung keine Rolle spielen, zeigt sich daran, dass sich die optischen Konstanten von Filmen, die auf (001)- und (111)-orientierten Substraten gewachsen wurden, nicht unterscheiden. So ergibt sich bei $\mathrm{La}_{0.5} \mathrm{Sr}_{0.5} \mathrm{MnO}_{3}$, das voll verspannt auf STO (001) und (111) aufwächst, ein Brechungsindex von $N_{(001)}=2.53(2)-i \cdot 0.61(1)$ für das Wachstum auf STO (001) und $N_{(111)}=2.52(2)-i \cdot 0.58(1)$ für das Wachstum auf STO (111). Man sieht, dass der mit der Absorption verbundene Imaginärteil $k$ monoton mit der Sr-Dotierung ansteigt, wobei die Steigung für $x \leq 0.4$ deutlich kleiner ist als für größere $x$. Der Realteil weist ein Minimum um $x=0.3$ auf und zeigt seine stärkste Änderung auch für $x>0.4$. Somit ist man bei der verwendeten Wellenlänge $(\lambda=632.8 \mathrm{~nm})$ besonders sensitiv auf Änderungen des Anteils der $\mathrm{Mn}^{4+}$-Ionen, wenn dieser bereits oberhalb von 0.4 liegt. Das komplizierte Verhalten von $n$ und $k$ als Funktion von $x$ lässt sich nicht durch eine einfache Mischung von $\mathrm{SrMnO}_{3}$ und $\mathrm{LaMnO}_{3}$ im Modell eines effektiven Mediums beschreiben. Wie in Abbildung $6.5 \mathrm{zu}$ sehen ist, zeigen die Werte, die sich aus dem Modell des effektiven Mediums nach Bruggeman ergeben, einen fast linearen Verlauf, der besonders für $n$ von den experimentellen Daten abweicht.

Die Dotierungsabhängigkeit ist also mit einer Verschiebung von Anregungsenergien verbunden. Bei der verwendeten Laserwellenlänge $(\lambda=632.8 \mathrm{~nm})$ tastet man eine Anregungsenergie von $\Delta E=1.96 \mathrm{eV}$ ab. In diesem spektralen Bereich werden typischerweise zwei Anregungen in mischvalenten Manganaten betrachtet: die Ladungstransfer-Anregung von den $O_{2 p^{-}}$in die $e_{g}$-Zustände $\left(O_{2 p} \rightarrow e_{g}, \mathrm{O}^{2-}+\mathrm{Mn}^{3+} \rightarrow \mathrm{O}^{-}+\mathrm{Mn}^{2+}\right.$ bzw. $\mathrm{O}^{2-}+\mathrm{Mn}^{4+} \rightarrow \mathrm{O}^{-}+\mathrm{Mn}^{3+}$ 26, 213, 214]) und d-d-Anregungen der Mn-Ionen. Die Ladungstransfer-Anregungen sind 
aufgrund von $\Delta l=1$ erlaubt für Dipolanregungen. Die d-d-Übergänge scheinen zunächst aufgrund der Auswahlregeln verboten. Jedoch sind die zur Erklärung der Spektren herangezogenen Übergänge Anregungen zwischen verschiedenen Mn-Ionen, die aufgrund der verschiedenen Symmetriezentren optisch erlaubt sind [27. Diese werden durch die p-d-Hybridisierung vermittelt, so dass Anregungen der $t_{2 g}$-Elektronen sehr unwahrscheinlich sind. Dementsprechend beobachtet man experimentell für $\mathrm{CaMnO}_{3}$, in dem wie bei $\mathrm{SrMnO}_{3}$ nur $\mathrm{Mn}^{4+}$-Ionen vorkommen, nur die bei $\Delta E=3.1-4.5 \mathrm{eV}$ zentrierte Ladungstransfer-Anregung [213]. In $\mathrm{La}_{1-\mathrm{x}} \mathrm{Ca}_{\mathrm{x}} \mathrm{MnO}_{3}$ werden durch die vorhandenen Elektronen auf den $e_{g}$-Niveaus d-d-Übergänge ermöglicht, so dass eine weitere Absorptionsbande in der optischen Leitfähigkeit erscheint, die bei $\Delta E=1.2-1.8 \mathrm{eV}$ zentriert ist 213 . Im gemischtvalenten Fall zieht man zur Erklärung dieser Bande einen Übergang von einem besetzten $e_{g}$-Orbital eines $\mathrm{Mn}^{3+}$-Ions in ein unbesetztes eines $\mathrm{Mn}^{4+}$-Ions heran $\left(\mathrm{Mn}^{3+}+\mathrm{Mn}^{4+} \rightarrow \mathrm{Mn}^{4+}+\mathrm{Mn}^{3+}\right)$. Die für diesen Übergang nötige Photonenenergie wird von der Jahn-Teller-Energie bestimmt 27, 213. Für reines $\mathrm{LaMnO}_{3}$ wird die Anregung eines $e_{g}$-Elektrons von einem $\mathrm{Mn}^{3+}{ }_{-}$Ion in ein unbesetztes $e_{g}$-Orbital eines anderen $\mathrm{Mn}^{3+}$-Ions $\left(\mathrm{Mn}^{3+}+\mathrm{Mn}^{3+} \rightarrow \mathrm{Mn}^{4+}+\mathrm{Mn}^{2+}\right)$ diskutiert [26. Bei Raumtemperatur werden die optischen Eigenschaften von $\mathrm{La}_{1-\mathrm{x}} \mathrm{Sr}_{\mathrm{x}} \mathrm{MnO}_{3}$ bei einer Energie $\Delta E=1.96 \mathrm{eV}$ also von der Überlagerung der d-d-Anregungen um $\Delta E=1.2-1.8 \mathrm{eV}$ und der LadungstransferAnregungen um $\Delta E=3.1-4.5 \mathrm{eV}$ bestimmt [213. Für $\mathrm{LaMnO}_{3}$ beobachtet man bei höheren Temperaturen, $T>800 \mathrm{~K}$, ein Verschwinden der Bande von d-d-Anregungen [215]. Da bei den höheren Temperaturen die Mn-Oktaeder bereits durch thermische Schwingungen in einer Jahn-Teller-Mode verzerrt werden, ist bei den Anregungen zwischen den Mn-Ionen keine Jahn-Teller-Energie mehr aufzubringen und das spektrale Gewicht verschiebt sich von der Region um $\Delta E=1.2-1.8 \mathrm{eV}$ zu kleineren Energien [215]. Damit wird das Anregungsspektrum bei der hier betrachteten Temperatur $T_{D e p} \approx 1208 \mathrm{~K}$ und der verwendeten Anregungsenergie $\Delta E=1.96 \mathrm{eV}$ von der $O_{2 p} \rightarrow e_{g}$-Anregung bestimmt, die sich hinsichtlich ihrer Position und ihres spektralen Gewichts wenig ändert [27, 215]. Dadurch ist die Temperaturabhängigkeit des Brechungsindexes von $\mathrm{SrMnO}_{3}$, wo die d-d-Anregungen aufgrund der fehlenden $e_{g}$-Elektronen nicht auftreten, deutlich schwächer als für $\mathrm{La}_{1-x} \mathrm{Sr}_{\mathrm{x}} \mathrm{MnO}_{3}$. Dies bestätigt sich auch hier experimentell bei der Betrachtung der Abkühlkurven der $\mathrm{La}_{1-\mathrm{x}} \mathrm{Sr}_{\mathrm{x}} \mathrm{MnO}_{3}$-Schichten nach der Deposition, wo bei SMO deutlich kleinere Änderungen zu sehen sind als bei $\mathrm{La}_{0.7} \mathrm{Sr}_{0.3} \mathrm{MnO}_{3}$. Die zunehmende Besetzung der $e_{g}$-Niveaus bei sinkender Sr-Dotierung führt zu einer kleineren Wahrscheinlichkeit der $O_{2 p} \rightarrow e_{g}$-Übergänge und einer Verschiebung des Zentrums der Absorption zu größeren Energien 213. Für $\Delta E=1.96 \mathrm{eV}$ sinkt so die optische Leitfähigkeit und damit die Absorption $k$ mit kleiner werdendem $x$. Die optischen Eigenschaften der mischvalenten Manganate werden hauptsächlich durch das Verhältnis zwischen der Zahl der $\mathrm{Mn}^{3+}$ - und $\mathrm{Mn}^{4+}$-Ionen bestimmt. Strukturelle Parameter wie die Bindungswinkel der Mn-Oktaeder haben nur geringen Einfluss, was sich durch die Ähnlichkeit der Spektren der optischen Leitfähigkeit für $\mathrm{RMnO}_{3}$ mit $\mathrm{R}=\mathrm{La}^{3+}, \mathrm{Pr}^{3+}, \mathrm{Nd}^{3+}, \mathrm{Sm}^{3+}, \mathrm{Eu}^{3+}$ zeigt 214. Somit lässt sich beim Wachstum von Heterostrukturen durch die Beobachtung der optischen Eigenschaften der wachsenden Lagen direkt auf die Valenz der Mn-Ionen schließen.

\subsection{Die ersten Monolagen: Durchmischung mit Substrat und Monolagenoszillationen}

Eine einfache numerische Simulation der in-situ Ellipsometrie, bei der man eine kontinuierliche Zunahme der Dicke eines $\mathrm{La}_{0.7} \mathrm{Sr}_{0.3} \mathrm{MnO}_{3}$ (LSMO)-Filmes auf einem $\mathrm{SrTiO}_{3}$-Substrat annimmt, führt zu starken Abweichungen von den gemessenen Daten. In Abbildung 6.6 

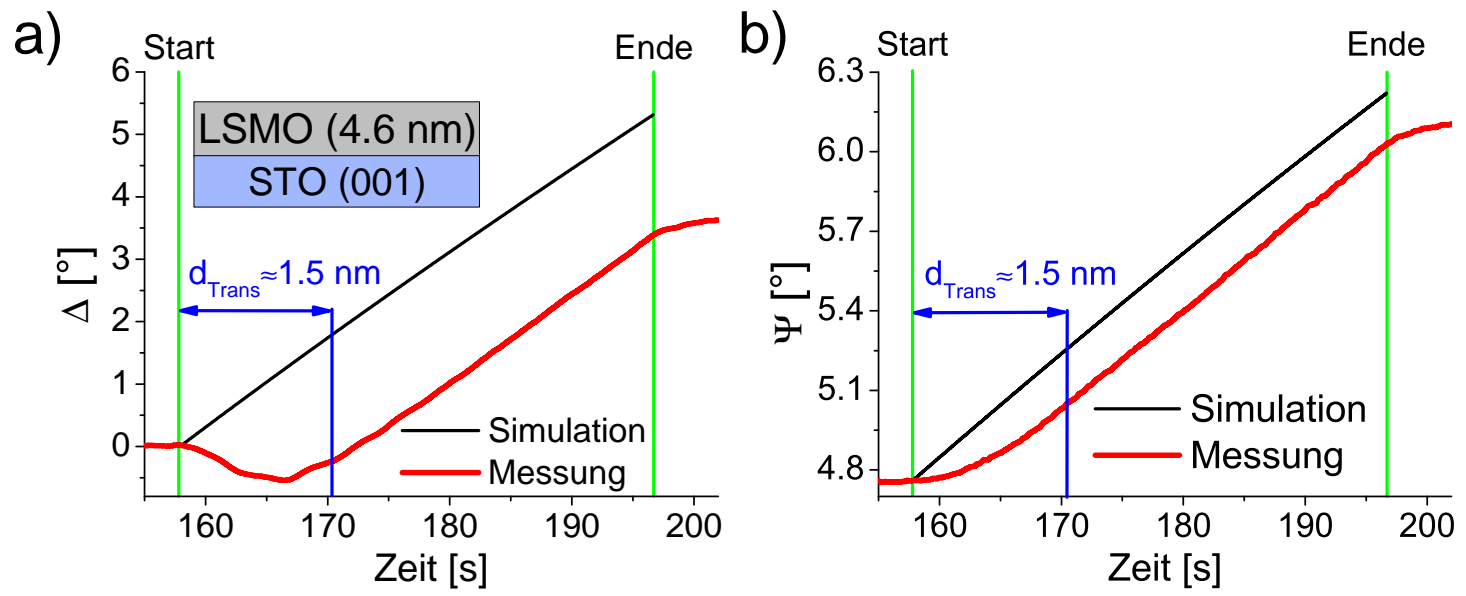

Abbildung 6.6.: Vergleich der gemessenen in-situ Ellipsometrie mit einer Simulation, bei der ein kontinuierliches Wachstum angenommen wird, für die Deposition einer LSMOSchicht auf einem unbehandelten STO (001)-Substrat. Nach der Übergangsregion $d_{\text {Trans }} \approx$ $1.5 \mathrm{~nm}$ verlaufen die gemessenen Daten und die Simulation parallel.

sieht man die in-situ Messungen von $(\Delta, \Psi)$ während des Wachstums einer LSMO-Schicht $\left(d_{L S M O}=4.6 \mathrm{~nm}\right)$ auf einem unbehandelten STO (001)-Substrat zusammen mit einer einfachen Simulation, für die der oben ermittelte Wert des Brechungsindexes von LSMO verwendet wurde (siehe Abbildung 6.4 b)). Für $\Delta$ und $\Psi$ sind deutliche Abweichungen zwischen den Messdaten und den simulierten Kurven auszumachen: In der Simulation zeigen $\Delta(t)$ und $\Psi(t)$ einen monotonen Anstieg mit einer fast konstanten Steigung. Dagegen zeigt sich bei den Messungen ein Übergangsbereich, dessen Breite sich über das deponierte Volumen der Präkursorlösung zu $d_{\text {Trans }} \approx 1.5 \mathrm{~nm}$ ergibt. In dieser Region offenbart sich für $\Delta(t)$ ein Minimum und für $\Psi(t)$ eine deutlich kleinere Steigung als in späteren Phasen der Deposition.

Wenn man $\Delta(t)$ in Abbildung 6.6 genauer untersucht, erkennt man besonders in der Anfangsphase eine weitere Struktur in den Messdaten. Diese kann man genauer analysieren, indem man die Messpunkte fortlaufend über jeweils 50 Punkte mittelt und diese gemittelte Größe, $\Delta_{A v}(t)$, von den Messpunkten subtrahiert (Abbildung 6.7 a), b)). Die Differenz $\Delta-\Delta_{A v}$ weist ein oszillierendes Verhalten auf, wobei die Schwingungsamplitude nach einer Depositionszeit von $\Delta t \approx 15 \mathrm{~s}$, was einer deponierten Schichtdicke von $d \approx 4 \mathrm{ML}$ entspricht, beginnt abzuklingen. Wenn man die Zeitpunkte $t\left(n_{E x}\right)$, an denen die Extrema auftreten als Funktion ihres Indexes $n_{E x}$ aufträgt, ergibt sich ein linearer Verlauf (Abbildung 6.7 c)). Der sich aus der Steigung dieser Geraden ergebende mittlere Abstand zwischen den Maxima bzw. Minima, $\Delta t=3.54(3) \mathrm{s}$, stimmt mit der Depositionszeit pro Monolage, $\Delta t(1 M L)_{X R R}=3.6(2) \mathrm{s}$, die sich aus der Gesamtdauer der Deposition und der nachträglich durch XRR bestimmten Gesamtschichtdicke ergibt, überein. Wenn man mit den Zeitintervallen $\Delta t(1 M L)$ von der Startzeit der Deposition ausgeht, folgt, dass die Maxima von $\Delta(t)-\Delta_{A v}(t)$ zu Zeitpunkten auftreten, wo gerade eine ganze Zahl von Monolagen deponiert wurde. Die darauf folgenden Minima erscheinen nach der Deposition einer weiteren halben Monolage (siehe Abbildung 6.7 b)). Diese Monolagenoszillationen sind also mit dem zweidimensionalen Inselwachstum verbunden. Wenn man LSMO-Filme mit unterschiedlichen Schichtdicken nach der Deposition mit dem Rastertunnelmikroskop (STM) untersucht, stellt man fest, dass für dünne Filme $\left(d_{L S M O} \lesssim 5 \mathrm{~nm}\right)$ eine Wachstumsmode mit zweidimensionalen Inseln dominiert, wogegen das Wachstum für dickere Filme eher einer step-flow-Wachstumsmode folgt (vergleiche Abbildung 6.8). Da bei einem step-flow-Wachstum die Oberfläche in jeder Phase des Wachstums 

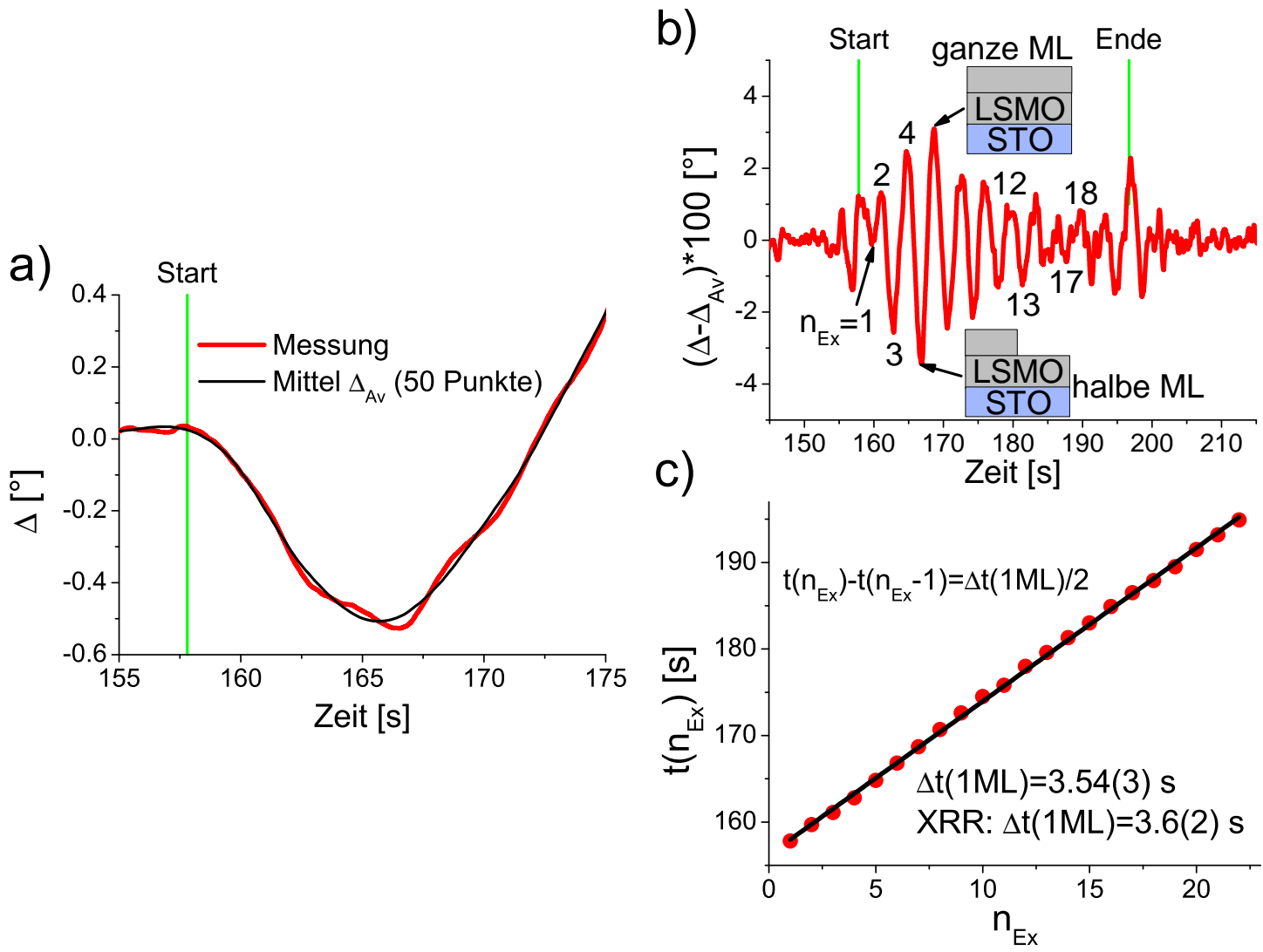

\begin{abstract}
Abbildung 6.7.: Oszillierendes Verhalten von $\Delta(t)$ bei der Deposition von LSMO $\left(d_{L S M O}=4.6 \mathrm{~nm}\right)$ auf STO (001)-Substrat: a) Gemessenes $\Delta(t)$ und dessen Mittel $\Delta_{A v}(t)$, für das fortlaufend der Mittelwert über 50 Punkte bestimmt wurde. b) Differenz $\Delta-\Delta_{A v}$ für die gesamte Depositionsdauer, mit $n_{E x}$ werden die lokalen Extrema durchnummeriert, die starken Ausschläge beim Start und Ende der Deposition sind Artefakte der Mittelung von $\Delta$. c) Darstellung der Zeitpunkte der Extrema als Funktion des Indexes $n_{E x}$, die Steigung der linearen Anpassung liefert die durchschnittliche Depositionszeit einer halben Monolage $\Delta t(1 M L) / 2$.
\end{abstract}

das gleiche Erscheinungsbild besitzt, kann man dort keine periodischen Oszillationen der optischen Eigenschaften erwarten. Somit folgt die Verminderung der Oszillationsamplitude in Abbildung 6.7 b) aus einem kontinuierlichen Übergang der Wachstumsmode von zweidimensionalem Inselwachstum zum step-flow-Wachstum.

Es ist nun zu begründen, wie sich die Messbarkeit der Monolagenoszillationen mit optischen Methoden ergibt. Eine halb bedeckte Oberfläche muss andere optische Eigenschaften besitzen als eine geschlossene Monolage. Ein einfacher Ansatz, bei dem man die optischen Eigenschaften einer mit einem Anteil $s$ bedeckten Oberfläche durch ein effektives Medium, das zu einem Anteil $s$ aus dem Material des Films und einem Teil $1-s$ aus Luft besteht, nähert, liefert auch ein oszillierendes Verhalten mit der Periode einer Monolage. Allerdings ergeben sich entgegen den oben gezeigten Messungen die Maxima von $\Delta-\Delta_{A v}$ bei einer halben Bedeckung, $s=0.5$, und nicht bei einer abgeschlossenen Monolage. Zudem ist die Amplitude der Oszillationen um einen Faktor $\approx 25$ größer als im hier durchgeführten Experiment. Dies deutet an, dass die lateralen Abmessungen der meisten Inseln zu groß sind für die Näherung durch ein effektives Medium. So ist in Abbildung 6.8 a) nur ein kleiner Teil der Probenoberfläche von Inseln bedeckt, für deren Durchmesser

$$
d<\lambda / 30=632.8 / 30 \mathrm{~nm} \approx 20 \mathrm{~nm}
$$


a)
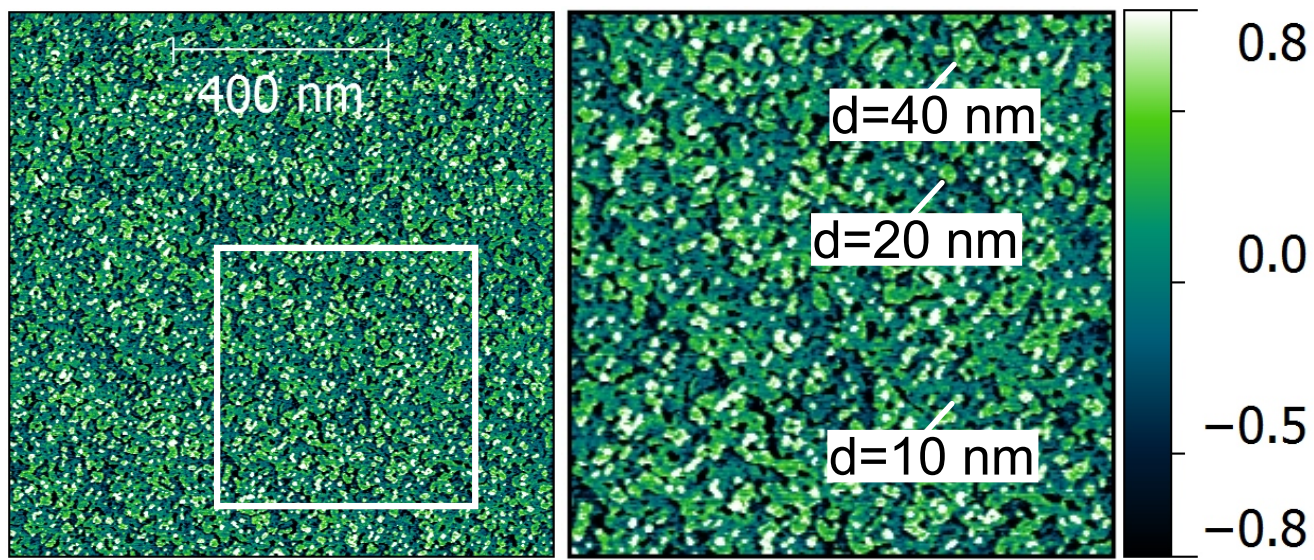

\section{$0.8 \mathrm{~nm}$}

b)

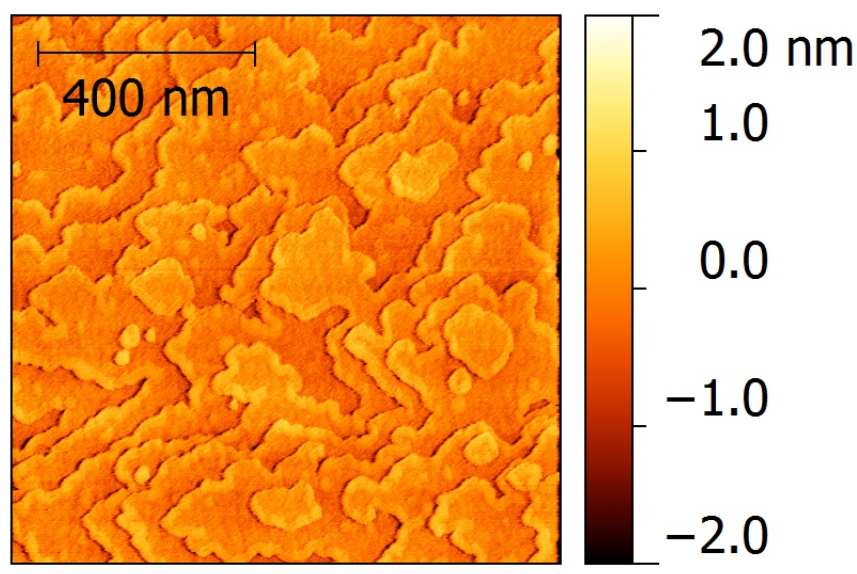

Abbildung 6.8.: Messungen der Oberflächenmorphologie mit dem STM für auf STO (001)Substraten gewachsene LSMO-Schichten verschiedener Dicke: a) $d_{L S M O} \approx 6 \mathrm{~nm}$, das rechte Bild zeigt eine Vergrößerung des weiß umrandeten Bereichs im linken Bild. Im vergrößerten Ausschnitt sind einzelne Inseln markiert und deren mittlere Durchmesser angegeben. b) $d_{L S M O} \approx 120 \mathrm{~nm}$.

gilt (vergleiche Abschnitt 4.3.3 und [156]). Ein Großteil des aufgebrachten Materials ist bereits zu den Kanten der Stufen diffundiert und bildet dort Strukturen, deren laterale Abmessungen $20 \mathrm{~nm}$ deutlich übersteigen. Bei der Homoepitaxie von STO wurden auch Oszillationen der optischen Eigenschaften mit der Periode einer Monolage beobachtet 148. Fei et al. erklärten diese durch eine andere Konzentration von Sauerstoffleerstellen an den Kanten der Inseln. In den Experimenten hier sind keine Sauerstoffleerstellen in den abgeschiedenen Filmen anzunehmen. Es besteht jedoch die Möglichkeit einer abweichenden chemischen Zusammensetzung im Bereich der Kanten der Inseln. Diese Randbereiche besitzen dann andere optische Eigenschaften. Dadurch dass das Volumen der Randbereiche mit dem Grad der Bedeckung $s$ oszilliert, ergeben sich dann die periodischen Variationen der optischen Eigenschaften. Eine mögliche Erklärung für eine solche chemisch inhomogene Verteilung ist in unterschiedlichen Mobilitäten der verschiedenen Adatome zu suchen.

Im Folgenden soll die starke Abweichung der gemessenen Verläufe von $\Delta(t)$ und $\Psi(t)$ von den einfachen theoretischen Berechnungen in der Anfangsphase der Deposition (vergleiche Abbildung 6.6 genauer betrachtet werden. Zur genaueren Anpassung der Messungen zerlegt man die gewachsene Schicht in einzelne Lagen mit dem Index $i_{M L}=1,2, \ldots, n_{M L}$, die jeweils 
a)

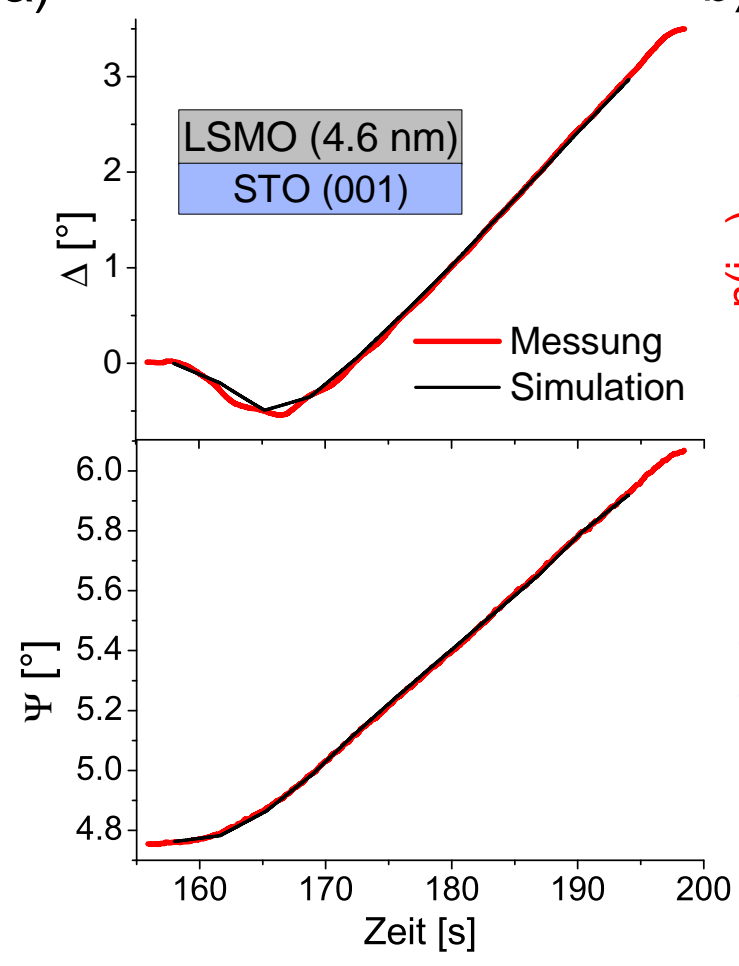

b)

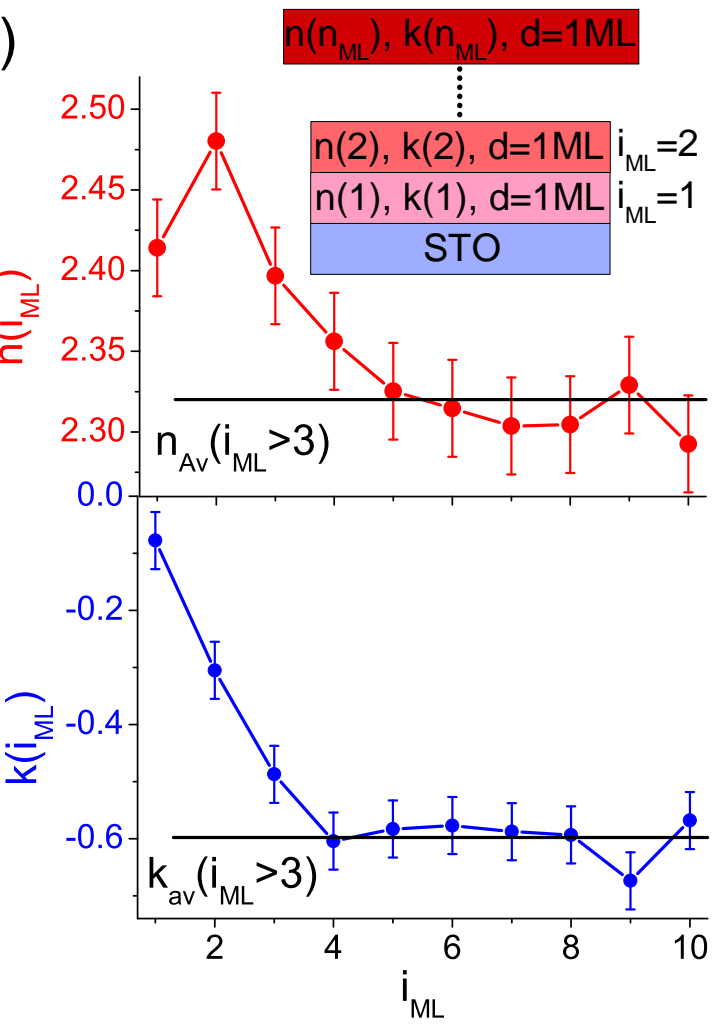

Abbildung 6.9.: Anpassung der gemessenen Verläufe $\Delta(t)$ und $\Psi(t)$ in der Anfangsphase der Deposition einer LSMO-Schicht auf einem unbehandelten STO (001)-Substrat durch die Zerlegung der Schicht in $n_{M L}$ Monolagen mit individuellen Werten für den Brechungsindex (vgl. b)): a) Gemessene $\Delta(t)$ und $\Psi(t)$ mit zugehörigen Anpassungen, b) Abhängigkeit von $n\left(i_{M L}\right)$ und $k\left(i_{M L}\right)$ als Funktion des Index der Lage $i_{M L}$.

die Dicke einer Monolage $d_{M L}$ und einen komplexen Brechungsindex $N\left(i_{M L}\right)=n\left(i_{M L}\right)+$ $i k\left(i_{M L}\right)$ aufweisen (siehe Abbildung 6.9 b)). Für diese Zerlegung kann man $n\left(i_{M L}\right)$ und $k\left(i_{M L}\right)$ iterativ durch numerische Inversion berechnen. In Abbildung 6.9 a) ist die sich so ergebende Anpassung für das Wachstum einer LSMO-Schicht $\left(d_{L S M O}=4.6 \mathrm{~nm}\right)$ auf einem STO (001)-Substrat dargestellt. Die daraus folgenden Abhängigkeiten der Brechungsindizes $n\left(i_{M L}\right)$ und $k\left(i_{M L}\right)$ sind in Abbildung $\left.6.9 \mathrm{~b}\right)$ aufgetragen. Man erkennt, dass $n$ und $k$ für $i_{M L} \geq 4 \mathrm{um}$ einen festen Wert schwanken, der ungefähr dem Brechungsindex einer vollen LSMO-Schicht entspricht. Für kleinere Werte von $i_{M L}$ zeigen $n$ und $k$ einen Abfall, wobei die Änderungen für $n$ wesentlich kleiner ausfallen als für $k$.

Es liegt nahe die Abweichungen der optischen Eigenschaften der ersten drei gewachsenen Monolagen von jenen einer reinen LSMO-Schicht der Durchmischung mit dem STO-Substrat zuzuschreiben. Darauf weist auch die Tatsache hin, dass $k$ zu Beginn nur einen kleinen Betrag besitzt. Da STO einen verschwindenden Imaginärteil des Brechungsindexes aufweist, sollte für eine Mischung von STO und LSMO eine kleinere Absorption zu registrieren sein als für reines LSMO. Die vierte aufgewachsene Monolage zeigt dann keine Beeinflussung aufgrund der Durchmischung mit dem Substrat. Die sich daraus ergebende Länge von $d_{\text {Mix }} \approx 1.2 \mathrm{~nm}$ gibt den Abstand von der Grenzfläche an, in dem man im aufgewachsenen LSMO-Film noch den Einfluss der chemischen Durchmischung mit dem Substrat registrieren kann. Dabei ist der Einfluss der eindiffundierten Ti-Ionen dominant, da der Betrag von $k$ kleiner als in LSMO ist. Zusätzliche Sr-Ionen aus dem Substrat würden durch die Veränderung 

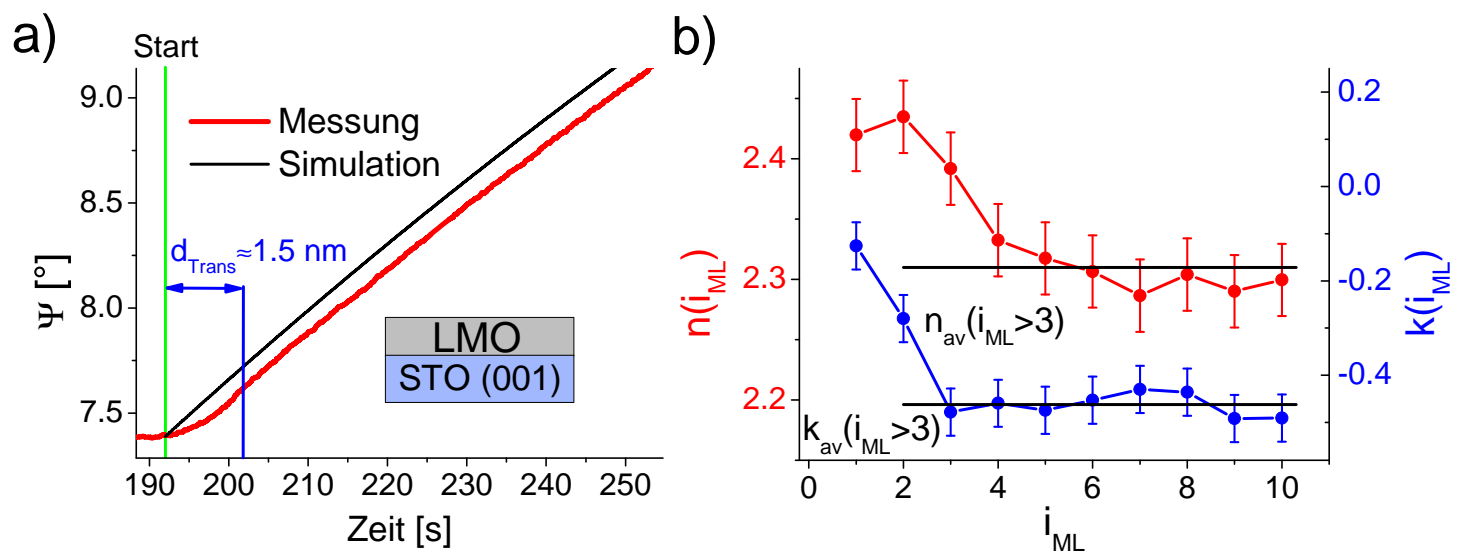

\begin{abstract}
Abbildung 6.10.: Anfangsphase der Deposition eines LMO-Filmes auf einem unbehandelten STO (001)-Substrat: a) Experimentelle Messung von $\Psi(t)$ und einfache Simulation, bei der kontinuierliches Wachstum angenommen wird. b) Abhängigkeit der analog zum Verfahren in Abbildung 6.9 bestimmten Brechungsindizes $n\left(i_{M L}\right)$ und $k\left(i_{M L}\right)$ von der Nummer der Monolage $i_{M L}$.
\end{abstract}

des $\mathrm{Mn}^{3+} / \mathrm{Mn}^{4+}$-Verhältnisses zu einer Vergrößerung des Betrages von $k$ führen (siehe auch Abbildung 6.4). Ex-situ kann man die chemische Durchmischung an der Grenzfläche mit Methoden wie XRR und STEM-EELS quantifizieren. Für die in Abbildung 6.9 gezeigte LSMOSchicht ergibt sich durch die Anpassung des XRR-Spektrums eine Rauigkeit zum Substrat von $\sigma_{S T O} / L S M O=0.6(1) \mathrm{nm}$. Wenn man das damit entstehende chemische Profil betrachtet, kann man ablesen, dass in der dritten deponierten Monolage noch etwa $5 \%$ des Materials vom Substrat zu registrieren sind und in der vierten Monolage weniger als 1\%. Da die XRRMessungen im Wesentlichen nur Informationen über die chemische Verteilung der La- und Sr-Ionen liefern, zieht man zum Vergleich außerdem Messungen mit STEM-EELS heran. Für LSMO-Filme, die bei den gleichen Bedingungen wie der in Abbildung 6.9 gezeigte Film mit der MAD hergestellt wurden, zeigt sich auch das Eindringen von Ti-Ionen bis einschließlich zur dritten aufgewachsenen Monolage der Manganat-Schicht 104. Selbst für LSMO-Filme, die mit anderen Methoden wie PLD abgeschieden wurden, ergibt sich bei STEM-EELSMessungen ein ähnlicher Befund hinsichtlich der chemischen Verteilung der Ti-Ionen 105. Somit liefert die in-situ gemessene Ellipsometrie Informationen über die chemischen Durchmischungen in Heterostrukturen.

Im Gegensatz zu den ex-situ Methoden kann man durch die in-situ Messungen außerdem klären, zu welchem Zeitpunkt während der Deposition es zur Durchmischung mit dem Substrat kommt. Die Messungen zeigen, dass das deponierte Material direkt beim Wachstum der jeweiligen Monolage mit dem Substrat reagiert. Dass die Breite des chemischen Durchmischungsprofils ähnlich ist für die in-situ Messung und die ex-situ Charakterisierungen, schließt außerdem Interdiffusionsprozesse beim weiteren Fortschreiten des Wachstums aus. Im Experiment lässt sich dies weiter bestätigen, wenn man die Rauigkeit der LSMO/STOGrenzfläche für LSMO-Filme unterschiedlicher Dicke und damit verschiedenen Depositionszeiten vergleicht: Für einen LSMO-Film mit $d_{L S M O}=6.1 \mathrm{~nm}\left(t_{D e p} \approx 0.8 \mathrm{~min}\right)$ ergibt sich mit $\sigma_{S T O / L S M O}=0.5(2) \mathrm{nm}$ ein ähnlicher Wert wie für einen deutlich dickeren Film mit $d_{L S M O}=37 \mathrm{~nm}\left(t_{D e p} \approx 5.1 \mathrm{~min}\right)$, wo eine Rauigkeit von $\sigma_{S T O} / L S M O=0.6(2) \mathrm{nm}$ registriert wird. Damit ist die LSMO/STO-Grenzfläche für die betrachteten Bedingungen und Depositionszeiten stabil und eine einfache Interdiffusion der beiden Materialien über die Zeit der Deposition kann ausgeschlossen werden. Das wird dadurch untermauert, dass sich 
a)

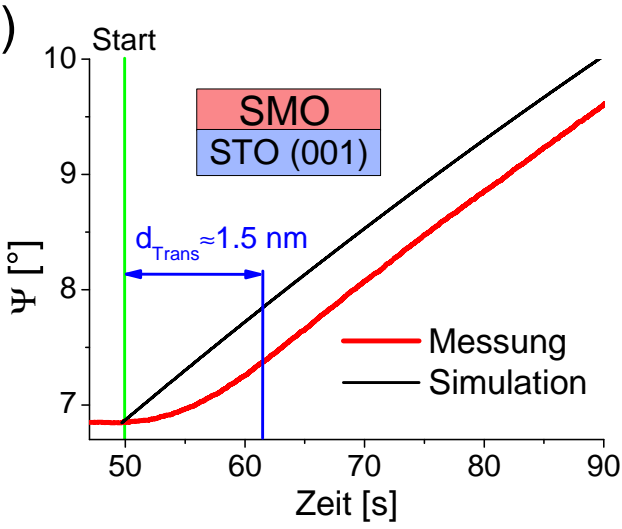

b)

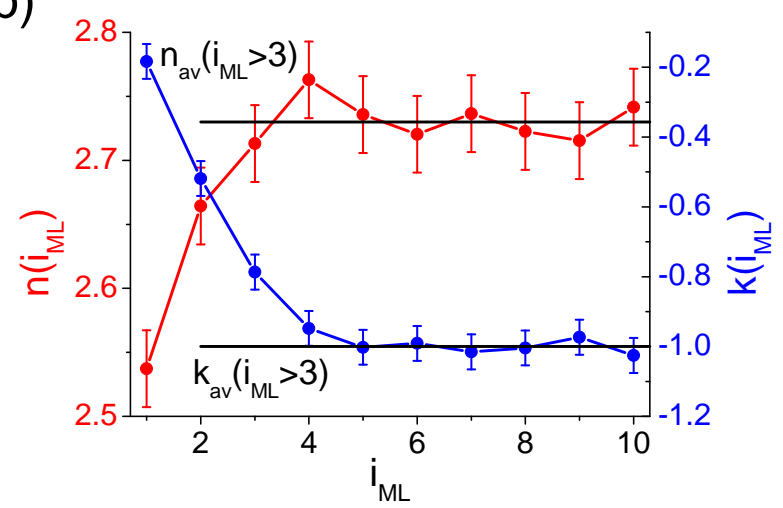

Abbildung 6.11.: Anfangsphase der Deposition eines SMO-Filmes auf einem unbehandelten STO (001)-Substrat: a) Experimentelle Messung von $\Psi(t)$ und einfache Simulation, bei der kontinuierliches Wachstum angenommen wird. b) Abhängigkeit der analog zum Verfahren in Abbildung 6.9 bestimmten Brechungsindizes $n\left(i_{M L}\right)$ und $k\left(i_{M L}\right)$ von der Nummer der Monolage $i_{M L}$.

auch bei einer gleichzeitigen Verringerung der Depositionstemperatur von $T_{\text {Dep }} \approx 930^{\circ} \mathrm{C}$ auf $T_{\text {Dep }} \approx 770^{\circ} \mathrm{C}$ und Verringerung der Depositionszeit pro Monolage von $\Delta t(1 M L) \approx 3.5 \mathrm{~s}$ auf $\Delta t(1 M L) \approx 0.1 \mathrm{~s}$ keine Veränderung von $\sigma_{S T O / L S M O}$ zeigt. Die hier dargestellte Phänomenologie für das Wachstum von LSMO auf STO ist nicht auf den Fall beschränkt, in dem STO als Substrat fungiert. Auch bei Übergittern aus STO und LSMO, die von Oleg Shapoval und Markus Michelmann deponiert wurden, beobachtet man für das Wachstum von LSMO auf STO ein analoges Verhalten der in-situ Ellipsometrie und eine identische Rauigkeit $\sigma_{S T O / L S M O}=0.6(1) \mathrm{nm}$ bei den anschließenden XRR-Messungen. Auch beim Wachstum von LSMO auf STO-Substraten, die entlang anderer Ebenen, der (110)- und (111)Ebene, geschnitten wurden, bemerkt man die Übergangsregion $d_{\text {Trans }}$ mit ähnlicher Breite und Ausprägung der Verläufe von $\Delta(t)$ und $\Psi(t)$ 97. Wenn man andere Zusammensetzungen des $\mathrm{La}_{1-\mathrm{x}} \mathrm{Sr}_{\mathrm{x}} \mathrm{MnO}_{3}$-Phasendiagrammes auf STO (001)-Substraten abscheidet, kann man die Analyse von Abbildung 6.9 analog ausführen und erhält ein vergleichbares Ergebnis: Die Brechungsindizes der ersten drei Monolagen unterscheiden sich vom Mittelwert der darauf folgenden Lagen $\left(i_{M L}>3\right)$, der dem Wert des reinen Materials entspricht. Dies ist für die beiden Materialien an den Enden des Phasendiagrammes, $\mathrm{LaMnO}_{3}$ (LMO) und $\mathrm{SrMnO}_{3}$ (SMO), in den Abbildungen 6.10 und 6.11 gezeigt. Ex-situ ermittelt man mittels XRR für SMO eine Rauigkeit von $\sigma_{S T O / S M O}=0.6(2) \mathrm{nm}$ zum Substrat, was der Rauigkeit $\sigma_{S T O / L S M O}$ von LSMO und STO entspricht. Für LMO-Filme beobachtet man nach Abschluss der Deposition deutlich größere Rauigkeiten. Außerdem hängt die Durchmischung mit dem STO-Substrat von der Dauer der Deposition ab, der diffuse Bereich der LMO/STO-Grenzfläche verbreitert sich bei Auslagerung der Probe unter Depositionsbedingungen. Durch diese Zeitabhängigkeit ist die Rauigkeit $\sigma_{S T O / L M O}$ bei gleicher Depositionsrate abhängig von der Schichtdicke, so ergibt sich für $d_{L M O}=27 \mathrm{~nm}\left(t_{D e p} \approx 4 \min \right) \sigma_{S T O / L M O}=1.4(2) \mathrm{nm}$ und für $d_{L M O}=50 \mathrm{~nm}$ $\left(t_{D e p}=7.5 \mathrm{~min}\right) \sigma_{S T O / L M O}=2.2(2) \mathrm{nm}$.

Mit den zuletzt aufgezählten Tatsachen kann man die Rolle der durch die polare Katastrophe verursachten Umverteilungen der Ladungen (siehe Abschnitt 3.1) bei der chemischen Diffusion an der Grenzfläche zwischen den Manganaten und STO beleuchten. Zur Motivation der polare Katastrophe wird davon ausgegangen, dass der an der Grenzfläche stattfindende Symmetriebruch der in Lagen angeordneten Ladungsdichten zu einem elektrischen Potential 
führt, das linear mit der Dicke des Filmes ansteigt. Eine Umverteilung von Ladungen wird dabei erst bei einer bestimmten kritischen Schichtdicke des Filmes beobachtet [9]. Aufgrund verschiedener experimenteller Befunde kommt die polare Katastrophe nicht als treibende Kraft für die Durchmischung der ersten drei aufgewachsenen Monolagen der Manganatfilme in Frage: Bei den hier gezeigten Messungen der in-situ Ellipsometrie erkennt man eine Abweichung der optischen Eigenschaften der aufwachsenden Manganatschichten schon beim Beginn der ersten Einheitszelle. Für das Wachstum von SMO auf STO (001) und dem Wachstum von Manganatfilmen auf STO (110)-Substraten erwartet man keine Umverteilungen von Ladungen aufgrund eines divergierenden elektrostatischen Potentials, da für diese Fälle die Abfolge von geladenen Lagen im Substrat und im Film identisch sind [97]. Die beobachteten Modifikationen der ersten drei gewachsenen Monolagen haben somit den Charakter einer Festkörperreaktion, welche aufgrund der modifizierten Bindungsenergien für die einzelnen Monolagen der Manganatschicht eintritt. Eine einzelne Monolage des Manganats, die sich auf dem STO-Substrat befindet, besitzt aufgrund der freien Oberfläche eine geringere Bindungsenergie, wodurch die erhöhte Entropie bei einer Durchmischung mit dem STO insgesamt die freie Enthalpie reduziert. Bei einer steigenden Schichtdicke wird diese Reaktion der Lage an der Oberfläche durch die steigende Entfernung zum STO-Substrat immer weiter gehemmt, bis sie bei der vierten Monolage komplett zum Erliegen kommt. Die Manganatlagen unterhalb der Oberfläche sind durch die dreidimensionale Bindung stabil gegen die Durchmischung mit dem Titanat.

Wenn man die Sr-Dotierung vermindert und sich im $\mathrm{La}_{1-\mathrm{x}} \mathrm{Sr}_{\mathrm{x}} \mathrm{MnO}_{3}-\mathrm{Phasendiagramm} \mathrm{in}$ Richtung LMO bewegt, ist es nicht mehr möglich die zur Vermeidung der polare Katastrophe nötigen Umverteilungen allein durch die Änderung der Valenz von Mn-Ionen zu bewerkstelligen. Dadurch kommt es zu einer Änderung der Valenz der Ti-Ionen $\left(\mathrm{T}^{4+} \rightarrow \mathrm{T}^{3+}\right)$ in der Nähe zur Grenzfläche, vergleichbar zur Situation von $\mathrm{LaAlO}_{3} / \mathrm{SrTiO}_{3}$-Heterostrukturen 61. Die räumliche Delokalisierung der Elektronen auf den Ti-Ionen führt dann zu einem Dipol, dessen Feld die Durchmischung der Kationen auf den A-Plätzen treibt. Dies bewirkt analog zu den $\mathrm{LaAlO}_{3} / \mathrm{SrTiO}_{3}$-Heterostrukturen eine Zunahme der Rauigkeit der Grenzflächen mit längerer Auslagerung bei Depositionsbedingungen [61. Somit kommt es für die niedrigen Sr-Dotierungen $x \lesssim 0.3$ neben der oben diskutierten Reaktion direkt beim Wachstum zusätzlich zu einer Interdiffusion der Materialien, welche durch die Ladungsumverteilung zwischen Grenz- und Oberfläche und dem dabei entstehenden Dipol im STO getrieben wird. In Abbildung 6.4 a) wird dies damit belegt, dass $\sigma_{L S M O / S T O}$ über einen großen Teil des Phasendiagrammes konstant bei $\sigma_{L S M O / S T O} \approx 0.6 \mathrm{~nm}$ liegt, um dann für $x \lesssim 0.3$ anzusteigen.

Aus den getätigten Folgerungen über die graduelle Veränderung der chemischen Zusammensetzung der deponierten Monolagen kann man Schlüsse für den Grund der beobachteten Änderung der Wachstumsmode vom zweidimensionalen Inselwachstum zum step-flow-Wachstum ziehen. Dieser Übergang wird getrieben durch eine Erhöhung der Beweglichkeit der Adatome mit steigender Schichtdicke. Wie in Abbildung 6.9 zu sehen ist, unterscheidet sich die chemische Zusammensetzung der wachsenden LSMO-Schicht bis einschließlich zur dritten Monolage vom Wert, der sich für das reine Material ergibt. Aufgrund dieser chemischen Inhomogenität ergibt sich für die Adatome eine komplizierte Energielandschaft bezüglich der Bewegung über die Oberfläche der zuletzt abgeschlossenen Monolage. Dies kann einerseits durch die Unterschiede in den Bindungsenergien für die Ti-O-Mn- und Mn-O-Mn-Bindungen motiviert werden. Andererseits unterdrücken die zusätzlichen Ti-Ionen in den LSMO-Lagen die Delokalisierung der $e_{g}$-Elektronen. Dadurch kann auch eine zufällige Akkumulation von La- bzw. Sr-Ionen zu lokalen Extrema in der Energielandschaft führen. Durch die Metallizität dickerer LSMO-Schichten werden diese Inhomogenitäten auf den A-Plätzen durch die mobilen 
$e_{g}$-Elektronen stärker abgeschirmt und die Energielandschaft ist homogener. Für das gezeigte Beispiel der auf dem STO (001)-Substrat gewachsenen LSMO-Schicht (siehe Abbildungen 6.7 und 6.9p wächst somit die fünfte Monolage auf einer Lage auf, die eine Zusammensetzung wie reines LSMO besitzt. Die Oszillationsamplitude ist für diese Lage bereits deutlich kleiner als zu Beginn $\left(n_{E x}=9,10\right.$ in Abbildung 6.7 b)).

In diesem Abschnitt wurde die Untersuchung der Abscheidung von einfachen Manganatfilmen auf STO-Substraten mit in-situ Ellipsometrie behandelt. Dabei konnte wie bei der in-situ Kontrolle mittels RHEED bei Depositionsmethoden wie PLD 216 218 ein Übergang von zweidimensionalem Inselwachstum zur step-flow-Wachstumsmode identifiziert werden. Dieser zeigte sich in einem Abklingen der Amplitude der Monolagenoszillationen, die sich bei der Betrachtung des in-situ gemessenen Verlaufs von $\Delta(t)$ zeigten. Weiterhin war es möglich Interdiffusionsprozesse zu identifizieren, die unmittelbar bei der Deposition auftreten und nicht durch einfache Diffusion erklärbar sind, sondern in Form einer Festkörperreaktion ablaufen, die sich mit steigender Schichtdicke selbst begrenzt. Die Änderung der Wachstumsmode wird durch diese Schichtdickenabhängigkeit der chemischen Zusammensetzung der aufwachsenden Manganatlagen induziert. Die Durchmischung der Filme aus $\mathrm{La}_{1-\mathrm{x}} \mathrm{Sr}_{\mathrm{x}} \mathrm{MnO}_{3}$ mit dem $\mathrm{SrTiO}_{3}-$ Substrat schreitet direkt bei der Deposition unabhängig von der Sr-Dotierung $x$ bis zu einer Dicke von drei Monolagen fort. Für $x \lesssim 0.3$ ergibt sich außerdem eine weitere Verbreiterung der durchmischten Grenzregion durch Diffusion, die durch die zur Vermeidung der polare Katastrophe stattfindenden Ladungsumverteilungen getrieben wird. 



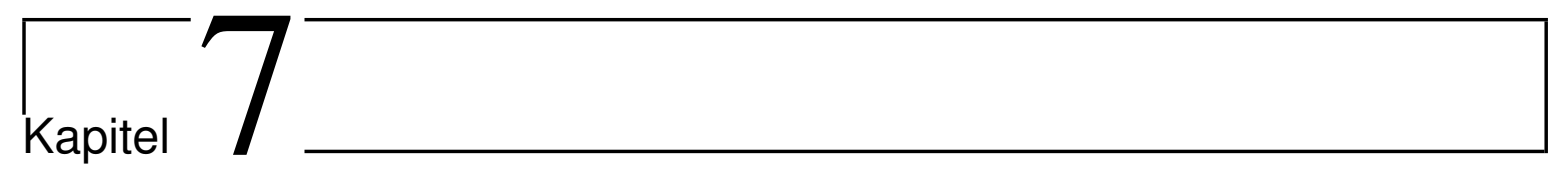

\section{Atomlagenepitaxie}

\subsection{Motivation}

Perowskite sind entlang der [001]-Richtung aus $\mathrm{AO}$ - und $\mathrm{BO}_{2}$-Ebenen aufgebaut (Abbildung 7.1). Im normalen MAD-Prozess werden mit gemischten Präkursorlösungen ganze $\mathrm{ABO}_{3}$ Einheitszellen aufgebracht. Verschiedene Experimente zeigen, dass es in Folge von Grenzbzw. Oberflächeneffekten zu Umverteilungen der Kationen auf den A- und B-Plätzen kommen kann [57, 58. Diese intrinsisch getriebenen Abweichungen von der von außen vorgegebenen Stöchiometrie finden teilweise auf der Längenskala einer halben Perowskitlage statt. So beobachtet man an der $\mathrm{La}_{2 / 3} \mathrm{Ca}_{1 / 3} \mathrm{MnO}_{3} / \mathrm{BaTiO}_{3}$-Grenzfläche eine reine CaO-Lage statt der stöchiometrischen $\mathrm{La}_{2 / 3} \mathrm{Ca}_{1 / 3} \mathrm{O}$-Lage [58. Als Auslöser für diese Rekonstruktionen wird zumeist die polare Katastrophe angeführt (vergleiche Abschnitt 3.1). Im Zusammenhang mit diesem Konzept ergibt sich für die LAO/STO-Grenzfläche eine starke Abhängigkeit von der Terminierung der STO-Oberfläche. Bei einem $\mathrm{TiO}_{2}$-Abschluss wird ein zweidimensionales Elektronengas registriert, für eine Bedeckung der Oberfläche mit einer SrO-Monolage nicht 17. 61. Zum genauen Studium von Effekten an Grenzflächen zwischen Perowskit-Oxiden ist es also erstrebenswert, Heterostrukturen mit der Kontrolle über eine halbe Monolage herzustellen.

Neben den Grenzflächen von einfachen Perowskiten ist diese Kontrolle auch entscheidend für die Synthese von oxidischen Lagenstrukturen. Diese Materialklasse zeigt eine Vielzahl an funktionalen Eigenschaften. Die Beispiele reichen von den Hochtemperatur-Supraleitern 219] über ermüdungsfreie Ferroelektrika, $\mathrm{SrBi}_{2} \mathrm{Ta}_{2} \mathrm{O}_{9} 220$, bis zu Photokatalysatoren, $\mathrm{K}_{4} \mathrm{Nb}_{6} \mathrm{O}_{17}$
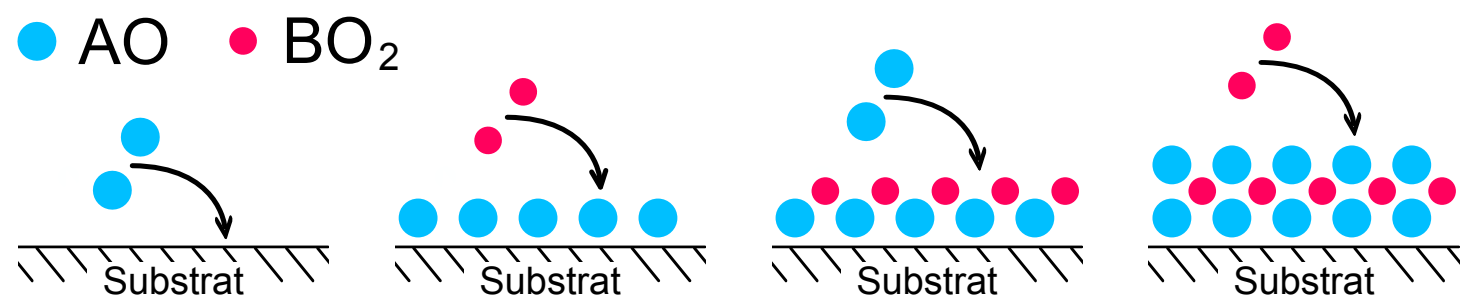

Abbildung 7.1.: Prinzip der MAD Atomlagenepitaxie (ALE): Aufbau eines Perowskites, $\mathrm{ABO}_{3}$, aus $\mathrm{AO}$ - und $\mathrm{BO}_{2}$-Lagen entlang der [001]-Richtung. 
221. Weiterhin sind die Ruddlesden-Popper (RP)-Strukturen, $\mathrm{Sr}_{\mathrm{n}+1} \mathrm{Ti}_{\mathrm{n}} \mathrm{O}_{3 \mathrm{n}+1}\left(\mathrm{SrO}\left(\mathrm{SrTiO}_{3}\right)_{\mathrm{n}}\right)$ 222, 223], zu nennen. Diese eignen sich als einstellbare Dielektrika mit geringem Verlust für Mikrowellen [224].

Die Synthese dieses Materialsystems mit einer einfachen Festkörperreaktion ist für $n \geq 3$ nicht mehr in Reinform möglich 225]. Die entstehenden Polykristalle sind mit RuddlesdenPopper-Titanaten, die einen anderen Wert von $n$ aufweisen, verunreinigt 225. Die Ursachen dafür sind die geringen Unterschiede in der freien Energie für die $\mathrm{SrO}\left(\mathrm{SrTiO}_{3}\right)_{n}$-Phasen mit verschiedenen Werten von $n$ [226]. Damit gibt es in der Energielandschaft viele lokale Minima und die Diffusion zur Bildung der reinen Phasen wird behindert. Durch die verlängerten Diffusionswege wird so der Ausschluss von Fremdphasen mit steigendem $n$ immer schwieriger zu erreichen. Mit MBE wurden RP STO-Filme mit $n=1-6$ abgeschieden. Dazu wurden abwechselnd Lagen aus $\mathrm{SrO}$ und $\mathrm{TiO}_{2}$ deponiert, um die gewünschte Sequenz zu erhalten. Es gibt auch Beispiele für die Herstellung von gestapelten Heterostrukturen mit PLD, z.B. $\mathrm{CaO}\left(\mathrm{CaMnO}_{3}\right)_{4-6}$ 227. (Bei der Herstellung dieser Strukturen wurden nicht durchgehend halbe Perowskitlagen aus $\mathrm{CaO}$ und $\mathrm{MnO}_{2}$ deponiert, sondern komplette Perowskitlagen von $\mathrm{CaMnO}_{3}$ und einzelne CaO-Lagen.). Bei diesen Vakuum-Depositionsmethoden erfolgt die Wachstumskontrolle durch RHEED (siehe Abschnitt 4.2).

Die hier verwendete MAD arbeitet bei deutlich anderen Bedingungen (Wachstumstemperatur, $T_{D e p}$, Sauerstoffpartialdruck, $p_{O_{2}}$ ). Mit Blick auf die potentiellen Anwendungen der komplexen Oxide mit Lagenstruktur stellt die MAD eine vorteilhafte Technologie zur industriellen Produktion dar. Die in den vorherigen Kapiteln vorgestellten Möglichkeiten der Wachstumsüberwachung mit in-situ Ellipsometrie werden auch für die MAD Atomlagenepitaxie (ALE) verwendet. Im Gegensatz zu der zuvor geschilderten Vorgehensweise bei der Deposition dünner Filme mit MAD wird bei der ALE nicht mit einer gemischten Lösung gearbeitet, in der sich die Präkursoren für die A- und B-Kationen befinden. Stattdessen löst man die Präkursoren für die A- bzw. B-Kationen getrennt auf. Mit diesen beiden Lösungen kann dann entweder eine $\mathrm{AO}$ - oder eine $\mathrm{BO}_{2}$-Lage deponiert werden (siehe Abbildung 7.1). Durch die entsprechende Abfolge der $\mathrm{AO}$ - und $\mathrm{BO}_{2}$-Lagen kann man so prinzipiell beliebige Lagenstrukturen abscheiden. Dabei sind allerdings die Stabilität der Lagen an der Oberfläche und eine zweidimensionale Wachstumsmode notwendige Kriterien.

Hier werden die ersten Versuche zur MAD ALE vorgestellt. Deshalb werden Materialien untersucht, die bereits häufig mit dem ursprünglichen MAD-Prozess hergestellt wurden und grundsätzlich ein zweidimensionales Wachstum zeigen. Das RP STO-System stellt dabei einen idealen Test für die Depositionsmethode dar. Aufgrund der ungeladenen $\mathrm{SrO}$ - und $\mathrm{TiO}_{2}$-Lagen muss man keine Instabilitäten beim Wachstum aufgrund von Coulomb-Potentialen erwarten.

Im ersten Abschnitt dieses Kapitels wird die Erzeugung von RP STO-Strukturen mit $n=$ 2,3,4 auf STO (001)-Substraten vorgestellt. Mit der in-situ Ellipsometrie kann man Defekte vorhersagen, die sich in den korrespondierenden TEM-Aufnahmen wiederfinden. Mit Blick auf die abweichenden Wachstumsbedingungen werden die Unterschiede zu den etablierten Depositionstechniken wie MBE und PLD diskutiert. Im Kontrast zu STO ergeben sich für die gemischt-valenten Perowskit-Manganate potentiell Verletzungen der Ladungsneutralität und der Aufbau von elektrostatischen Potentialen beim Wachstum mit ALE. Im zweiten Abschnitt wird mit Blick auf diese Problematik die Epitaxie von Heterostrukturen aus $\left(\mathrm{SrMnO}_{3}\right)_{1} /\left(\mathrm{LaMnO}_{3}\right)_{2}$ untersucht. Dabei wird aufgezeigt, dass eine Abweichung von der formalen Stöchiometrie in den beiden ersten halben Monolagen das Wachstum entscheidend beeinflusst. 


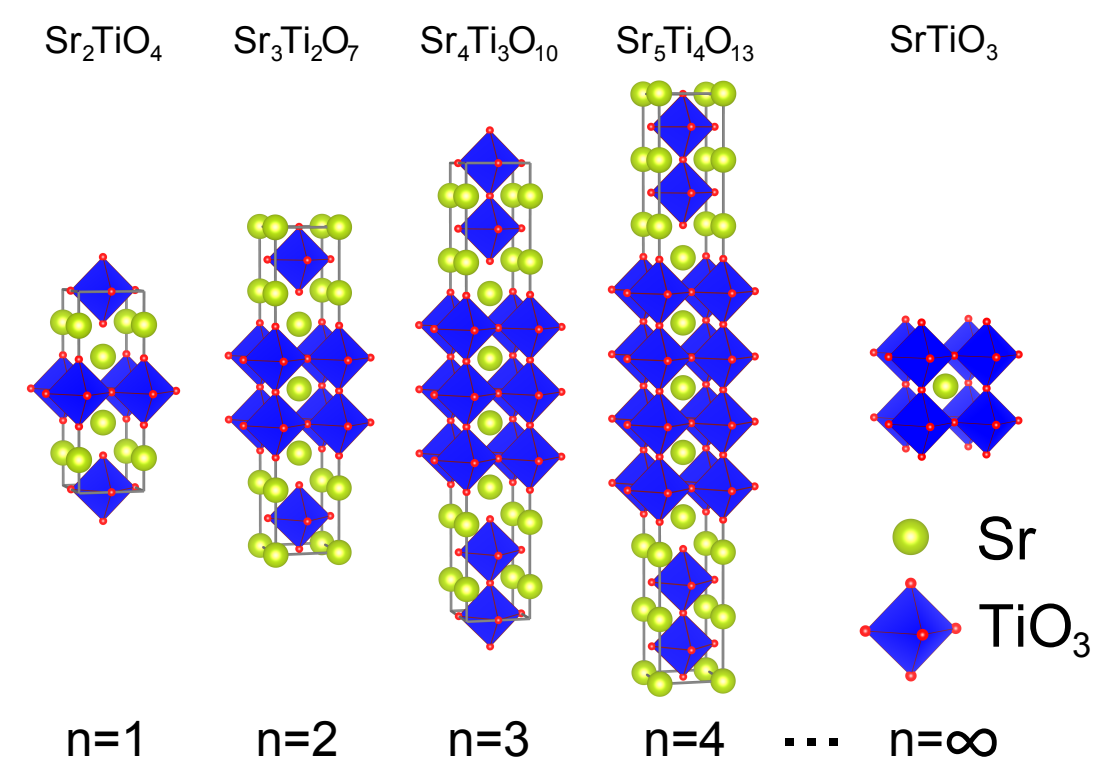

Abbildung 7.2.: Einheitszellen der Ruddlesden-Popper Phase von STO, $\mathrm{Sr}_{n+1} \mathrm{Ti}_{n} \mathrm{O}_{3 \mathrm{n}+1}$ für $n=1-4, \infty$ (nach 228, 229 gezeichnet mit VESTA 21]).

\section{2. $\mathrm{Sr}_{\mathrm{n}+1} \mathrm{Ti}_{n} \mathrm{O}_{3 \mathrm{n}+1}$ Ruddlesden-Popper Phase}

\subsubsection{Einführung}

In Abbildung 7.2 sind die Einheitszellen der RP STO-Phase für $n=1-4, \infty$ dargestellt. Nach $n$ Lagen des Perowskits $\mathrm{SrTiO}_{3}$ folgt eine zusätzliche rocksalt-Lage aus SrO. Durch die SrO-Lage kommt es zu einer Verschiebung der folgenden STO-Lagen um den Vektor $[1 / 2$ 1/2 0]. Diesem Konstruktionsprinzip folgend gibt es neben dem namensgebenden Materialsystem $\mathrm{Sr}_{\mathrm{n}+1} \mathrm{Ti}_{\mathrm{n}} \mathrm{O}_{3 \mathrm{n}+1}$ auch andere Beispiele wie $\mathrm{Sr}_{\mathrm{n}+1} \mathrm{Ru}_{\mathrm{n}} \mathrm{O}_{3 \mathrm{n}+1}\left[229\right.$ oder $(\mathrm{SrO})\left(\mathrm{La}_{1-\mathrm{x}} \mathrm{Sr}_{\mathrm{x}} \mathrm{MnO}_{3}\right)_{\mathrm{n}}$ 230,231 .

In diesem Abschnitt wird das Vorgehen bei der Herstellung von RP STO-Strukturen mit MAD ALE beschrieben. Zunächst erfolgen experimentelle Vorarbeiten, bei denen man die korrekte $\mathrm{Sr} /$ Ti-Stöchiometrie mit in-situ Ellipsometrie ermittelt und die Depositionsmengen für eine Monolage bestimmt. Darauf wird die Herstellung der RP STO-Filme beschrieben und die dabei aufgenommenen Daten zur in-situ Ellipsometrie diskutiert. Die hergestellten Strukturen werden mit XRD und TEM analysiert, wobei die Defekte in der Abfolge der $\mathrm{SrTiO}_{3}-\mathrm{SrO}-$ Lagen erfasst werden. In der abschließenden Diskussion werden die strukturellen Messungen mit den Daten der in-situ Ellipsometrie korreliert und ein Modell der Wachstumsvorgänge erstellt. Mit Blick auf die verschiedenen Depositionsbedingungen erfolgt eine vergleichende Betrachtung mit anderen Depositionstechniken wie MBE.

Die in diesem Abschnitt gezeigten Ergebnisse wurden in Zusammenarbeit mit der Gruppe von Johan Verbeeck der Universität Antwerpen gewonnen. Dabei ist insbesondere Ricardo Egoavil zu nennen, der die hoch aufgelösten TEM-Aufnahmen erstellte und auswertete. Die Resultate wurden bereits veröffentlicht 232 und es erfolgen anknüpfende Forschungen zur Herstellung von STO RP-Strukturen auf anderen Substraten wie LSAT und $\mathrm{DyScO}_{3}$ in der Masterarbeit von Fryderyk Lyzwa, wobei Vladimir Roddatis vom Institut für Materialphysik der Universität Göttingen TEM-Aufnahmen dieser Heterostrukturen erstellte. 


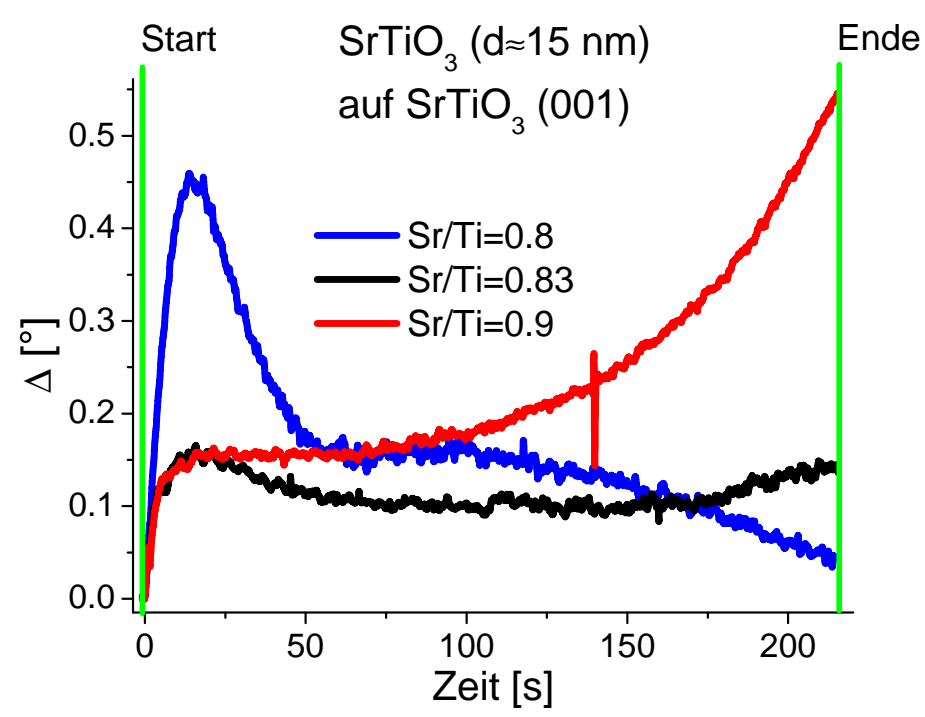

\begin{abstract}
Abbildung 7.3.: Ermittlung der korrekten molaren Verhältnisse von den Sr- und TiPräkursoren zur Herstellung stöchiometrischer STO-Filme auf STO (001)-Substraten: Im konventionellen MAD-Prozess werden Mischlösungen aus Sr- und Ti-Präkursoren mit molaren Verhältnissen $n(\mathrm{Sr}) / n(\mathrm{Ti})(\mathrm{Sr} / \mathrm{Ti})$ erstellt und auf STO-Substraten abgeschieden. Die resultierenden Verläufe von $\Delta(t)$ für das Wachstum von Filmen mit einer Dicke von $d \approx 15 \mathrm{~nm}$ sind geplottet.
\end{abstract}

\title{
7.2.2. Experimentelle Vorbereitungen
}

Die RP STO-Heterostrukturen werden aus Monolagen von $\mathrm{SrO}$ und $\mathrm{TiO}_{2}$ aufgebaut. Somit muss man für die verwendeten Depositionsbedingungen die korrekte Sr/Ti-Stöchiometrie einstellen und das Volumen der Präkursorlösung bestimmen, das man dosieren muss, um eine Monolage zu bedecken. Für die hier vorgestellten Experimente werden kommerzielle Präkursoren, $\mathrm{Sr}$ (acetylacetonat) $)_{2}$ und $\mathrm{Ti}$ (isoprop) $)_{2}$ (tetramethylheptadionat) $)_{2}$, verwendet ${ }^{21}$. Diese werden in Dimethylformamid (DMF) mit einer Konzentration von $0.02 \mathrm{~mol} / \mathrm{l}$ gelöst.

Der Faktor $c_{S r / T i}$ beziffert das molare Verhältnis von Sr- und Ti-Ionen in der Präkursorlösung, das im abgeschiedenen Film zu einem stöchiometrischen molaren Verhältnis, $n(S r) / n(T i)=$ 1, führt. Wie es in Abschnitt 4.1 beschrieben wurde, erfolgt die Bestimmung der Korrekturfaktoren durch einen iterativen Prozess, bei dem die Qualität der deponierten Filme einbezogen wird. In Abschnitt 6.1 wurde dargelegt, dass die in-situ Ellipsometrie beim Wachstum von LSMO-Filmen Hinweise auf die Korrektheit der Stöchiometrie liefern kann. Dieses Prinzip kann bei der Homoepitaxie von STO sehr anschaulich angewendet werden. Bei einem korrekten molaren Verhältnis der Sr- und Ti-Ionen gibt es keinen Unterschied in den optischen Eigenschaften des STO-Substrates und dem STO-Film. Eine Abweichung der Messgrößen $(\Delta, \Psi)$ von einer waagerechten Linie während des Filmwachstums zeigt also einen Fehler der Stöchiometrie an. Je nachdem in welche Richtung $(\Delta(t), \Psi(t))$ von der Waagerechten abweicht, kann man einen Ti- bzw. Sr-Überschuss feststellen.

In Abbildung 7.3 sind die Verläufe von $\Delta(t)$ für verschiedene molare Verhältnisse der Sr- und Ti-Präkursoren dargestellt. Man erkennt, dass $\Delta(t)$ für $\mathrm{Sr} / \mathrm{Ti}=0.83$ einen fast waagerechten

\footnotetext{
${ }^{21}$ Zum Teil sind kommerziell keine Präkursoren mit ausreichender Qualität verfügbar. In diesen Fällen stellte Serhiy Demeshko vom Institut für Anorganische Chemie der Universität Göttingen Sr-Präkursoren für unsere Arbeitsgruppe her.
} 


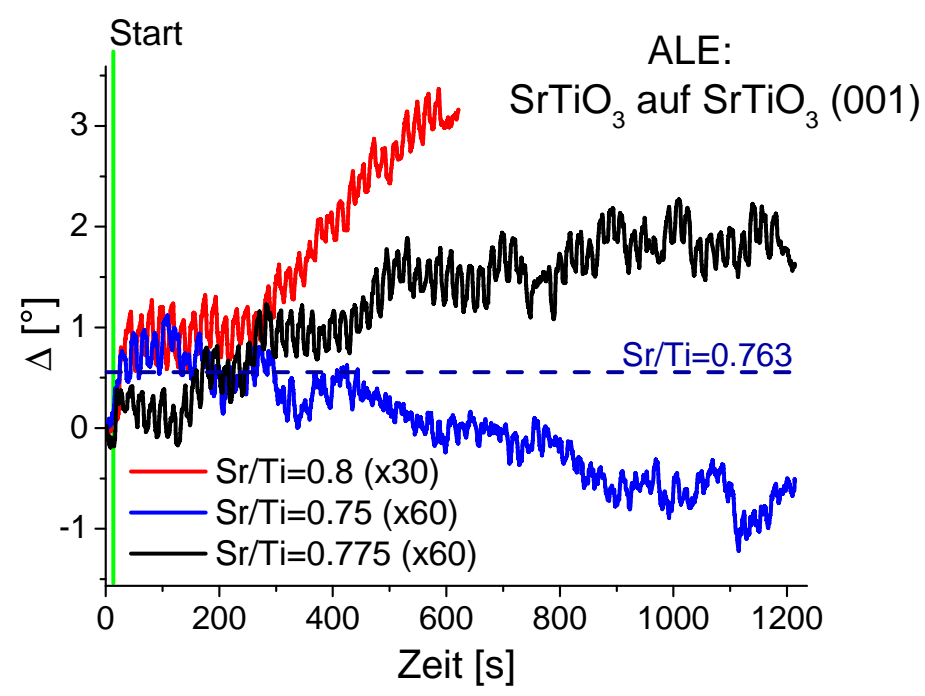

\begin{abstract}
Abbildung 7.4.: Zur Ermittlung der korrekten Sr/Ti-Stöchiometrie für MAD ALE beim homoepitaktischen Wachstum von STO (001): Sr- und Ti-Lösungen gleicher Molarität von $0.02 \mathrm{~mol} / \mathrm{l}$ werden abwechselnd dosiert. Von der Ti-Lösung dosiert man ein Volumen von V $\left(\mathrm{TiO}_{2}\right)=11 \mu \mathrm{l}$. Das Volumen der Sr-Lösung wird zu Bestimmung der Sr/TiStöchiometrie variiert. Dabei gibt $\mathrm{Sr} / \mathrm{Ti}$ den Quotienten der dosierten Volumina $c_{S r / T i}^{V}=$ $\mathrm{V}(\mathrm{SrO}) / \mathrm{V}\left(\mathrm{TiO}_{2}\right)$ an und die Zahl in Klammern die Zahl der Wiederholungen der Sr- und Ti-Pulse. Für einen mittleren Wert $\mathrm{Sr} / \mathrm{Ti}=0.763$ kann man einen waagerechten Verlauf von $\Delta(t)$ erwarten.
\end{abstract}

Verlauf zeigt. Dagegen folgt für einen Sr-Überschuss ( $\mathrm{Sr} / \mathrm{Ti}=0.9)$ ein Drift zu größeren Werten von $\Delta$ und für Ti-Überschuss ( $\mathrm{Sr} / \mathrm{Ti}=0.8$ ) zu kleineren. Auch externe Charakterisierungen von Filmen, deren Stöchiometrie mit diesem Verfahren eingestellt wurde, demonstrieren die Korrektheit der Zusammensetzung 233. In TEM-Aufnahmen kann man keine Fremdphasen ausmachen. Insbesondere sieht man keine Ruddlesden-Popper-Defekte (eingeschobene vertikale oder horizontale SrO-Lagen) [233, die sich bei einem Überangebot von Sr-Ionen ausbilden 234.

Die gemessenen $\Delta(t)$-Kurven für verschiedene Verhältnisse der Volumina von den Sr- und TiLösungen, $c_{S r / T i}^{V}=\mathrm{V}(\mathrm{SrO}) / \mathrm{V}\left(\mathrm{TiO}_{2}\right)(\mathrm{Sr} / \mathrm{Ti})$, sind in Abbildung 7.4 gezeigt. Es ergibt sich eine ähnliche Tendenz wie für die Situation der Sr/Ti Mischlösungen (vgl. Abbildung 7.3): Für einen Sr-Überschuss $(\mathrm{Sr} / \mathrm{Ti}=0.8,0.775)$ driftet $\Delta(t)$ zu größeren Werten, wogegen für einen Ti-Überschuss ( $\mathrm{Sr} / \mathrm{Ti}=0.75)$ ein Drift in die negative Richtung folgt. Für $\mathrm{Sr} / \mathrm{Ti}=0.763 \mathrm{kann}$ man näherungsweise einen waagerechten Verlauf erwarten ${ }^{22}$ Unter Beachtung des Signal-zuRausch-Verhältnisses bei der in-situ Messung von $\Delta$ lässt sich der Korrekturfaktor, $c_{S r / T i}$ bzw. $c_{S r / T i}^{V}$, mit der oben geschilderten Herangehensweise bis zu einer Genauigkeit von etwa $1.5 \%$ festlegen.

Nachdem die korrekte Sr/Ti-Stöchiometrie ermittelt worden ist, muss das Volumen, das zur Abscheidung einer Monolage $\mathrm{TiO}_{2}$ dosiert werden muss, $\mathrm{V}\left(\mathrm{TiO}_{2}\right)$, bestimmt werden. Daraus folgt dann direkt die Menge zur Deposition einer Monolage SrO:

$$
\mathrm{V}(\mathrm{SrO})=c_{S r / T i}^{V} \cdot \mathrm{V}\left(\mathrm{TiO}_{2}\right)
$$

\footnotetext{
${ }^{22}$ Die Sr/Ti-Stöchiometrie entspricht nicht exakt den Werten für das konventionelle Verfahren, da bei den in Abbildung 7.3 gezeigten Experimenten eine andere Charge der Sr- und Ti-Präkursoren verwendet wurde als für die Experimente in Abbildung 7.4. Durch Unterschiede in der Menge des Kristallwassers können die molaren Gewichte der Präkursoren differieren. Bei der Angabe der nominellen Sr/Ti-Verhältnisse wird immer von der Summenformel, die der Hersteller angibt, ausgegangen, um die Molmassen zu ermitteln.
} 
Bei homoepitaktischen STO-Filmen kann man die Schichtdicke aufgrund des geringen chemischen Kontrastes nicht durch XRR-Messungen gewinnen. Deshalb zieht man $\mathrm{Ba}_{\mathrm{x}} \mathrm{Sr}_{1-\mathrm{x}} \mathrm{TiO}_{3^{-}}$ Filme mit $x=0.3$ heran, die einen ausreichenden Kontrast zum STO-Substrat zeigen. Man stellt Filme dieses Materials mit dem konventionellen MAD-Prozess auf STO-Substraten her und ermittelt deren Dicke dann mit XRR. Mit dem bekannten Volumen der deponierten Präkursorlösung folgt dann $\mathrm{V}\left(\mathrm{TiO}_{2}\right)=11 \mu \mathrm{l}$.

Die nach den Erläuterungen oben gewonnen Parameter, $\mathrm{V}\left(\mathrm{TiO}_{2}\right)$ und $c_{S r / T i}^{V}$, werden verwendet, um die im Folgenden präsentierten RP STO-Strukturen herzustellen.

\subsubsection{Wachstum und in-situ Ellipsometrie}

In Abbildung 7.5 sieht man die in-situ Ellipsometrie für das Wachstum einer RP STO $(\mathrm{n}=4)$ Struktur. Die Deposition wird auf einem Ti-terminierten STO (001)-Substrat mit der Abscheidung einer doppelten Lage $\mathrm{SrO}$ gestartet und endet mit einer $\mathrm{TiO}_{2}$-Lage. Hier wird nur das Signal von $\Delta(t)$ vorgestellt, da man für $\Psi(t)$ nur kleine Änderungen sieht, die im Rauschen untergehen. Bei jedem Sr-Puls zeigt $\Delta$ einen Anstieg und bei jedem Ti-Puls einen Abfall. Aufgrund der insgesamt Sr-reichen Zusammensetzung der RP STO ( $\mathrm{n}=4)$-Struktur zeigt $\Delta(t)$ in Abbildung 7.5 a) insgesamt einen leichten Anstieg (vergleiche Abschnitt 7.2.2). Entsprechend der Bildung von STO heben sich die Änderungsamplituden bei der aufeinanderfolgenden Abscheidung einer Sr- und Ti-Lage gegenseitig fast auf.

Die durchschnittliche $\mathrm{Sr} / \mathrm{Ti}$-Oszillationsamplitude $\Delta$ STO für die vier konsekutiven STOLagen zeigt für $r=1$ einen deutlich kleineren Wert als für die späteren Perioden. Außerdem sieht man während der Deposition der vier aufeinander folgenden STO-Lagen im Mittel einen leichten Anstieg, der einen leichten Sr-Überschuss andeutet. Die Änderung von $\Delta$ nach der zweifachen SrO-Lage, $\Delta 2 \mathrm{SrO}$, ist auch nicht konstant für alle $r$, sondern nimmt von $r=1$ bis $r=5$ kontinuierlich zu und streut dann um den Mittelwert $\Delta 2 \mathrm{SrO}(r>5)=1.1(1)^{\circ}$ (Inset von Abbildung 7.5 b)). Nach der Deposition nimmt man mit dem Rasterkraftmikroskop die Oberflächenmorphologie auf, die mit $\sigma_{R M S}=0.28 \mathrm{~nm}$ nur kleine Rauigkeiten aufweist (siehe Inset von Abbildung 7.5 a)).

\subsubsection{Strukturelle Charakterisierung}

In den XRD-Spektren der RP STO $(n=2,3,4)$-Filme sieht man ausschließlich Maxima, die (001)-Ebenen zuzuordnen sind (Abbildung 7.6). Dies deutet auf ein epitaktisches Wachstum hin, bei dem die c-Achse der RP STO-Strukturen senkrecht auf der (001)-Ebene des Substrates steht. Die lineare Anpassung von $\sin \theta$ für die Positionen der Beugungsmaxima liefert mit dem Bragg-Gesetz die Gitterebenenabstände in c-Richtung, die gut zu den Literaturwerten passen 222, 223, 226]. Insbesondere sieht man in den Spektren keine Beiträge von Phasen mit anderem $n$.

Um lokale Informationen über die Defektstruktur zu erhalten, werden TEM-Aufnahmen des Filmes aus RP STO mit $n=4$ betrachtet. Diese wurden von Ricardo Egoavil und Vladimir Roddatis angefertigt. In der HAADF-Übersicht erkennt man ein epitaktisches Wachstum des RP STO (n=4)-Filmes auf dem STO (001)-Substrat. Die mit dem TEM ermittelte Schicht- 

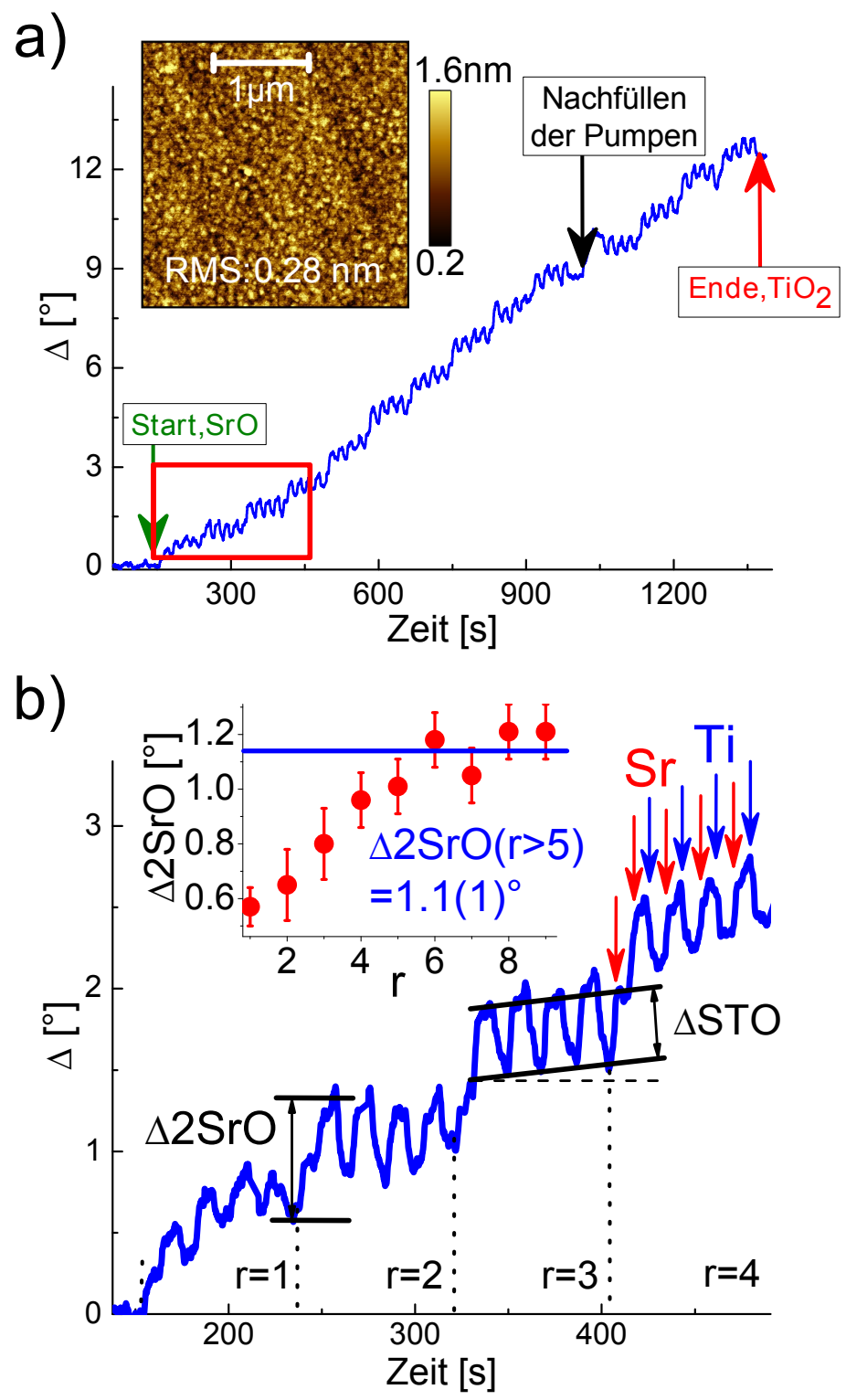

\begin{abstract}
Abbildung 7.5.: In-situ Ellipsometrie beim Wachstum von RP STO (n=4) auf einem Titerminierten STO (001)-Substrat: a) $\Delta(t)$ für alle 14 Wiederholungen der $\mathrm{SrO}\left(\mathrm{SrTiO}_{3}\right)_{4}{ }^{-}$ Einheitszelle. Das Inset zeigt eine Aufnahme der Oberfläche des resultierenden Filmes mit dem Rasterkraftmikroskop. b) Vergrößerte Darstellung des roten Kastens aus a). $\Delta 2 \mathrm{SrO}$ bezeichnet die Änderung von $\Delta$ nach der Deposition einer doppelten Lage $\mathrm{SrO}$ und $\Delta \mathrm{STO}$ die durchschnittliche Oszillationsamplitude bei der Abscheidung der vier konsekutiven $\mathrm{SrTiO}_{3}$ Lagen. Im Inset ist $\Delta 2 \mathrm{SrO}$ als Funktion des Indexes $r$, mit dem die $\mathrm{SrO}\left(\mathrm{SrTiO}_{3}\right)_{4}$-Einheiten durchnummeriert werden, dargestellt. Der Mittelwert für alle $r>5, \Delta 2 \mathrm{SrO}(r>5)=$ $1.1(1)^{\circ}$, ist angegeben.
\end{abstract}

dicke beträgt etwa $22 \mathrm{~nm}$, was um etwa eine Einheitszelle der $\mathrm{Sr}_{5} \mathrm{Ti}_{4} \mathrm{O}_{13}$-Struktur von der theoretischen Filmdicke $d_{\text {calc }}=n_{R P} \cdot 3.6 \mathrm{~nm}=25.2 \mathrm{~nm}$ abweicht (siehe Abbildung 7.7 a)). Die rocksalt-SrO-Lagen erkennt man an der horizontalen Verschiebung der angrenzenden STO-Einheitszellen um eine halbe Gitterkonstante. Durch die Aufnahme von TEM EELSKarten für $\mathrm{Sr}$ und $\mathrm{Ti}$ kann man die $\mathrm{SrO}\left(\mathrm{SrTiO}_{3}\right)_{4}$-Sequenz noch präziser verifizieren: In Abbildung 7.8 sieht man einen Ausschnitt von Abbildung 7.7 a), in dem die angestrebte STO-SrO-Lagerung vorliegt. In den EELS-Linienprofilen kann man deutlich die Sequenz von 


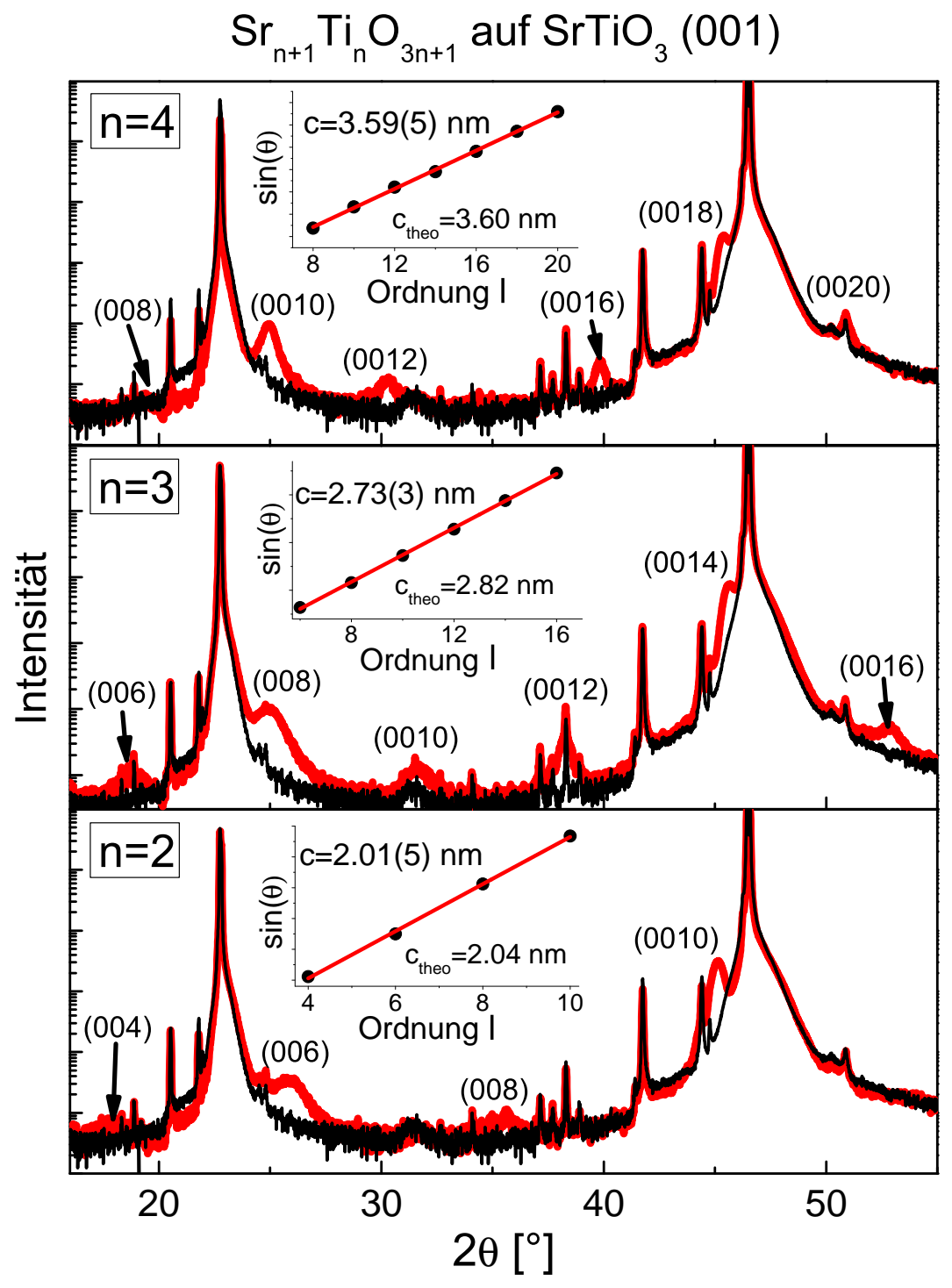

Abbildung 7.6.: XRD-Spektren für Filme von RP STO $(n=2,3,4), \mathrm{Sr}_{n+1} \mathrm{Ti}_{n} \mathrm{O}_{3 \mathrm{n}+1}$, auf STO (001)-Substraten: Neben den Spektren der Filme (rot) wird auch das Spektrum eines reinen STO-Substrates (schwarz) geplottet, um die Peaks zu identifizieren, die aus einer Verunreinigung der $\mathrm{Cu}-\mathrm{K}_{\alpha}$-Strahlung resultieren. In den Insets werden die $\sin \theta$-Werte für die Beugungsmaxima als Funktion der Ordnung $l$ dargestellt. Aus diesen ergeben sich mit dem Bragg-Gesetz die angegeben Gitterparameter in c-Richtung $c$ die mit den theoretischen Größen $c_{\text {theo }}$ 222, 223, 226. verglichen werden.

4 STO-Lagen, die durch eine zusätzliche SrO-Lage separiert sind, erkennen. Da im Bereich der doppelten SrO-Lagen keine Intensität an der Ti-Kante erkennbar ist, ist davon auszugehen, dass sich die SrO-Lage über die gesamte Dicke der TEM-Lamelle $\left(d_{T E M} \approx 10-20 \mathrm{~nm}\right)$ erstreckt.

Um die enthaltenen Defekte zu erfassen, betrachtet man die HAADF-Übersicht in Abbildung 7.7 a) mit einer Fläche $A_{T E M}=22 \cdot 49 \mathrm{~nm}^{2}$. Die Grenzfläche zwischen Film und Substrat ist nicht scharf definiert auf der Längenskala einer Einheitszelle von STO. Die Positionen der ersten horizontalen Lagen von $\mathrm{SrO}$ variieren um etwa vier STO-Einheitszellen entlang der c-Achse. Neben der gewünschten SrO-STO-Abfolge für RP STO $(n=4)$ gibt es auch andere 


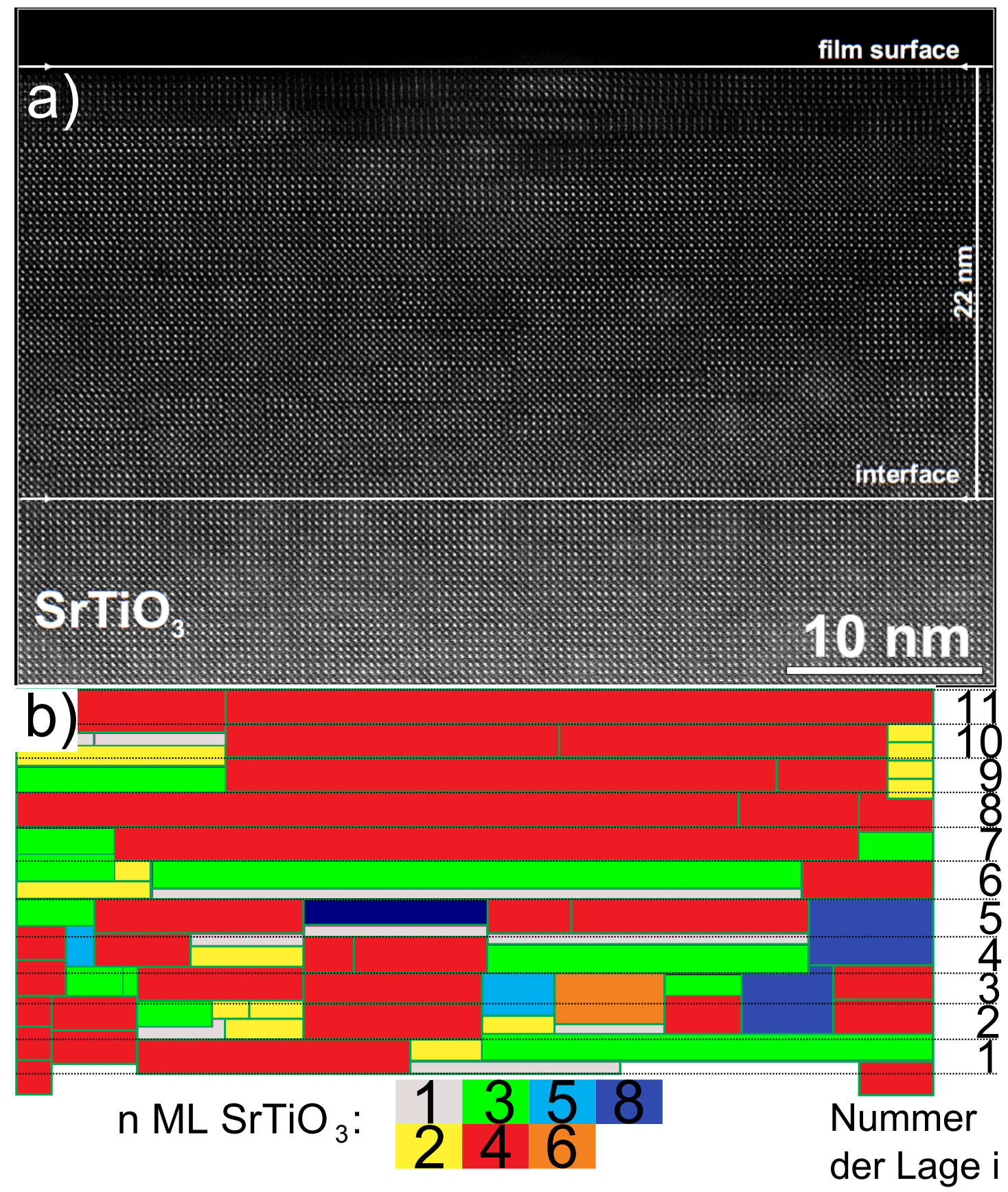

Abbildung 7.7.: Analyse des Netzwerkes aus SrO-Lagen in RP STO (n=4)-Film auf STO (001): a) HAADF-Übersicht, der Bereich des Filmes ist abgegrenzt und seine Schichtdicke angegeben. b) Identifikation der eingeschobenen SrO-Lagen für Aufnahme aus a), durch horizontale und vertikale Linien werden die doppelten SrO-lagen nachgezeichnet. Zwischen den SrO-Lagen befinden sich Blöcke aus n Lagen STO. Man kennzeichnet diese Blöcke entsprechend der Zahl der enthaltenen Lagen von STO mit der unten abgebildeten Farbskala. Für die spätere statistische Auswertung teilt man die Struktur in Lagen $i=1-11$ ein. (Die TEM-Aufnahme und die Identifikation der STO-Blöcke wurden von Ricardo Egoavil durchgeführt.). 


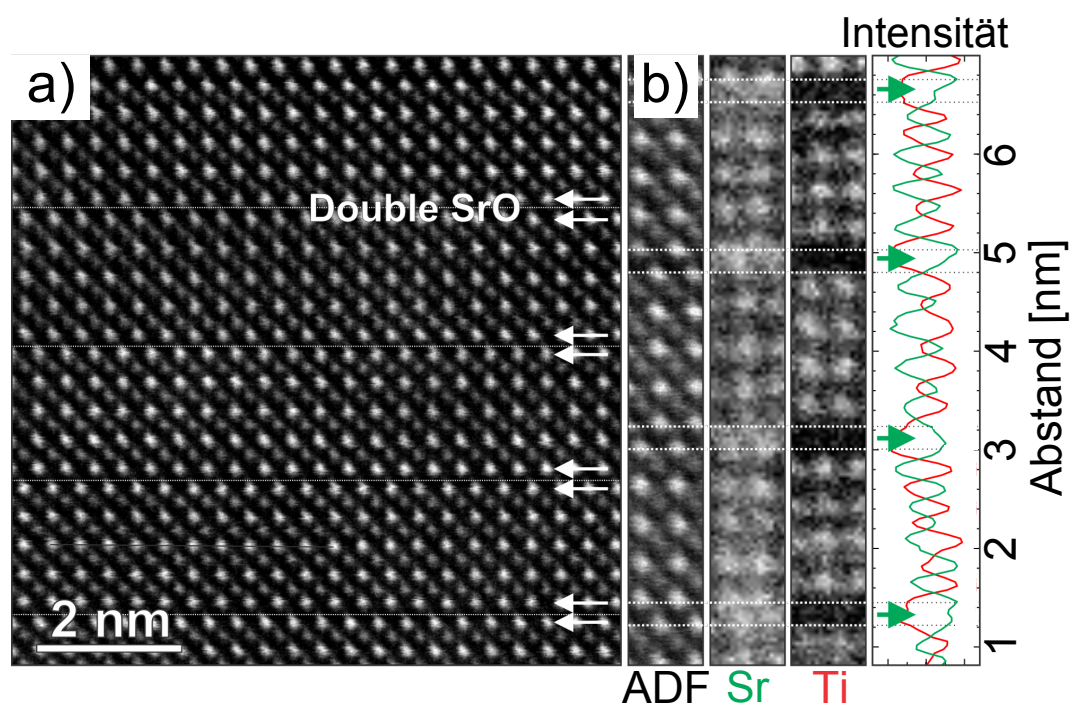

Abbildung 7.8.: TEM-Analyse von Ausschnitt aus Abbildung 7.7 a), in dem eine perfekte Lagerung der STO und SrO-Lagen entsprechend der Struktur von RP STO (n=4) vorliegt: a) HAADF-Aufnahme, die Sr-Ionen erscheinen aufgrund der größeren Gesamtelektronendichte heller als die Ti-Ionen. Die Positionen der doppelten SrO-Lagen wurden mit weißen Pfeilen markiert. b) Linien-Profile über die vier doppelten SrO-Lagen aus a). ADF: annular dark field, Sr: EELS an der $\mathrm{Sr}-\mathrm{M}_{4 ; 5}$-Kante, Ti: EELS an der Ti $-\mathrm{L}_{2 ; 3}$-Kante (Die TEM-Aufnahmen wurden von Ricardo Egoavil angefertigt und ausgewertet.).

a)

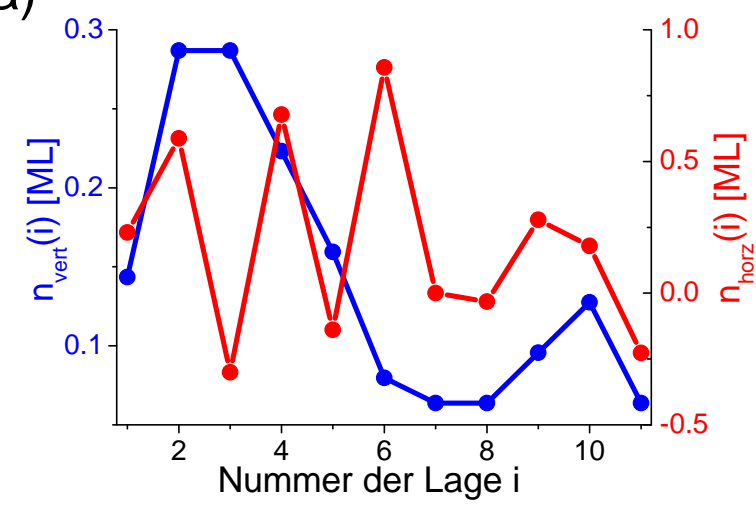

b)

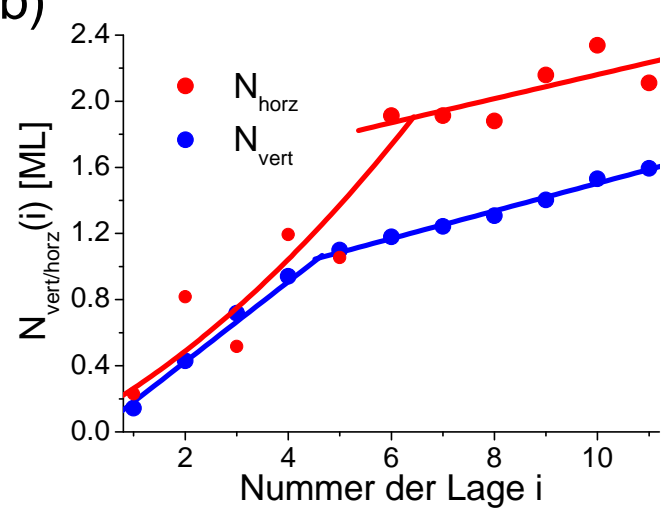

Abbildung 7.9.: Zur statistischen Auswertung von Abbildung 7.7 b): a) Zahl der zusätzlichen vertikalen, $n_{\text {vert }}(i)$, und horizontalen, $n_{\text {hor }}(i)$, SrO-Lagen als Funktion des Indexes $i$. b) Summen von $n_{\text {vert }}, n_{\text {hor } z}$ aus a): $N_{\text {vert }, \text { horz }}(i)=\sum_{k=1}^{i} n_{\text {vert }, \text { hor } z}(k)$ (Die eingezeichneten Linien dienen der Orientierung.).

Periodizitäten mit $n=1-8$. Die horizontalen SrO-Lagen verschieben die STO-Blöcke entlang der a- und b-Achse um $c_{S T O} / 2 \approx 0.195 \mathrm{~nm}$ und entlang der c-Achse um $c_{S r O} \approx 0.24 \mathrm{~nm}\left(c_{S T O}\right.$ und $c_{S r O}$ aus $\left.[222,223,226]\right)$. Vertikale SrO-Ebenen werden eingefügt, um die Verschiebungen zwischen den STO-Blöcken auszugleichen, die bei ungleichen SrO-STO-Sequenzen entstehen. Durch den Unterschied zwischen $c_{S T O} / 2$ und $c_{S r O}$ ergeben sich im Netzwerk aus den durch SrO-Ebenen eingeschlossenen STO-Einheiten elastische Verspannungen.

Zur statistischen Auswertung der Verteilung der Stapelfehler identifiziert man in Abbildung 7.7 a) die verschiedenen STO-Blöcke und kennzeichnet diese entsprechend der Zahl der enthaltenen STO-Lagen (Abbildung 7.7 b)). Die Größe der abgegrenzten Rechtecke entspricht maßstabsgerecht der Ausdehnung der korrespondierenden Areale in Abbildung 7.7 a). Der 
Anteil von RP STO $(\mathrm{n}=4)$ an der gezeigten Fläche $A_{T E M}$ entspricht etwa $60 \%$. Man kann deutlich erkennen, dass die Konzentration der Defekte in der Nähe zum Substrat deutlich größer ist als an der Oberfläche.

Diese Phänomenologie bewertet man quantitativ, indem man die Probe in $i=1-11$ Lagen mit der Dicke einer $\mathrm{SrO}\left(\mathrm{SrTiO}_{3}\right)_{4}$-Zelle teilt (vergleiche Abbildung 7.7 b)). In jeder Lage $i$ quantifiziert man die Defekte durch die Abweichung von der gewünschten Sr/Ti-Stöchiometrie. Im durch die Lage $i$ abgegrenzten Bereich stellt man die Zahl der vertikalen, $n_{\text {vert }}(i)$, und horizontalen, $n_{\text {horz }}^{*}(i)$, SrO-Lagen fest. $n_{\text {vert }}$ und $n_{\text {hor } z}^{*}$ werden auf eine volle Monolage, die sich für den gemessenen Bereich über eine Breite von $49 \mathrm{~nm}$ erstreckt, normiert. Da in der perfekten RP STO ( $\mathrm{n}=4)$-Struktur eine horizontale SrO-Lage in jeder Lage mit Index $i$ vorkommen würde, zieht man diese in jeder Lage ab und erhält so $n_{\text {horz }}(i)=n_{\text {hor }}^{*}(i)-1 \mathrm{ML}$.

$n_{\text {vert }}$ fällt als Funktion von $i$ kontinuierlich ab und erreicht bei $i=6$ dann ein fast konstantes Niveau (siehe Abbildung 7.9a)). $n_{\text {hor } z}$ oszilliert sehr stark für $i=1-6$, was daraus resultiert, dass die Länge der horizontalen SrO-Lagen im Bereich der Breite der HAADF-Aufnahme liegt. Deshalb betrachtet man zusätzlich die Summen

$$
N_{\text {vert }, \text { horz }}(i)=\sum_{k=1}^{i} n_{\text {vert }, \text { horz }}(k)
$$

um einen Eindruck von der akkumulierten Unstöchiometrie zu gewinnen. Für $N_{\text {vert,horz }}(i)$ sieht man so eine klare Änderung der Steigung bei $i=6$ (siehe Abbildung 7.9 b)). In den ersten $i=1-6$ Lagen ergibt sich insgesamt ein Überschuss von

$$
\sum_{k=1}^{6}\left(n_{\text {vert }}(k)+n_{\text {horz }}(k)\right)=3.1 \mathrm{ML}
$$

SrO, was einem Sr-Überschuss von $\sim 10 \%$ gleichkommt. Für die folgenden $i=6-11$ Lagen ist dieser Überschuss stark reduziert und liegt bei

$$
\sum_{k=6}^{11}\left(n_{\text {vert }}(k)+n_{\text {hor }}(k)\right)=0.6 \mathrm{ML}
$$

einem Sr-Überschuss von $\sim 2.4 \%$. Diese verringerte Konzentration an Defekten in der oberen Hälfte der Heterostruktur macht sich auch in einem erhöhten Anteil der gewünschten RP STO (n=4)-Phase bemerkbar. Dieser beträgt in den letzten $i=7-11$ Lagen $85 \%$.

\subsubsection{Diskussion}

$\mathrm{Zu}$ Beginn soll ausgeschlossen werden, dass sich die hier vorgestellten RP STO-Strukturen durch eine einfache Festkörperreaktion der aufgebrachten Sr- und Ti-Ionen gebildet haben. In einem zusätzlichen Experiment erstellt man eine gemischte Lösung der Sr- und TiPräkursoren, wobei die molaren Verhältnisse entsprechend der mittleren Sr/Ti-Stöchiometrie in der RP STO ( $n=4)$-Struktur gewählt wurden. Bei der Deposition dieser Lösung auf ein STO (001)-Substrat entstand ein Film, dessen XRD-Spektrum keine Peaks einer RP STO-Phase enthielt. Es entstand also STO mit zufällig verteilten SrO-Lagen. Bei den in der Literatur vorkommenden Studien zur Synthese von RP STO-Kristallen über eine Festkörperreaktion wurden außerdem deutlich andere Herstellungsparameter gewählt als hier. Dort wurden die Kristalle bei $T_{F K} \approx 1240^{\circ} \mathrm{C}$ über mehrere Wochen gesintert 225. 
Für die bei der MAD ALE vorherrschenden Bedingungen ist die bulk-Diffusion der SrIonen zu vernachlässigen. Der Diffusionskoeffizient für Sr in undotiertem STO beträgt bei $T_{\text {Dep }} \approx 930^{\circ} \mathrm{C} D(1208 \mathrm{~K}) \approx 1.2 \cdot 10^{-26} \mathrm{~m}^{2} \mathrm{~s}^{-1}[60$, womit die Diffusionslänge bei einer Depositionsdauer in der Größenordnung $t_{M A D} \approx 1 \mathrm{~h}$ bei $\sqrt{2 D t_{M A D}} \approx 0.01 \mathrm{~nm}$ liegt. Experimentell lässt sich diese Tatsache dadurch bestätigen, dass man eine RP STO-Heterostruktur bei $T_{D e p}$ für eine Stunde auslagert und die Röntgenspektren vor und nach dem Auslagern betrachtet. Dabei kann man keine signifikanten Unterschiede ausmachen.

Neben der Diffusion im bulk kann es aber noch zu Reaktionen an der Oberfläche kommen. Diese wurden bereits im Abschnitt 6.3 für das Wachstum von Manganaten auf STO beobachtet. In der Tat berichten Szot et al. eine Anreicherung von $\mathrm{SrO}$ auf oder einige Monolagen unterhalb der Oberfläche von STO (001), das unter oxidierenden Bedingungen $\left(T=900{ }^{\circ} \mathrm{C}\right.$, $p_{\mathrm{O}_{2}}=200$ torr) ausgelagert wurde [235]. Als treibende Kraft wird dabei eine erhöhte Versetzungsdichte an der Oberfläche angeführt 235. Zudem werden die Versetzungen als Diffusionskanäle benannt. Da bei der MAD ALE mit $T_{D e p} \approx 930{ }^{\circ} \mathrm{C}$ und $p_{O_{2}} \approx 160$ torr ähnliche Bedingungen vorherrschen, lässt sich auch die beobachtete Mikrostruktur des RP STO (n=4)Filmes auf eine derartige Eigenschaft des Substrates zurückführen. Nahe zum STO-Substrat stören Diffusionsvorgänge die Abfolge der $\mathrm{SrO}$ - und $\mathrm{TiO}_{2}$-Lagen. In den späteren Lagen verschwindet der Einfluss des Substrates und das Wachstum ist nur wenig gestört.

Die Messungen der in-situ Ellipsometrie geben Hinweise auf Diffusionsvorgänge zu Beginn der Deposition. $\Delta 2 \mathrm{SrO}$ steigt für die ersten $r=1-5$ kontinuierlich an, um dann zu sättigen. Eine solche verkleinerte Amplitude von $\Delta$ beim Wachstum der doppelten SrO-Lage kann man nicht mit einem Aufrauen identifizieren. Eine raue SrO-Schicht würde im Rahmen der Näherung durch ein effektives Medium einen kleineren effektiven Brechungsindex aufweisen als $\mathrm{SrO}$, wodurch $\Delta$ einen stärkeren Anstieg zeigen würde. Bei einer teilweisen Durchmischung von SrO mit dem STO-Substrat ist allerdings eine kleinere Änderung von $\Delta$ zu erwarten als beim Wachstum eines geschlossenen SrO-Filmes. Die Mischlage aus STO und der eindiffundierten Sr-Atome hat in der Näherung durch ein effektives Medium einen Brechungsindex, der zwischen dem Wert für SrO und STO liegt. Durch den geringeren Unterschied zum STO-Substrat trägt eine derartige Mischlage dann deutlich weniger zu $\Delta$ bei als ein reiner SrO-Film.

In Abbildung 7.10 ist ein Modell der Vorgänge beim Wachstum dargestellt, das die SrDiffusionsvorgänge berücksichtigt. Die erste zweifache SrO-Lage, die auf das Ti-terminierte Substrat aufgebracht wird, diffundiert teilweise in das STO und bildet dort horizontale und vertikale RP-Defekte (Abbildung 7.10 a)-b)). In der Folge wird $\mathrm{TiO}_{2}$ abgeschieden. Das $\mathrm{SrO}$ im STO reagiert dabei mit der Bildung von STO (Abbildung $7.10 \mathrm{~b}$ )-c)). Das Auftreten dieses Prozesses erklärt auch die kleine Amplitude von $\Delta$ STO zu Beginn. Für $r=1$ kommt es nicht zu einem Wechsel zwischen einer SrO- und $\mathrm{TiO}_{2}$-Terminierung, sondern die Heterostruktur behält durch die Diffusionsvorgänge vorwiegend einen SrO-Abschluss (siehe Abbildung 7.10 b)-e)). Wenn die Heterostruktur nach dem $\mathrm{TiO}_{2}$-Puls aus SrO-terminiertem STO besteht und nach dem SrO-Puls aus STO mit RP-Defekten (Abbildung 7.10 c)-d)), ist die Änderung der optischen Eigenschaften deutlich kleiner als bei einer festen Änderung der Terminierung zwischen $\mathrm{SrO}$ und $\mathrm{TiO}_{2}$ in den späteren Phasen.

Die geschilderten Bewegungen der Sr-Ionen führen zu einer Verschiebung der im TEM sichtbaren Grenzfläche der gesamten Heterostruktur. Daraus resultiert der Unterschied zwischen der aus der TEM-Aufnahme ermittelten Filmdicke und der Vorhersage. Durch lokale Variatio- 


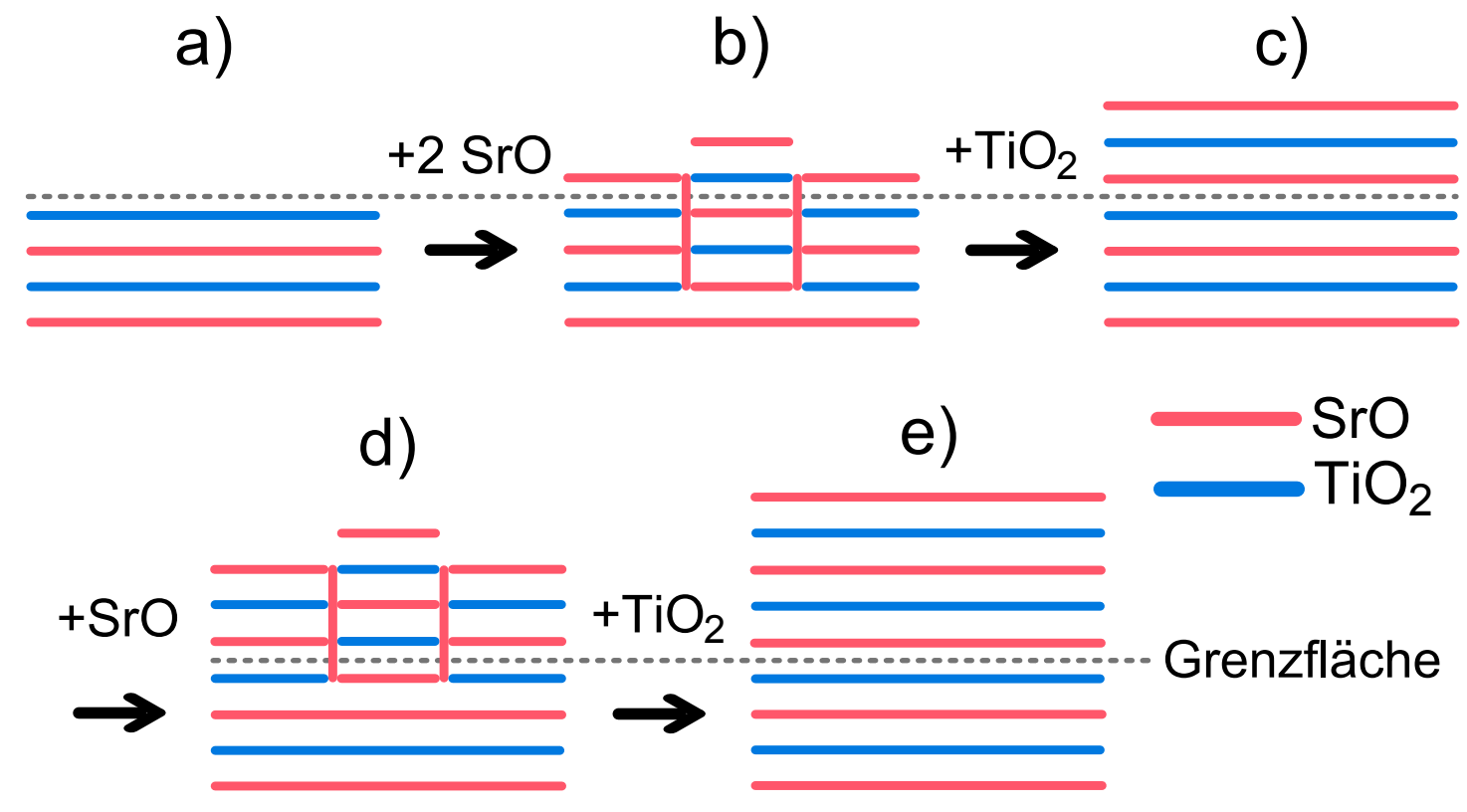

\begin{abstract}
Abbildung 7.10.: Modell der Vorgänge zu Beginn des Wachstums von RP STO auf Titerminiertem STO (001)-Substrat: a)-b) Auf das Ti-terminierte Substrat wird eine zweifache Lage von SrO aufgebracht. Dabei diffundiert ein Teil der Sr-Ionen oberflächlich in das STO-Substrat und bildet horizontale und vertikale RP-Defekte. b)-c) Eine $\mathrm{TiO}_{2}$-Lage wird deponiert. Das $\mathrm{SrO}$ in den RP-Defekten bewegt sich an die Oberfläche und bildet mit dem $\mathrm{TiO}_{2}$ eine STO-Lage mit SrO-Abschluss. c)-d) Bei der Abscheidung einer einfachen SrOLage erfolgt die erneute Bildung von RP-Defekten an der Oberfläche. d)-e) Die RP-Defekte reagieren wiederum mit dem dosierten $\mathrm{TiO}_{2}$, so dass STO mit Sr-Terminierung entsteht.
\end{abstract}

nen der SrO-Beweglichkeit resultiert zudem die undefinierte Position der ersten zusätzlichen SrO-Lagen. Die in Abbildung 7.10 skizzierten Vorgänge bewirken eine Anreicherung von SrO an der Oberfläche des Films vergleichbar zu den Prozessen, die Szot et al. vorschlagen. Diese oberflächliche Erhöhung der SrO-Konzentration ist dann die Ursache des erhöhten Aufkommens an SrO-Lagen im ersten Teil der RP STO $(n=4)$-Heterostruktur (vergleiche Abschnitt 7.2 .4 .

Im weiteren Verlauf der Deposition sinkt die Mobilität der SrO-Lagen. Für $r>5$ sättigt $\Delta 2 \mathrm{SrO}$ bei dem Mittelwert für $r=6-14$, womit man annehmen kann, dass für $r>5$ die aufgebrachten doppelten SrO-Lagen nicht mehr in das Substrat diffundieren. Im zweiten Teil der Heterostruktur sind die SrO-Lagen also nicht mehr so beweglich wie im ersten. Es gibt keine treibende Kraft mehr, die eine Bewegung der Sr-Ionen zur Oberfläche hervorruft. Dies wird an der plötzlichen Abnahme der Steigungen von $N_{\text {hor }}, N_{\text {vert }}$ bei $i=6$ verdeutlicht (siehe Abbildung 7.9 b)).

Man kann über die Ursachen der Demobilisierung der SrO-Lagen nur spekulieren. Zum einen können die Versetzungen in der Oberfläche des STO-Substrates nach dem Wachstum des ersten Teils der Heterostruktur ausgeheilt sein, so dass diese nicht mehr die Sr-Diffusion treiben können. Andererseits hat sich in der ersten Hälfte der RP STO (n=4)-Struktur ein STO-SrO-Netzwerk gebildet. In diesem ergibt sich durch $c_{S T O} / 2 \neq c_{S r O}$ eine komplizierte Landschaft der elastischen Energie. Beim Einbau zusätzlicher SrO-Lagen ist es so für das System schwierig ein neues Energieminimum zu finden, da eine gleichzeitige Bewegung vieler Lagen notwendig ist. 

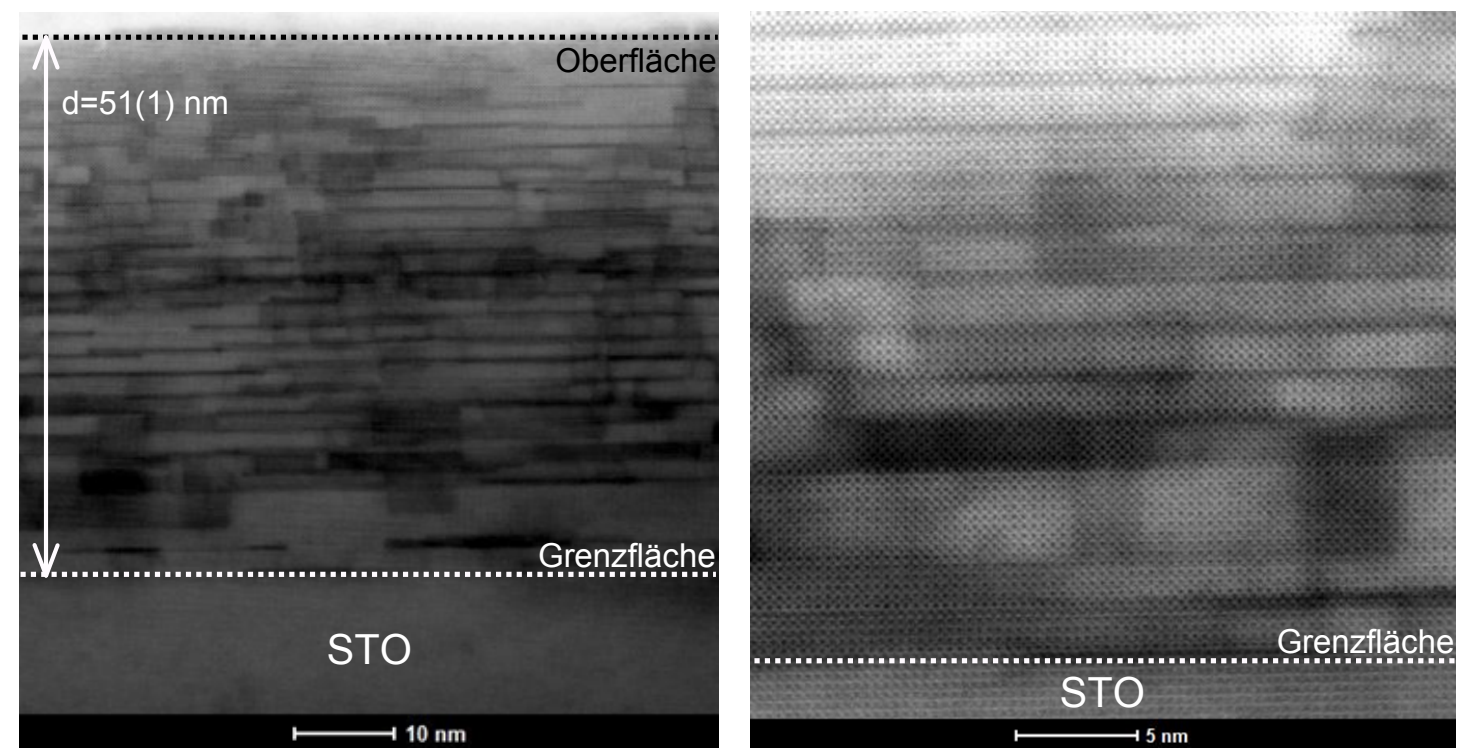

Abbildung 7.11.: STEM ADF-Aufnahmen von RP STO ( $\mathrm{n}=4)$-Heterostruktur auf STO (001)-Substrat. Rechts: Vergrößerte Darstellung von Bereich nahe zum Substrat (Der Film wurde von Fryderyk Lyzwa mit MAD ALE abgeschieden und die TEM-Aufnahmen von Vladimir Roddatis angefertigt.).
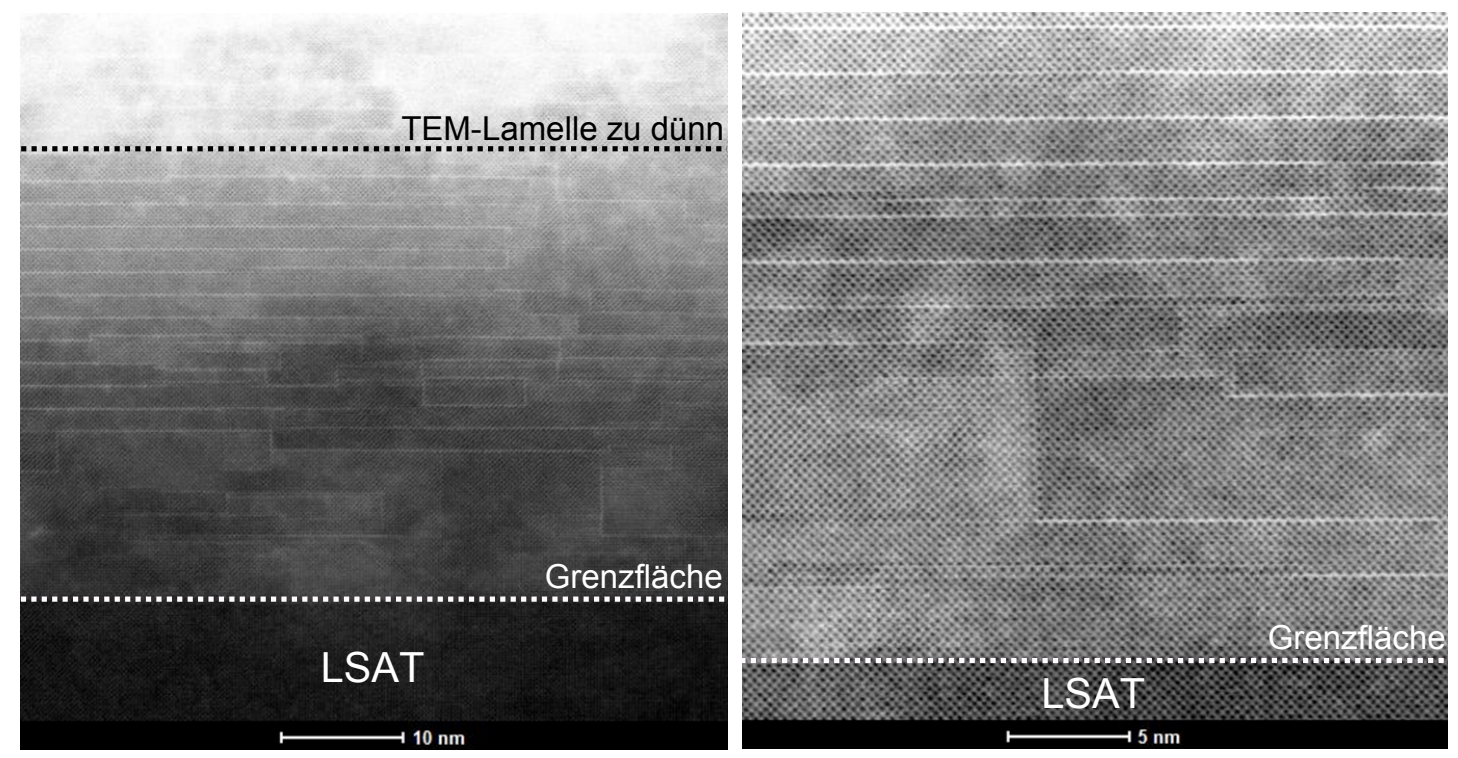

Abbildung 7.12.: STEM ADF-Aufnahmen von RP STO ( $\mathrm{n}=4)$-Heterostruktur auf LSAT (001)-Substrat. Rechts: Vergrößerte Darstellung von Bereich nahe zum Substrat (Der Film wurde von Fryderyk Lyzwa mit MAD ALE abgeschieden und die TEM-Aufnahmen von Vladimir Roddatis angefertigt.).

Im weiteren Verlauf der Deposition bestimmt dann allein die Stöchiometrie der dosierten Sr-,Ti-Präkursoren die Sr/Ti-Stöchiometrie im Film. Der leichte Drift von $\Delta$ nach oben beim Wachstum der vier STO-Lagen ist so mit dem Sr-Überschuss von $2.4 \%$ zu verbinden, der sich bei der Auswertung der TEM-Aufnahme ergibt.

Mit dieser Modellvorstellung über das Wachstum der RP STO-Strukturen durch MAD ALE wurden die nachfolgenden Experimente, die von Fryderyk Lyzwa im Rahmen seiner Masterarbeit durchgeführt wurden, motiviert. Dabei wurden wie hier RP STO (n=4)-Strukturen 
mittels MAD ALE abgeschieden. Allerdings wurden deutlich dickere Filme $(d \approx 50 \mathrm{~nm})$ aufgewachsen, um die Struktur fern vom gestörten Bereich nahe zum Substrat zu betrachten. Die RP STO $(\mathrm{n}=4)$-Strukturen mit $d \approx 50 \mathrm{~nm}$ wurden auf STO (001)- und LSAT (001)Substraten aufgewachsen.

In Abbildung 7.11 sieht man eine STEM ADF-Aufnahme der RP STO (n=4)-Struktur auf STO (001) ${ }^{23}$ Deutlich erkennt man Störungen der SrO-STO-Abfolge in der Nähe zum Substrat. Dieser gestörte Bereich erstreckt sich ähnlich wie in Abbildung 7.7 über eine Dicke von $\sim 10 \mathrm{~nm}$. Daran anschließend herrscht die angestrebte Sequenz mit vier STO-Lagen zwischen den zusätzlichen SrO-Ebenen bis hin zur Oberfläche des Filmes vor. Beim Wachstum auf LSAT zeigt sich ein ähnliches Bild mit undefinierter STO-SrO-Abfolge in einer Region mit $d \sim 10 \mathrm{~nm}$ in der Nähe zum Substrat und einer folgenden Zone, in der durchgehende horizontale SrO-Lagen anzutreffen sind (Abbildung 7.12). Die zuvor geschilderten Diffusionsvorgänge finden also auch beim LSAT-Substrat statt. Dies wird plausibel, wenn man die Fülle von Perowskiten betrachtet, in die bereits zusätzliche rocksalt-Lagen eingebracht wurden, z.B. $\mathrm{SrRuO}_{3}$ 229], $\mathrm{La}_{1-\mathrm{x}} \mathrm{Sr}_{\mathrm{x}} \mathrm{MnO}_{3}$ 230, 231, $\mathrm{CaMnO}_{3}$ 227].

Zum Schluss wird die hier eingeführte MAD Atomlagenepitaxie (ALE) mit den etablierten Depositionstechniken wie MBE verglichen. Der Anteil von $85 \%$ der RP STO ( $\mathrm{n}=4)$-Phase im oberen Teil der Heterostruktur aus Abbildung 7.7 ist nur etwas kleiner als ein Anteil von 94\%, welcher sich für Filme ergibt, die mit MBE hergestellt wurden [226]. Für die Schichten, die mit MBE hergestellt wurden, gibt es keine Berichte über eine gestörte Region in der Nähe zum Substrat [226, 228, 236]. Dies ist womöglich eine Folge der anderen Wachstumsbedingungen $\left(T_{\text {Dep }} \approx 750^{\circ} \mathrm{C}, p_{O_{2}} \approx 2 \cdot 10^{-7}\right.$ torr $)$, die zu einer teilweisen Reduktion von STO führen. Nach Szot et al. folgen daraus andere Reaktionen an der Oberfläche von STO (001) 235]: Es kommt zu einer Anreicherung von $\mathrm{TiO}_{\mathrm{x}}$ statt $\mathrm{SrO}$. Bei MAD ALE lässt sich die Beweglichkeit der SrO-Lagen derzeit nicht durch eine reduzierte Depositionstemperatur vermindern. Denn die Pyrolyse des verwendeten Ti-Präkursors läuft für $T \leq 900{ }^{\circ} \mathrm{C}$ nicht mehr in der geeigneten Art und Weise ab, die für ein epitaktisches Wachstum notwendig ist.

Hier wurde die korrekte $\mathrm{Sr} / \mathrm{Ti}$-Stöchiometrie unter Anwendung der in-situ Ellipsometrie ermittelt. Das Verhältnis der dosierten Volumina der Sr- und Ti-Lösung wurde so eingestellt, dass die Änderung von $\Delta$ nach aufeinanderfolgenden Sr- und Ti-Pulsen verschwindet. Die erreichbare Genauigkeit dieser Methode wurde auf $\sim 1.5 \%$ abgeschätzt. Diese Strategie ist vergleichbar mit der Methode, die von Haeni et al. angewendet wird, um die SrO- und $\mathrm{TiO}_{2^{-}}$ Flüsse bei der $\mathrm{MBE}$ zu kalibrieren [237]. Die $\mathrm{SrO}$ - und $\mathrm{TiO}_{2}$-Dosen können damit bis zu einer Präzision von $\sim 1 \%$ festgelegt werden.

\footnotetext{
${ }^{23}$ Die Positionen der doppelten SrO-Lagen sind hier nur verschwommen zu sehen, was auf eine Substratstufe innerhalb der TEM-Lamelle zurückzuführen ist.
} 


\section{3. $\left(\left(\mathrm{SrMnO}_{3}\right)_{1} /\left(\mathrm{LaMnO}_{3}\right)_{2}\right)_{\mathrm{m}}$ : Rolle der ersten Lagen}

\subsubsection{Einführung}

Bei den im vorherigen Kapitel vorgestellten STO-RP-Strukturen lag unter den oxidierenden Bedingungen der MAD mit $\mathrm{Ti}^{4+}$ eine feste Valenz der Übergangsmetallionen vor. Des Weiteren sind die SrO- und $\mathrm{TiO}_{2}$-Lagen elektrisch neutral. Beim Wachstum von gemischtvalenten Manganaten mittels MAD ALE ergibt sich im Gegensatz dazu eine veränderliche ManganValenz zwischen $\mathrm{Mn}^{3+}$ und $\mathrm{Mn}^{4+}$. Außerdem sind die $\mathrm{MnO}_{2^{-}}$und LaO-Lagen nicht mehr elektrisch neutral, so dass sich beim Wachstum elektrostatische Potentiale aufbauen können (vergleiche Abschnitt 3.1). Mit der in-situ Ellipsometrie kann man dabei Umverteilungen der Elektronen auf den Mn-Ionen erfassen. Die Qualität der mit MAD ALE hergestellten Manganate lässt sich anschließend durch elektrische und magnetische Messungen verifizieren, so dass man die in-situ Messungen direkt mit den funktionalen Eigenschaften verknüpfen kann.

In diesem Abschnitt betrachtet man das Wachstum der in Abbildung 7.13 dargestellten Heterostruktur von $\left(\mathrm{SrMnO}_{3}\right)_{1} /\left(\mathrm{LaMnO}_{3}\right)_{2}$ auf Ti-terminierten STO (001)-Substraten. Bei den hier durchgeführten Experimenten wird die Einheitszelle aus Abbildung $7.13 m=18$-mal wiederholt. Insgesamt werden so $6 \cdot m=108$ halbe Perowskit-Lagen abgeschieden. Hier soll im Speziellen untersucht werden, welchen Einfluss eine lokale Abweichung von der korrekten Stöchiometrie besitzt. Man variiert dabei nur die deponierten Volumina für die ersten beiden halben Perowskit-Lagen (erste SrO- und $\mathrm{MnO}_{2}$-Lage). Für die restlichen 106 Lagen dosiert man die stöchiometrischen Mengen der La-, Sr- und Mn-Lösungen. Die Bestimmung der stöchiometrischen Mengen wird in Abschnitt 7.3.2 beschrieben. Für die LMO-Lagen kann man aufgrund der oxidierenden Bedingungen nicht ausschließen, dass ein La-Defizit eingebaut wird (vergleiche Abschnitte 3.3.3 und 9.2.1). Dieses liegt jedoch im Bereich weniger Prozent 114116] und ist damit wesentlich kleiner als die absichtlich eingeführten lokalen Abweichungen von der idealen Stöchiometrie. Die folgenden Ausführungen fokussieren sich auf zwei Proben: Bei der ersten Probe wird für die erste SrO-Lage das 1.5-fache der stöchiometrischen Menge deponiert $(\mathrm{f}(\mathrm{Mn} / \mathrm{Sr})=0.6)$ und bei der zweiten Probe für die erste $\mathrm{MnO}_{2}$-Lage das 1.6-fache $(\mathrm{f}(\mathrm{Mn} / \mathrm{Sr})=1.6)$. Die verbleibenden Lagen sind für beide Proben identisch.

In diesem Abschnitt werden zunächst die zum Wachstum dieser Strukturen nötigen experimentellen Vorbereitungen besprochen. Dann werden die Messungen der in-situ Ellipsometrie für die beiden Proben mit $\mathrm{f}(\mathrm{Mn} / \mathrm{Sr})=0.6$ bzw. $\mathrm{f}(\mathrm{Mn} / \mathrm{Sr})=1.6$ detailliert verglichen. Nach der Deposition wurden die strukturellen Eigenschaften mit XRD und XRR untersucht und

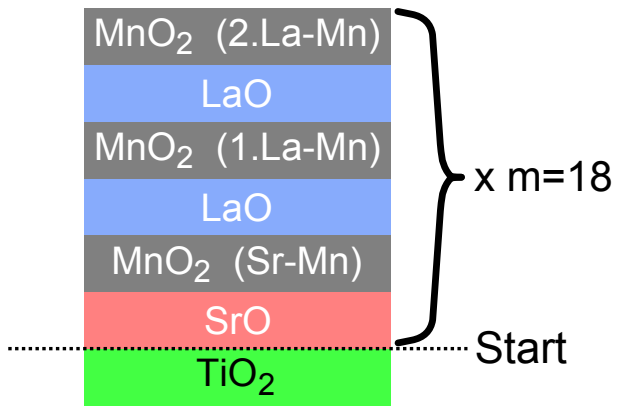

\footnotetext{
Abbildung 7.13.: Illustration der Heterostruktur von $\left(\mathrm{SrMnO}_{3}\right)_{1} /\left(\mathrm{LaMnO}_{3}\right)_{2}$, die durch abwechselnde Deposition von $\mathrm{SrO}-, \mathrm{MnO}_{2}$ - und LaO-Lagen auf einem Ti-terminierten STO (001)-Substrat mit MAD ALE hergestellt wird. Die $\left(\mathrm{SrMnO}_{3}\right)_{1} /\left(\mathrm{LaMnO}_{3}\right)_{2}$-Einheit wird dabei $m=18$-mal wiederholt.
} 
die Oberflächenmorphologie mittels STM abgetastet. Außerdem werden die elektrischen und magnetischen Eigenschaften beider Heterostrukturen präsentiert. In der abschließenden Diskussion werden anhand der in-situ Messungen Modelle für das Wachstum entwickelt und aufgezeigt, wie sich die Abweichungen von der korrekten Stöchiometrie in den ersten SrOund $\mathrm{MnO}_{2}$-Lagen auf das Wachstum auswirken.

\subsubsection{Experimentelle Vorbereitungen}

Wie bei den STO-RP-Strukturen muss man vor der Deposition der Heterostrukturen aus Manganaten die nötigen Volumina V (X) der Präkursorlösung bestimmen, die zur Abscheidung einer Monolage des Elementes X notwendig ist. Unter Verwendung von Mischlösungen aus Sr- und Mn- bzw. La- und Mn-Präkursoren ergeben sich die Korrekturfaktoren $C_{S r / M n}=1.37$ bzw. $C_{L a / M n}=1.35$ (vergleiche Abschnitt 4.1). V (SrO) und V(LaO) folgen dann mit diesen Faktoren aus $\mathrm{V}\left(\mathrm{MnO}_{2}\right)$ (vergleiche Abschnitt 7.2.2), dem Volumen der Mn-Präkursorlösung, das zur Deposition einer Monolage von $\mathrm{MnO}_{2}$ notwendig ist. Im konventionellen MAD-Prozess deponiert man dazu $\mathrm{La}_{0.7} \mathrm{Sr}_{0.3} \mathrm{MnO}_{3}$-Filme unter Verwendung unterschiedlicher Volumina der Präkursorlösung. Durch die Auswertung von XRR-Spektren dieser Filme folgt dann unter Kenntnis der dosierten Präkursormenge V $\left(\mathrm{MnO}_{2}\right)=6.9 \mu \mathrm{l}$. In Abschnitt 8.2.3 wurde gezeigt, dass die abgeschiedene Schichtdicke von LSMO(x)-Filmen nicht von der Sr-Dotierung $x$ abhängt, sondern allein durch die Zahl der dosierten Mn-Ionen bestimmt wird. Des Weiteren ergibt sich, dass die Korrekturfaktoren, $C_{S r / M n}$ und $C_{L a / M n}$, für das gesamte LSMO(x)-Phasendiagramm anwendbar sind. Damit ist eine gegenseitige Beeinflussung der Präkursoren bei der gemeinsamen Lösung in DMF auszuschließen und die Korrekturfaktoren lassen sich vom konventionellen MAD-Prozess auf die MAD ALE übertragen. Für die ALE werden die einzelnen Präkursoren, $\mathrm{Sr}$ (acetylacetonat) ${ }_{2}$, La (acetylacetonat) ${ }_{3}$ und Mn (acetylacetonat) ${ }_{2}$, mit einer Konzentration von $0.02 \mathrm{~mol} / \mathrm{l}$ in DMF gelöst. Man terminierte die STO (001)-Substrate entsprechend dem Verfahren in Abschnitt 4.4 .2 mit einem $\mathrm{TiO}_{2}$-Abschluss. Die Deposition erfolgt bei einer Substrattemperatur von $T_{D e p}=930^{\circ} \mathrm{C}$ und mit einer Rate von $p_{D e p} \approx 0.5-0.6 \mathrm{ML} / \mathrm{s}$. Die Wartezeit zwischen den Präkursorpulsen wurde so eingestellt, dass das Aerosol in der Kammer komplett abgesaugt worden ist, bevor das nächste Material deponiert wird.

Aus den individuellen Lösungen der La-, Sr-, und Mn-Präkursoren werden im Anschluss die Heterostrukturen aus $\left(\mathrm{SrMnO}_{3}\right)_{1} /\left(\mathrm{LaMnO}_{3}\right)_{2}$ abgeschieden. V $(\mathrm{X})$ bezeichnet die stöchiometrische Menge, die nach den oben durchgeführten Prozeduren für die Deposition einer Monolage des Materials X notwendig ist. Für die jeweils erste Lage des Materials X wird nicht diese stöchiometrische Menge dosiert, sondern $\mathrm{V}_{0}(\mathrm{X})$. Bei den hier vorgestellten Experimenten erfolgt die Eichung der Länge der Depositionsleitungen durch die Beobachtung des Wachstums mit in-situ Ellipsometrie (vergleiche Abschnitt 4.4). Dadurch ergibt sich ein Fehler von $\sigma\left(V_{0}(X)\right)=0.4 \mu \mathrm{l}$ bei der nachträglichen Bestimmung von $\mathrm{V}_{0}(\mathrm{X})$ über die Auswertung der in-situ Messungen. In den folgenden Darstellungen werden zwei Depositionsvorgänge verglichen, die sich nur hinsichtlich $\mathrm{V}_{0}(\mathrm{Mn})$ und $\mathrm{V}_{0}(\mathrm{Sr})$ unterscheiden. Bei der ersten La-Lage gilt in beiden Fällen $\mathrm{V}_{0}(\mathrm{La})=\mathrm{V}(\mathrm{La})$. Zur einfacheren Differenzierung dieser beiden Proben wird der Quotient

$$
\mathrm{f}(\mathrm{Mn} / \mathrm{Sr})=\frac{\mathrm{V}^{0}(\mathrm{Mn})}{\mathrm{V}(\mathrm{Mn})} \cdot \frac{\mathrm{V}(\mathrm{Sr})}{\mathrm{V}^{0}(\mathrm{Sr})}
$$

eingeführt, mit dem die Proben im Folgenden bezeichnet werden. Für die erste Probe folgt aus $\mathrm{V}_{0}(\mathrm{Sr}) / \mathrm{V}(\mathrm{Sr})=1.5$ und $\mathrm{V}_{0}(\mathrm{Mn}) / \mathrm{V}(\mathrm{Mn})=0.9 \mathrm{f}(\mathrm{Mn} / \mathrm{Sr})=0.6$ und für die zweite Probe aus $\mathrm{V}_{0}(\mathrm{Sr}) / \mathrm{V}(\mathrm{Sr})=1.0$ und $\mathrm{V}_{0}(\mathrm{Mn}) / \mathrm{V}(\mathrm{Mn})=1.6 \mathrm{f}(\mathrm{Mn} / \mathrm{Sr})=1.6$ (siehe Tabelle 7.1). 
a)

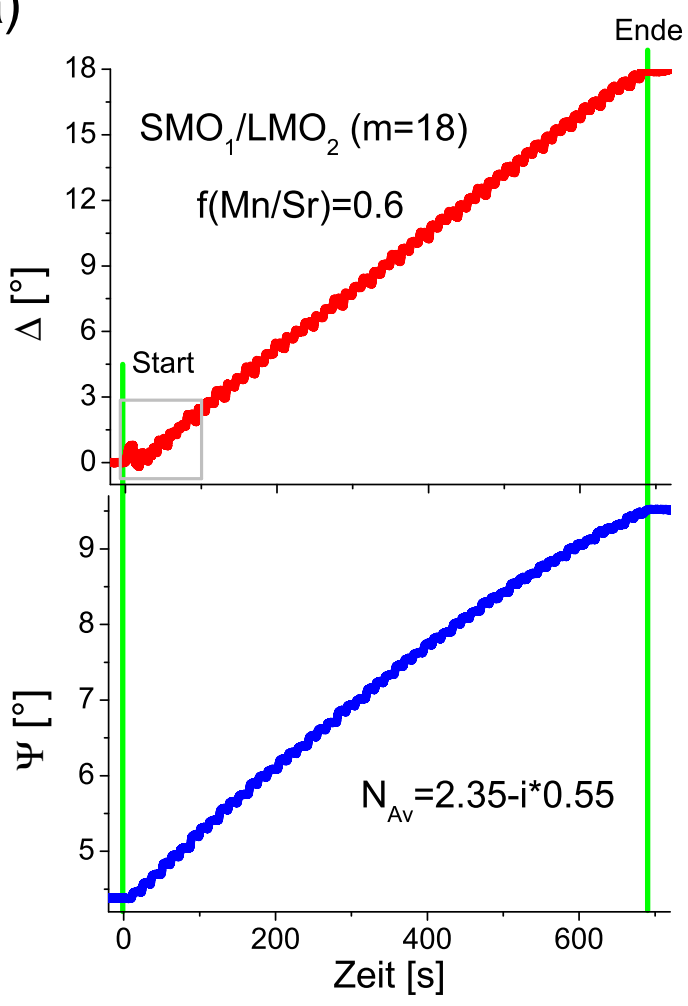

b)

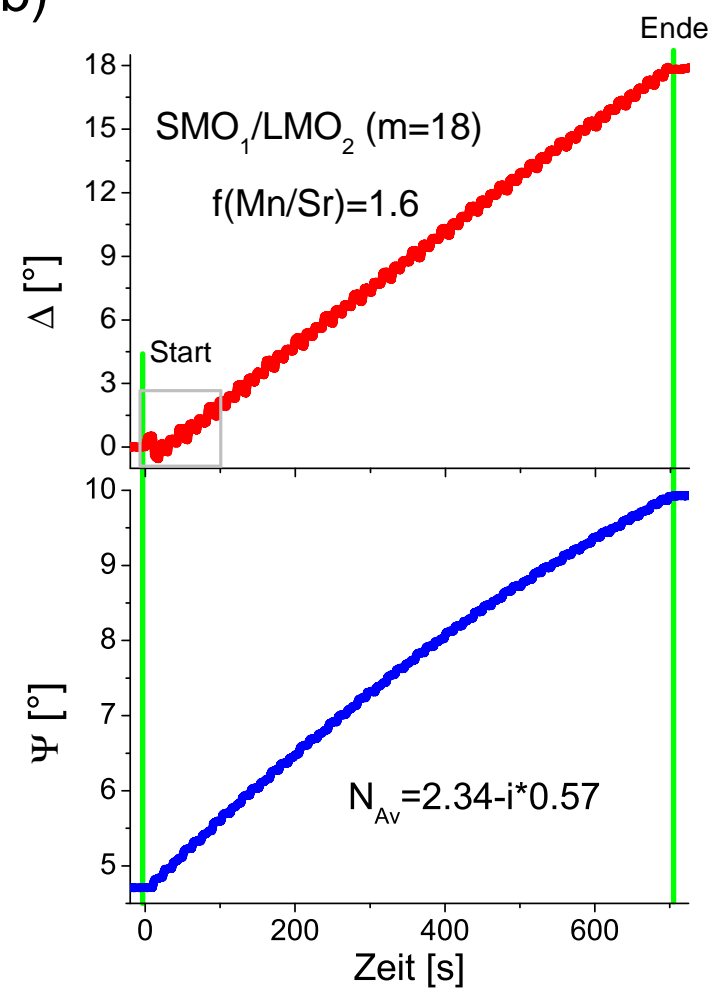

Abbildung 7.14.: Überblick der in-situ Ellipsometrie von $\mathrm{SMO}_{1} / \mathrm{LMO}_{2}(m=18)$ Heterostruktur auf STO (001)-Substraten für a) $\mathrm{f}(\mathrm{Mn} / \mathrm{Sr})=0.6$ und b) $\mathrm{f}(\mathrm{Mn} / \mathrm{Sr})=1.6$. Aus den Werten von $\Delta, \Psi$ am Ende der Deposition ergeben sich die durchschnittlichen Brechungsindizes $N_{A v}$ die in den Diagrammen vermerkt sind.

\subsubsection{In-situ Ellipsometrie}

In Abbildung 7.14 ist eine Übersicht der in-situ Messungen beim Wachstum der Proben mit $\mathrm{f}(\mathrm{Mn} / \mathrm{Sr})=0.6$ und $\mathrm{f}(\mathrm{Mn} / \mathrm{Sr})=1.6$ gezeigt. Die Einhüllende von $\Delta(t), \Psi(t)$ entspricht dem Verlauf, der sich für einen einfachen $\operatorname{LSMO}(\mathrm{x}=0.33)$-Film ergibt. Die durchschnittlichen Brechungsindizes sind mit $N_{A v}=2.35(2)-i \cdot 0.55(1)$ für $\mathrm{f}(\mathrm{Mn} / \mathrm{Sr})=0.6$ und $N_{A v}=$ $2.34(2)-i \cdot 0.57(1)$ für $\mathrm{f}(\mathrm{Mn} / \mathrm{Sr})=1.6$ fast identisch und stimmen mit dem Brechungsindex einer einfachen $\operatorname{LSMO}(\mathrm{x}=0.33)$-Schicht überein (vergleiche Abschnitt 6.2). Dies deutet eine vorwiegend homogene Verteilung der $e_{g}$-Elektronen in der LMO/SMO-Heterostruktur an (vergleiche Abschnitt 6.2 und 9.3.2.

Im Detail zeigen sich aber Unterschiede im zeitlichen Verlauf von $\Delta$ und $\Psi$, wie man in den Abbildungen 7.15 und 7.16, die den Verlauf von $\Delta(t)$ zu Beginn zeigen, erkennen kann. Deutlich kann man einen längeren ersten Sr-Puls für $\mathrm{f}(\mathrm{Mn} / \mathrm{Sr})=0.6$ und einen längeren ersten Mn-Puls für $\mathrm{f}(\mathrm{Mn} / \mathrm{Sr})=1.6$ erkennen. Beide Proben zeigen in den Pausen ein relaxierendes Verhalten in $\Delta(t)$. Für $\mathrm{f}(\mathrm{Mn} / \mathrm{Sr})=1.6$ sind die Änderungen in den Pausen kleiner als für $\mathrm{f}(\mathrm{Mn} / \mathrm{Sr})=0.6$. Des Weiteren folgt das Vorzeichen der Änderungen in den Pausen bei $\mathrm{f}(\mathrm{Mn} / \mathrm{Sr})=1.6$ dem Vorzeichen der Änderungen während der Dosierung der Präkursoren. Es fällt auf, dass die Relaxation nach der Deposition einer $\mathrm{MnO}_{2}$-Lage deutlich ausgeprägter ist als nach einer SrO- oder LaO-Lage, wo der Verlauf fast waagerecht ist. Bei f $(\mathrm{Mn} / \mathrm{Sr})=0.6$ zeigt sich ein anderes Bild: Es sind ausgeprägte Änderungen des Signals in den Pausen zu erkennen und überdies besitzt die Steigung von $\Delta(t)$ in den Pausen teilweise ein anderes Vorzeichen als während der zuvor stattfindenden Präkursorpulse. 


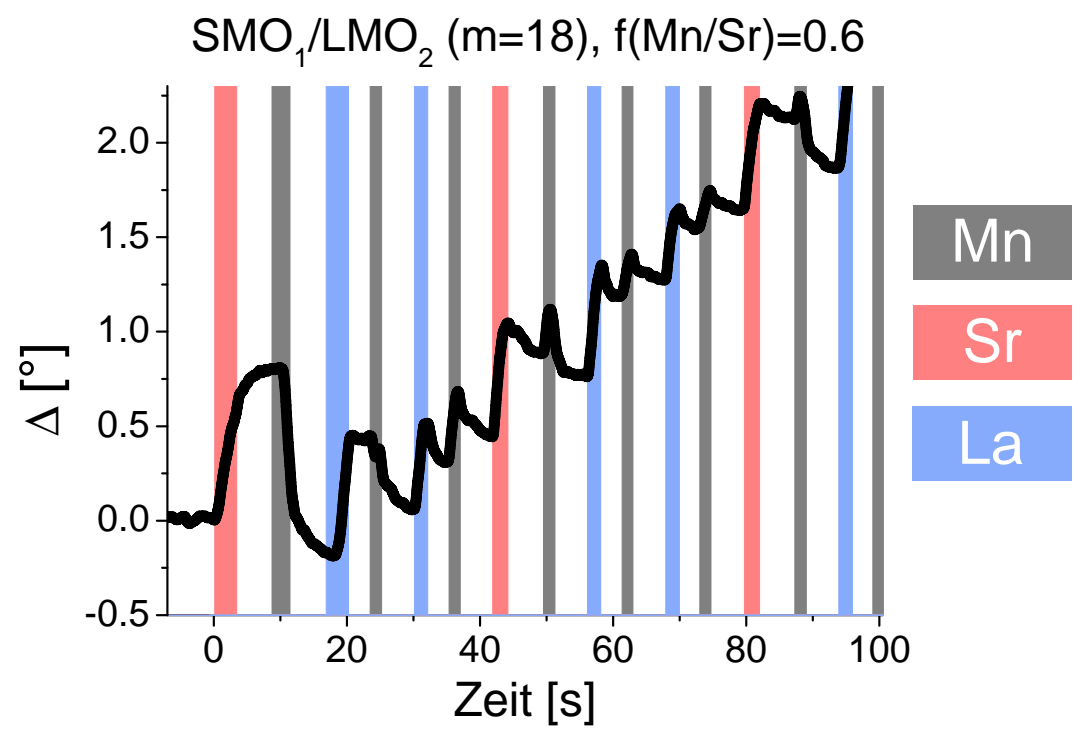

Abbildung 7.15.: Vergrößerte Darstellung des grau umrandeten Bereichs aus Abbildung 7.14 a). Die Zeitintervalle, in denen eine Dosierung der Mn-, Sr- und La-Präkursoren stattfindet, sind entsprechend der Farbskala im rechten Teil des Bildes hinterlegt. In den weiß unterlegten Bereichen wurde keine Lösung dosiert.

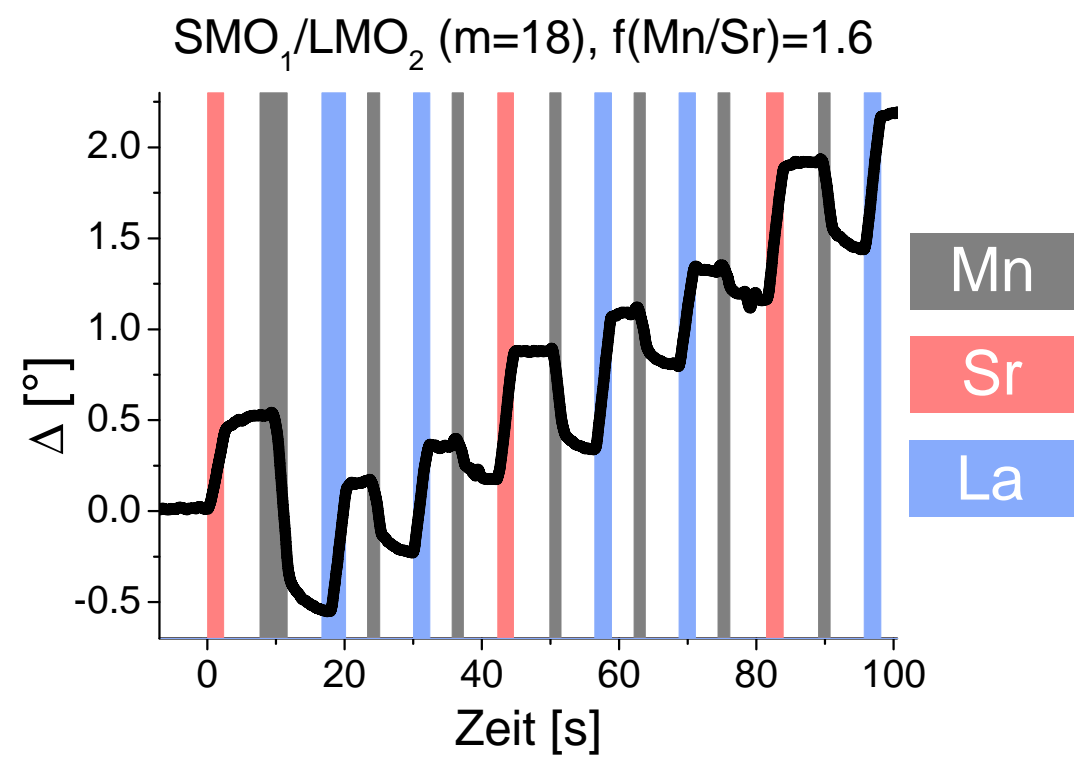

Abbildung 7.16.: Vergrößerte Darstellung des grau umrandeten Bereichs aus Abbildung 7.14 b). Die Zeitintervalle, in denen eine Dosierung der Mn-, Sr- und La-Präkursoren stattfindet, sind entsprechend der Farbskala im rechten Teil des Bildes hinterlegt. In den weiß unterlegten Bereichen wurde keine Lösung dosiert.

Zur quantitativen Auswertung der in-situ Ellipsometrie definiert man in Abbildung 7.17 die Größen $\Delta^{D}(\mathrm{X})$ und $\Delta^{R}(\mathrm{X})$, mit denen die Änderungen von $\Delta(t)$ während der Dosierung der Präkursoren und in den Depositionspausen beschrieben werden. $\Delta^{T}(\mathrm{X})=\Delta^{D}(\mathrm{X})+\Delta^{R}(\mathrm{X})$ ist die gesamte Änderung von $\Delta$ bis zur Dosierung des nächsten Präkursors. Für $\Psi(t)$ sind die Änderungen im Vergleich zum Rauschen zu klein, um eine systematische Übersicht zu erhalten. 


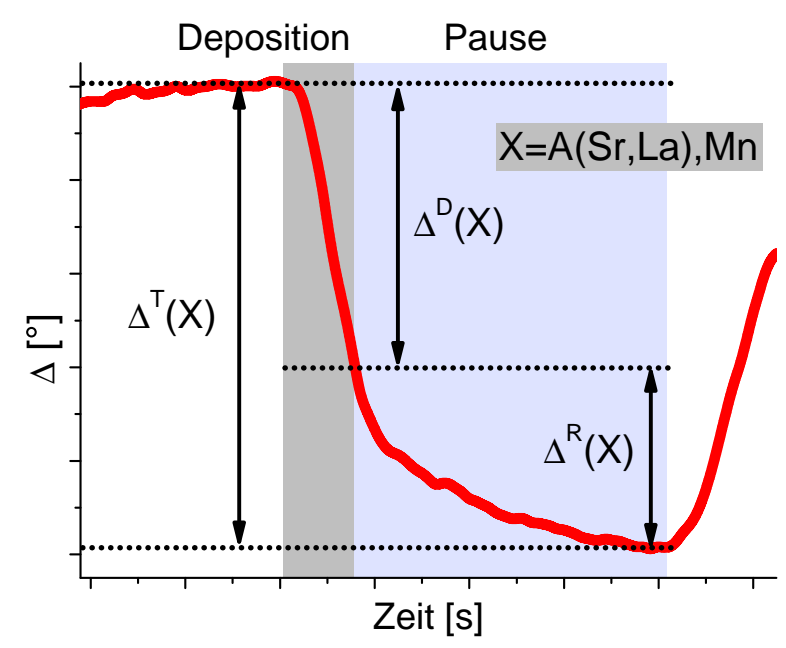

\begin{abstract}
Abbildung 7.17.: Zur quantitativen Auswertung von $\Delta(t)$ : Die Änderungen während der Dosierung der Präkursorlösungen, $\Delta^{D}(\mathrm{X})$, in der Pause zwischen den Präkursorpulsen, $\Delta^{R}(\mathrm{X})$, und die gesamte Änderung, $\Delta^{T}(\mathrm{X})=\Delta^{D}(\mathrm{X})+\Delta^{R}(\mathrm{X})$, werden aus dem Verlauf von $\Delta(t)$ abgelesen. Dabei steht $\mathrm{X}$ für das dosierte Element: die A-Plätze, Sr und La, sowie Mn auf dem B-Platz.
\end{abstract}

In Abbildung 7.18 sind diese drei Größen für die Mn-Pulse als Funktion der Nummer der $\mathrm{MnO}_{2}$-Lage, $i_{M n}=1-54$, dargestellt. $\Delta^{D}(\mathrm{Mn})$ zeigt für $\mathrm{f}(\mathrm{Mn} / \mathrm{Sr})=1.6$ einen sehr periodischen Verlauf mit lokalen Minima bei der $\mathrm{MnO}_{2}$-Lage nach dem Sr-Puls ( $\mathrm{Sr}-\mathrm{Mn}$ ) und lokalen Maxima bei der $\mathrm{MnO}_{2}$-Lage nach dem zweiten La-Puls (2. La-Mn). Für $\mathrm{f}(\mathrm{Mn} / \mathrm{Sr})=0.6$ ist diese Periodizität nur schwach ausgeprägt, $\Delta^{D}(\mathrm{Mn})$ schwankt als Funktion von $i_{M n}$ eher zufällig. Des Weiteren fällt auf, dass $\Delta^{D}(\mathrm{Mn})$ bei $\mathrm{f}(\mathrm{Mn} / \mathrm{Sr})=1.6$ für alle $i_{M n}$ negativ ist, wogegen $\Delta^{D}(\mathrm{Mn})$ für $\mathrm{f}(\mathrm{Mn} / \mathrm{Sr})=0.6$ bei $i_{M n}=10$ einen Vorzeichenwechsel erfährt. In den Pausen verhält sich die Probe mit $\mathrm{f}(\mathrm{Mn} / \mathrm{Sr})=1.6$ ähnlich wie während der Präkursorpulse. Es ist die gleiche Periodizität zu erkennen mit Minima und Maxima an den gleichen Positionen wie bei $\Delta^{D}(\mathrm{Mn})$. Im Mittel ist $\Delta^{R}(\mathrm{Mn})$ wesentlich kleiner als $\Delta^{D}(\mathrm{Mn})$ und macht etwa $1 / 3$ der totalen Änderung $\Delta^{T}(\mathrm{Mn})$ aus. Auch für $\mathrm{f}(\mathrm{Mn} / \mathrm{Sr})=0.6$ zeigt $\Delta^{R}(\mathrm{Mn})$ ausgeprägte Oszillationen als Funktion von $i_{M n}$. Die Extrema dieser Schwingungen befinden sich an den gleichen Stellen wie bei $\mathrm{f}(\mathrm{Mn} / \mathrm{Sr})=1.6$. Allerdings reproduziert sich das Schema $\operatorname{der} \Delta^{R}(\mathrm{Mn})$ mit den aufeinander folgenden Lagen, Sr-Mn, 1. La-Mn und 2. La-Mn nicht so präzise wie für $\mathrm{f}(\mathrm{Mn} / \mathrm{Sr})=1.6$. In der Addition resultiert dann $\Delta^{T}(\mathrm{Mn})$. Diese Größe zeigt für $\mathrm{f}(\mathrm{Mn} / \mathrm{Sr})=1.6$ eine sich wiederholende Abfolge, bei der $\Delta^{T}(\mathrm{Mn})$ von der Sr-Mn-Lage über die erste La-Mn-Lage bis zur zweiten La-Mn-Lage kontinuierlich ansteigt. Die Oszillationsamplitude, die Differenz zwischen $\Delta^{T}(\mathrm{Mn})$ für die Sr-Mn-Lage und $\Delta^{T}(\mathrm{Mn})$ für die 2. La-Mn-Lage, sinkt mit steigendem $i_{M n}$ langsam ab. Im Mittel liegt diese Amplitude für $\mathrm{f}(\mathrm{Mn} / \mathrm{Sr})=1.6$ bei $0.25^{\circ}$, für $\mathrm{f}(\mathrm{Mn} / \mathrm{Sr})=0.6$ ist diese mit $0.17^{\circ}$ kleiner. Außerdem ist die periodische Wiederholung der Oszillationen nicht so reproduzierbar wie bei $\mathrm{f}(\mathrm{Mn} / \mathrm{Sr})=1.6$. So ist $\Delta^{T}(\mathrm{Mn})$ für die 1. La-Mn-Lage bei $\mathrm{f}(\mathrm{Mn} / \mathrm{Sr})=1.6$ stets signifikant kleiner als für die 2. La-Mn-Lage. Dies ist bei $\mathrm{f}(\mathrm{Mn} / \mathrm{Sr})=0.6$ nicht mehr der Fall, zum Teil ist dort $\Delta^{T}(\mathrm{Mn})$ für die 1. La-Mn-Lage innerhalb des Messfehlers gleich oder sogar größer als für die 2. LaMn-Lage.

Auch bei der Betrachtung der Änderung von $\Delta$ beim Aufbringen von SrO- und LaO-Lagen auf die A-Plätze zeigen sich periodische Abläufe entsprechend dem Depositionsschema. $\Delta^{D}(\mathrm{~A})$ zeigt für $\mathrm{f}(\mathrm{Mn} / \mathrm{Sr})=0.6$ und $\mathrm{f}(\mathrm{Mn} / \mathrm{Sr})=1.6$ eine ähnliche Ausprägung der Oszillationen sowohl mit Blick auf die Positionen der Extrema als auch hinsichtlich der Amplitude der 


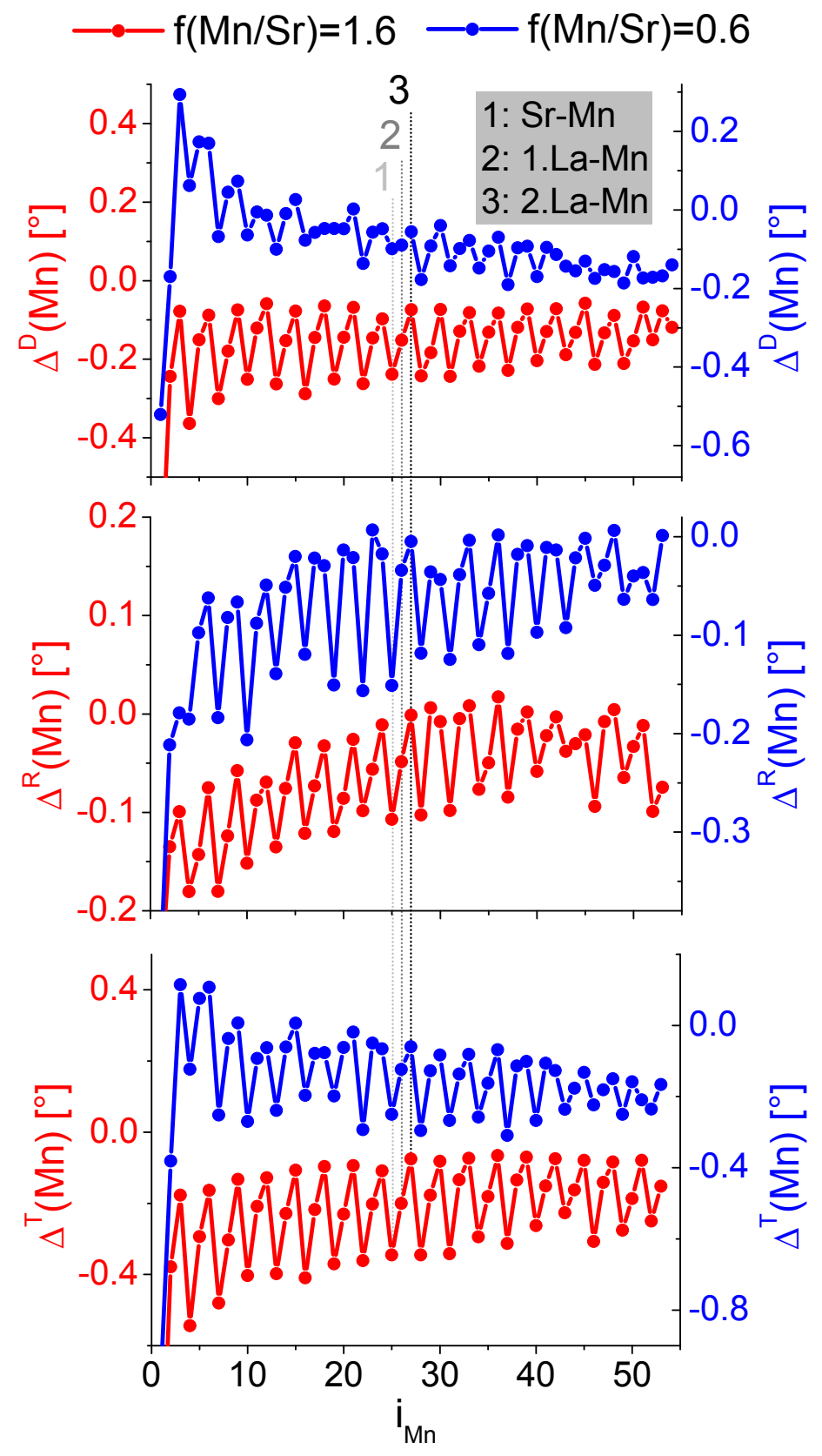

Abbildung 7.18.: $\Delta^{D}(\mathrm{Mn}), \Delta^{R}(\mathrm{Mn})$ und $\Delta^{T}(\mathrm{Mn})=\Delta^{D}(\mathrm{Mn})+\Delta^{R}(\mathrm{Mn})$ für $\mathrm{f}(\mathrm{Mn} / \mathrm{Sr})=0.6$ und $\mathrm{f}(\mathrm{Mn} / \mathrm{Sr})=1.6$ als Funktion der Nummer der deponierten $\mathrm{MnO}_{2^{-}}$ Lage, $i_{M n}=1-3 \cdot m=1-54$. Mit den eingezeichneten Linien werden die unterschiedlichen $\mathrm{MnO}_{2}$-Lagen in einer Periode identifiziert (vergleiche Abbildung 7.13).

Oszillationen. Das Minimum dieser Schwingungen von $\Delta^{D}(\mathrm{~A})$ zeigt sich bei der Deposition der 2. La-Lage, das Maximum bei der Deposition der 1. La-Lage (siehe Abbildung 7.19). Für $\mathrm{f}(\mathrm{Mn} / \mathrm{Sr})=1.6$ ist in $\Delta^{R}(\mathrm{~A})$ keine Struktur der periodischen Abfolge von $\Delta^{D}(\mathrm{~A}) \mathrm{zu}$ erkennen. Insgesamt ist $\Delta^{R}(\mathrm{~A})$ für $\mathrm{f}(\mathrm{Mn} / \mathrm{Sr})=1.6 \mathrm{sehr}$ klein und macht etwa $10 \%$ von $\Delta^{T}(\mathrm{~A})$ aus. Für $\mathrm{f}(\mathrm{Mn} / \mathrm{Sr})=0.6$ haben die Änderungen in der Pause einen deutlich größeren Anteil an $\Delta^{T}(\mathrm{~A})$. Überdies besitzt $\Delta^{R}(\mathrm{~A})$ für $i_{A} \lesssim 20$ ein Vorzeichen entgegengesetzt zu $\Delta^{D}(\mathrm{~A})$. In der Addition resultiert wieder $\Delta^{T}(\mathrm{~A})$. Wie für $\Delta^{T}(\mathrm{Mn})$ zeigt sich auch hier eine mit $i_{A}$ abnehmende Oszillationsamplitude. Wiederum ist die durchschnittliche Amplitude 


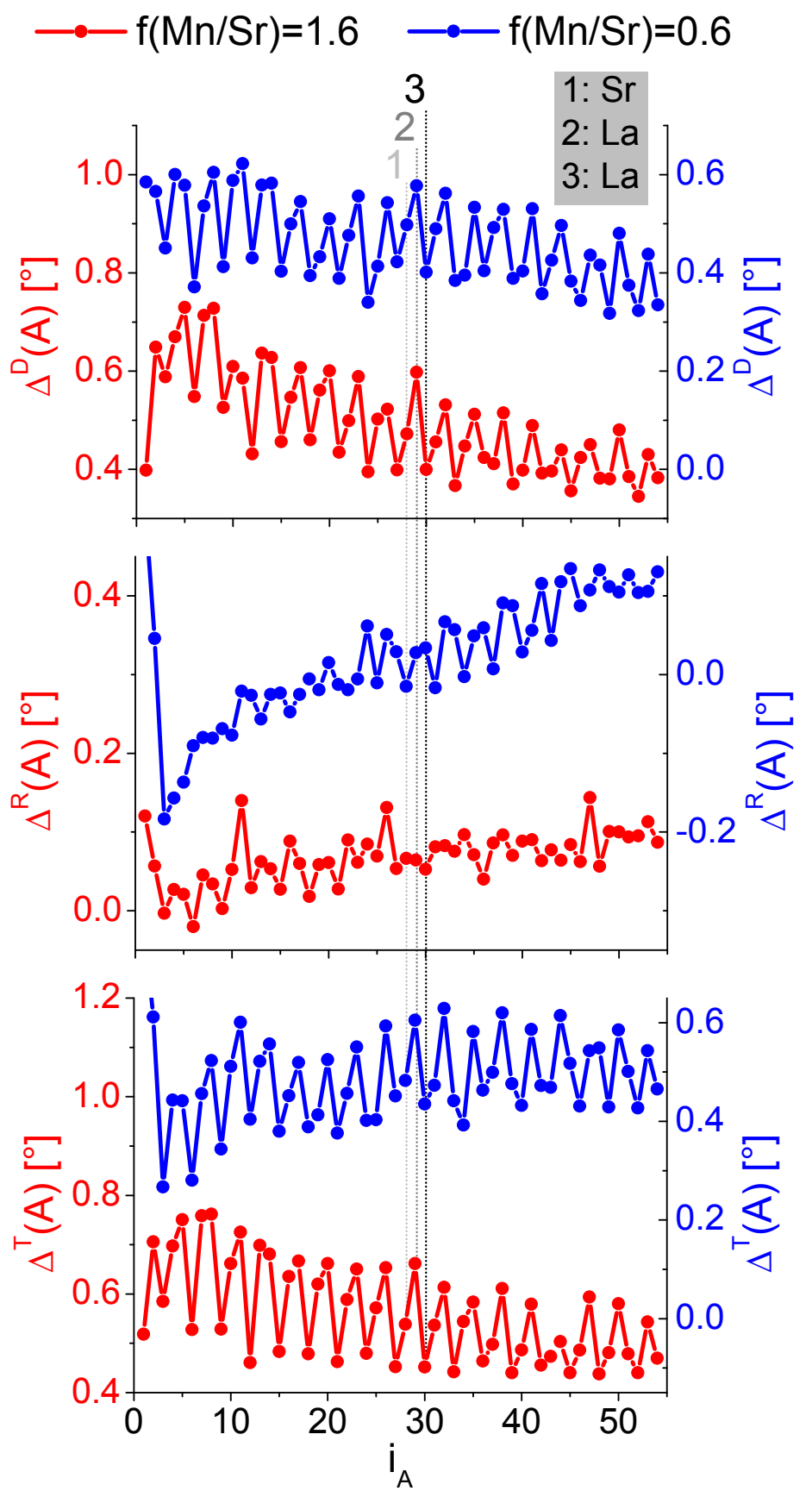

Abbildung 7.19.: $\Delta^{D}(\mathrm{~A}), \Delta^{R}(\mathrm{~A})$ und $\Delta^{T}(\mathrm{~A})=\Delta^{D}(\mathrm{~A})+\Delta^{R}(\mathrm{~A})$ mit $\mathrm{A}=\mathrm{Sr}$, La für $\mathrm{f}(\mathrm{Mn} / \mathrm{Sr})=0.6$ und $\mathrm{f}(\mathrm{Mn} / \mathrm{Sr})=1.6$ als Funktion der Nummer der deponierten A-PlatzLagen $(\mathrm{SrO}, \mathrm{LaO}), i_{A}=1-3 \cdot m=1-54$. Mit den eingezeichneten Linien werden die Positionen der verschiedenen Elemente ( $\mathrm{Sr}$ und $\mathrm{La}$ ) auf den A-Plätzen für eine Periode markiert.

der Oszillationen für $\mathrm{f}(\mathrm{Mn} / \mathrm{Sr})=1.6$ mit $0.17^{\circ}$ größer als für $\mathrm{f}(\mathrm{Mn} / \mathrm{Sr})=0.6$ mit $0.13^{\circ}$. Es fällt außerdem wie bei $\Delta^{T}(\mathrm{Mn})$ auf, dass die Oszillationen für $\mathrm{f}(\mathrm{Mn} / \mathrm{Sr})=1.6$ regelmäßiger verlaufen als für $\mathrm{f}(\mathrm{Mn} / \mathrm{Sr})=0.6$.

Die Kombination von $\Delta^{T}(\mathrm{Mn})$ und $\Delta^{T}(\mathrm{~A})$ ist in Abbildung 7.20 als Funktion des Indexes $i=1-108$, mit dem die aufgebrachten Lagen durchgezählt werden, gezeigt. In der Übersicht in Abbildung 7.20 a) bestätigt sich der Eindruck eines periodischen Verlaufs von $\Delta^{T}$. Für 

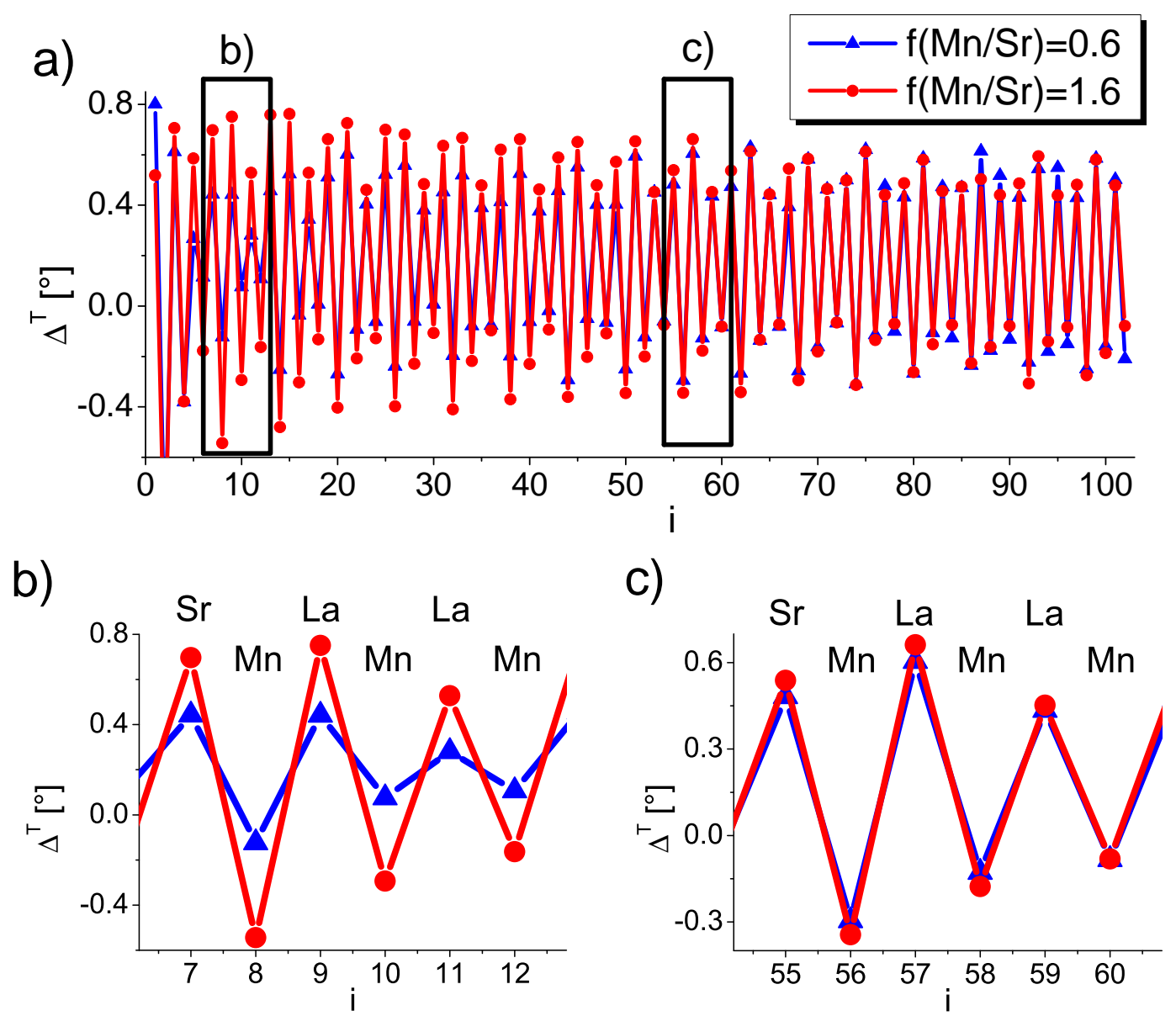

Abbildung 7.20.: Zusammenfassende Darstellung von $\Delta^{T}(M n)$ aus Abbildung 7.18 und $\Delta^{T}(A)$ aus Abbildung 7.19 für $\mathrm{f}(\mathrm{Mn} / \mathrm{Sr})=0.6$ und $\mathrm{f}(\mathrm{Mn} / \mathrm{Sr})=1.6$ : a) Übersicht der ganzen Depositionsdauer, $i=1-108$ nummeriert alle deponierten Lagen. b) und c) sind Vergrößerungen der markierten Bereiche in a).

$\mathrm{f}(\mathrm{Mn} / \mathrm{Sr})=1.6$ besitzen die Oszillationen zu Beginn die größte Amplitude, die dann mit steigendem $i$ langsam abfällt. Für die andere Probe mit $\mathrm{f}(\mathrm{Mn} / \mathrm{Sr})=0.6$ ist die Amplitude zu Beginn wesentlich kleiner als für $\mathrm{f}(\mathrm{Mn} / \mathrm{Sr})=1.6$ (siehe Abbildung $7.20 \mathrm{~b}$ )). Im weiteren Verlauf der Deposition vergrößert sich diese Amplitude allerdings und erreicht dann für $i \gtrsim 55$ ein ähnliches Niveau wie für $\mathrm{f}(\mathrm{Mn} / \mathrm{Sr})=1.6$ (Abbildung 7.20 c)).

\subsubsection{Strukturelle Charakterisierung}

Die deutlichen Kontraste in der in-situ Ellipsometrie zwischen den beiden vorgestellten Proben schlagen sich auch in deren strukturellen Eigenschaften nieder. Zunächst soll die Oberflächenmorphologie betrachtet werden (Abbildung 7.21): Für $\mathrm{f}(\mathrm{Mn} / \mathrm{Sr})=1.6$ zeigt sich ein zweidimensionales Wachstum mit klar erkennbaren Terrassen, die aus dem Fehlschnitt des Substrates resultieren. Es ist eher ein zweidimensionales Inselwachstum zu beobachten als eine step-flow-Wachstumsmode. Die RMS-Rauigkeit ist mit $\sigma_{R M S}=0.38 \mathrm{~nm}$ gering. Für $\mathrm{f}(\mathrm{Mn} / \mathrm{Sr})=0.6$ ist die Wachstumsmode nicht mehr zweidimensional, man erkennt Inseln mit einer Höhe von etwa $5 \mathrm{~nm}$, woraus eine deutlich größere RMS-Rauigkeit von $\sigma_{R M S}=1.0 \mathrm{~nm}$ folgt. Die Inseln sind entlang von parallelen Linien aufgereiht. Vermutlich verlaufen diese Li- 
a)

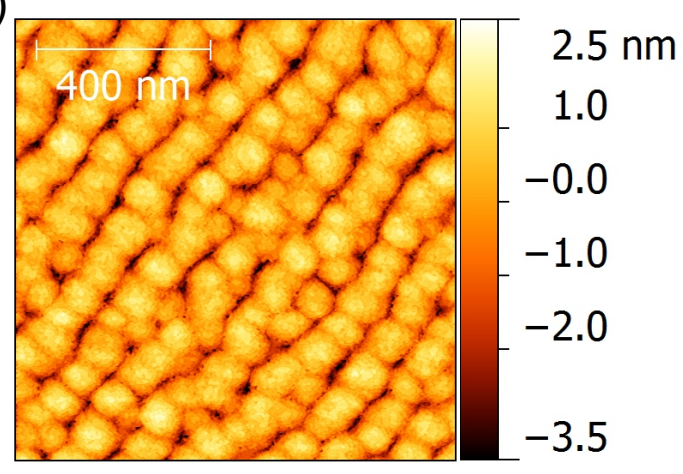

b)

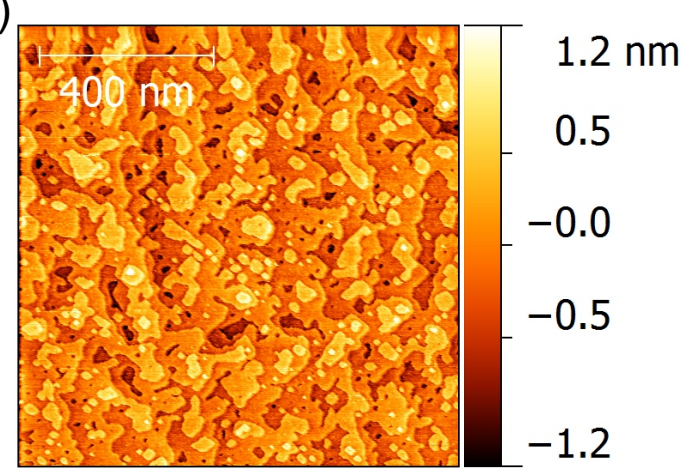

Abbildung 7.21.: STM-Aufnahmen der Oberflächenmorphologien für die $\mathrm{SMO}_{1} / \mathrm{LMO}_{2}$ $(m=18)$-Heterostruktur mit a) $\mathrm{f}(\mathrm{Mn} / \mathrm{Sr})=0.6$ und b) $\mathrm{f}(\mathrm{Mn} / \mathrm{Sr})=1.6$.

a)

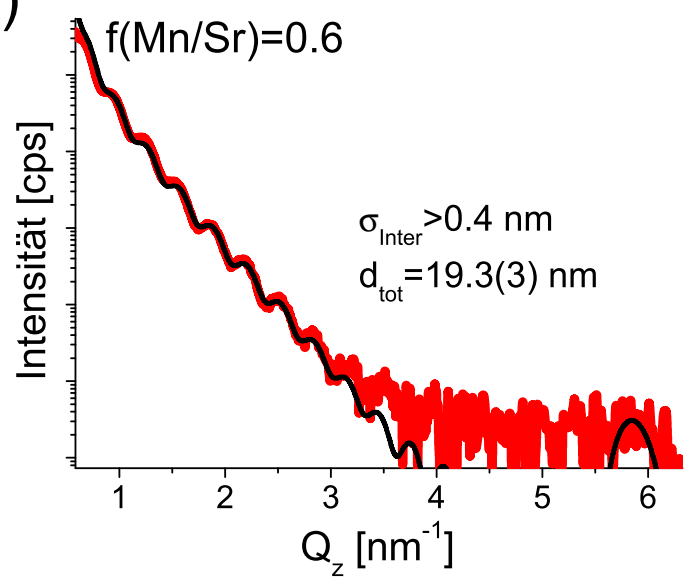

b)

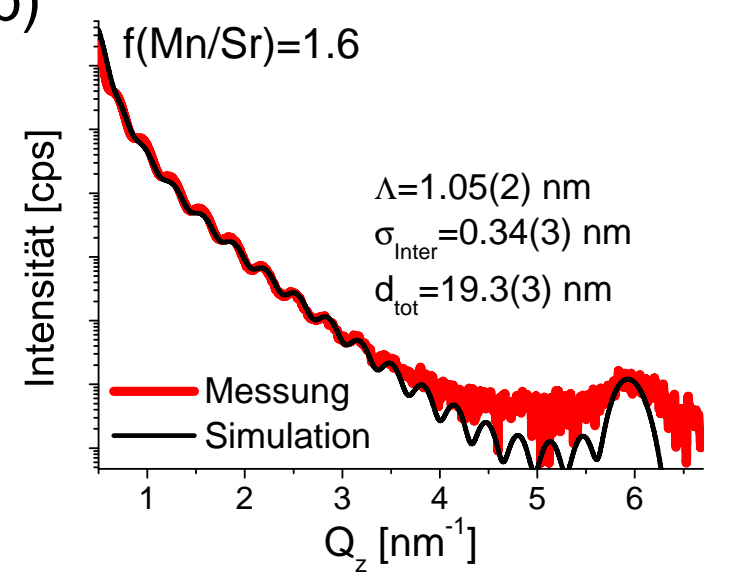

Abbildung 7.22.: XRR-Spektren der $\mathrm{SMO}_{1} / \mathrm{LMO}_{2}(m=18)$-Heterostrukturen mit a) $\mathrm{f}(\mathrm{Mn} / \mathrm{Sr})=0.6$ und b) $\mathrm{f}(\mathrm{Mn} / \mathrm{Sr})=1.6$. Aus der Anpassung der Messungen mit ReMagX ergeben sich die strukturellen Parameter der Übergitter, die in den Diagrammen angegeben sind: $\sigma_{\text {Inter }}$ - mittlere Rauigkeit der SMO/LMO-Grenzflächen, $d_{t o t}$ - Gesamtdicke der Struktur und $\Lambda$ - Dicke der $\mathrm{SMO}_{1} / \mathrm{LMO}_{2}$-Einheiten.

nien parallel zu den Kanten der Substratstufen. Beim Wachstum besitzen die Adatome eine zu kleine Mobilität, so dass sich Inseln in der Mitte der Terrassen bilden (vergleiche Abschnitt 4.4.2).

Auch die Auswertung der chemischen Modulation der Übergitter mit XRR-Messungen zeigt deutliche Kontraste (Abbildung 7.22). Für $\mathrm{f}(\mathrm{Mn} / \mathrm{Sr})=1.6$ ist ein Übergitterpeak erkennbar, über den man die mittlere Rauigkeit der LMO/SMO-Grenzflächen von $\sigma_{\text {Inter }}=0.34(3) \mathrm{nm}$ abschätzen kann. Mit dem Fehlerintervall liegt diese im Bereich der Grenzflächenrauigkeit, die man für ein $\mathrm{LMO}_{2} / \mathrm{SMO}_{1}$-SL, das mit dem konventionellen MAD-Prozess gewachsen wurde, erhält 238. Beide Proben besitzen eine identische Gesamtschichtdicke von $d_{t o t}=19.3(3) \mathrm{nm}$. Dies ist aufgrund der geringen Unterschiede bei den deponierten Präkursormengen zu erwarten. Der Unterschied der beiden Heterostrukturen liegt ja nur in der ersten Perowskit-Lage. Die Doppellagendicke $\Lambda=1.05(2) \mathrm{nm}$ wird für $\mathrm{f}(\mathrm{Mn} / \mathrm{Sr})=1.6$ auch durch die Betrachtung eines XRD-Spektrums bestätigt (siehe Abbildung 7.23). Der experimentell ermittelte Wert von $\Lambda$ ist um etwa $9 \%$ kleiner als der theoretische Wert $\Lambda^{\text {theo }}=1.16 \mathrm{~nm}$. Der Übertrag bei dem ALE-Prozess ist also kleiner als beim konventionellen MAD-Prozess, mit dem das Prä- 


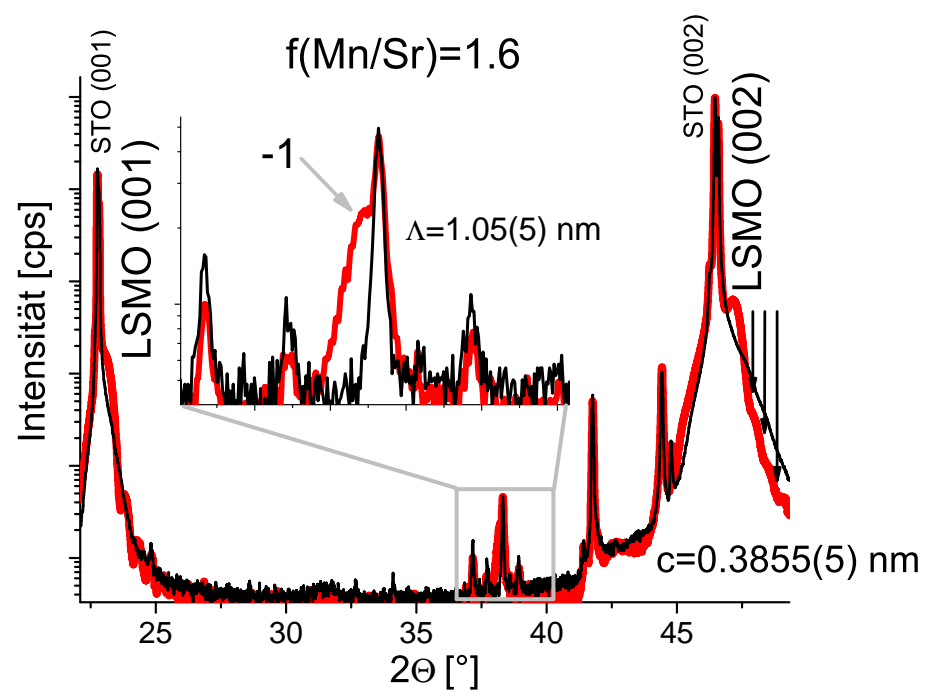

\begin{abstract}
Abbildung 7.23.: XRD-Spektrum für $\mathrm{SMO}_{1} / \mathrm{LMO}_{2} \quad(m=18)$-Struktur mit $\mathrm{f}(\mathrm{Mn} / \mathrm{Sr})=1.6$ : Zur Identifikation der Fremdpeaks ist neben dem gemessenen Spektrum der Heterostruktur (rot) auch das Spektrum eines blanken STO (001)-Substrates (schwarz) gezeigt. Im Inset ist der Satellitenpeak des (002)-Peaks zusammen mit der Bilagendicke $\Lambda$ gezeigt. Die Pfeile markieren die Positionen der Maxima der Laue-Oszillationen um den (002)-Peak. Aus der Position der Beugungsmaxima resultiert der im Diagramm gezeigte Gitterebenenabstand in c-Richtung.
\end{abstract}

kursorvolumen zur Deposition einer Monolage $\mathrm{MnO}_{2}$ ermittelt wurde. Der Gitterparameter ist für beide Proben identisch und liegt bei $c=0.3855(5) \mathrm{nm}$. Das entspricht der c-Achse einer teilweise entspannten $\operatorname{LSMO}(0.3)$-Schicht mit $d \approx 30 \mathrm{~nm}[97$.

\title{
7.3.5. Magnetische und elektrische Eigenschaften
}

Im Folgenden werden die magnetischen und elektrischen Eigenschaften der beiden betrachteten Proben verglichen. Aus den in Abbildung 7.24 a) gezeigten $\rho(T)$-Kurven werden die Temperaturen des Metall-Isolator-Überganges, $T_{M I}$, ermittelt. Für $\mathrm{f}(\mathrm{Mn} / \mathrm{Sr})=1.6$ liegt $T_{M I}=$ $330 \mathrm{~K}$ deutlich über $T_{M I}=285 \mathrm{~K}$ für $\mathrm{f}(\mathrm{Mn} / \mathrm{Sr})=0.6$. Des Weiteren ist der Metall-IsolatorÜbergang für $\mathrm{f}(\mathrm{Mn} / \mathrm{Sr})=1.6$ schärfer, d.h. der maximale Wert von TCR ist höher als für $\mathrm{f}(\mathrm{Mn} / \mathrm{Sr})=0.6$. Auch der spezifische Widerstand bei $T=5 \mathrm{~K}, \rho(5 \mathrm{~K})$, ist für $\mathrm{f}(\mathrm{Mn} / \mathrm{Sr})=1.6$ um einen Faktor von etwa 2 kleiner als bei $\mathrm{f}(\mathrm{Mn} / \mathrm{Sr})=0.6$. Innerhalb des Messfehlers stimmt $\rho(5 \mathrm{~K})$ für $\mathrm{f}(\mathrm{Mn} / \mathrm{Sr})=0.6$ mit den Messungen einfacher $\mathrm{La}_{0.7} \mathrm{Sr}_{0.3} \mathrm{MnO}_{3}$-Filme vergleichbarer Schichtdicke überein 97. In weiteren Experimenten wurden zusätzliche Heterostrukturen abgeschieden, bei denen ebenso Variationen der dosierten Volumina $V_{0}(X)$ für die ersten Lagen vorgenommen wurden (siehe Tabelle 7.1). Im Inset von Abbildung 7.24 b) sieht man die Entwicklung von $T_{M I}$ mit $\mathrm{f}(\mathrm{Mn} / \mathrm{Sr})$. $T_{M I}$ zeigt eine ansteigende Tendenz als Funktion von $\mathrm{f}(\mathrm{Mn} / \mathrm{Sr})$. Für diese Proben mit unterschiedlichem $\mathrm{f}(\mathrm{Mn} / \mathrm{Sr})$ ergeben sich unter Beachtung der Fehlerintervalle identische Werte des komplexen Brechungsindexes, $N=n-i \cdot k$ (siehe Tabelle 7.1).

Auch die magnetischen Eigenschaften bestätigen den Trend der elektrischen Messungen: Für $\mathrm{f}(\mathrm{Mn} / \mathrm{Sr})=1.6$ ist die Curie-Temperatur größer als für $\mathrm{f}(\mathrm{Mn} / \mathrm{Sr})=0.6$ (Abbildung 7.25). Außerdem ist der Betrag der Steigung von $M(T)$ bei $T \lesssim T_{C}$ für $\mathrm{f}(\mathrm{Mn} / \mathrm{Sr})=1.6$ wesentlich größer als für $\mathrm{f}(\mathrm{Mn} / \mathrm{Sr})=0.6$. Der magnetische Übergang ist bei $\mathrm{f}(\mathrm{Mn} / \mathrm{Sr})=0.6$ also 
a)

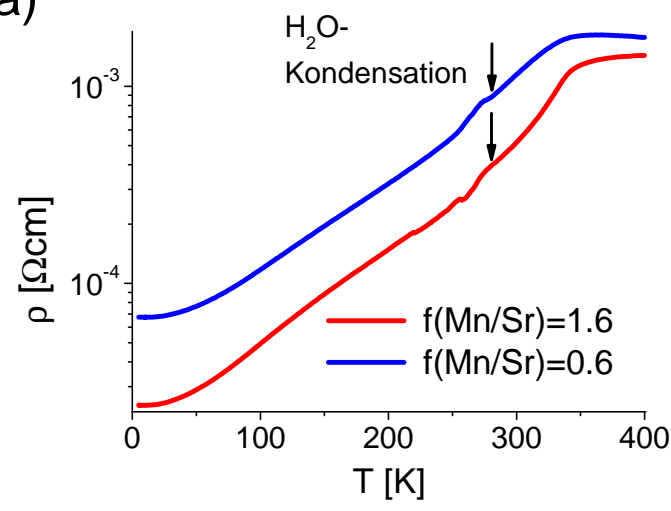

b)

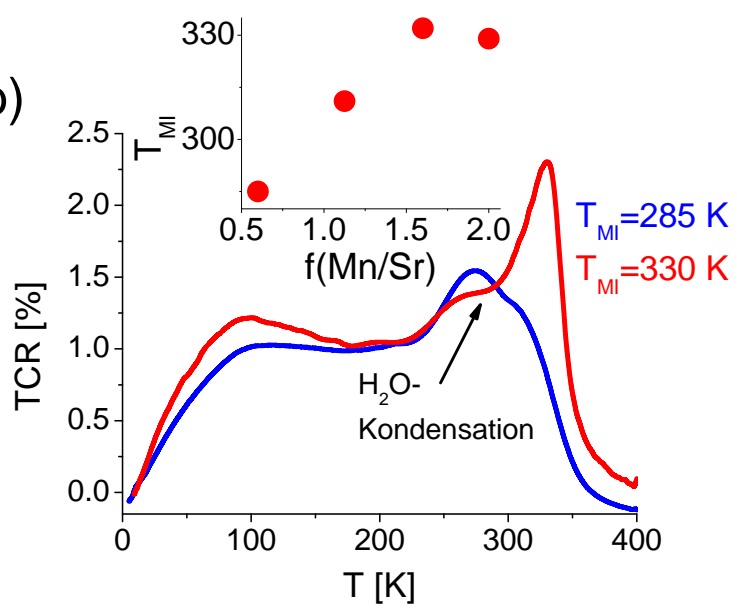

Abbildung 7.24.: Elektrische Eigenschaften der $\mathrm{SMO}_{1} / \mathrm{LMO}_{2}(m=18)$-Heterostrukturen für $\mathrm{f}(\mathrm{Mn} / \mathrm{Sr})=0.6$ und $\mathrm{f}(\mathrm{Mn} / \mathrm{Sr})=1.6$ : a) Temperaturabhängigkeit des spezifischen Widerstandes $\rho, \mathrm{b})$ Darstellung von TCR als Funktion der Temperatur zur Bestimmung der im Diagramm vermerkten Metall-Isolator-Übergangstemperaturen $T_{M I}$. Im Inset ist $T_{M I}$ in Abhängigkeit von $\mathrm{f}(\mathrm{Mn} / \mathrm{Sr})$ für verschiedene Proben dargestellt (vergleiche Tabelle 7.1). Durch Kondensation und Gefrieren von Wasser im PPMS treten in den $\rho(T)$ - und TCR $(T)$ Kurven zusätzliche Besonderheiten auf, die entsprechend markiert wurden.

\begin{tabular}{ccccccc}
\hline$\frac{\mathrm{V}^{0}(\mathrm{Sr})}{\mathrm{V}(\mathrm{Sr})}$ & $\frac{\mathrm{V}^{0}(\mathrm{La})}{\mathrm{V}(\mathrm{La})}$ & $\frac{\mathrm{V}^{0}(\mathrm{Mn})}{\mathrm{V}(\mathrm{Mn})}$ & $\mathrm{f}(\mathrm{Mn} / \mathrm{Sr})$ & $T_{M I}[\mathrm{~K}]$ & $n$ & $k$ \\
\hline 1.50 & 1.00 & 0.90 & $0.60(4)$ & 285 & 2.35 & 0.55 \\
\hline 1.00 & 1.00 & 1.60 & $1.6(1)$ & 332 & 2.34 & 0.57 \\
\hline 0.80 & 1.00 & 0.90 & $1.1(1)$ & 311 & 2.35 & 0.54 \\
\hline 0.80 & 0.80 & 1.60 & $2.0(2)$ & 329 & 2.34 & 0.56 \\
\hline
\end{tabular}

Tabelle 7.1.: Elektrische und magnetische Eigenschaften von $\mathrm{SMO}_{1} / \mathrm{LMO}_{2}-\mathrm{SL}$ in Abhängigkeit von den relativen Stöchiometrien der Anfangslagen, $\frac{\mathrm{V}^{0}(\mathrm{X})}{\mathrm{V}(\mathrm{X})}$ mit $\mathrm{X}=\mathrm{Sr}, \mathrm{La}, \mathrm{Mn}$. Für die gezeigten Größen gelten die folgenden Fehlerintervalle: $\sigma\left(\frac{\mathrm{V}^{0}(\mathrm{X})}{\mathrm{V}(\mathrm{X})}\right)=0.05, \sigma\left(T_{M I}\right)=2 \mathrm{~K}$, $\sigma(n)=0.02, \sigma(k)=0.01$. Wie oben wird der Koeffizient $\mathrm{f}(\mathrm{Mn} / \mathrm{Sr})=\frac{\mathrm{V}^{0}(\mathrm{Mn})}{\mathrm{V}(\mathrm{Mn})} \cdot \frac{\mathrm{V}(\mathrm{Sr})}{\mathrm{V}^{0}(\mathrm{Sr})}$ berechnet, dessen Fehler durch Fehlerfortpflanzung ermittelt wird.

unschärfer. Dies deutet darauf hin, dass die FM-Ordnung in dieser Probe nicht im gesamten Volumen bei der gleichen Temperatur eintritt. Es gibt Bereiche mit unterschiedlichen Curie-Temperaturen. Die Überlagerung der magnetischen Momente dieser Bereiche führt dann zu einer Verbreiterung des FM-Überganges der gesamten Probe. $T_{C}=346(1) \mathrm{K}$ für $\mathrm{f}(\mathrm{Mn} / \mathrm{Sr})=1.6$ ist vergleichbar mit $T_{C}=349(1) \mathrm{K}$, das sich für ein LMO/SMO $(n=1)$ SL ergibt (siehe Abschnitt 9.4). Die magnetischen Eigenschaften bei $T=5 \mathrm{~K}, M_{\text {sat }}$ und $H_{C}$, sind für beide Proben identisch (Abbildung $7.25 \mathrm{~b}$ )). Das Sättigungsmoment, $M_{\text {sat }}=$ 3.8 (2) $\mu_{\mathrm{B}} / \mathrm{Mn}$ entspricht dem theoretischen Wert für eine volle Ausrichtung aller Spins auf den Mn-Ionen. Das Koerzitivfeld $H_{C}=25$ Oe ist vergleichbar mit den Werten, die sich für einfache LSMO(0.3)-Filme ergeben [97]. 
a)

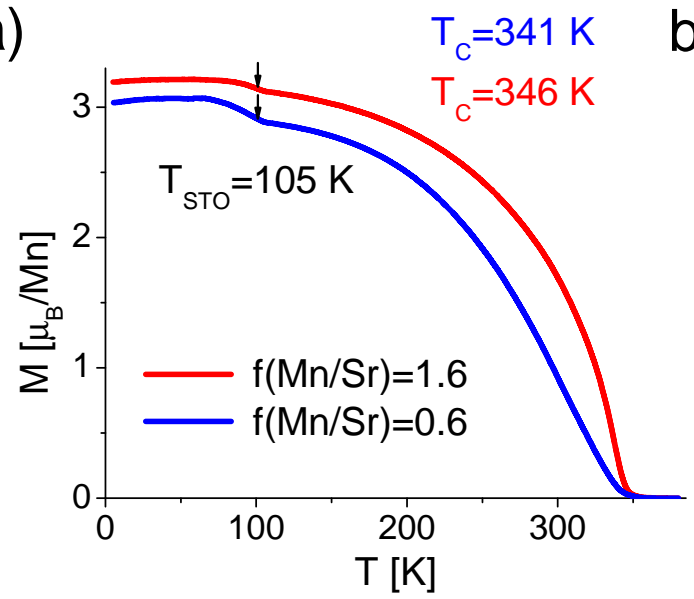

b)

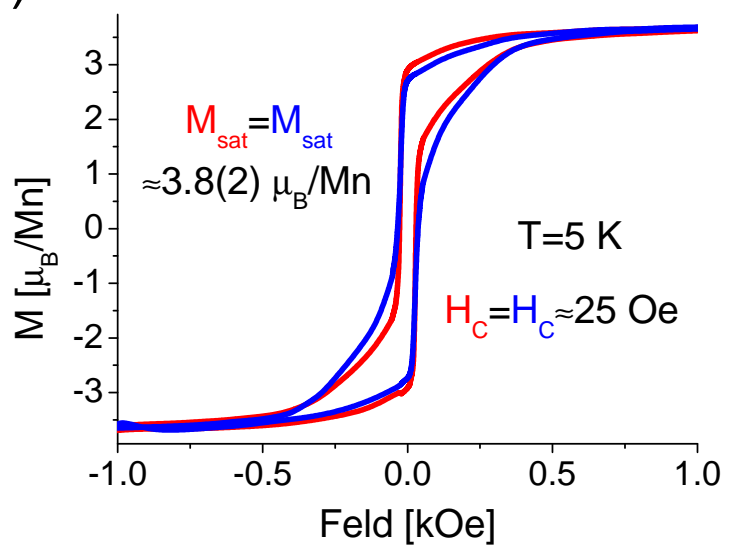

Abbildung 7.25.: Magnetische Eigenschaften der $\mathrm{SMO}_{1} / \mathrm{LMO}_{2}$-SL: a) $M(T)$-Kurven, die Curie-Temperaturen $T_{C}$ sind eingezeichnet $\left(T_{S T O}=105 \mathrm{~K}\right.$ bezeichnet die Temperatur des strukturellen Überganges vom STO-Substrat), b) $M(H)$-Kurven bei $T=5 \mathrm{~K}$, die Koerzitivfelder $H_{C}$ und die Sättigungsmagnetisierungen $M_{\text {sat }}$ sind im Diagramm gegeben.

\subsubsection{Diskussion}

Insgesamt zeigen die im vorigen Abschnitt gezeigten Resultate eine drastische Änderung der strukturellen, elektrischen und magnetischen Eigenschaften der Heterostrukturen aus $\mathrm{SMO}_{1} / \mathrm{LMO}_{2}$ bei Modifikationen der $\mathrm{Sr} / \mathrm{Mn}$-Stöchiometrie in der ersten Perowskit-Lage. Zunächst soll ausgeschlossen werden, dass es zu einer einfachen Festkörperreaktion auf dem Substrat kommt, bei der sich alle abgeschiedenen Kationen durchmischen. Im Vergleich zu einer stöchiometrischen Probe ergibt sich für die Probe mit $\mathrm{f}(\mathrm{Mn} / \mathrm{Sr})=0.6$ ein Sr-Überschuss von etwa $2.3 \%$. Bei der Herstellung einer gemischten LSMO( $\mathrm{x}=0.33)$-Schicht mit dem konventionellen MAD-Prozess entspricht dies etwa dem dreifachen des Fehlers beim Abwiegen der Präkursoren. Auch für die Probe mit $\mathrm{f}(\mathrm{Mn} / \mathrm{Sr})=1.6$ ist der sich ergebende Mn-Überschuss mit $1.5 \%$ vernachlässigbar klein. Diese kleinen Modifikationen der durchschnittlichen Zusammensetzung können also nicht die Unterschiede der magnetischen und elektrischen Eigenschaften erklären.

Auch die Messungen der in-situ Ellipsometrie sind nicht im Einklang mit einer homogenen Durchmischung der zugeführten Kationen. In Abbildung 7.26 sind die experimentellen Daten von $\Delta^{T}$ (vergleiche Abbildung 7.20 a) ) für die Struktur mit $\mathrm{f}(\mathrm{Mn} / \mathrm{Sr})=1.6$ zusammen mit einer numerischen Simulation gezeigt. Für die Simulation wird eine homogene Verteilung der Sr- und La-Ionen angenommen. Die relativen Anteile der Ionen auf den A-Plätzen bestimmen dann die mittlere Valenz der Mn-Ionen und damit auch den durchschnittlichen Brechungsindex der Heterostruktur in der Simulation. Die berechneten Datenpunkte weichen von den Messdaten ab. Zum einen ist die Oszillationsamplitude in der Simulation deutlich größer als im Experiment. Andererseits verschwinden in der Simulation mit steigendem $i$ die Unterschiede der Werte von $\Delta^{T}$ für die Deposition der 1. und 2. La-Lage.

Das relaxierende Verhalten in den Depositionspausen deutet das Vorhandensein von Reaktionen bzw. Umverteilungen der aufgebrachten Materialien an. Da sich die LSMO(x)-Perowskite aus der Mischlösung im konventionellen MAD-Prozess ergeben, kann man diese PerowskitManganate als die thermodynamisch bevorzugten Produkte der in den Pausen stattfindenden Reaktionen ansehen. Für $\mathrm{f}(\mathrm{Mn} / \mathrm{Sr})=0.6$ sind diese Reaktionen besonders in der ersten Depo- 


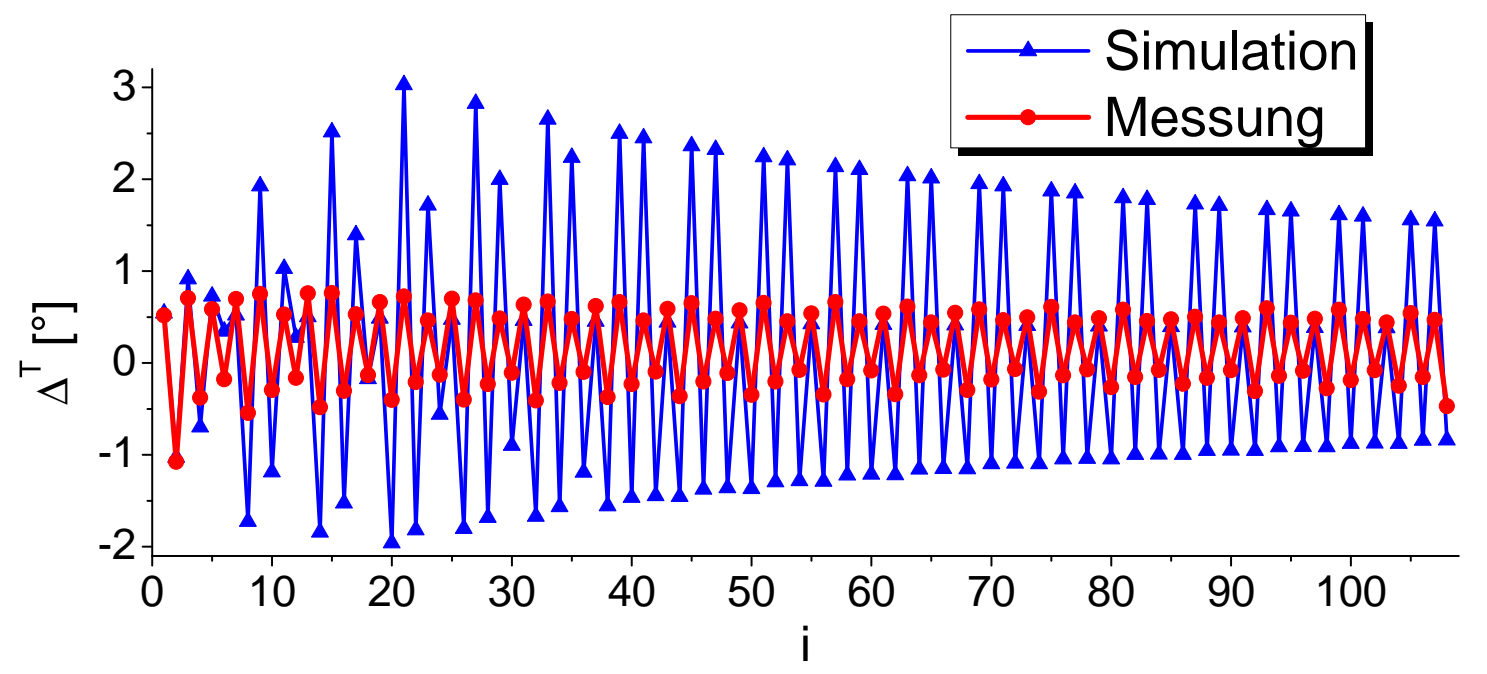

Abbildung 7.26.: Vergleich der Messdaten von $\Delta^{T}$ für die Probe mit $\mathrm{f}(\mathrm{Mn} / \mathrm{Sr})=1.6$ und einer Simulation, bei der man eine homogene Verteilung der $e_{g}$-Elektronen über die gesamte Heterostruktur annimmt.

sitionsphase ( $i \lesssim 20$, vgl. Abbildung 7.15$)$ in den Pausen noch nicht vollständig abgeschlossen. Dies ist daran zu erkennen, dass die Vorzeichen der Änderungen von $\Delta$ in den Pausen für zwei aufeinander folgende Lagen identisch sind (Abbildung 7.15). Für f $(\mathrm{Mn} / \mathrm{Sr})=1.6$ sind die stattfindenden Prozesse in den Pausen weitestgehend abgeschlossen, was man an dem fast waagerechten Verlauf von $\Delta(t)$ am Ende der Pausen erkennen kann (Abbildung 7.16). Auffällig ist, dass man bei $\mathrm{f}(\mathrm{Mn} / \mathrm{Sr})=1.6$ nur Relaxationen in den Pausen nach den Mn-Pulsen sehen kann, also beim Abschluss einer vollen Perowskit-Lage (Die Deposition wurde mit SrO auf einem $\mathrm{TiO}_{2}$-terminierten STO-Substrat begonnen.).

Die nicht abgeschlossene Bildung der vollen Perowskit-Einheitszellen für $\mathrm{f}(\mathrm{Mn} / \mathrm{Sr})=0.6$ bedeutet das Vorhandensein von Blöcken der einfachen Oxide, $\mathrm{SrO}, \mathrm{La}_{2} \mathrm{O}_{3}$ und $\mathrm{Mn}_{3} \mathrm{O}_{4}$ auf der Oberfläche. Aufgrund der strukturellen Unterschiede zur Unterlage mit Perowskit-Struktur kann man von einer verminderten Beweglichkeit dieser Einheiten im Vergleich zu Inseln aus Perowskit-Manganat ausgehen. Daraus resultiert die Bildung von Inseln in der Mitte der vom Substrat vorgegebenen Terrassen (Abbildung 7.21 a)). Auch die magnetischen und elektrischen Eigenschaften werden durch die unvollständige Ausbildung der Perowskit-Einheitszellen beeinträchtigt. Am Anfang der Deposition von der Heterostruktur mit $\mathrm{f}(\mathrm{Mn} / \mathrm{Sr})=0.6$ bildet sich das Manganat aus dreidimensionalen Blöcken der einfachen Oxide. Daraus resultiert eine ungleichmäßige Verteilung der Sr- und La-Kationen auf den A-Plätzen, was zu einer Zunahme der Coulomb-Streuung der $e_{g}$-Elektronen und damit Abnahme von $T_{C}, T_{M I}$ führt.

Nach diesen groben Überlegungen zu den Informationen, die sich aus den Ellipsometriemessungen gewinnen lassen, soll im Folgenden genauer darauf eingegangen werden, wie es zur Ausformung des oszillierenden Verhaltens in $\Delta^{T}(\mathrm{~A}), \Delta^{T}(\mathrm{Mn})$ kommt (siehe Abbildungen 7.18 bis 7.20$)$. Für $\mathrm{f}(\mathrm{Mn} / \mathrm{Sr})=1.6$ sind diese Oszillationen vom Anfang bis zum Ende des Depositionsvorganges in der gleichen Ausprägung vorhanden. Am Ende der Deposition kann man mit dem STM ein zweidimensionales Inselwachstum nachweisen (Abbildung 7.21 b)). Aufgrund der Regelmäßigkeit der in-situ Messungen kann man so auf eine Oberfläche mit zweidimensionalen Inseln bei jedem Abschluss mit $\mathrm{MnO}_{2}$ schließen. Abweichungen von diesem Wachstum würden sich in den Messungen der Ellipsometrie zeigen, wie es bereits in den Abschnitten 4.3.3, 6.1 und 9.3.1 dargelegt wurde. 
Wie bereits zuvor erwähnt wurde, ist nach dem Aufbringen einer $\mathrm{MnO}_{2}$-Lage von der thermodynamisch getriebenen Bildung einer vollen Perowskit-Lage auszugehen. Allerdings kann man den Zustand nach der Deposition der Lagen für den A-Platz aus LaO bzw. SrO nicht mit dieser Argumentation erfassen. SrO passt mit seiner rocksalt-Struktur zum Perowskit-Gitter, wie man es schon im Abschnitt über die STO RP-Strukturen gesehen hat. Theoretische und experimentelle Untersuchungen zeigen die Formation von durchgehenden SrO-Lagen auf Ti-terminierten STO (001) Substraten [239, 240]. Für La ist die Situation allerdings komplizierter, da die LaO-Lage in der Perowskit-Struktur eine formale Ladung von +1 aufweist. Unter der Voraussetzung der Ladungsneutralität der gesamten Heterostruktur kann man zwei verschiedene Szenarien ausmachen, die sich nach der Dosierung der La-Lösung ergeben: Zum einen kann sich das einfache Oxid, $\mathrm{La}_{2} \mathrm{O}_{3}$, bilden. Aufgrund der strukturellen Unterschiede zum Perowskit würde dies in Inseln aufwachsen und dann im folgenden Depositionsschritt mit den deponierten Mn-Ionen reagieren. Alternativ kann sich die geladene Perowskit-Lage, $(\mathrm{LaO})^{+}$, bilden, wobei zum Erhalt der Ladungsneutralität eine Änderung der Ladungen auf den Mn-Ionen stattfinden muss. Für jede $(\mathrm{LaO})^{+}$-Einheit wird im Mittel ein zusätzliches $e_{g}$-Elektron hinzugefügt.

Bei der Betrachtung der $\Delta^{T}$ (A) fällt der Unterschied zwischen der 1. und 2. LaO-Lage auf (siehe Abbildungen 7.19 und 7.20). Diese reproduzierbare Differenz lässt sich nicht durch das einfache Wachstum von $\mathrm{La}_{2} \mathrm{O}_{3}$-Inseln erklären, da man dann für beide La-Pulse die gleiche Änderung erhalten müsste. Es besteht aber die Möglichkeit, dass ein Teil der aufgebrachten La-Atome $\mathrm{La}_{2} \mathrm{O}_{3}$-Inseln bildet und der andere Teil die Oberfläche mit einer $(\mathrm{LaO})^{+}$-Lage benetzt. Tatsächlich beobachteten Achutharaman et al. bei der Herstellung von $\mathrm{La}_{0.67} \mathrm{Ca}_{0.33} \mathrm{MnO}_{3}$-Filmen mit MBE, dass das RHEED-Beugungsbild des wachsenden Manganat-Filmes nach der Deposition der Atome auf den A-Plätzen (La, Ca) unschärfer ist als nach der Deposition der Mn-Atome 241]. Dies würde für die Entstehung von Clustern aus einfachen Oxiden sprechen, die aufgrund der daraus folgenden unvollständigen Bedeckung der Oberfläche zu einer Reduktion der Intensität der Beugungsreflexe führen würden. Hier kann eine quantitative Anpassung der in-situ Messungen genauere Informationen zu den Wachstumsprozessen liefern.

Bei der numerischen Simulation geht man ähnlich vor wie in Abschnitt 9.3.2 Allerdings werden hier die Änderungen der Mn-Valenzen beim Aufbringen halber Perowskit-Lagen betrachtet. Bei der Deposition einer $(\mathrm{SrO})^{0}$-Lage kommt es zu keinen Änderungen der Valenzen der Mn-Ionen. Für die Simulation nimmt man an, dass die dosierte La-Lösung vollständig in eine $(\mathrm{LaO})^{+}$-Schicht umgesetzt wird. Dabei erhöht sich die Zahl der $e_{g}$-Elektronen gemäß der Ladungsneutralität. Die mittlere Zahl der $e_{g}$-Elektronen auf jeder $\mathrm{MnO}_{2}$-Lage, $n\left(e_{g}\right)$, klingt mit wachsender Entfernung $z$ in Wachstumsrichtung exponentiell ab (vergleiche Abschnitt 9.3.2):

$$
n(z)=n_{0} \cdot e^{-|z| / L_{T F}}
$$

Da hier auch halbe Perowskit-Lagen $(\mathrm{SrO}, \mathrm{LaO})$ auf der Oberfläche auftreten, fließen in die Simulation zusätzlich die Brechungsindizes von $\mathrm{SrO}$ und $\mathrm{LaO}, N(\mathrm{SrO})$ und $N(\mathrm{LaO})$, ein. Damit ergeben sich drei freie Parameter, die man durch die Anpassung der Daten von $\Delta^{T}$ für die Probe mit $\mathrm{f}(\mathrm{Mn} / \mathrm{Sr})=1.6$ erhält: $N(\mathrm{SrO})$ und $N(\mathrm{LaO})$ sowie die charakteristische Länge für die Delokalisierung der $e_{g}$-Elektronen, $L_{T F}$. Für SrO kann man den Wert für die bulk-Phase, $N(\mathrm{SrO})=1.862(\lambda=656 \mathrm{~nm})[242$, als Ausgangspunkt für den Fit verwenden. Für die elektrische geladenen $(\mathrm{LaO})^{+}$-Lagen gibt es kein bulk-Äquivalent, so dass man auch vom Wert für SrO ausgeht. 

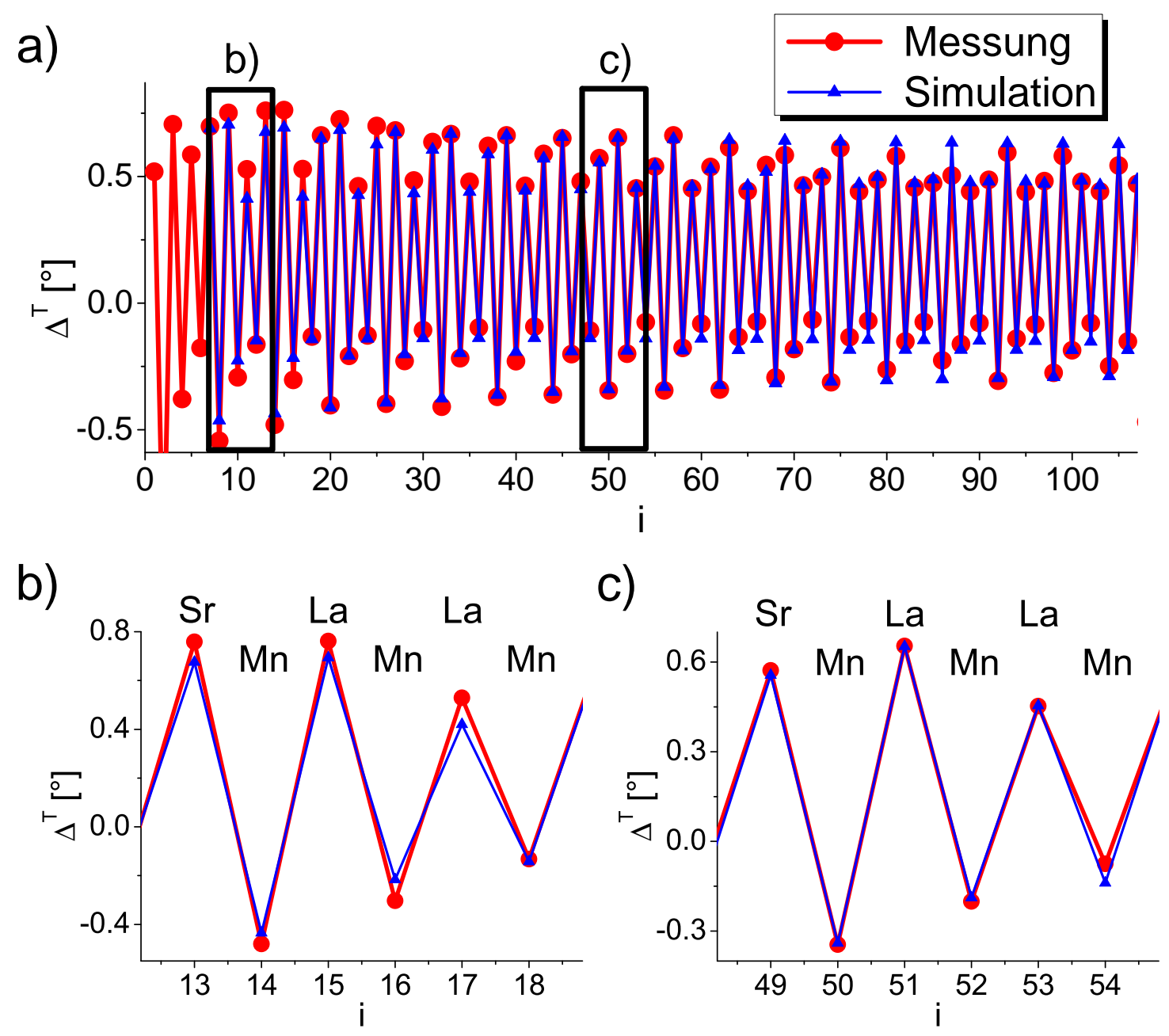

\begin{abstract}
Abbildung 7.27.: Numerische Anpassung der Messdaten für die Probe mit $\mathrm{f}(\mathrm{Mn} / \mathrm{Sr})=1.6$ (Abbildung 7.20): Die Simulation erfolgte gemäß den Erläuterungen im Text und Abbildung 7.28 Die beste Anpassung ergibt sich für die folgende Wahl der freien Parameter: $N(\mathrm{SrO})=$ $1.8(2), N(\mathrm{LaO})=1.9(2)$ und $L_{T F}=0.33(4) \mathrm{nm}$. Innerhalb der jeweiligen Fehlerintervalle verändert sich der Mittelwert über die Beträge der Differenzen zwischen den Messpunkten und der Simulation um den Messfehler von $\Delta^{T}$, welcher bei $\sim 0.02^{\circ}$ liegt. In Teil a) ist eine Übersicht für alle $i=1-108$ dargestellt und in b) und c) sieht man Vergrößerungen der schwarz umrandeten Bereiche in a).
\end{abstract}

In Abbildung 7.27 ist die beste numerische Anpassung der Daten von Abbildung 7.20 a) gezeigt, wobei man die ersten $i=1-6$ Datenpunkte nicht bei der Anpassung berücksichtigt hat. Im Besonderen fällt auf, dass die simulierten Daten die Positionen der lokalen Minima und Maxima richtig reproduzieren. Auch die graduelle Abnahme der Oszillationsamplitude mit der Zahl der deponierten Lagen, $i$, wird korrekt nachgebildet. Für $i=19-102$ liegt die mittlere Abweichung der simulierten Punkte von den Messpunkten bei $\sim 0.029^{\circ}$. Dies kommt dem Messfehler von $\sim 0.02^{\circ}$ für $\Delta^{T}$, welcher sich durch das Rauschen von $\Delta$ und die Zeitabstände zwischen den gemessenen Werten der $\Delta(t)$-Kurve ergibt, sehr nahe.

Abbildung 7.28 zeigt die Zustände der Probenoberfläche bei der Simulation des Wachstums einer $\mathrm{SMO}_{1} / \mathrm{LMO}_{2}$-Einheit. Aufgrund der Periodizität des Übergitters wiederholt sich dieses Schema im Verlauf der Deposition $m=18$-mal. Deutlich erkennt man die Veränderungen der Elektronenverteilung beim Hinzufügen von $\mathrm{MnO}_{2}$ - und LaO-Lagen. Wenn die SrO-Lage 

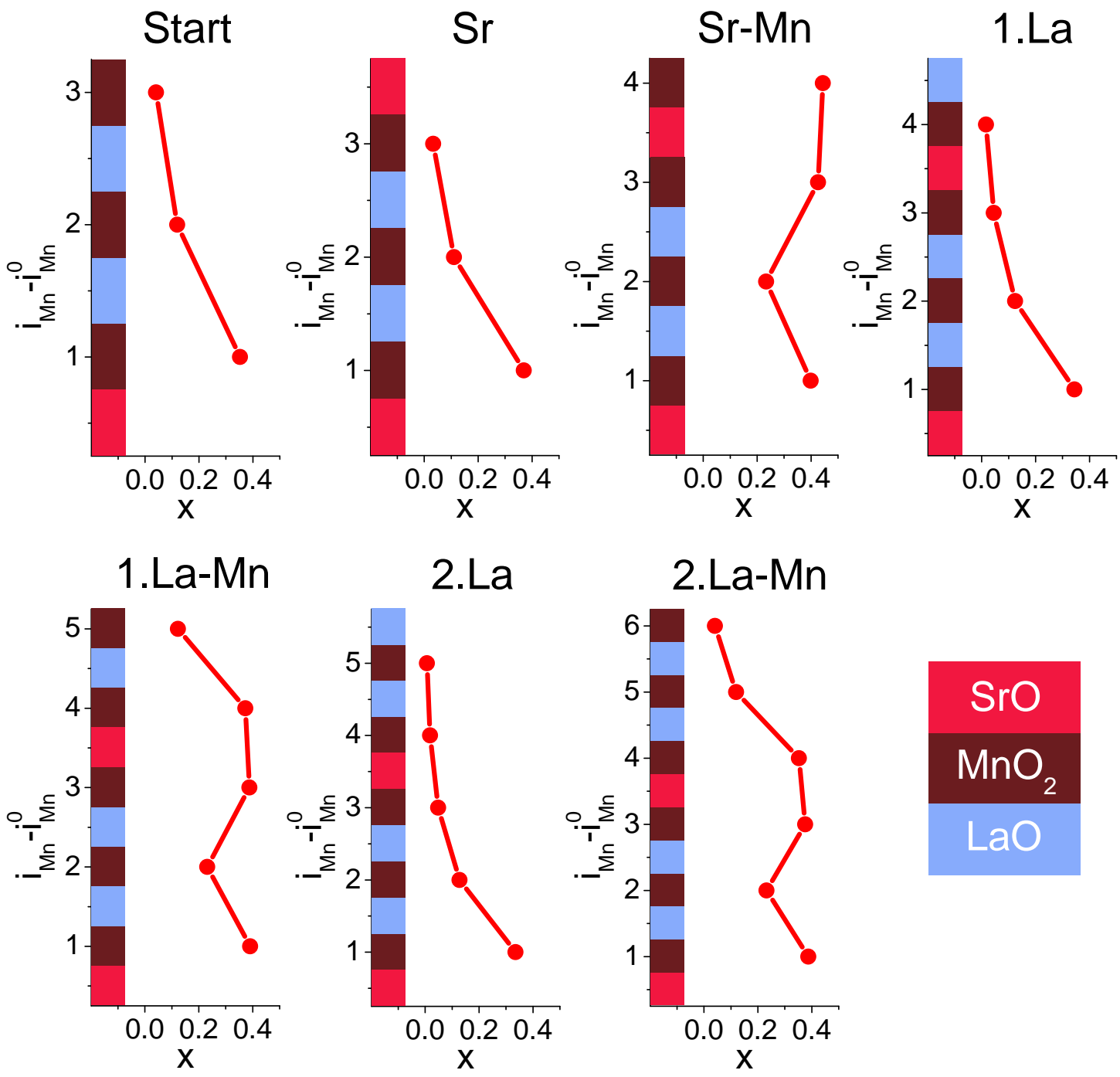

Abbildung 7.28.: Entwicklung der Besetzung der $e_{g}$-Niveaus, $n\left(e_{g}\right)=1-x$, in den $\mathrm{MnO}_{2}$-Lagen bei der Deposition einer $\mathrm{SMO}_{1} / \mathrm{LMO}_{2}$-Einheit mit MAD ALE, die zu der in Abbildung 7.27 gezeigten Simulation von $\Delta^{T}(i)$ führt. Mit den Überschriften der einzelnen Diagramme wird die zuletzt deponierte Lage bezeichnet (vergleiche Abbildung 7.13). Die Delokalisierung der $e_{g}$-Elektronen wird mit Gleichung 7.6 beschrieben, wobei hier $L_{T F}=$ $0.33 \mathrm{~nm}$ verwendet wurde. Mit $i_{M n}$ werden die $\mathrm{MnO}_{2}$-Lagen durchnummeriert und $i_{M n}^{0}$ ist die Zahl der vor $i_{M n}-i_{M n}^{0}=1$ deponierten $\mathrm{MnO}_{2}$-Lagen in der kompletten Heterostruktur.

hinzugefügt wird, erfolgt keine Änderung. Die charakteristische Länge für die Delokalisierung der Elektronen hat bei der besten Anpassung einen Wert von $L_{T F}=0.33(4) \mathrm{nm}$, was den Ergebnissen in Abschnitt 9.3.2 und theoretischen Überlegungen entspricht (siehe Abschnitt 3.124

Insgesamt bestätigen die Simulationen der in-situ Ellipsometrie das Bild eines lagenweisen (layer-by-layer) Wachstums der Probe mit $\mathrm{f}(\mathrm{Mn} / \mathrm{Sr})=1.6$. Speziell für die LaO-Lagen kann eine Bildung des einfachen Oxids, $\mathrm{La}_{2} \mathrm{O}_{3}$, ausgeschlossen werden, da dies sich nicht in der

\footnotetext{
${ }^{24} \mathrm{Im}$ Gegensatz zu den LMO/SMO-SL in Abschnitt 9.3.2 gibt es bei den hier betrachteten $\mathrm{SMO}_{1} / \mathrm{LMO}_{2}$ Heterostrukturen eine Durchmischung der Sr- und La-Ionen, die zur Delokalisierung der $e_{g}$-Elektronen beiträgt. Dadurch ist der hier ermittelte Wert von $L_{T F}$ nicht allein auf elektronische Effekte zurückzuführen, sondern enthält auch Anteile der Durchmischung der Kationen auf den A-Plätzen.
} 


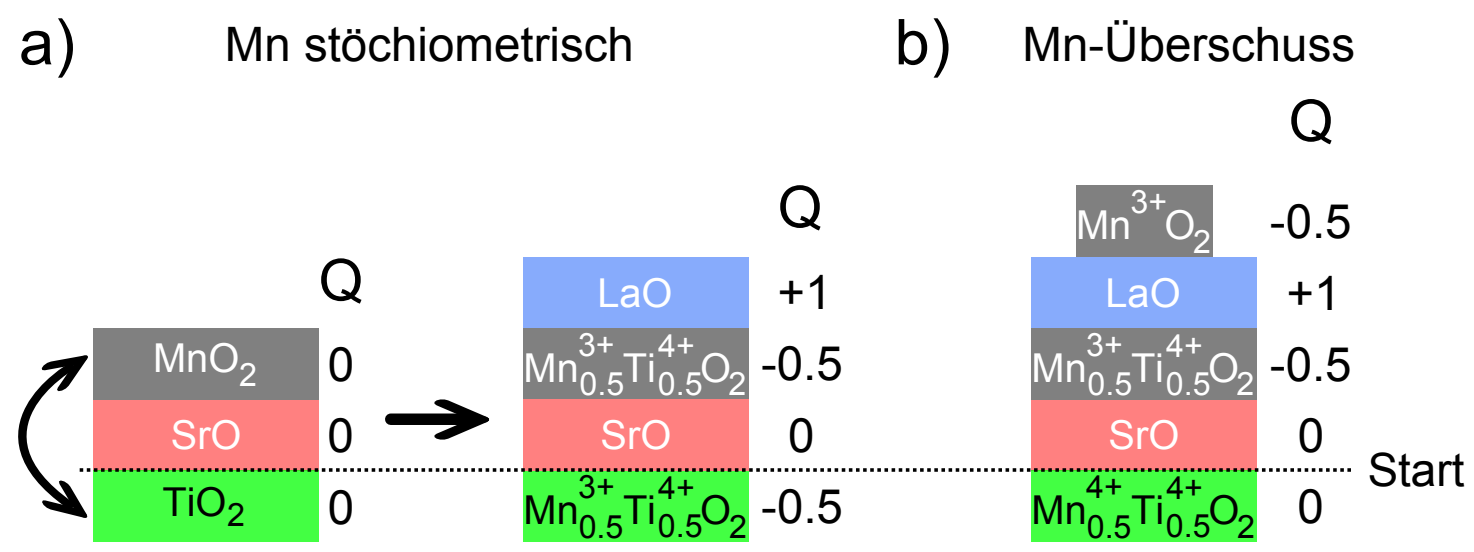

Abbildung 7.29.: Formale Ladungen $Q$ der ersten $i=1-3$ Lagen auf dem Ti-terminiertem STO-Substrat für eine stöchiometrische Menge der Kationen a) und einen Mn-Überschuss von einer halben Lage b). Man nimmt vereinfachend an, dass die Hälfte der Mn-Ionen in das STO diffundiert und auf der ersten $\mathrm{TiO}_{2}$-Lage verbleibt.

Verteilung der $e_{g}$-Elektronen niederschlagen würde. Für die Struktur mit f $(\mathrm{Mn} / \mathrm{Sr})=0.6$ beobachtet man eine reduzierte Oszillationsamplitude in den ersten Phasen der Deposition (Abbildung 7.20 b)). Damit weicht das Wachstum dieser Probe zu Beginn vom lagenweisen Wachstum $\mathrm{ab}$, das sich für $\mathrm{f}(\mathrm{Mn} / \mathrm{Sr})=1.6$ ergibt. Dies wird auch dadurch bestätigt, dass für $\mathrm{f}(\mathrm{Mn} / \mathrm{Sr})=0.6$ der Anteil von $\Delta^{R}(\mathrm{Mn} / \mathrm{A})$ an $\Delta^{T}(\mathrm{Mn} / \mathrm{A})$ deutlich größer ist als für $\mathrm{f}(\mathrm{Mn} / \mathrm{Sr})=1.6$. In der ersten Phase bilden sich also Blöcke der einfachen Oxide auf der Oberfläche. Die Pausenzeiten sind aber zu kurz, um eine vollständige Reaktion zum Perowskit-Manganat zu erreichen. In den späteren Depositionsphasen $(i \gtrsim 55$, vgl. Abbildung 7.20 reicht die Zeit der Pausen aus, damit die Materialien zum thermodynamisch stabilen Perowskit reagieren können. Allerdings sind auch in diesem Abschnitt die Änderungen in den Pausen deutlich ausgeprägter als für $\mathrm{f}(\mathrm{Mn} / \mathrm{Sr})=1.6$. Dies resultiert aus den dreidimensionalen Inseln auf der Oberfläche, die die Mobilität der Adatome einschränken.

Nach den obigen Betrachtungen kann man annehmen, dass für $\mathrm{f}(\mathrm{Mn} / \mathrm{Sr})=1.6$ das lagenweise Wachstum über den gesamten Depositionsprozess besteht. Aus der Betrachtung der Anteile von $\Delta^{R}(\mathrm{Mn} / \mathrm{A})$ an $\Delta^{T}(\mathrm{Mn} / \mathrm{A})$ kann man dann schließen, dass sich die AO-Lagen während der Dosierung der Präkursoren ausbilden, wogegen dies für die $\mathrm{MnO}_{2}$-Lagen zuerst nur teilweise geschieht. In den Pausen nach den Mn-Pulsen verteilen sich die Mn-Ionen dann komplett über die Oberfläche. Die Oberflächendiffusion ist für die La- und Sr-Ionen also wesentlich schneller als für die Mn-Ionen.

Nachdem man aus den Beobachtungen der in-situ Ellipsometrie eine Vorstellung der Wachstumsprozesse entwickelt hat, soll diskutiert werden, wie die Änderungen der Stöchiometrie der ersten Lagen das anschließende Wachstum bestimmen können. Dazu berücksichtigt man die formalen Ladungen der individuellen Lagen der wachsenden Heterostruktur. Die hier hergestellten Übergitter beginnen mit einer $\mathrm{SrMnO}_{3}$-Lage. Damit wird ein Sprung im elektrostatischen Potential an der Grenzfläche zwischen Manganat und Titanat vermieden [106. Würde man im ALE-Verfahren mit einer $\mathrm{LaMnO}_{3}$-Lage beginnen, ergäbe sich außerdem eine weitere Problematik: Beim Aufbringen der La-Ionen auf das Ti-terminierte Substrat müsste es bei der Bildung einer $(\mathrm{LaO})^{+}$-Lage zur Bildung von $\mathrm{Ti}^{3+}$-Ionen kommen, um die Ladungsneutralität zu erhalten. Da bei den Wachstumsbedingungen der MAD eine $\mathrm{Ti}^{4+}$-Valenz bevorzugt wird, ergibt sich eine treibende Kraft zur Bildung von $\mathrm{La}_{2} \mathrm{O}_{3}$-Blöcken statt einer $(\mathrm{LaO})^{+}$-Lage. 
In der Tat zeigen Experimente von Felix Massel, dass das Wachstum von $\mathrm{LaMnO}_{3}$-Filmen mit MAD ALE instabil ist und eher einer Festkörperreaktion aller aufgebrachten Atome entspricht 243 .

Wie in Abschnitt 6.3 gezeigt wurde, kommt es beim Wachstum von Manganaten auf STOSubstraten zu einer Durchmischung der Kationen. Für die erste $\mathrm{SrO}$ - und $\mathrm{MnO}_{2}$-Lage ist diese Durchmischung nicht kritisch, da beide aufgebrachten Lagen wie die Lagen im STO-Substrat elektrisch neutral sind. Beim Aufbringen der folgenden $(\mathrm{LaO})^{+}$-Lage kommt es dann aber zum Aufbau von Coulomb-Potentialen, wenn Ti die thermodynamisch bevorzugte Valenz von 4+ behält (Abbildung 7.29 a)). Für die Probe mit $\mathrm{f}(\mathrm{Mn} / \mathrm{Sr})=0.6$ folgt daraus eine treibende Kraft zum Wachstum von $\mathrm{La}_{2} \mathrm{O}_{3}$-Inseln statt einer $(\mathrm{LaO})^{+}$-Lage. Des Weiteren kann der SrÜberschuss durch den Einbau von RP-Defekten die Mobilität der Adatome herabsetzen. Für $\mathrm{f}(\mathrm{Mn} / \mathrm{Sr})=1.6$ verhindern die zusätzlichen Mn-Ionen den Aufbau von Coulomb-Potentialen beim Wachstum der LaO-Lage, da die zusätzlichen $\mathrm{Mn}^{3+}$-Ionen die Ladungsneutralität herstellen (Abbildung 7.29 b) ${ }^{25}$.

\subsection{Zusammenfassung}

In diesem Kapitel wurden die ersten Experimente zur Atomlagenepitaxie (ALE) mit MAD präsentiert. Entlang der (001)-Richtung lassen sich die Perowskite $\mathrm{ABO}_{3}$ in Lagen von $\mathrm{AO}$ und $\mathrm{BO}_{2}$ zerlegen. Im Gegensatz zum konventionellen MAD-Prozess werden bei der ALE die Kationen auf den A- und B-Plätzen aus getrennten Präkursorlösungen auf das Substrat gebracht, wodurch man dieser Zerlegung in $\mathrm{AO}-$ und $\mathrm{BO}_{2}$-Lagen entspricht. Hier führt man dieses Prinzip der MAD ALE am Beispiel von Strukturen aus der $\mathrm{SrTiO}_{3}$-SrO RuddlesdenPopper-Phase, $\mathrm{Sr}_{\mathrm{n}+1} \mathrm{Ti}_{\mathrm{n}} \mathrm{O}_{3 \mathrm{n}+1}(n=2-4)$, und Manganat-Strukturen aus $\mathrm{SMO}_{1} / \mathrm{LMO}_{2}$ vor.

Mit MAD ALE wurden STO RP-Strukturen mit $n=2-4$ auf STO (001)-Substrate deponiert. Hochaufösende TEM-Aufnahmen und Rötgenbeugung zeigen, dass die angestrebte SrO-STO-Abfolge die Struktur der jeweiligen Probe dominiert. Die in-situ Ellipsometrie erlaubt die Ermittlung der korrekten Sr/Ti-Stöchiometrie mit einer Genauigkeit von 1.5\%. Für $n=4$ kann man die Messungen der in-situ Ellipsometrie mit der in den TEM-Aufnahmen sichtbaren Defektverteilung korrelieren. In der Nähe zum STO-Substrat befinden sich zusätzliche horizontale und vertikale SrO-Lagen, deren Zahl sich nach 5-6 Wiederholungen der $\mathrm{SrO} /\left(\mathrm{SrTiO}_{3}\right)_{4}$-Einheit deutlich verringert. Diese Defekte werden mit der Diffusion von SrOLagen in die Oberfläche des Substrates begründet.

Bei den Heterostrukturen aus $m=18$ Wiederholungen von $\mathrm{SMO}_{1} / \mathrm{LMO}_{2}$ wurde untersucht, wie sich das Wachstum verändert, wenn man die Stöchiometrie der ersten Perowskit-Lage modifiziert. Dabei betrachtet man insbesondere zwei Proben: Für die erste Probe verwendet man in der ersten SrO-Lage das 1.5-fache und für die zweite Probe in der ersten $\mathrm{MnO}_{2}$-Lage das 1.6-fache der stöchiometrischen Menge. In den restlichen Lagen werden die stöchiometrischen Mengen für Sr, La und Mn verwendet. Die Probe mit Mn-Überschuss zeigt ein zweidimensionales Wachstum und die magnetischen und elektrischen Eigenschaften entsprechen denen, die sich für vergleichbare Proben mit dem konventionellen MAD-Prozess ergeben. Beim SrÜberschuss bilden sich dreidimensionale Inseln und die Übergangstemperaturen, $T_{C}$ und $T_{M I}$,

\footnotetext{
${ }^{25}$ Beim Aufbringen der La-Ionen muss es zu einer Umverteilung der Ti- und Mn-Ionen kommen, so dass ein lokaler Ladungsausgleich erreicht wird. Damit kann es im Gegensatz zur vereinfacht gezeichneten Situation in Abbildung 7.29 b) dazu kommen, dass Ti-Ionen an die Oberfläche der Struktur gelangen.
} 
sind reduziert. Die numerische Anpassung der in-situ Ellipsometrie zeigt die Änderung der Mn-Valenzen mit jeder aufgebrachten Lage. Damit wird für die Probe mit Mn-Überschuss belegt, dass die aufgebrachten Sr-, La- und Mn-Ionen lagenweise aufwachsen. Für die Probe mit Sr-Überschuss weist die Zeitabhängigkeit der Ellipsometriemessungen in den Depositionspausen darauf hin, dass sich die Perowskit-Struktur durch eine Festkörperreaktion der einfachen Oxide ergibt. Den starken Kontrast zwischen den beiden behandelten Proben begründet man mit elektrostatischen Potentialen, die sich bei der Probe ohne Mn-Überschuss aufgrund der Ti/Mn-Durchmischung mit dem STO-Substrat aufbauen. Bei der anderen Probe wird dies durch die zusätzlichen Mn-Ionen vermieden. 
$\prod_{\text {Kapitel }} \mathbf{Q}$

\section{Exchange bias in $\mathrm{La}_{0.7} \mathrm{Sr}_{0.3} \mathrm{MnO}_{3} / \mathrm{La}_{1-\mathrm{x}} \mathrm{Sr}_{\mathrm{x}} \mathrm{MnO}_{3}(\mathrm{x}=0.6-1.0)$ Heterostrukturen}

\subsection{Motivation}

In der Theorie wurde exchange bias (EB) als Kopplungsphänomen an der Grenzfläche zwischen Ferromagneten (FM) und Antiferromagneten (AFM) vorgestellt, wo frustrierte Austauschwechselwirkungen in der Grenzregion für die Modifikation der magnetischen Hysterese verantwortlich sind. Wenn man Grenzflächen zwischen komplexen Oxiden betrachtet, ist die magnetische Kopplung von Materialien mit verschiedenen Typen magnetischer Ordnung nur ein Teilaspekt der auftretenden Effekte. Vor den magnetischen Kopplungen sind die Veränderungen der chemischen Zusammensetzung und der Ladungen der Übergangsmetallionen zu berücksichtigen. Diese haben einen großen Einfluss auf die magnetische Ordnung von Übergangsmetallperowskiten (siehe z.B. Abbildung 2.5). Zudem ist bei 3d-Übergangsmetallionen der orbitale Freiheitsgrad von großer Bedeutung. Durch epitaktische Verspannungen bewirkte orbitale Polarisationen können Veränderungen der magnetischen Kopplungen an Grenzflächen auftreten (siehe Abschnitt 3.2).

Es gibt eine Vielzahl von Perowskit-Oxiden mit verschiedenen Ausprägungen magnetischer Ordnung. Aufgrund ihrer ähnlichen Struktur kann man aus diesen Materialien qualitativ hochwertige Heterostrukturen mit scharfen Grenzflächen herstellen. Experimentell wird so exchange bias für diverse Materialkombinationen von Oxiden, die ferromagnetisch und antiferromagnetisch ordnen, beobachtet: $\mathrm{SrRuO}_{3} / \mathrm{SrMnO}_{3}$ 244, $\mathrm{La}_{0.7} \mathrm{Sr}_{0.3} \mathrm{MnO}_{3} / \mathrm{BiFeO}_{3}$ 245247] und $\mathrm{La}_{2 / 3} \mathrm{Ca}_{1 / 3} \mathrm{MnO}_{3} / \mathrm{La}_{1 / 3} \mathrm{Ca}_{2 / 3} \mathrm{MnO}_{3} 248$ 250. Zusätzlich gibt es Fälle, in denen ferromagnetische Materialien intrinsisch oder unter Kombination mit Materialien ohne kollektive magnetische Ordnung exchange bias aufweisen: Bei der Kombination von $\mathrm{La}_{0.7} \mathrm{Sr}_{0.3} \mathrm{MnO}_{3}$ mit dem diamagnetischen Isolator $\mathrm{SrTiO}_{3}$ wird das EB mit dem Einbau von Sauerstoffleerstellen, die zusammen mit epitaktischer Verspannung zu einem antiferromagnetischen Grundzustand der Manganat-Lagen in der Nähe der Grenzflächen führen [50, 251, begründet. Das EB, das bei der Kombination des ferromagnetischen Isolators $\mathrm{LaMnO}_{3-\delta}$ mit dem paramagneti-

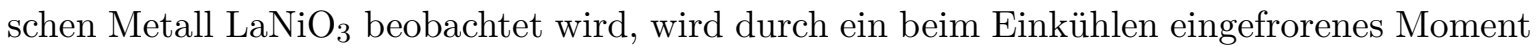


a)

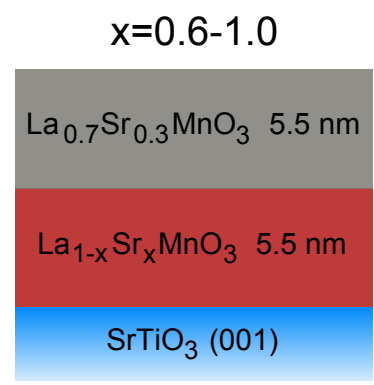

b)

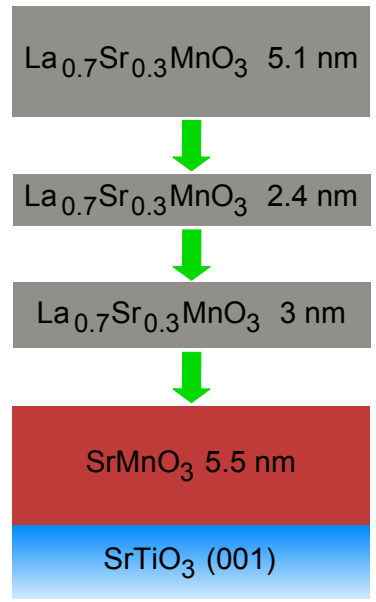

c)

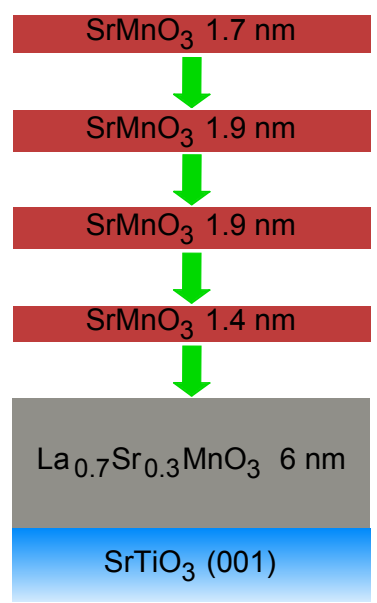

Abbildung 8.1.: Darstellung der studierten Heterostrukturen: a) Doppellagen aus $\mathrm{La}_{0.7} \mathrm{Sr}_{0.3} \mathrm{MnO}_{3}$ (LSMO) und $\mathrm{La}_{1-\mathrm{x}} \mathrm{Sr}_{\mathrm{x}} \mathrm{MnO}_{3}$ mit $x=0.6-1.0$ (LSMO(x)). Beide Lagen besitzen eine gleiche Schichtdicke von $5.5 \mathrm{~nm}$. b) Auf ein $\mathrm{SrTiO}_{3}$ (STO) (001)-Substrat wird zunächst eine $\mathrm{SrMnO}_{3}(\mathrm{SMO})-\operatorname{Schicht}\left(d_{S M O} \approx 5.5 \mathrm{~nm}\right)$ aufgebracht. Dann werden auf dieselbe Probe nach und nach LSMO-Lagen mit den in der Abbildung gegebenen Schichtdicken aufgebracht. Damit erhält man eine Serie von SMO/LSMO-Doppellagen mit steigender Schichtdicke $d_{L S M O}$ der LSMO-Lage. c) Analog zu b) wird auf ein STO (001)-Substrat zunächst eine LSMO-Schicht $\left(d_{L S M O} \approx 6 \mathrm{~nm}\right)$ deponiert, woraufhin nach und nach SMOSchichten deponiert werden, so dass eine Serie von LSMO/SMO-Bilagen mit steigender Dicke $d_{S M O}$ der SMO-Lage entsteht.

auf den Ni-Ionen begründet 252,253 . Insgesamt wird das EB in den zuvor aufgeführten Heterostrukturen durch eine eingefrorene Spinkonfiguration mit nichtverschwindender Nettomagnetisierung, welche sich durch die Frustration der FM- und AFM- Wechselwirkungen an der Grenzfläche einstellt, in Verbindung gebracht. Zum Teil manifestieren sich diese magnetischen Momente in einer Verschiebung der $M(H)$-Kurven entlang der Magnetisierungsachse 244, 251. Es gibt aber auch theoretische Überlegungen, die dem speziellen Charakter der Bindungen an den Grenzflächen zwischen komplexen Oxiden Rechnung tragen. Bei einer nicht-kollinearen Ausrichtung der Spins an der Grenzfläche zwischen einem Antiferromagneten des G-Typs und einem Ferromagneten wird EB auf Basis der Dzyaloshinskii-MoriyaWechselwirkung vorhergesagt 254].

Hier wird die Kopplung zwischen dem ferromagnetischen Metall $\mathrm{La}_{0.7} \mathrm{Sr}_{0.3} \mathrm{MnO}_{3}$ (LSMO, $T_{C}=370 \mathrm{~K}\left[38 \mid\right.$ ) und dem Isolator $\mathrm{SrMnO}_{3}$ (SMO) mit einer antiferromagnetischen Ordnung des G-Typs $\left.\left(T_{N}=260 \mathrm{~K}, 40\right]\right)$ betrachtet. Im Rahmen dieser Arbeit wurden Untersuchungen an LSMO/SMO/LSMO-Trilagen vorgenommen, welche in 255] publiziert wurden. Daran anschließend wurden die in diesem Kapitel dargestellten Studien vorgenommen, um die Ursachen der beobachteten Phänomenologie des EB aufzuklären. Die betrachteten Doppellagen wurden ausnahmslos auf $\mathrm{SrTiO}_{3}$ (STO) mit einem Schnitt entlang der (001)-Ebene deponiert. Auf Substraten mit anderen Orientierungen, z.B. (111), zeigt SMO eine raue Morphologie (vergleiche Kapitel 9), so dass es nicht möglich ist einen systematischen Vergleich für verschiedene Grenzebenen vorzunehmen. Der Einfluss des Typs der antiferromagnetischen Ordnung wird mit Bilagen aus LSMO und $\mathrm{La}_{1-\mathrm{x}} \mathrm{Sr}_{\mathrm{x}} \mathrm{MnO}_{3}$ festgestellt (siehe Abbildung 8.1 a)). Durch die Variation der Sr-Dotierung von $x=0.6-1.0 \mathrm{kann}$ man für das bulk-Material Ordnungen des A-, C- und G-Typs einstellen (siehe Abbildung 2.5). Indem man getrennt die 
a)

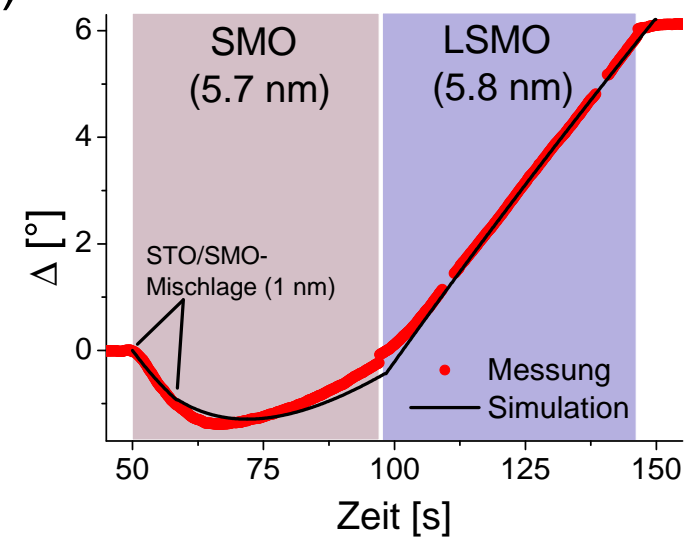

b)

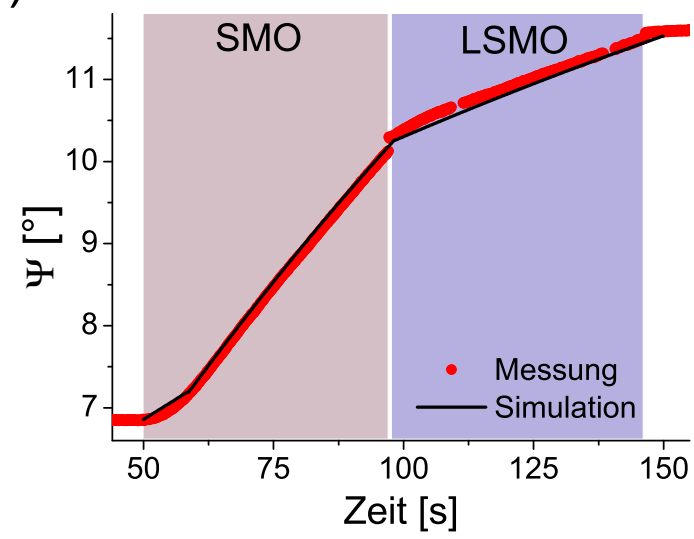

Abbildung 8.2.: In-situ Ellipsometrie während des Wachstums einer SMO/LSMO-Bilage auf einem STO (001)-Substrat. Neben den gemessenen Kurven von $\Delta(t)$ in a) und $\Psi(t)$ in b) ist auch ein numerisch berechneter Verlauf gezeigt, aus dem sich die Schichtdicken $d_{S M O}=5.7 \mathrm{~nm}$ für SMO und $d_{L S M O}=5.8 \mathrm{~nm}$ für LSMO ergeben (Die Depositionspause zwischen LSMO und SMO wurde zur besseren Übersicht entfernt).

Dicke des ferromagnetischen LSMO und antiferromagnetischen SMO variiert, kann man die Abhängigkeit des EB vom Verspannungszustand der Schichten und den Einfluss der magnetischen Anisotropie des Antiferromagneten abschätzen (siehe Abbildung 8.1 b) und c)). Da die verwendeten Substrate keinen festen Fehlschnitt aufweisen und die magnetischen Eigenschaften durch Stärke und Ausrichtung des Fehlschnitts beeinflusst werden [256, deponiert man diese Doppellagen jeweils auf denselben Substraten und erhöht sukzessive die Dicke von LSMO bzw. SMO (vergleiche Abbildung 8.1 b) und c)).

Zunächst wird die Herstellung der untersuchten Heterostrukturen beschrieben und die Prozesse beim Wachstum diskutiert. Bei der Charakterisierung wird ein Schwerpunkt auf die chemische Qualität der Grenzflächen gelegt, da diese obligatorisch für die Interpretation von Grenzflächeneffekten ist. Außerdem wird der Verspannungszustand als Funktion der Schichtdicken analysiert. Aus den Daten der in-situ Ellipsometrie werden Rückschlüsse auf die Umverteilung der Ladungen auf den Mn-Ionen beim Wachstum gezogen. Im darauf folgenden Abschnitt wird die Phänomenologie des bei den hergestellten Proben erscheinenden EB aufgeführt. Mit Blick auf diese experimentellen Daten wird schließlich ein Bild für den magnetischen Zustand der Grenzfläche entwickelt und die beobachtete Phänomenologie des EB begründet.

\subsection{Probenherstellung und Charakterisierung}

\subsubsection{In-situ Ellipsometrie}

Die Heterostrukturen wurden auf unbehandelten STO (001)-Substraten bei einer Temperatur von $T_{\text {Dep }}=930^{\circ} \mathrm{C}$ und mit einer Rate von $p_{\text {Dep }} \approx 7 \mathrm{~nm} / \mathrm{min}$ deponiert. Die richtige Stöchiometrie der Präkursoren für die $\operatorname{LSMO}(\mathrm{x})$-Filme ergab sich durch die Mischung der Zusammensetzungen für SMO- und LMO-Filme. Außerdem wurde angenommen, dass der Übertrag von der Präkursorlösung in die gewachsene Schicht proportional zur Zahl der deponierten Mn-Atome ist und damit nicht von der Sr-Dotierung abhängt. 
a)

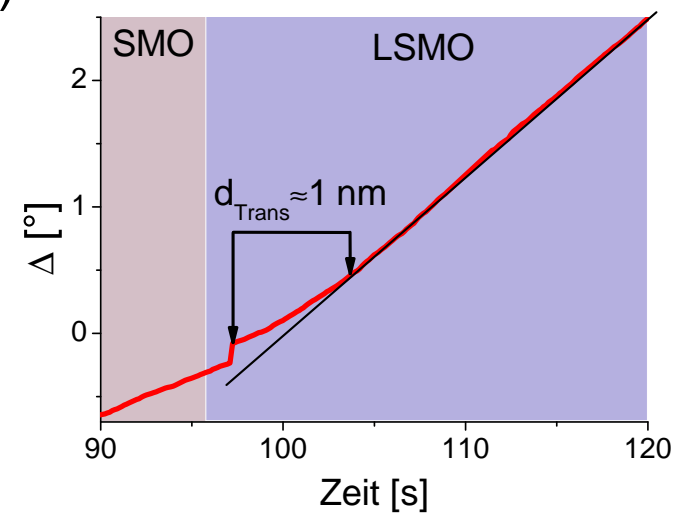

b)

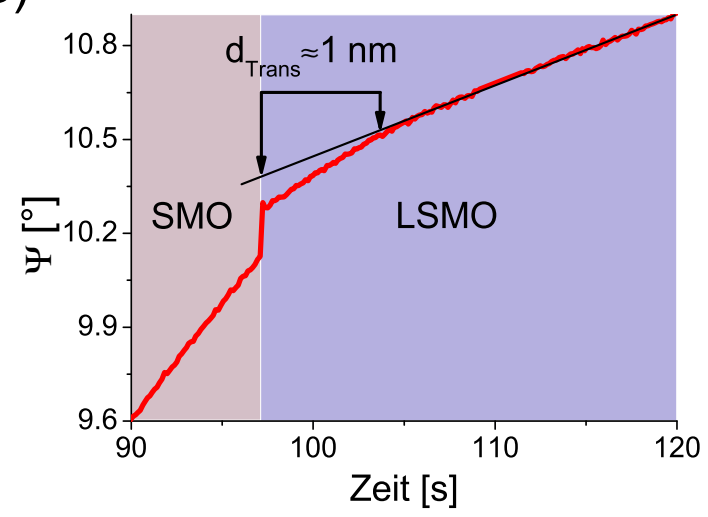

Abbildung 8.3.: Vergrößerte Darstellungen von $\Delta(t)$ und $\Psi(t)$ aus Abbildung 8.2 zeigen eine Übergangsregion $d_{\text {Trans }} \approx 1 \mathrm{~nm}$ zu Beginn des Wachstums von LSMO.

In Abbildung 8.2 ist die in-situ Ellipsometrie der Deposition einer SMO/LSMO ${ }^{26}$ Doppellage gezeichnet. Für die dazu angefertigte numerische Simulation von $\Delta(t)$ und $\Psi(t)$ wurden die Brechungsindizes von LSMO und SMO verwendet (siehe Abbildung 6.4) und angenommen, dass zu Beginn eine SMO-STO-Mischlage mit einem Brechungsindex $N_{M i x}=2.47-i \cdot 0.25$ und einer Dicke $d_{M i x}=1 \mathrm{~nm}$ aufwächst. Die sich dadurch ergebenden Werte für die Schichtdicken $d_{S M O}=5.7 \mathrm{~nm}$ für $\mathrm{SMO}$ und $d_{L M S O}=5.8 \mathrm{~nm}$ für LSMO stimmen gut mit den Werten überein, die sich nach der Deposition durch XRR ergeben: $d_{L S M O}=5.8 \mathrm{~nm}$ und $d_{S M O}=5.6 \mathrm{~nm}$. Bei genauerer Betrachtung der Deposition zu Beginn der LSMO-Lage fällt eine Übergangsregion $d_{\text {Trans }} \approx 1 \mathrm{~nm}$, also etwa $2-3$ Monolagen (ML) LSMO, auf, in dem die simulierten Kurven von $\Delta(t)$ und $\Psi(t)$ von den gemessenen Kurven abweichen (Abbildung 8.3). Dies ist nicht mit einer einfachen Veränderung der Depositionsrate zu Beginn der LSMO-Lage zu erklären, da die Steigung von $\Delta(t)$ kleiner und von $\Psi(t)$ größer ist als später. Auch bei einer unterbrochenen Deposition desselben Materials ergibt sich keine Änderung der Steigung von $\Delta(t)$ oder $\Psi(t)$, wenn ein weiterer Abschnitt der Schicht deponiert wird (vergleiche Abschnitt 4.4.5). Somit zeigt die Messung eine Reaktion der beiden unterschiedlichen Materialien an der Grenzfläche. Die Übergangsregion ist auch für die anderen Sr-Dotierungen $x=1.0-0.6$ in den LSMO(x)/LSMO-Bilagen (vgl. Abbildung 8.1 a)) zu beobachten. Die Breite $d_{\text {Trans }}$ ist dabei bis $x=0.8$ weiter im Bereich von $2-3$ ML. Für kleinere Sr-Dotierungen verkleinert sich die Breite der Übergangsregion, was allerdings mit den zu kleinen Unterschieden zwischen den Brechungsindizes von LSMO und LSMO(x) für $x \leq 0.7$ zu erklären ist. Eine genauere Betrachtung dieser Übergangsregion erfolgt weiter unten, nachdem die chemische Qualität der Grenzfläche zwischen den Manganaten qualifiziert wurde.

\subsubsection{Oberflächenmorphologie}

Durch die Betrachtung der Oberflächenmorphologien nach der Deposition kann man auf die vorherrschende Wachstumsmode schließen. Wie die Abbildungen 8.4 bis 8.6 zeigen, wachsen die LSMO- und LSMO(x)-Filme mit einer Mode zwischen zweidimensionalem Inselwachstum und step-flow-Wachstum. Die sichtbaren Stufen in den STM-Aufnahmen weisen innerhalb der experimentellen Unsicherheit die Höhe einer Monolage $\Delta z=0.4(2) \mathrm{nm}$ auf. In Abbil-

\footnotetext{
${ }^{26}$ Eine entsprechende Untersuchung der LSMO/SMO-Grenzfläche ist hier nicht möglich, da die SMO-Lagen der hier betrachteten LSMO/SMO-Doppellagen nicht an einem Stück deponiert wurden (vergleiche Abbildung 8.1 c)). Diese mehrfachen Depositionsvorgänge verhindern eine genaue Analyse der in-situ Ellipsometrie.
} 
a)

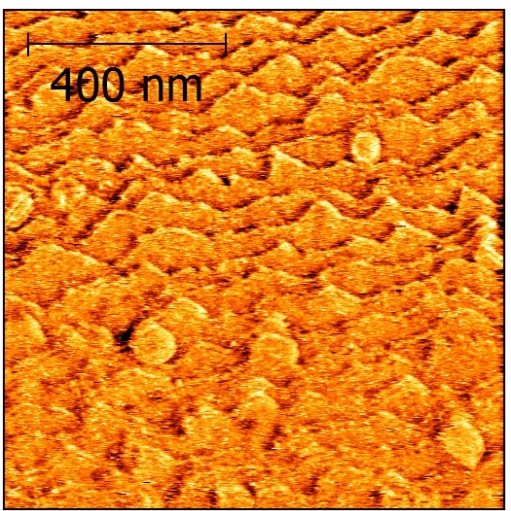

c)

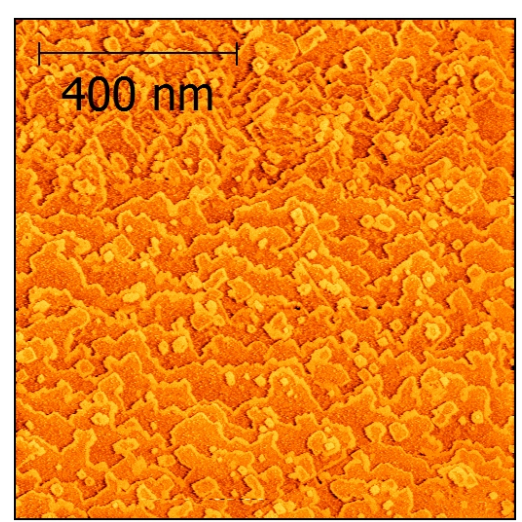

b)
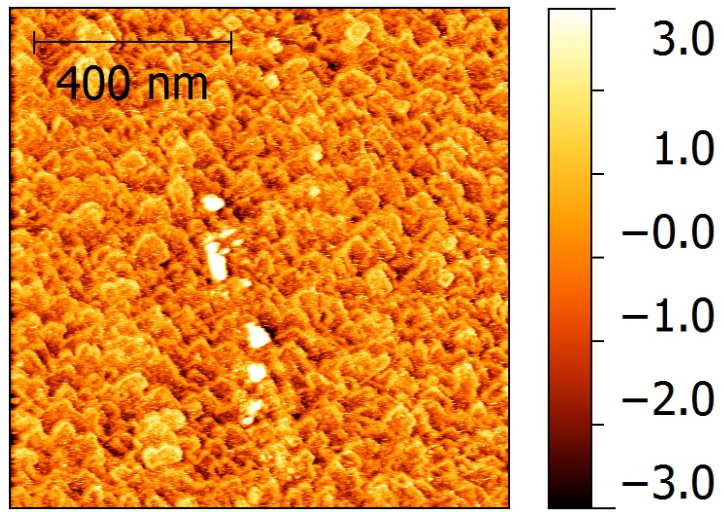

d)
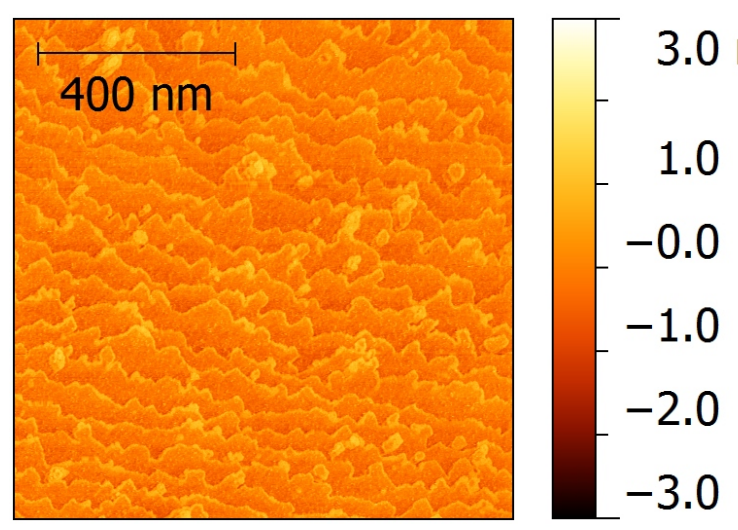

1.0

$-0.0$

$-1.0$

$-2.0$

$-3.0$

$3.0 \mathrm{~nm}$

Abbildung 8.4.: Mit STM gemessene Oberflächenmorphologien der SMO $\left(d_{S M O} \approx\right.$ $5.5 \mathrm{~nm}) /$ LSMO $\left(d_{L S M O}\right)$-Heterostrukturen für verschiedene Werte von $d_{L S M O}$ (vgl. Abbildung 8.1 b)): a) $d_{L S M O}=0 \mathrm{~nm}$, b) $d_{L S M O}=3 \mathrm{~nm}$, c) $d_{L S M O}=5.4 \mathrm{~nm}$, d) $d_{L S M O}=$ $10.5 \mathrm{~nm}$.

dung 8.4 a) bis d) kann man verfolgen, dass sich die zu Beginn bestehende step-flow-Mode des SMO-Films auch beim darauf wachsenden LSMO-Film fortsetzt, wobei die Stufenbreite unverändert bleibt, es kommt also nicht zu step-bunching, bei dem sich mehrere Terrassen vereinigen und Stufen mit dem Vielfachen einer Gitterkonstanten entstehen. Bei der Serie von LSMO $\left(d_{L S M O} \approx 6 \mathrm{~nm}\right) / \mathrm{SMO}\left(d_{S M O}\right)$-Heterostrukturen wächst der LSMO-Film zunächst mit sehr kleinen Inseln zweidimensional auf (siehe Abbildung 8.5 a)), was auf eine geringe Mobilität der Adatome auf den Terrassen schließen lässt27. Wird auf der LSMO-Schicht dann SMO deponiert, so ergibt sich eine step-flow-Mode (Abbildung 8.5 b)-d)). Dies legt nahe, dass die Mobilität für der Adatome auf SMO deutlich größer ist als für LSMO. Durch das Vorhandensein von zufällig verteilten Kationen auf den A-Plätzen in LSMO ergeben sich aufgrund der Coulomb-Energie für die Adatome komplizierte Energielandschaften mit lokalen Minima, wodurch die Bewegung behindert wird. In SMO ist dies nicht der Fall, so dass die Adatome schnell genug zu den Kanten der Stufen diffundieren können. Bei der dicksten SMOSchicht erkennt man das Auftreten von senkrecht zueinander verlaufenden Spannungsrissen und einen beginnenden Übergang zum Inselwachstum, das zur Relaxation der aufgebauten epitaktischen Verspannungen dient (Abbildung 8.5 d)).

\footnotetext{
${ }^{27}$ Für die in Abbildung 8.5 gezeigte Probenserie ist die Breite der durch das Substrat induzierten Stufen mit etwa $80 \mathrm{~nm}$ deutlich größer als für die in Abbildung 8.4 gezeigte Probe, wo die Stufenbreite $50 \mathrm{~nm}$ beträgt. Bei den breiteren Stufen ist die step-flow-Mode empfindlicher auf eine Verringerung der Mobilität der Adatome auf den Stufen.
} 
a)

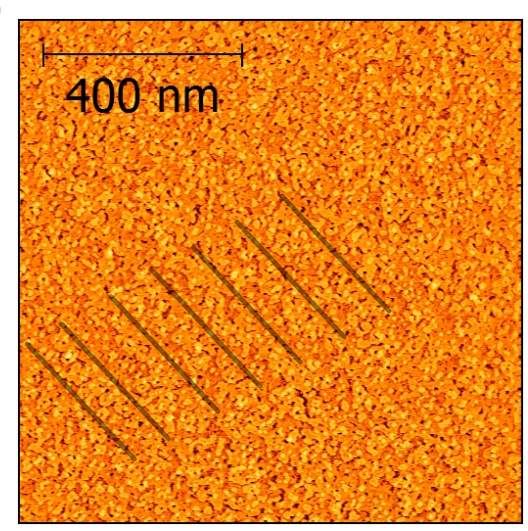

c)

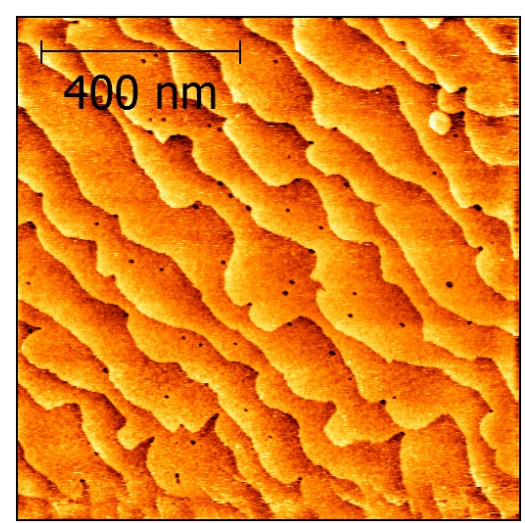

b)

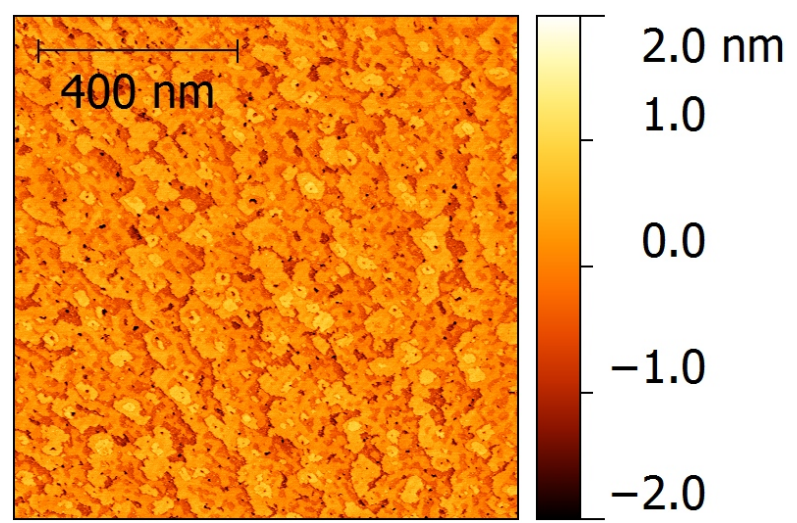

d)

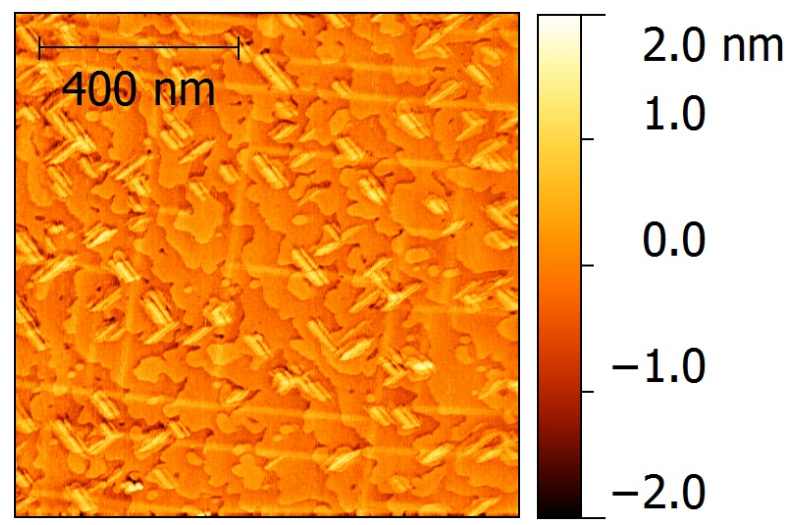

Abbildung 8.5.: Mit STM gemessene Oberflächenmorphologien der $\operatorname{LSMO}\left(d_{L S M O} \approx\right.$ $6 \mathrm{~nm}) / \mathrm{SMO}\left(d_{S M O}\right)$-Heterostrukturen für verschiedene $d_{S M O}(\operatorname{vgl}$. Abbildung 8.1 c) $\left.): \mathrm{a}\right)$ $d_{S M O}=0 \mathrm{~nm}$ (Durch die kleinen Inseln wird die Identifikation der Substratstufen erschwert. Deshalb sind zur Orientierung Linien parallel zu den Stufenkanten eingezeichnet.), b) $d_{S M O}=1.4 \mathrm{~nm}$, c) $d_{S M O}=5.2 \mathrm{~nm}$, d) $d_{S M O}=6.9 \mathrm{~nm}$.

\subsubsection{Schärfe der Grenzflächen: Röntgenreflektometrie}

Das zuvor gesehene zweidimensionale Wachstum ist eine Grundvoraussetzung für scharfe Grenzflächen. Mit einer Analyse der XRR-Spektren kann man außerdem die Durchmischung der Kationen aufgrund von Diffusion bestimmen. Da die Reflexion der Röntgenstrahlung von der Gesamtelektronendichte in den jeweiligen Schichten bestimmt wird, sind die gemessenen Spektren direkt mit der Verteilung der Kationen verbunden. Die Umverteilung der Elektronen auf den Mn-Ionen ist nicht auflösbar, da sich die Streufaktoren der $\mathrm{Mn}^{3+}$ - und $\mathrm{Mn}^{4+}$-Ionen bei der verwendeten $\mathrm{Cu}-K_{\alpha}$-Strahlung nur wenig unterscheiden 196, 202. Damit ist man also insbesondere sensitiv auf die Verteilung der $\mathrm{La}^{3+}$ - und $\mathrm{Sr}^{2+}-$ Ionen an der Grenzfläche zwischen SMO (bzw. LSMO(x)) und LSMO. Die gezeigten Anpassungen der XRR-Spektren wurden mit dem Programm ReMagX [165] numerisch ausgeführt. In Abbildung 8.7 sieht man die Reflektivitäten für zwei Proben aus der Serie mit konstanter Dicke der SMO-Lage $\left(d_{S M O} \approx 5.5 \mathrm{~nm}\right)$ und veränderlicher Dicke der LSMO-Lage. Durch den schwachen chemischen Kontrast zwischen dem STO-Substrat und SMO sind $d_{S M O}$ und $\sigma_{S M O / S T O}$ mit größeren Fehlern behaftet als die anderen Parameter. An den in Tabelle 8.2 aufgeführten Werten für diese Abfolge von Proben kann man feststellen, dass das wiederholte Beschichten und damit verbundene mehrmalige Aufheizen derselben Probe nicht zu zusätzlichen Durchmischungen in 
a)

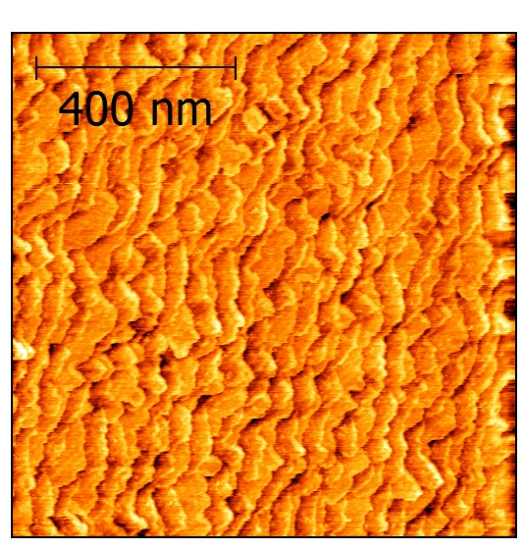

b)
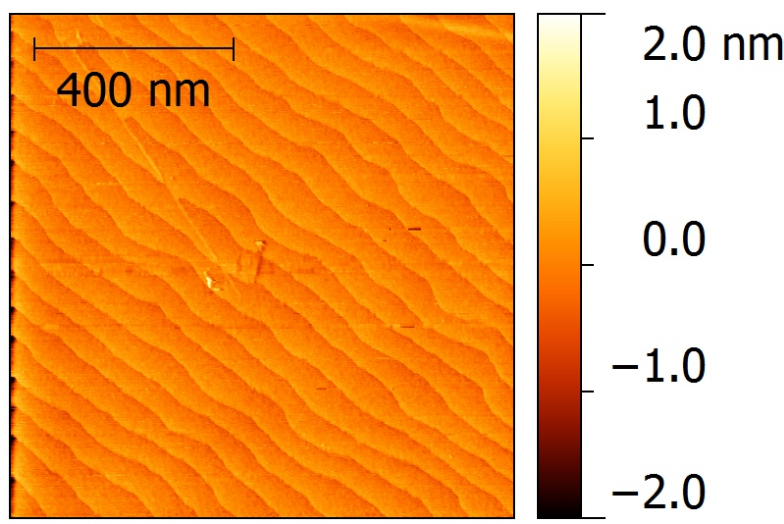

Abbildung 8.6.: Mit dem STM gemessene Oberflächenmorphologie von a) $\operatorname{LSMO}(\mathrm{x}=0.7) / \mathrm{LSMO}-\mathrm{Bilage}$ und $\mathrm{b}) \quad \mathrm{SMO} / \mathrm{LSMO} / \mathrm{SMO}$-Trilage (siehe Abbildung 8.17 .

a)

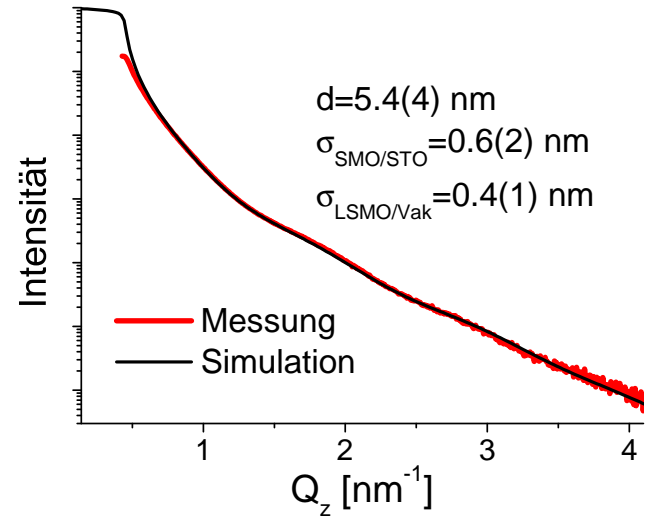

b)

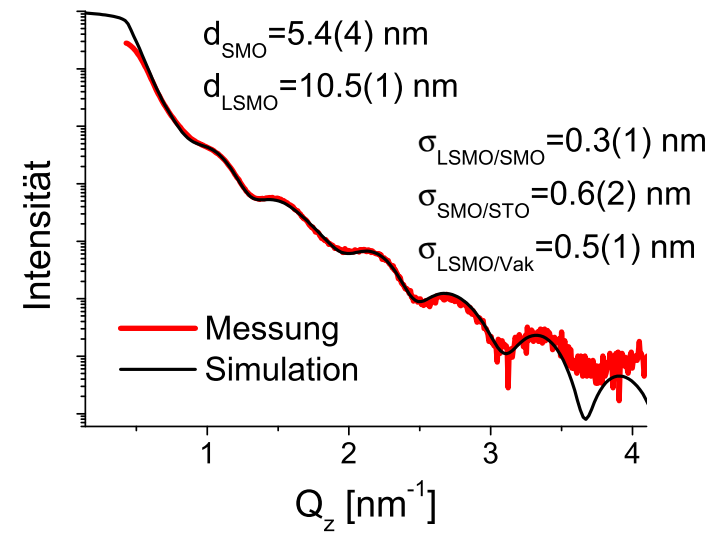

Abbildung 8.7.: XRR-Messungen und zugehörige Anpassungen für SMO ( $d_{S M O} \approx$ $5.5 \mathrm{~nm}) /$ LSMO $\left(d_{L S M O}\right)$-Heterostrukturen: a) $\left.d_{L S M O}=0 \mathrm{~nm}, \mathrm{~b}\right) d_{L S M O}=10.5 \mathrm{~nm}$.

der Heterostruktur führt, da $d_{S M O}, \sigma_{S M O / S T O}$ und $\sigma_{L S M O / S M O}$ konstant sind als Funktion von $n$. Die gleiche Aussage ergibt sich auch für die Probenserie, bei der man bei einer unveränderten LSMO-Lage weitere SMO-Schichten deponiert (siehe Abbildung 8.8 und Tabelle 8.2). Für beide Typen, die SMO/LSMO- und die LSMO/SMO- Heterostrukturen, ergibt sich eine relativ diffuse Manganat-Titanat-Grenzfläche mit einer Rauigkeit von $\sigma \approx 0.5-0.6 \mathrm{~nm}$. Die hier untersuchte Grenzfläche zwischen SMO und LSMO ist mit $\sigma \approx 0.2-0.3 \mathrm{~nm}$ sehr abrupt. Ein ähnliches Bild ergibt sich auch für die Heterostrukturen LSMO(x)/LSMO mit variabler Sr-Dotierung (siehe Abbildung 8.9 und Tabelle 8.3). Die Rauigkeit zwischen den Manganat-Lagen schwankt in einem Bereich von $\sigma \approx 0.1-0.3 \mathrm{~nm}$, wobei keine Korrelation mit der Sr-Dotierung $x$ besteht. Diese zufälligen Schwankungen sind vermutlich auf verschieden geschnittene Substrate zurückzuführen. Die daraus resultierenden Variationen der Wachstumsmode führen zu Änderungen der chemischen Profile. Zur Veranschaulichung der Schärfe einer Grenzfläche mit $\sigma_{L S M O / L S M O(x)}=0.2 \mathrm{~nm}$ betrachtet man die Grenzfläche zwischen SMO und LSMO: Wenn man sich von der Grenzfläche eine halbe Gitterkonstante tiefer bewegt, beträgt das Sr/La-Verhältnis 0.85 (statt 1.0 in SMO), und wenn man sich eine halbe Gitterkonstante nach oben bewegt, 0.4 (statt 0.3 in LSMO). Entfernt man sich dann 


\begin{tabular}{cccccc}
\hline$n$ & $d_{L S M O}$ & $\sigma_{L S M O / S M O}$ & $\sigma_{L S M O}(S M O) / V a k$ & $\sigma_{S M O / S T O}$ & $d_{S M O}$ \\
\hline 0 & - & - & $0.4(1)$ & $0.6(2)$ & $5.4(4)$ \\
\hline 1 & $3.0(3)$ & $0.3(1)$ & $0.4(1)$ & $0.6(2)$ & $5.4(4)$ \\
\hline 2 & $5.4(2)$ & $0.3(1)$ & $0.4(1)$ & $0.6(2)$ & $5.4(4)$ \\
\hline 3 & $10.5(1)$ & $0.3(1)$ & $0.5(1)$ & $0.6(2)$ & $5.4(4)$ \\
\hline
\end{tabular}

Tabelle 8.1.: Strukturelle Eigenschaften der SMO/LSMO-Bilagen nach n Beschichtungen mit LSMO (vgl. Abbildung 8.1 b)), die sich durch die Anpassung der XRR-Spektren ergeben. $\sigma_{L S M O(S M O) / V a k}$ bezeichnet die Rauigkeit von LSMO(SMO) zur Luft und $\sigma_{S M O / S T O}$ die Rauigkeit zwischen der SMO-Schicht und dem STO-Substrat (Für alle Größen ist die Einheit $\mathrm{nm})$.

a)

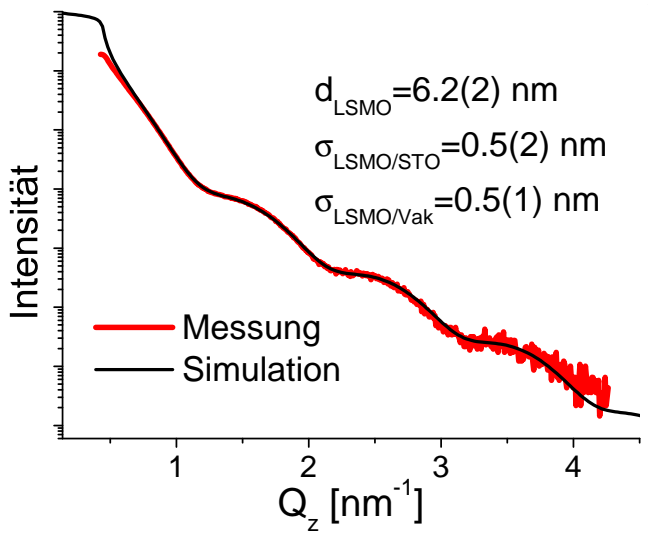

b)

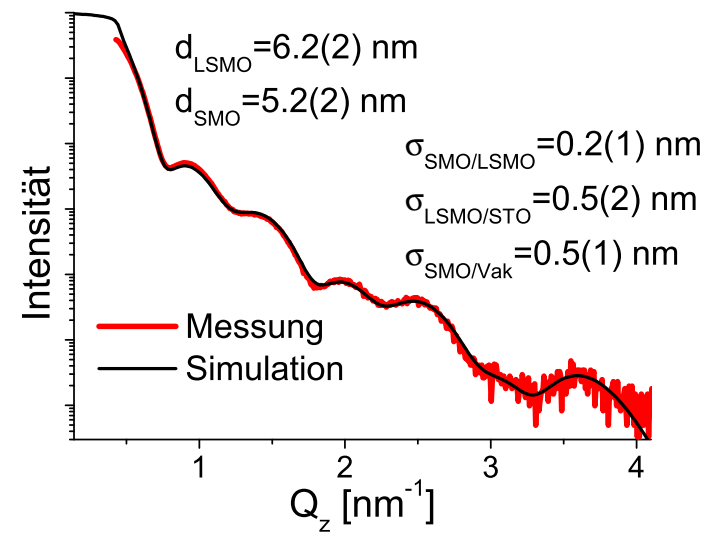

Abbildung 8.8.: XRR-Messungen und zugehörige Anpassungen für LSMO $\left(d_{L S M O} \approx\right.$ $6 \mathrm{~nm}) / \mathrm{SMO}\left(d_{S M O}\right)$-Heterostrukturen: a) $\left.d_{S M O}=0 \mathrm{~nm}, \mathrm{~b}\right) d_{S M O}=5.2 \mathrm{~nm}$.

noch eine weitere Gitterkonstante von der Grenzfläche, so ist der Einfluss der anderen Schicht zu vernachlässigen. Neben den Rauigkeiten der Grenzflächen $\sigma_{L S M O / L S M O(x)}$ ist in Tabelle 8.3 auch die Gesamtschichtdicke $d_{\text {tot }}$ der $\operatorname{LSMO}(\mathrm{x}) / \mathrm{LSMO}-H e t e r o s t r u k t u r$ als Funktion von $x$ aufgeführt. Innerhalb der Messungenauigkeit weichen die Werte von $d_{\text {tot }}$ nur um wenige Prozent vom Mittelwert $d_{t o t}^{a v}=11 \mathrm{~nm}$ ab. Zudem zeigt $d_{t o t}$ keinen Trend mit der Dotierung $x$. Damit bestätigt sich die eingangs aufgeführte Annahme, dass die deponierte Schichtdicke einfach proportional zur Zahl der Mn-Ionen in der dosierten Lösung ist und keine Abhängigkeit von der Sr-Dotierung aufweist.

\begin{tabular}{cccccc}
\hline$n$ & $d_{S M O}$ & $\sigma_{S M O / L S M O}$ & $\sigma_{S M O(L S M O) / V a k}$ & $\sigma_{L S M O / S T O}$ & $d_{L S M O}$ \\
\hline 0 & - & - & $0.5(1)$ & $0.5(2)$ & $6.2(2)$ \\
\hline 1 & $1.4(2)$ & $0.2(1)$ & $0.5(1)$ & $0.5(2)$ & $6.2(2)$ \\
\hline 2 & $3.3(2)$ & $0.2(1)$ & $0.5(1)$ & $0.5(2)$ & $6.2(2)$ \\
\hline 3 & $5.2(2)$ & $0.2(1)$ & $0.5(1)$ & $0.5(2)$ & $6.2(2)$ \\
\hline 4 & $6.9(2)$ & $0.2(1)$ & $0.5(1)$ & $0.5(2)$ & $6.2(2)$ \\
\hline
\end{tabular}

Tabelle 8.2.: Strukturelle Eigenschaften der LSMO/SMO-Bilagen nach n Beschichtungen mit SMO (vgl. Abbildung 8.1 c) (Für alle Größen ist die Einheit nm). 
a)

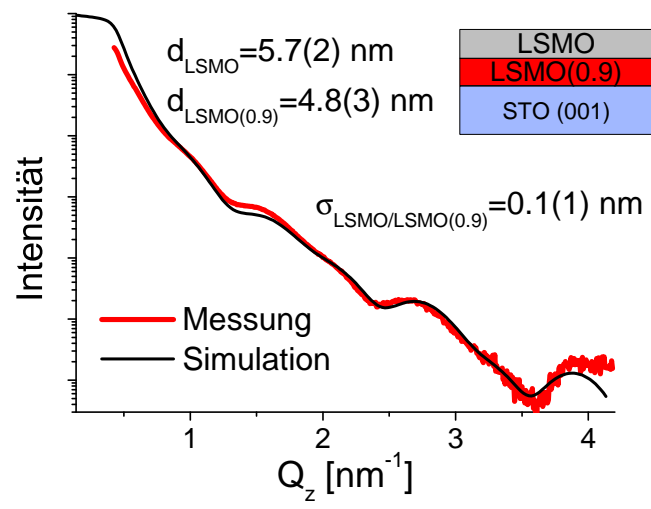

c)

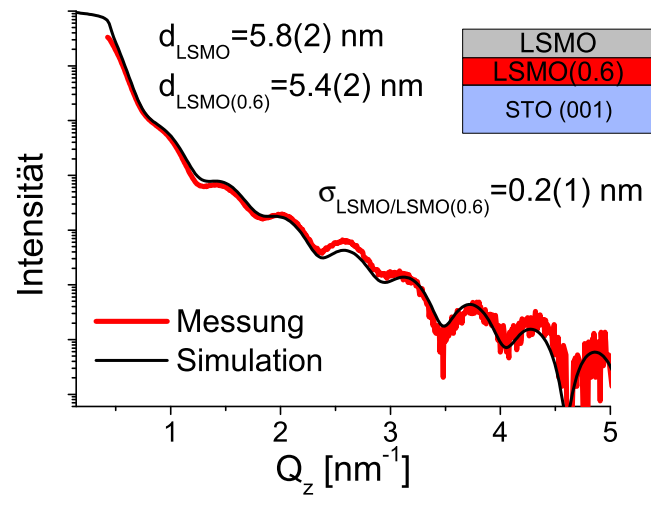

b)

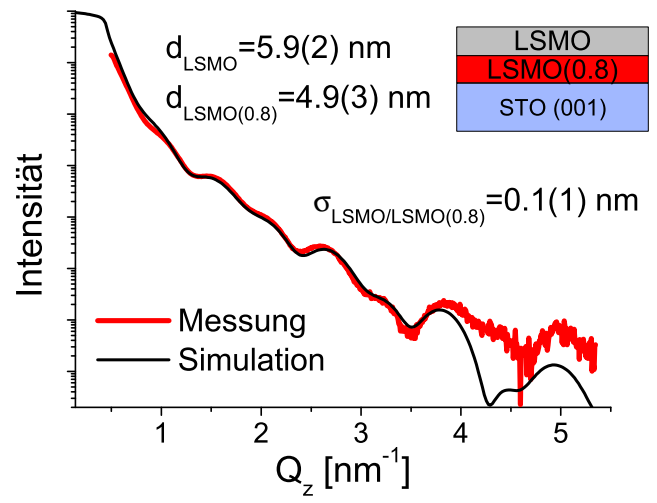

d)

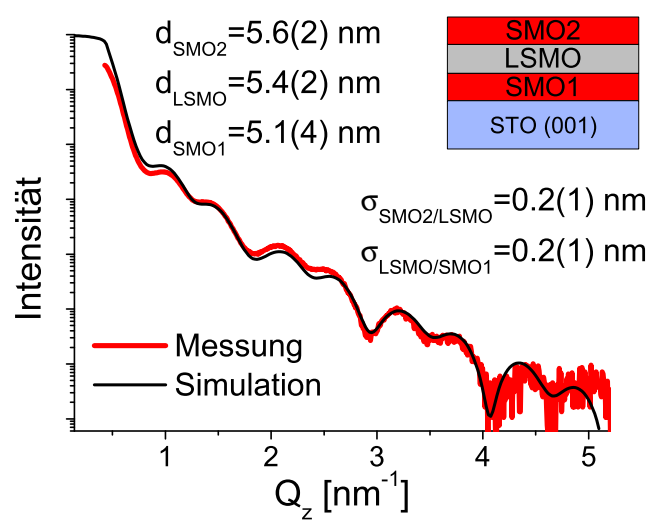

Abbildung 8.9.: XRR-Messungen und zugehörige Anpassungen für LSMO(x)/LSMOBilagen (vgl. Abbildung 8.1 a)): a) $x=0.9$, b) $x=0.8$, c) $x=0.6$ und SMO/LSMO/SMOTrilage d).

\begin{tabular}{ccc}
\hline$x$ & $d_{\text {tot }}$ & $\sigma_{\text {LSMO/LSMO }}(x)$ \\
\hline 1.0 & $10.5(5)$ & $0.2(2)$ \\
\hline 0.95 & $11.5(4)$ & $0.3(2)$ \\
\hline 0.9 & $10.5(4)$ & $0.1(1)$ \\
\hline 0.8 & $10.7(3)$ & $0.1(1)$ \\
\hline 0.7 & $11.2(3)$ & $0.2(2)$ \\
\hline 0.6 & $11.2(3)$ & $0.2(1)$ \\
\hline
\end{tabular}

Tabelle 8.3.: Strukturelle Parameter der $\operatorname{LSMO}(\mathrm{x}) / \mathrm{LSMO}-$ Heterostrukturen für $x=1.0-$ 0.6 , die sich aus den Anpassungen der XRR-Spektren ergeben: $d_{t o t}$ bezeichnet die Summe der Schichtdicken der LSMO(x)- und LSMO-Lage, $\sigma_{L S M O / L S M O(x)}$ die Grenzflächenrauigkeit zwischen den verschieden dotierten Manganaten (Für alle Größen ist die Einheit nm).

\subsubsection{Epitaktische Verspannung: Röntgendiffraktion}

Die XRD-Spektren der Heterostrukturen zeigen nur Peaks, die mit (001) indiziert werden können (siehe Abbildung 8.10 a)). Ein direktes Ablesen der Gitterkonstanten anhand der Position der Beugungsmaxima wird durch den vorhandenen Untergrund um die Peaks des STO-Substrates, der durch die Bremsstrahlung verursacht wird, erschwert. Indem man das Spektrum eines unbeschichteten Substrates subtrahiert, kann man den Untergrund eliminieren und erhält die Beugungsstruktur der Heterostruktur (Abbildung 8.10). Zur Analyse der 
a)

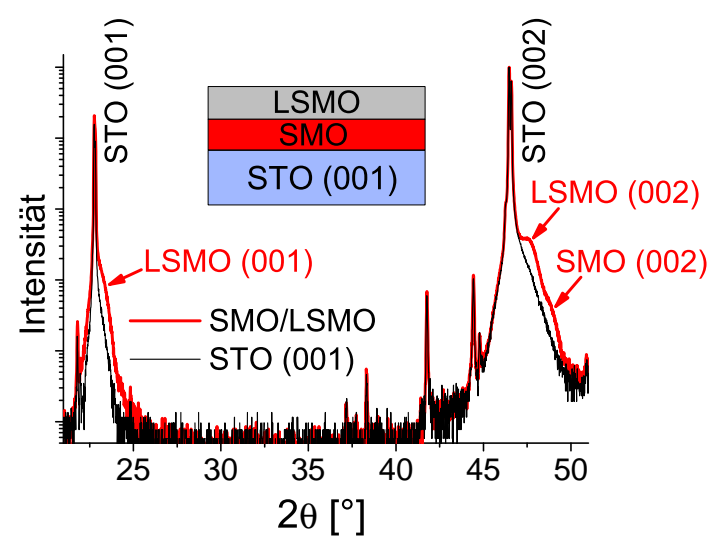

b)

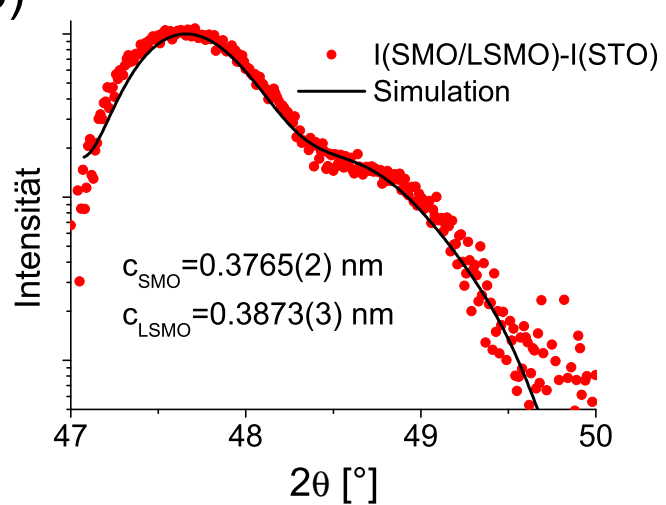

Abbildung 8.10.: Zur Auswertung der Gitterparameter der c-Achse für die hergestellten Heterostrukturen am Beispiel einer SMO/LSMO-Bilage: Man subtrahiert das XRDSpektrum eines unbeschichteten STO (001)-Substrates vom Spektrum des Filmes auf dem Substrat (siehe a)). An das resultierende Spektrum der Differenz passt man ein simuliertes Spektrum an, was die genauen Gitterparameter der einzelnen Filme liefert (siehe b)).

Gitterkonstanten zieht man nun nur die (002)-Peaks heran. Bei den (001)-Peaks ist der störende Einfluss des Peaks vom Substrat zu stark. Zudem ist die Intensität des (001)-Peaks für LSMO(x) mit großer Sr-Dotierung aufgrund des Strukturfaktors deutlich reduziert. Wegen der kleinen Schichtdicke beeinflussen sich die breiten Beugungsmaxima der Schichten, so dass es je nach relativer Lage der Peaks zu Verschiebungen der Positionen oder Veränderung der relativen Intensitäten kommt. Deshalb werden die Gitterparameter entlang der c-Achse durch die Anpassung von numerisch berechneten Beugungsspektren ermittelt. Die Simulationen erfolgen mit einem selbst geschriebenen Programm, das gemäß den in Abschnitt 5.2 .1 beschriebenen Prinzipien arbeitet. Die Schichtdicken werden mit den aus den XRR-Messungen bestimmten Werten festgelegt, so dass zur Anpassung der XRD-Spektren nur die Gitterparameter variiert werden. Dabei ist neben der absoluten Position der Maxima auch deren relative Intensität zu beachten, um die korrekten Gitterparameter zu erhalten (Abbildung 8.10 b)).

Wenn ein dünner Film, dessen Gitterstruktur vereinfachend als kubisch mit einer Gitterkonstanten $c_{0}$ angenommen wird, durch das Wachstum auf einem Substrat mit dem Gitterparameter $a_{\text {Sub }}$ elastisch verspannt wird, ergibt sich die resultierende Gitterkonstante $c$ senkrecht zur Filmebene zu 181

$$
c=c_{0}-\frac{2 \nu}{1-\nu}\left(a_{\text {Sub }}-c_{0}\right)
$$

Dabei wird angenommen, dass die Gitterkonstante des dünnen Filmes in der Ebene dem Wert des Substrates entspricht. Das Poisson-Verhältnis $\nu$ liegt nach verschiedenen Messungen in der Literatur in der Nähe von $\nu=1 / 3$ [107, 257, 258. Damit erhält man vereinfachend:

$$
c=2 c_{0}-a_{S u b}
$$

Für $\mathrm{La}_{0.7} \mathrm{Sr}_{0.3} \mathrm{MnO}_{3}$ ist die pseudokubische Gitterkonstante $a_{L S M O}=0.3875 \mathrm{~nm}$ [121], wodurch bei einem voll verspannten Film auf STO eine c-Achse von $c_{L S M O}^{\text {strain }}=0.3845 \mathrm{~nm}$ folgt. Das kubische SMO hat einen Gitterparameter von $a_{S M O}=0.3805 \mathrm{~nm}[212$, so dass ein voll verspannter Film eine c-Achse von $c_{S M O}^{\text {strain }}=0.3705 \mathrm{~nm}$ aufweisen solltt 28

\footnotetext{
${ }^{28}$ Bei diesen rein elastischen Betrachtungen werden Modifikationen der Stöchiometrie bzw. Mn-Valenz, die sich durch Grenzflächeneffekte ergeben, vernachlässigt. Diese können sich aber auch auf den Gitterparameter auswirken (siehe z.B. 107]). Zudem zeigen in letzter Zeit vorgenommene Untersuchungen, dass sich in der Nähe von Grenzflächen (in den ersten 2-7 Monolagen) Verkippungsstrukturen der Mn-O-Oktaeder einstellen können, die zu c-Achsenparametern führen, welche nicht konsistent mit diesen einfachen elastischen Modellen sind [259]. $c_{L S M O}^{\text {strain }}$ und $c_{S M O}^{\text {strain }}$ sind somit nur grobe Werte, die sich ohne Beachtung dieser Grenzflächeneffekte ergeben. Für Schichtdicken $d_{L S M O} \gtrsim 10 \mathrm{~nm}$ stimmen diese einfachen Berechnungen allerdings gut mit dem Experiment überein 258.
} 

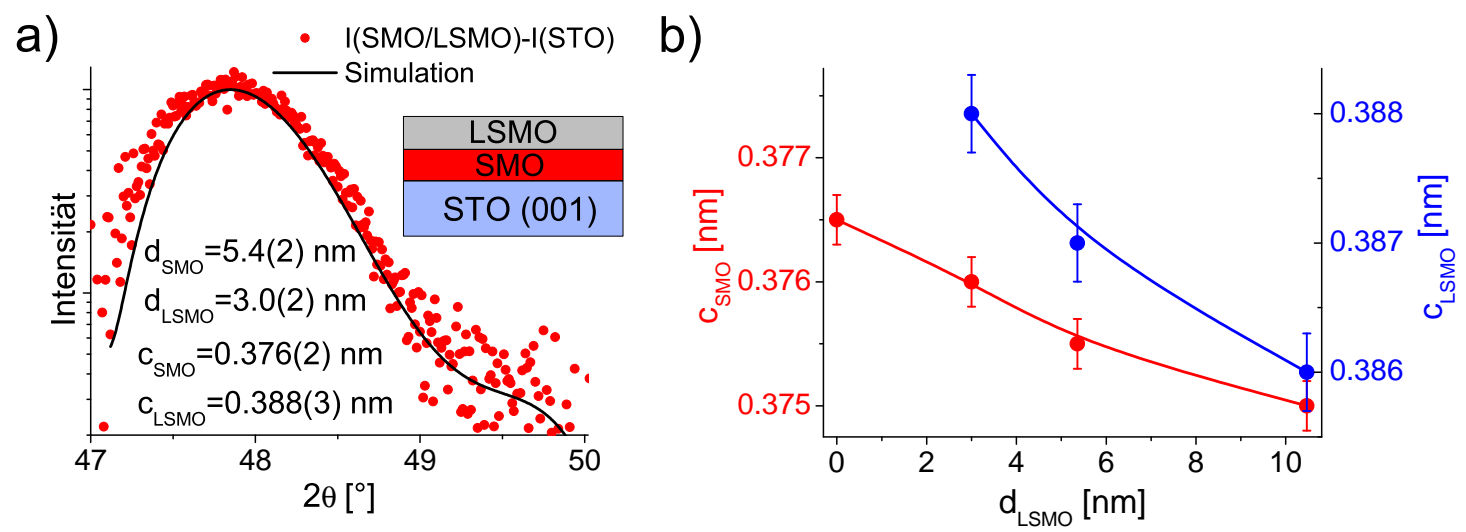

Abbildung 8.11.: Gitterparameter der $\operatorname{SMO}\left(d_{S M O} \approx 5.5 \mathrm{~nm}\right) / \mathrm{LSMO}\left(d_{L S M O}\right)$ Heterostrukturen: a) Simulation des XRD-Spektrums für $d_{L S M O}=3 \mathrm{~nm}$, b) Entwicklung der Gitterparameter $c_{S M O}$ der SMO-Schicht und $c_{L S M O}$ der LSMO-Schicht als Funktion von $d_{L S M O}$.
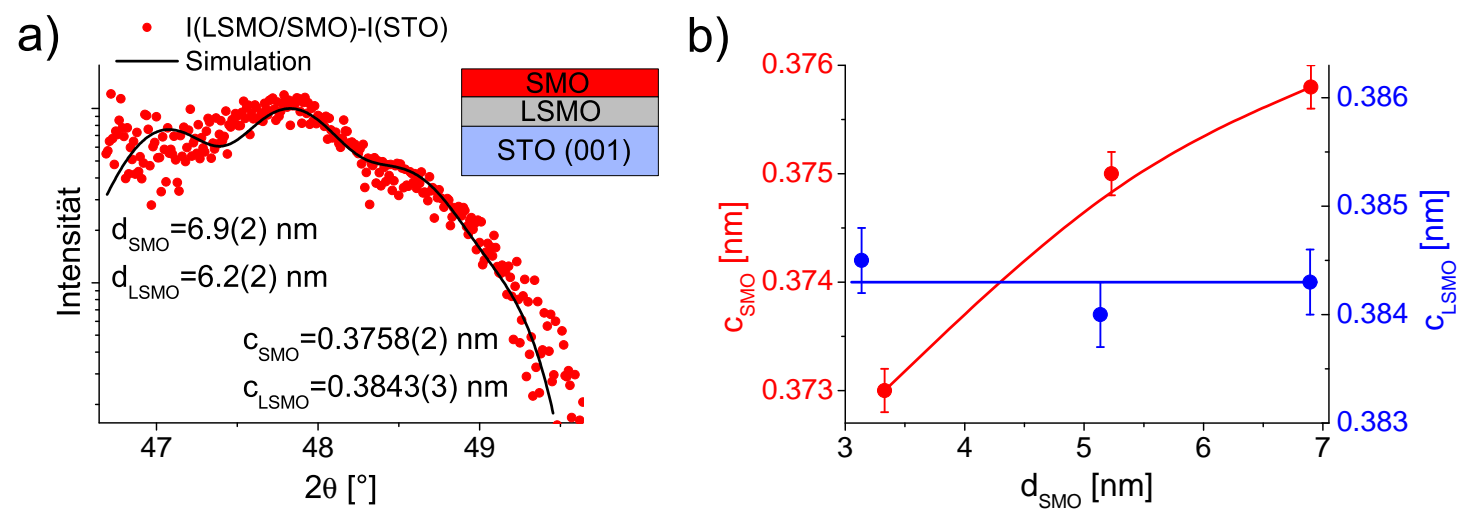

Abbildung 8.12.: Gitterparameter der LSMO $\left(d_{L S M O} \approx 6 \mathrm{~nm}\right) / \mathrm{SMO}\left(d_{S M O}\right)$ Heterostrukturen: a) Simulation des XRD-Spektrums für $d_{S M O}=6.9 \mathrm{~nm}$, b) Entwicklung der Gitterparameter $c_{S M O}$ der SMO-Schicht und $c_{L S M O}$ der LSMO-Schicht als Funktion von $d_{S M O}$.

Die auf dem bereits stark entspannten SMO-Film gewachsene dünne LSMO-Schicht mit $d_{L S M O}=3.0 \mathrm{~nm}$ zeigt eine c-Achse, die größer ist als die bulk-Gitterkonstante. Auf den LSMO-Film wirkt also eine durch den entspannten SMO-Film verursachte Druckspannung. Mit steigender Dicke der LSMO-Schicht führt die Minimierung der elastischen Energie zu einem gleichzeitigen Abfall von $c_{L S M O}$ und $c_{S M O}$. Die Gitterkonstante in der Ebene wird dann stärker von LSMO dominiert als von SMO (siehe Abbildung 8.11). Für die LSMO/SMOHeterostrukturen bleibt die Gitterkonstante des LSMO-Films unverändert bei $c_{L S M O} \approx$ $0.3843(5) \mathrm{nm}$ unter Variation von $d_{S M O}$. Der LSMO-Film, der direkt auf STO gewachsen ist, zeigt also einen voll verspannten Zustand mit dem theoretisch erwarteten Wert. $c_{S M O}$ vergrößert sich mit steigender Schichtdicke $d_{S M O}$, was die für $d_{S M O}=6.9 \mathrm{~nm}$ schon im STM angedeutete Tendenz zur Entspannung des Filmes aufzeigt (siehe Abbildung 8.12). Die Breite der Beugungsmaxima der simulierten Spektren und der gemessenen Daten sind identisch (Abbildungen 8.10 b), 8.12 a) und 8.11 a)). Man kann so annehmen, dass die c-Achse über die Dicke der Schichten konstant ist. Die zur Entspannung eingebrachten Versetzungen wandern vollständig an die Grenzflächen. 


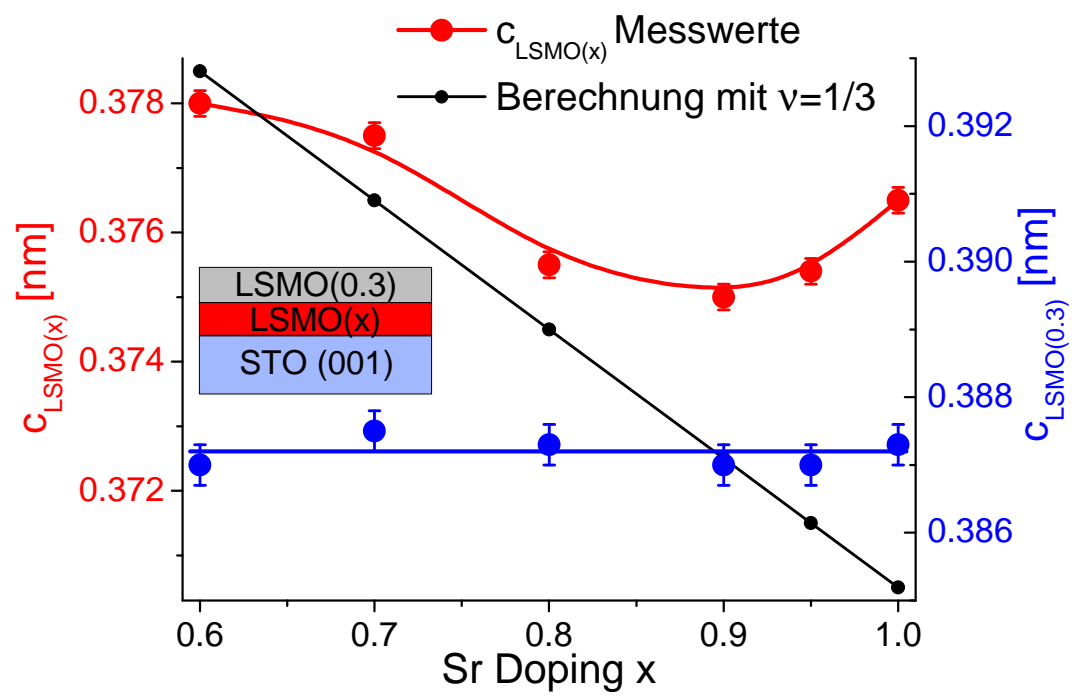

\begin{abstract}
Abbildung 8.13.: Entwicklung der Gitterparameter $c_{L S M O(x)}$ der Lage aus LSMO(x) und $c_{L S M O(0.3)}$ der Lage aus $\operatorname{LSMO}(0.3)$ in $\operatorname{LSMO}(\mathrm{x}) / \operatorname{LSMO}(0.3)$-Bilagen als Funktion der SrDotierung $x=1.0-0.6$. Zum Vergleich der Messwerte von $c_{L S M O(x)}$ sind berechnete Werte für vollständig verspannte Schichten aus LSMO(x) gezeigt, wobei man ein Poisson-Verhältnis von $\nu=1 / 3$ annimmt.
\end{abstract}

In den LSMO(x)/LSMO-Doppellagen kann man beobachten, wie sich die LSMO(x)-Lagen mit steigender Sr-Dotierung $x$ immer mehr von einem voll verspannten Zustand entfernen. Dies ergibt sich durch den Vergleich von $c_{L S M O(x)}$ mit dem theoretischen Verlauf, den man unter der Annahme von $\nu=1 / 3$ und einer linear abnehmenden pseudokubischen Gitterkonstanten des bulk-Materials 29

$$
a_{L S M O(x)}=\frac{a_{S M O}-a_{L S M O}}{0.7} \cdot(x-0.3)+a_{L S M O}
$$

erhält (vergleiche Abbildung 8.13). Für $x \geq 0.7$ ist $c_{L S M O(x)}$ deutlich größer als der Wert bei voller Verspannung. Der LSMO-Film, der auf diesen LSMO(x)-Lagen wächst, zeigt unabhängig von der Dotierung der LSMO(x)-Lage eine Gitterkonstante um $c_{L S M O}=0.3873(5) \mathrm{nm}$, was der pseudokubischen Gitterkonstanten des bulk-Materials entspricht. Die LSMO-Filme an der Oberfläche befinden sich also in einem entspannten Zustand.

\title{
8.2.5. Simulation der in-situ Ellipsometrie
}

Nach der Betrachtung der Wachstumsmode, der chemischen Qualität der Grenzflächen und der strukturellen Eigenschaften der Schichten kann man die in der in-situ Ellipsometrie beobachtete Übergangsregion beim Wachstum von LSMO auf SMO (Abbildung 8.3) genauer analysieren. Aufgrund der STM-Aufnahmen und der chemischen Qualität der Grenzflächen kann man annehmen, dass der SMO-Film eine geschlossene Oberfläche besitzt, die den atomaren Terrassen des Substrates entspricht. Aufgrund der scharfen Grenzfläche wird eine diffuse Vermischung der La- und Sr- Kationen an der Grenzfläche in den folgenden Betrachtungen vernachlässigt. Die Messungen der Ellipsometrie liefern zu den XRR-Spektren komplementäre Informationen, da die Verteilung der Kationen auf den A-Plätzen keinen direkten Einfluss

\footnotetext{
${ }^{29}$ Die Annahme einer kubischen Struktur ist mit Blick auf die Gitterstruktur von bulk-Einkristallen, die für $x=0.6-0.85$ eine tetragonale Struktur mit $(c-a) / a \approx \pm 0.4 \%$ zeigen 38, gerechtfertigt.
} 

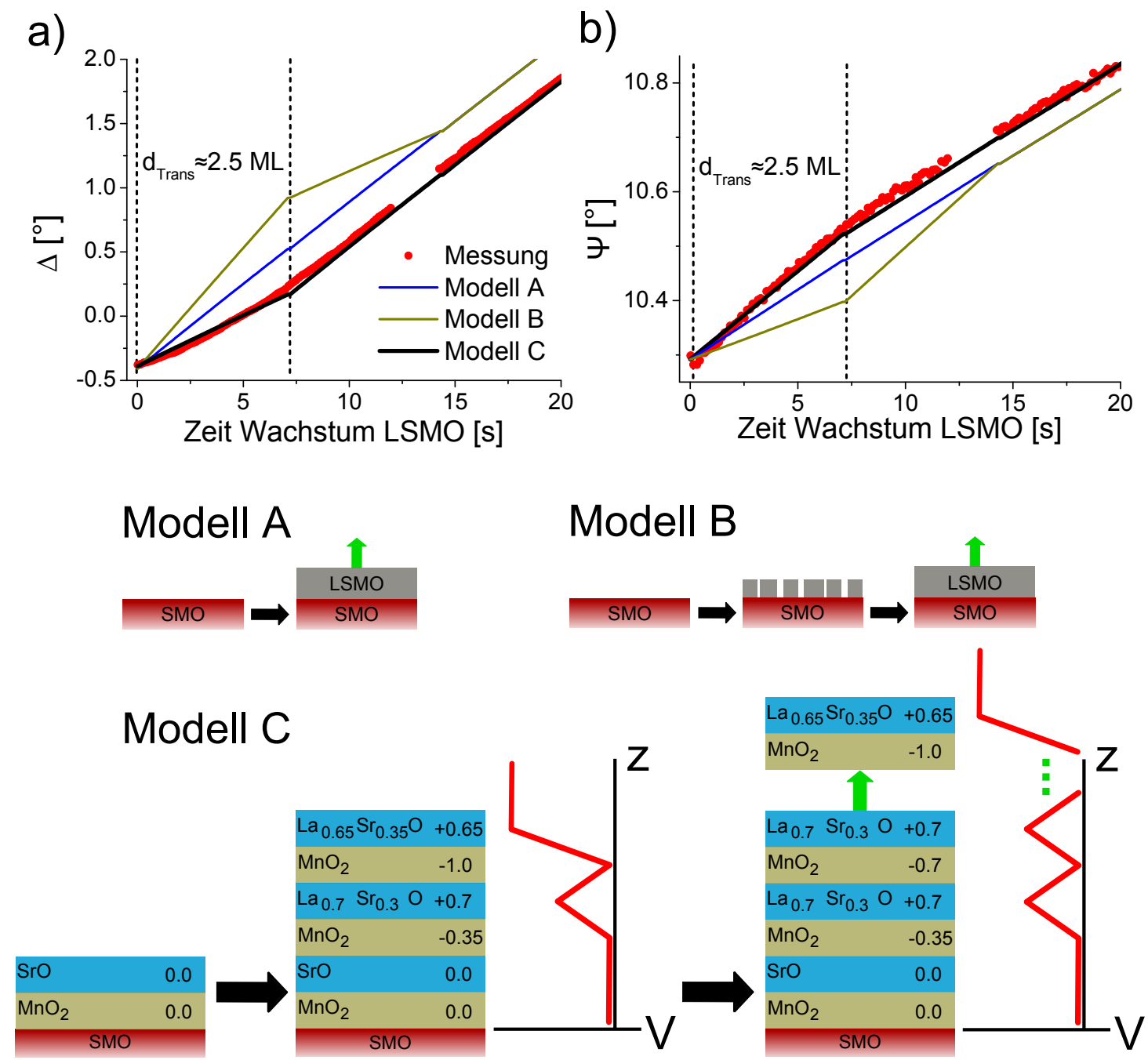

Modell B

Abbildung 8.14.: Numerische Simulation der ersten Phase des Wachstums von LSMO auf SMO (siehe Abbildung 8.3 und die damit verbundenen Modelle des Wachstums: In a) und b) sind die gemessenen Verläufe von $\Delta(t)$ und $\Psi(t)$ zusammen mit den simulierten Kurven, die den in c) gezeigten Modellen A, B und C entsprechen, dargestellt. Bei Modell A wird ein kontinuierliches Wachstum der LSMO-Schicht angenommen. Bei Modell B nimmt man an, dass der LSMO-Film zunächst rau aufwächst und nach einer deponierten Menge, die einer Schichtdicke von $d_{\text {Trans }} \approx 1 \mathrm{~nm}$ entspricht, die Rauigkeiten aufgefüllt werden und der Film kontinuierlich weiterwächst (Für die Simulation wurde aufgenommen, dass der LSMO-Film zu Beginn mikroskopisch rau ist und zu einem Teil von 20\% aus Leerstellen besteht. Der Brechungsindex dieses rauen Filmes ergibt sich über die Näherung als effektives Medium. Dieser raue Film wächst dann bis zu einer Dicke von $\left.1.25 \cdot d_{\text {Trans }}.\right)$. Die Simulation entsprechend Modell C passt am besten zu den Messdaten. Bei diesem nimmt man eine Umverteilung der Ladungen auf den Mn-Ionen in den LSMO-Lagen zur Vermeidung der polaren Katastrophe an. Die Zahlen hinter der jeweiligen chemischen Formel der AO- und $\mathrm{BO}_{2}$-Lagen bezeichnen deren elektrischen Ladungszustand. Durch die Umverteilung der Ladungen zwischen den Mn-Ionen ergibt sich ein als Funktion des Abstandes $z$ von der Grenzfläche oszillierendes elektrostatisches Potential $V$. 
hat und die Verteilung der Valenzen der Mn-Ionen bestimmend ist (siehe Abschnitt 6.2). In Abbildung 8.14 a) und b) sind die zeitlichen Verläufe $\Delta(t)$ und $\Psi(t)$ zusammen mit numerischen Simulationen, die den gezeigten Modelle A, B und C folgen, gezeigt. Das einfachste Modell A, bei dem der LSMO-Film kontinuierlich auf SMO aufwächst, führt zu linearen Verläufen von $\Delta$ und $\Psi$, womit man die Messungen nicht nachbilden kann. Auch ein zunächst raues Wachstum des LSMO-Filmes, das sich nach einer deponierten Menge, die bei kontinuierlichem Wachstum zu einer Schichtdicke von $d_{\text {Trans }} \approx 1 \mathrm{~nm}$ führt, in ein kontinuierliches Wachstum umwandelt, kann die gemessenen Daten nicht erklären (Modell B in Abbildung 8.14).

Aufgrund der Mischvalenz der Manganate, die bei den vorliegenden Depositionsbedingungen zwischen $\mathrm{Mn}^{3+}$ und $\mathrm{Mn}^{4+}$ variieren können, kann man neben den zuvor aufgeführten geometrischen Veränderungen beim Wachstum der Schicht auch eine Umverteilung der $e_{g^{-}}$ Elektronen auf den Mn-Ionen annehmen. Eine einfache Delokalisierung der Elektronen an der LSMO/SMO-Grenzfläche ist konsistent mit den Messungen von $\Delta(t)$, für $\Psi(t)$ ergibt sich dabei jedoch ein linearer Verlauf wie bei Modell A, der nicht konsistent mit den Messungen ist. Durch die wechselnde elektrische Ladung der $\mathrm{MnO}_{2^{-}}$und $\mathrm{La}_{0.7} \mathrm{Sr}_{0.3} \mathrm{O}$-Lagen in LSMO kommt es, wie in Abschnitt 3.1 beschrieben, zu einer Divergenz des elektrostatischen Potentials, wenn keine weitere Umverteilung von Ladungen geschieht 105, 106. Diese Tatsache wird für Modell C berücksichtigt: Auf der mit SrO terminierten SMO-Lage wächst das LSMO mit zwei Lagen gleichzeitig auf, wobei die $e_{g}$-Elektronen von der Lage an der Grenzfläche zur Lage an der Oberfläche transferiert werden (Zur vollständigen Aufhebung der Divergenz des Potentials bei einer dickeren Schicht muss eine weitere Anpassung erfolgen, die hier durch eine kleine Änderung der Stöchiometrie, $\mathrm{La}_{0.7} \mathrm{Sr}_{0.3} \mathrm{O} \rightarrow \mathrm{La}_{0.65} \mathrm{Sr}_{0.35} \mathrm{O}$, in der Lage an der Oberfläche bewerkstelligt wird). Bei weiterem Wachstum der LSMO-Lage verbleiben diese Konfigurationen in den Lagen an der Oberfläche und der Grenzfläche, so dass sich ein oszillierender Verlauf des elektrostatischen Potentials $V$ ergibt, der an der Oberfläche der Probe in einem Potentialsprung endet. Mit den Brechungsindizes aus Abbildung 6.4 für Mangantfilme mit unterschiedlichem $\mathrm{Mn}^{3+} / \mathrm{Mn}^{4+}$-Verhältnis kann man $\Delta(t)$ und $\Psi(t)$ für Modell $\mathrm{C}$ simulieren. Es zeigt sich eine gute Übereinstimmung mit den experimentellen Daten (siehe Abbildung 8.14 a) und b)). Es ist anzumerken, dass man in diesem Fall aus den Messungen der Ellipsometrie nicht schließen kann, ob die Elektronen von der Grenzfläche zur Oberfläche oder umgekehrt umverteilt werden. Ein Transfer der Elektronen von der Oberfläche zur Grenzfläche würde aber zu einem divergierenden Potential führen und kann so ausgeschlossen werden. Bei einer $\mathrm{MnO}_{2}$-Terminierung von der SMO-Lage wäre die sich ergebende Umverteilung der Mn-Ladungen inkonsistent mit den gemessenen Daten. Eine selbstorganisierte Einstellung der Terminierung bei dem Wachstum von Perowskiten wurde von Rijnders et al. bei dem Wachstum von $\mathrm{SrRuO}_{3}$ auf $\mathrm{SrTiO}_{3}$-Substraten beobachtet 217, 218]: Nach der Deposition der $\mathrm{SrRuO}_{3}$-Filme stellte man eine SrO-Terminierung der Oberfläche fest, obwohl die Substrate zuvor mit der $\mathrm{TiO}_{2}$-Lage terminiert worden waren. Während des Wachstums kam es also zu einem Wechsel des Abschlusses der Perowskit-Lagen entgegen der durch das Substrat vorgegebenen Reihenfolge. Auch bei einer zusätzlichen Delokalisierung der Elektronen an der Grenz- bzw. Oberfläche würde bei der für Modell $\mathrm{C}$ gezeigten Ladungsverteilung kein divergierendes Potential auftreten 61. Wenn bei der Delokalisierung aber mehr als 0.1 $e_{g}$-Elektronen pro Einheitszelle in der $\mathrm{MnO}_{2}$-Lage der SMO Schicht auftreten, ergeben sich signifikante Abweichungen von den Messdaten. 

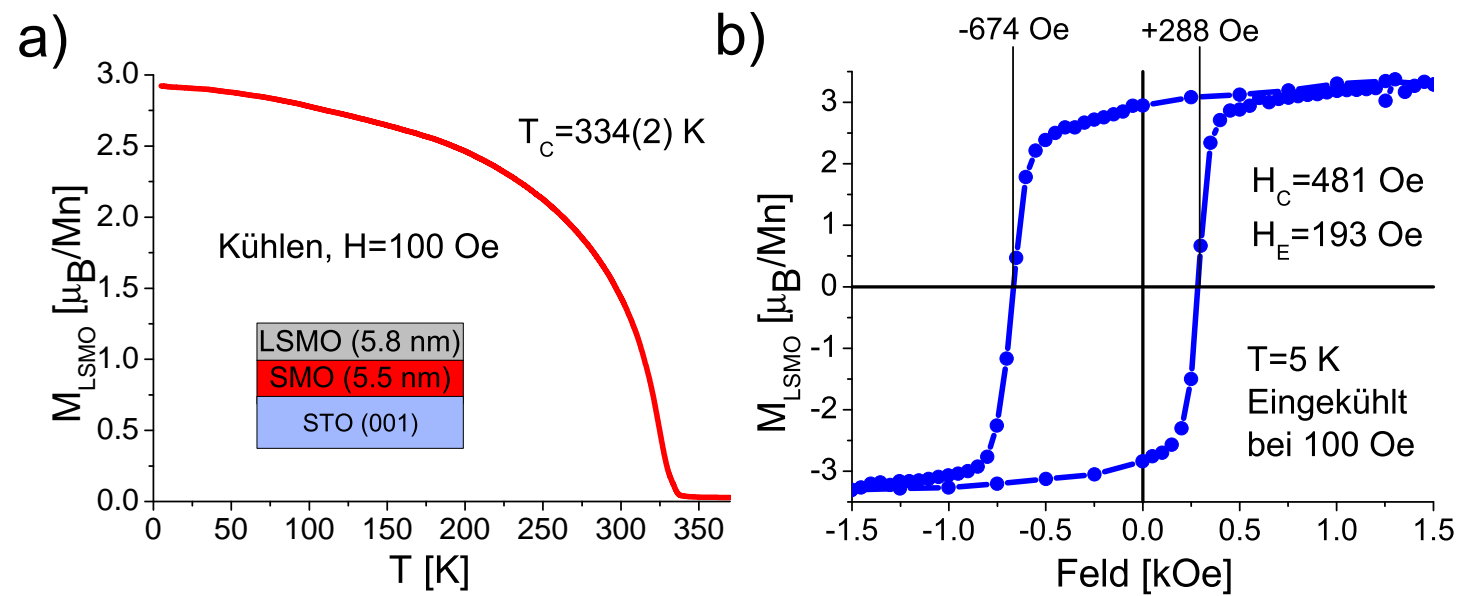

\begin{abstract}
Abbildung 8.15.: Magnetische Eigenschaften einer SMO/LSMO-Doppellage: a) $M_{L S M O}(T)$ und b) $M_{L S M O}(H)$ bei $T=5 \mathrm{~K}$ nach Einkühlen mit $H=+100$ Oe (Die zugehörigen Werte für die Amplitude des exchange bias $H_{E}$ und das Koerzitivfeld $H_{C}$ sind im Graphen angegeben.).
\end{abstract}

\title{
8.3. Magnetische Eigenschaften
}

Das magnetische Feld wurde für alle gezeigten Messungen entlang der [100]-Richtung der STO (001)-Substrate angelegt und lag in der Ebene des Filmes. Zur Messung des exchange bias (EB) wurden die Proben mit einem angelegten Magnetfeld von $H=+100$ Oe von $T=370 \mathrm{~K}>T_{C}$ eingekühlt. Dabei wurden die gezeigten Temperaturabhängigkeiten $M(T)$ der Magnetisierung aufgenommen. Die Kühlrate bewegte sich dabei in einem Bereich von $p=3-8 \mathrm{~K} / \mathrm{min}$. In diesem Bereich war keine Abhängigkeit des EB von der Kühlrate festzustellen. Eine Normierung der magnetischen Momente auf die vorhandenen Mn-Ionen erfolgt mit den oben ermittelten Schichtdicken. Dabei bezeichnet $M_{L S M O}$ das auf die Mn-Ionen in der LSMO-Schicht normierte Moment. Bei der Messung der $M(H)$-Kurven wurde das maximal angelegte Feld $H_{\max }$ so gewählt, dass sich die Hystereseschleife schließt, um minorloop-Effekte auszuschließen [124, 125].

Anhand einer SMO/LSMO-Bilage soll die grundsätzliche Phänomenologie des hier beobachteten exchange bias aufgezeigt werden. Beim Einkühlen beobachtet man einen ferromagnetischen Übergang mit einer Curie-Temperatur $T_{C}=334(2) \mathrm{K}$ (siehe Abbildung 8.15 a)). Dieser Wert ist deutlich größer als $T_{C}=315(5) \mathrm{K}$ für eine einfache LSMO-Schicht ähnlicher Dicke auf STO (001) und in der Nähe von $T_{C}=345(5) \mathrm{K}$, der sich als Sättigungswert für dickere LSMO-Schichten $\left(d_{L S M O} \gtrsim 15 \mathrm{~nm}\right)$ auf STO (001)-Substraten ergibt $97,105,107,216$. Dies wird durch die zusätzliche SMO-Schicht verursacht, welche zu einem entspannten Wachstum der LSMO-Schicht führt. Außerdem ist die Grenzfläche zwischen SMO und LSMO wesentlich schärfer als zwischen LSMO und STO, so dass die Degradation an der Grenzfläche verhindert wird. Nach dem Einkühlen mit einem angelegten Feld von $H=+100$ Oe beobachtet man in der $M(H)$-Kurve ein positives exchange bias, eine Verschiebung in die Feldrichtung entgegen der Richtung des angelegten Feldes beim Kühlen (Abbildung 8.15 b)). Aus den Werten $H_{C}^{+}$ und $H_{C}^{-}$für die beiden Nulldurchgänge der $M(H)$-Kurve definiert man die Amplitude des exchange bias $H_{E}$ und das Koerzitivfeld $H_{C}$ [124, 125:

$$
H_{E}=-\frac{H_{C}^{+}+H_{C}^{-}}{2}, H_{C}=\frac{H_{C}^{+}-H_{C}^{-}}{2}
$$


a)

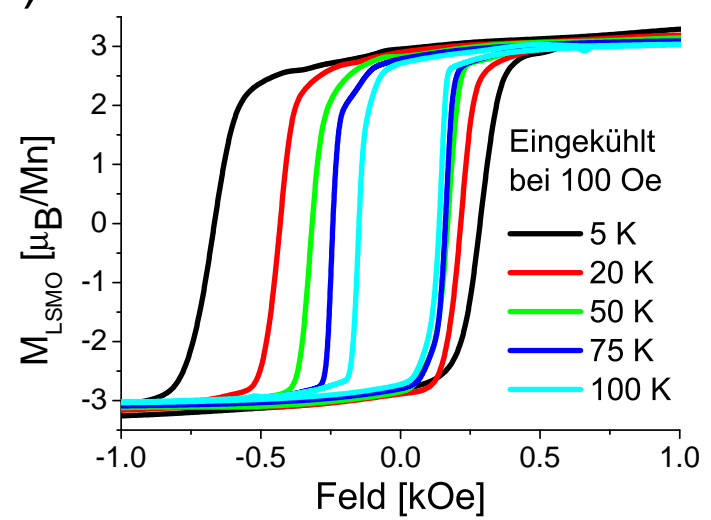

b)

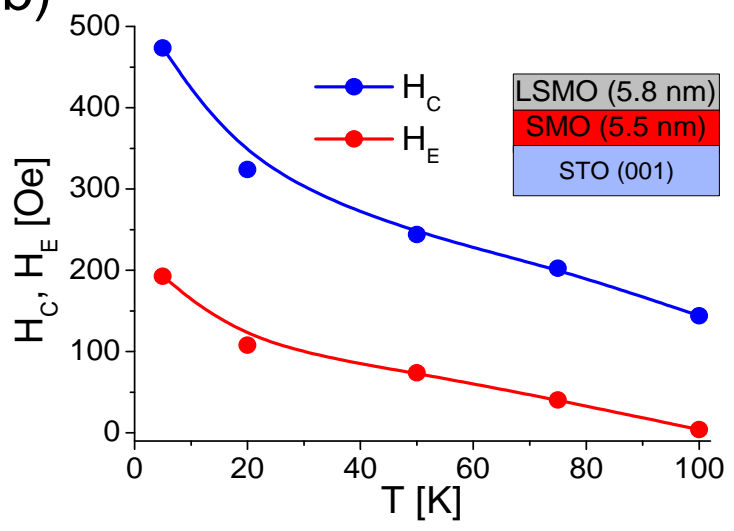

Abbildung 8.16.: Veränderung der $M(H)$-Hysterese mit der Temperatur für eine SMO/LSMO-Bilage, nachdem die Probe zunächst bei $H=+100$ Oe auf $T=5 \mathrm{~K}$ gekühlt wurde: a) $M_{L S M O}(H)$-Kurven bei verschiedenen Temperaturen, wobei diese nach dem Einkühlen in der Reihenfolge steigender Temperaturen gemessen wurden. In b) sind die aus a) ermittelten $H_{C}(T)$ und $H_{E}(T)$ gezeigt.

a)

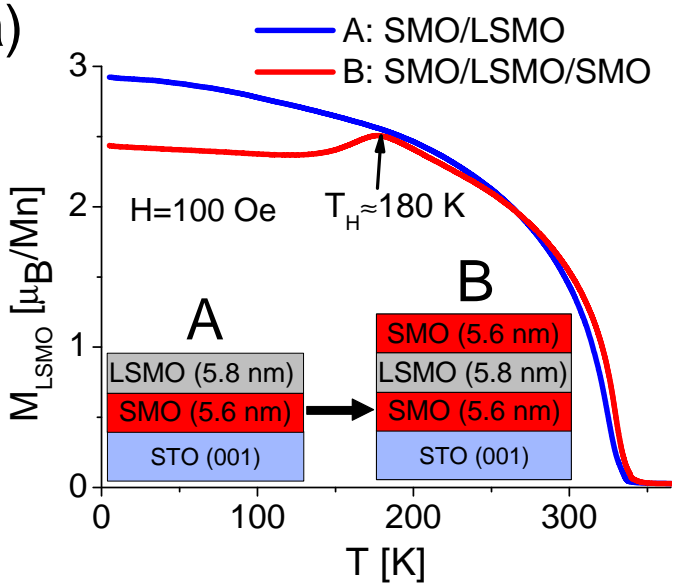

b)

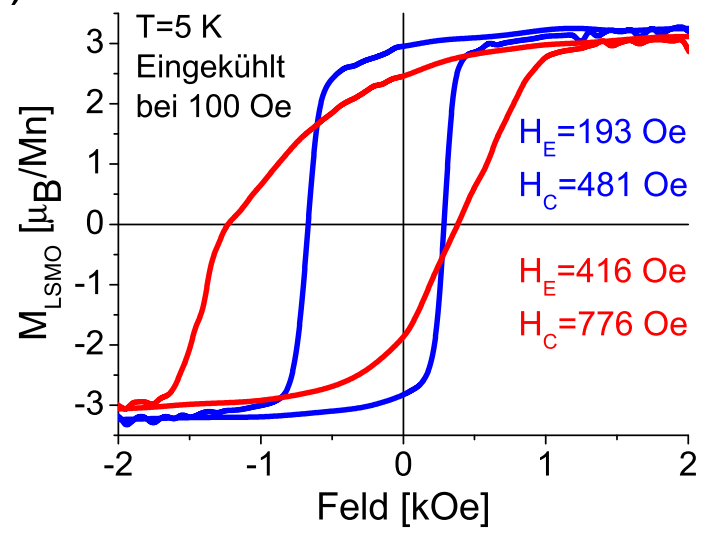

Abbildung 8.17.: Effekt einer zusätzlich deponierten SMO-Lage auf die SMO/LSMOHeterostruktur: a) $M_{L S M O}(T)$ und $M_{L S M O}(H)$ bei $T=5 \mathrm{~K}$ nach Einkühlen mit $H=$ +100 Oe.

Die auf die Mn-Ionen in der LSMO-Schicht normierte Sättigungsmagnetisierung liegt mit $M_{L S M O}^{\text {sat }}=3.3(1) \mu_{\mathrm{B}} / \mathrm{Mn}$ etwas unterhalb dem Wert von $M_{\text {sat }}=3.7 \mu_{\mathrm{B}} / \mathrm{Mn}$, der sich theoretisch bei der Ausrichtung aller Spins in der LSMO-Schicht ergeben würde. Die Abweichung entspricht etwa 1 - 2 unmagnetischen $\mathrm{MnO}_{2}$-Lagen in der LSMO-Schicht (Bei einer Dicke $d_{L S M O}=5.8 \mathrm{~nm}$ reduziert jede unmagnetische Lage das durchschnittliche Moment pro Mn-Ion $M_{L S M O}$ um $\Delta M_{L S M O}=0.25 \mu_{\mathrm{B}} / \mathrm{Mn}$.). Mit steigender Temperatur nehmen $H_{E}$ und $H_{C}$ ab, wobei sich das in Richtung des Kühlfeldes liegende $H_{C}^{+}$deutlich schwächer mit der Temperatur ändert als $H_{C}^{-}$. Bei $T \approx 100 \mathrm{~K}$ verschwindet $H_{E}$, der Wert von $H_{C}$ liegt aber weiterhin deutlich über dem Koerzitivfeld einer einfachen LSMO-Schicht auf STO $(001) H_{C}^{L S M O}(T=5 \mathrm{~K}) \approx 50$ Oe für $d_{L S M O}=6 \mathrm{~nm}$ (siehe Abbildung 8.16). Wird auf die SMO/LSMO-Doppellage eine weitere SMO-Lage aufgebracht (siehe Abbildung 8.17), so kommt es zu starken Veränderungen des magnetischen Verhaltens. Die Umkehr der Magnetisierung durch ein äußeres Feld geschieht in einem deutlich größeren Feldintervall und zeigt einen stark asymmetrischen Verlauf, die $M(H)$-Kurve ist nicht mehr nur einfach entlang der 

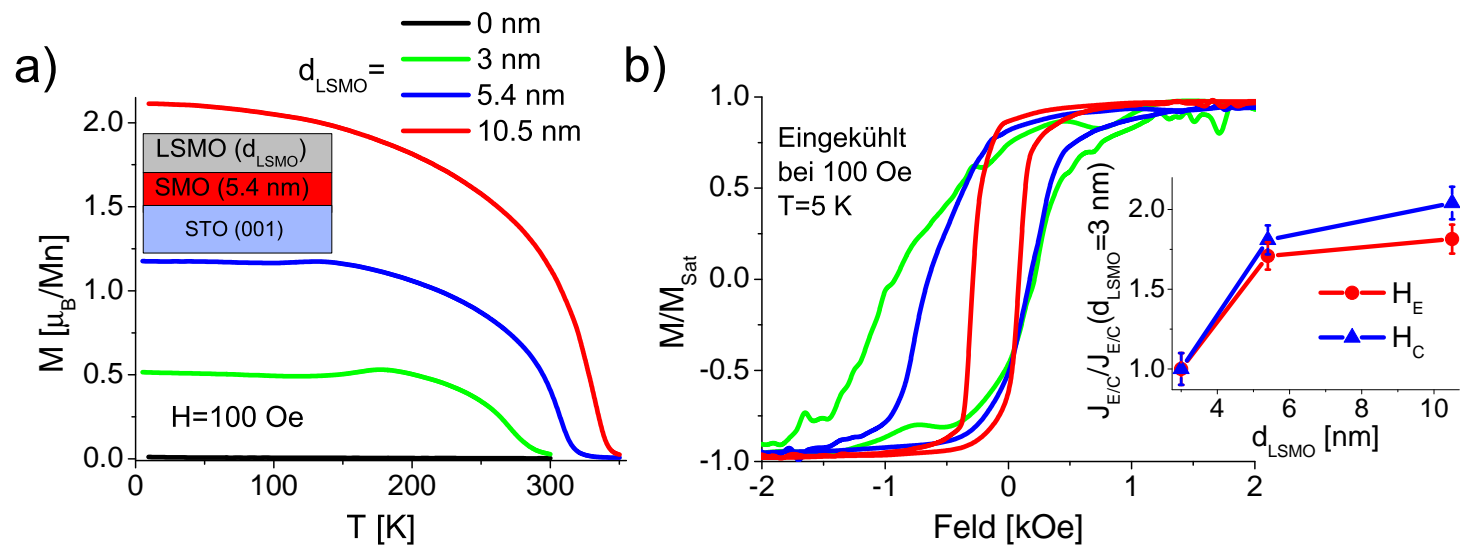

Abbildung 8.18.: Entwicklung der magnetischen Eigenschaften der SMO $\left(d_{S M O} \approx\right.$ $5.5 \mathrm{~nm}) / \mathrm{LSMO}\left(d_{L S M O}=0-10.5 \mathrm{~nm}\right)$-Bilagen: a) Temperaturabhängigkeit der Magnetisierung, wobei das magnetische Moment auf alle Mn-Ionen in der Heterostruktur normiert wurde, b) $M(H)$-Kurven nach Einkühlen im Magnetfeld $H=+100$ Oe. Das Inset von b) zeigt die Abhängigkeit des Produktes $J_{E / C}=H_{E / C} M_{L S M O} d_{L S M O}$, wobei eine Normierung auf den Wert für $d_{L S M O}=3.0 \mathrm{~nm}$ vorgenommen wurde, von $d_{L S M O}$.

Feldachse verschoben. Durch die zusätzliche SMO-Lage kommt es näherungsweise zu einer Verdopplung von $H_{C}$ und $H_{E}$. Diese Veränderung der magnetischen Hysterese wirkt sich auch auf den Verlauf von $M(T)$ aus, wo man bei $T_{H} \approx 180 \mathrm{~K}$ ein Maximum erkennt.

Für die in Abbildung $8.1 \mathrm{~b})$ gezeigte Serie von SMO $\left(d_{S M O} \approx 5.5 \mathrm{~nm}\right) / \mathrm{LSMO}\left(d_{L S M O}=\right.$ $0-10.5 \mathrm{~nm}$ )-Bilagen ergibt sich das in Abbildung 8.18 dargestellte magnetische Verhalten. Die zunächst deponierte SMO-Lage zeigt kein magnetisches Moment. Für die darauf deponierte LSMO-Schicht ergibt sich eine mit der Schichtdicke ansteigende Curie-Temperatur. Die Curie-Temperatur $T_{C}=290(2) \mathrm{K}$ der dünnsten Schicht mit $d_{L S M O}=3.0 \mathrm{~nm}$ ist etwa $20 \mathrm{~K}$ größer als für einfache LSMO-Schichten auf STO (001) mit gleicher Schichtdicke [105]. Dies ist eine Folge des durch die SMO-Lage veränderten Verspannungszustandes (vergleiche Abbildung 8.11) und der Tatsache, dass die Grenzfläche zwischen SMO und LSMO chemisch wesentlich schärfer ist als zwischen STO und LSMO. Die charakteristischen Felder $H_{E}, H_{C}$ nehmen mit $d_{L S M O}$ ab. Wie es in der Theorie beschrieben wurde, sind $H_{E / C}$ aufgrund der an der Grenzfläche lokalisierten Kopplung zwischen dem Ferromagneten und dem Antiferromagneten umgekehrt proportional zum magnetischen Moment der ferromagnetischen Schicht $m=M_{F M} t_{F M}$. Wenn man nun das Produkt $J_{E / C}=H_{E / C} M_{L S M O} d_{L S M O}$ betrachtet, erhält man ein Maß für die Stärke der an den Ferromagneten koppelnden gepinnten bzw. rotierbaren Momente an der Grenzfläche zum Antiferromagneten. Im Inset von Abbildung 8.18 b) sind diese Produkte als Funktion von $d_{L S M O}$ dargestellt, wobei eine Normierung auf den jeweiligen Wert für $d_{L S M O}=3.0 \mathrm{~nm}$ vorgenommen wurde. Ausgehend von $d_{L S M O}=3.0 \mathrm{~nm}$ verdoppelt sich $J_{E / C}$ zunächst und bleibt dann für die beiden größeren Schichtdicken etwa konstant.

Bei den LSMO $\left(d_{L S M O} \approx 6 \mathrm{~nm}\right) / \operatorname{SMO}\left(d_{S M O}=0-6.9 \mathrm{~nm}\right)$-Heterostrukturen erhöht sich die Curie-Temperatur $T_{C}$ der LSMO-Lage nach der Deposition der ersten SMO-Lage von $320 \mathrm{~K}$ auf $327 \mathrm{~K}$ und ändert sich danach bei weiterer Vergrößerung von $d_{S M O}$ nur um $1-2 \mathrm{~K}$. Ebenso steigt das Sättigungsmoment bei $T=5 \mathrm{~K}$ mit der Deposition der ersten SMO-Lage um 0.3(1) $\mu_{\mathrm{B}} / \mathrm{Mn}$ und zeigt bei Erhöhung von $d_{S M O}$ keine weiteren Änderungen. Umgerechnet auf die Dicke der LSMO-Schicht entspricht diese Änderung dem magnetischen Moment einer $\mathrm{MnO}_{2}$-Lage. Dies deutet auf das Vorhandensein einer toten Lage an der Oberfläche der 


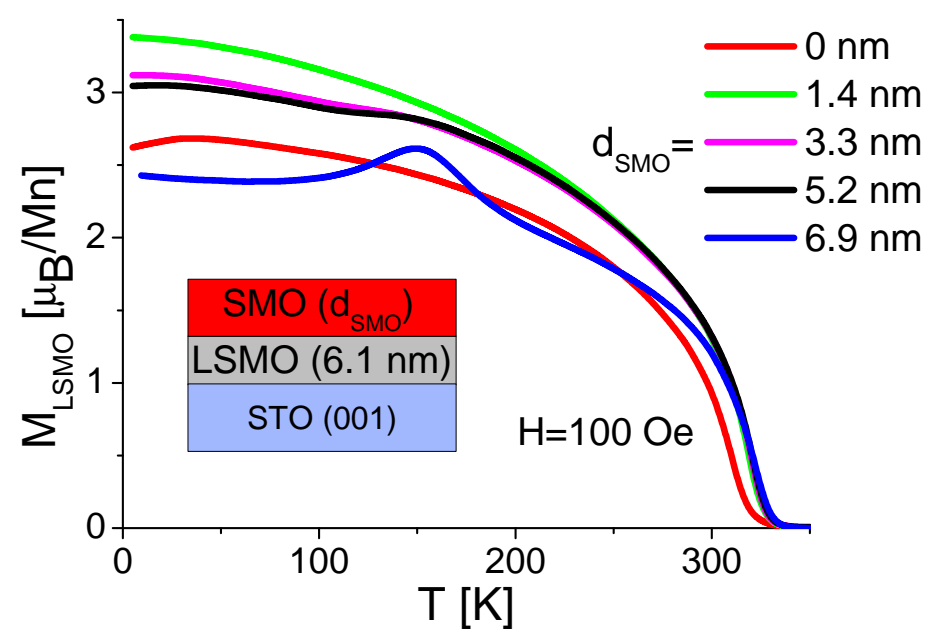

Abbildung 8.19.: Temperaturabhängigkeit der Magnetisierung für die LSMO $\left(d_{L S M O} \approx\right.$ $6 \mathrm{~nm}) / \mathrm{SMO}\left(d_{S M O}=0-6.9 \mathrm{~nm}\right)$-Bilagen. Die Magnetisierung $M_{L S M O}$ wurde auf die MnIonen in der LSMO-Lage normiert.

isolierten LSMO-Schicht hin, welche durch die Deposition einer SMO-Lage magnetisch wird. Ähnlich wie bei der SMO/LSMO/SMO-Dreifachschicht (vergleiche Abbildung 8.17 a)) zeigt sich für $d_{S M O}=6.9 \mathrm{~nm}$ ein Maximum in der $M(T)$-Kurve. Nachdem die Heterostrukturen mit einem angelegten Feld $H=+100$ Oe auf $T=5 \mathrm{~K}$ eingekühlt worden sind, wurden die $M(H)$-Kurven aufgenommen. In Abbildung 8.20 ist die Änderung der $M(H)$-Kurven mit steigender Schichtdicke $d_{S M O}$ illustriert: Die betrachtete Heterostruktur wurde auf einem STO-Substrat mit relativ kleinem Fehlschnitt aufgewachsen (siehe Abbildung 8.5). Dadurch wird die Umkehr der Magnetisierung durch die biaxiale Anisotropie dominiert, die sich aus der Verspannung durch das STO-Substrat ergibt. Da die leichten Richtungen dann entlang der $\langle 110\rangle$-Richtungen liegen [260, 261] und die Messung entlang der [100]-Richtung erfolgt, ergibt sich eine relativ kleine Remanenz $M_{R} \approx M_{S} / \sqrt{2}$. Nach der ersten Beschichtung mit SMO vergrößert sich das Koerzitivfeld $H_{C}$ deutlich und die Remanenz liegt näher an der Sättigungsmagnetisierung. Die $M(H)$-Kurve zeigt nicht mehr den Einfluss der biaxialen Anisotropie, sondern den einer uniaxialen, wobei das angelegte Feld in Richtung der leichten Achse verläuft. Mit Erhöhung von $d_{S M O}$ vergrößert sich das $H_{C}$ weiter und es tritt ein ausgeprägtes exchange bias auf. Man erkennt außerdem, dass die Umkehr der Magnetisierung in einem wesentlich breiteren Feldbereich stattfindet als für die kleineren Werte von $d_{S M O}$. Mit weiteren Depositionen von SMO steigt $H_{C}$ stetig an, während $H_{E}$ wieder abfällt. Zudem wird die Steigung, mit der die $M(H)$-Kurve die Feldachse bei $H_{C}^{+/-}$schneidet, kleiner. Für die größte Schichtdicke $d_{S M O}=6.9 \mathrm{~nm}$ fällt auf, dass sich der Verlauf der $M(H)$-Kurve für steigende und fallende Magnetisierung deutlich unterscheidet. Dies ist in ähnlicher Form auch für die SMO/LSMO/SMO-Heterostruktur zu erkennen (siehe Abbildung 8.17). Für den Teil der Kurve, wo die Magnetisierung fällt, d.h. entgegen das Einkühlfeld gedreht wird, ergibt sich eine deutliche Änderung der Steigung von $M(H)$ in der Nähe des Koerzitivfeldes. Dagegen zeigt sich bei der Drehung in die andere Richtung keine plötzliche Steigungsänderung. Zusammenfassend sind im unteren Teil von Abbildung 8.20 die Änderungen von $H_{C / E}$ mit der Schichtdicke $d_{S M O}$ dargestellt. Das Koerzitivfeld $H_{C}$ nimmt näherungsweise linear mit $d_{S M O}$ zu. Die Amplitude des exchange bias $H_{E}$ zeigt dagegen ein nichtmonotones Verhalten mit einem Maximum zwischen $d_{S M O}=3.3 \mathrm{~nm}$ und $d_{S M O}=5.4 \mathrm{~nm}$.

In den LSMO(x)/LSMO-Bilagen (siehe Abbildung 8.1 a)) zeigen die Curie-Temperatur $T_{C}$ und die Sättigungsmagnetisierung $M_{\text {Sat }}$ der LSMO-Schicht keine Abhängigkeit von der SrDotierung $x$. Beide Werte schwanken zufällig mit $\Delta T_{C}=6 \mathrm{~K}$ und $\Delta M_{\text {Sat }}=0.3 \mu_{\mathrm{B}} / \mathrm{Mn}$ um 

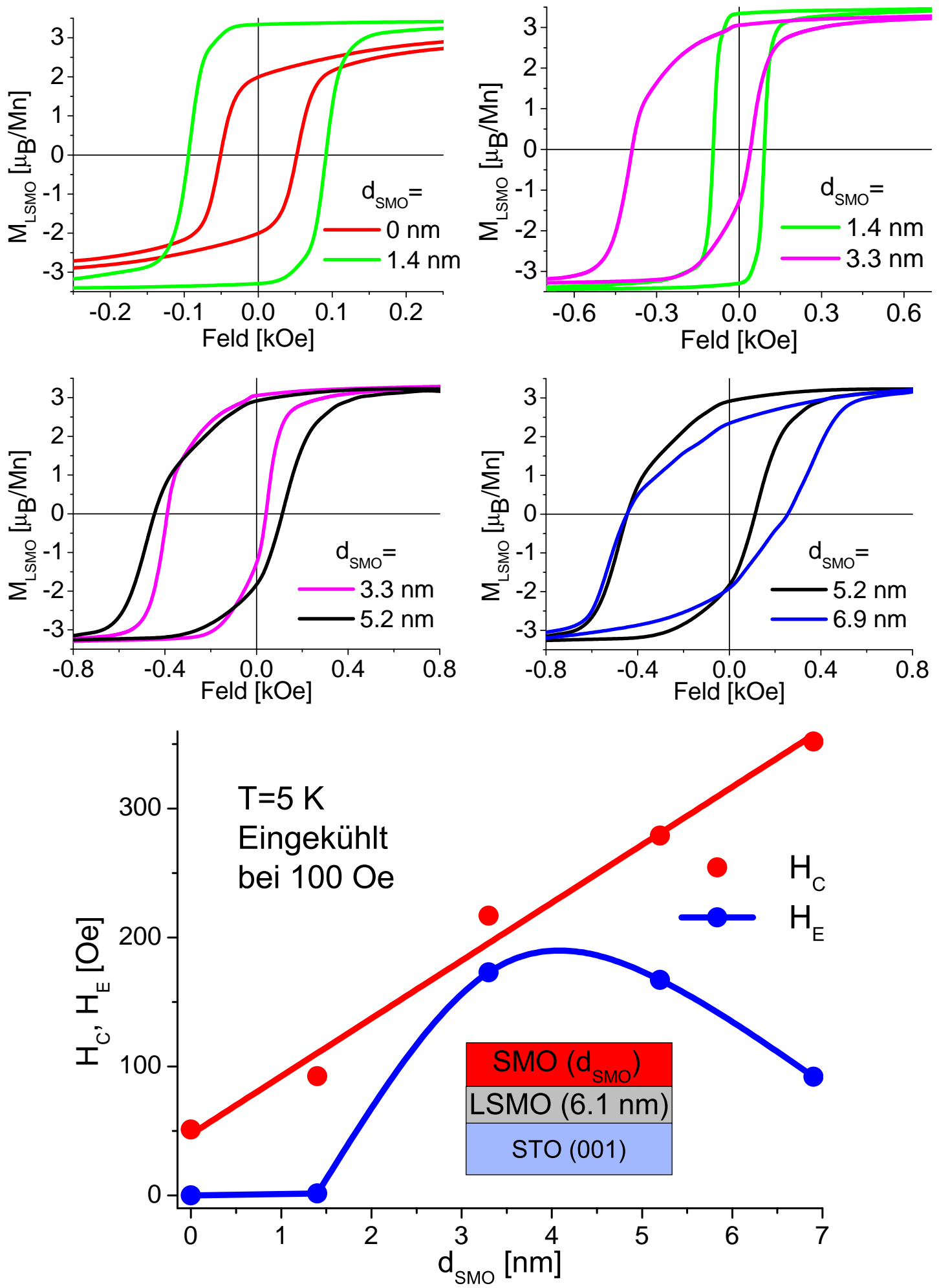

Abbildung 8.20.: Magnetische Hysterese der LSMO $\left(d_{L S M O} \approx 6 \mathrm{~nm}\right) / \mathrm{SMO}\left(d_{S M O}=\right.$ $0-6.9 \mathrm{~nm}$ )-Bilagen bei $T=5 \mathrm{~K}$ nach Einkühlen mit $H=+100$ Oe: Die gezeigten Paare von $M(H)$-Kurven zeigen, wie sich das magnetische Verhalten der Bilagen bei jeder Erhöhung von $d_{S M O}$ verändert (oben). Aus den $M(H)$-Kurven werden $H_{C / E}$ abgelesen und sind unten als Funktion von $d_{S M O}$ gezeichnet $\left(H_{C}\left(d_{S M O}\right)\right.$ wurde linear angepasst, die Verbindung der Datenpunkte von $H_{E}\left(d_{S M O}\right)$ erfolgt zur besseren Darstellung). 


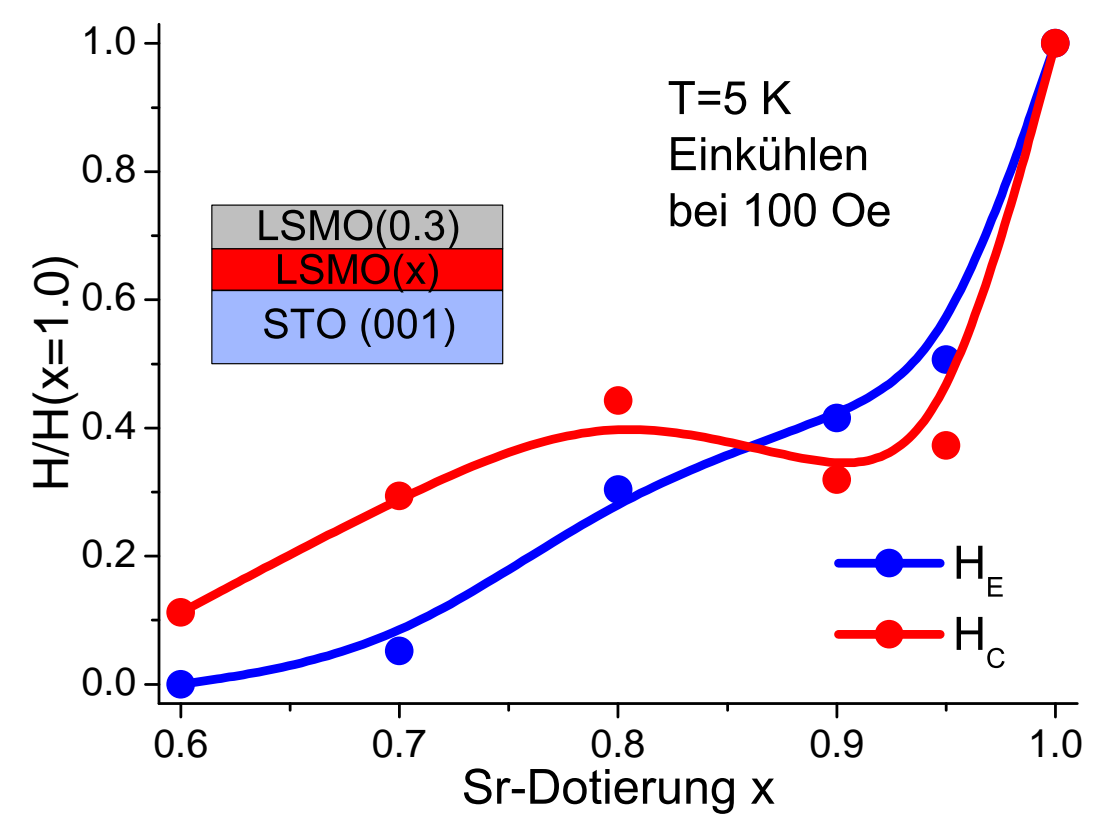

Abbildung 8.21.: LSMO(x)/LSMO-Heterostrukturen: Abhängigkeit des Koerzitivfeldes $H_{C}$ und der exchange bias-Amplitude $H_{E}$ von der Sr-Dotierung $x$ bei $T=5 \mathrm{~K}$ nach Einkühlen mit $H=+100$ Oe. Die jeweiligen Feldwerte wurden auf die Werte für $x=1$ normiert.

mittlere Werte von $T_{C}=335 \mathrm{~K}$ und $M_{S a t}=3.2 \mu_{\mathrm{B}} / \mathrm{Mn}$. Dies ergibt sich wahrscheinlich durch kleine Abweichungen der Schichtdicken und Unterschiede beim Fehlschnitt der Substrate, was zu Veränderungen der Wachstumsmode führt. Die LSMO(x)-Lagen tragen somit nicht zum messbaren magnetischen Moment bei und man kann annehmen, dass sich diese für alle $x$ antiferromagnetisch ordnen ${ }^{30}$ Als Funktion der Temperatur zeigen $H_{C / E}$ ein ähnliches Verhalten wie in den SMO/LSMO-Doppellagen und man beobachtet ein Verschwinden von $H_{E}$ für $T \approx 100 \mathrm{~K}$. Wenn man die Werte von $H_{E}$ und $H_{C}$ bei $T=5 \mathrm{~K}$ betrachtet, fällt sofort auf, dass $H_{E}$ und $H_{C}$ für $x=1$ etwa doppelt so groß sind wie für die anderen Dotierungen (siehe Abbildung 8.21). Die Signifikanz der Werte für $x=1$ zeigt sich in den ähnlichen Werten von $H_{C / E}$, die sich für die weiter oben gezeigten Heterostrukturen ergeben: SMO $(5.5 \mathrm{~nm}) / \mathrm{LSMO}(5.8 \mathrm{~nm})$ (Abbildung 8.15), SMO (5.4 nm)/LSMO (5.4 nm) (Abbildung 8.18) und LSMO $(6.1 \mathrm{~nm}) / \mathrm{SMO}(5.2 \mathrm{~nm})$ (Abbildung 8.20). Mit fallendem $x$ verkleinern sich $H_{E}$ und $H_{C}$ dann kontinuierlich und bei $x=0.6$ verschwindet $H_{E}$ und $H_{C}$ nimmt den gleichen Wert an wie für eine einfache LSMO-Schicht auf STO $(001)$ mit $d_{L S M O} \approx 5.5 \mathrm{~nm}$.

\subsection{Diskussion}

Die oben vorgestellten Multilagen aus LSMO und LSMO(x) weisen sehr scharfe Grenzflächen zwischen den unterschiedlichen Manganaten auf. Die beobachteten Rauigkeiten von $\sigma_{L S M O / L S M O(x)}=0.2-0.3 \mathrm{~nm}$ sind vergleichbar mit Werten, die sich bei der Herstellung von Übergittern aus Manganaten mit anderen Methoden ergeben. Bhattacharya et al. deponierten Übergitter aus LMO und SMO mittels Molekularstrahlepitaxie, wobei sie durch die Anpassung von XRR-Spektren eine Rauigkeit von $\sigma_{L M O / S M O}=0.23 \mathrm{~nm}$ bestimmen konnten

\footnotetext{
${ }^{30}$ Durch den Fehler bei der Auswertung der magnetischen Momente pro Mn-Ion kann es nicht ausgeschlossen werden, dass die $\operatorname{LSMO}(\mathrm{x})$-Schichten ein magnetisches Moment besitzen, das dem einer Monolage in der LSMO-Schicht entspricht.
} 
[111. Die Grenzflächen zwischen verschiedenen Manganaten sind deutlich abrupter als der Übergang zwischen Titanat (STO-Substrat) und einer Manganat-Schicht. Dies verdeutlicht, dass die unterschiedliche Besetzung der B-Plätze die treibende Kraft für die Reaktion der Manganat-Lagen mit dem Titanat-Substrat darstellt.

Dass es bei den mehrmaligen Beschichtungen der SMO/LSMO- und LSMO/SMO-Bilagen zu keinem Austausch der Sr- und La-Kationen an der Grenzfläche kommt, der zu einer Veränderung von $H_{E}$ führt, kann man durch den Vergleich der verschiedenen Varianten der hergestellten SMO/LSMO-Bilagen überprüfen (vergleiche Abbildung 8.1 a)-c)). Man betrachtet die Heterostrukturen, für die $d_{S M O} \approx d_{L S M O} \approx 5.5 \mathrm{~nm}$ gilt. Bei der in Abbildung $8.1 \mathrm{a}$ ) gezeigten Struktur ergibt sich dies nach einem einmaligen Depositionsvorgang, wogegen diese Schichtdicken für die LSMO/SMO- (Abbildung 8.1 c)) bzw. SMO/LSMO-Bilagen (Abbildung 8.1 b)) nach vier bzw. drei Depositionsprozeduren erreicht wurden. Bei gleichbleibender Sättigungsmagnetisierung der ferromagnetischen Lage, $M_{F M}$, ist $H_{E}$ aufgrund der an der Grenzfläche lokalisierten Kopplung umgekehrt proportional zur Schichtdicke des Ferromagneten $t_{F M}: H_{E} \propto \frac{1}{t_{F M}}$ 11. Da die Schichtdicke von LSMO in diesen Heterostrukturen etwas variiert, wird der zum Vergleich herangezogene Wert $H_{E}(T=5 \mathrm{~K})$ somit mit einem Faktor $s=\frac{d_{L S M O}}{5.5 \mathrm{~nm}}$ skaliert, um die Vergleichbarkeit zu verbessern. So ergeben sich folgende Werte von $s H_{E}$ für die drei Typen von Heterostrukturen: 198 Oe für die LSMO/SMO-Bilage, die sich nach vier Beschichtungen ergibt (Abbildung 8.1 c)), 208 Oe für die SMO/LSMOBilage, die sich nach einem Depositionsvorgang ergibt (Abbildung 8.1 a)), und 238 Oe für die SMO/LSMO-Heterostruktur, für die drei Beschichtungen erfolgten (Abbildung 8.1 b)). Diese ähnlichen Werte von $s H_{E}$ zeigen, dass das wiederholte Auslagern der Heterostrukturen bei Depositionsbedingungen keine Veränderungen der magnetischen Kopplungen an den SMO/LSMO-Grenzflächen bewirkt. Die beobachteten Unterschiede sind vermutlich mit Unterschieden des Verspannungszustandes in Verbindung zu bringen, der aus kleinen Differenzen von $d_{S M O}$ und $d_{L S M O}$ folgt.

Um den hier vorliegenden Mechanismus, der zum exchange bias führt, zu diskutieren, bietet sich eine Betrachtung von $H_{E}$ und $H_{C}$ in Abhängigkeit von der Schichtdicke des Antiferromagneten $t_{A F M}$ an. Hier ergibt sich als Funktion der Schichtdicke von SMO $d_{S M O}$ ein Maximum in $H_{E}$ bei $d_{S M O} \approx 4-12 \mathrm{ML} 31$, das Koerzitivfeld $H_{C}$ steigt monoton an (siehe Abbildung 8.20). Dieser nichtmonotone Verlauf von $H_{E}\left(d_{S M O}\right)$ widerspricht der einfachen theoretischen Vorhersage nach dem Modell von Meiklejohn und Bean, das die Anisotropie des Antiferromagneten $K_{A F M}$ berücksichtigt. Entsprechend Gleichung 3.9 erwartet man dort, dass sich $H_{E}\left(t_{A F M}\right)$ monoton ansteigend seinem asymptotischen Grenzwert nähert. Das dieser Theorie entsprechende Verhalten wird zum Beispiel von Kobrinskii et al. in $\mathrm{La}_{1 / 3} \mathrm{Ca}_{2 / 3} \mathrm{MnO}_{3} / \mathrm{La}_{2 / 3} \mathrm{Ca}_{1 / 3} \mathrm{MnO}_{3}$-Bilagen [246] und von Huijben et al. in Bilagen aus LSMO und $\mathrm{BiFeO}_{3}$ 249 registriert. Hier gibt es ebenso wie in den in der Theorie vorgestellten Modellen eine kritische Schichtdicke des Antiferromagneten $t_{A F M}^{c r i t}$, unterhalb der man kein exchange bias feststellen kann. Diese liegt für die LSMO/SMO-Bilagen im Bereich $t_{A F M}^{c r i t}=4-8 \mathrm{ML}$. Auch die sehr dünne SMO-Lage $\left(d_{S M O}=3-4 \mathrm{ML}\right)$ hat bereits einen starken Einfluss auf die $M(H)$-Kurve bei tiefen Temperaturen. Sie verursacht eine deutliche Vergrößerung von $H_{C}$ und unterdrückt die biaxiale Anisotropie, die sich in der LSMO-Schicht durch epitaktische Verspannungen ausbildet. Dies illustriert das Vorhandensein von antiferromagnetischer Ordnung in diesem ultradünnen SMO-Film und wirft die Frage auf, ob bei antiferromagnetischen Manganaten wie SMO die Zahl der magnetisch toten Lagen kleiner

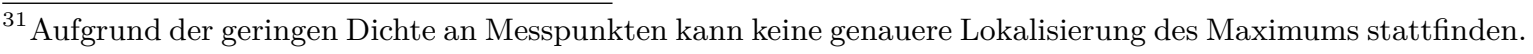


ist als bei ferromagnetischen wie LSMO. Das Vorhandensein von magnetisch toten Lagen, die keine antiferromagnetische Ordnung aufweisen, würde zu einer Erhöhung von $t_{A F M}^{c r i t}$ führen, die nicht einfach mit dem Verhältnis zwischen $J_{I F}$ und $K_{A F M}$ zusammenhängt (siehe Abschnitt 3.3.4).

Die Néel-Temperatur $T_{N}$ dieser dünnen antiferromagnetischen Filme kann man über die Modifikation der magnetischen Eigenschaften des ferromagnetischen Filmes abschätzen. Wie es in Abschnitt 3.3.4 der Theorie erläutert wurde, liegt die minimale Temperatur $T_{B}$, für die man noch EB beobachten kann, deutlich unterhalb der Néel-Temperatur $T_{N}$, da die Anisotropie des AFM, $K_{A F M}$, kontinuierlich mit steigender Temperatur abnimmt. Das Koerzitivfeld zeigt aber theoretisch bei Unterschreiten von $T_{N}$ einen sofortigen Anstieg. Damit kann man durch die Betrachtung von $H_{C}(T)$ Rückschlüsse auf $T_{N}$ ziehen. Für die hier betrachteten SMO/LSMO-Doppellagen zeigen die detaillierten Messungen von $H_{C}(T)$, dass die Néel-Temperatur der SMO-Filme mit einer Dicke $d_{S M O} \approx 5.5 \mathrm{~nm}$ mindestens oberhalb von $100 \mathrm{~K}$ liegt (siehe Abbildung 8.16 b)). Eine bessere Abschätzung nach unten erlaubt die $M(T)$-Kurve, die beim Kühlen im konstanten Feld für eine SMO/LSMO/SMO-Dreifachlage gemessen wurde (siehe Abbildung 8.17 a)). Der plötzliche Einbruch der Magnetisierung bei $T_{H} \approx 180 \mathrm{~K}$ kann darauf zurückgeführt werden, dass die beiden antiferromagnetischen SMOLagen einen zusätzlichen Anisotropieterm beitragen. Die leichte Richtung dieser Anisotropie liegt dabei nicht in Richtung des angelegten Feldes. Bei $T_{H} \approx 180 \mathrm{~K}$ ist die Anisotropie dann so groß, dass das externe Feld die Magnetisierung der LSMO-Schicht nicht mehr komplett parallel zu $H_{\text {ext }}$ ausrichten kann. Damit ist $T_{H} \approx 180 \mathrm{~K} \leq T_{N}$ die untere Grenze für die NéelTemperatur von mindestens einer der beiden SMO-Lagen in der SMO/LSMO/SMO-Trilage.

Bei Studien von exchange bias zwischen Manganaten verschiedener Dotierung in der Literatur ist die Dicke der antiferromagnetischen Manganatschichten zumeist deutlich größer als hier [246, 249, 262, 263. Das häufig behandelte System aus $\mathrm{La}_{1 / 3} \mathrm{Ca}_{2 / 3} \mathrm{MnO}_{3}$ und $\mathrm{La}_{2 / 3} \mathrm{Ca}_{1 / 3} \mathrm{MnO}_{3}$ zeigt mit $t_{A F M}^{c r i t} \approx 5 \mathrm{~nm}[249]$ eine deutlich größere kritische Schichtdicke des Antiferromagneten als das hier dargestellte LSMO/SMO-System. Andere Arbeitsgruppen, die das EB von ferromagnetischen Manganatschichten im Kontakt mit SMO beobachten, verwenden mit $d_{S M O} \approx 50 \mathrm{~nm}$ deutlich größere Schichtdicken als hier und betrachten nur eine Heterostruktur mit einer festen Schichtdicke von SMO [262, 263. $\mathrm{SrMnO}_{3}$ kommt nicht nur in der kubischen Modifikation vor, sondern auch in einer hexagonalen. Aufgrund des großen Toleranzfaktors $t=1.05$ stellt sich letztere bei einer bulk-Synthese von $\mathrm{SrMnO}_{3}$ unter atmosphärischen Bedingungen ein [264. Des Weiteren gibt es mit dem orthorhombischen $\mathrm{SrMnO}_{2.5}$ eine Variante, die sich unter reduzierenden Bedingungen einstellen kann. Da in $\mathrm{SrMnO}_{2.5}$ die Mn-Ionen mit einer Valenz von 3+ vorliegen, ist die Gitterkonstante dieses Materials größer als von $\mathrm{SrMnO}_{3}$. Dadurch sind epitaktische Verspannungen eine treibende Kraft zum Einbau von Sauerstoffleerstellen in SMO [54. Bei den Studien von Ding et al. ist der Gitterebenenabstand der SMO-Schicht entlang der c-Achse deutlich größer als der kubische Gitterparameter $a_{S M O}^{c u b}=0.3805 \mathrm{~nm}$, wobei die in dieser Arbeit betrachteten Heterostrukturen wie hier auf STO (001)-Substraten deponiert wurden. Dies schreiben die Autoren einer hexagonalen Kristallstruktur des SMO-Films zu [263]. Alternativ würde auch der Einbau von Sauerstoffleerstellen diese angehobene Gitterkonstante erklären. Bei den hier gezeigten SMO-Filmen ist davon auszugehen, dass die kubische Variante von $\mathrm{SrMnO}_{3}$ aufwächst. Einerseits wird der Einbau von Sauerstoffleerstellen durch den hohen Sauerstoffpartialdruck bei der Deposition und Abkühlung der Filme vermieden [212. Zum anderen sieht man in den Spektren der Röntgendiffraktion vom SMO nur Reflexe, die der Orientierung des STO (001)-Substrates entsprechen, und der Atomabstand entlang der c-Achse deutet einen epitaktisch verzerrten Zustand an, der mit zunehmender Filmdicke relaxiert. Auch die in Kapitel 9 


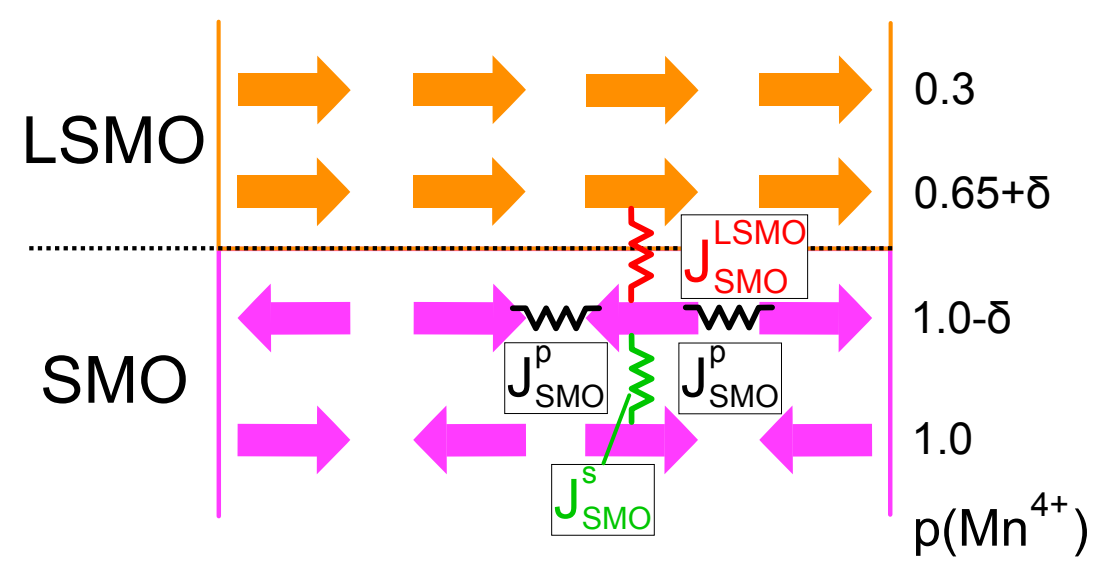

\begin{abstract}
Abbildung 8.22.: Illustration der magnetischen Wechselwirkungen eines Spins im SMO mit den benachbarten Spins im LSMO und SMO: $J_{S M O}^{p}$ bezeichnet die Wechselwirkung mit den SMO-Spins in der Ebene parallel zur Grenzfläche, $J_{S M O}^{s}$ mit dem in Richtung senkrecht zur Grenzfläche benachbarten Spin im SMO und $J_{S M O}^{L S M O}$ mit dem Spin im LSMO. p $\left(\mathrm{Mn}^{4+}\right)$ ist der Anteil an $\mathrm{Mn}^{4+}$-Ionen in der jeweiligen $\mathrm{MnO}_{2}$-Lage. Die Vermeidung der polaren Katastrophe sorgt für eine Akkumulation von $\mathrm{Mn}^{4+}$-Ionen an der Grenzfläche (vergleiche Abbildung 8.14. Zudem sorgt eine Delokalisierung der $e_{g}$-Elektronen für eine Veränderung $\delta<0.1$ von $\mathrm{p}\left(\mathrm{Mn}^{4+}\right)$ an der Grenzfläche.
\end{abstract}

aufgeführten TEM-Messungen von LMO/SMO-Übergittern unterstützen das Vorliegen einer kubischen Struktur von SMO.

In Doppellagen aus antiferromagnetischem IrMn und ferromagnetischem Co beobachtet man ein Maximum im Verlauf von $H_{E}\left(t_{A F M}\right)$ [265]. Dies wird von Ali et al. im domain state Modell begründet: Proportional zur Filmdicke des Antiferromagneten steigt die Energie einer Domänenwand, so dass sich die Zahl der Wände mit steigender Dicke der IrMn-Lage verkleinert. Damit verringert sich auch die Zahl der eingefrorenen Momente im Antiferromagneten, die für das EB verantwortlich sind 265]. Die Domänenwände bewegen sich durch die Kopplung zum Ferromagneten teilweise durch den Antiferromagneten und sorgen dadurch für eine Erhöhung des Koerzitivfeldes. Da mit zunehmender Dicke des Antiferromagneten auch die Zahl dieser beweglichen Domänenwände abfällt, verringert sich das Koerzitivfeld $H_{C}$ kontinuierlich mit steigendem $t_{A F M}$ [265]. Dagegen steigt hier das Koerzitivfeld monoton mit steigender Dicke des SMO-Films. Für die theoretische Beschreibung im Rahmen des domain state Modells ist eine Unordnung der magnetischen Austauschkonstanten zwischen den Momenten auf den magnetischen Ionen essentiell. Im Beispiel der IrMn-Schicht folgt diese aus den unterschiedlichen Wechselwirkungen der Ir-Mn- und Mn-Mn-Bindungen [265]. Für die hier untersuchten SMO-Lagen ist eine solche Unordnung, die sich über die ganze Dicke der antiferromagnetischen Schicht erstreckt, nicht zu konstruieren. Aufgrund der scharfen Grenzfläche zum LSMO befindet sich auf den A- und B-Plätzen jeweils nur eine Atomsorte, so dass es nicht zu zufälligen Akkumulationen einzelner Atomsorten kommen kann. Diese würden durch eine lokale Veränderung der Mn-O-Mn-Bindungswinkel oder eine zufällige Variation des Coulomb-Potentials der Kationen auf dem A-Platz eine räumliche Variation der Austauschkonstanten verursachen.

Während eine Unordnung der magnetischen Austauschwechselwirkungen zwischen den MnIonen innerhalb der SMO-Schicht auszuschließen ist, ergibt sich ein solcher Zustand automatisch durch den Symmetriebruch an der SMO/LSMO-Grenzfläche. Die SMO-Lage hat auch bei den starken epitaktischen Verspannungen durch das STO-Substrat im Grundzustand eine 
antiferromagnetische Ordnung des G-Typs [80] (vergleiche Abbildung 3.3). Nach den GKARegeln ist die Austauschwechselwirkung zwischen den leeren $e_{g}$-Orbitalen der $\mathrm{Mn}^{4+}$-Ionen immer antiferromagnetisch. Die durch die Verspannung bewirkte energetische Absenkung der $d_{x^{2}-y^{2}}$-Orbitale führt nur dazu, dass die Kopplung innerhalb der (001)-Ebenen stärker ist als entlang der c-Achse. Mit der Auswertung der in-situ Ellipsometrie kommt man zu dem Ergebnis, dass der Anteil an $\mathrm{Mn}^{4+}$-Ionen in der $\mathrm{MnO}_{2}$-Grenzlage des LSMO zum SMO etwa 0.6 beträgt (siehe Abschnitt 8.2.5). Für eine epitaktisch verspannte Schicht mit einem $\mathrm{Mn}^{4+}$-Anteil von 0.6 erwartet man theoretisch und auch nach den Experimenten hier eine antiferromagnetische Ordnung des A-Typs [80]. Da die $\mathrm{Mn}^{4+}$-Dotierung von 0.6 nur in einer einzelnen $\mathrm{MnO}_{2}$-Lage vorliegt, besteht die Frage, ob diese Lage ferromagnetisch oder antiferromagnetisch an den Hauptteil der ferromagnetischen LSMO-Schicht koppelt. Wenn man die LSMO-Lage $\left(d_{L S M O} \approx 6.0 \mathrm{~nm}\right)$ mit SMO beschichtet, steigt die Curie-Temperatur und die Sättigungsmagnetisierung $M_{\text {sat }}$. Die absolute Änderung des Momentes entspricht dabei etwa dem Beitrag von 1-2 Monolagen $\mathrm{MnO}_{2}$. Daraus kann man schließen, dass die Grenzlagen zum SMO dann ferromagnetisch zum Hauptteil der LSMO-Schicht ausgerichtet sind. Der verringerte Wert von $M_{\text {sat }}$ ohne SMO an der Oberfläche ist auf das Fehlen von magnetischer Ordnung an der freien Oberfläche zurückzuführen.

Damit ergibt sich das in Abbildung 8.22 gezeigte Bild von der magnetischen Grenzfläche zwischen SMO, dem Antiferromagneten des G-Typs, und dem ferromagnetischen LSMO. An der Grenzfläche treten dabei gegensätzliche magnetische Kopplungen auf. Entsprechend der Darstellung in Abbildung 8.22 erfährt ein Spin der SMO-Schicht folgende Kopplungen an der Grenzfläche: Innerhalb der SMO-Lage koppelt er mit $J_{S M O}^{p}$ an vier andere SMO-Spins, die bei einem Antiferromagneten des G-Typs alle antiparallel zum betrachteten Spin stehen. $J_{S M O}^{p}$ wird einerseits wie im bulk-Material vom Superaustausch bestimmt, andererseits kommt es an der Grenzfläche zum LSMO zu einem Ladungstransfer $\delta$ der $e_{g}$-Elektronen. Dieser führt zu einem zusätzlichen Anteil des Doppelaustausches in $J_{S M O}^{p}$, der über die in der Ebene liegenden $d_{x^{2}-y^{2}}$-Orbitale vermittelt wird. Durch die epitaktische Verspannung wird die Entartung der $e_{g}$-Orbitale aufgehoben, so dass der Austausch senkrecht zur Grenzfläche über die

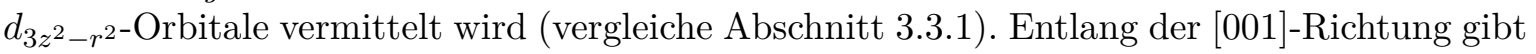
es so eine Kopplung zu einem Spin im SMO $J_{S M O}^{S}$, die hauptsächlich durch den Superaustausch gegeben ist, und die Kopplung zum LSMO $J_{S M O}^{L S M O}$, die aufgrund des ferromagnetisch metallischen Zustandes im LSMO eine parallele Orientierung der Spins gemäß des Doppelaustausches unterstützt (siehe Abbildung 8.23). Der in Abbildung 8.22 herausgestellte Spin ist also frustrierten magnetischen Kopplungen ausgesetzt.

Durch die zufällige Verteilung der La- und Sr-Ionen auf den A-Plätzen im LSMO ergibt sich zudem eine lokale Variation der jeweiligen Kopplungskonstanten auf atomarer Skala. Die leicht verschiedenen Ionenradion der $\mathrm{Sr}^{2+}$ - und $\mathrm{La}^{3+}$-Ionen bewirken Unterschiede der Mn-O-Mn-Bindungswinkel und damit des Hopping-Integrals $t_{d d}$, das die Stärke von Superund Doppelaustausch bestimmt. Des Weiteren zeigt das von den Ionen auf den A-Plätzen beeinflusste Coulomb-Potential eine räumliche Inhomogenität. Da die Abschirmlänge in LSMO im Bereich einer Monolage liegt [106], folgt daraus eine Modifikation des Ladungstransfers der $e_{g}$-Elektronen und der magnetischen Kopplungen an der LSMO/SMO-Grenzfläche. Nach Radu und Zabel führt diese Koinzidenz von frustrierten magnetischen Wechselwirkungen und einer räumlichen Unordnung der Austauschkonstanten zu einem Spin-Glas-Zustand an der FM/AFM-Grenzfläche 11. Theoretische Berechnungen zeigen, dass man in diesem Modell ein Maximum im Verlauf von $H_{E}\left(t_{A F M}\right)$ erwarten kann. Zudem ergibt sich für $t_{A F M}>t_{A F M}^{c r i t}$ ein kontinuierlicher Anstieg des Koerzitivfeldes [11]. Dies entspricht dem hier experimentell ermittelten Verhalten (siehe Abbildung 8.20 . Allerdings gibt es Abweichungen für $t_{A F M}<t_{A F M}^{\text {crit }}$ und Unterschiede im Verhältnis $H_{E} / H_{C}$. Dies ist auf die stark vereinfachten Annahmen des 


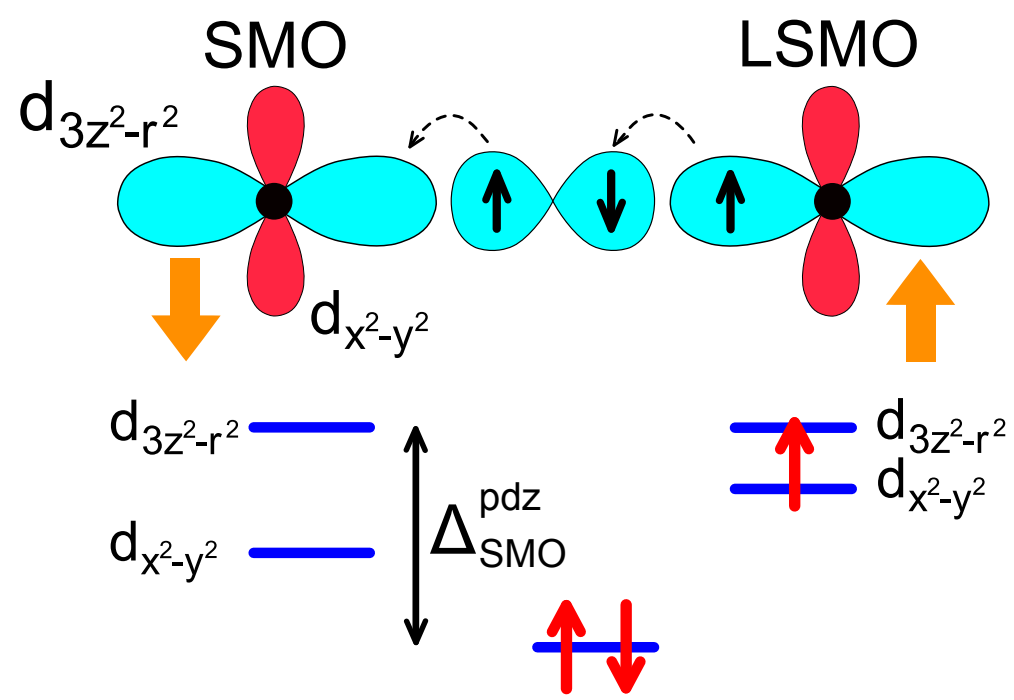

\begin{abstract}
Abbildung 8.23.: Genauere Betrachtung von $J_{S M O}^{L S M O}$ (vergleiche Abbildung 8.22): Es erfolgt Doppelaustausch entlang der $d_{3 z^{2}-r^{2}}$-Orbitale, der eine parallele Stellung der $t_{2 g^{-}}$ Rumpfspins in LSMO und SMO favorisiert. Durch die epitaktische Zugspannung werden die $d_{x^{2}-y^{2}}$-Orbitale, die in der Ebene parallel zur Grenzfläche liegen, energetisch abgesenkt. Im LSMO ist diese Aufspaltung durch die kleinere Verzerrung und den homogenen ferromagnetisch metallischen Zustand deutlich kleiner als im SMO, wo $\Delta_{S M O}^{p d z}$ die Energielücke

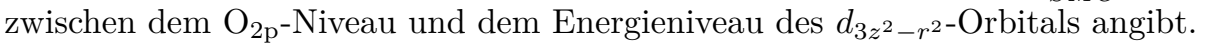

Modells zurückzuführen, das nur von wenigen Parametern abhängt und nicht berücksichtigt, dass diese, insbesondere für die hier untersuchten Materialsysteme, von der Schichtdicke des Antiferromagneten $t_{A F M}$ abhängen können.

Das Vorhandensein von magnetisch toten Lagen in der SMO-Schicht würde die Abweichungen im Bereich $t_{A F M}<t_{A F M}^{c r i t}$ erklären. Dadurch würde $K_{A F M}$ für sehr dünne SMO-Schichten rapide sinken, so dass sich die Spins leichter mit der Magnetisierung der LSMO-Schicht drehen. Somit beobachtet man entgegen der theoretischen Vorhersagen von Radu und Zabel keinen starken Abfall von $H_{C}$, nachdem $t_{A F M}$ die kritische Schichtdicke $t_{A F M}^{\text {crit }}{ }_{\text {überschritten hat. Für }}$ $t_{A F M}>t_{A F M}^{c r i t}$ dreht sich der Hauptteil der antiferromagnetischen Schicht nicht mehr mit dem angekoppelten Ferromagneten, so dass sich im Spin-Glas-Zustand an der Grenzfläche eingefrorene Momente bilden können. Radu und Zabel charakterisieren dieses Spin-Glas mit dem Parameter $f$, wobei sich für $f=0$ alle Spins mit dem Ferromagneten drehen und keine eingefrorenen Momente mehr existieren (siehe Abschnitt 3.3.4). Durch die starken Korrelationen mit dem Gitter kann man für die hier studierten Multilagen aus Manganaten erwarten, dass sich die Eigenschaften des Spin-Glases und damit der Parameter $f$ als Funktion von $d_{S M O}$ verändern. Wie es aus Abbildung 8.12 b) ersichtlich wird, entspannt der SMO-Film mit zunehmender Schichtdicke. Durch die verkleinerte tetragonale Verzerrung des Gitters nimmt der Energieunterschied zwischen dem $d_{3 z^{2}-r^{2-}}$ und dem $d_{x^{2}-y^{2}}$ Orbital ab, wodurch sich auch die Energielücke $\Delta_{S M O}^{p d z}$ zwischen dem $\mathrm{O}_{2 \mathrm{p}}$-Niveau und dem $d_{3 z^{2}-r^{2}}$-Orbital verringert (vergleiche Abbildung 8.23). Über Gleichung 2.3 ist $\Delta_{S M O}^{p d z}$ direkt mit dem Transfermatrixelement $t_{d d}$ verknüpft, so dass der Betrag von $J_{S M O}^{L S M O}$ mit $d_{S M O}$ anwächst. Durch diese verstärkte Ankopplung an die LSMO-Schicht gibt es im Spin-Glas an der Grenzfläche mehr Momente, die sich mit der LSMO-Schicht drehen, und weniger Momente, die fest bleiben. Im Modell von Radu und Zabel entspricht dies einer Vergrößerung von $J_{I F}$ bei einer gleichzeitigen Verkleinerung von $f$ (vergleiche Abschnitt 3.3.4). 
Im Spin-Glas-Modell tragen diese beiden Veränderungen des magnetischen Zustandes der Grenzfläche zusammen zu einer Erhöhung der effektiven Anisotropie, $K_{S G}^{e f f}=(1-f) J_{I F}$ (Gleichung 3.11), der drehbaren Spins an der Grenzfläche und damit zu einem Anstieg des Koerzitivfeldes bei. Im Bezug auf die Amplitude des exchange bias $H_{E}$ haben die Veränderungen von $f$ und $J_{I F}$ einen gegensätzlichen Effekt. $H_{E}$ ist proportional zur effektiven Kopplung, $J_{I F}^{e f f}=f J_{I F}$ (Gleichung 3.11). Demzufolge können sich die individuellen Änderungen von $f$ und $J_{I F}$ kompensieren, so dass sich $H_{E}$ nur wenig ändert. Dies ist hier für die Erhöhung der Schichtdicke von $d_{S M O} \approx 3.3 \mathrm{~nm}$ auf $d_{S M O} \approx 5.2 \mathrm{~nm}$ zu beobachten, wo sich $H_{E}$ nur wenig ändert (siehe Abbildung 8.20). Für eine weitere Vergrößerung auf $d_{S M O} \approx 6.9 \mathrm{~nm}$ überwiegt die Veränderung des magnetischen Zustandes an der Grenzfläche und damit die Verringerung von $f$, so dass $H_{E}$ absinkt. Die drehbaren Momente an der LSMO/SMO-Grenzfläche besitzen eine gewisse Verteilung hinsichtlich ihrer effektiven Anisotropie $K_{S G}^{\text {eff }}$ und ihrer leichten Richtungen, die man mit dem Winkel $\theta_{K S G}$ zwischen der individuellen leichten Achse des Moments und der leichten Achse des Antiferromagneten ausdrückt (vergleiche Gleichung 3.10). Bei zunehmender Kopplungsstärke nimmt die Zahl der drehbaren Momente zu und infolgedessen auch die Breite der Verteilungen von $K_{S G}^{e f f}$ und $\theta_{K S G}$. Daraus resultiert die Verbreiterung des Bereichs, in dem die Umkehr der Magnetisierung stattfindet, und die Verringerung der Remanenz, wenn man $d_{S M O}$ erhöht (siehe Abbildung 8.20 .

Da die magnetischen Kopplungen an der Grenzfläche stattfinden, sind die Amplitude des exchange bias $H_{E}$ und das Koerzitivfeld $H_{C}$ theoretisch umgekehrt proportional zum magnetischen Moment $m=M_{F M} t_{F M}$ der ferromagnetischen Schicht. Diese Relation wurde in der Literatur für viele verschiedene Systeme experimentell bestätigt [11, 124, 125]. Hier wurden SMO/LSMO-Doppellagen verglichen, die bei einer konstanten Dicke der SMO-Schicht $\left(d_{S M O} \approx 5.5 \mathrm{nmn}\right)$ LSMO-Filme verschiedener Dicke enthielten (siehe Abbildung 8.1 b)). Man sieht, dass die mit $m$ multiplizierten charakteristischen Felder, $J_{E / C}=m H_{C / E}$, für die kleinste Schichtdicke deutlich abfallen. Für die beiden dickeren LSMO-Filme ergeben sich sehr ähnliche Werte, die die an der Grenzfläche lokalisierte Kopplung bestätigen (siehe Inset von Abbildung 8.18 b)). Die dünnste LSMO-Lage mit $d_{L S M O} \approx 3.0 \mathrm{~nm}$ weist mit $M_{s a t} \approx 2.0(1) \mu_{\mathrm{B}} / \mathrm{Mn}$ bereits eine deutlich reduzierte Sättigungsmagnetisierung und verkleinerte Curie-Temperatur $T_{C} \approx 290(5) \mathrm{K}$ auf. Infolgedessen induziert diese dünne LSMO-Schicht kleinere Veränderungen des magnetischen Zustandes der SMO-Schicht an der Grenzfläche als die dickeren LSMOSchichten. Der Zustand der Spins an der Grenzfläche ist so näher an der Konfiguration, die sich für einen idealen Antiferromagneten des G-Typs ergibt. Aufgrund der magnetisch kompensierten Grenzfläche erwartet man für diesen Idealzustand kein exchange bias und auch keine Vergrößerung des Koerzitivfeldes.

Nach der Diskussion der Übergitter aus reinem SMO und LSMO soll nachfolgend der Effekt der La-Dotierung der SMO-Schicht beleuchtet werden. Man sieht einen plötzlichen Einbruch von $H_{E}$ und $H_{C}$ auf etwa die Hälfte der Werte bei $x=1.0$, sobald man der SMO-Schicht eine kleine Menge von La-Ionen zufügt (siehe Abbildung 8.21). Für diesen Dotierungsbereich findet man im Phasendiagramm von $\mathrm{La}_{1-\mathrm{x}} \mathrm{Sr}_{\mathrm{x}} \mathrm{MnO}_{3}$ um $x=0.95$ einen rapiden Übergang von einem Antiferromagneten des G-Typs zu einem des C-Typs (siehe Abbildung 2.5). Verkleinert sich die Sr-Dotierung dann weiter, so sieht man eine stetige Abnahme von $H_{C}$ und $H_{E}$, zu der man keine Entsprechung im bulk-Phasendiagramm finden kann. Um das Verhalten für $x \geq 0.95$ zu erklären, muss zunächst untersucht werden, welcher Typ antiferromagnetischer Ordnung in den LSMO(x)-Filmen vorliegt. Dadurch dass die beiden $e_{g}$-Orbitale in der bulkPhase fast entartet sind, ergibt sich eine starke Empfindlichkeit der magnetischen Ordnung gegenüber epitaktischer Verspannung [38, 80]. Die hier vorliegenden LSMO(x)-Filme stehen alle unter einer starken Zugspannung durch das STO-Substrat. Unter der Annahme eines 


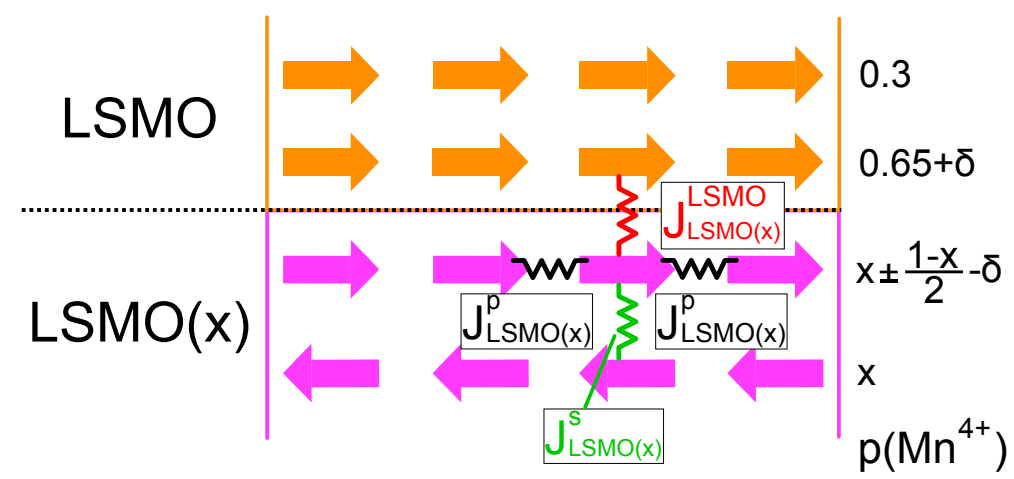

\begin{abstract}
Abbildung 8.24.: Magnetische Wechselwirkungen an der Grenzfläche zwischen $\mathrm{La}_{1-\mathrm{x}} \mathrm{Sr}_{\mathrm{x}} \mathrm{MnO}_{3}(\mathrm{LSMO}(\mathrm{x}))$, das eine antiferromagnetische Ordnung des A-Typs zeigt und ferromagnetischem $\mathrm{La}_{0.7} \mathrm{Sr}_{0.3} \mathrm{MnO}_{3}$ (LSMO): $J_{L S M O(x)}^{p}$ bezeichnet die Kopplung zu den benachbarten Spins in der Ebene parallel zur Grenzfläche, $J_{L S M O(x)}^{s}$ die Kopplung zum Nachbarspin in [001]-Richtung und $J_{L S M O(x)}^{L S M O}$ die Kopplung zum benachbarten Spin der LSMO-Schicht. $\mathrm{p}\left(\mathrm{Mn}^{4+}\right)$ bezeichnet den Anteil der $\mathrm{Mn}^{4+}$-Ionen in der jeweiligen $\mathrm{MnO}_{2}{ }^{-}$ Lage. Zur Vermeidung der polaren Katastrophe ergibt sich für die LSMO-Lage an der Grenzfläche ein Wert $\mathrm{p}\left(\mathrm{Mn}^{4+}\right)=0.65$ (siehe Abbildung 8.14). Für die LSMO(x)-Lage ergibt sich in Abhängigkeit der Terminierung des STO-Substrates $\mathrm{p}\left(\mathrm{Mn}^{4+}\right)=\mathrm{x} \pm \frac{1-\mathrm{x}}{2}$. Durch die Delokalisierung der $e_{g}$-Elektronen der ferromagnetisch metallischen LSMO-Schicht ergibt sich zudem eine Modifikation von $\mathrm{p}\left(\mathrm{Mn}^{4+}\right)$ um $\delta$.
\end{abstract}

Poisson-Verhältnisses von $\nu=1 / 3$ und den bekannten Gitterparametern für bulk-Manganate [38 kann man mit den Werten für die Gitterparameter in c-Richtung aus Abbildung 8.13 die Tetragonalität $c / a$ der gewachsenen Schichten abschätzen. Für $x \leq 0.95$ ergibt sich damit $0.96 \leq c / a \leq 0.97$. Nach dem theoretischen Phasendiagramm von Fang et al. würde eine Verzerrung dieser Größenordnung eine klare Bevorzugung der antiferromagnetischen Ordnung des A-Typs verursachen 80. Auch die Experimente an bulk-Einkristallen zeigen, dass schon kleine Tetragonalitäten in der Größenordnung von $(c-a) / a \approx \pm 0.4 \%$ den Ausschlag zwischen einer antiferromagnetischen Ordnung des A- und C-Typs geben können [38]. Somit ist hier für die LSMO(x)-Filme mit $x=0.6-0.95$ von einer antiferromagnetischen Ordnung des A-Typs auszugehen.

Zur Vermeidung der polaren Katastrophe beim Wachstum des LSMO(x)-Filmes auf dem STO-Substrat ergibt sich ein Ladungstransfer, der abhängig von der Terminierung des STOSubstrates zu einer Änderung von $\mathrm{p}\left(\mathrm{Mn}^{4+}\right)$ um $\pm \frac{1-\mathrm{x}}{2}$ in der obersten $\mathrm{MnO}_{2}$-Lage der LSMO(x)-Schicht führt (siehe Abbildung 8.24). Durch die kompensierte Spinkonfiguration an der Grenzfläche ergeben sich die frustrierten magnetischen Kopplungen nicht direkt an der Grenzfläche, sondern eine Lage tiefer innerhalb der LSMO(x)-Lage. Der LSMO-Film ist in einem ferromagnetisch metallischen Zustand und wächst unverspannt auf der $\operatorname{LSMO}(\mathrm{x})$ Schicht auf. Letzteres zeigt sich durch den gemessenen Gitterebenenabstand entlang der [001]Richtung, der der pseudokubischen Gitterkonstanten entspricht (siehe Abbildung 8.13). Im LSMO sind also die $e_{g}$-Orbitale entartet. Dadurch ergibt sich mit dem Doppelaustausch eine ferromagnetische Austauschkopplung $J_{\operatorname{LSMO}(x)}^{L S M O}$ an die $\operatorname{LSMO}(\mathrm{x})$-Lage.

Durch die epitaktische Verspannung liegt in der LSMO(x)-Lage eine grundsätzliche Präferenz der $d_{x^{2}-y^{2}}$-Orbitale vor, die den Antiferromagnetismus des A-Typs bewirkt. Wie es schon in der Theorie besprochen wurde, kann es an Grenzflächen zu einer Veränderung der orbitalen Polarisation kommen (siehe Abschnitt 3.2). Für LSMO-Filme auf STO-Substraten wird dies 
einer verbesserten Delokalisierung der $e_{g}$-Elektronen zugeschrieben [83]. Auch hier kommt es zu einer leichteren Delokalisierung der $e_{g}$-Elektronen und damit Reduktion der kinetischen Energie, wenn auf den Mn-Ionen in der Grenzlage des LSMO(x)-Filmes das $d_{x^{2}-y^{2}}$ und

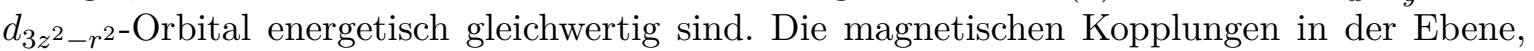
$J_{L S M O(x)}^{p}$, (siehe Abbildung 8.24 bleiben auch bei dieser veränderten orbitalen Polarisation ferromagnetische Austauschkopplungen gemäß des Doppelaustausches. Dagegen ergibt sich für $J_{L S M O(x)}^{s}$ eine Konkurrenz zwischen ferromagnetischem Doppelaustausch und antiferromagnetischem Superaustausch. Die zufälligen Besetzungen der A-Plätze mit Sr- und La-Ionen können durch ihre lokalen Modifikationen des Gitters und des Coulomb-Potentials die Art und Stärke der insgesamt resultierenden magnetischen Kopplung vorgeben. Für die Spins in der zweiten $\mathrm{MnO}_{2}$-Lage ergibt sich also ein Spin-Glas-Zustand, der für das exchange bias verantwortlich ist.

Mit sinkender Sr-Dotierung $x$, d.h. steigender Zahl von $e_{g}$-Elektronen in der LSMO(x)Schicht, verstärkt sich der Doppelaustausch zwischen der LSMO-Schicht und der obersten $\mathrm{MnO}_{2}$-Lage im LSMO(x)-Film. Diese stärkere Delokalisierung der Ladungsträger an der LSMO/LSMO(x)-Grenzfläche sorgt außerdem dafür, dass in der Grenzlage der LSMO(x)Schicht mehr Elektronen die $d_{3 z^{2}-r^{2}}$-Orbitale besetzen ${ }^{32}$. Durch diese verstärkte Kopplung der ferromagnetischen Schicht an den Antiferromagneten dreht sich ein größerer Teil der magnetischen Momente im Spin-Glas an der Grenzfläche mit dem Ferromagneten. Im SpinGlas-Modell des exchange bias folgt daraus die beobachtete Verkleinerung von $H_{E}$ (siehe Abbildung 8.21). Die zugleich stattfindende Abnahme von $H_{C}$ muss mit einer sinkenden Anisotropie der drehbaren Momente verbunden sein. Mit sinkender Sr-Dotierung $x$ treten in bulk-Kristallen von $\operatorname{LSMO}(\mathrm{x})$ die antiferromagnetischen Ordnungen zunehmend in Konkurrenz zu einer dreidimensionalen ferromagnetischen Ordnung. Für $x \approx 0.6$ mündet diese Konkurrenz in einer Koexistenz von ferromagnetischer und antiferromagnetischer Phase im Grundzustand [38, 39]. Diese beiden konkurrierenden magnetischen Wechselwirkungen sorgen dann für eine leichtere Drehbarkeit der rotierbaren Momente und damit für die Abnahme von $H_{C}$ mit sinkendem $x$.

\subsection{Zusammenfassung}

In diesem Kapitel wurde das magnetische Verhalten von $\mathrm{La}_{1-\mathrm{x}} \mathrm{Sr}_{\mathrm{x}} \mathrm{MnO}_{3} / \mathrm{La}_{0.7} \mathrm{Sr}_{0.3} \mathrm{MnO}_{3}$ $(\operatorname{LSMO}(\mathrm{x}) / \mathrm{LSMO})-$ Heterostrukturen mit $x=0.6-1.0$ untersucht. Mittels metallorganischer Aerosoldeposition (MAD) konnten qualitativ hochwertige Mehrfachlagen deponiert werden, die ein zweidimensionales Wachstum entsprechend einer step-flow-Wachstumsmode aufweisen. Hinsichtlich der Verteilung von La- und Sr-Ionen sind die Grenzflächen zwischen den verschiedenen Manganaten chemisch scharf mit einer Rauigkeit $\sigma \approx 0.2 \mathrm{~nm}$. Dies ist vergleichbar mit der Qualität von Grenzflächen, die mit anderen Methoden wie Molekularstrahlepitaxie präpariert wurden [111]. Durch die numerische Anpassung der gemessenen in-situ Ellipsometrie stellt man beim Wachstum von $\mathrm{La}_{0.7} \mathrm{Sr}_{0.3} \mathrm{MnO}_{3}$ auf $\mathrm{SrMnO}_{3}(\mathrm{SMO})$ einen Transfer von Elektronen zur Oberfläche der LSMO-Schicht fest. Dadurch beträgt der Anteil von $\mathrm{Mn}^{4+}$ Ionen $\mathrm{p}\left(\mathrm{Mn}^{4+}\right) \approx 0.6$ in der ersten $\mathrm{MnO}_{2}$-Lage, die auf die $\mathrm{SrMnO}_{3}$-Schicht trifft. Dieser Ladungstransfer wird mit der Vermeidung der polaren Katastrophe in Verbindung gebracht.

\footnotetext{
${ }^{32} \mathrm{Da}$ sich die Tetragonalität für $x \leq 0.95$ in einem relativ schmalen Bereich $0.96 \leq c / a \leq 0.97$ bewegt, ist die durch die epitaktische Verspannung begründete Umbesetzung der $e_{g}$-Orbitale nur schwach abhängig von der Sr-Dotierung.
} 
Für $x=0.7-1.0$ beobachtet man in diesen Heterostrukturen eine Vergrößerung des Koerzitivfeldes und exchange bias. Mit der Sr-Dotierung $x$ verändert sich der Typ der antiferromagnetischen Ordnung der hier untersuchten verspannten LSMO(x)-Filme. Der Übergang von einer Ordnung des G-Typs für $x=1.0$ zu einer Ordnung des A-Typs für $x \leq 0.95$ wird von einer drastischen Abnahme des Koerzitivfeldes $H_{C}$ und der Amplitude des exchange bias $H_{E}$ begleitet. Die frustrierten magnetischen Kopplungen an der Grenzfläche zwischen dem Ferromagneten und den antiferromagnetischen Schichten führen zu einem Spin-GlasZustand der Grenzfläche, woraus das exchange bias resultiert. Für LSMO/SMO-Doppellagen zeigt sich ein Maximum von $H_{E}$ als Funktion der Schichtdicke des Antiferromagneten $d_{S M O}$, wogegen $H_{C}$ kontinuierlich ansteigt. Diese Phänomenologie wird dadurch erklärt, dass der SMO-Film mit steigender Schichtdicke seine epitaktischen Verspannungen abbaut, wodurch sich die energetische Aufspaltung der beiden $e_{g}$-Orbitale reduziert. Damit verändert sich die magnetische Ankopplung an die ferromagnetische Schicht über den Doppelaustausch, was den Spin-Glas-Zustand der Grenzfläche modifiziert. 



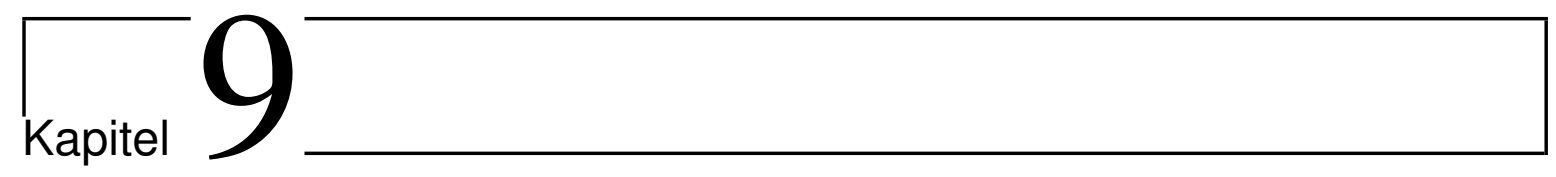

\section{$\mathrm{LaMnO}_{3} / \mathrm{SrMnO}_{3}$-Übergitter}

\subsection{Motivation}

Wie es bereits in der Theorie angeklungen ist, haben Übergitter (SL) aus $\mathrm{LaMnO}_{3}$ (LMO) und $\mathrm{SrMnO}_{3}(\mathrm{SMO})$ eine Vielzahl an theoretischen und experimentellen Studien angeregt. Dieses Materialsystem ist ein Prototyp für das Studium von Grenzflächeneffekten, bei denen durch das Zusammenspiel der Freiheitsgrade der Ladungen und der Orbitale die magnetischen und elektrischen Eigenschaften bestimmt werden. Für die bisherigen experimentellen Studien in der Literatur wurden die Übergitter mit den typischen Depositionsmethoden wie gepulster Laserdeposition (PLD) und Molekularstrahlepitaxie (MBE) präpariert (siehe Kapitel 3). Besonders mit Blick auf die Sauerstoffpartialdrücke weichen die Wachstumsbedingungen dieser Methoden deutlich von den Wachstumsbedingungen der metallorganischen Aerosol Deposition (MAD) ab. Im Vergleich zur PLD gibt es zudem gravierende Unterschiede bei den kinetischen Energien der auf das Substrat treffenden Teilchen: Bei der PLD ergibt sich durch die Beschleunigung der Ionen im Plasma eine sehr breite Energieverteilung mit einer mittleren Energie von einigen eV. Dagegen sind die Teilchenenergien bei der MBE und MAD moderat und liegen im Bereich von $\sim 0.1 \mathrm{eV}$ 266. Des Weiteren findet die Abscheidung von Material bei der PLD in einem sehr kurzen Zeitintervall $\Delta t \sim 1 \mu$ s statt, so dass es nach jedem Laserpuls zu Relaxationsprozessen kommt, bei der die hochenergetischen Teilchen thermalisieren und diffundieren. Bei der Thermalisierung der hochenergetischen Ionen kann es dabei zum Einbau von Nichtgleichgewichtsdefekten kommen [266. Dies kann zum einen zu Unterschieden hinsichtlich der Defekte innerhalb der individuellen LMO- und SMO-Schichten führen (siehe Abschnitt 3.1 und zum anderen eine andere Qualität der Grenzflächen hervorrufen. Von May et al. wurde beobachtet, dass nanoskalige Rauigkeiten an den LMO/SMO-Grenzflächen zu einer Unterdrückung der ferromagnetischen Ordnung führen können [13, 14]. Wie es in den vorherigen Kapiteln aufgezeigt wurde, wird die Wachstumsmode der Perowskit-Manganate bei der MAD von step-flow-Wachstum dominiert. Damit wären nanoskalige Rauigkeiten der LMO/SMO-Grenzflächen auszuschließen.

In Kapitel 6 wurde der Zusammenhang zwischen der Mn-Valenz und dem Brechungsindex von $\mathrm{La}_{1-\mathrm{x}} \mathrm{Sr}_{\mathrm{x}} \mathrm{MnO}_{3}$-Filmen aufgezeigt. Dies wurde dann in Kapitel 8 verwendet, um unter Ausnutzung der beim Wachstum aufgenommenen in-situ Ellipsometrie die Umverteilung der $e_{g}$-Elektronen an der Grenzfläche zwischen $\mathrm{La}_{0.7} \mathrm{Sr}_{0.3} \mathrm{MnO}_{3}$ und $\mathrm{SrMnO}_{3}$ zu analysieren. Für 
die hier betrachteten LMO/SMO-SL kann man ebenso eine derartige Möglichkeit zur Analyse der Ladungsumverteilungen an den Grenzflächen erwarten. Durch die periodische Wiederholung der LMO/SMO-Grenzflächen und den starken Kontrast zwischen LMO und SMO sollten diese Betrachtungen begünstigt werden, so dass man auf die Thomas-Fermi-Abschirmlänge $L_{T F}$ schließen kann (vergleiche Abschnitt 3.1).

Orbitale Freiheitsgrade haben einen starken Einfluss auf die magnetischen Eigenschaften von Perowskit-Manganaten (siehe Abschnitt 3.3.1). Diese werden hier dadurch beleuchtet, dass die LMO/SMO-SL auf verschiedenen Substraten, nämlich $\mathrm{SrTiO}_{3}$ (STO) (001), (111) und $\left(\mathrm{La}_{0.3} \mathrm{Sr}_{0.7}\right)\left(\mathrm{Al}_{0.65} \mathrm{Ta}_{0.35}\right) \mathrm{O}_{3}$ (LSAT) (001) gewachsen werden.

In diesem Kapitel werden zunächst die magnetischen Eigenschaften einfacher $\mathrm{La}_{1-\mathrm{x}} \mathrm{Sr}_{\mathrm{x}} \mathrm{MnO}_{3}$ (LSMO(x))-Filme, die mit der MAD auf STO (001)- und STO (111)-Substraten aufgewachsen wurden, dargestellt. Anschließend wird das Wachstum der LMO/SMO-SL auf STO (001)- und STO (111)-Substraten beschrieben. Durch die numerische Anpassung der gemessenen in-situ Ellipsometrie kann man Modelle für das Wachstum dieser Filme konstruieren. Für das Wachstum auf STO (111) kann man auf eine Durchmischung der La- und Sr-Atome schließen, welche sich auch in hochauflösenden TEM-Messungen wiederfindet. Auf STO (001)-Substraten ergeben sich chemisch scharfe LMO/SMO-Grenzflächen. Mit der in-situ Ellipsometrie kann man dann die Delokalisierung der $e_{g}$-Elektronen in jeder Phase des Wachstums quantifizieren. Dann erfolgt ein Vergleich der magnetischen Eigenschaften der auf diesen beiden Substratorientierungen gewachsenen Übergitter für verschiedene Dicken der LMO/SMO-Doppellagen. Für STO (001) beobachtet man zwei ferromagnetische Phasen. Die Phase mit der höheren Curie-Temperatur $T_{C}$ wird genauer behandelt und ihre magnetischen Eigenschaften charakterisiert. Durch die zusätzliche Betrachtung eines LMO/SMO-Übergitters auf einem LSAT (001)-Substrat wird schließlich die Rolle der orbitalen Polarisation bei der Ausbildung der beiden ferromagnetischen Phasen geklärt.

\subsection{Vorbereitung: Einfache $\mathrm{La}_{1-x} \mathrm{Sr}_{x} \mathrm{MnO}_{3}-$ Filme}

\subsubsection{Die Ausgangsmaterialien $\mathrm{SrMnO}_{3}$ und $\mathrm{LaMnO}_{3}$}

Bei der MAD ergeben sich Korrekturfaktoren zwischen der Stöchiometrie der Metallionen in der Präkursorlösung und in der deponierten Schicht (vergleiche Abschnitt 4.1). Diese Faktoren $C_{S r / M n}$ bzw. $C_{L a / M n}$ gewinnt man für $\mathrm{SrMnO}_{3}$ bzw. $\mathrm{LaMnO}_{3}$ durch Optimierung des Wachstums, welches durch die Oberflächenmorphologie von dünnen Filmen dieser Materialien qualifiziert wird. Bei Abweichungen von der idealen Zusammensetzung ergeben sich Störungen des Wachstums. Diese manifestieren sich in der Bildung von Ausscheidungen an der Oberfläche oder induzieren die vermehrte Bildung von zwei- bzw. dreidimensionalen Inseln aufgrund einer herabgesetzten Mobilität der Adatome. Für den Einbau in hochwertige Heterostrukturen ist eine zweidimensionale Wachstumsmode, die im besten Fall einer step flow-Wachstumsmode entspricht, unabdingbar. Durch systematische Variation ergeben sich dann die optimalen Werte von $C_{S r / M n}$ bzw. $C_{L a / M n}$.

In den Abbildungen $9.1 \mathrm{~b}$ ) und $9.2 \mathrm{~b}$ ) erkennt man sowohl für LMO als auch für SMO eine zweidimensionale Wachstumsmode, die eine Mischform zwischen zweidimensionalem Inselwachstum und step-flow-Wachstum darstellt. Aufgrund der großen Fehlpassung zwischen dem Kristallgitter von SMO und STO wird dabei nur eine sehr dünne Schicht mit $d_{S M O} \approx 5.5 \mathrm{~nm}$ 


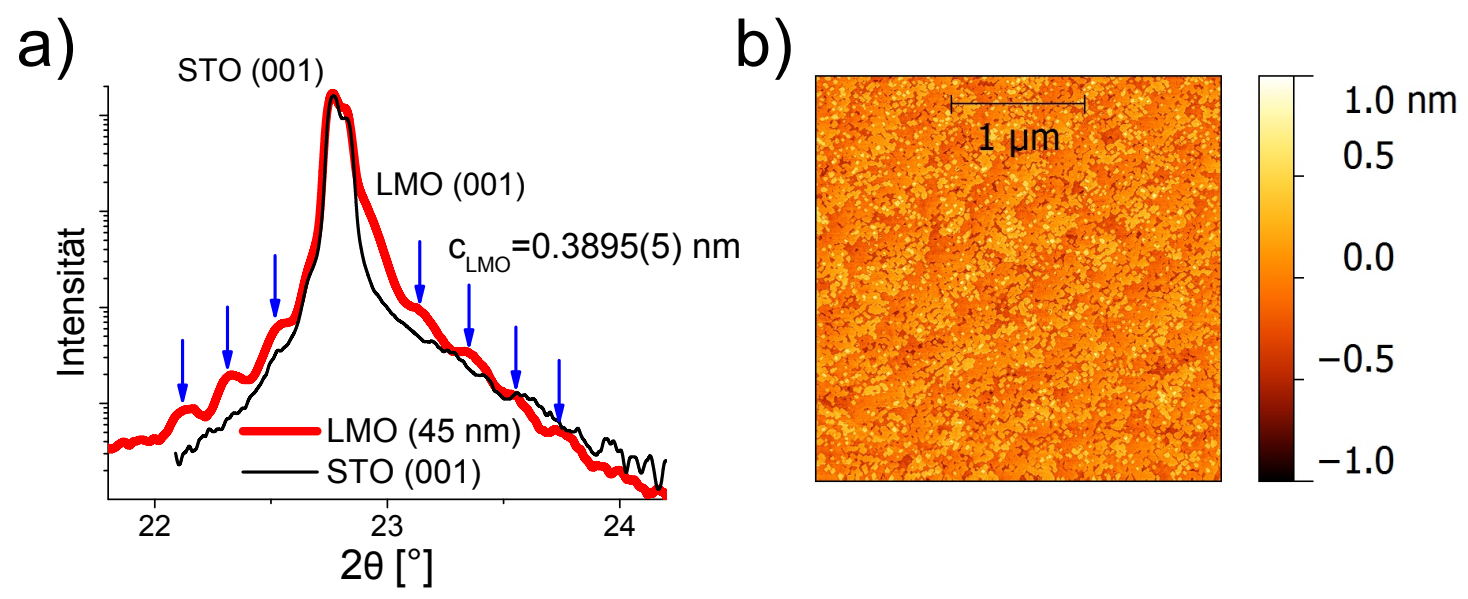

Abbildung 9.1.: Strukturelle und morphologische Eigenschaften von dünnen $\mathrm{LaMnO}_{3}$ (LMO)-Filmen auf $\mathrm{SrTiO}_{3}$ (STO) (001)-Substraten: a) XRD-Spektrum eines LMO-Filmes $(d \approx 45 \mathrm{~nm})$ um den (001)-Peak des STO-Substrates (Die Positionen der Maxima der LaueOszillationen sind mit blauen Pfeilen markiert). b) STM-Aufnahme eines LMO-Filmes mit $d \approx 35 \mathrm{~nm}$.
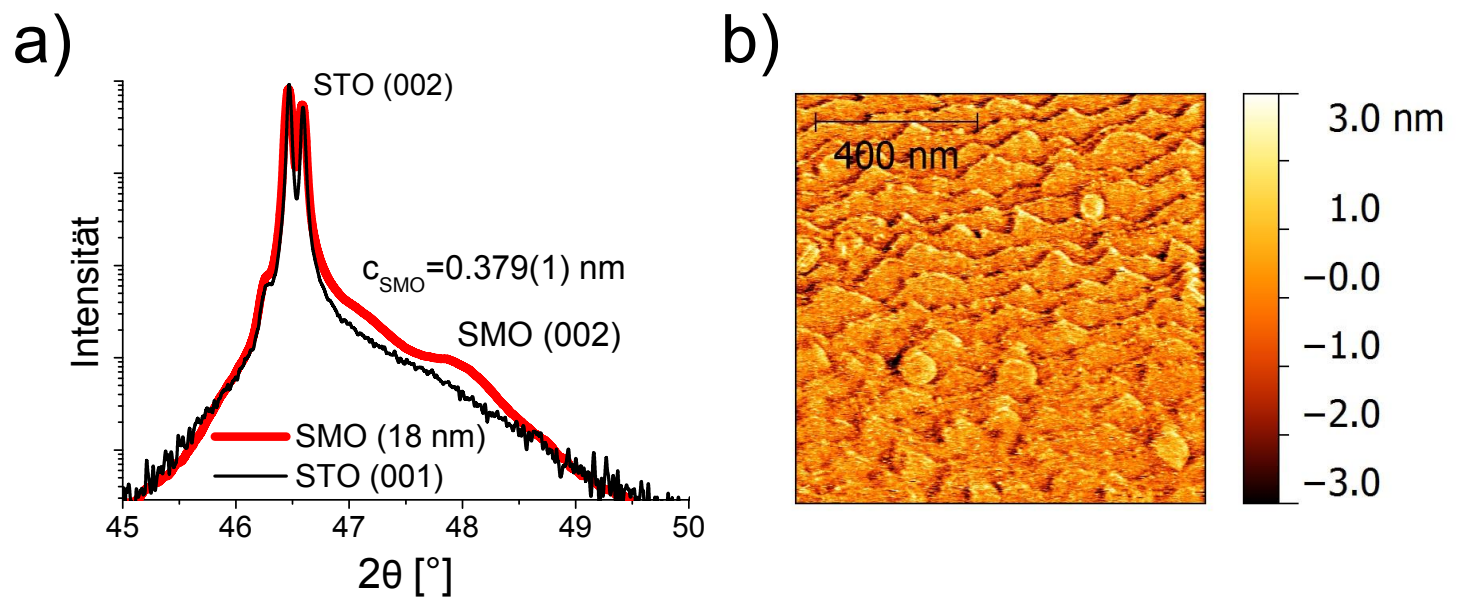

\begin{abstract}
Abbildung 9.2.: Strukturelle und morphologische Eigenschaften von dünnen $\mathrm{SrMnO}_{3}$ (SMO)-Filmen auf $\mathrm{SrTiO}_{3}$ (STO) (001)-Substraten: a) XRD-Spektrum eines SMO-Filmes $(d \approx 18 \mathrm{~nm})$ um den (002)-Peak von STO. b) STM-Aufnahme eines SMO-Filmes mit $d \approx 5.5 \mathrm{~nm}$ (Das verstärkte Rauschen erklärt sich durch die geringe Leitfähigkeit des SMOFilmes).
\end{abstract}

herangezogen, da dickere Schichten zum Abbau der epitaktischen Spannungen aufrauen (siehe Abschnitt 8.2.2). Auf STO (111) zeigt auch LMO ein sehr glattes Wachstum mit Stufen mit der Höhe einer Monolage (ML). Allerdings raut SMO auch bei kleinen Schichtdicken stark auf. Für einen SMO-Film der Dicke $d \approx 8 \mathrm{~nm}$ beobachtet man so Inseln mit einer Höhe von etwa $2 \mathrm{~nm}$ an der Oberfläche 238].

Da der Gitterparameter von LMO hier in der Nähe der Gitterkonstanten von STO liegt, kann man die Position des Beugungsmaximums in Abbildung 9.1 nicht direkt ablesen, sondern nur durch die zusätzliche Betrachtung der Laue-Fringes ermitteln. Der (001)-Peak des LMOFilmes befindet sich dann genau in der Mitte zwischen zwei Laue-Maxima der Ordnung $+n$ und $-n$. So ergibt sich $c_{L M O}=0.3895(5) \mathrm{nm}$ für den Gitterebenenabstand des LMO-Filmes senkrecht zur Filmebene. Mit $\nu=1 / 3$ folgt dann $a_{L M O}=0.3900(5) \mathrm{nm}$ für die pseudokubi- 

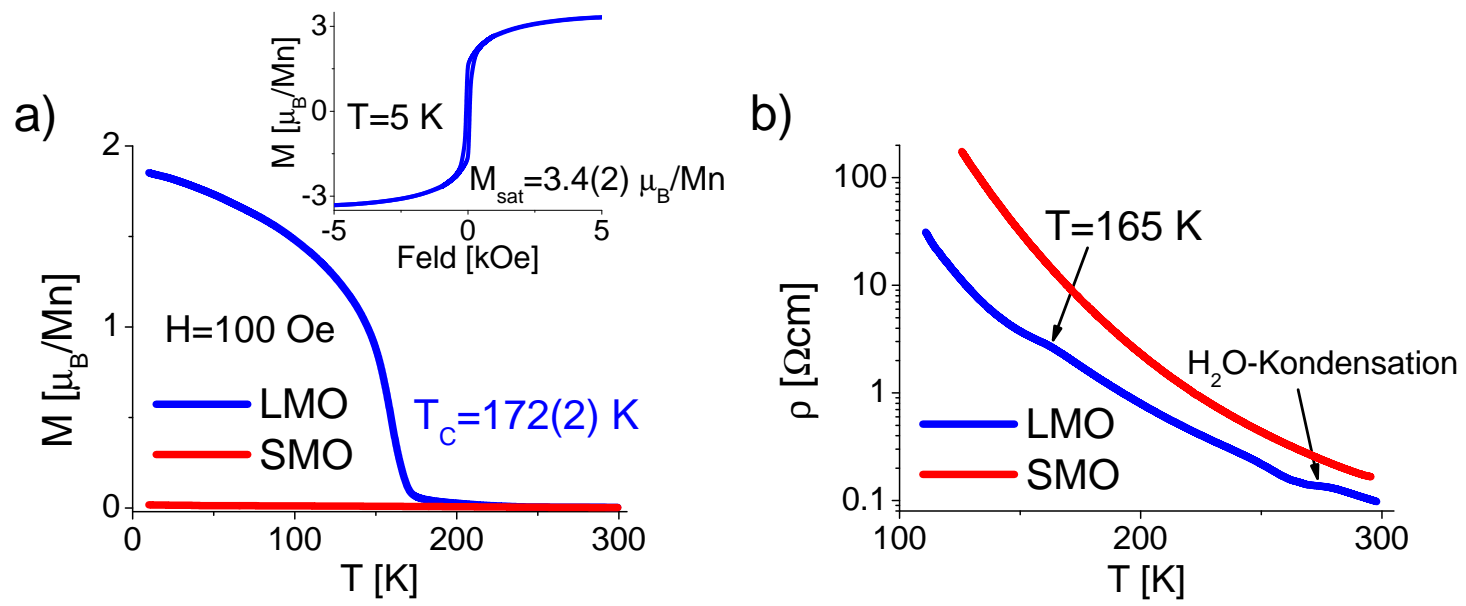

\begin{abstract}
Abbildung 9.3.: Magnetische und elektrische Eigenschaften von LMO- und SMO-Filmen auf STO (001)-Substraten: a) $M(T)$-Messungen, das Inset zeigt die $M(H)$-Hysterese des LMO-Filmes bei $T=5 \mathrm{~K}$ und den zugehörigen Wert für die Sättigungsmagnetisierung $M_{\text {sat }}$. b) Spezifischer Widerstand $\rho$ als Funktion der Temperatur $T$ (Bei $T \approx 273 \mathrm{~K}$ ergibt sich eine Anomalie durch das Kondensieren und Gefrieren von Wasser auf der Probe.)
\end{abstract}

sche Gitterkonstante der deponierten Schicht (vergleiche Abschnitt 8.2.4). Dies entspricht dem Wert der unstöchiometrischen, rhomboedrischen Modifikation von LMO, die sich bei großen Sauerstoffpartialdrücken ergibt 114 116. Damit geht eine $\mathrm{Mn}^{3+} / \mathrm{Mn}^{4+}$-Mischvalenz einher, die zu einem ferromagnetischen (FM)-Übergang bei $T_{C}=172(2) \mathrm{K}$ führt (siehe Abbildung 9.3 a)). Bei tiefen Temperaturen $(T=5 \mathrm{~K})$ ergibt sich mit einer Sättigungsmagnetisierung von $M_{\text {sat }}=3.4(2) \mu_{\mathrm{B}} / \mathrm{Mn}$ eine fast vollständige Ausrichtung der magnetischen Momente, die in einer Magnetisierung von $M_{\text {sat }}^{\text {theo }}=4.0 \mu_{\mathrm{B}} / \mathrm{Mn}$ resultieren würde. Die FM-Ordnung wird von einem Wendepunkt im Verlauf von $\rho(T)$ begleitet (siehe Abbildung 9.3 b)).

Der Gitterebenenabstand $c_{S M O}=0.379(1) \mathrm{nm}$ (siehe Abbildung 9.2 eines dicken $\left(d_{S M O} \approx\right.$ $18 \mathrm{~nm}$ ) und damit fast vollständig entspannten SMO-Filmes kommt dem Wert der kubischen und vollständig oxidierten Variante von $\mathrm{SrMnO}_{3}$ mit $a_{S M O}=0.3805 \mathrm{~nm}[212$ sehr nahe. Die stark mit Sauerstoffleerstellen beladenen Variante $\mathrm{SrMnO}_{2.5}$ würde aufgrund der Mn-Valenz von $\mathrm{Mn}^{3+}$ eine deutlich größere Gitterkonstante zeigen [53, 54]. Die hier hergestellten SMOFilme zeigen keine Anzeichen von ferromagnetischer Ordnung und ein isolierendes Verhalten von $\rho(T)$ (siehe Abbildung 9.3). Die hier gezeigten magnetischen und elektrischen Eigenschaften dünner LMO- und SMO-Filme auf STO (001)-Substraten ergeben sich in der gleichen Ausprägung auch beim Wachstum auf STO (111)-Substraten (siehe nächster Abschnitt).

\title{
9.2.2. Magnetismus von $\mathrm{La}_{1-x} \mathrm{Sr}_{x} \mathrm{MnO}_{3}$-Filmen auf $\mathrm{SrTiO}_{3}(001)$ - und (111)-Substraten
}

Für die spätere Interpretation der magnetischen Eigenschaften der LMO/SMO-SL ist es vorteilhaft, die magnetischen Eigenschaften von Perowskit-Manganaten in Abhängigkeit des Verhältnisses zwischen $\mathrm{Mn}^{3+}$ - und $\mathrm{Mn}^{4+}$-Ionen zu kennen. Da epitaktische Verspannungen die Existenzbereiche von ferro- und antiferromagnetischen Phasen verschieben können (vergleiche Abschnitt 3.3.1), werden für diese Betrachtungen dünne LSMO(x)-Filme auf STO (001)und STO (111)-Substraten herangezogen. Von Danny Schwarzbach wurden im Rahmen seiner Masterarbeit $\mathrm{La}_{1-\mathrm{x}} \mathrm{Sr}_{\mathrm{x}} \mathrm{MnO}_{3}(\mathrm{LSMO}(\mathrm{x}))$-Filme mit einer Dicke von $d \approx 40 \mathrm{~nm}$ auf die 
a)

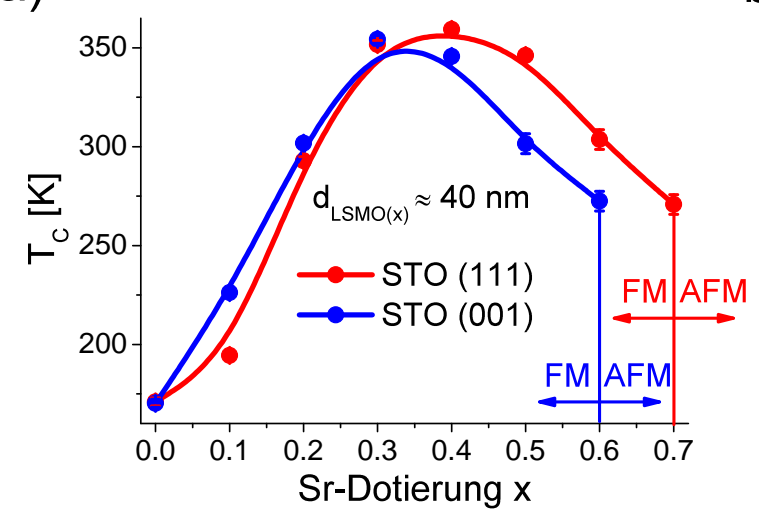

b)

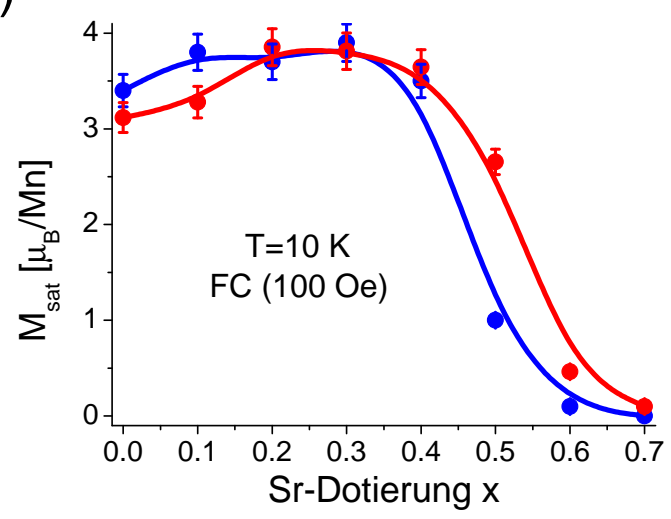

Abbildung 9.4.: Magnetische Eigenschaften von $\operatorname{La}_{1-\mathrm{x}} \mathrm{Sr}_{\mathrm{x}} \mathrm{MnO}_{3}(\mathrm{LSMO}(\mathrm{x}))$-Filmen $(d \approx$ $40 \mathrm{~nm}$ ) auf STO (001)- und STO (111)-Substraten: a) Curie-Temperatur $T_{C}$. Für $x>0.6$ zeigen die Filme auf STO (001) keinen ferromagnetischen (FM) Anteil mehr, bei STO (111) für $x>0.7$. b) Sättigungsmagnetisierung bei $T=10 \mathrm{~K}$ als Funktion der Sr-Dotierung $x$ (Daten aus der Masterarbeit von Danny Schwarzbach entnommen [267]).

beiden erwähnten Typen von Substraten abgeschieden und die magnetischen Eigenschaften untersucht [267]. Für STO (111) ist der Existenzbereich von ferromagnetischen Anteilen in den Filmen um etwa $\Delta x=0.1$ breiter als für STO (001). Insgesamt ergeben sich für STO (111) größere Curie-Temperaturen $T_{C}$ und Sättigungsmomente $M_{\text {sat }}$ als für STO (001) (siehe Abbildung 9.4). Für $x=0-0.4$ liegt ein fast komplett ferromagnetischer Grundzustand vor, der für größere Sr-Dotierungen einem Mischzustand weicht, bei dem $M_{\text {sat }}(T=10 \mathrm{~K})$ unter dem theoretischen Wert $M_{\text {theo }}=(4-x) \mu_{\mathrm{B}} / \mathrm{Mn}$ liegt.

Auf STO (001) liegen bis $x=0.6$ voll verspannte Filme vor, bei STO (111) relaxiert die Verspannung schon ab $x=0.5$ teilweise [267, so dass das Phasendiagramm dem der bulkMaterialien ähnelt (vergleiche Abbildung 2.5). Auf STO (001) bewirkt die durch die Verspannung aufgehobene orbitale Entartung eine Bevorzugung der A-AFM-Ordnung gegenüber einer FM-Ordnung (vergleiche Abschnitt 3.3.1. 


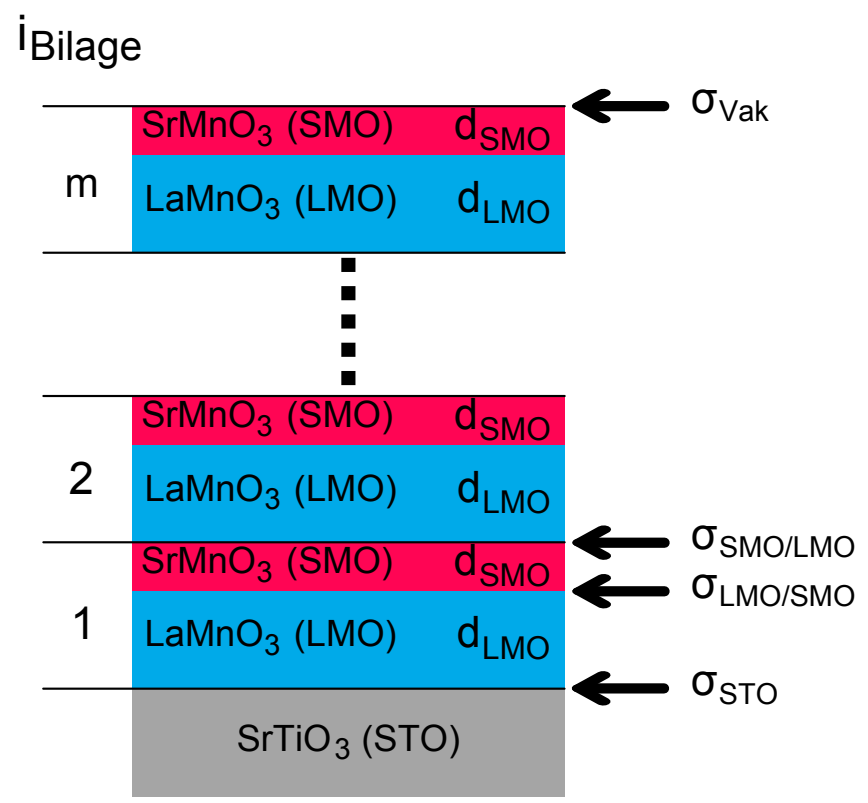

\begin{abstract}
Abbildung 9.5.: Schema der hergestellten LMO/SMO-SL: Auf ein STO-Substrat werden Doppellagen aus LMO der Dicke $d_{L M O}$ und SMO der Dicke $d_{S M O}$ aufgebracht. Diese LMO/SMO-Doppellagen werden mit $i_{\text {Bilage }}$ durchgezählt und m-fach wiederholt. Mit $\sigma_{S T O}$ wird die Rauigkeit zum Substrat und mit $\sigma_{V a k}$ die der Oberfläche bezeichnet. Für die LMO/SMO-Grenzflächen gibt es zwei Rauigkeiten: $\sigma_{L M O / S M O}$ und $\sigma_{S M O / L M O}$ je nachdem, welche der beiden Grenzflächen man in Wachstumsrichtung betrachtet.
\end{abstract}

\title{
9.3. Wachstum von $\mathrm{LaMnO}_{3} / \mathrm{SrMnO}_{3}$-Übergittern
}

Gemäß dem Schema in Abbildung 9.5 wurden Übergitter aus LMO und SMO auf die verschiedenen Substrate abgeschieden. Zur Vergleichbarkeit mit den Studien von May et al. wurden die Schichtdicken $d_{L M O}$ und $d_{S M O}$ so gewählt, dass $d_{L M O} / d_{S M O} \approx 3$ gilt. Die Dicken von LMO und SMO werden mit einem Parameter $n$ skaliert, so dass $d_{L M O}=2 n \mathrm{ML}$ und $d_{S M O}=\frac{2}{3} n$ ML gilt. Die Dicke einer LMO/SMO-Doppellage ergibt sich dann zu:

$$
\Lambda=d_{L M O}+d_{S M O}=n\left(2+\frac{2}{3}\right) \mathrm{ML}
$$

Aus gemessenen XRD- und XRR-Spektren kann man die exakten Werte von $\Lambda$ ermitteln und daraus den genauen Wert von $n$ errechnen. Übergitter, bei denen man mit einer LMOSchicht auf dem STO-Substrat beginnt, werden als LMO/SMO-SL bezeichnet und Übergitter, bei denen man mit einer SMO-Lage startet, als SMO/LMO-SL.

Die STO (001)-Substrate wurden gemäß der Routine aus Abschnitt 4.4 .2 vorbehandelt und damit $\mathrm{TiO}_{2}$-terminiert. Die STO (111)- und LSAT (001)-Substrate wurden im vom Hersteller bereitgestellten Zustand verwendet. Das Wachstum der in diesem Kapitel vorgestellten Heterostrukturen erfolgte bei einer Depositionstemperatur $T_{D e p}=930^{\circ} \mathrm{C}$ und mit einer Depositionsrate $p_{D e p}=0.09-0.14 \mathrm{~nm} / \mathrm{s}$. Die Depositionspausen zwischen den individuellen LMOund SMO-Lagen wurden so eingestellt, dass kein weiteres Wachstum durch das verbleibende Aerosol mehr zu beobachten war, bevor die Abscheidung der nächsten Lage begonnen wurde (vergleiche Abschnitt 4.4.5). Die Pausenzeiten wurden mit der Schichtdicke der jeweils zuvor deponierten Lage skaliert und nehmen typischerweise Werte von $\Delta t_{\text {Pause }}=d_{L M O} / d_{1 M L} \cdot 2 \mathrm{~s}$ bzw. $\Delta t_{\text {Pause }}=d_{S M O} / d_{1 M L} \cdot 2 \mathrm{~s}$ an. Bei den hier gezeigten Darstellungen der in-situ Ellipsometrie wurden die Pausenabschnitte zur besseren Übersicht entfernt. 
a)

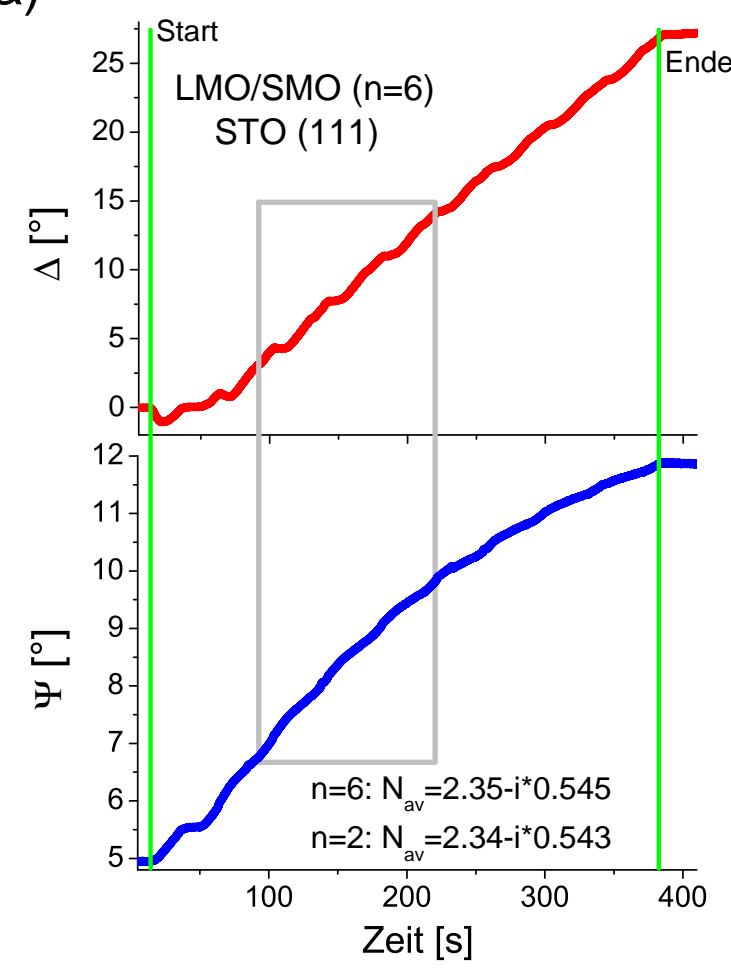

b)

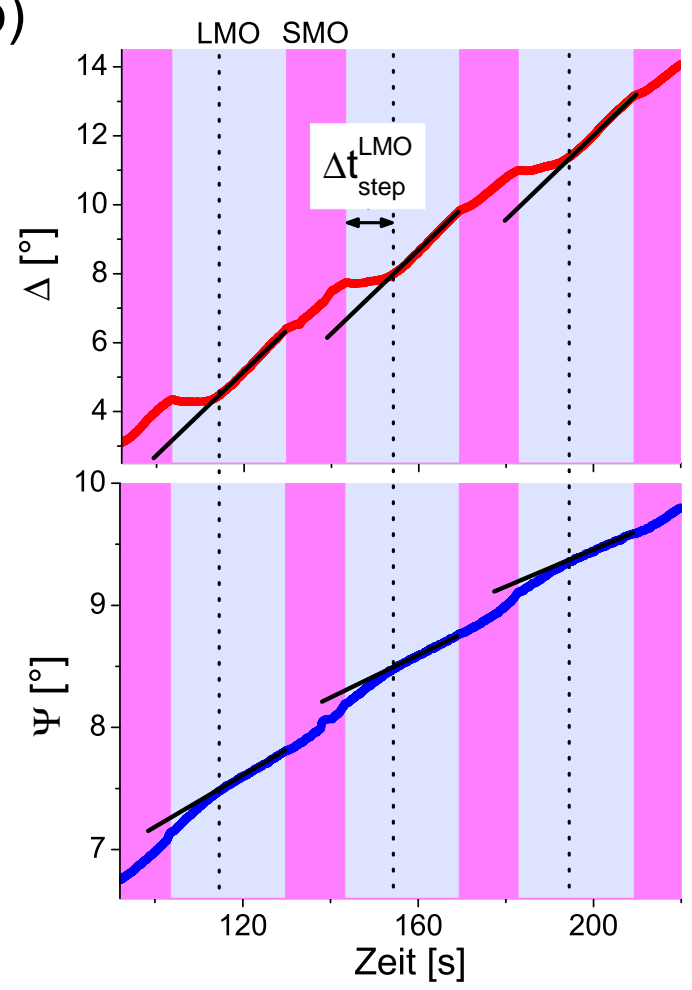

Abbildung 9.6.: In-situ Ellipsometrie beim Wachstum eines LMO/SMO-Übergitters auf STO (111) mit $n \approx 6$ und $m=9$ : a) Überblick über $\Delta(t)$ und $\Psi(t)$ für die gesamte Depositionsdauer. Aus den Endwerten von $(\Delta, \Psi)$ lässt sich der mittlere Brechungsindex $N_{a v}$ bestimmen. Dieser ist für das dargestellte Übergitter und eine Probe mit $n \approx 2$ im Diagramm angegeben. b) Vergrößerte Darstellung des grau umrandeten Bereichs in a). Innerhalb der Wachstumsphase der LMO-Schicht kommt es nach einem Zeitintervall $\Delta t_{\text {step }}^{L M O}$ zu einer gleichzeitigen Steigungsänderung von $\Delta(t)$ und $\Psi(t)$.

Entlang der (001)-Richtung sind die Abstände zwischen benachbarten Gitterebenen und damit die Dicke einer Monolage um einen Faktor $\sqrt{3}$ größer als entlang der (111)-Richtung. Um für LMO/SMO-SL, die auf STO (001)- und STO (111)-Substraten gewachsen wurden, eine ähnliche Separation der La- und Sr-Ionen zu erreichen, wählt man dann unterschiedliche Werte von $n$. Für den Wert $n=n_{(001)}$ einer Heterostruktur auf dem (001)-Substrat wählt man so zum Vergleich $n_{(111)}=2 n_{(001)} \approx \sqrt{3} n_{(001)}$ für die Strukturen auf Substraten, die entlang der (111)-Ebene geschnitten wurden. Insgesamt wurden dann Übergitter mit verschiedenen Werten von $n$ hergestellt: Für STO (001) in einem Bereich $n_{(001)}=1-7$ und für STO (111) $n_{(111)}=2-6$.

\subsection{1. $\mathrm{SrTiO}_{3}$ (111): Sr/La-Durchmischung}

In Abbildung 9.6 a) sieht man die in-situ Ellipsometrie für das Wachstum eines LMO/SMOÜbergitters auf STO (111) mit $n \approx 6$ und $m=9$. Aus den Werten von $\Delta$ und $\Psi$ nach Abschluss der Deposition lässt sich der durchschnittliche Brechungsindex $N_{a v}(n=6)=$ 2.35(1) $-i \cdot 0.54(1)$ der kompletten Heterostruktur bestimmen. Dieser ist identisch mit dem Brechungsindex eines Übergitters mit einer kleineren Dicke der LMO/SMO-Doppellagen $(n \approx$ 2 ), für das man $N_{a v}(n=2)=2.34(2)-i \cdot 0.54(1)$ erhält. Diese Werte kommen dem einer 
a)

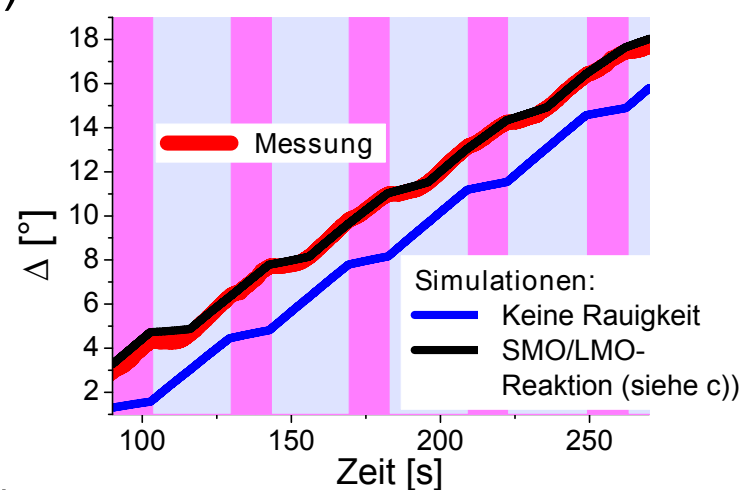

c)
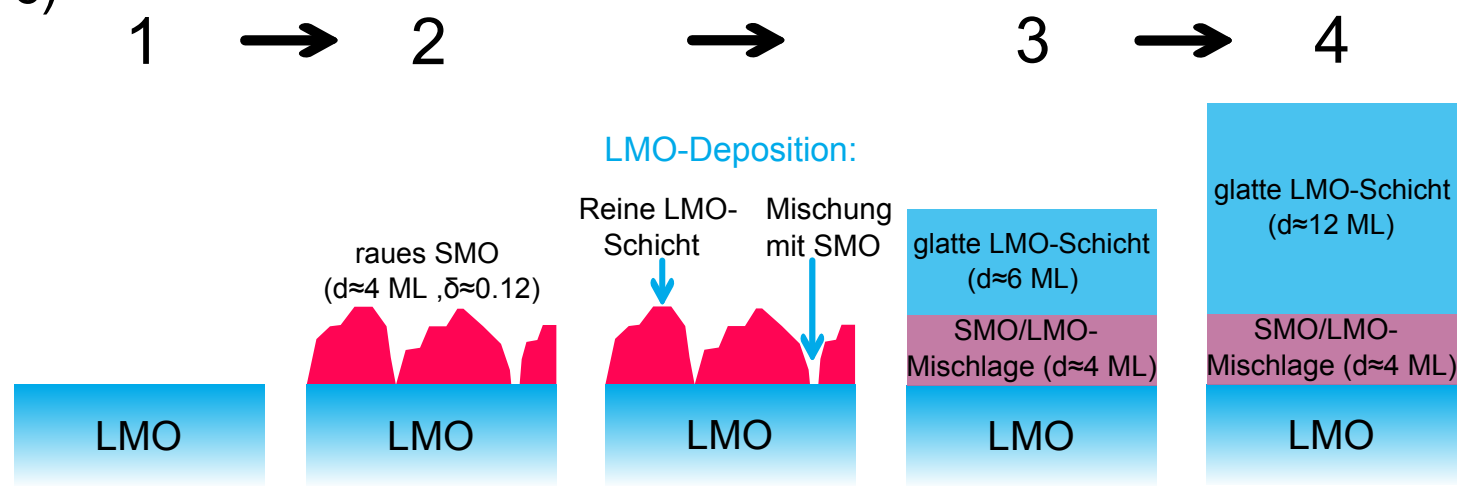

LMO-Deposition:

Abbildung 9.7.: Numerische Anpassung der in-situ Ellipsometrie des in Abbildung 9.6 dargestellten LMO/SMO-Übergitters mit $n \approx 6$ auf STO (111): a) Gemessener Verlauf von $\Delta(t)$ mit einem simulierten Verlauf entsprechend dem Modell in Teil c) und einer Simulation ohne Reaktion zwischen LMO und SMO, b) vergrößerter Ausschnitt von a), c) Wachstumsmodell, das von einem Aufrauen der SMO-Schicht und einer anschließenden Durchmischung mit LMO ausgeht.

durchmischten LSMO ( $\mathrm{x}=0.25$ )-Schicht sehr nahe (siehe Abschnitt 6.2). In Abschnitt 6.2 wurde gezeigt, dass der in der Näherung eines effektiven Mediums (EMA) als Mischung von LMO und SMO berechnete Brechungsindex von $\operatorname{LSMO}(\mathrm{x})$ deutlich vom experimentell ermittelten Wert $N_{L S M O(x)}$ abweicht. Die hier festgestellte Gleichheit von $N_{a v}(n=6)$ und $N_{a v}(n=2)$ lässt sich also nicht durch die Bildung eines effektiven Mediums aus LMO und SMO verstehen, sondern muss aus einer Delokalisierung der $e_{g}$-Elektronen über die komplette Heterostruktur mit $n=6$ resultieren.

Eine detaillierte Betrachtung der zeitlichen Verläufe von $\Delta$ und $\Psi$ erlaubt Rückschlüsse auf die Wachstumsprozesse. Bei den in Abbildung 9.6 b) gezeigten Verläufen von $\Delta(t)$ und $\Psi(t)$ erkennt man beim Wachstum der LMO-Lagen eine Steigungsänderung, die nach dem Zeitintervall $\Delta t_{\text {step }}^{L M O}$ einsetzt. Dies entspricht einer gewachsenen Schichtdicke von etwa $\Delta d_{\text {step }}^{L M O}=4 \mathrm{ML}$ von insgesamt $d_{L M O} \approx 12 \mathrm{ML}$ für eine komplette LMO-Lage. Die Änderung der Steigung ist dann nach einer gewachsenen Dicke von etwa $6 \mathrm{ML}$ abgeschlossen, wo $\Delta(t), \Psi(t)$ wieder linear verlaufen (siehe Abbildung 9.7 b), Markierung 3).

Wenn man die gemessenen Daten mit einer einfachen Simulation des Wachstums von LMO und SMO, bei der keine Reaktionen an den Grenzflächen stattfinden, vergleicht, erkennt man starke Abweichungen (siehe Abbildung 9.7 a),b)). Zunächst zeigt sich in der Simulation eine konstante Steigung während des Wachstums der LMO-Lage und überdies verläuft $\Delta(t)$ beim 

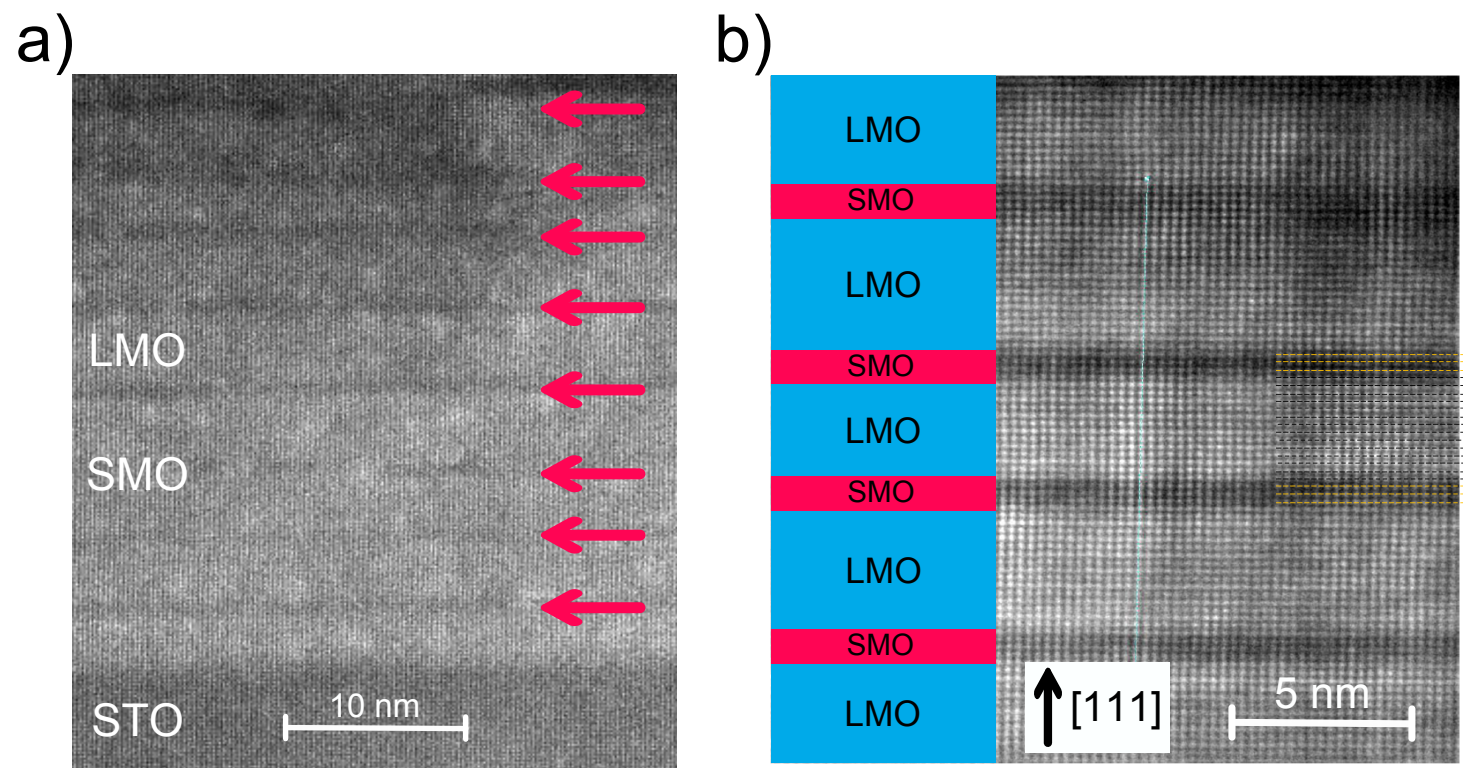

Abbildung 9.8.: HAADF-STEM-Aufnahmen des LMO/SMO-Übergitters $(n \approx 6)$ auf STO (111) (siehe Abbildungen 9.6 und 9.7): a) Übersicht über die gesamte Dicke der Heterostruktur, die roten Pfeile markieren die Positionen der SMO-Lagen (die SMO-Lage an der Oberfläche ist aufgrund der Beschädigung der TEM-Lamelle nicht zu sehen), b) Vergrößerung von a) (Die TEM-Aufnahmen wurden von Vladimir Roddatis angefertigt).

Wachstum der SMO-Lage wesentlich flacher als in der Messung 33 Wie eingangs erwähnt, zeigt sich für LMO ein zweidimensionales Wachstum, wogegen SMO auf STO (111) aufraut. XRRMessungen der LMO/SMO-SL auf STO (111) deuten auf erhöhte Rauigkeiten, $\sigma_{L M O / S M O}$ und $\sigma_{S M O / L M O}$, hin, die eine numerische Simulation der gemessenen Spektren erschweren 238 .

Mit diesen Informationen entwickelt man ein Wachstumsmodell für die LMO/SMO-SL auf STO (111), um die gemessenen Daten der in-situ Ellipsometrie nachzubilden. In Übereinstimmung mit Abbildung 9.7 c) wächst auf einer glatten LMO-Schicht SMO mit nanoskaligen Rauigkeiten auf. Diese werden mit der Näherung des effektiven Mediums behandelt. Die SMO-Schicht wird als eine Mischung von Luft mit einem Anteil von $\delta=0.12$ und SMO angenommen, um die experimentellen Daten am besten nachzubilden ${ }^{34}$. Durch das Aufrauen vergrößert sich die Steigung von $\Delta(t)$ deutlich im Vergleich zur einfachen Simulation (Abbildung 9.7 b)). Dann beginnt die Deposition der LMO-Lage mit einer gesamten Dicke von $d_{L M O}=12 \mathrm{ML}$. Ein Teil des abgeschiedenen LMO mischt sich dann mit der rauen SMOSchicht, wogegen der verbleibende Anteil einen glatten Film auf dem SMO bildet (vergleiche Abbildung 9.7 c) $2 \rightarrow 3$ ). Dieser Prozess schreitet fort bis eine Menge von LMO aufgebracht wurde, die einer Schichtdicke von $6 \mathrm{ML}$ entspricht. Dann liegt an der Oberfläche ein glatter LMO-Film vor, unter dem sich eine LMO/SMO-Mischlage befindet. Zum Abschluss werden dann die restlichen $6 \mathrm{ML}$ von LMO deponiert.

\footnotetext{
${ }^{33}$ Für $\Psi(t)$ fallen die Änderungen deutlich kleiner aus als für $\Delta(t)$, so dass aufgrund der besseren Darstellbarkeit nur die Messungen und Simulationen von $\Delta(t)$ gezeigt werden.

${ }^{34}$ Entgegen der Simulation zeigt sich im Experiment kein komplett linearer Verlauf von $\Delta(t)$ beim Wachstum von SMO. Dies ist womöglich damit zu verbinden, dass das Aufrauen des Filmes in verschiedenen Phasen abläuft.
} 
Die Resultate des geschilderten Wachstumsmodells finden sich auch in HAADF-Aufnahmen des LMO/SMO-Übergitters, die von Vladimir Roddatis angefertigt wurden, wieder. In Abbildung 9.8 wird die LMO/SMO-Durchmischung dadurch verdeutlicht, dass der Kontrast zwischen LMO und SMO deutlich schwächer ist als zwischen LMO und dem STO-Substrat. Aufgrund der ähnlichen Gesamtelektronendichte von SMO und STO sollte der Kontrast bei idealem Wachstum identisch sein. Da die Dicke der TEM-Lamelle in einem Bereich von $d_{T E M}=10-20 \mathrm{~nm}$ liegt, kann man die Skala der Durchmischung der La- und Sr-Ionen auf eine Längenskala deutlich unterhalb von $d_{T E M}$ einschränken.

Das Aufrauen der SMO-Lagen bei diesen kleinen Schichtdicken $d_{S M O} \approx 0.9 \mathrm{~nm}$ kann man nicht allein durch den Abbau der epitaktischen Verzerrungen erklären. Mit der Formel von Hirsch kann man die minimale Filmdicke abschätzen, für die es energetisch günstiger ist, Versetzungen in den Film einzubauen, die die Fehlpassung zum Substrat ausgleichen 268. Für den hier betrachteten Fall liegt diese kritische Schichtdicke bei $d_{C} \approx 0.8 \mathrm{~nm}$. Ab dieser Schichtdicke besteht also eine treibende Kraft die ersten Versetzungen einzubauen und den Film zu relaxieren. Das hier beobachtete Wachstum von SMO mit Inseln, deren laterale Abmessungen im Bereich von $10 \mathrm{~nm}$ liegen, erfordert aufgrund des Bruches der Bindungen an den Oberflächen eine deutlich größere Energie als der Einbau der Versetzungen. Damit muss es neben den epitaktischen Verzerrungen eine andere treibende Kraft für das Aufrauen der SMO-Lagen geben.

Entlang der [001]-Richtung kann man SMO in einzelne SrO- und $\mathrm{MnO}_{2}$-Ebenen zerlegen, die jeweils ungeladen sind. Für diesen Schnitt ergibt sich also wie beim STO kein elektrostatisches Potential. Wird die Oberfläche aber entlang der (111)-Ebene geschnitten, so tragen die einzelnen Lagen aus $\mathrm{Mn}^{4+}$ und $\mathrm{Sr}^{2+} \mathrm{O}_{3}^{2-}$ formale Ladungen von \pm 4 pro Einheitszelle 97 . Wie in Abschnitt 3.1 beschrieben, ergibt sich daraus ein elektrostatisches Potential, welches durch Rekonstruktionen an der Grenz- und Oberfläche reduziert werden kann. Aufgrund des Fehlens von $e_{g}$-Elektronen im SMO kann dies nicht durch eine Veränderung der Mn-Valenzen erreicht werden. Alternativ können auch unstöchiometrische Rekonstruktionen an der Oberund Grenzfläche für einen Reduktion des elektrostatischen Potentials sorgen. Schon bei kleinen Filmdicken übersteigt die Stärke des elektrischen Potentials die Aktivierungsenergie für Diffusion (siehe Abschnitt 3.1), so dass strukturelle Modifikationen auch mit Blick auf die Kinetik denkbar sind. An den freien Oberflächen der SMO-Inseln kann es dann leicht zu einer Interdiffusion mit den nachfolgend deponierten La-Ionen kommen. 

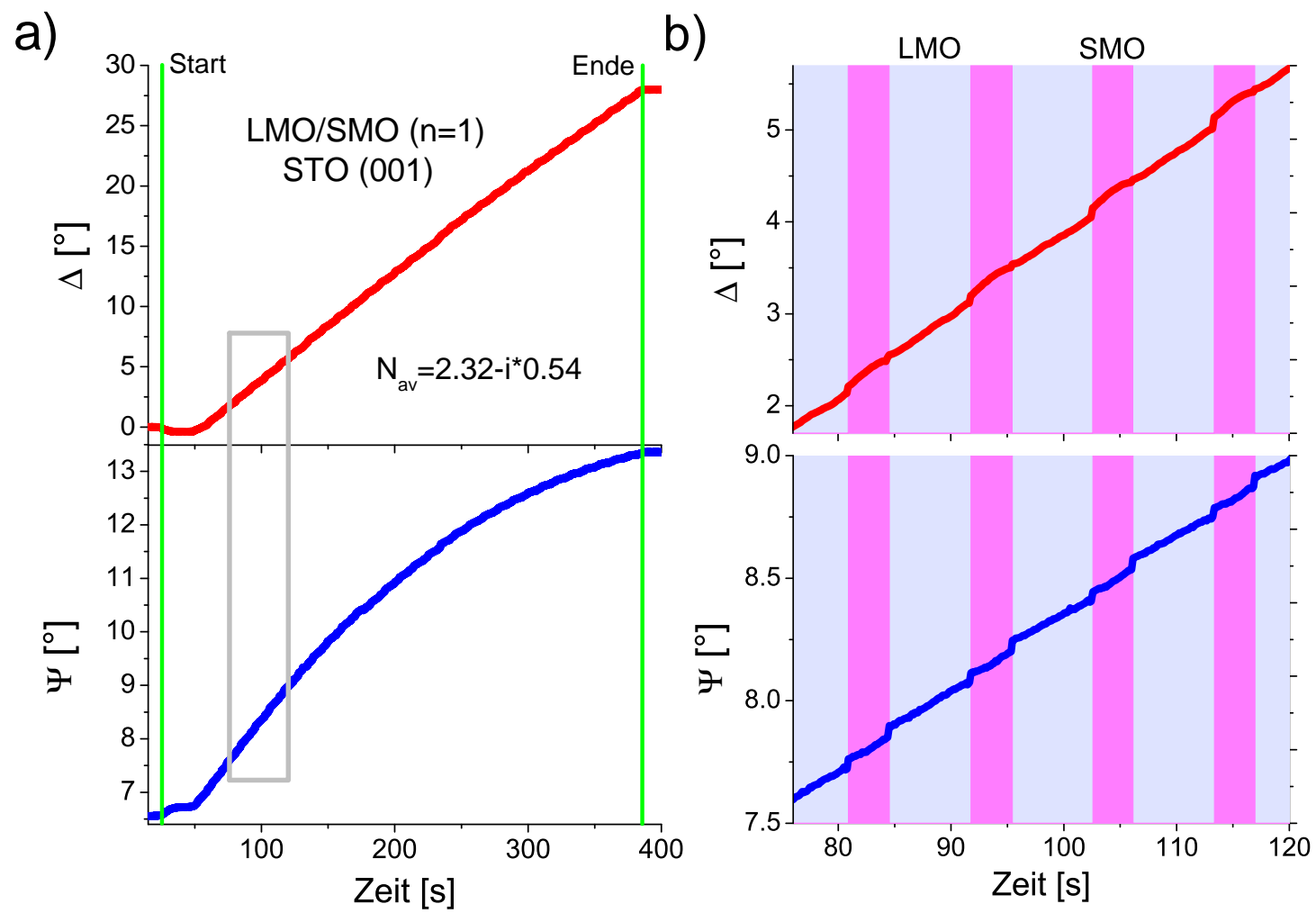

\begin{abstract}
Abbildung 9.9.: In-situ Ellipsometrie beim Wachstum eines LMO/SMO-Übergitters $(n \approx$ $1, d_{L M O} \approx 2 \mathrm{ML}, d_{S M O} \approx 2 / 3 \mathrm{ML}, m=32$ ) auf STO (001): a) Übersicht der gesamten Depositionsdauer, der durchschnittliche Brechungsindex $N_{a v}$ berechnet mit den Werten von $\Delta$ und $\Psi$ am Ende der Deposition ist im Diagramm angegeben. b) Vergrößerte Darstellung des grau umrandeten Bereichs in a). (Die erkennbaren Sprünge in $\Delta(t)$ und $\Psi(t)$ beim Übergang zwischen LMO und SMO resultieren aus der Entfernung der Pausenabschnitte.)
\end{abstract}

\title{
9.3.2. $\mathrm{SrTiO}_{3}(001)$ : Beobachtung der Delokalisierung von $\mathrm{e}_{\mathrm{g}}$-Elektronen durch in-situ Ellipsometrie
}

Im diesem Abschnitt untersucht man die in-situ Messungen der Ellipsometrie beim Wachstum von LMO/SMO-Übergittern auf STO (001)-Substraten. Zunächst werden die EllipsometrieMessungen für $n=1,2,3$ phänomenologisch betrachtet und verglichen. Dann wird die chemische Qualität der LMO/SMO-Grenzflächen mit XRR-, XRD- und TEM-Messungen qualifiziert. Mit diesen experimentellen Befunden entwickelt man dann ein Modell für die Umverteilung der $e_{g}$-Elektronen während des Wachstums.

Für das LMO/SMO-SL mit $n \approx 1$ ergibt sich mit $N_{a v}=2.32(2)-i \cdot 0.54(1)$ (siehe Abbildung 9.9 a)) ein durchschnittlicher Brechungsindex, der dem Wert der im vorherigen Abschnitt vorgestellten Übergitter auf STO (111) entspricht. Dieses Übergitter weist also eine ähnlich homogene Verteilung der $e_{g}$-Elektronen auf.

Bei einer Erhöhung der Bilagendicke, $\Lambda$, für $n \approx 2$ weicht der durchschnittliche Brechungsindex $N_{a v}=2.34(2)-i \cdot 0.56(1)$ schon leicht vom Wert für das Übergitter mit $n \approx 1 \mathrm{ab}$ (siehe Abbildung 9.10). Des Weiteren ist eine deutliche Steigungsänderung in $\Delta(t)$ während des Wachstums der SMO-Lagen zu erkennen (Für $\Psi(t)$ ist diese auch vorhanden aber nur schwach ausgeprägt.). Im Bereich der SMO-Lage verläuft $\Delta(t)$ zunächst mit einer ähnlichen Steigung wie im Bereich von LMO, um dann deutlich abzuflachen (siehe Abbildung 9.10 

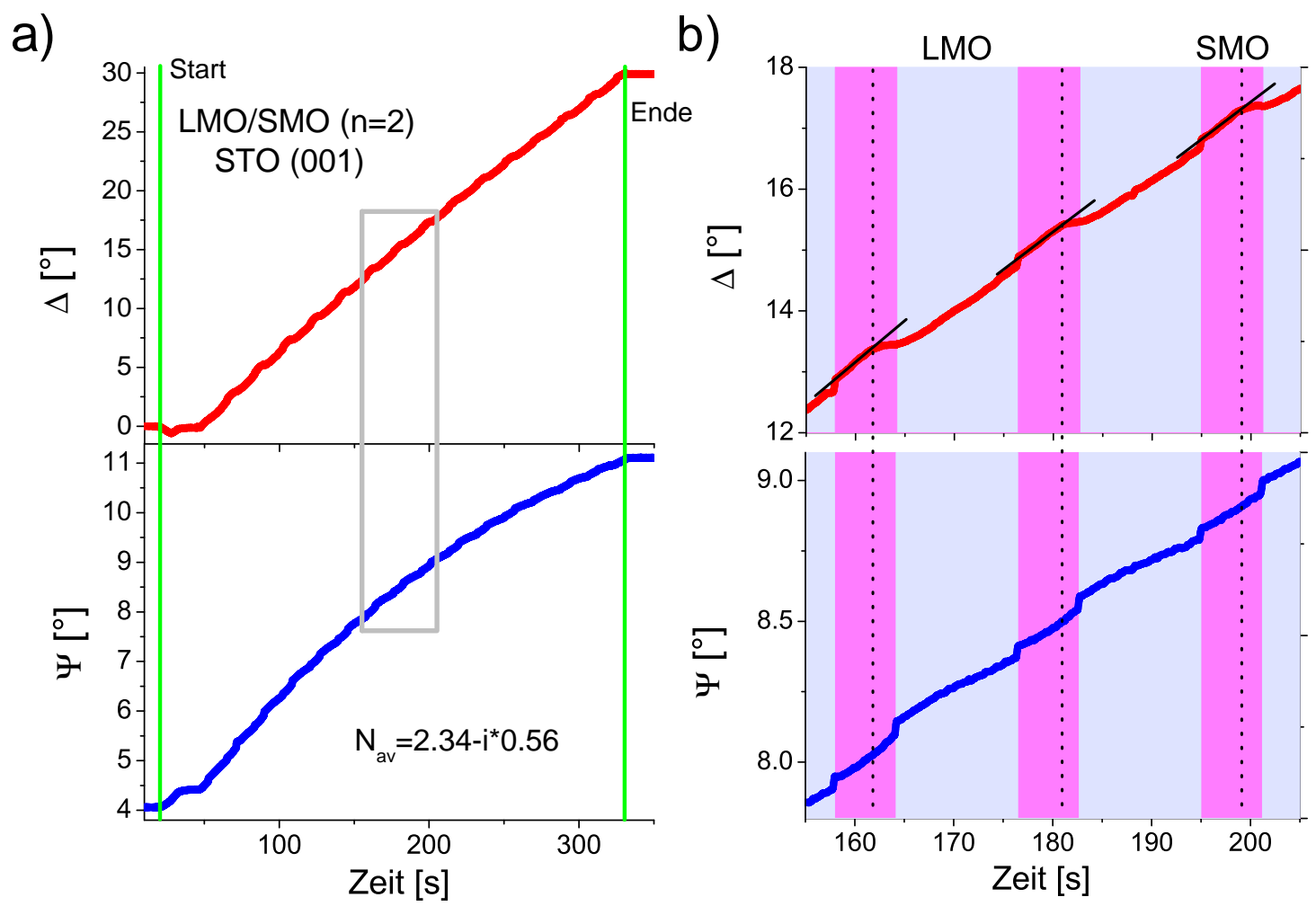

Abbildung 9.10.: In-situ Ellipsometrie beim Wachstum eines LMO/SMO-Übergitters $\left(n \approx 2, d_{L M O} \approx 4 \mathrm{ML}, d_{S M O} \approx 4 / 3 \mathrm{ML}, m=16\right)$ auf STO (001): a) Übersicht über gesamte Depositionsdauer, der durchschnittliche Brechungsindex $N_{a v}$ der gesamten Heterostruktur, der sich aus den Endwerten von $\Delta(t)$ und $\Psi(t)$ ergibt, ist im Diagramm angegeben. b) Vergrößerte Darstellung des grau umrandeten Bereiches aus a), die Positionen der Steigungsänderung in $\Delta(t)$ und $\Psi(t)$ bei der Deposition von SMO sind markiert.

b)). Diese Steigungsänderung setzt nach einem charakteristischen Zeitintervall $\Delta t_{\text {step }}^{S M O}$ ein (vergleiche Abbildung 9.11 a)). Dividiert man dieses durch die Depositionszeit pro Monolage SMO, $\Delta t_{M L}^{S M O}$, so ergibt sich die Zahl der deponierten Monolagen, bis zum Eintreten der Steigungsänderung

$$
n_{\text {step }}^{S M O}=\frac{\Delta t_{\text {step }}^{S M O}}{\Delta t_{M L}^{S M O}}
$$

Wie man in Abbildung 9.11 b) erkennen kann, schwankt diese Größe mit dem Verlauf des Wachstums der Heterostruktur um einen Mittelwert in der Nähe von $n_{\text {step }}^{S M O}=1$. Nach dem Wachstum einer Monolage SMO kommt es also zu einer Änderung der Steigung von $\Delta(t)$.

Für $n \approx 3$ beobachtet man einen fast stufenartigen Verlauf in $\Delta(t)$ beim Wachstum der SMO-Lagen und eine sichtbare Steigungsänderung bei $\Psi(t)$ (siehe Abbildung $9.12 \mathrm{~b}$ )). Der durchschnittliche Brechungsindex $N_{a v}=2.36(2)-i \cdot 0.59(1)$ weicht signifikant vom Wert für $n \approx 1$ ab. Für $n \approx 3$ bewegt sich $N_{a v}$ in die Richtung des Brechungsindexes, der sich für eine Mischung von LMO und SMO im Rahmen der Näherung durch ein effektives Medium ergibt (vergleiche Abbildung 6.5). Dies deutet darauf hin, dass für das Übergitter mit $n \approx 3$ eine inhomogene Verteilung der $e_{g}$-Elektronen vorliegt. Dies steht im Gegensatz zum LMO/SMOSL auf STO (111) aus dem vorherigen Abschnitt, wo man für $n \approx 6$ und damit einen ähnlichen Wert von $\Lambda$ eine homogene Verteilung annehmen kann. 

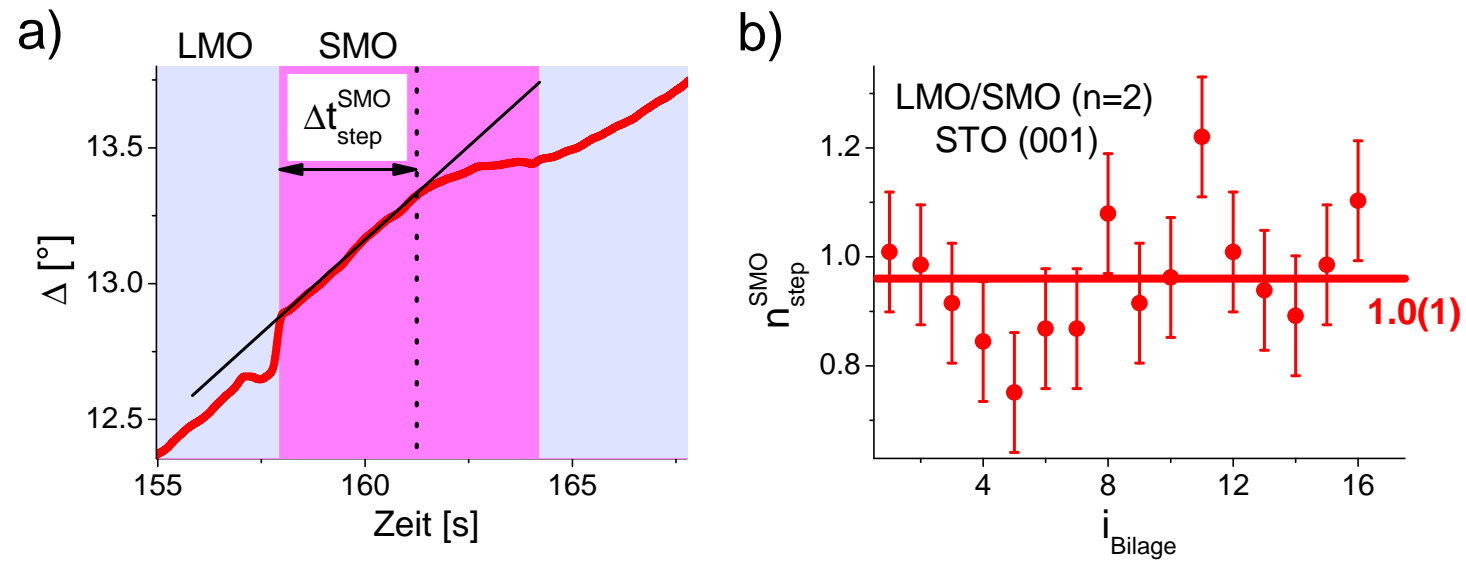

\begin{abstract}
Abbildung 9.11.: Zur Steigungsänderung in $\Delta(t)$ beim Wachstum des LMO/SMOÜbergitters $\left(n \approx 2\right.$, vergleiche Abbildung 9.10): a) $\Delta t_{\text {step }}^{S M O}$ bezeichnet das Zeitintervall, nach dem $\Delta(t)$ beginnt vom zunächst linearen Verlauf abzuweichen. b) $n_{\text {step }}^{S M O}=\frac{\Delta t_{s t e p}^{S M O}}{\Delta t_{M L}^{S M O}}$ als Funktion des Indexes der LMO/SMO-Bilage $i_{\text {Bilage }}$.
\end{abstract}

Der Verlauf von $\Delta(t)$ ist für jede deponierte SMO/LMO-Doppellage sehr ähnlich aber nicht identisch. Es zeigen sich für die ersten 2-3 Wiederholungen im Detail Abweichungen vom Verhalten, das sich in den späteren Phasen des Wachstums einstellt und zu einem reproduzierbaren Verlauf von $\Delta(t)$ führt (siehe Abbildung $9.12 \mathrm{~b}$ )). Im Inset von Abbildung $9.12 \mathrm{a}$ ) sieht man die Deposition der zweiten Wiederholung der SMO/LMO-Bilage. $\Delta(t)$ verhält sich dabei ähnlich wie für das LMO/SMO-SL $(n \approx 6)$ auf STO (111) (Abbildung 9.6 b)), was eine Durchmischung der La- und Sr-Atome an den Grenzflächen der ersten SMO/LMO-Bilagen andeutet.

Wie man in Abbildung 9.13 a) erkennen kann, ergibt sich nach der charakteristischen Zeit $\Delta t_{\text {step }}^{S M O}$ eine stufenartige Änderung der Steigung von $\Delta(t)$ und simultan eine wahrnehmbare Änderung der Steigung in $\Psi(t)$. Wie für $n \approx 2$ streut auch hier $n_{\text {step }}^{S M O}$ um einen Mittelwert, der sehr nahe bei einer Monolage liegt (Abbildung $9.13 \mathrm{~b}$ )). Insgesamt zeigen sich also Hinweise darauf, dass die hier beobachteten Eigenheiten bei der in-situ Ellipsometrie für Übergitter auf STO (001) mit der Verteilung der $e_{g}$-Elektronen zusammenhängen. Vor der Entwicklung eines Modells zur Beschreibung der Verteilung der $e_{g}$-Elektronen in den verschiedenen Phasen des Wachstums sollen nachfolgend die strukturellen Eigenschaften der LMO/SMO (bzw. SMO/LMO)-SL mit $n \approx 3$ auf STO (001) dargelegt werden.

Bei der Untersuchung der Oberflächenmorphologien mit dem STM erkennt man eine zweidimensionale Wachstumsmode zwischen Insel- und step flow-Wachstum. Bei dieser Mode zeichnen sich in den Aufnahmen deutlich die durch den Fehlschnitt der Substrate verursachten Terrassen ab. Zudem zeigt sich diese Morphologie unabhängig davon, ob die Heterostruktur mit SMO oder LMO abschließt (siehe Abbildung 9.14).

Die chemische Qualität der vergrabenen Grenzflächen wird mit Methoden der Röntgenbeugung und Transmissionselektronenmikroskopie (TEM) weiter dargestellt. XRD- und XRRMessungen zeigen chemisch scharfe LMO/SMO-Grenzflächen mit $\sigma_{L M O} / S M O=0.1-0.2 \mathrm{~nm}$ (siehe Abbildung 9.15). Aus der mittleren Dicke der LMO/SMO-Bilagen von $\Lambda=3.32(4) \mathrm{nm}$ ergibt sich der genaue Wert von $n \approx 3.2$. Durch die numerische Anpassung des XRDSpektrums ergeben sich die individuellen Gitterparameter der LMO- und SMO- Lagen senkrecht zur Substratebene. Mit $c_{L M O}=0.394(2) \mathrm{nm}$ und $c_{S M O}=0.365(7) \mathrm{nm}$ zeigt sich ein 
a)

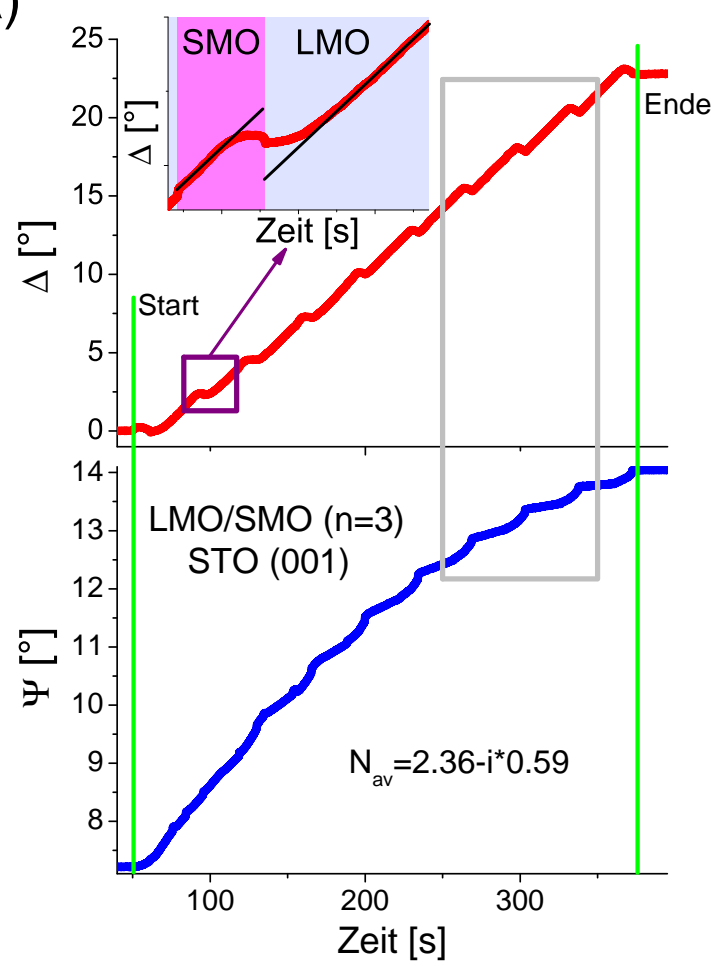

b)

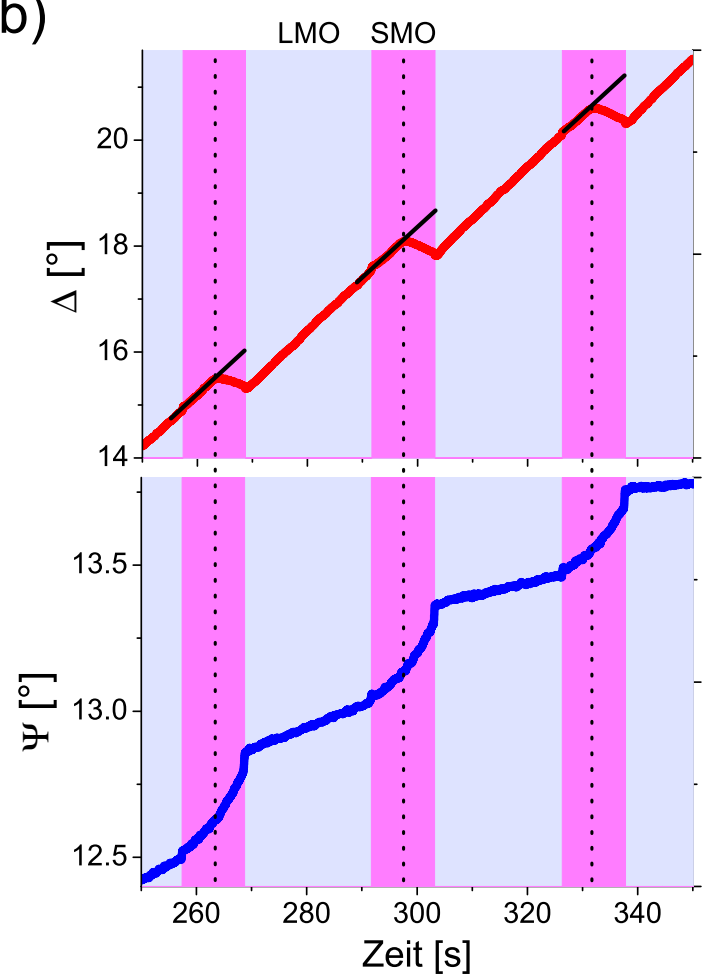

Abbildung 9.12.: In-situ Ellipsometrie von SMO/LMO-SL $\left(n \approx 3, d_{L M O} \approx 6 \mathrm{ML}\right.$, $\left.d_{S M O} \approx 2 \mathrm{ML}, m=16\right)$ auf STO (001): a) Übersicht der gesamten Depositionsdauer, der aus den Endwerten von $\Delta(t)$ und $\Psi(t)$ berechnete durchschnittliche Brechungsindex $N_{a v}$ ist im Diagramm angegeben. Das Inset zeigt $\Delta(t)$ für das Wachstum der zweiten Doppellage aus SMO und LMO. b) Vergrößerte Darstellung des grau umrandeten Bereiches in a), die Positionen, an denen sich die Steigung von $\Delta(t)$ während des Wachstums der SMO-Lagen ändert, sind markiert.

sehr starker Unterschied zwischen den individuellen Gitterparametern. Der Gitterparameter für die LMO-Lagen ist deutlich größer als jener, der sich für eine einfache LMO-Schicht auf STO ergibt (siehe Abschnitt 9.2.1). Aus dem Gitterebenenabstand in c-Richtung für die LMO-Lagen, $c_{L M O}=0.394(2) \mathrm{nm}$, berechnet man unter der Annahme einer vollständigen Verspannung durch das STO-Substrat und $\nu=1 / 3$ die pseudokubische Gitterkonstante $a_{L M O}=0.392(2) \mathrm{nm}$. Diese liegt zwischen dem Wert der rhomboedrischen Phase, die für einzelne LMO-Filme auf STO realisiert wird, und dem Wert für das stöchiometrische, orthorhombische LMO 122. Der Gitterebenenabstand der SMO-Lagen kommt dem Wert $c=0.3705 \mathrm{~nm}$, den man für einen voll verspannten SMO-Film auf STO erwarten würde $\left(a_{S M O}=0.3805 \mathrm{~nm}, \nu=1 / 3\right)$, sehr nahe. Damit ist hier von stöchiometrischen SMO auszugehen. Bei einem reduzierten Sauerstoffgehalt würde der Gitterebenenabstand aufgrund der höheren Konzentration von $\mathrm{Mn}^{3+}$-Ionen größer ausfallen.

Die Auswertung der Röntgenspektren hat gezeigt, dass die LMO/SMO-Grenzflächen im Mittel chemisch sehr abrupt sind. Durch die Betrachtung von STEM-Aufnahmen kann man etwaige lokale Variationen erfassen. In Abbildung 9.16 ist eine HAADF-STEM-Übersicht des LMO/SMO-Übergitters $(n \approx 3, m=11)$ dargestellt. Man erkennt für $i_{\text {Bilage }}=3-11$ einen deutlichen Kontrast zwischen den SMO- und LMO-Lagen, der sich aus den Unterschieden der Gesamtelektronendichte ergibt. Die ersten beiden SMO-Lagen $\left(i_{\text {Bilage }}=1,2\right)$ sind etwas 
a)

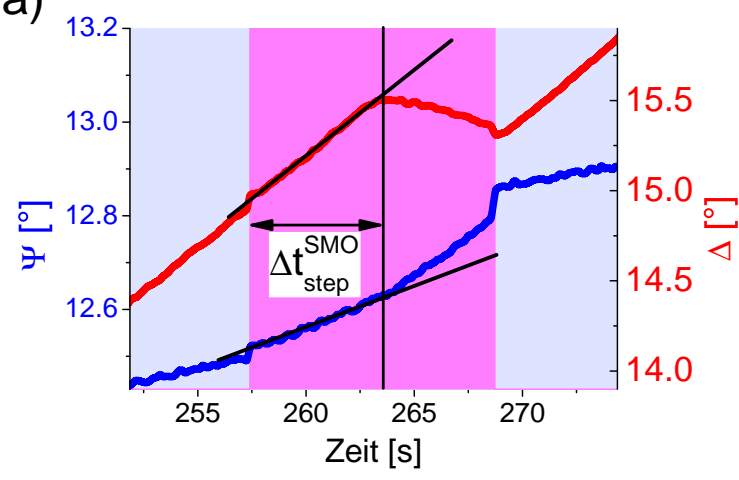

b)

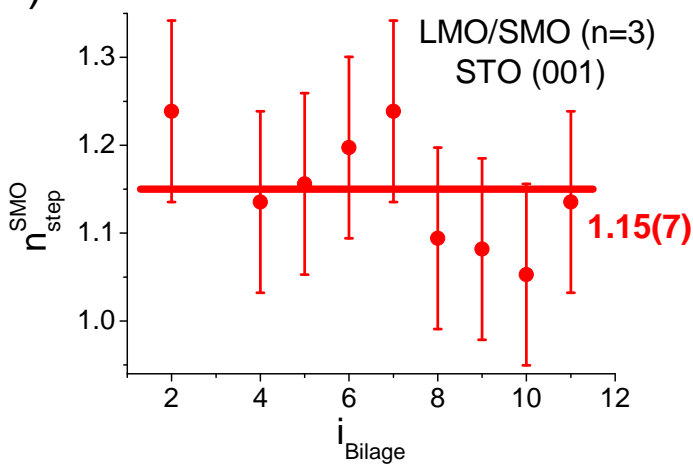

Abbildung 9.13.: Detailansicht von $\Delta(t), \Psi(t)$ beim Wachstum des SMO/LMOÜbergitters $\left(n \approx 3, d_{L M O} \approx 6 \mathrm{ML}, d_{S M O} \approx 2 \mathrm{ML}\right)$ aus Abbildung 9.12 a) Stufenartige Änderung der Steigung von $\Delta(t), \Psi(t)$ beim Wachstum von SMO nach $\Delta t_{\text {step }}^{S M O}$, b) $n_{\text {step }}^{S M O}$ als Funktion der Nummer der Doppellage $i_{\text {Bilage }}$.

a)

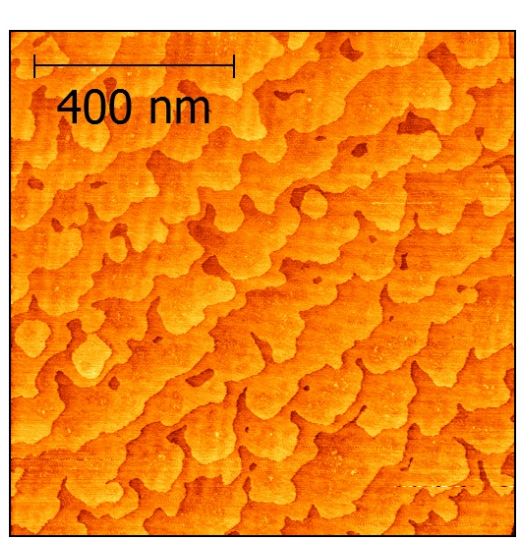

b)

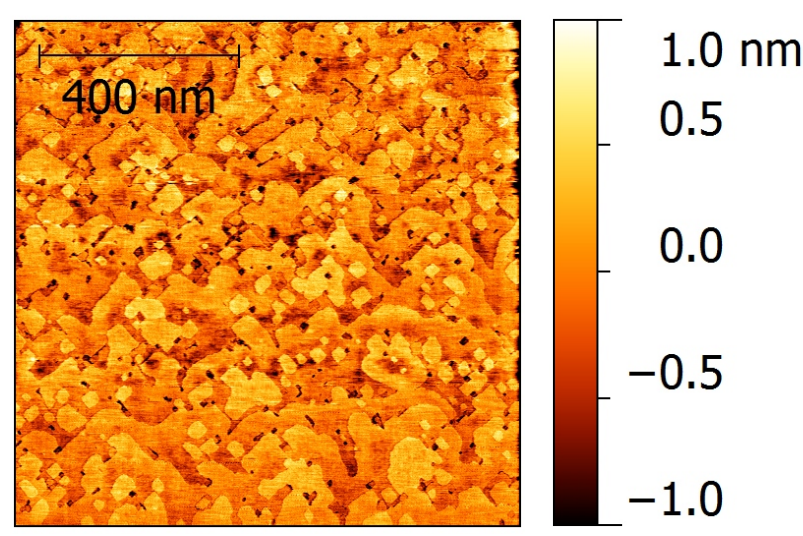

Abbildung 9.14.: Oberflächenmorphologien von LMO/SMO-Übergittern auf STO (001)Substraten $(n \approx 3)$ gemessen mit dem STM: a) LMO/SMO, $m=11$ (SMO an der Oberfläche), b) SMO/LMO, $m=16$ (LMO an der Oberfläche).

schwieriger auszumachen als die folgenden, was auf eine stärkere chemische Durchmischung in diesen Lagen hindeutet. Des Weiteren kann man feststellen, dass die SMO-Lagen über die gesamte Breite von $400 \mathrm{~nm}$ keine größeren Unterbrechungen zeigen.

In der höher aufgelösten HAADF-STEM-Aufnahme bestätigt sich das zuvor geschilderte Bild. Die chemische Durchmischung der La- und Sr-Ionen in den ersten beiden Wiederholungen der SMO/LMO-Doppellagen deutet sich bereits in der in-situ Ellipsometrie an (vergleiche Inset von Abbildung $9.12 \mathrm{a}$ )). Dies ist vermutlich mit einer geringeren Mobilität der Adatome für $i_{\text {Bilage }}=1,2$ zu erklären (vergleiche Abschnitt 6.3). In der Anfangsphase wird dadurch die Oberflächenmorphologie der individuellen Lagen von kleinen Inseln gekennzeichnet. Bei einer solchen Beschaffenheit der Oberfläche kann es dann beim Aufbringen der folgenden Materiallage durch Diffusionsprozesse an der Oberfläche leicht zu einer chemischen Durchmischung kommen. Für $i_{\text {Bilage }}>2$ bilden sich an der Oberfläche deutlich größere Inseln, so dass eine Durchmischung der Kationen an der Oberfläche nicht mehr ins Gewicht fällt. Im Gegensatz zur Oberfläche ist die Diffusion innerhalb der Heterostruktur durch die dreidimensionale Bindung stark gehemmt. Dies wurde auch schon in Abschnitt 8.2.3 belegt: Ein mehrmaliges Auslagern von Multilagen aus Manganaten bei den hier verwendeten Depositionsbedingungen führte zu keinen Veränderungen der Grenzflächen zwischen den einzelnen Manganat-Schichten. 
a)

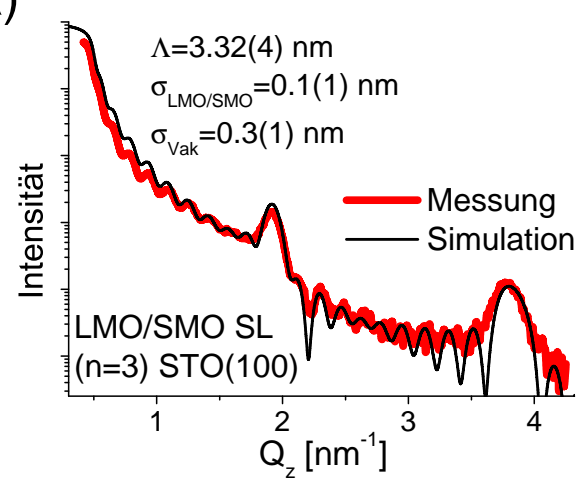

b)

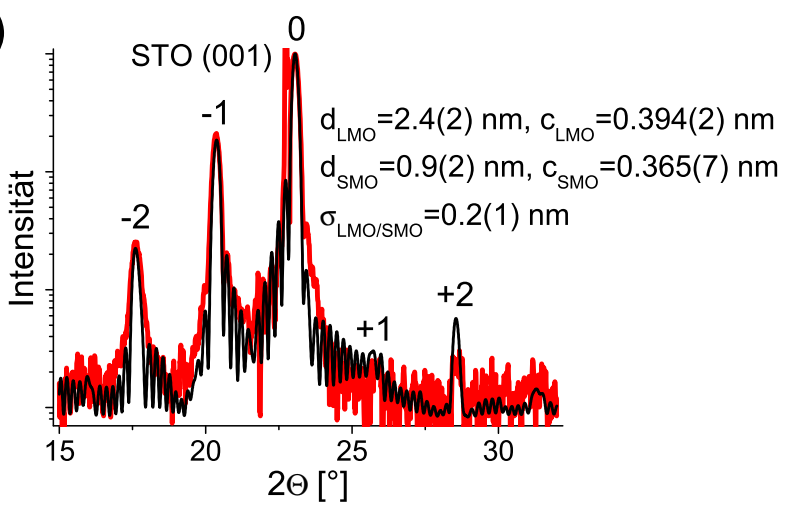

Abbildung 9.15.: Ermittlung der strukturellen Eigenschaften von LMO/SMO-SL $(n \approx 3$, $m=11$ ) auf STO (001) durch Analyse der Röntgenspektren: a) Durch die numerische Anpassung des XRR-Spektrums mit ReMagX [165] ergeben sich die Bilagendicke $\Lambda$ und die Rauigkeiten $\sigma_{L M O} / S M O$ und $\sigma_{V a k}$. b) Aus der numerischen Anpassung des XRD-Spektrums folgen Werte für die individuellen Schichtdicken $d_{L M O}, d_{S M O}$ und Gitterparameter $c_{L M O}$, $c_{S M O}$ sowie die Rauigkeit $\sigma_{L M O / S M O}$.

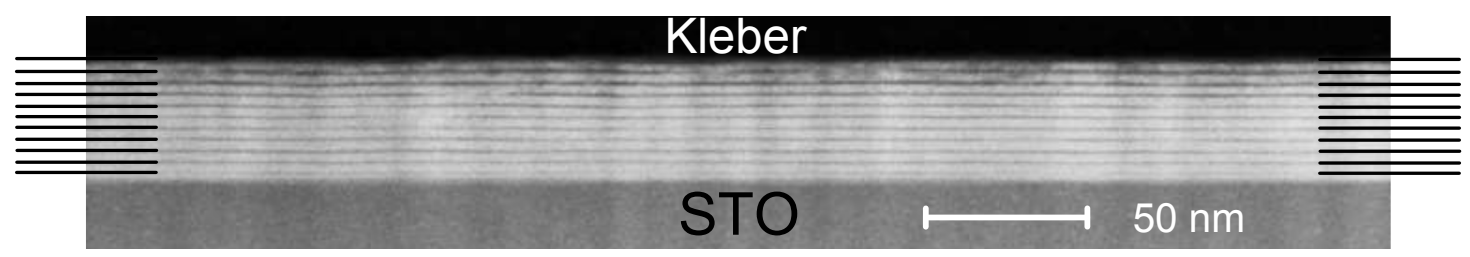

Abbildung 9.16.: HAADF-STEM-Übersicht von LMO/SMO-SL $(n \approx 3, m=11)$ auf STO (001): Die SMO-Lagen weisen eine geringere Gesamtelektronendichte auf und erscheinen so dunkler als die LMO-Lagen. Die Positionen der SMO-Lagen sind durch schwarze Linien an den Rändern gekennzeichnet (Aufnahme erstellt von Ricardo Egoavil).

Eine präzisere Übersicht über die Verteilung der La-Ionen im Übergitter ergibt sich durch eine STEM-EELS-Karte an der La-Kante, die in Abbildung 9.17 dargestellt ist. Auch hier ist ein klarer Kontrast zwischen den LMO- und SMO-Lagen zu erkennen, der für $i_{\text {Bilage }}=$ 1,2 etwas schwächer erscheint als für $i_{\text {Bilage }}>2^{35}$. In der Vergrößerung der STEM-EELSKarte kann man die Schichtdicken von LMO und SMO abzählen. Diese liegen mit $d_{S M O}=$ $2 \mathrm{ML}$ und $d_{L M O}=6-7 \mathrm{ML}$ im erwarteten Bereich. An den LMO/SMO-Grenzflächen sieht man ein größeres La-Signal im SMO (rote Pfeile in Abbildung 9.17) als an den SMO/LMOGrenzflächen. Da in dem betrachteten Übergitter die Dicke einer LMO/SMO-Bilage mit $\Lambda=3.3 \mathrm{~nm} \approx 8.6 \mathrm{ML}$ keine ganze Zahl von Atomlagen darstellt (vergleiche Abbildung 9.15), kann dies nicht aus den Stufen des Substrates resultieren. Also ist dieses kleine La-Signal innerhalb der SMO-Lagen einer leichten La/Sr-Interdiffusion zuzuordnen.

Unter Berücksichtigung der strukturellen Charakterisierungen der LMO/SMO-SL entwickelt man im Folgenden ein Wachstumsmodell, mit dem man die gemessene in-situ Ellipsometrie numerisch erfassen kann. Mit Blick auf die Oberflächenmorphologie nimmt man ein step-flowWachstum an. Damit muss man im Gegensatz zum Wachstum auf STO (111)-Substraten kein Aufrauen der Filme über die Näherung mit einem effektiven Medium (EMA) berücksichtigen.

\footnotetext{
$\overline{{ }^{35} \text { Die LMO/SMO-Grenzflächen für } i_{\text {Bilage }}}=9-11$ erscheinen im Bild etwas unschärfer. Dies ergibt sich daraus, dass sich die TEM-Lamelle bei der Messung dieses Bereichs nicht mehr perfekt im Fokus des Elektronenstrahls befand. Die reduzierte Auflösung in diesem Bereich macht sich auch durch die fehlende atomare Auflösung der La-Atome in diesem Bereich bemerkbar.
} 


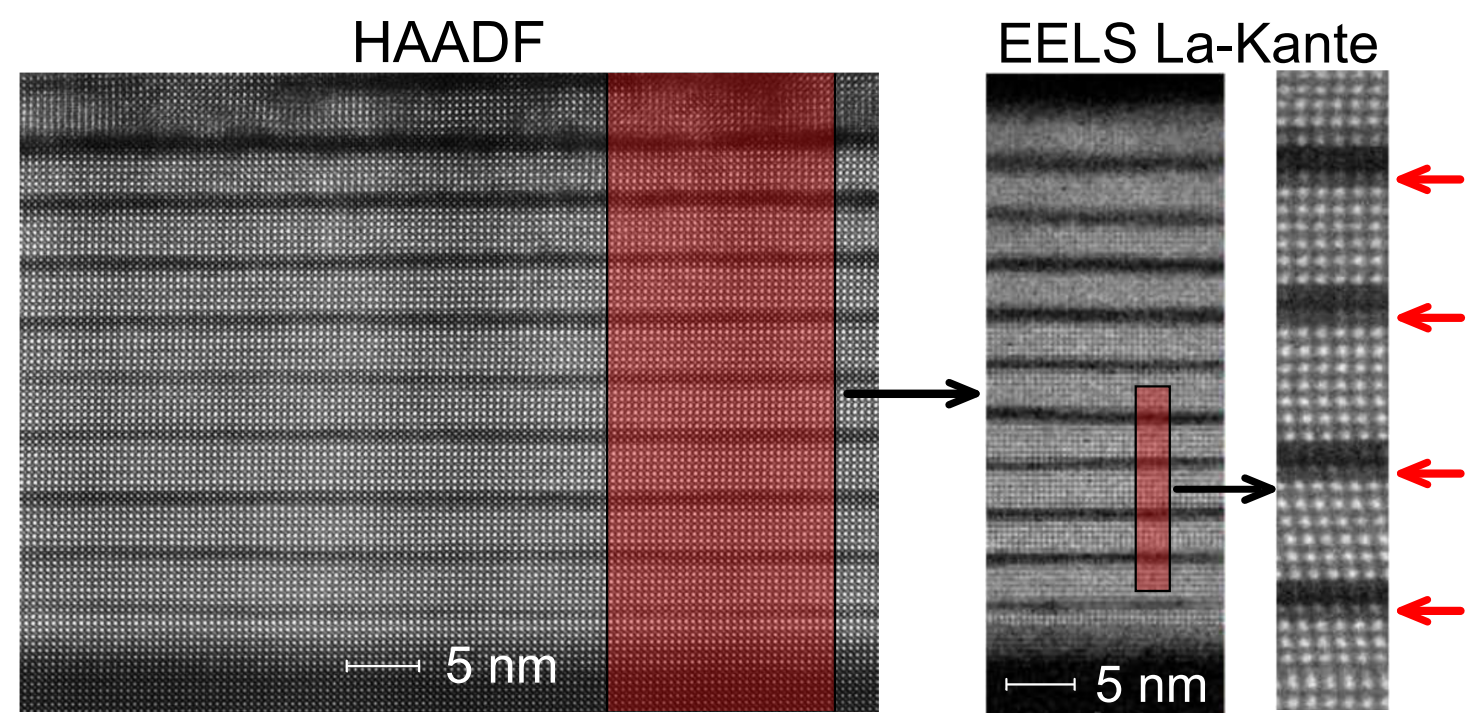

\begin{abstract}
Abbildung 9.17.: Hochauflösende HAADF-Aufnahme von LMO/SMO-SL ( $n \approx 3, m=11$ ) auf STO (001) und STEM-EELS Karte an der La-Kante für den rot eingefärbten Bereich. Die roten Pfeile neben der EELS-Karte zeigen auf die LMO/SMO-Grenzflächen, an denen eine geringe La-Konzentration in den SMO-Lagen festzustellen ist. An den SMO/LMOGrenzflächen ist dies nicht erkennbar (Bilder von Ricardo Egoavil aufgenommen).
\end{abstract}

Da die mittlere chemische Durchmischung sehr klein ausfällt, wird sie in den folgenden Betrachtungen vernachlässigt. Die Effekte an den Grenzflächen werden von der Delokalisierung der $e_{g}$-Elektronen bestimmt, welche mit einer Thomas-Fermi-Abschirmlänge $L_{T F}$ quantifiziert wird (vergleiche Abschnitt 3.1). In Abbildung 9.18 wird das Prinzip zur numerischen Behandlung dieser Umverteilung der Ladungen auf den Mn-Ionen illustriert. Die LMO/SMOSL werden in einzelne Lagen, die jeweils die Dicke einer einzelnen Perowskit-Lage besitzen, zerlegt. Diesen wird eine separate Dotierung $x_{i}$, welche dem Anteil der $\mathrm{Mn}^{4+}$-Ionen entspricht, zugeordnet. In Abschnitt 6.2 wurde dargelegt, dass der Brechungsindex eines dünnen Filmes aus einem Perowskit-Manganat von dem Verhältnis zwischen $\mathrm{Mn}^{3+}$ - und $\mathrm{Mn}^{4+}$-Ionen abhängt. Damit setzt man für den Brechungsindex einer solchen Lage mit der Dotierung $x_{i}$ den Wert $N\left(L S M O\left(x_{i}\right)\right)$ für einen einfachen Film aus $\operatorname{LSMO}\left(x_{i}\right)$ an. Mit jeder abgeschiedenen Monolage verändert sich die Verteilung der Elektronen auf den Mn-Ionen. Dabei klingt die mittlere Zahl der Elektronen in jeder Monolage gemäß

$$
n(z)=n_{0} \cdot e^{-|z| / L_{T F}}
$$

mit wachsender Entfernung $z$ entlang der Wachstumsrichtung ab (Der Vorfaktor $n_{0}$ dient zur Normierung und wird so gewählt, dass die Gesamtzahl von Elektronen bei der Umverteilung konstant bleibt.). Dies ist in Abbildung 9.18 b) für das Wachstum einer Monolage SMO auf LMO demonstriert.

Mit dem beschriebenen Formalismus erfolgt nun die numerische Simulation der in-situ Ellipsometrie des SMO/LMO-Übergitters $(n \approx 3, m=16)$ aus Abbildung 9.12 $\beta$. Dabei wird eine Bilage des Übergitters aus SMO $(d \approx 2 \mathrm{ML}) / \mathrm{LMO}(d \approx 6 \mathrm{ML})$ herangezogen. Mit jeder deponierten Monolage verändert sich die Verteilung der $e_{g}$-Elektronen, wie es in Abbildung 9.18 b) illustriert wurde. Bei der numerischen Anpassung wird eine Änderung von $L_{T F}$ im

\footnotetext{
${ }^{36}$ Hier werden nur die Daten zu $\Delta(t)$ gezeichnet. Für $\Psi(t)$ ergeben sich ähnliche Ergebnisse, die aber wegen der deutlich kleineren Amplitude der Änderungen und des größeren Rauschanteils weniger deutlich darstellbar sind.
} 


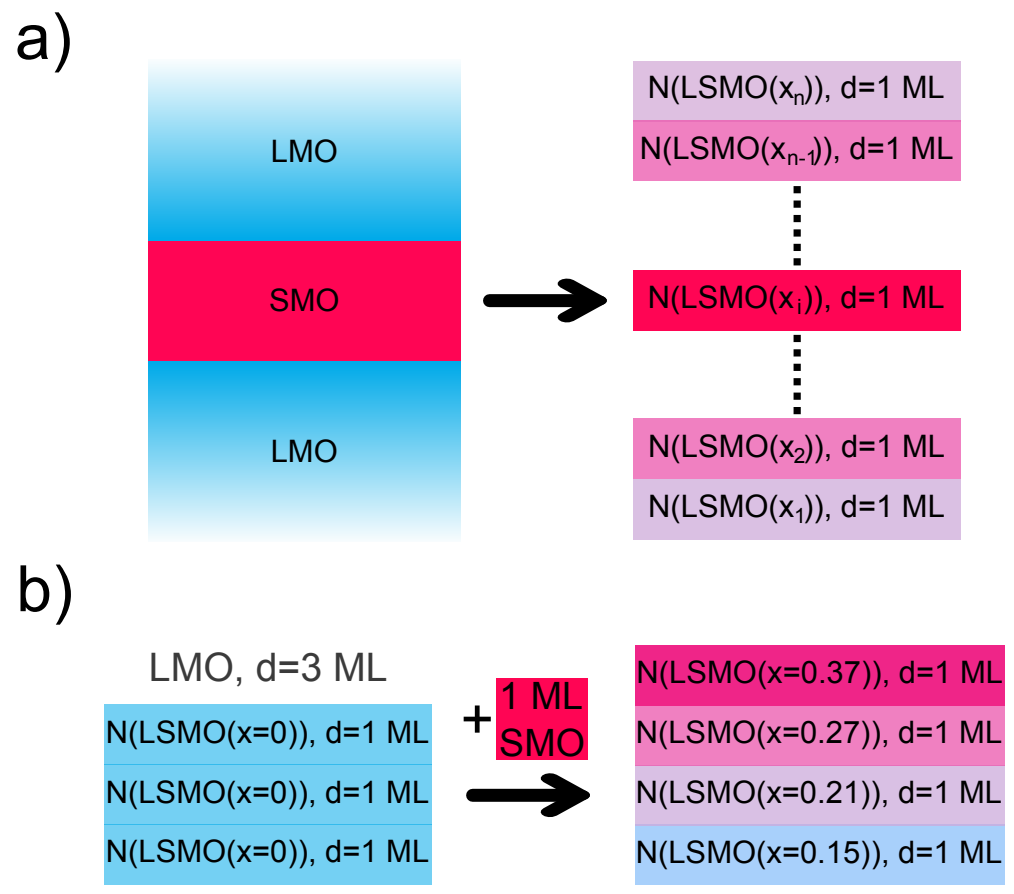

\begin{abstract}
Abbildung 9.18.: Illustration des Verfahrens zur numerischen Behandlung der in-situ Ellipsometrie von LMO/SMO-Übergittern auf STO (001): a) Zerlegung von LMO/SMO-SL in Monolagen $(d=1 \mathrm{ML})$ mit variablen Anteil von $\mathrm{Mn}^{4+}$-Ionen $x_{i}$. Durch den Wert von $x_{i}$ wird der zugeordnete Brechungsindex festgelegt, der sich über den Wert für $\operatorname{LSMO}\left(x_{i}\right)$, $N\left(\operatorname{LSMO}\left(x_{i}\right)\right)$, ergibt. b) Das Hinzufügen einer Lage SMO auf die LMO-Lagen setzt eine Umverteilung der Ladungen auf den Mn-Ionen gemäß Gleichung 9.3 in Gang (In diesem Beispiel ist $L_{T F}=1.4 \mathrm{~nm}$.).
\end{abstract}

Verlauf der Deposition erlaubt. Wenn man die gemessenen Daten mit einer einfachen Simulation, bei der kein Ladungstransfer stattfindet, vergleicht, sieht man deutliche Abweichungen. Insbesondere der stufenartige Verlauf beim Wachstum der SMO-Schicht wird nicht reproduziert (siehe Abbildung 9.19). Wenn man eine Delokalisierung der $e_{g}$-Elektronen zulässt, kann man die gemessenen Daten sehr gut durch die numerische Simulation nachbilden. Die Evolution der Elektronenverteilung, die zu der in Abbildung 9.19 gezeichneten Kurve führt, ist in Abbildung 9.20 dargestellt.

Von der vorherigen Doppellage liegt zu Beginn eine LMO-Schicht an der Oberfläche vor. Auf diese werden dann $2 \mathrm{ML}$ SMO deponiert. Dabei ist $L_{T F}$ mit $L_{T F}(1)=1.4(7) \mathrm{nm} \mathrm{zu}$ Beginn sehr hoch und nimmt dann für die zweite SMO-Lage auf $L_{T F}(2)=0.7(2) \mathrm{nm}$ ab. Daran anschließend beginnt die Abscheidung von LMO. Aus Symmetriegründen zeigen dann die $\mathrm{MnO}_{2}$-Lagen bei $i_{M L}=3$ und $i_{M L}=5$ eine sehr ähnliche Besetzung der $e_{g}$-Niveaus. Diese Aufteilung der Elektronen zwischen diesen beiden Lagen wird auch zur Vermeidung der polaren Katastrophe erforderlich $733^{37}$. Nach dem Aufbringen der ersten LMO-Lage verkleinert sich $L_{T F}$ auf einen Wert $L_{T F}(3)=0.37(6) \mathrm{nm}$, der etwa der Dicke einer Monolage entspricht. Auch im weiteren Verlauf des Wachstums verbleibt $L_{T F}$ dann bei diesem Wert.

Die Signifikanz der ermittelten Längenskalen für $L_{T F}$ zeigt sich über die Begutachtung von Simulationen, die sich unter Variation der Parameter $L_{T F}(i)$ ergeben (siehe Abbildung 9.21). Aus dieser systematischen Variation der $L_{T F}(i)$ ergeben sich dann die in Abbildung 9.20 angegebenen Fehlerintervalle.

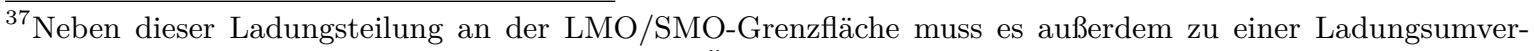
teilung zwischen der Oberfläche des gesamten Übergitters und der ersten SMO/LMO- bzw. STO/LMOGrenzfläche kommen, um die Divergenz des elektrostatischen Potentials vollständig zu unterbinden (siehe Abschnitt 3.1.
} 


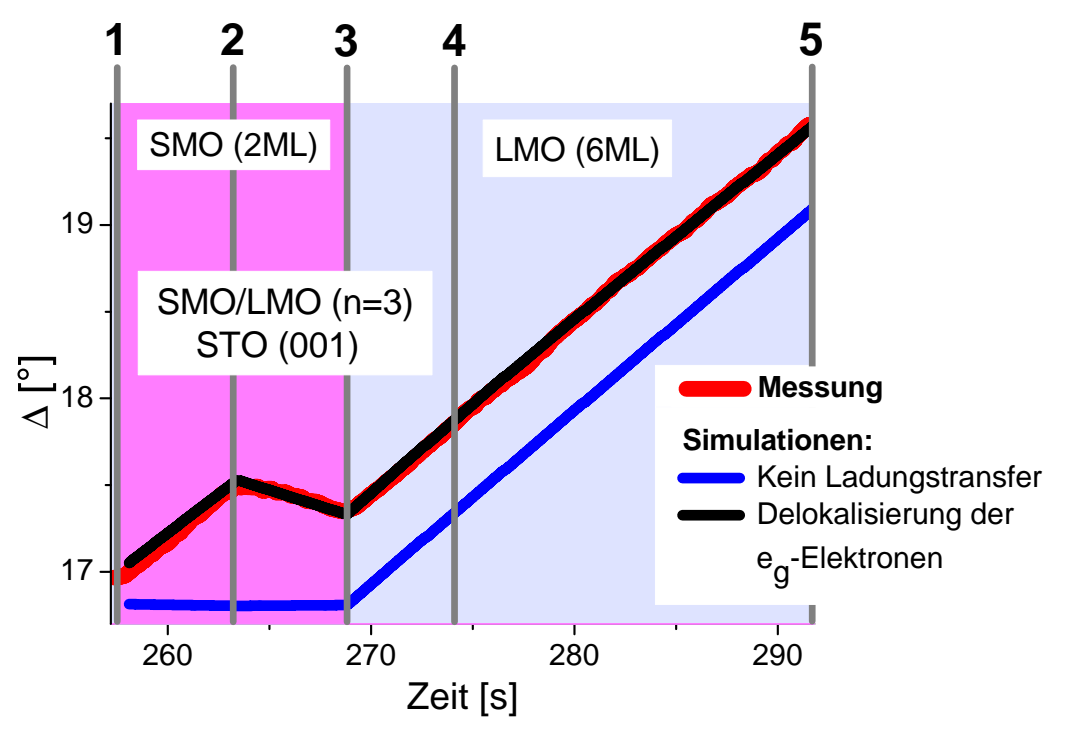

Abbildung 9.19.: Numerische Anpassung der in-situ Ellipsometrie für das Wachstum einer
SMO/LMO-Bilage des SMO/LMO-Übergitters $(n \approx 3, m=16)$ aus Abbildung 9.12 Neben
den Messdaten ist eine einfache Simulation mit den Brechungsindizes für LMO und SMO
gezeigt, bei der kein Ladungstransfer in Betracht gezogen wurde. Die beste Anpassung der
Messung ergibt sich durch das in Abbildung 9.20 gezeigte Schema des Ladungstransfers, wo
den hier markierten Phasen 1-5 entsprechende Besetzungen der $e_{g}$-Niveaus zugeordnet sind.

Für die beste Anpassung der experimentellen Daten muss eine Änderung von $L_{T F}$ während der Deposition erlaubt werden. Dies ist ein Hinweis auf einen Zusammenhang zwischen der Oberfläche der Heterostruktur und der Delokalisierung der Elektronen. An der Oberfläche ist eine Besetzung der $d_{3 z^{2}-r^{2}}$-Orbitale energetisch stark bevorzugt 84 . Es gibt somit bei Phase 2 in Abbildung 9.20 eine zusätzliche treibende Kraft, die eine Anreicherung von Elektronen in der SMO-Lage unterstützt. Es ist energetisch günstiger, wenn die mit den $\mathrm{Mn}^{3+}$-Ionen einhergehenden Jahn-Teller-Verzerrungen in der Lage an der Oberfläche als in tieferen Lagen vorliegen. Nach der Deposition einer weiteren SMO-Lage reduziert sich $L_{T F}$, da die Besetzung der $\mathrm{MnO}_{2}$-Lage an der Oberfläche durch das Coulomb-Potential behindert wird. Durch das Aufbringen der LMO-Lage verschwindet dieses zusätzliche treibende Element, da die Oberfläche dann automatisch stark mit Elektronen besetzt ist.

Die starke Delokalisierung der Elektronen nach der Deposition der ersten SMO-Lage könnte die Ursache für die leichte La/Sr-Interdiffusion der LMO/SMO-Grenzfläche sein (vergleiche Abbildung 9.17). Wie bei der $\mathrm{LaAlO}_{3} / \mathrm{STO}$-Grenzfläche führt die über 3-4 ML delokalisierte Elektronenwolke der $e_{g}$-Elektronen zu einem elektrischen Dipol, der durch Verschiebung der Sr- und La-Ionen ausgeglichen werden kann [61].

Es ist davon auszugehen, dass die hier für Depositionsbedingungen konstruierte Verteilung der $e_{g}$-Elektronen bei tieferen Temperaturen $\left(T_{C / N}<T \lesssim 400 \mathrm{~K}\right)$ ähnlich verläuft ${ }^{38}$. Die thermische Aufweichung der Fermi-Verteilung beträgt bei $T_{\text {Dep }}=930^{\circ} \mathrm{C} k_{B} T_{D e p} \approx 0.1 \mathrm{eV}$. Dies ist deutlich kleiner als die typische Jahn-Teller-Energie $E_{J T} \approx 0.7 \mathrm{eV}$ (siehe Abschnitt 2.2 und die Coulomb-Energie, die bei einer Ladungsseparation um eine Perowskit-Lage bezahlt werden muss $\Delta E_{C} \sim 1 \mathrm{eV}$. Infolge von $E_{J T}>k_{B} T$ kommt es nicht zu einer thermisch getriebenen Umbesetzung der $e_{g}$-Orbitale und einer damit einhergehenden Änderung von $t_{d d}$

\footnotetext{
${ }^{38} \mathrm{Im}$ Grundzustand $(T \approx 5 \mathrm{~K})$ kann eine kollektive magnetische Ordnung $t_{d d}$ und damit die Delokalisierung zusätzlich beeinflussen.
} 

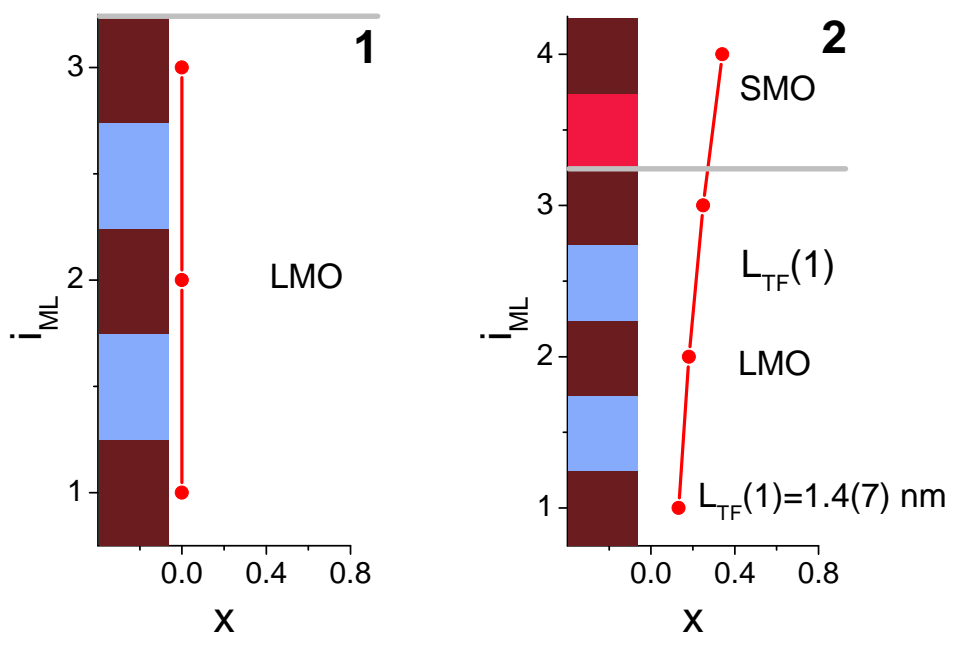

2: $\quad \mathrm{L}_{\mathrm{TF}}(1)=1.4(7) \mathrm{nm}$

3: $\quad \mathrm{L}_{\mathrm{TF}}(2)=0.7(2) \mathrm{nm}$

4-5: $\mathrm{L}_{\mathrm{TF}}(3)=0.37(6) \mathrm{nm}$
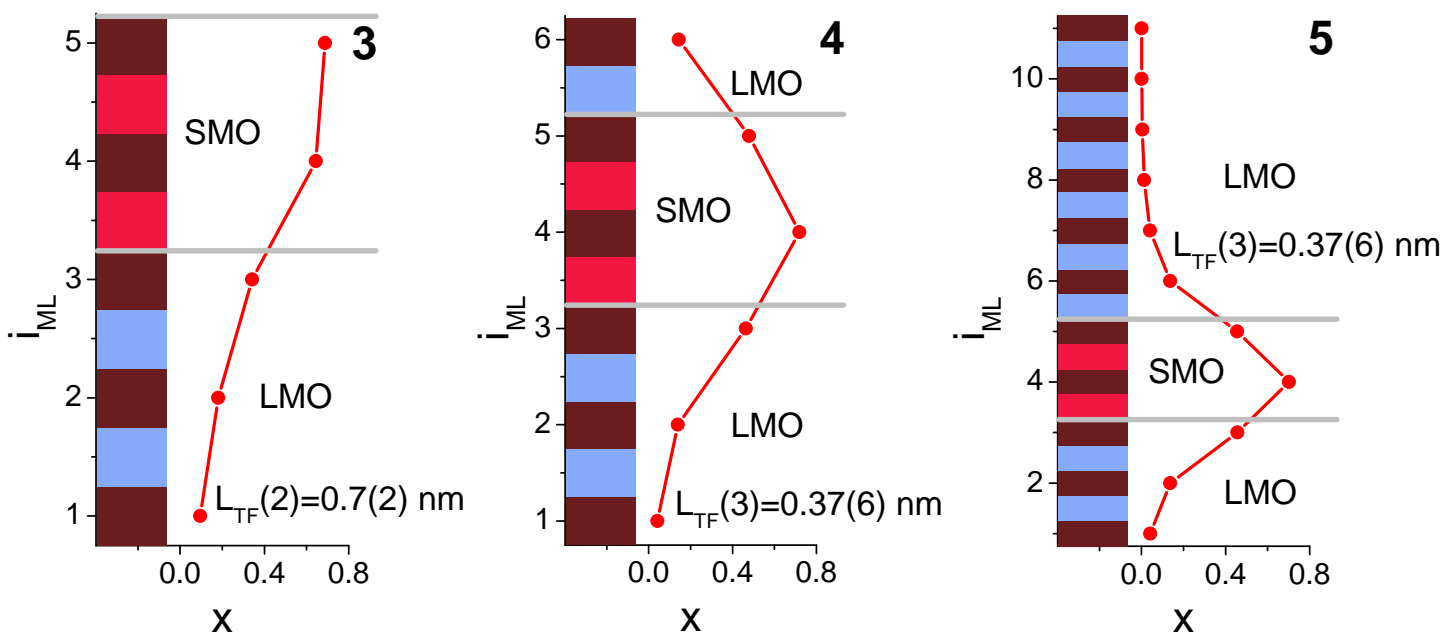

Abbildung 9.20.: Besetzung der $e_{g}$-Niveaus, $n\left(e_{g}\right)=1-x$, in den Phasen 1-5, die zu der in Abbildung 9.19 gezeigten numerischen Simulation führen. Die Elektronenverteilungen ergeben sich durch Anwendung von Gleichung 9.3, wobei $L_{T F}$ in Abhängigkeit von der jeweiligen Depositionsphase die im Diagramm vermerkten Werte annimmt.

(vergleiche Abschnitte 2.3 und 3.1). Auch die thermische Ausdehnung treibt nur kleine Änderungen der orbitalen Umbesetzung. Die linearen, thermischen Ausdehnungskoeffizienten sind für STO [269] und $\operatorname{LSMO}(\mathrm{x}=0.3)$ [270] sehr ähnlich:

$$
\begin{aligned}
\alpha_{S T O}(T & =300-2000 \mathrm{~K})=1.1 \cdot 10^{-5} K^{-1} \\
\alpha_{L S M O}(T & =300-1300 \mathrm{~K})=1.2 \cdot 10^{-5} K^{-1}
\end{aligned}
$$

Der kleine Unterschied der thermischen Ausdehnungen

$$
\left(\alpha_{L S M O}-\alpha_{S T O}\right) \cdot\left(T_{D e p}-300 \mathrm{~K}\right) \approx 0.1 \%
$$

führt nur zu minimalen Änderungen des Verspannungszustandes der Manganat-Lagen und kann damit die Besetzung der $e_{g}$-Orbitale nur geringfügig beeinflussen. Wegen $k_{B} T<\Delta E_{C}$ nimmt $L_{T F}$ nach dem Abkühlen keinen deutlich anderen Wert an als bei Depositionsbedingungen (vergleiche Abschnitt 3.1). 


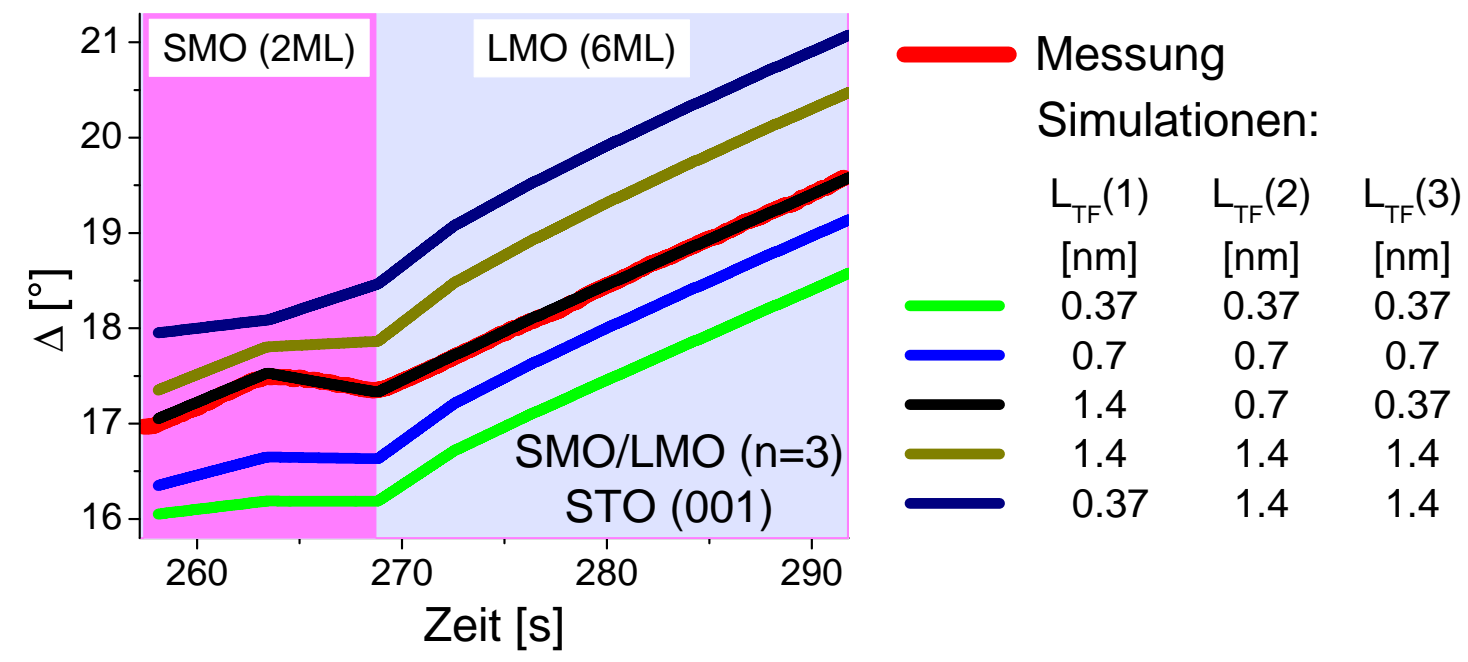

Abbildung 9.21.: Zur Signifikanz der ermittelten Werte $L_{T F}(i=1-3)$ (vergleiche Abbildung 9.20): Im Diagramm sind die simulierten Verläufe von $\Delta(t)$ für verschieden ausgewählte Werte von $L_{T F}(i)$ gezeigt, um sie mit den Messdaten und der in Abbildung 9.19 gezeichneten Simulation zu vergleichen (Zur besseren Übersicht wurden die Kurven entlang $\Delta$ verschoben.).

\subsection{Magnetismus der $\mathrm{LaMnO}_{3} / \mathrm{SrMnO}_{3}$-Übergitter}

In diesem Abschnitt soll zunächst ein systematischer Vergleich der LMO/SMO-SL, die auf STO-Substraten der (001)- und (111)-Orientierung gewachsen wurden, erfolgen. Für die Heterostrukturen auf STO (001) beobachtet man die Koexistenz zweier unabhängiger ferromagnetischer Phasen. Die Phase mit der kleineren Curie-Temperatur, die Tieftemperaturphase (LTP), folgt qualitativ dem Verhalten, das in der Literatur für diese Art von Übergittern berichtet wird. Deshalb wird für den Vergleich mit den Übergittern auf STO (111) diese Phase herangezogen. Die zweite Phase mit der größeren Ordnungstemperatur, die Hochtemperaturphase (HTP), wird dann im darauf folgenden Abschnitt genauer beleuchtet.

Die hier gezeigten magnetischen Messungen wurden entsprechend den Beschreibungen in Abschnitt 5.4 mittels SQUID-Magnetometrie ausgeführt. Soweit nicht anders beschrieben, lag das von außen angelegte Magnetfeld in der Ebene des Filmes und entlang der [100]Richtung für STO/LSAT (001)- bzw. entlang der [1̄12]-Richtung für STO (111)-Substrate. Die $M(T)$-Kurven, aus denen die Curie-Temperatur $T_{C}$ ermittelt wird, wurden beim Kühlen mit einer Rate von $3 \mathrm{~K} / \mathrm{min}$ aufgenommen. Das magnetische Moment der Filme wurde auf die gesamte Zahl der Mn-Ionen normiert.

\subsubsection{Vergleich $\mathrm{SrTiO}_{3}(111)$ - und (001)-Substrat}

Da der Abstand zwischen zwei Monolagen entlang der (111)-Richtung mit $c_{(111)}=\frac{1}{\sqrt{3}} c_{(001)}$ deutlich kleiner ist als entlang der (001)-Richtung mit $c_{(001)}$, muss man zum Vergleich der Heterostrukturen auf den verschieden geschnittenen Substraten unterschiedliche Werte für $n$ heranziehen, um ähnliche Dicken $\Lambda$ der LMO/SMO-Doppellagen zu erreichen. Für ein Übergitter auf STO (001) mit $n_{(001)}$ wählt man zum Vergleich so ein Übergitter auf STO (111) mit $n_{(111)}=2 \cdot n_{(001)}$. Durch eine entsprechende Wahl der Zahl von Wiederholungen $m$ haben die hier vorgestellten Strukturen eine Gesamtdicke von $d_{t o t}=m \cdot \Lambda=36 \pm 4 \mathrm{~nm}$. 

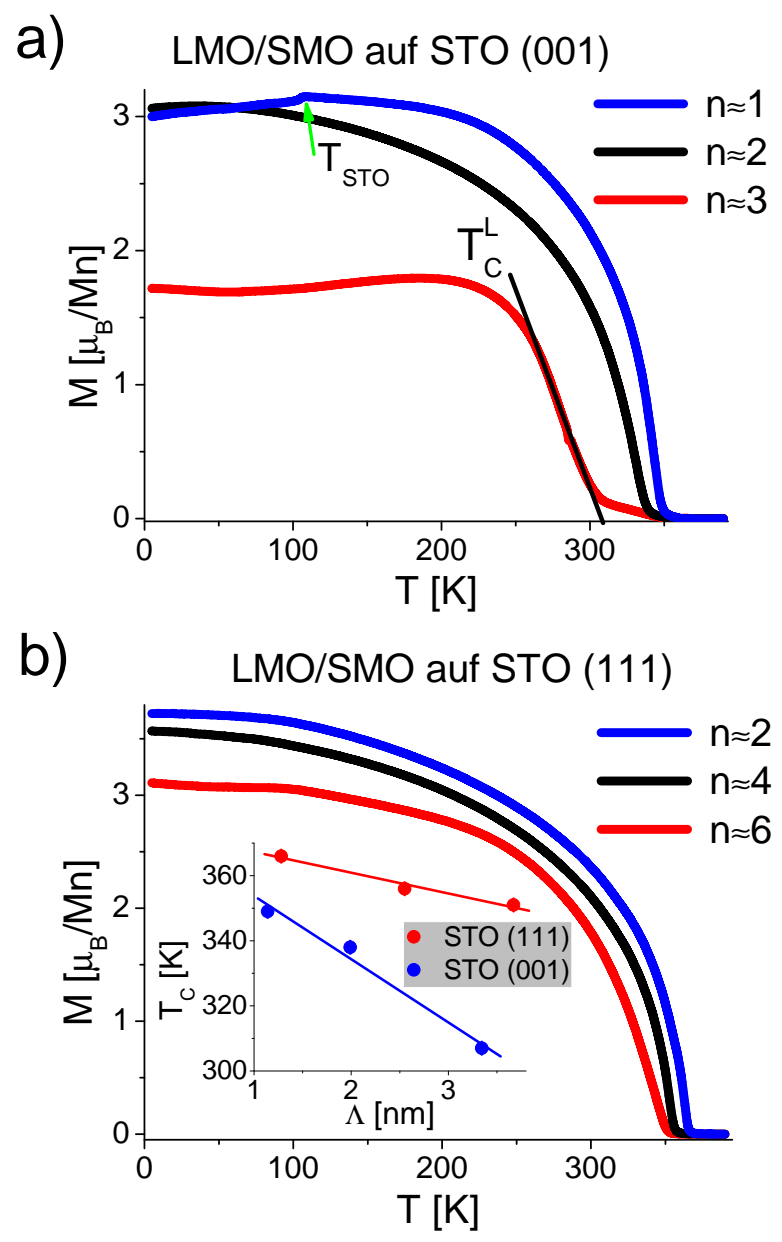

\begin{abstract}
Abbildung 9.22.: Vergleich der magnetischen Eigenschaften von LMO/SMO-Übergittern auf STO (001)- und STO (111)-Substraten, $M(T)$-Messungen: a) STO (001) für $n \approx 1-3$ und $d_{t o t}=m \cdot \Lambda=36 \pm 4 \mathrm{~nm}$. Die Temperatur des strukturellen Überganges des STOSubstrates, $T_{S T O}=105 \mathrm{~K}$, und die zur Ermittlung der Curie-Temperatur der Tieftemperaturphase, $T_{C}^{L}$ herangezogene Gerade an den Wendepunkt sind eingezeichnet. b) STO (111) $n \approx 2-6$ und $d_{t o t}=m \cdot \Lambda=36 \pm 3 \mathrm{~nm}$. Das Inset zeigt die Entwicklung von $T_{C}$ mit der Dicke der Bilagen $\Lambda$.
\end{abstract}

Die in Abbildung 9.22 gezeichneten Übergitter weisen alle einen ferromagnetischen Übergang auf. Die Curie-Temperaturen für $n=1$ (STO (001)) und $n=2$ (STO (111)) stimmen mit jenen von einfachen Filmen aus $\operatorname{LSMO}(\mathrm{x}=0.3)$ mit Schichtdicken von $d=30-40 \mathrm{~nm}$ überein 97, 267. Bei der Vergrößerung von $\Lambda$ kommt es zu einer Abnahme von $T_{C}$. Diese fällt für die Übergitter auf STO (001) wesentlich ausgeprägter aus als für die Strukturen auf STO (111). Bei der Verdreifachung von $\Lambda$ reduziert sich $T_{C}$ um $\Delta T_{C} \approx 40 \mathrm{~K}$ für STO (001) und um $\Delta T_{C} \approx 15 \mathrm{~K}$ für STO (111) (Inset Abbildung $9.22 \mathrm{~b}$ )). Bei der $M(T)$ )-Kurve für $n \approx 3$ fällt außerdem das Vorhandensein eines zweiten Überganges auf, der im nächsten Abschnitt behandelt wird.

Nach dem Einkühlen der Proben auf $T=5 \mathrm{~K}$ werden $M(H)$-Kurven aufgenommen. Für $n \approx$ 1,2 zeigen die Heterostrukturen auf STO (001)-Substraten schmale Hystereseschleifen, deren Koerzitivfeld mit $H_{C}=8,14$ Oe im Bereich der Ergebnisse für einfache LSMO-Filme liegt [97. Auch das magnetische Moment in Sättigung $M_{\text {sat }}$ stimmt für diese beiden Heterostrukturen mit dem Wert überein, der sich bei der vollen Ausrichtung aller Mn-Spins ergibt (siehe Inset 

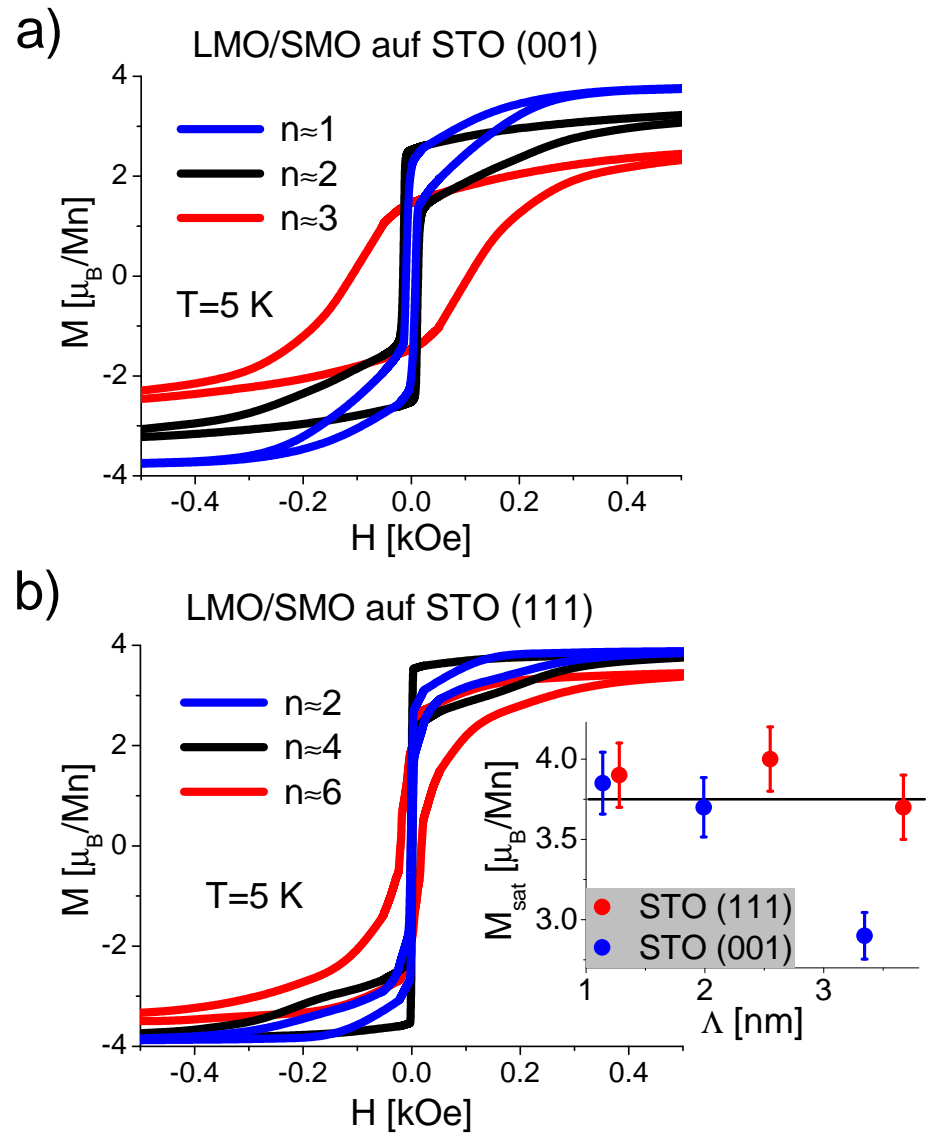

\begin{abstract}
Abbildung 9.23.: Vergleich der magnetischen Eigenschaften von LMO/SMO-Übergittern auf STO (001)- und STO (111)-Substraten, $M(H)$-Hysteresekurven bei $T=5 \mathrm{~K}$ : a) STO (001) für $n \approx 1-3$ und $d_{t o t}=m \cdot \Lambda=36 \pm 4 \mathrm{~nm}$. b) STO (111) $n \approx 2-6$ und $d_{t o t}=m \cdot \Lambda=$ $36 \pm 3 \mathrm{~nm}$. Das Inset zeigt das magnetische Moment in Sättigung, $M_{\text {sat }}$, als Funktion der Bilagendicke (Der theoretische Wert von $M_{s a t}=3.75 \mu_{\mathrm{B}} / \mathrm{Mn}$ für die volle Ausrichtung aller Spins ist eingezeichnet.).
\end{abstract}

9.23 b)). Erhöht sich $\Lambda$ weiter auf $n=3$, so sieht man, dass sich das Moment in Sättigung auf $2.9 \mu_{\mathrm{B}} / \mathrm{Mn}$ reduziert und das Koerzitivfeld drastisch auf $H_{C}(5 \mathrm{~K})=110$ Oe ansteigt. $M_{\text {sat }}=3.2 \mu_{\mathrm{B}} / \mathrm{Mn}$ ist der Wert, der sich für die vollständige Ausrichtung der Spins in allen LMO-Lagen und in jeweils einer Monolage der SMO-Schichten ergeben würde.

Die Übergitter auf STO (111) weisen für $n \approx 2-4$ sehr kleine Koerzitivfelder mit $H_{C}(5 \mathrm{~K}) \approx$ 2 Oe auf, die man auch für einfache $\operatorname{LSMO}(\mathrm{x}=0.3)$-Filme erhält 97 . Bei $n \approx 6$ ergibt sich ein leichter Anstieg auf $H_{C} \approx 20$ Oe. Im Gegensatz zu den Übergittern auf STO (001) beobachtet man aber keinen signifikanten Einbruch der Sättigungsmagnetisierung für die größte Bilagendicke (siehe Inset Abbildung $9.23 \mathrm{~b}$ )). Es ist also davon auszugehen, dass für die hier untersuchten Übergitter auf STO (111) ein homogener ferromagnetischer Zustand vorliegt.

Die Ursache dieser Unterschiede im magnetischen Zustand der Übergitter auf STO (001) und (111) ist zunächst in der Verteilung der $e_{g}$-Elektronen zu suchen. Die Durchmischung der La- und Sr-Ionen für das Wachstum auf STO (111) unterstützt eine homogene Verteilung der $e_{g}$-Elektronen. Durch die in-situ Ellipsometrie ergeben sich bereits Hinweise darauf, dass die $e_{g}$-Elektronen für die Übergitter auf STO (111) deutlich homogener verteilt sind als auf STO (001). Für STO (111) ist der durchschnittliche Brechungsindex der Heterostruktur 
unabhängig von der Dicke der Bilagen $\Lambda$. Beim Wachstum auf STO (001) zeigt sich ein deutlicher Unterschied in $N_{A v}$ für $n \approx 1$ und $n \approx 3$. Die Simulation der Verteilung der $e_{g^{-}}$ Elektronen aus dem gemessenen Verlauf der in-situ Ellipsometrie für $n \approx 3$ zeigt überdies, dass in der $\mathrm{MnO}_{2}$-Lage zwischen zwei SrO-Lagen ein Anteil von $x_{S r O}^{\mathrm{SrO}} \approx 0.75 \mathrm{Mn}^{4+}$-Ionen vorliegt und in den $\mathrm{MnO}_{2}$-Lagen zwischen einer SrO- und LaO-Lage ein Anteil von $x_{S r O}^{\mathrm{LaO}} \approx$ 0.45 (siehe Abbildung 9.20). Nach dem magnetischen Phasendiagramm für Filme auf STO (001) erwartet man für $x_{S r O}^{S r O}$ einen AFM- und für $x_{S r O}^{\mathrm{LaO}}$ einen FM-Grundzustand (vergleiche Abbildung 9.4). Man würde also ein Sättigungsmoment von etwa $3.2 \mu_{\mathrm{B}} / \mathrm{Mn}$ erwarten. Bei den Untersuchungen hier ergibt sich mit $2.9 \mu_{\mathrm{B}} / \mathrm{Mn}$ ein etwas kleinerer Wert, der möglicherweise durch eine unvollständige Ausrichtung der Spins in den LMO-Lagen erklärt werden kann. Auch das erhöhte Koerzitivfeld ist konsistent mit der FM-AFM-Koexistenz.

In der Literatur findet man ebenso den hier festgestellten Einbruch des ferromagnetischen Momentes beim Übergang von $n=2$ auf $n=3$ [111. In den Experimenten von Bhattacharya et al. zeigen die LMO/SMO-Übergitter allerdings eine deutlich kleinere Magnetisierung, auch für $n=1$ liegt diese nur bei $M_{\text {sat }}(10 \mathrm{~K})=3.2 \mu_{\mathrm{B}} / \mathrm{Mn}$ und für $n=3$ bei $M_{\text {sat }}(10 \mathrm{~K})=$ $1.9 \mu_{\mathrm{B}} / \mathrm{Mn}$. Dies ist ein Hinweis auf eine andere Struktur und Art von Defekten, die sich in solchen mit MBE abgeschiedenen Heterostrukturen ausbilden. Sauerstoffleerstellen würden über das veränderte $\mathrm{Mn}^{3+} / \mathrm{Mn}^{4+}$-Verhältnis und Störungen des magnetischen Austausches aufgrund fehlender Mn-O-Mn-Brücken Einfluss auf die magnetischen Eigenschaften nehmen.

Mit den hier durchgeführten Studien ist nicht abschließend zu klären, ob die magnetischen Eigenschaften der Übergitter auf STO (111) allein aus der durch die chemische Durchmischung hervorgerufenen homogenen Verteilung der $e_{g}$-Elektronen resultieren. In der TEM-Aufnahme ist noch ein Kontrast zwischen den LMO- und SMO-Lagen sichtbar, so dass man nicht von einer kompletten Durchmischung der La- und Sr-Ionen ausgehen kann (siehe Abbildung 9.8). Eine weitere Ursache für eine homogene Verteilung der $e_{g}$-Elektronen folgt aus der geometrischen Anordnung der 3d-Orbitale an den Grenzflächen. Die Delokalisierung der $e_{g}$-Elektronen kann nicht allein in den (111)-Ebenen verlaufen sondern hat immer eine Komponente senkrecht dazu. Dadurch ist die charakteristische Delokaliserungslänge in c-Richtung womöglich größer als beim Wachstum auf STO (001)-Substraten. Genauere Aussagen wären nach einer präzisen Analyse der Verteilung von La- und Sr-Kationen in den Übergittern mittels EELS möglich. Damit könnte man die elektronischen Effekte, die zur Delokalisierung beitragen von einer einfachen Durchmischung der Kationen auf den A-Plätzen trennen.

Auch mit Blick auf den Magnetismus kann man Auswirkungen von der Geometrie der 3dOrbitale erwarten. Im Gegensatz zum Wachstum auf STO (001) verursachen die epitaktischen Verspannungen beim Wachstum auf STO (111) keine Aufhebung der Entartung der 3d-Orbitale der Mn-Ionen. Daraus resultiert eine Stabilisierung des ferromagnetischen Grundzustandes, was sich in den Phasendiagrammen für einfache LSMO(x)-Filme zeigt (Abbildung 9.4). Zusätzlich ist die magnetische Kopplung senkrecht zur Wachstumsrichtung deutlich stärker als für eine (001)-Orientierung, da jeweils drei Mn-O-Mn-Bindungen zur benachbarten Monolage bestehen [252]. In einer ohne Grenzflächeneffekte antiferromagnetisch ordnenden Lage kann so durch die Ankopplung an einen Ferromagneten eine ferromagnetische Ordnung der Spins induziert werden. Bei dem Wachstum in (001)-Orientierung wäre diese Beeinflussung an der Grenzfläche weniger effektiv, da der Antiferromagnet in der Ebene vier Bindungen besitzt und senkrecht dazu nur eine, so dass der Ferromagnet die Bilanz der Austauschwechselwirkungen deutlich weniger manipulieren kann. 


\subsubsection{Neue ferromagnetische Phase?}

Nachdem im vorigen Abschnitt die Evolution der magnetischen Eigenschaften für $n=1-3$ beleuchtet wurde, soll hier die zweite FM-Phase, die für $n \gtrsim 3$ erscheint, eingeführt werden. In Abbildung 9.24 ist die grundsätzliche magnetische Phänomenologie eines LMO/SMOÜbergitters mit $n=3$ dargestellt. In der Auftragung von $M(T)$ sieht man zwei magnetische Übergänge. Die zugehörigen Ordnungstemperaturen werden anhand der Tangenten an den Wendepunkten bei $T_{1}, T_{2}$ definiert (Bestimmung von $T_{1}, T_{2}$ im Inset von Abbildung 9.24 e)). Bei $T_{C}^{H}=342(2) \mathrm{K}$ ordnet die Hochtemperaturphase (HTP) und bei $T_{C}^{L}=282 \mathrm{~K}$ die Tieftemperaturphase (LTP).

An den in Abbildung 9.24 e) markierten Temperaturen wurden $M(H)$-Kurven aufgenommen, die in Abbildung 9.24 a)-d) abgebildet sind. Für $T_{C}^{L}<T=300 \mathrm{~K}<T_{C}^{H}$ erkennt man eine einfache Hysterese mit $H_{C}^{H}=23$ Oe. Wenn man durch $T_{C}^{L}$ kühlt, erscheint ein weiteres sehr kleines Koerzitivfeld von $H_{C}^{L}=2$ Oe. Das Moment dieser Tieftemperaturphase wächst dann deutlich an und übersteigt den Anteil der HTP. Bei $T=5 \mathrm{~K}$ schließlich sind die beiden Phasen nicht mehr zu unterscheiden und es ergibt sich ein einfaches Koerzitivfeld.

Zunächst soll ausgeschlossen werden, dass die HTP aus Effekten an den Rändern und Grenzflächen der gesamten Heterostruktur resultiert. Dass die HTP nicht an den Rändern des beschichteten Bereichs auf dem Substrat lokalisiert ist, kann man durch den Vergleich der Hystereseschleife für eine komplette Probe und jener eines Bruchstückes, welches keine Randabschnitte enthält (vergleiche Abbildung 9.25, prüfen. In Abbildung 9.25 erkennt man eine gute Übereinstimmung dieser beiden Kurven. Insbesondere bleibt das Moment der HTP komplett erhalten (Leichte Abweichungen ergeben sich aus dem diamagnetischen Untergrund, der bei dem kleinen Probenstück deutlich stärker ins Gewicht fällt.).

Das Moment der HTP skaliert außerdem mit der Zahl von Wiederholungen der LMO/SMOBilagen. Auf ein LMO/SMO-SL $(n=3, m=11)$ (Struktur A) werden nach erfolgter magnetischer Charakterisierung weitere $m=5$ Wiederholungen der LMO/SMO-Bilage abgeschieden (Struktur B). Wie es in Abschnitt 8.2.3 gezeigt wurde, kommt es durch diesen mehrfachen Depositionsprozess nicht zu Veränderungen der chemischen Qualität der Grenzflächen. In Abbildung 9.26 a) betrachtet man die $M(H)$-Kurven für die Strukturen A und B bei $T=280 \mathrm{~K}$, wo HTP und LTP gleichermaßen zu erkennen sind. Wenn man die Messwerte von $M(H)$ für Struktur A entsprechend der Zahl der Wiederholungen mit dem Faktor $\frac{16}{11}$ multipliziert, ergibt sich eine Kurve, die mit der $M(H)$-Kurve für Struktur B deckungsgleich ist (Abbildung $9.26 \mathrm{~b})$ ).

Das Vorhandensein der HTP lässt sich damit nicht einfach durch Randeffekte erklären. Nach diesen Betrachtungen sollen im Folgenden die magnetischen Eigenschaften der HTP im Detail behandelt werden.

Im Zusammenhang mit dem Fehlschnitt der STO (001)-Substrate ergibt sich eine starke uniaxiale Anisotropie der HTP. In Abbildung 9.27 sieht man $M(H)$-Kurven für LMO/SMOHeterostrukturen auf STO-Substraten mit verschiedenen Werten für den Fehlschnitt $\alpha_{\text {cut }}$. Für die gezeigten Messungen wurden Temperaturen ausgewählt, bei denen die HTP und LTP mit ähnlichen magnetischen Momenten zu beobachten sind. Dadurch kann man die Anisotropie beider Phasen gleichzeitig studieren. Bei einem kleinen Wert von $\alpha_{c u t}=0.1^{\circ}$ kann man nicht unterscheiden, ob das Feld parallel zu den Substratstufen (EA) oder senkrecht (HA) dazu verläuft (siehe Abbildung 9.27 a)). Für den größeren miscut $\alpha_{c u t}=0.21^{\circ}$ ergibt sich 
a)

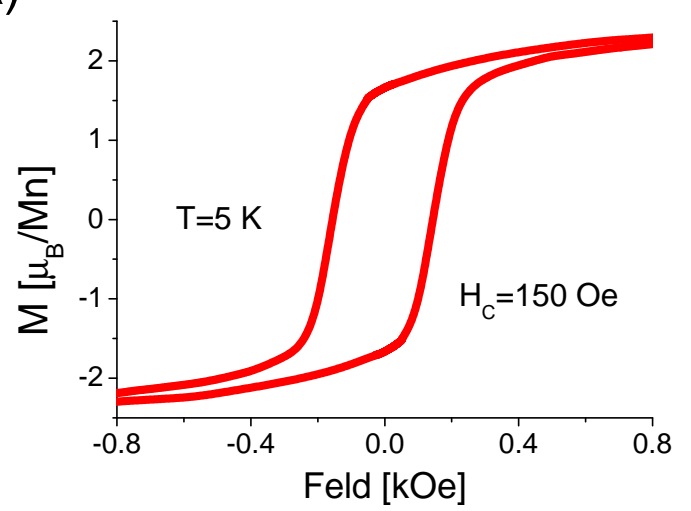

c)

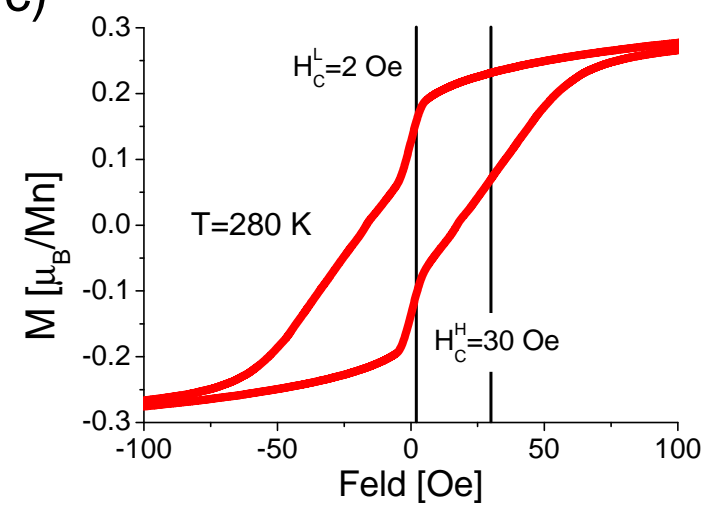

b)

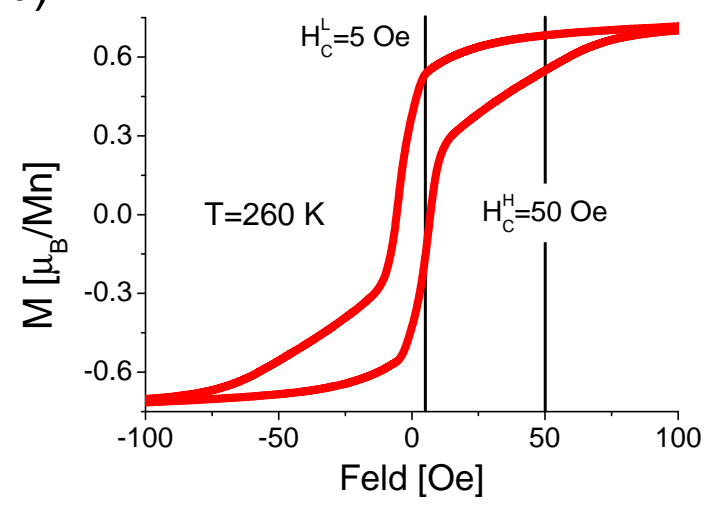

d)

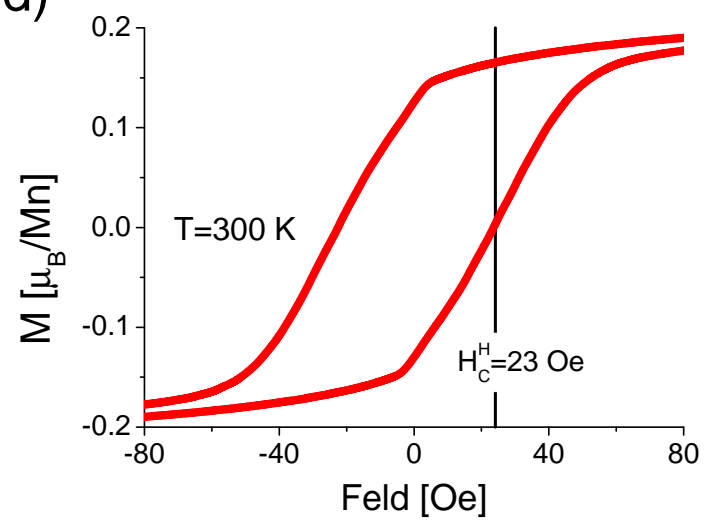

e)

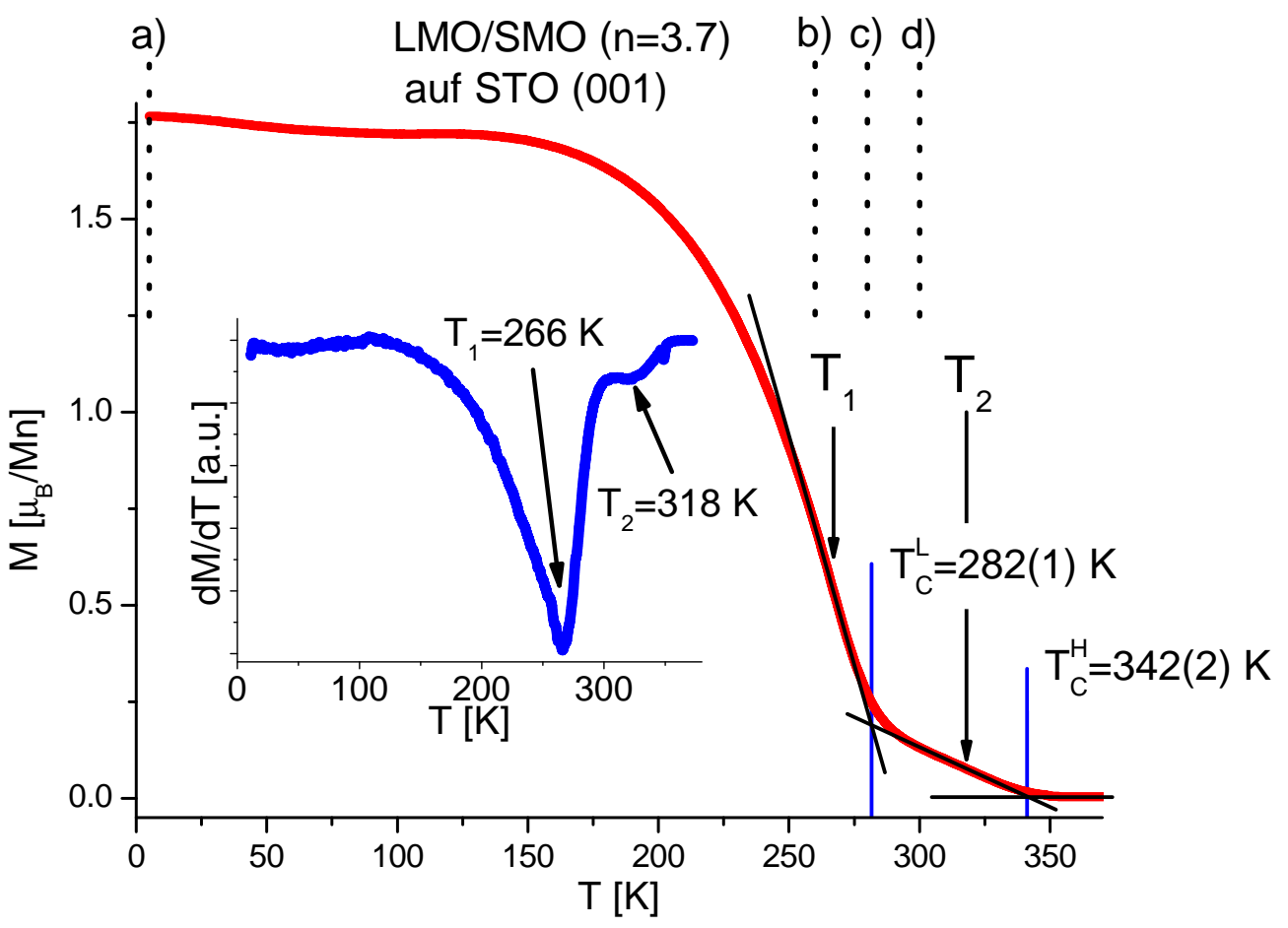

Abbildung 9.24.: Magnetische Eigenschaften eines LMO/SMO-Übergitters $(n \approx 3.7$, $m=16)$ im Detail: a)-d) $M(H)$-Kurven für verschiedene Temperaturen, die in den Diagrammen angegebenen Koerzitivfelder wurden anhand der Maxima von $\frac{d M}{d H}$ ermittelt. e) $M(T)$-Kurve, die Übergangstemperaturen der Hochtemperaturphase (HTP), $T_{C}^{H}$, und Tieftemperaturphase (LTP), $T_{C}^{L}$, wurden über Tangenten an den Wendepunkten bei $T_{1}, T_{2}$ bestimmt. Das Inset zeigt $\frac{d M}{d T}$ zur Ermittlung der Positionen von $T_{1}, T_{2}$. 


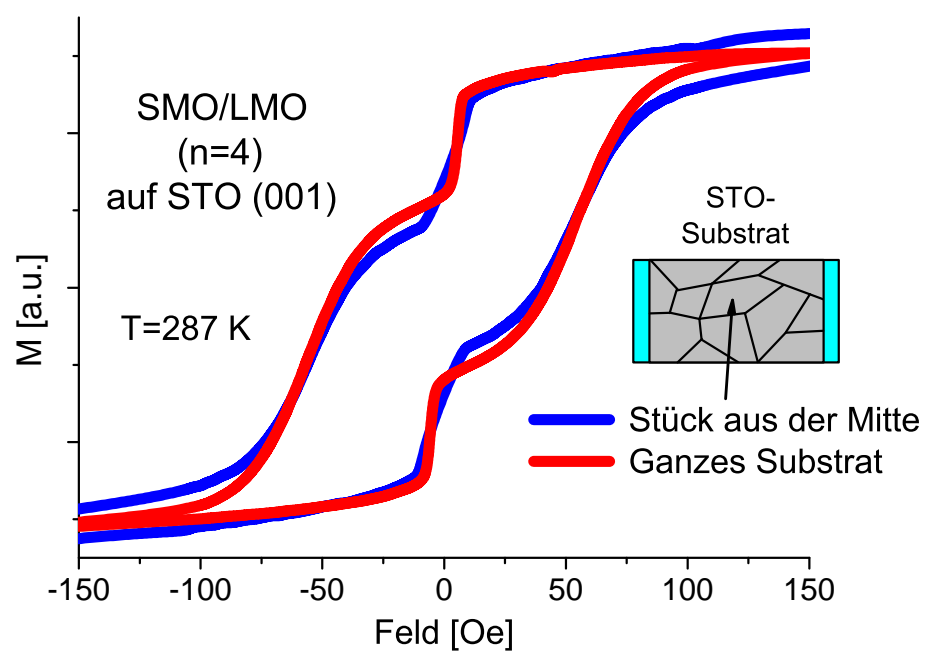

Abbildung 9.25.: $M(H)$-Messung von Bruchstück aus STO (001)-Substrat für SMO/LMO-SL $(n=4, m=16)$ bei $T=287 \mathrm{~K}$, wo HTP und LTP mit ähnlicher Amplitude sichtbar sind.

a)

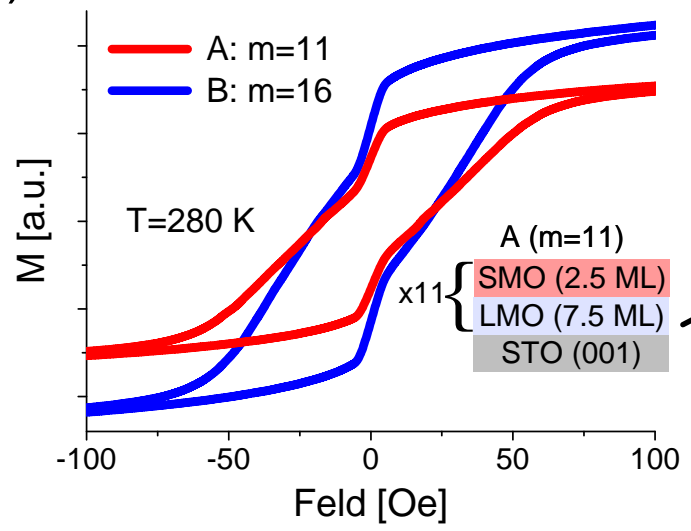

b)

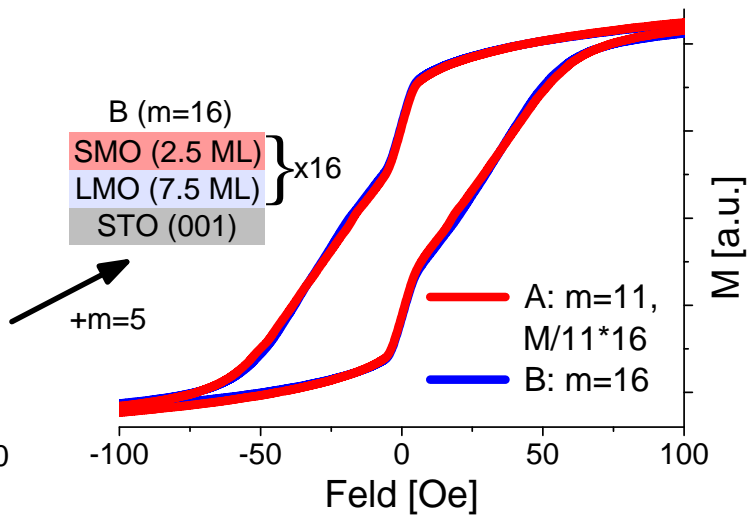

Abbildung 9.26.: Skalierung des magnetischen Momentes der HTP mit der Zahl der Bilagen $m$ : Auf ein LMO/SMO-SL $(n=3.7, m=11)$ werden weitere $\Delta m=5$ Wiederholungen der LMO/SMO-Bilage gewachsen. Die $M(H)$-Kurve für $m=11$ in a) lässt sich durch den Faktor $\frac{16}{11}$ skalieren, so dass sie fast deckungsgleich mit der Kurve für $m=16$ ist (siehe b)).

ein starker Kontrast zwischen diesen beiden Ausrichtungen des Feldes. Es zeigt sich das deutliche Bild einer uniaxialen Anisotropie mit der leichten Richtung parallel zu den Stufen des Substrates (Abbildung $9.27 \mathrm{~b}$ )). Interessanterweise hat die HTP eine sehr ausgeprägte Anisotropie, wogegen diese bei der LTP nur schwer auszumachen ist.

Über die $M(H)$-Messungen bis zur Sättigung kann man durch Integration die Anisotropiekonstante $K$ abschätzen [271]:

$$
K=\mu_{0}\left(\int_{0}^{M_{s a t}} H_{H A}(M) d M-\int_{0}^{M_{s a t}} H_{E A}(M) d M\right)
$$

Dabei ist $H_{H A}(M)$ bzw. $H_{E A}(M)$ das nötige Feld zum Erreichen der Magnetisierung $M$ bei Ausrichtung des Feldes entlang der HA bzw. EA. Die Integration erfolgt von $M=0$ bei $-H_{C}$ (für die EA) bis $M=+M_{\text {sat }}$. Aus dieser Prozedur ergibt sich dann $K=2.0(2) \cdot 10^{4} \frac{\mathrm{J}}{\mathrm{m}^{3}}$. In 

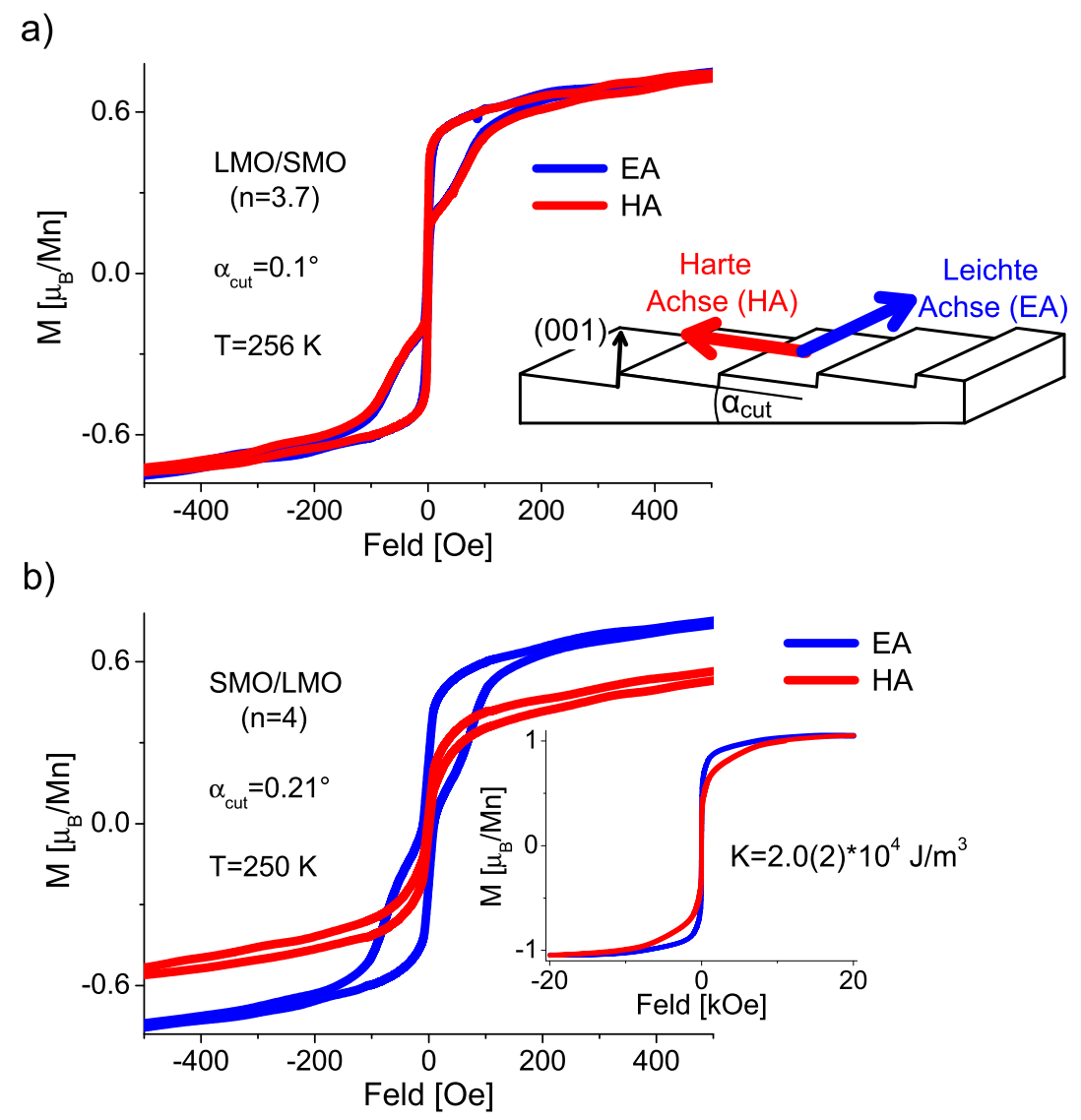

\begin{abstract}
Abbildung 9.27.: Magnetische Anisotropie der HTP in Bezug zum Fehlschnitt des Substrates $\alpha_{c u t}$ : Die leichte Achse (EA) verläuft parallel zu den Stufen des Substrates, die schwere Achse (HA) senkrecht dazu (siehe Inset Teil a)). a) Für $\alpha_{c u t}=0.1^{\circ}$ zeigt sich kein signifikanter Unterschied zwischen der Magnetisierung entlang HA oder EA. b) Für $\alpha_{c u t}=0.21^{\circ}$ ergibt sich ein starker Unterschied zwischen der Magnetisierung entlang der HA und EA für die HTP. Das Inset zeigt die beiden Kurven bis zur Sättigung. Die Anisotropiekonstante $K$ ergibt sich daraus über Gleichung 9.6
\end{abstract}

der Literatur wird auch für einfache LSMO-Filme auf STO (001)-Substraten eine uniaxiale Anisotropie beobachtet 256, 260, 272]. Dabei ist die EA ebenfalls parallel zu den Kanten der Substratstufen ausgerichtet. Als Ursache für diese Anisotropie wird vor allem die Minimierung der magnetostatischen Energie angeführt [272]. Allerdings sind die Anisotropiekonstanten deutlich kleiner als jene, die sich hier für die HTP ergibt. Selbst unter Verwendung von vizinalen Substraten mit $\alpha_{c u t}=10^{\circ}$ ist die Anisotropieenergiedichte mit $K \approx 2 \cdot 10^{3} \frac{\mathrm{J}}{\mathrm{m}^{3}}$ eine Größenordnung kleiner als hier 272]. Die berechnete Anisotropiekonstante übersteigt sogar die Formanisotropie einer dünnen Schicht, die wie im Inset von Abbildung 9.27b) eine Sättigungsmagnetisierung von $M_{\text {sat }}=1 \mu_{\mathrm{B}} / \mathrm{Mn}$ besitzt $[23$ :

$$
K=\frac{1}{2} \mu_{0} M_{\text {sat }}^{2} \sim 1.7 \cdot 10^{4} \frac{\mathrm{J}}{\mathrm{m}^{3}}
$$

Als Funktion der Temperatur fällt $H_{C}^{H}$ linear ab. Dabei liegt der Schnittpunkt der FitGeraden durch die Messpunkte deutlich oberhalb von $T_{C}^{H}$. $H_{C}^{L}$ lässt sich durch einen exponentiellen Abfall approximieren, der für einen Spin-Glas-Zustand einer FM/AFM-Grenzfläche typisch ist 273. Das Verhalten von $H_{C}^{H}(T)$ zeigt keine Änderung beim Unterschreiten von $T_{C}^{L}$. Dieses Fehlen einer magnetischen Kopplung zwischen HTP und LTP macht sich auch bei der 


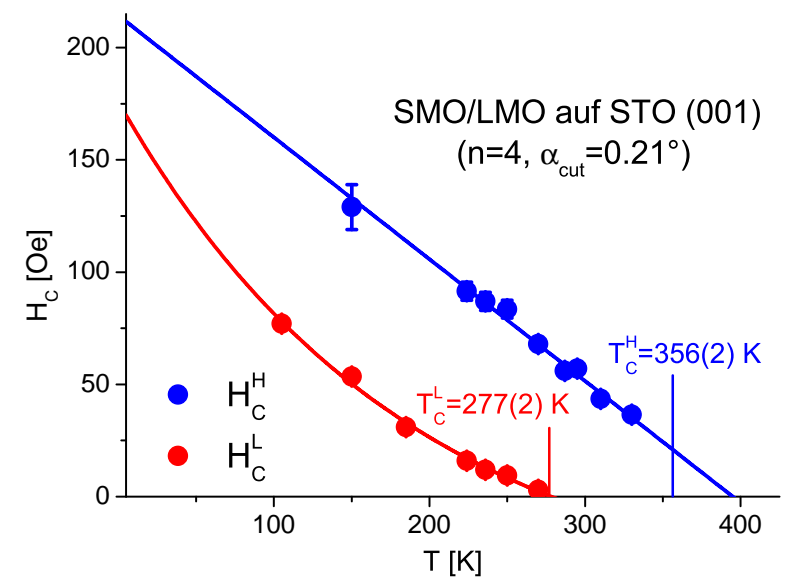

\begin{abstract}
Abbildung 9.28.: Temperaturabhängigkeit der Koerzitivfelder von der HTP, $H_{C}^{H}$, und der LTP, $H_{C}^{L}$, für SMO/LMO-SL $(n=4, m=16)$ auf einem STO (001)-Substrat mit Fehlschnitt $\alpha_{c u t}=0.21^{\circ}$ (Vergleiche Abbildung $\left.9.27 \mathrm{~b}\right)$ ). $H_{C}^{H}(T)$ wurde mit einem linearen Fit angepasst und $H_{C}^{L}$ mit einem exponentiellen Abfall der Form $H_{C}(T)=H_{C}^{0} \exp (-\alpha T)$.
\end{abstract}

Messung von minor-loops bemerkbar. Zur Aufnahme einer minor-loop sättigt man die Probe zunächst im positiven Feld und reduziert dieses dann auf $H_{m}$, für das $-H_{C}^{H}<H_{m}<-H_{C}^{L}$ gilt. Von $H_{m}$ fährt man das Feld dann wieder bis zur Sättigung hoch. Wenn man diese minorloop mit einer kompletten Hysterese vergleicht, sieht man keinen Unterschied bei der Lage von $H_{C}^{L}$. Die magnetische Umkehr der LTP ist also unabhängig von der Ausrichtung der HTP.

In Abbildung 9.29 ist die Entwicklung der magnetischen Eigenschaften von HTP und LTP in Abhängigkeit von $n$ dargestellt. Die Curie-Temperatur der LTP, $T_{C}^{L}$, fällt mit steigender Bilagendicke $\Lambda$ ab. $T_{C}^{H}$ dagegen streut zufällig um einen Wert $T_{C}^{H} \sim 345 \mathrm{~K}$ ohne erkennbare Abhängigkeit von $n$. Wie schon in Abschnitt 9.4.1 beschrieben wurde, fällt das Sättigungsmoment $M_{\text {sat }}(5 \mathrm{~K})$ für $n \geq 3$ deutlich unter den Wert $M_{\text {mix }}=3.75 \mu_{\mathrm{B}} / \mathrm{Mn}$, der sich für eine volle Ausrichtung aller Mn-Spins in der Heterostruktur ergibt. Für $3 \leq n \leq 4$ bewegt sich $M_{\text {sat }}$ zwischen $M_{L M O+1 S M O}$ und $M_{L M O} . M_{L M O}$ ergibt sich für eine Magnetisierung aller LMO-Schichten mit $M_{\text {sat }}=3.4 \mu_{\mathrm{B}} / \mathrm{Mn}$ (vergleiche Abbildung 9.3). $M_{L M O+1 S M O}$ erhält man, wenn man die Spins in allen $\mathrm{MnO}_{2}$-Lagen der LMO-Schichten und jeweils einer $\mathrm{MnO}_{2}$-Lage der SMO-Schichten ausrichtet:

$$
M_{L M O+1 S M O}=\underbrace{\frac{2 \cdot M_{m i x}}{2+\frac{2}{3}}}_{M_{L M O}}+\underbrace{\frac{M_{m i x}}{n \cdot\left(2+\frac{2}{3}\right)}}_{M_{1 S M O}}
$$

Die Definition von $M_{L M O+1 S M O}$ wird durch Abbildung 9.20 Teil 5 motiviert: Im LMO/SMOSL gibt es zwei $\mathrm{MnO}_{2}$-Lagen an der LMO/SMO-Grenzfläche, die von einer SrO- und einer LaO-Lage eingeschlossen werden. Für diese beiden Grenzlagen und alle $\mathrm{MnO}_{2}$-Lagen, die an beiden Seiten von LaO-Lagen umschlossen werden, nimmt man eine volle Ausrichtung der Spins auf den Mn-Ionen an. Die Spins auf den $\mathrm{MnO}_{2}$-Lagen, die auf beiden Seiten von SrOLagen eingeschlossen werden, tragen nicht zum Sättigungsmoment bei. Da jeweils eine der beiden $\mathrm{MnO}_{2}$-Grenzlagen zur SMO-Schicht gehört und die andere zur LMO-Schicht, wird zur Berechnung von $M_{L M O+1 S M O}$ nur eine Monolage der SMO-Schichten herangezogen. Bei weiterer Erhöhung von $n$ fällt $M_{\text {sat }}(5 \mathrm{~K})$ auch unter $M_{L M O}$, so dass in diesen Heterostrukturen von einer unvollständigen Ausrichtung der Spins in den LMO-Lagen auszugehen ist. 

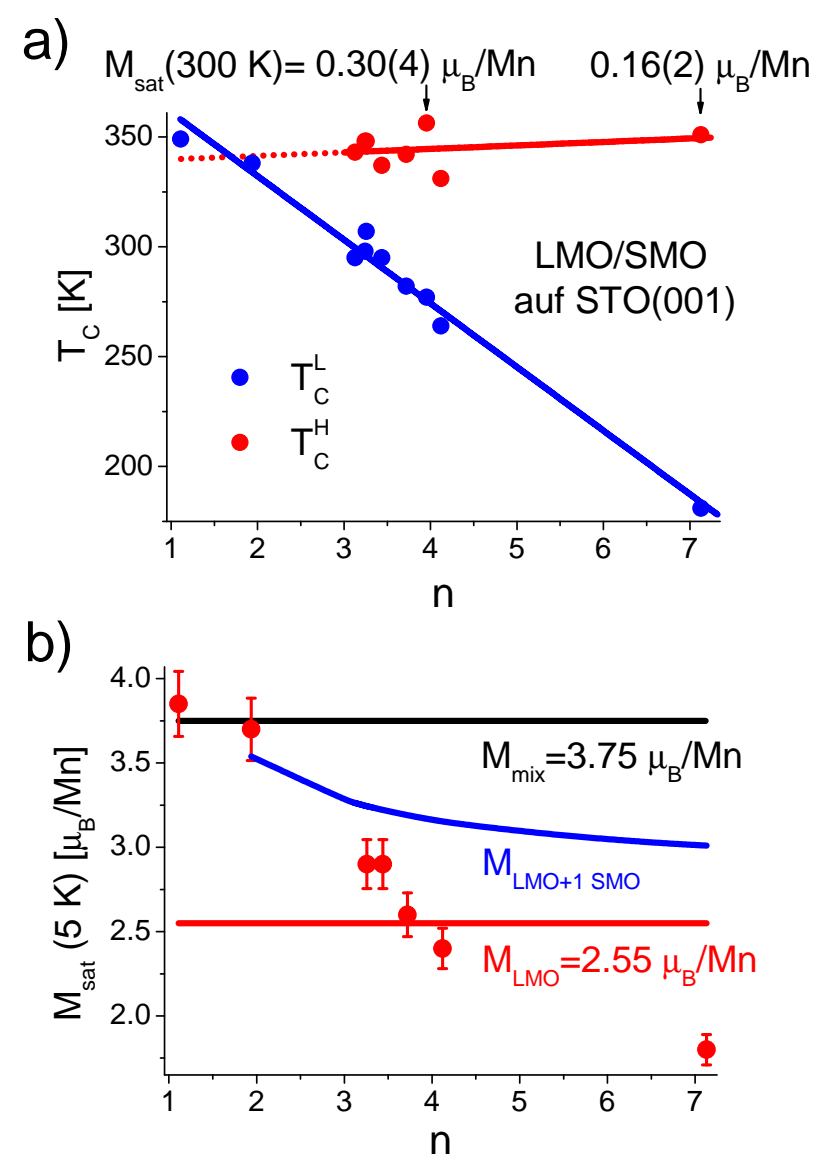

\begin{abstract}
Abbildung 9.29.: Zusammenfassende Darstellung der magnetischen Eigenschaften der LMO/SMO-SL als Funktion von $n$ : a) Entwicklung der Curie-Temperaturen der HTP, $T_{C}^{H}$, und LTP, $T_{C}^{L}$, als Funktion von $n$. Die magnetischen Momente in Sättigung für Übergitter mit $n=4.0$ und $n=7.1$ bei $T=300 \mathrm{~K}$ sind angegeben. b) Sättigungsmomente bei $T=5 \mathrm{~K}$, die eingezeichneten Linien zeigen die Momente für volle Ausrichtung aller Spins auf den Mn-Ionen $\left(M_{m i x}\right)$, Ausrichtung aller Spins in den LMO-Schichten und jeweils einer Monolage der SMO-Schichten $\left(M_{L M O+1 S M O}\right)$, und Magnetisierung der LMO-Lagen mit $M_{\text {sat }}=3.4 \mu_{\mathrm{B}} / \mathrm{Mn}$ gemäß den Daten in Abbildung $9.3\left(M_{L M O}\right)$.
\end{abstract}

Durch die Überlagerung der Hysteresekurven ist es nicht möglich, Informationen über das magnetische Moment der HTP bei $T=5 \mathrm{~K}$ zu gewinnen. Deshalb zieht man Messungen bei höheren Temperaturen $T_{C}^{L}<T<T_{C}^{H}$ heran. Damit wird das magnetische Moment allein von der HTP gegeben. Wenn die HTP an Grenzflächen lokalisiert ist, muss das auf alle Mn-Ionen in der Heterostruktur normierte magnetische Moment umgekehrt proportional zur Dicke der LMO/SMO-Bilagen $\Lambda$ bzw. $n$ verlaufen. In Abbildung 9.29 a) sind die Sättigungsmomente bei $T=300 \mathrm{~K}$ für eine Probe mit $n=4.0$ und $n=7.1$ aufgeführt. Diese beiden Heterostrukturen weisen vergleichbare Werte von $T_{C}^{H}$ auf: $T_{C}^{H}=351(2) \mathrm{K}$ für $n=7.1$ und $T_{C}^{H}=356(2) \mathrm{K}$ für $n=4.0$. Damit werden die Werte für $M_{\text {sat }}(300 \mathrm{~K})$ nicht durch stark verschiedene Ordnungstemperaturen verfälscht. Die etwaigen Unterschiede, die aus der kleinen Diskrepanz zwischen $T_{C}^{H}(n=4.0)$ und $T_{C}^{H}(n=7.1)$ resultieren, verschwinden in den Fehlerintervallen von $M_{\text {sat }}$ (für das SL mit $n=7.1$ ist $M_{\text {sat }}(T=270 \mathrm{~K})=0.21 \mu_{\mathrm{B}} / \mathrm{Mn}$.). Die Bildung der Verhältnisse belegt die Einhaltung der oben erwähnten Skalenrelation $M_{\text {sat }}(300 \mathrm{~K}) \propto \frac{1}{n}$ :

$$
\frac{n=7.1}{n=4.0} \approx 1.88, \frac{M_{\text {sat }}(n=4.0)}{M_{\text {sat }}(n=7.1)}=\frac{0.30(4)}{0.16(2)}=1.8(3)
$$


a)

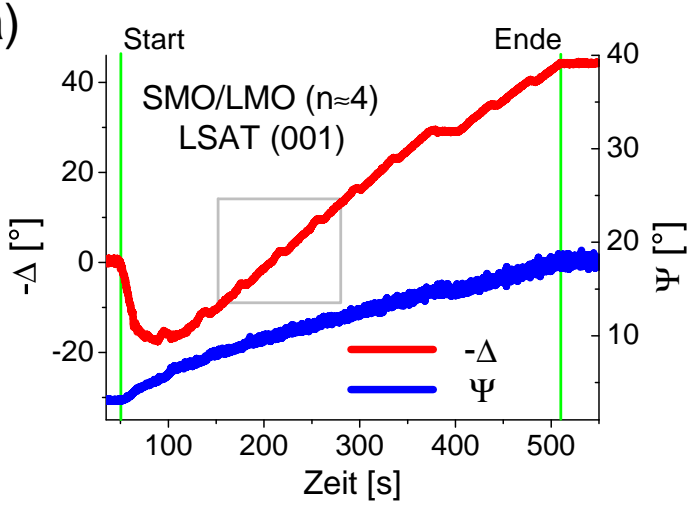

c)

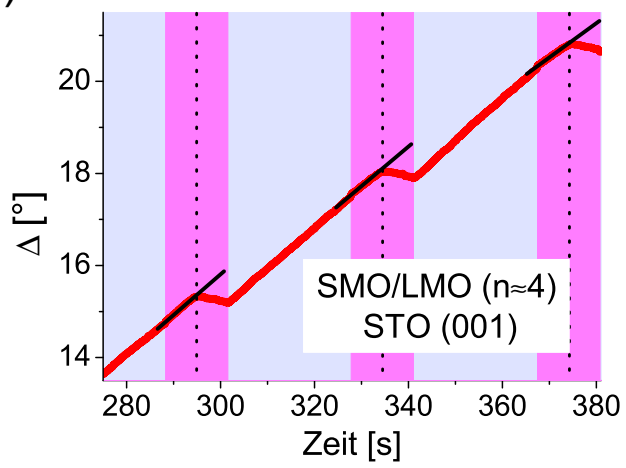

b)

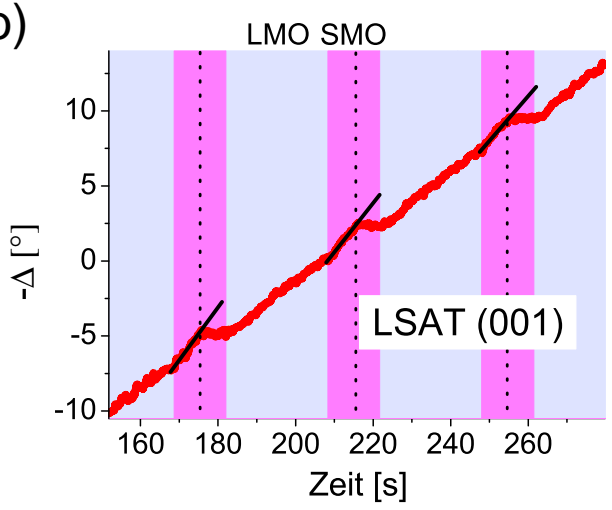

d)

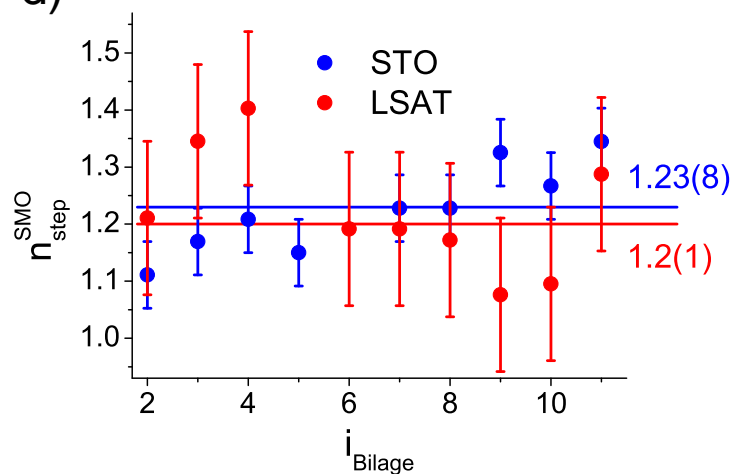

Abbildung 9.30.: In-situ Ellipsometrie beim Wachstum von SMO/LMO-SL $(n \approx 4$, $\left.d_{L M O} \approx 8 \mathrm{ML}, d_{S M O} \approx 2.67 \mathrm{ML}, m=11\right)$ auf einem STO (001)- und einem LSAT (001)Substrat: a) Übersicht von $-\Delta(t)$ und $\Psi(t)$ beim Wachstum auf LSAT (001), b) Vergrößerte Darstellung des grau umrandeten Bereiches in a), die zeitlichen Positionen der Steigungsänderungen beim Wachstum von SMO sind markiert. c) Ausschnitt aus Verlauf von $\Delta(t)$ für das Wachstum auf STO (001), die Positionen der Steigungsänderungen beim Wachstum der SMO-Lagen sind eingezeichnet. d) Zahl der gewachsenen Monolagen SMO, nach der es zu einer Änderung der Steigung kommt, $n_{\text {step }}^{S M O}$ (vergleiche Gleichung 9.2, als Funktion von $i_{\text {Bilage }}$. Die Mittelwerte sind im Diagramm vermerkt (Durch die größere Rauschamplitude beim Wachstum auf LSAT sind die Fehlerintervalle größer als bei STO. Außerdem kann der Punkt bei $i_{\text {Bilage }}=5$ aufgrund einer Messbereichsumschaltung nicht ausgewertet werden.).

Da die orbitalen Freiheitsgrade einen großen Einfluss auf die magnetischen Kopplungen in Manganaten nehmen, wird zusätzlich das magnetische Verhalten von LMO/SMO-SL auf LSAT (001)-Substraten untersucht. Der Gitterparameter von LSAT ist mit $a_{L S A T}=0.3869 \mathrm{~nm}$ deutlich kleiner als für STO mit $a_{S T O}=0.3905 \mathrm{~nm}$. Außerdem erlaubt die gute Qualität der verfügbaren LSAT-Substrate eine ähnlich perfekte Epitaxie wie auf STO (001)-Substraten. Im Folgenden sollen so zwei SMO/LMO-SL mit fast identischer Dicke der Bilagen $(\Lambda=4.2 \mathrm{~nm}$, $n \approx 4)$ und gleicher Zahl der Wiederholungen $(m=11)$, die auf einem STO (001)- und LSAT (001)-Substrat deponiert wurden, verglichen werden. Dazu wird zunächst sichergestellt, dass die chemische Schärfe der Grenzflächen identisch ist. Dann wird der unterschiedliche Verspannungszustand herausgestellt, um schließlich die Unterschiede des magnetischen Verhaltens aufzuzeigen.

In Abbildung 9.30 werden die Messungen der in-situ Ellipsometrie für das Wachstum auf LSAT und STO verglichen. Durch den anderen Brechungsindex des LSAT-Substrates differiert der grobe Verlauf von $\Delta(t)$ und $\Psi(t)$ deutlich von jenem, der sich für das Wachstum auf einem STO-Substrat ergibt (vergleiche Abbildung 9.12 a)). Im Detail zeigen sich aber 
a)

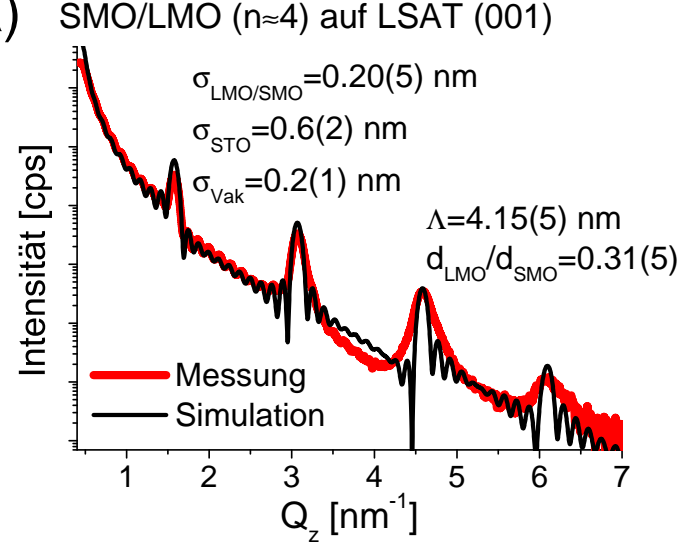

b) SMO/LMO ( $\mathrm{n} \approx 4)$ auf STO (001)

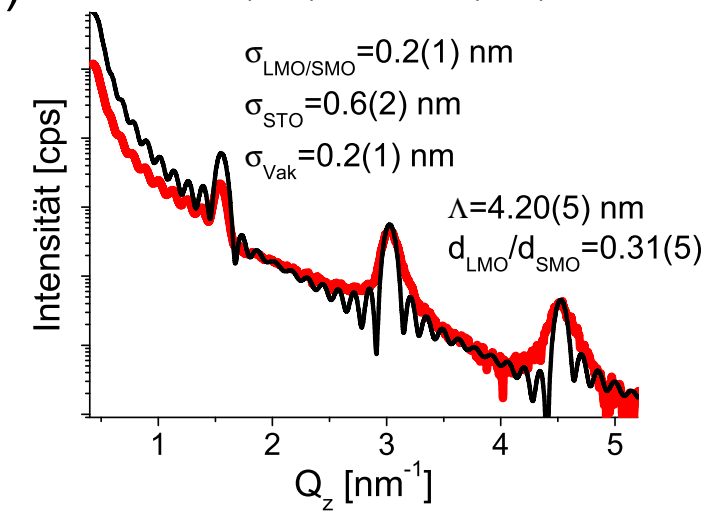

Abbildung 9.31.: Charakterisierung der chemischen Qualität der SMO/LMOGrenzflächen für SMO/LMO-SL $(n \approx 4, m=11)$ auf a) LSAT (001)- und b) STO (001)Substrat anhand von XRR-Messungen. Die Fit-Parameter der numerischen Anpassungen mit ReMagX 165. sind in den Diagrammen gegeben.
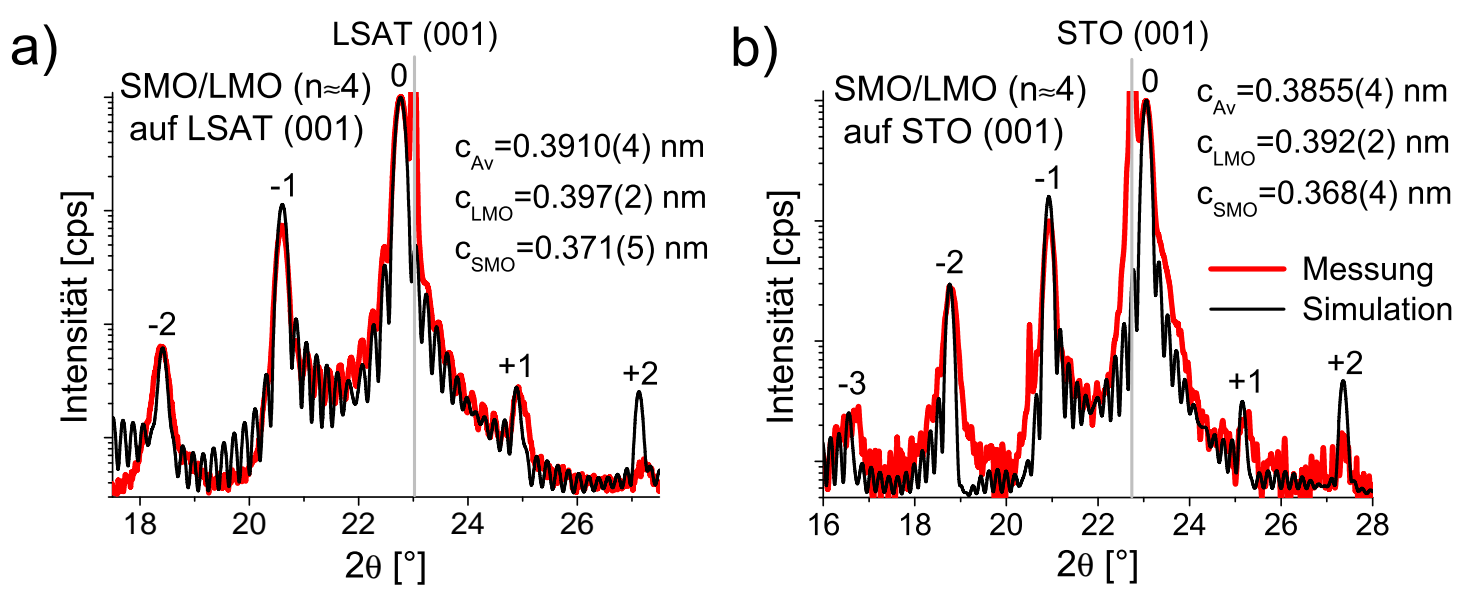

Abbildung 9.32.: XRD-Spektren für SMO/LMO-SL $(n \approx 4, m=11)$ auf a) LSAT (001)und b) STO (001)-Substrat. Die numerische Anpassung der Messdaten liefert Werte für den durchschnittlichen Gitterparameter $c_{A v}$ und die individuellen Gitterabstände entlang der (001)-Richtung für SMO, $c_{S M O}$, und LMO, $c_{L M O}$.

ähnliche Strukturen. In Abbildung 9.30 b) sieht man beim Wachstum der SMO-Lagen eine Steigungsänderung, $\Delta(t)$ hat einen stufenartigen Verlauf. Dies geschieht analog zum Verhalten beim Wachstum auf STO (siehe Abbildung 9.30 c)). Wenn man $n_{\text {step }}^{\text {SMO }}$ (siehe Gleichung 9.2 als Funktion von $i_{\text {Bilage }}$ aufträgt, erkennt man, dass diese Größe für beide Substrate um einen mit Blick auf die Fehlerintervalle identischen Wert streut (Abbildung 9.30 d)). Es ist also davon auszugehen, dass die in-situ Ellipsometrie beim Wachstum auf LSAT von ähnlichen Delokalisierungsvorgängen bestimmt wird wie beim Wachstum auf STO (001) und keine Durchmischung der Kationen auf den A-Plätzen stattfindet. Die genauen Längenskalen der Delokalisierung würden sich nur durch eine detaillierte Simulation von $\Delta(t)$ ergeben, was hier durch die vergrößerte Rauschamplitude erschwert wird.

Mit der Anpassung der XRR-Spektren gelingt eine Abschätzung der chemischen Modulation in beiden Übergittern. Innerhalb des Fehlers sind die strukturellen Parameter, $\Lambda, d_{L M O} / d_{S M O}$ und insbesondere $\sigma_{L M O / S M O}$ für beide SL identisch (siehe Abbildung 9.31). 

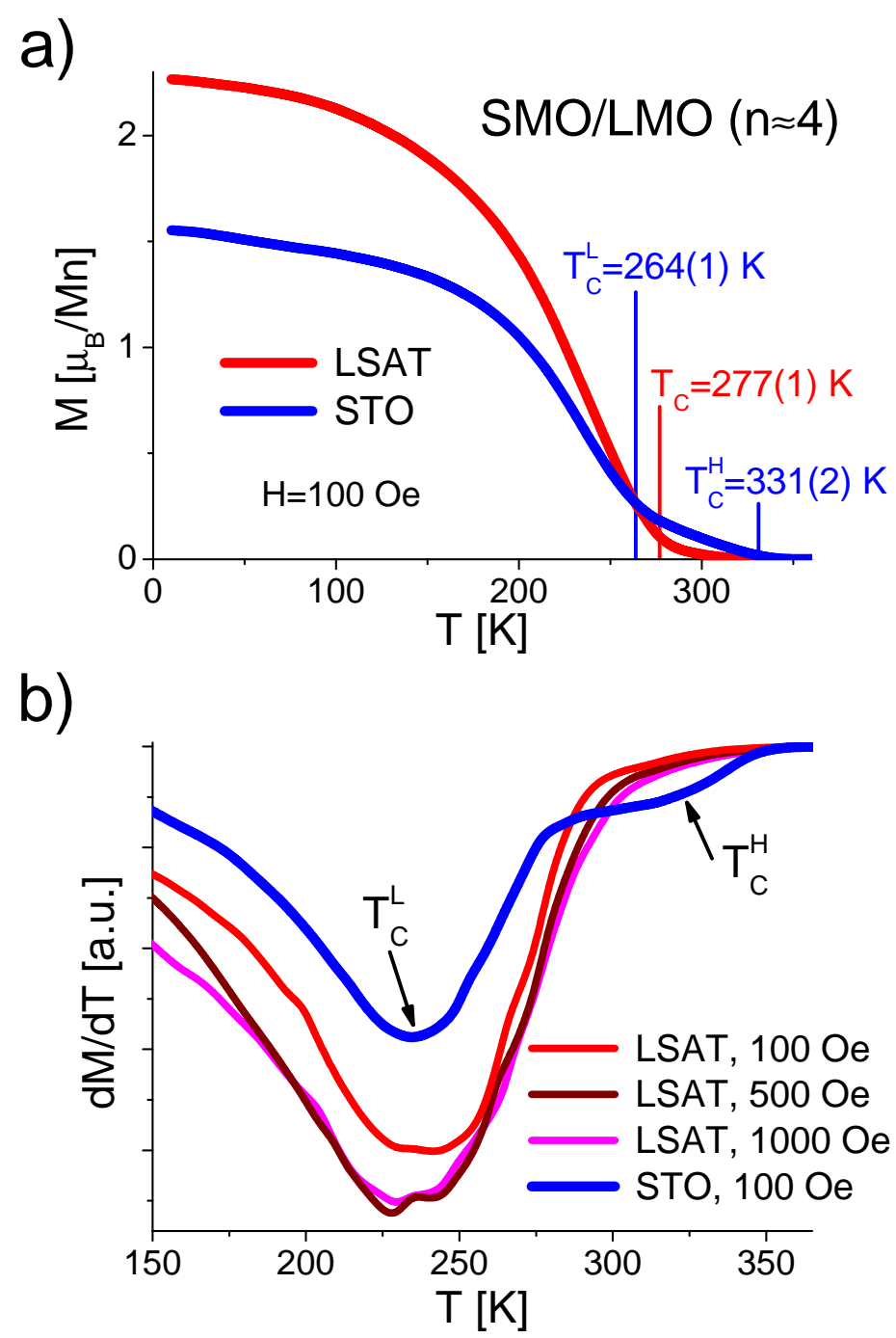

\begin{abstract}
Abbildung 9.33.: Temperaturabhängigkeit der Magnetisierung für SMO/LMO-SL $(n \approx$ $4, m=11)$ auf STO (001)- und LSAT (001)-Substrat: a) $M(T)$-Kurven, die CurieTemperaturen sind angegeben. b) $\frac{d M}{d T}$-Kurven zur Bestimmung der Curie-Temperaturen. Für STO sind zwei Maxima in $\frac{d M}{d T}$ markiert, für LSAT zeigt sich auch für verschiedene Felder nur ein einfaches Maximum.
\end{abstract}

In den XRD-Spektren des Übergitters auf LSAT kann man wie beim Wachstum auf STO nur Beugungsmaxima des Typs (001) ausmachen, was auf ein epitaktisches Wachstum hindeutet. Wie man in den XRD-Spektren sieht, resultiert aus dem Unterschied zwischen $a_{L S A T}$ und $a_{S T O}$ ein stark verschiedener Verspannungszustand (siehe Abbildung 9.32). Durch die verstärkte Druckspannung auf die LMO-Lagen und reduzierte Zugspannung auf die SMO-Lagen sind $c_{S M O}$ und $c_{L M O}$ für das Wachstum auf LSAT deutlich größer als beim Wachstum auf STO.

Die Abweichungen im Verspannungszustand schlagen sich auch in den Ausprägungen des Magnetismus nieder. In Abbildung 9.33 sind die $M(T)$-Kurven für die beiden Proben gezeigt. Beim Wachstum auf STO sind deutlich zwei magnetische Übergänge bei $T_{C}^{L}=264(1) \mathrm{K}$ und $T_{C}^{H}=331(2) \mathrm{K}$ auszumachen. Für das SL auf dem LSAT-Substrat ist dies nicht der Fall und es erscheint ein einfacher Ordnungsübergang mit $T_{C}=277(1) \mathrm{K}$. Diese Phänomenologie wird bei der Begutachtung von $\frac{d M}{d T}$ verdeutlicht. $\frac{d M}{d T}$ besteht für das SL auf STO (001) aus einer 

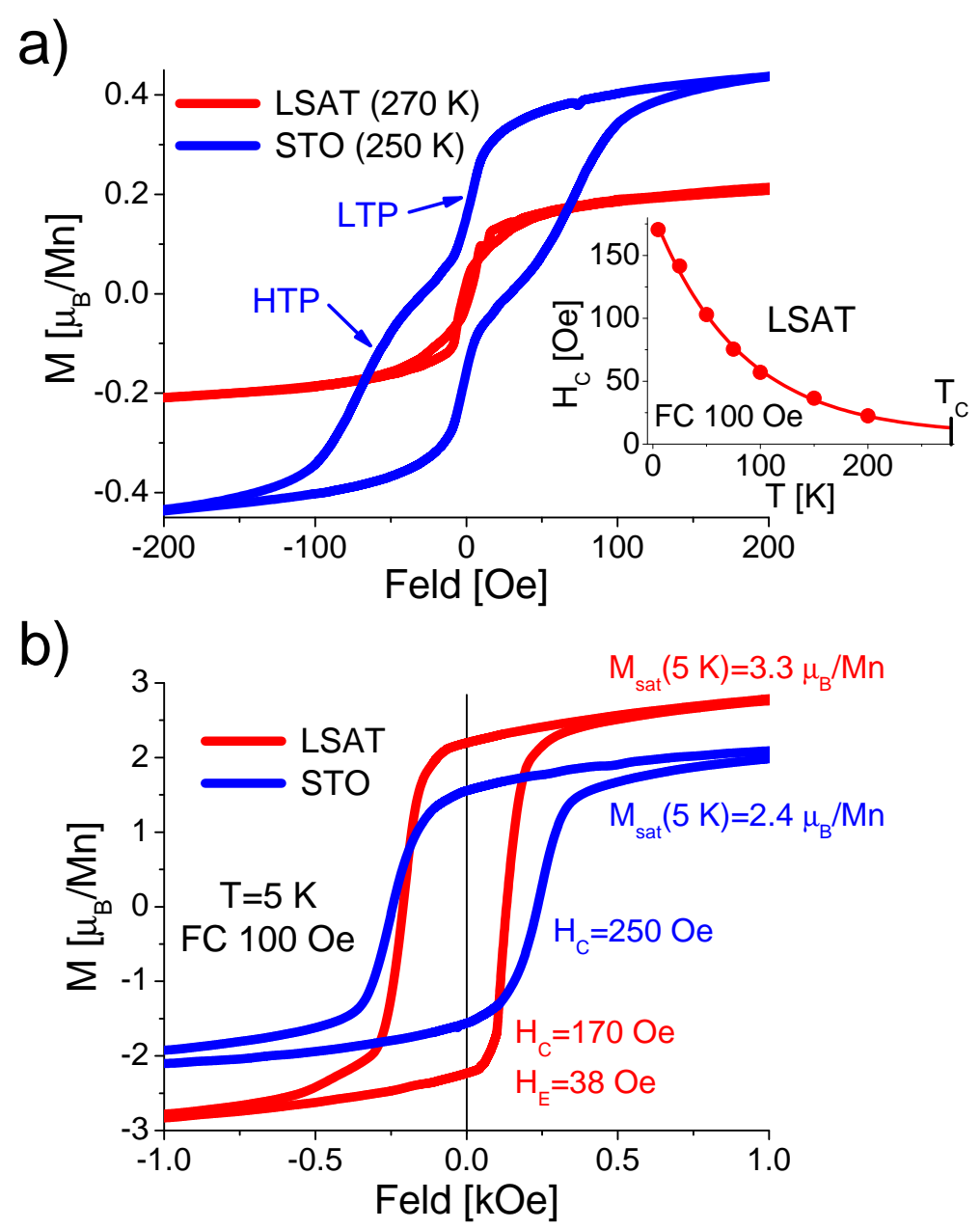

\begin{abstract}
Abbildung 9.34.: Feldabhängigkeit der Magnetisierung für SMO/LMO-SL $(n \approx 4, m=$ 11) auf STO (001)- und LSAT (001)-Substrat: a) $M(H)$-Kurven bei Temperaturen kurz unterhalb von $T_{C}^{L}$ bzw. $T_{C}$. Das Inset zeigt die Temperaturabhängigkeit des Koerzitivfeldes $H_{C}$ für das SL auf LSAT. b) $M(H)$-Kurven bei $T=5 \mathrm{~K}$, die Koerzitivfelder und die Amplitude des exchange bias (EB) $H_{E}$ sowie die Werte für das Sättigungsmoment $M_{s a t}$ sind im Diagramm angegeben.
\end{abstract}

Überlagerung zweier Maxima. Beim SL auf LSAT sieht man nur ein Maximum, unabhängig von der Wahl des von außen angelegten Feldes (siehe Abbildung 9.33 b)). Das Fehlen des zweiten Maximums in $\frac{d M}{d T}$ kann nicht durch den Einfluss einer starken Anisotropie der HTP erklärt werden. Bei der Curie-Temperatur würde die Anisotropieenergie verschwinden, so dass das externe Feld zu einer kompletten Ausrichtung einer möglicherweise vorhandenen HTP führen würde, auch wenn das Feld senkrecht zur leichten Richtung angelegt werden würde.

Für das SL auf LSAT fehlt also die HTP. Diese Tatsache spiegelt sich auch in den Feldabhängigkeiten der Magnetisierung wieder (siehe Abbildung 9.34 a)). Beim Wachstum auf STO besteht die $M(H)$-Kurve aus der bekannten Überlagerung zweier Hystereseschleifen mit verschiedenen Koerzitivfeldern. Das SL auf LSAT weist nur eine einfache Hysterese auf (Da die Ordnungstemperatur der LTP mit $T_{C}^{L}=264(1) \mathrm{K}$ deutlich unterhalb $T_{C}=277(1) \mathrm{K}$ für das SL auf LSAT liegt, wird hier für das SL auf STO eine Messung bei einer niedrigeren Temperatur als für LSAT gezeigt.). Als Funktion der Temperatur verhält sich $H_{C}$ so wie bei 


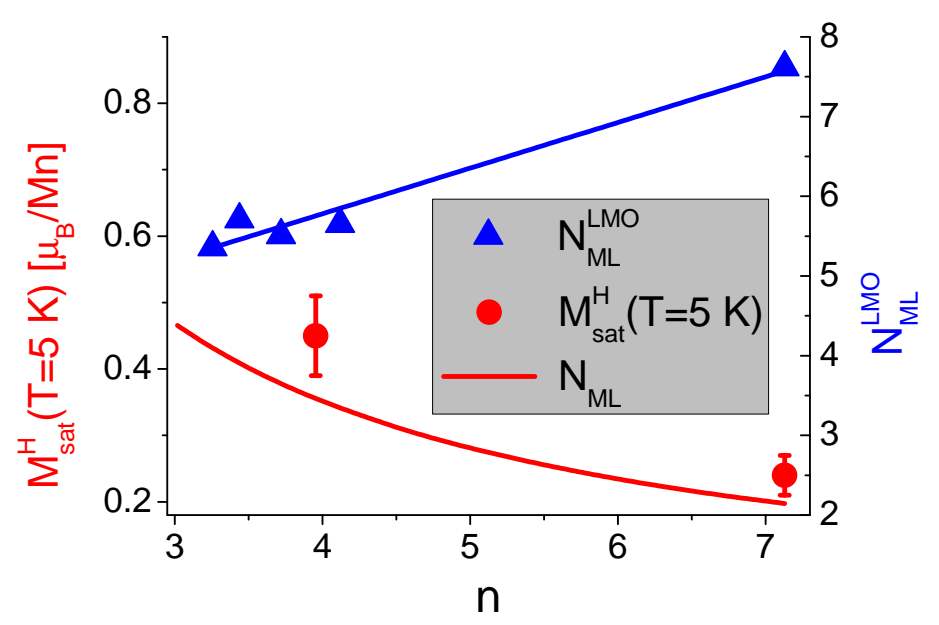

Abbildung 9.35.: Abschätzung der Sättigungsmagnetisierung der HTP bei $T=5 \mathrm{~K}$, $M_{\text {sat }}^{H}(T=5 \mathrm{~K})$, (Die rote Linie zeigt die theoretischen Werte für die Magnetisierung $N_{M L}$, die sich ergibt, wenn in jeder LMO/SMO-Einheit eine mit $3.75 \mu_{\mathrm{B}} / \mathrm{Mn}$ magnetisierte $\mathrm{MnO}_{2^{-}}$ Lage vorliegt.) und formale Zahl der $\mathrm{MnO}_{2}$-Lagen im LMO, die mit einer Magnetisierung von $4.0 \mu_{\mathrm{B}} / \mathrm{Mn}$ versehen sind, $N_{M L}^{L M O}$.

der LTP für ein SL auf STO (vergleiche Abbildung 9.28). $H_{C}$ fällt mit steigender Temperatur exponentiell ab. Auch bei tiefen Temperaturen besteht eine Diskrepanz zwischen den Übergittern auf STO und LSAT. Zum einen ist in den $M(H)$-Kurven für LSAT ein exchange bias (EB) zu erkennen, das für das STO-Substrat fehlt. Andererseits ist die Sättigungsmagnetisierung für das SL auf LSAT mit $M_{\text {sat }}(5 \mathrm{~K})=3.3(2) \mu_{\mathrm{B}} / \mathrm{Mn}$ deutlich höher als für das SL auf STO mit $M_{\text {sat }}(5 \mathrm{~K})=2.4(2) \mu_{\mathrm{B}} / \mathrm{Mn} . M_{\text {sat }}(5 \mathrm{~K})=3.3 \mu_{\mathrm{B}} / \mathrm{Mn}$ liegt in dem Bereich, den man für eine volle Ausrichtung der Spins in den LMO-Lagen und in jeweils einer Monolage der SMO-Schichten erwartet $\left(M_{L M O+1 S M O}\right.$, vergleiche Abbildung 9.29).

\subsection{Diskussion}

In diesem Abschnitt soll ausschließlich der magnetische Zustand der LMO/SMO-SL mit $n \geq 3$ auf STO (001)-Substraten diskutiert werden. Die für diese Heterostrukturen beobachtete Koexistenz zweier ferromagnetischer Phasen wurde in der Literatur zuvor noch nicht beobachtet. Das Verhalten der SL auf STO (111) wurde bereits in Abschnitt 9.4 behandelt. In diesem Abschnitt wurden auch die SL auf STO (001)-Substraten mit $n \leq 2$ besprochen. Die in-situ Ellipsometrie beim Wachstum der LMO/SMO-SL auf STO (001) wurde in Abschnitt 9.3.2 interpretiert. Die dabei gewonnene Verteilung der $e_{g}$-Elektronen soll in diesem Abschnitt zur Erklärung des magnetischen Zustandes dieser SL herangezogen werden.

Zunächst sollen die experimentellen Befunde bezüglich der magnetischen Eigenschaften der LMO/SMO-SL mit $n \geq 3$ zusammengefasst werden. Man beobachtet zwei ferromagnetische Signaturen, die Hochtemperaturphase (HTP) mit der Curie-Temperatur $T_{C}^{H}$ und die Tieftemperaturphase (LTP) mit $T_{C}^{L}<T_{C}^{H} \cdot T_{C}^{H}$ zeigt keine systematische Abhängigkeit von $n$ und schwankt zufällig um $T_{C}^{H} \sim 345 \mathrm{~K} \pm 10 \mathrm{~K}$. Dagegen fällt $T_{C}^{L}$ zwischen $n \approx 3$ und $n \approx 7$ um $\Delta T \approx 120 \mathrm{~K}$ deutlich ab. 
Bei $T=300 \mathrm{~K}>T_{C}^{L}$ kann man die Magnetisierung der HTP, $M_{\text {sat }}^{H}$, bestimmen und sieht, dass $M_{\text {sat }}^{H}(T=300 \mathrm{~K}) \propto \frac{1}{n}$ gilt. Diese Skalenrelation passt zu einem magnetischen Moment, das an den LMO/SMO-Grenzflächen der SL lokalisiert ist. Denn der Anteil dieser Grenzlagen am Gesamtvolumen der Struktur sinkt ebenfalls mit $\frac{1}{n}$ ab. Um eine grobe Abschätzung der Sättigungsmagnetisierung der HTP bei $T=5 \mathrm{~K}, M_{\text {sat }}^{H}(T=5 \mathrm{~K})$, zu gewinnen, betrachtet man eine einfache LSMO-Schicht auf STO (001) mit $T_{C} \sim T_{C}^{H}$. Für diese nimmt $M_{\text {sat }}$ von $T=300 \mathrm{~K}$ bis $T=5 \mathrm{~K}$ um einen Faktor von $1.5 \mathrm{zu}: M_{\text {sat }}(T=5 \mathrm{~K}) \approx 1.5 \cdot M_{\text {sat }}(T=300 \mathrm{~K})$. Unter Ausnutzung dieser Abschätzung folgt für die im vorigen Abschnitt gezeigten Beispiele: $M_{\text {sat }}(T=5 \mathrm{~K})=0.45(6) \mu_{\mathrm{B}} / \mathrm{Mn}$ für $n=4$ und $M_{\text {sat }}(T=5 \mathrm{~K})=0.24(3) \mu_{\mathrm{B}} / \mathrm{Mn}$ für $n=7.1$ (vergleiche Abbildung 9.35). Wenn sich an jeder zweiten LMO/SMO-Grenzfläche eine mit $3.75 \mu_{\mathrm{B}} / \mathrm{Mn}$ magnetisierte $\mathrm{MnO}_{2}$-Lage befindet, ergibt sich für die Struktur mit $n=4$ eine theoretische Sättigungsmagnetisierung von $M_{M L}=0.35 \mu_{\mathrm{B}} / \mathrm{Mn}$ und für $n=$ 7.1 ist $M_{M L}=0.2 \mu_{\mathrm{B}} / \mathrm{Mn}$ (Abbildung 9.35). Man berechnet nun den Quotienten $N_{M L}=$ $M_{\text {sat }}^{H}(T=5 \mathrm{~K}) / M_{M L}$ für $n=4,7.1$, der formal der Zahl der mit $3.75 \mu_{\mathrm{B}} / \mathrm{Mn}$ magnetisierten $\mathrm{MnO}_{2}$-Lagen pro Wiederholung der LMO/SMO-Einheiten entspricht. Für $n=4$ resultiert damit $N_{M L}=1.3(2) \mathrm{ML}$ und für $n=7.1 N_{M L}=1.2(2) \mathrm{ML}$. Das magnetische Moment der HTP kommt also dem Moment einer mit $3.75 \mu_{\mathrm{B}} / \mathrm{Mn}$ aufmagnetisierten $\mathrm{MnO}_{2}$-Lage in jeder LMO/SMO-Doppellage nahe. Auch für die LTP berechnet man, welcher Zahl von $\mathrm{MnO}_{2^{-}}$ Lagen man die gemessene Sättigungsmagnetisierung zuordnen kann. Gemäß den Ausführungen zum Moment der HTP zieht man von den Messwerten der gesamten Sättigungsmagnetisierung, $M_{\text {sat }}(5 \mathrm{~K})$ (Abbildung $9.29 \mathrm{~b}$ )) den Wert ab, der dem Moment einer mit $3.75 \mu_{\mathrm{B}} / \mathrm{Mn}$ magnetisierten $\mathrm{MnO}_{2}$-Lage in jeder LMO/SMO-Bilage entspricht. Dann berechnet man, wie vielen $\mathrm{MnO}_{2}$-Lagen $N_{M L}^{L M O}$ mit einer Magnetisierung von $4.0 \mu_{\mathrm{B}} / \mathrm{Mn}$ diese Magnetisierung entspricht. In Abbildung 9.35 sind die resultierenden Werte von $N_{M L}^{L M O}$ gezeigt. Von $n \approx 3$ bis $n \approx 7.1$ bewegt sich $N_{M L}^{L M O}$ im Bereich von $5-7$ mit einer ansteigenden Tendenz als Funktion von $n$.

Im Zusammenhang mit dem Fehlschnitt der STO-Substrate registriert man eine starke uniaxiale Anisotropie der HTP für $\alpha_{c u t}=0.2^{\circ}$. Dabei liegt die leichte Richtung entlang der Kanten der Terrassen des Substrates und die schwere Richtung senkrecht dazu. In den hier durchgeführten Messungen weist die LTP keine entsprechende uniaxiale Anisotropie auf. Der im Inset von Abbildung 9.27 b) gezeigte Wert für die Energiedichte der magnetischen Anisotropie bezieht sich auf das gesamte Volumen der Heterostruktur. Unter der Annahme, dass die HTP auf eine Monolage pro LMO/SMO-Bilage konzentriert ist und die HTP alleine für die messbare Anisotropie verantwortlich ist, multipliziert man $K$ mit der Zahl der Monolagen in jeder LMO/SMO-Doppellage. Dadurch ergibt sich die auf das Volumen der HTP bezogene Anisotropieenergiedichte $K_{H T P} \approx 2.1(2) \cdot 10^{5} \frac{\mathrm{J}}{\mathrm{m}^{3}}$. Diese Energiedichte ist groß im Vergleich zu den Literaturwerten für die in-plane Anisotropie von dünnen Manganatfilmen. In der Literatur gibt es auch Beispiele von uniaxialer Anisotropie, die dem Fehlschnitt des Substrates folgt [256, 260, 272]. Allerdings ist die Anisotropieenergiedichte deutlich kleiner. Sogar für LSMO-Filme auf vizinalen STO-Substraten $\left(\alpha_{c u t}=10^{\circ}\right)$ wird nur ein Wert von $K \approx 2 \cdot 10^{3} \frac{\mathrm{J}}{\mathrm{m}^{3}}$ festgestellt 272 . Die Größenordnung von $K_{H T P}$ ist dagegen vergleichbar mit der Formanisotropie eines dünnen Manganatfilmes, der mit $3.75 \mu_{\mathrm{B}} / \mathrm{Mn}$ magnetisiert ist:

$$
K_{F}=\frac{1}{2} \mu_{0} M_{\text {sat }}^{2} \approx 2.4 \cdot 10^{5} \frac{\mathrm{J}}{\mathrm{m}^{3}}
$$

Nach der Behandlung der individuellen Eigenschaften von HTP und LTP wird die Kopplung dieser Phasen besprochen. Man kann für die HTP und LTP getrennte Koerzitivfelder ausmachen und sieht keine Modifikation der Hysteresekurve der LTP beim Durchfahren einer 
minor-loop, bei der es nicht zur magnetischen Umkehr der HTP kommt. Damit besteht keine messbare Austauschkopplung zwischen diesen beiden Phasen 39

Im Folgenden soll ausgeschlossen werden, dass experimentelle Artefakte zur Ausbildung der HTP führen. Da $T_{C}^{H} \sim 345 \mathrm{~K}$ in der Nähe der Curie-Temperatur eines dünnen Filmes der Mischphase $\operatorname{LSMO}(\mathrm{x}=0.25)$ liegt, könnte man annehmen, dass sich die HTP aus der Durchmischung der Kationen auf den A-Plätzen ergibt. In der Darstellung der Ergebnisse wurde bereits gezeigt, dass die HTP nicht in den Randbereichen der beschichteten Fläche auf dem Substrat lokalisiert ist (vergleiche Abbildung 9.25). In den TEM-Aufnahmen des LMO/SMOSL erkennt man, dass die ersten beiden LMO/SMO-Grenzflächen unscharf sind hinsichtlich der Verteilung der Sr- und La-Ionen (Abbildungen 9.16 und 9.17). Nach den Experimenten zur Skalierung des magnetischen Momentes der HTP mit $m$ können auch diese chemisch durchmischten Bereiche nicht verantwortlich für die HTP sein (siehe Abbildung 9.26). Das Skalierungsexperiment zeigt außerdem, dass die HTP in jeder LMO/SMO-Bilage gleichermaßen auftritt. Auch die starke uniaxiale Anisotropie der HTP lässt sich schwer mit einer Durchmischung der Ionen in Einklang bringen. Selbst eine sehr anisotrope Ausprägung der Interdiffusion kann nicht zu einer solch starken Anisotropie führen. Dies kann man an folgendem hypothetischen Beispiel verdeutlichen: Man nimmt an, dass die Diffusion entlang der Stufenkanten erhöht ist, so dass sich beim Wachstum des LMO/SMO-SL Ebenen parallel zu den Stufenkanten und senkrecht zur Filmebene bilden, in denen die Sr- und La-Ionen stark durchmischt sind. Eine solche Ebene würde die hier beobachtete Anisotropie mit der leichten Richtung entlang der Stufenkanten besitzen. Allerdings wäre die Anisotropieenergie kleiner als die Formanisotropie eines einfachen Filmes. Denn diese hypothetischen Ebenen sind von magnetischen Materialien innerhalb des SL umgeben, wodurch die magnetische Dipolenergie herabgesetzt wird. Aufgrund dieser Ausführungen und mit Blick auf die TEM-Aufnahmen (Abbildungen 9.16 und 9.17), die nur eine schwache Sr/La-Durchmischung zeigen, kann man ausschließen, dass die HTP durch eine Interdiffusion der Sr- und La-Ionen verursacht wird.

Bei den in der Literatur vorkommenden Studien gibt es keine Berichte über das Auftreten zweier FM Phasen in LMO/SMO-SL. Es werden nur einfache Hystereseschleifen und eine einzelne Übergangstemperatur beobachtet $1119,120,274$. Im Gegensatz zu den Experimenten hier wurden die Heterostrukturen in der Literatur mit Vakuum-Methoden wie PLD 120 und MBE 119, 274 abgeschieden. Bei diesen Methoden unterscheiden sich die Wachstumsbedingungen, die Depositionstemperatur $T_{D e p}$ und der $\mathrm{O}_{2} / \mathrm{O}_{3}$-Partialdruck, deutlich von $T_{D e p} \approx 930{ }^{\circ} \mathrm{C}$ und $p_{O_{2}} \sim 0.21$ bar bei der MAD. In Kapitel 3 ist es bereits angeklungen, dass die Stöchiometrie der Ausgangsmaterialien, SMO und LMO, von den Depositionsbedingungen beeinflusst wird. SMO kann nicht nur in der kubischen Variante, $\mathrm{SrMnO}_{3}$, vorliegen, sondern bei einer hohen Konzentration von Sauerstoffleerstellen auch in $\mathrm{SrMnO}_{2.5}$ umgewandelt werden. Dies wird zusätzlich durch die epitaktischen Zugspannungen beim Wachstum auf STO-Substraten begünstigt. Die in der Literatur gezeigten einfachen SMO-Filme auf STO (001)-Substraten zeigen aber ähnliche Eigenschaften wie die hier präsentierten Filme. In beiden Fällen ist kein ferromagnetisches Moment messbar. Der Gitterebenenabstand für Filme mit $d_{S M O} \sim 20 \mathrm{~nm}$ liegt mit $c=0.378 \mathrm{~nm}$ 113, 275 in einem ähnlichen Bereich wie hier (vergleiche Abbildung $9.2 \mathrm{a}$ )). Der spezifische Widerstand bei $T=200 \mathrm{~K} \rho(200 \mathrm{~K}) \approx 5 \Omega \mathrm{cm}$ [276] ist vergleichbar mit $\rho(200 \mathrm{~K}) \approx 3 \Omega \mathrm{cm}$ hier (Abbildung $9.3 \mathrm{~b}$ )).

\footnotetext{
${ }^{39}$ Die HTP ist nur für $n=7.1$ bis $T=5 \mathrm{~K}$ von der LTP zu trennen, für kleinere Werte $(n \approx 3-4)$ kann man die HTP und LTP schon bei $T^{\text {sep }} \sim 150-180 \mathrm{~K}$ nicht mehr in der $M(H)$-Kurve separieren. Damit kann man für $n=7.1$ eine magnetische Austauschkopplung zwischen HTP und LTP im gesamten zugänglichen Temperaturbereich ausschließen, für $n=3-4$ nur im Bereich $T^{\text {sep }}<T<T_{C}^{L}$.
} 
Für einfache LMO-Filme weichen die mit der MAD präparierten Proben allerdings deutlich von den Filmen anderer Gruppen ab. In Abschnitt 3.3.3 wurde beschrieben, dass das stöchiometrische $\mathrm{LaMnO}_{3}$ bei hohen Sauerstoffpartialdrücken $\left(p_{O_{2}} \approx 0.2-1\right.$ bar $)$ nicht stabil ist. Es bildet sich eine unstöchiometrische Phase, die La-Leerstellen aufweist und ferromagnetisch ordnet. Auch die mit MBE hergestellten LMO-Filme zeigen eine teilweise ferromagnetische Ordnung [119, 274]. Dies wird von den Autoren ebenfalls einem La-Defizit zugeschrieben und angedeutet, dass sich das La-Defizit mit einer Erhöhung des Sauerstoffpartialdruckes vergrößert. Daraus folgt dann eine Erhöhung des ferromagnetischen Momentes 274. Durch den atmosphärischen Sauerstoffpartialdruck zeigen die mit der MAD abgeschiedenen Filme einen Zustand, der einem größeren La-Defizit entspricht als die Filme, die mit MBE hergestellt wurden. Der Gitterparameter in c-Richtung ist hier mit $c=0.3895(5) \mathrm{nm}$ (Abbildung 9.1 a)) deutlich kleiner als $c=0.393-0.396 \mathrm{~nm}$ [113, 275] für Filme, die mit MBE auf STO deponiert wurden. Auch die Curie-Temperatur $T_{C}=172(2) \mathrm{K}$ und das Sättigungsmoment $M_{\text {sat }}(5 \mathrm{~K})=3.4(2) \mu_{\mathrm{B}} / \mathrm{Mn}$ sind höher als $T_{C}=135 \mathrm{~K}$ und $M_{\text {sat }}(5 \mathrm{~K})=1 \mu_{\mathrm{B}} / \mathrm{Mn}$ [274 für Strukturen, die mit MBE abgeschieden wurden. Die stärkere FM Ordnung sorgt für eine deutliche Erniedrigung des spezifischen Widerstandes auf $\rho(200 \mathrm{~K})=0.5 \Omega \mathrm{cm}$ (Abbildung 9.3 b)) im Gegensatz zu $\rho(200 \mathrm{~K})=8 \Omega \mathrm{cm} 276$.

Neben den beiden Materialien am Ende des LSMO(x)-Phasendiagrammes ergeben sich auch Abweichungen für $\operatorname{LSMO}(\mathrm{x}=0.33-0.4)$-Filme, die im bulk einen homogenen FMM-Zustand zeigen. Bei den mit MAD hergestellten Filmen stimmt das Sättigungsmoment $M_{\text {sat }}(T=5 \mathrm{~K})$ von Filmen aus diesen Materialien $(d \approx 40 \mathrm{~nm})$ mit den theoretischen Erwartungen überein (vergleiche Abbildung 9.4 b) und [97, 267]). Im Rahmen der Studien zu LMO/SMO-SL in der Literatur wurden zum Vergleich durchmischte LSMO(x)-Filme hergestellt, deren La/SrVerhältnis den durchschnittlichen Anteilen im LMO/SMO-SL entspricht 119, 120, 274]. Die Sättigungsmomente dieser Referenzproben $(d \sim 20-40 \mathrm{~nm})$ liegen deutlich unter dem theoretischen Wert für eine volle Ausrichtung aller Spins auf den Mn-Ionen. Für LMSO(x=0.4)Filme auf STO (001) wird von Yamada et al. ein Sättigungsmoment von $3.2 \mu_{\mathrm{B}} / \mathrm{Mn}$ und auf LSAT (001) $3.0 \mu_{\mathrm{B}} / \mathrm{Mn}$ festgestellt [120]. Auch bei $\operatorname{LSMO}(\mathrm{x}=0.33)$-Filmen auf STO (001) ist $M_{\text {sat }}(T=10 \mathrm{~K})$ mit $3.0-3.2 \mu_{\mathrm{B}} / \mathrm{Mn}$ wesentlich kleiner als der theoretische Wert $3.67 \mu_{\mathrm{B}} / \mathrm{Mn}$ 119,274 .

Dieser Trend setzt sich auch für die LMO/SMO-SL fort. In $\mathrm{LMO}_{2} / \mathrm{SMO}_{1}$-SL erwartet man aufgrund von $a b$ initio Rechnungen [117] und XAS/XLD-Messungen [113] eine homogene Verteilung der $e_{g}$-Elektronen. Damit würde sich für diese SL ein homogener FMM-Zustand mit $M_{\text {sat }}=3.67 \mu_{\mathrm{B}} / \mathrm{Mn}$ ergeben. Bei den hier deponierten LMO/SMO $(n=1)$-SL sieht man genau das theoretisch erwartete Verhalten (siehe Inset von Abbildung 9.23 b)). Die mit MBE hergestellten $\mathrm{LMO}_{2} / \mathrm{SMO}_{1}-\mathrm{SL}$ besitzen aber mit 2.8-3.0 $\mu_{\mathrm{B}} / \mathrm{Mn}$ eine kleinere Magnetisierung in Sättigung [119, 274]. Die Curie-Temperaturen dieser Strukturen liegen nur $\Delta T \approx 10 \mathrm{~K}$ unter der Ordnungstemperatur der hier gezeigten LMO/SMO $(n=1)$-SL. Diese abweichenden magnetischen Eigenschaften lassen sich durch den Einbau von Sauerstoffleerstellen erklären. Derartige Defekte modifizieren zunächst das Verhältnis von $\mathrm{Mn}^{3+}$ - und $\mathrm{Mn}^{4+}$-Ionen. Des Weiteren werden Mn-O-Mn-Bindungen unterbrochen, was die Itineranz der $e_{g}$-Elektronen behindert. Huijben et al. stellen LSMO-Filme auf STO (001)-Substraten unter Variation der $\mathrm{O}_{2}$-Hintergrundatmosphäre mittels PLD her [85]. Bei Unterschreiten eines kritischen Wertes von $p_{\mathrm{O}_{2}}$ kommt es dabei zu einer Halbierung von $M_{\text {sat }}$, wogegen $T_{C}$ nur um $\Delta T \sim 10-15 \mathrm{~K}$ abfällt 85 .

Neben der globalen Konzentration von Defekten ist für die magnetische Phänomenologie der LMO/SMO-SL auch die strukturelle Qualität der Grenzflächen entscheidend. Bei den Depo- 
sitionsbedingungen der MBE ist die Mobilität der Adatome geringer als bei den Bedingungen der MAD. Daraus folgt eine Oberflächenmorphologie, bei der sich eine Vielzahl kleiner Inseln $(D \sim 5-30 \mathrm{~nm})$ auf den Terrassen sammelt 275]. Bei den mit MAD hergestellten Heterostrukturen gibt es nur in der Nähe zum Substrat eine Region, in der die Mobilität der Adatome durch eine Ti/Mn-Durchmischung reduziert ist und sich kleine zweidimensionale Inseln $(D \sim 10-40 \mathrm{~nm})$ bilden. In größerer Entfernung dominiert eine Wachstumsmode zwischen dem Wachstum großer zweidimensionaler Inseln $\left(D \sim 1 / 2 \cdot w_{T e r r}, w_{T e r r}\right.$ : Breite der durch den Fehlschnitt verursachten Substratstufen) und step-flow-Wachstum (vergleiche Abschnitt 6.3, 8.2.2 und 9.3.2). Diese unterschiedlichen Wachstumsmoden verursachen eine differierende Qualität der LMO/SMO-Grenzflächen. May et al. beobachten eine Asymmetrie der Grenzflächen in LMO/SMO-SL 13, 14. Die Grenzfläche, bei der LMO auf SMO folgt (SMO/LMO), ist schärfer als jene, bei der SMO auf LMO wächst (LMO/SMO). Für die LMO/SMO-Grenze sieht man Täler einer Breite von etwa $10 \mathrm{~nm}$ und einer Tiefe von 2 Monolagen an der Oberfläche der LMO-Lagen. Die hier vorgestellten LMO/SMO-SL zeigen auch eine scharfe SMO/LMO-Grenze. Für die andere Grenzfläche (LMO/SMO) sieht man keine Täler wie May et al., sondern eher eine diffuse Durchmischung der La- und Sr-Ionen (siehe Abbildung 9.17). May et al. sehen diese strukturelle Asymmetrie der Grenzflächen als Ursache für eine ungleiche Ausbildung der ferromagnetischen Ordnung an den beiden Grenzflächen an. Es wird eine deutliche Reduktion des magnetischen Momentes an der unscharfen Grenzfläche beobachtet 13, 14.

Insgesamt weisen die getätigten Betrachtungen darauf hin, dass in den hier abgeschiedenen LMO/SMO-SL eine andere Ausprägung an Defekten vorliegt als in den Heterostrukturen, die mit PLD und MBE fabriziert wurden. Daraus ergeben sich schon bei den globalen magnetischen Eigenschaften $\left(M_{s a t}, T_{C}\right)$ deutliche Unterschiede. Insbesondere im Hinblick auf die strukturellen Unterschiede der LMO/SMO-Grenzflächen sind deshalb auch Abweichungen der lokalen magnetischen Kopplungen in den LMO/SMO $(n \geq 3)$-SL zu erwarten.

Abschließend soll ein Modell für den magnetischen Zustand der LMO/SMO ( $n \geq 3)$-SL vorgestellt und diskutiert werden. Ein wichtiger Anhaltspunkt dafür ist die Entwicklung des magnetischen Zustandes der SL bei der Modifikation des Verspannungszustandes, der sich beim Wachstum auf einem LSAT (001)-Substrat ergibt. Es kommt zum Verschwinden der HTP und $M_{\text {sat }}(5 \mathrm{~K})$ entspricht innerhalb des experimentellen Fehlers $M_{L M O+1 S M O}$, also der Magnetisierung, die man für eine volle Ausrichtung der Spins in den LMO-Lagen und jeweils einer $\mathrm{MnO}_{2}$-Lage in den SMO-Schichten erwartet (Abbildungen 9.33 und 9.34). In Abschnitt 3.2 wurde erläutert, wie epitaktische Verspannungen die Freiheitsgrade der 3dOrbitale bei oxidischen Heterostrukturen beeinflussen. Daraus ergeben sich dann zum Teil drastische Konsequenzen für die magnetischen Wechselwirkungen (Abschnitt 3.3.1).

Hier wird nun erörtert, wie die abweichenden Verspannungszustände der SL auf STO und LSAT die lokalen Besetzungswahrscheinlichkeiten der beiden $e_{g}$-Orbitale, $d_{x^{2}-y^{2}}$ und $d_{3 z^{2}-r^{2}}$, beeinflussen. Die Anpassungen der XRD-Messungen der beiden Strukturen zeigen, dass in beiden Fällen ein voll verspannter Zustand der einzelnen SMO- bzw. LMO-Lagen vorliegt (Abbildung 9.32). Man folgert dies, indem man aus den angepassten Parametern $c_{L M O}, c_{S M O}$ die pseudokubischen Gitterkonstanten $a_{L M O}, a_{S M O}$ des unverspannten Materials gemäß der Gleichung $(\nu=1 / 3)$

$$
a_{L M O / S M O}=\frac{c_{L M O / S M O}+a_{S u b}}{2}
$$

berechnet (vergleiche Gleichung 8.1). Für beide Substrate erhält man bei dieser Rechnung Werte von $a_{L M O / S M O}$, die innerhalb des Messfehlers identisch sind: 


\section{LMO/SMO (n=3) STO(001)}

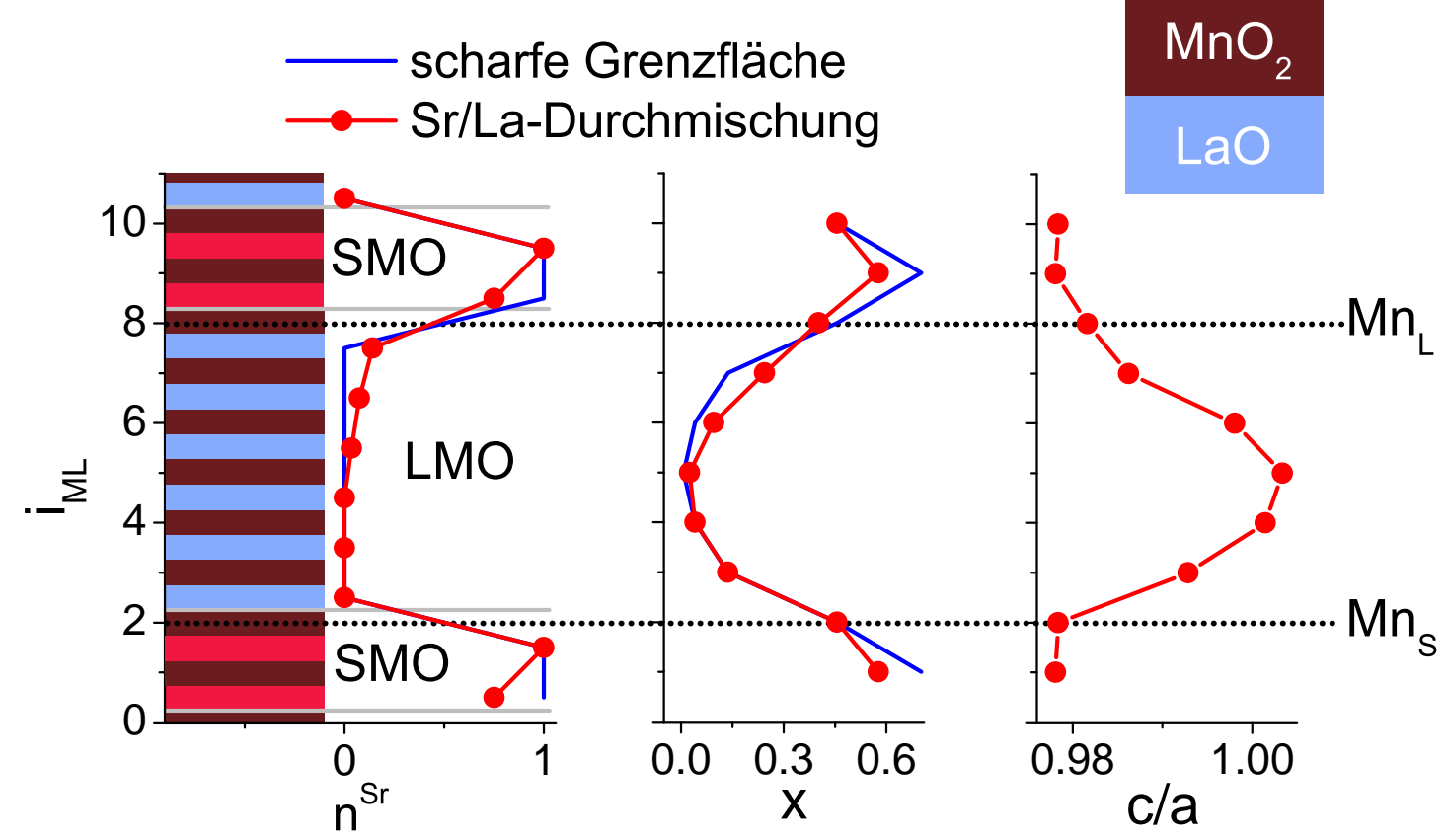

Abbildung 9.36.: Verteilung der $e_{g}$-Elektronen $n\left(e_{g}\right)=1-x$ und lokale tetragonale Verzerrung $c / a$ in LMO/SMO $(n=3)$-SL unter Berücksichtigung der Verteilung der SrIonen $n^{S r}$ als Funktion der Nummer der $\mathrm{MnO}_{2}$-Lage $i_{M L}$. Die $\mathrm{MnO}_{2}$-Lagen an den beiden Grenzflächen wurden mit $\mathrm{Mn}_{\mathrm{S}}$ bzw. $\mathrm{Mn}_{\mathrm{L}}$ markiert.

- LSAT: $a_{L M O}=0.392(1) \mathrm{nm}, a_{S M O}=0.379(3) \mathrm{nm}$

- STO: $a_{L M O}=0.391(1) \mathrm{nm}, a_{S M O}=0.379(2) \mathrm{nm}$

$a_{S M O}$ passt zum Gitterparameter der kubischen bulk-Phase und $a_{L M O}$ stimmt mit dem Wert überein, der sich für das LMO/SMO-SL $(n=3)$ ergibt (vergleiche Abbildung 9.15).

Mit Hilfe der aufgenommenen in-situ Ellipsometrie errechnete man die Verteilung der $e_{g^{-}}$ Elektronen an den SMO/LMO-Grenzflächen. Aus der Variation der durchschnittlichen MnValenz in jeder $\mathrm{MnO}_{2}$-Lage folgt auch eine lokale Modulation der Gitterverspannungen, da das $\mathrm{Mn}^{3+}$-Ion deutlich größer ist als das $\mathrm{Mn}^{4+}$-Ion. Die orbitale Polarisation in jeder $\mathrm{MnO}_{2}$-Lage ist dann mit der lokalen tetragonalen Verzerrung, die durch das Verhältnis $c / a$ quantifiziert wird, verknüpft. Zur Abschätzung von $c / a$ zieht man neben der mittleren Mn-Valenz in jeder $\mathrm{MnO}_{2}$-Lage auch die Stöchiometrie der Kationen auf den beiden benachbarten AO-Lagen heran. Damit kann man dann durch den Vergleich mit entsprechenden bulk-Materialien einen Wert für den pseudokubischen Gitterparameter $a_{p}$ der unverspannten Struktur errechnen.

In den TEM-EELS-Aufnahmen der LMO/SMO-Heterostruktur stellt man eine Durchmischung der LMO/SMO-Grenzfläche fest (Abbildung 9.17). Man berücksichtigt diese Umverteilung der La- und Sr-Ionen bei der Bestimmung der lokalen Mn-Valenz. Wenn man die zu Abbildung 9.17 gehörigen Intensitätsprofile inspiziert, stellt man fest, dass der Anteil von La-Ionen in der SrO-Lage an der LMO/SMO-Grenzfläche etwa 0.25 beträgt. Im Rahmen der Auflösung der TEM-EELS-Aufnahme an der La-Kante kann man nicht feststellen, wie sich die Sr-Ionen in den LMO-Lagen verteilen. Deshalb kann man ausschließen, dass die Sr-Ionen in der LaO-Lage an der Grenzfläche lokalisiert bleiben. In Abschnitt 9.3 .2 wurde motiviert, 
dass die starke Delokalisierung der $e_{g}$-Elektronen beim Aufbringen der ersten SMO-Lage zu dieser Sr/La-Durchmischung an der LMO/SMO-Grenzfläche führt. Man nimmt somit eine Verteilung der Sr-Ionen an, die der in Abbildung 9.20 Phase 2 dargestellten Umverteilung der $e_{g}$-Elektronen entspricht (siehe Abbildung 9.36). Die in das LMO eingedrungenen Sr-Atome modifizieren das lokale Verhältnis der $\mathrm{Mn}^{3+}$ - und $\mathrm{Mn}^{4+}$-Ionen, so dass ein asymmetrisches Dotierungsprofil $x\left(i_{M L}\right)$ entsteht. Diese Änderung der Verteilung der $e_{g}$-Elektronen liegt im Fehler der in-situ Ellipsometrie, da N (LSMO(x)) nur kleine Änderungen für den Dotierungsbereich $x=0.0-0.4$ zeigt.

Mit den Verteilungen der Kationen auf den A-Plätzen und der Elektronen auf den Mn-Ionen kann man $a_{p}$ und $c / a$ abschätzen: Die $\mathrm{MnO}_{2}$-Lage bei $i_{M L}$ hat einen Anteil $x\left(i_{M L}\right)$ von $\mathrm{Mn}^{4+}$-Ionen und die benachbarten AO-Lagen haben einen mittleren Anteil Sr-Ionen von

$$
n_{a v}^{S r}\left(i_{M L}\right)=\frac{1}{2}\left(n^{S r}\left(i_{M L}-1 / 2\right)+n^{S r}\left(i_{M L}+1 / 2\right)\right)
$$

Zur Abschätzung von $a_{p}$ zieht man zunächst die lineare Relation

$$
a_{L S M O(x)}=a_{L M O}+\left(a_{S M O}-a_{L M O}\right) \cdot x
$$

heran (vergleiche Abschnitt 8.2.4), wobei man $a_{L M O}$ und $a_{S M O}$ aus der Anpassung der XRDSpektren der LMO/SMO-SL gewonnen hat. Wenn $n_{a v}^{S r}\left(i_{M L}\right) \neq x\left(i_{M L}\right)$, muss man eine zusätzliche Deformation des Gitters einbeziehen, die sich durch die verschiedenen Ionenradien von $\mathrm{Sr}^{2+}$ - und $\mathrm{La}^{3+}$ ergibt $\left(r\left(L a^{3+}\right)=0.136 \mathrm{~nm}, r\left(S r^{2+}\right)=0.144 \mathrm{~nm}\right.$ für die zwölffache Koordination der Perowskit-Struktur [277]). Dazu zieht man $\mathrm{CaMnO}_{3}$ mit $a_{C M O}=0.373 \mathrm{~nm}$ 278 heran, da $r\left(\mathrm{Ca}^{2+}\right)=0.144 \mathrm{~nm}$ [277] fast identisch mit $r\left(\mathrm{La}^{3+}\right)$ ist. Die Änderung von $a_{p}$ bei $n_{a v}^{S r} \neq x$ wird dann durch Addition von $\left(a_{S M O}-a_{C M O}\right) \cdot\left(n_{a v}^{S r}-x\right)$ abgeschätzt. Insgesamt ergibt sich $a_{p}$ für jede Monolage dann durch folgende Gleichung:

$$
a_{p}=a_{L M O}+x \cdot\left(a_{S M O}-a_{L M O}\right)+\left(n_{a v}^{S r}-x\right) \cdot\left(a_{S M O}-a_{C M O}\right)
$$

Dann wird die volle Verspannung durch das Substrat berücksichtigt, wodurch der Atomabstand in der Ebene auf den des Substrates fixiert wird, $a=a_{S u b}$. Mit der Querkontraktion unter der Annahme von $\nu=1 / 3$ folgt der Gitterparameter $c$ senkrecht zur Wachstumsrichtung und damit die tetragonale Verzerrung $c / a$ (siehe Abbildung 9.36).

Auch für das Wachstum auf dem LSAT-Substrat kann man den Verlauf von $c / a$ abschätzen, wenn man die Gitterkonstante in der Ebene auf $a_{L S A T}$ fixiert und $c$ dann mit Gleichung 8.1 errechnet. Dabei verwendet man die für die Heterostrukturen auf STO ermittelten Daten für die Verteilung der $e_{g}$-Elektronen und Kationen auf den A-Plätzen (Abbildung 9.36). $c / a$ ist so deutlich größer als für das Wachstum auf STO. Der Großteil der $\mathrm{MnO}_{2}$-Lagen wird Druckspannungen ausgesetzt. Die beiden $\mathrm{MnO}_{2}$-Lagen an den Grenzflächen, $\mathrm{Mn}_{\mathrm{S}}$ und $\mathrm{Mn}_{\mathrm{L}}$, besitzen eine fast kubische Struktur $(c / a \approx 1.0)$. Damit sind die $e_{g}$-Orbitale in diesen beiden Lagen gleichmäßig besetzt und der Energiegewinn $t_{d d}$ bei Delokalisierung der $e_{g}$-Elektronen unabhängig von der Richtung, in der sich die Elektronen bewegen. Da $t_{d d}$ entlang der cRichtung somit größer ist als für die Heterostrukturen auf STO, kann man eine stärkere Delokalisierung der $e_{g}$-Elektronen an den LMO/SMO-Grenzflächen erwarten (vergleiche Abschnitt 3.1 ${ }^{40}$. Die LMO-Lagen mit einem kleinen Anteil von $\mathrm{Mn}^{4+}$-Ionen werden starken Druckspannungen ausgesetzt, die nach theoretischen Rechnungen 117, 118 zur Stabilisierung der FM-Ordnung führen. Außerdem koppeln diese LMO-Lagen dann bevorzugt an die

\footnotetext{
${ }^{40}$ Hier konnte die Verteilung der $e_{g}$-Elektronen beim Wachstum auf LSAT nicht ermittelt werden, weil die Messungen der in-situ Ellipsometrie zu verrauscht sind (Abbildung 9.30).
} 


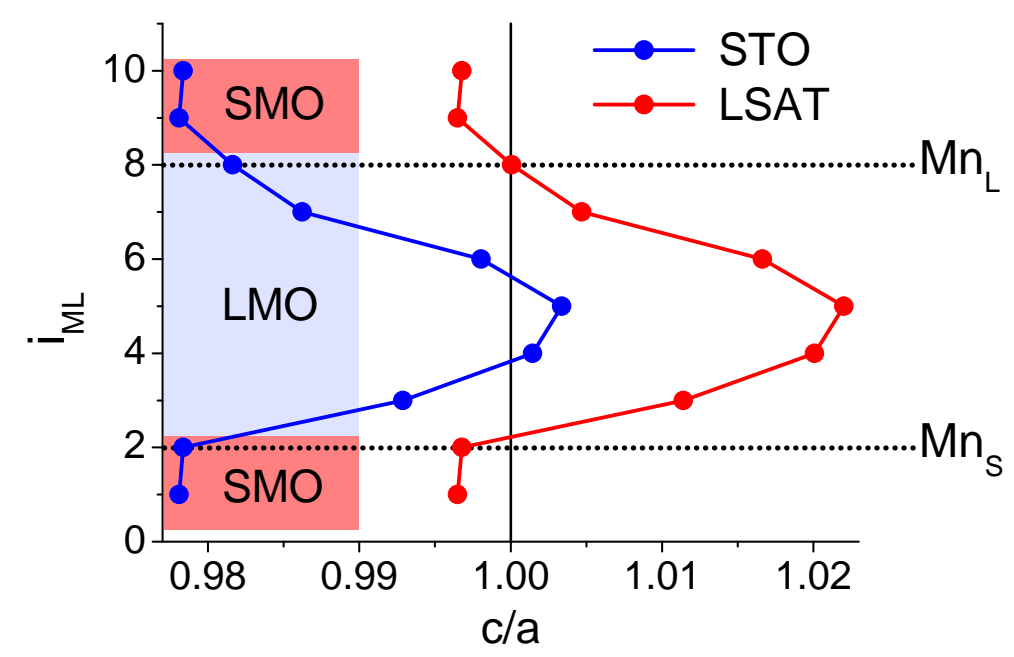

Abbildung 9.37.: Verlauf der tetragonalen Verzerrung $c / a$ (vergleiche Abbildung 9.36 für eine LMO/SMO $(n=3)$-Heterostruktur beim Wachstum auf STO und LSAT.

benachbarten $\mathrm{MnO}_{2}$-Lagen, für die man aufgrund des günstigen Anteils an $\mathrm{Mn}^{4+}$-Ionen eine FM-Ordnung erwartet. Dadurch stellt sich in dem LMO/SMO-SL auf LSAT ein FM-Zustand ein, der sich über alle $\mathrm{MnO}_{2}$-Lagen in LMO und den beiden Lagen an den Grenzflächen erstreckt. Die $\mathrm{MnO}_{2}$-Lagen, die beidseitig von SrO eingeschlossen werden, ordnen antiferromagnetisch und vermitteln das exchange bias.

Beim Wachstum auf STO steht die Struktur unter Zugspannungen. Die daraus resultierende Bevorzugung der $d_{x^{2}-y^{2}}$-Orbitale schwächt die Kopplung parallel zur Wachstumsrichtung. Damit kann sich im Gegensatz zur Struktur auf LSAT ein magnetisch inhomogener Zustand ausbilden. Die beiden $\mathrm{MnO}_{2}$-Lagen an den SMO/LMO-Grenzflächen, $\mathrm{Mn}_{\mathrm{S}}$ und $\mathrm{Mn}_{\mathrm{L}}$, unterscheiden sich hinsichtlich ihres Anteils von $\mathrm{Mn}^{4+}$-Ionen $\mathrm{x}$ und der Stärke der tetragonalen Verzerrung $c / a$. Des Weiteren differiert die Verteilung der Sr- und La-Ionen in ihrer jeweiligen Nachbarschaft und die Eigenschaften $(x, c / a)$ der angrenzenden $\mathrm{MnO}_{2}$-Lagen (Abbildung 9.36).

Mit Blick auf diese Unterschiede inspiziert man im Folgenden die magnetischen Kopplungen dieser beiden $\mathrm{MnO}_{2}$-Ebenen. Zur benachbarten $\mathrm{MnO}_{2}$-Lage, die zu beiden Seiten von $\mathrm{SrO}$ eingeschlossen wird $\left(i_{M L}=1,9\right.$, vergleiche Abbildung 9.36), ist die magnetische Kopplung schwach. Durch die starken epitaktischen Verzerrungen $(c / a \sim 0.98)$ werden vorwiegend die $d_{x^{2}-y^{2}}$-Orbitale besetzt, so dass ein AFM Superaustausch wirkt. Wie in Abschnitt 8.4 diskutiert, ergibt sich im Zusammenhang der energetischen Aufspaltung der $e_{g}$-Niveaus eine Vergrößerung der Energiedifferenz $\Delta_{p d}$ zwischen den $d_{3 z^{2}-r^{2}}$ und $O_{2 p^{-}}$Orbitalen. Dies vermindert $t_{d d}$ und damit die Amplitude der Austauschwechselwirkung $J_{S E}$, welche zu klein ist, um die magnetische Ordnung des Ferromagneten zu beeinflussen. Die Kopplung zum AFM macht sich wie in Kapitel 8 nur in der Modifikation der $M(H)$-Kurven (exchange bias, Erhöhung von $H_{C}$ ) bemerkbar.

Innerhalb der $\mathrm{MnO}_{2}$-Ebenen, $\mathrm{Mn}_{\mathrm{L}}$ und $\mathrm{Mn}_{\mathrm{S}}$, ergibt sich eine $\mathrm{FM}$ Austauschwechselwirkung über die bevorzugt besetzten $d_{x^{2}-y^{2}}$-Orbitale. In der mit $\mathrm{Mn}_{\mathrm{S}}$ markierten Lage zeigen die Srund La-Ionen nur eine schwache Durchmischung. Durch das damit einhergehende CoulombPotential wird der Austausch entlang der c-Richtung zusätzlich unterdrückt. Bei $\mathrm{Mn}_{\mathrm{L}}$ erlaubt die diffuse Verteilung der Kationen eine Bewegung der $e_{g}$-Elektronen senkrecht zur Wachstumsrichtung. Die geordnete Konfiguration der Sr- und La-Ionen für $\mathrm{Mn}_{\mathrm{S}}$ verstärkt außerdem 


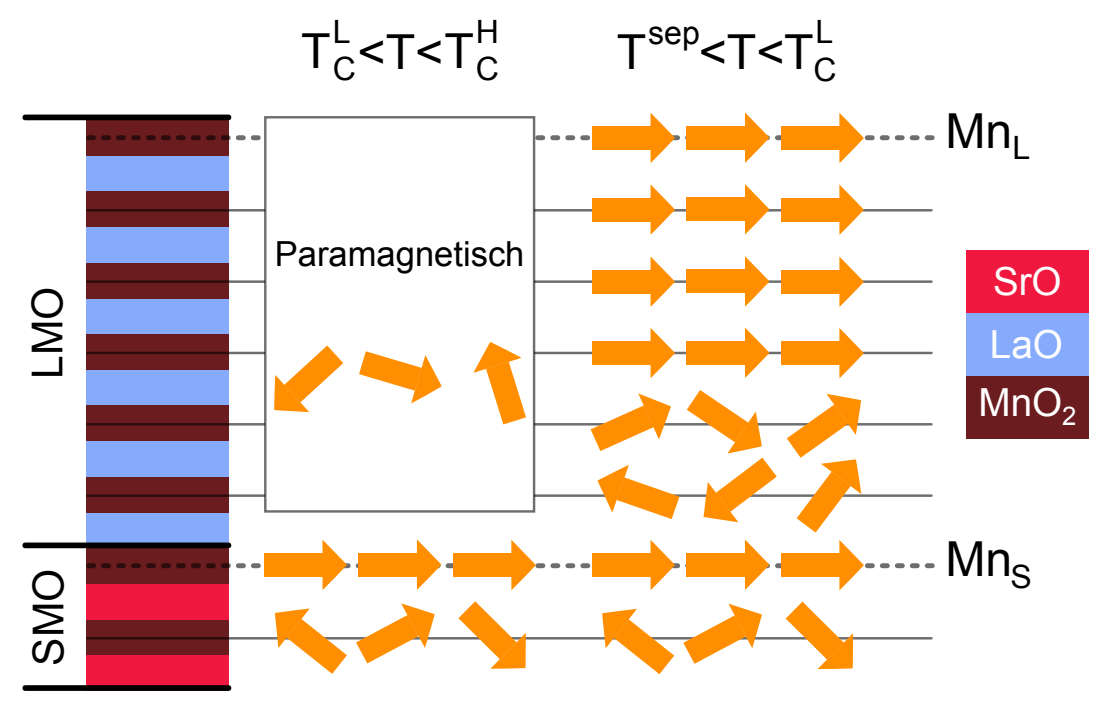

\begin{abstract}
Abbildung 9.38.: Modellvorstellung des magnetischen Zustandes in einem LMO/SMO $(n=3)$-SL auf STO (vergleiche Abbildung 9.36). $T_{C}^{L}<T<T_{C}^{H}$ : Es liegt eine ferromagnetische Ordnung der Spins auf der mit $\mathrm{Mn}_{\mathrm{S}}$ markierten $\mathrm{MnO}_{2}$-Lage vor, in den restlichen $\mathrm{MnO}_{2}$-Lagen zeigen die Spins keine kollektive Ordnung. $T^{\text {sep }}<T<T_{C}^{L}$ : (Für $T<T^{s e p}$ kann man in der $M(H)$-Kurve nicht mehr zwischen der HTP und LTP unterscheiden.) Neben den Spins auf der mit $\mathrm{Mn}_{\mathrm{S}}$ markierten Lage entsteht auch eine ferromagnetische Ordnung in den Lagen um die $\mathrm{MnO}_{2}$-Lage $\mathrm{Mn}_{\mathrm{L}}$. Die ferromagnetische Ordnung erstreckt sich dabei nicht über alle $\mathrm{MnO}_{2}$-Lagen im LMO, sondern wird durch Bereiche ohne kollektive Ordnung unterbrochen, so dass es keine effektive Austauschkopplung zwischen den beiden ferromagnetischen Bereichen gibt.
\end{abstract}

den Doppelaustausch innerhalb der $\mathrm{MnO}_{2}$-Ebenen. Bei einer zufälligen Platzierung der Kationen auf den A-Plätzen wie bei $\mathrm{Mn}_{\mathrm{L}}$ wird die Itineranz der Elektronen durch Variationen des lokalen Coulomb-Potentials und der Bindungswinkel gestört. Entsprechend dieses Prinzips erreichten May et al. eine Steigerung von $T_{N}$ um $\sim 70 \mathrm{~K}$ bei einer lagenweisen Anordnung der La- und Sr-Ionen in $\mathrm{La}_{1 / 3} \mathrm{Sr}_{2 / 3} \mathrm{MnO}_{3}[15$.

In der $\mathrm{Mn}_{\mathrm{S}}$-Lage findet der Austausch vorwiegend über die $d_{x^{2}-y^{2}}$-Orbitale in der Ebene statt. Sadoc et al. argumentieren, dass eine Konzentration der $e_{g}$-Elektronen in der Ebene eines dünnen Filmes zu einer Verstärkung der resultierenden FM Austauschwechselwirkung führt [279]. Bei der zweidimensionalen Konfiguration eines Dünnfilmes ist der Austausch in der Ebene ausschlaggebend für die Curie-Temperatur. Die Kopplungen in c-Richtung haben aufgrund der kleinen Zahl von magnetischen Momenten in dieser Richtung einen vernachlässigbaren Einfluss auf die Ordnungstemperatur [279]. Somit ordnen die Spins in der $\mathrm{Mn}_{\mathrm{S}}$-Lage bei einer größeren Temperatur ferromagnetisch als die Spins auf der $\mathrm{Mn}_{\mathrm{L}}$-Lage, wo die $e_{g}$-Elektronen weniger stark in der Ebene gebunden sind. Die HTP kann man mit diesen Überlegungen in der $\mathrm{Mn}_{\mathrm{S}}$-Lage lokalisieren (siehe Abbildung 9.38). Auch in der $\mathrm{Mn}_{\mathrm{L}}$-Lage ergibt sich eine FM Ordnung, deren Curie-Temperatur $T_{C}^{L}$ aufgrund der obigen Überlegungen deutlich kleiner ist als für $\mathrm{Mn}_{\mathrm{S}}$. Die Verteilung der Sr-Ionen und die Ankopplung an die $\mathrm{Mn}_{\mathrm{L}}$-Lage führt da$\mathrm{zu}$, dass auch die $\mathrm{MnO}_{2}$-Lagen innerhalb der LMO-Schicht $\left(i_{M L}<8\right.$, Abbildung 9.36) eine FM Ordnung ausbilden, wenn die Temperatur weiter reduziert wird (Abbildung 9.38). Diese Lagen bei $\mathrm{Mn}_{\mathrm{L}}$ ordnet man der LTP zu. 
In der hier betrachteten Struktur mit $n=3$ sind HTP und LTP für $T>T^{\text {sep }}$ magnetisch entkoppelt, man kann getrennte Signaturen in den $M(H)$-Kurven ausmachen. Es gibt also keine durchgängige FM Ausrichtung der Spins zwischen $\mathrm{Mn}_{\mathrm{S}}$ und $\mathrm{Mn}_{\mathrm{L}}$ (siehe Abbildung 9.38). Für $i_{M L}=3-4$ ist der Effekt der Ankopplung an $\mathrm{Mn}_{\mathrm{L}}$ zu schwach, um eine ferromagnetische Ordnung zu induzieren. Die Kopplung zur $\mathrm{Mn}_{\mathrm{S}}$-Lage ist nach der Diskussion weiter oben zu schwach, um die FM Ordnung in der benachbarten $\mathrm{MnO}_{2}$-Lage zu verursachen. Wenn man LMO/SMO-SL mit größerem $n$ betrachtet, verbreitert sich der Bereich in der Mitte der LMO-Schicht, wo man keine FM Ordnung mehr feststellen kann. Überdies besteht dieser Bereich auch bei tiefen Temperaturen, so dass man die magnetische Entkopplung von HTP und LTP auch bei $T=5 \mathrm{~K}$ feststellen kann. Die unvollständige magnetische Ordnung der Spins in den LMO-Lagen erkennt man auch in Abbildung 9.35. $N_{M L}^{L M O}$ steigt nur wenig mit $n$ an, so dass die Breite des Bereichs ohne FM Ordnung mit $n$ ansteigt. Das Moment der LMO-Lagen in den SL liegt deutlich unter dem Wert, der sich im Vergleich mit dem einfachen LMO-Film ergibt (vergleiche Abbildung 9.29). Eine mögliche Ursache liegt in einer anderen Defektkonzentration (La-Leerstellen) der LMO-Lagen in den SL. Darauf weist auch der Unterschied der Gitterparameter für den einfachen Film und den LMO-Lagen im SL hin. Die andere Konzentration von Defekten verschiebt den magnetischen Grundzustand der LMO-Lagen in den SL in Richtung einer AFM Ausrichtung der Spins.

Die asymmetrische Verteilung der $\mathrm{La}^{3+}$ - und $\mathrm{Sr}^{2+}$-Ionen um die $\mathrm{Mn}_{\mathrm{S}}$-Lage modifizieren das Kristallfeld der Mn-Ionen in dieser Lage. Durch diese reduzierte Symmetrie des Kristallfeldes wird das Bahnmoment der 3d-Elektronen nicht mehr komplett gequencht wie in einer oktaedrischen Umgebung. Dieses Bahnmoment kann über die Spin-Bahn-Kopplung die im Experiment beobachtete starke Anisotropie der HTP bewirken. Damit sich ein Zusammenhang mit den Stufen des Substrates ergibt, muss man annehmen, dass sich die Struktur der $\mathrm{Mn}_{\mathrm{S}}$-Lage im Einklang zur Ausrichtung der Stufen ändert.

\subsection{Zusammenfassung}

In diesem Kapitel wurden Übergitter (SL) aus $\mathrm{LaMnO}_{3}$ (LMO) und $\mathrm{SrMnO}_{3}$ (SMO) auf STO (001)- und STO (111)-Substraten behandelt. Die Schichtdicke der LMO-Lagen ist jeweils dreimal so groß wie die Dicke der SMO-Lagen und man variiert die Gesamtdicke der LMO/SMODoppellagen in einem Bereich von $\Lambda \approx 1 \mathrm{~nm}$ bis $\Lambda \approx 7 \mathrm{~nm}$. Mit den Messungen der in-situ Ellipsometrie kann man bereits beim Wachstum Hinweise auf die strukturellen und elektrischen Eigenschaften der SL gewinnen. Beim Wachstum auf den (111)-Substraten beobachtet man ein Aufrauen der wachsenden SMO-Lagen, woraus eine Durchmischung der La- und Sr-Ionen resultiert. Diese kann man auch in der anschließenden Charakterisierung mit dem TEM feststellen. Im Gegensatz dazu ist das Wachstum auf den (001)-Substraten ungestört und man kann bei der Inspektion der chemischen Qualität der Grenzflächen nur eine leichte Durchmischung der Kationen registrieren. Interessanterweise sieht man eine asymmetrische Rauigkeit. Wenn man LMO auf SMO wächst (SMO/LMO-Grenzfläche), kann man mit dem TEM keine La/Sr-Interdiffusion erkennen. Wächst dagegen SMO auf LMO (LMO/SMO), so sieht man einen Austausch der Kationen auf den A-Plätzen. Die gemessenen Daten der insitu Ellipsometrie für das Wachstum der LMO/SMO-SL auf STO (001) kann man mit einem Modell anpassen, das die Delokalisierung der $e_{g}$-Elektronen über die Grenzflächen berücksichtigt. Die charakteristische Länge für die Unschärfe der Ladungsverteilung $L_{T F}$ verändert sich im Verlauf der Deposition einer LMO/SMO-Bilage im Bereich $L_{T F}=0.4-1.4 \mathrm{~nm}$. Die starke Delokalisierung der $e_{g}$-Elektronen $\left(L_{T F}=1.4 \mathrm{~nm}\right)$ beim Aufbringen der ersten SMO- 
Lage ist eine mögliche treibende Kraft für die Unschärfe der LMO/SMO-Grenzflächen. Nach Abschluss der Deposition einer vollständigen SMO/LMO-Bilage ergibt sich $L_{T F} \approx 0.4 \mathrm{~nm}$, was mit theoretischen Rechnungen für diese Art von Heterostrukturen übereinstimmt.

Die SL auf STO (111) besitzen für $\Lambda \approx 1-3 \mathrm{~nm}$ einen homogenen FM Grundzustand, bei dem alle Spins auf den Mn-Ionen ferromagnetisch geordnet sind. Für die Strukturen auf STO (001) ist dies nur für $\Lambda \approx 1,2 \mathrm{~nm}$ der Fall. Wird die Dicke der LMO/SMO-Bilagen weiter erhöht $(\Lambda \gtrsim 3 \mathrm{~nm})$, sieht man einen deutlichen Abfall der Sättigungsmagnetisierung. Dies bedeutet, dass mindestens eine $\mathrm{MnO}_{2}$-Lage pro LMO/SMO-Doppellage nicht an der kollektiven FM Ordnung teilnimmt. Überdies sieht man für diese Übergitter zwei Curie-Temperaturen und zwei ferromagnetische Beiträge in den $M(H)$-Kurven: die Hochtemperaturphase (HTP) mit $T_{C}^{H} \sim 345 \mathrm{~K} \pm 10 \mathrm{~K}$ und die Tieftemperaturphase (LTP), deren Curie-Temperatur mit steigendem $\Lambda$ kontinuierlich abfällt, von $T_{C}^{L} \approx 300 \mathrm{~K}$ für $\Lambda \approx 3 \mathrm{~nm}$ auf $T_{C}^{L} \approx 180 \mathrm{~K}$ für $\Lambda \approx 7 \mathrm{~nm}$. Die HTP und LTP sind magnetisch entkoppelt, so dass man getrennte Koerzitivfelder in $M(H)$ ausmachen kann. Das magnetische Moment der HTP skaliert mit der Zahl der Wiederholungen von den LMO/SMO-Einheiten und ist umgekehrt proportional zu $\Lambda$. Außerdem entspricht der Absolutwert des magnetischen Momentes der HTP ungefähr dem Moment einer $\mathrm{MnO}_{2}$-Lage pro LMO/SMO-Doppellage. Dies deutet darauf hin, dass die HTP ein Grenzflächenphänomen ist. Im Zusammenhang mit dem Fehlschnitt der Substrate zeigt die HTP eine starke uniaxiale Anisotropie, deren leichte Richtung parallel zu den Kanten der Substratstufen verläuft. Für einen Fehlschnitt $\alpha_{c u t}=0.2^{\circ}$ kann man eine Anisotropieenergiedichte von $K_{H T P} \approx 2 \cdot 10^{5} \frac{\mathrm{J}}{\mathrm{m}^{3}}$ abschätzen, welche die typischen Literaturwerte für die Anisotropie dünner Manganatfilme um zwei Größenordnungen übersteigt. Beim Wachstum von LMO/SMO-SL auf LSAT (001), das mit $a_{L S A T}=0.3869 \mathrm{~nm}$ eine kleinere Gitterkonstante besitzt als STO $\left(a_{S T O}=0.3905 \mathrm{~nm}\right)$, verschwindet die HTP. Die HTP erscheint nicht bei anderen Studien von LMO/SMO-SL in der Literatur. Bei der MAD ist der Sauerstoffpartialdruck wesentlich höher als bei den typischen Vakuum-Methoden wie MBE und PLD. Daraus ergeben sich andere Defekte in den Heterostrukturen, wie Sauerstoff- bzw. La-Leerstellen, die zu einer Veränderung des magnetischen Verhaltens führen. Zur Erklärung der magnetischen Phänomenologie der HTP wird ein Modell vorgeschlagen, bei dem die HTP auf jeweils einer $\mathrm{MnO}_{2}$-Lage an den chemisch scharfen SMO/LMO-Grenzflächen lokalisiert ist und die LTP sich von den unscharfen LMO/SMO-Grenzflächen ins LMO ausdehnt. Die starken epitaktischen Verspannungen beim Wachstum auf STO und die Ordnung der Sr- und La-Ionen zwingen die $e_{g}$-Elektronen der HTP in die $d_{x^{2}-y^{2}}$ Orbitale, so dass der Austausch mit den benachbarten $\mathrm{MnO}_{2}$-Lagen sehr schwach ist. In der Mitte der LMO-Schichten ist die FM Ordnung aufgrund des kleinen Anteils von $\mathrm{Mn}^{4+}$-Ionen unterdrückt, so dass die HTP und LTP magnetisch entkoppelt sind. 

$\Gamma_{\text {a ex }} 10$

\section{Zusammenfassung und Ausblick}

Die Grenzflächen komplexer Oxide sind ein faszinierendes Forschungsgebiet mit großem technologischen Potential. Da sich die elektronischen Grenzflächeneffekte auf sehr kleinen Längenskalen im Bereich weniger Monolagen abspielen können, ergeben sich in diesem Feld hohe Anforderungen an die Depositionsmethoden. Diese müssen es ermöglichen, die Chemie der Grenzflächen auf atomarer Skala zu definieren. Die in dieser Arbeit behandelten PerowskitOxide weisen eine komplexe Defektchemie auf, die stark von den Depositionsbedingungen beeinflusst wird. Insbesondere der vorherrschende Sauerstoffpartialdruck $p_{\mathrm{O}_{2}}$ hat einen wesentlichen Einfluss auf die chemische Zusammensetzung und damit die funktionalen Eigenschaften dieser Materialien. Damit steht die metallorganische Aerosol-Deposition (MAD), die bei atmosphärischen Bedingungen arbeitet $\left(p_{\mathrm{O}_{2}} \approx 0.21 \mathrm{bar}\right)$, im Kontrast zu den etablierten Vakuum-Methoden wie gepulster Laserdeposition (PLD) und Molekularstrahlepitaxie (MBE).

Diese Arbeit lässt sich grob in zwei Blöcke unterteilen. Zum einen wurde eine neue MADAnlage mit Wachstumskontrolle durch in-situ Ellipsometrie aufgebaut. Mit dieser kann man vollautomatisch oxidische Heterostrukturen mit hoher Präzision und Reproduzierbarkeit abscheiden. Andererseits wurden die magnetischen Wechselwirkungen in Heterostrukturen aus verschiedenen Manganaten des $\mathrm{La}_{1-\mathrm{x}} \mathrm{Sr}_{\mathrm{x}} \mathrm{MnO}_{3}$-Phasendiagrammes untersucht.

Die in-situ Ellipsometrie erleichtert die Auflösung der Vorgänge, die bei der Deposition von Heterostrukturen ablaufen. Schon für einfache $\mathrm{La}_{1-\mathrm{x}} \mathrm{Sr}_{\mathrm{x}} \mathrm{MnO}_{3}(\mathrm{LSMO}(\mathrm{x}))$-Schichten auf $\mathrm{SrTiO}_{3}$ (STO)-Substraten kann man mit Hilfe der in-situ Ellipsometrie interessante Erkenntnisse zum Wachstum gewinnen. Aus den sichtbaren Monolagenoszillationen kann man auf ein Wachstum in zweidimensionalen Inseln schließen. In den ersten 3 aufwachsenden Monolagen des Manganatfilmes kommt es zu einer Reaktion mit dem STO-Substrat. Für $x \geq 0.2$ gibt es anschließend keine weiteren Diffusionsvörgänge, die Festkörperreaktion ist abgeschlossen. Diese selbstlimitierende Reaktion wird durch den Unterschied der Kationen auf den B-Plätzen getrieben. Bei kleinen Sr-Dotierungen, $x<0.2$, treiben polare Effekte eine weitere Interdiffusion über die gesamte Depositionsdauer. Beim Wachstum von $\mathrm{LaMnO}_{3}(\mathrm{LMO}) / \mathrm{SrMnO}_{3}$ (SMO)-Übergittern (SL) auf STO (111)-Substraten konnte man mit der in-situ Ellipsometrie ein Aufrauen der SMO-Schichten und die anschließende Durchmischung mit LMO identifizieren. 
Unter Anwendung der Ellipsometrie konnte man nicht nur einfache Modifikationen der Chemie oder Morphologie erfassen, sondern auch elektronische Eigenschaften wie die Valenzen der Übergangsmetallionen. Damit liefert die Ellipsometrie komplementäre Informationen zu RHEED-Messungen, die nur die Gesamtelektronendichte an der Oberfläche erfassen können. Bei den Depositionsbedingungen der $\operatorname{MAD}\left(p_{\mathrm{O}_{2}} \approx 0.21 \mathrm{bar}, T_{D e p} \approx 930^{\circ} \mathrm{C}\right)$ ist der Brechungsindex von $\operatorname{LSMO}(\mathrm{x}), x=0-1$, durch die mittlere Mn-Valenz bestimmt. Man kann so während des Wachstums von Manganat-Strukturen die Umverteilung der $e_{g}$-Elektronen auf den Mn-Ionen nachvollziehen. Dazu passt man die gemessenen Verläufe der in-situ Ellipsometrie unter Verwendung der zuvor ermittelten Brechungsindizes von LSMO(x) numerisch an.

Dieses Prinzip wurde hier an verschiedenen Systemen vorgeführt. In SMO/LSMO(0.3)-Bilagen auf STO (001) rekonstruiert man einen Elektronentransfer innerhalb der LSMO(0.3)-Lagen von der SMO/LSMO(0.3)-Grenzfläche zur Filmoberfläche. Dieser Transfer führt zur Vermeidung der polaren Katastrophe. Bei der Atomlagenepitaxie von (LMO) $)_{2} /(\mathrm{SMO})_{1}$-SL auf STO (001) kann man die Umverteilung der Elektronen nach der Deposition jeder einzelnen $\mathrm{LaO}$ - und $\mathrm{MnO}_{2}$-Lage erfassen. Daraus kann man auf ein lagenweises Wachstum der halben Perowskitlagen schließen. Speziell die formal geladenen $(\mathrm{LaO})^{+}$-Lagen können durch eine Valenzänderung der bereits abgeschiedenen $\mathrm{MnO}_{2}$-Lagen aufwachsen. Es kommt nicht zur Bildung von elektrisch neutralen $\mathrm{La}_{2} \mathrm{O}_{3}$-Inseln.

In LMO/SMO-SL delokalisieren die $e_{g}$-Elektronen an den LMO/SMO-Grenzflächen über eine Länge von $L_{T F}$. Diese charakteristische Länge kann man durch die Anpassung der in-situ Ellipsometrie ermitteln und beobachtet, dass sich $L_{T F}$ im Verlauf der Deposition der SMOSchichten ändert. Von $L_{T F} \approx 1.4 \mathrm{~nm}$ für die erste SMO-Monolage sinkt $L_{T F}$ auf $0.7 \mathrm{~nm}$ ab bei der Abscheidung der zweiten. Wenn auf die zwei Monolagen SMO LMO abgeschieden wird, sinkt $L_{T F}$ auf den während der Deposition der LMO-Schicht konstanten Wert $L_{T F} \approx$ $0.4 \mathrm{~nm}$ ab. Diese Variation wird darauf zurückgeführt, dass die Elektronen bevorzugt die MnIonen an der Oberfläche besetzen. Der anfänglich große Wert von $L_{T F}$ treibt die in TEM EELS-Aufnahmen erkennbare Interdiffusion der Sr- und La-Ionen, so dass die SMO/LMOGrenzfläche (LMO folgt auf SMO) chemisch schärfer ist als die LMO/SMO-Grenze (SMO folgt auf LMO).

In dieser Arbeit wurde das Prinzip der MAD Atomlagenepitaxie (ALE) eingeführt. Im Gegensatz zum konventionellen MAD-Prozess werden dabei keine kompletten Perowskitlagen $\mathrm{ABO}_{3}$ aufgebracht, sondern $\mathrm{AO}-$ und $\mathrm{BO}_{2}$-Lagen, aus denen das Perowskit entlang der [001]-Richtung aufgebaut ist. Die MAD ALE wurde am Beispiel der gestapelten SrO-STO Ruddlesden-Popper-Strukturen und Übergittern aus (LMO) $)_{2} /(\mathrm{SMO})_{1}$ erprobt. Es wurden $\mathrm{SrO}\left(\mathrm{SrTiO}_{3}\right)_{\mathrm{n}}$-Strukturen mit $n=2-4$ abgeschieden. Stapelfehler in der SrO-STO-Abfolge sind vorwiegend in der Nähe zum STO-Substrat lokalisiert. Diese Fehler führt man auf Interdiffusionsvorgänge mit dem STO (001)-Substrat zurück. Diese Vorgänge kommen nach einer deponierten Materialstärke von $\sim 10 \mathrm{~nm}$ zum Erliegen. Auch in der Ellipsometrie kann man diesen Übergangsbereich ausmachen. Überdies dient die Ellipsometrie als Instrument zur Einstellung der korrekten Stöchiometrie der Sr- und Ti-Präkursoren mit einer Genauigkeit von $\sim 1.5 \%$. Beim SrO-STO-System liegen elektrisch neutrale $\mathrm{SrO}$ - und $\mathrm{TiO}_{2}$-Lagen vor. In den Manganaten gibt es LaO- und $\mathrm{MnO}_{2}$-Lagen mit nichtverschwindenden formalen Ladungen. Dadurch kann es während des Wachstums zum Aufbau von Coulomb-Potentialen kommen, die ein lagenweises Wachstum verhindern. Im Experiment kann man Abweichungen von einer zweidimensionalen Wachstumsmode an einem relaxierenden Verhalten der in-situ Ellipsometrie während der Depositionspausen erkennen. Das Perowskit bildet sich in diesem Fall durch 
eine Festkörperreaktion aus SrO-, LaO-, und $\mathrm{Mn}_{3} \mathrm{O}_{4}$-Blöcken. Hier wurde demonstriert, dass sich eine zweidimensionale Wachstumsmode durch einen Mn-Überschuss in der ersten $\mathrm{MnO}_{2}$ Lage herbeiführen lässt. Die zusätzlichen Mn-Ionen verhindern durch ihre variable Valenz den Aufbau eines elektrostatischen Potentials.

Es wurde systematisch die magnetische Kopplung zwischen ferromagnetischen (FM) und antiferromagnetischen (AFM) Manganat-Schichten anhand von $\operatorname{LSMO}(0.3) / \mathrm{LSMO}(\mathrm{x})$-Bilagen, $x=0.6-1.0$, untersucht. Für $x>0.6$ zeigt die FM LSMO(0.3)-Schicht ein erhöhtes Koerzitivfeld und eine Verschiebung der Hystereseschleife entlang der Feldachse (exchange bias (EB)). Als Funktion der Sr-Dotierung zeigen die durch das STO (001)-Substrat verspannten LSMO (x)-Filme einen Übergang vom Antiferromagneten des G-Typs (G-AFM) $(x>0.95)$ zum Antiferromagneten des A-Typs (A-AFM) $(x \leq 0.95)$. Bei diesem Übergang zum AAFM ergibt sich eine Halbierung der EB-Amplitude im Vergleich zum G-AFM. Für das LSMO(0.3)/SMO-System wurde zudem die Abhängigkeit des magnetischen Verhaltens von den Schichtdicken der beiden Materialien studiert. Man sieht schon für sehr dünne SMOFilme mit einer Dicke von 4 Monolagen einen deutlichen Einfluss auf die Feldumkehr der LSMO(0.3)-Schicht. Diese dünne SMO-Schicht zeigt also bereits eine AFM Ordnung. Mit zunehmender Schichtdicke des SMO-Filmes ergibt sich eine Entspannung der epitaktischen Verzerrungen und gleichzeitig eine Abnahme der EB-Amplitude. Man erklärt die beobachtete Phänomenologie des EB mit einem Spin-Glas an der FM/AFM-Grenzfläche, welches aus den frustrierten magnetischen Kopplungen resultiert. Bei Änderungen der tetragonalen Verzerrung der Manganat-Filme kommt es zu einer Modifikation der Balance zwischen den magnetischen Kopplungen in der Filmebene und senkrecht dazu. Dadurch verändern sich die Eigenheiten des Spin-Glases an der Grenzfläche und damit das magnetische Verhalten der Heterostruktur.

Die LMO/SMO-SL auf STO (001)-Substraten zeigen eine zuvor noch nicht beobachtete Phänomenologie. Hier wurden LMO/SMO-SL mit einer Bilagen-Periode $\Lambda \approx 1-7 \mathrm{~nm}$ studiert. Die Dicke der LMO-Lagen war dabei dreimal so groß wie die Dicke der SMO-Lagen. Für $\Lambda \approx 1-2 \mathrm{~nm}$ beobachtet man einen homogenen FM Zustand. Wenn man die Dicke der Lagen weiter auf $\Lambda \gtrsim 3 \mathrm{~nm}$ vergrößert, nimmt mindestens eine $\mathrm{MnO}_{2}$-Lage pro LMO/SMO-Doppellage nicht an der kollektiven FM Ordnung teil. Des Weiteren fallen zwei Curie-Temperaturen und die Existenz zweier Koerzitivfelder auf. Die Tieftemperaturphase (LTP) hat eine Curie-Temperatur $T_{C}^{L} \approx 180-300 \mathrm{~K}$, die kontinuierlich mit $\Lambda$ abfällt, die Hochtemperaturphase (HTP) hat eine Curie-Temperatur $T_{C}^{H}=345 \mathrm{~K} \pm 10 \mathrm{~K}$, die keine systematische Abhängigkeit von $\Lambda$ besitzt. Die getrennt sichtbaren Koerzitivfelder deuten auf eine magnetische Entkopplung dieser beiden Phasen hin. Der Absolutwert des magnetischen Momentes der HTP entspricht etwa dem Moment einer $\mathrm{MnO}_{2}$-Lage pro LMO/SMO-Doppellage. Außerdem skaliert das Moment mit der Zahl der LMO/SMO-Einheiten und ist umgekehrt proportional zu $\Lambda$. Dies ist ein Hinweis auf den Grenzflächencharakter der HTP. Im Zusammenhang mit dem Fehlschnitt der Substrate zeigt die HTP eine starke uniaxiale Anisotropie, deren leichte Richtung parallel zu den Kanten der Substratstufen verläuft. Es wird eine ungewöhnlich große Anisotropieenergiedichte $K_{H T P} \approx 2 \cdot 10^{5} \frac{\mathrm{J}}{\mathrm{m}^{3}}$ registriert, welche die typischen Literaturwerte für dünne Manganatfilme um zwei Größenordnungen übersteigt. Beim Wachstum auf $\left(\mathrm{La}_{0.3} \mathrm{Sr}_{0.7}\right)\left(\mathrm{Al}_{0.65} \mathrm{Ta}_{0.35}\right) \mathrm{O}_{3}$ (LSAT), das eine kleinere Gitterkonstante als STO besitzt, verschwindet die HTP. Andere Gruppen berichten nicht von einer HTP in LMO/SMOSL. Die Ursache dafür ist in den abweichenden Depositionsbedingungen zu suchen. Bei der MAD ist der Sauerstoffpartialdruck größer als bei den typischen Vakuum-Methoden wie MBE und PLD. Daraus ergeben sich andere Defekte in den Heterostrukturen wie Sauerstoff- bzw. La-Leerstellen, die das magnetische Verhalten beeinflussen. Zur Erklärung der HTP stellt man 
ein Modell vor, bei dem die HTP an der chemisch scharfen SMO/LMO-Grenzfläche lokalisiert ist und die LTP sich von der chemisch unschärferen LMO/SMO-Grenzfläche ins LMO ausdehnt. Das STO-Substrat führt zu epitaktischen Zugspannungen, die die $e_{g}$-Elektronen in den an den SMO/LMO-Grenzflächen befindlichen $\mathrm{MnO}_{2}$-Lagen auf die $d_{x^{2}-y^{2}}$-Orbitale zwingen. Dadurch ist der Austausch zu den in c-Richtung benachbarten $\mathrm{MnO}_{2}$-Lagen sehr schwach und der magnetische Austausch findet vorwiegend in der Ebene statt. Dieser zweidimensionale Austausch wird durch die Ordnung der Sr- und La-Ionen unterstützt. In der Mitte der LMO-Schichten ist die FM-Ordnung aufgrund des kleinen Anteils von $\mathrm{Mn}^{4+}$-Ionen unterdrückt, so dass es zur Entkopplung der HTP und LTP kommt.

Es finden bereits anknüpfende Untersuchungen statt, bei denen die hier etablierten Techniken und Erkenntnisse angewendet werden: Von Alexander Belenchuk und Vitaly BruchmannBamberg wird der Einfluss von zusätzlich eingeschobenen SrO-Lagen, die einen RuddlesdenPopper-Defekt bilden (vergleiche Abschnitt 7.2), auf die magnetischen Eigenschaften von $\mathrm{Pr}_{0.7} \mathrm{Ca}_{0.3} \mathrm{MnO}_{3}(\mathrm{PCMO}) / \mathrm{SrTiO}_{3}$ (STO)-Übergittern (SL) untersucht. Sven Esser und Marius Keunecke greifen die Ergebnisse von den LMO/SMO-SL auf und nehmen erweiterte Experimente vor, bei denen das Doppelperowskit $\mathrm{La}_{2} \mathrm{CoMnO}_{6}$ (LMCO) in die LMO/SMOHeterostrukturen eingebracht wird. Durch die Kationen-Ordnung der $\mathrm{Co}^{2+}$ - und $\mathrm{Mn}^{4+}$ Ionen im LMCO kommt es an der LMCO/SMO-Grenzfläche nicht zu einem Transfer der $e_{g}$-Elektronen wie an der LMO/SMO-Grenzfläche. Dadurch wird die Hochtemperaturphase in LMCO/SMO-SL vollständig unterdrückt [280].

Abgesehen von diesen bereits angestoßenen Vorhaben bieten sich weitere technische Verbesserungen und Erweiterungen der MAD mit in-situ Ellipsometrie an. Zunächst ist es sinnvoll, die in-situ Ellipsometrie nicht nur bei einer Wellenlänge zu betreiben, sondern so zu erweitern, dass die ellipsometrischen Messgrößen über einen breiten Spektralbereich erfasst werden können. Damit könnte man eine höhere Genauigkeit bei der Beobachtung von Änderungen der Mn-Valenzen erreichen, indem man Photonenenergien auswählt, für die sich die optischen Eigenschaften der Manganate mit der Mn-Valenz stark ändern. Eine derartige Erweiterung ist auch interessant für die sauerstoffarme MAD [135, 238, wo man die vom Sauerstoffpartialdruck abhängige Valenz der Übergangsmetallionen, z.B. in $\mathrm{Fe}_{3} \mathrm{O}_{4}$ oder $\mathrm{Sr}_{2} \mathrm{CrMoO}_{6}$ überwachen könnte. Mit der in-situ Ellipsometrie ermittelte man die charakteristische Länge $L_{T F}$, über die Elektronen an Grenzflächen delokalisieren. Dies wurde hier bisher nur an Heterostrukturen aus Manganaten vorgeführt. Zukünftig könnte man diese Längenskala auch in Übergittern mit anderen 3d-Ionen wie Co ermitteln. An Grenzflächen zwischen Perowskiten mit verschiedenen Ionen auf den B-Plätzen kann es zu Redox-Reaktionen kommen, z.B. $\mathrm{Co}^{3+}+\mathrm{Mn}^{3+} \rightarrow \mathrm{Co}^{2+}+\mathrm{Mn}^{4+}$ für $\mathrm{LaMnO}_{3}$ und $\mathrm{LaCoO}_{3}$. Die Längenskala solcher Reaktionen konnte bisher nur ex-situ mit Röntgenabsorptionsspektroskopie (XAS) erfasst werden [76. Mit der MAD mit in-situ Ellipsometrie könnte man diese Reaktionen schon beim Wachstum beobachten.

Es bieten sich weitere Freiheitsgrade an, um das Spin-Glas an der Grenzfläche zwischen FM und AFM Manganaten zu manipulieren. Hier wurde die Dotierung der AFM Lage zwischen $x=0.6$ und $x=1.0$ variiert. Zusätzlich könnte man bei fester Dotierung der AFM Lage, z.B. $x=1.0$, die Sr-Dotierung des Ferromagneten in einem Bereich $x \approx 0.2-0.5$ ändern. Des Weiteren kann man die orbitalem Freiheitsgrade direkt durch das Wachstum auf einem anderen Substrat beeinflussen. Bei den LMO/SMO-SL hat sich bereits gezeigt, dass das Wachstum auf LSAT zu einer drastischen Modifikation des magnetischen Verhaltens führt, da die magnetischen Austauschwechselwirkungen in c-Richtung intensiviert werden. Der scharfe Übergang vom G-AFM zum A-AFM für $x \leq 0.95$ führt zu einer drastischen 
Abnahme des EB. Es ist vorstellbar, dass man eine solch kleine Dotierungsänderung auch durch ein elektrisches Feld erreichen kann, wenn man die AFM La ${ }_{1-x} \mathrm{Sr}_{\mathrm{x}} \mathrm{MnO}_{3}$-Lage auf einem Ferroelektrikum wächst. Damit könnte man durch ein elektrisches Feld die Art der AFM Ordnung einstellen. Über das messbare EB einer auf der $\mathrm{La}_{1-\mathrm{x}} \mathrm{Sr}_{\mathrm{x}} \mathrm{MnO}_{3}$-Lage deponierten $\mathrm{La}_{0.7} \mathrm{Sr}_{0.3} \mathrm{MnO}_{3}$-Schicht kann man diese Änderungen dann registrieren. Als Resultat erhält man dann eine Kopplung zwischen dem elektrischen Feld und der Magnetisierung.

In den LMO/SMO-SL beobachtet man die interessante Phänomenologie der Koexistenz von LTP und HTP. Zukünftig kann man die Eigenschaften der HTP weiter charakterisieren. Insbesondere die ungewöhnlich hohe Anisotropieenergie sollte genauer untersucht werden, indem man die LMO/SMO-SL auf einer Serie von STO (001)-Substraten mit variablem Fehlschnitt aufwächst. Man kann weitere LMO/SMO-SL abscheiden, in denen man die La/SrStöchiometrie verändert oder andere Kationen auf den A-Plätzen einführt. Mit diesen zusätzlichen Experimenten wäre es möglich, die Aussagen des hier vorgestellten Modells weiter zu überprüfen. Eine leichte Sr-Dotierung, $x \approx 0.1$, der LMO-Lagen müsste beispielsweise dazu führen, dass die LTP und HTP nicht mehr getrennt wahrnehmbar sind, da in der Mitte der $\operatorname{LSMO}(x \approx 0.1)$-Schichten dann auch eine FM Ordnung vorherrschen würde. Außerdem könnte man eine künstliche Durchmischung der Sr- und La-Ionen an beiden Grenzflächen einstellen. Damit sollte man ebenfalls nur noch eine FM Phase erkennen können, da die an der scharfen Grenzfläche vermutete HTP dann verschwindet. Wenn man dem SMO die kleineren $\mathrm{Ca}^{2+}$-Ionen zufügt, kann man an der scharfen SMO/LMO-Grenzfläche eine Unordnung der Mn-O-Mn-Bindungswinkel induzieren, woraus eine Reduktion von $T_{C}^{H}$ folgen würde.

Die Grenzflächen von komplexen Oxiden bieten also auch in Zukunft ein aktives Forschungsgebiet mit einer Vielzahl an grundlegenden Fragestellungen. Mit der in dieser Arbeit aufgebauten MAD-Anlage steht ein ideales Instrument bereit, um diese anzugehen. 

Teil IV.

Anhang 



\section{Abkürzungsverzeichnis}

\begin{tabular}{|c|c|}
\hline $\mathrm{ADF}$ & Annular Dark Field \\
\hline AFM & Antiferromagnet/antiferromagnetisch \\
\hline ALE & Atomlagenepitaxie \\
\hline BTO & $\mathrm{BaTiO}_{3}$ \\
\hline DMF & Dimethylformamid \\
\hline EA & Leichte Achse \\
\hline EB & Exchange Bias \\
\hline EELS & Electron Energy Loss Spectroscopy \\
\hline EMA & Effective Medium Approximation \\
\hline FC & Field Cooled \\
\hline $\mathbf{F M}$ & Ferromagnet/ferromagnetisch \\
\hline FMM & Ferromagnetisch metallisch \\
\hline HA & Schwere Achse \\
\hline HAADF & High Angle Annular Dark Field \\
\hline НTP & Hochtemperaturphase \\
\hline LAO & $\mathrm{LaAlO}_{3}$ \\
\hline LCMO & $\mathrm{La}_{2 / 3} \mathrm{Ca}_{1 / 3} \mathrm{MnO}_{3}$ \\
\hline LMO & $\mathrm{LaMnO}_{3}$ \\
\hline LSAT & $\left(\mathrm{La}_{0.3} \mathrm{Sr}_{0.7}\right)\left(\mathrm{Al}_{0.65} \mathrm{Ta}_{0.35}\right) \mathrm{O}_{3}$ \\
\hline LSMO & $\mathrm{La}_{0.7} \mathrm{Sr}_{0.3} \mathrm{MnO}_{3}$ \\
\hline
\end{tabular}




$\begin{array}{ll}\text { LSMO(x) } & \text { La }_{1-\mathrm{x}} \mathrm{Sr}_{\mathrm{x}} \mathrm{MnO}_{3} \\ \text { LTP } & \text { Niedrigtemperaturphase } \\ \text { MAD } & \text { Metallorganische Aerosol Deposition } \\ \text { MBE } & \text { Molekularstrahlepitaxie } \\ \text { ML } & \text { Monolage } \\ \text { PEM } & \text { Photoelastischer Modulator } \\ \text { PLD } & \text { Gepulste Laserdeposition } \\ \text { PPMS } & \text { Physical Property Measurement System } \\ \text { RDS } & \text { Reflectance Difference Spectroscopy } \\ \text { RHEED } & \text { Reflection High-Energy Electron Diffraction } \\ \text { RP } & \text { Ruddlesden-Popper } \\ \text { SL } & \text { Übergitter } \\ \text { SMO } & \text { SrMnO } \\ \text { SQUID } & \text { Superconducting Quantum Interference Device } \\ \text { STEM } & \text { Scanning Transmissionselektronenmikroskopie } \\ \text { STM } & \text { Rastertunnelmikroskop } \\ \text { STO } & \text { SrTiO } \\ \text { TEM } & \text { Transmissionselektronenmikroskopie } \\ \text { XRD } & \text { Röntgendiffraktion } \\ \text { XRR } & \text { Röntgenreflektometrie } \\ & \end{array}$




\section{Literatur}

[1] Herbert Kroemer. Quasi-electric fields and band offsets: teaching electrons new tricks (2000), www.nobelprize.org/nobel_prizes/physics/laureates/2000/kroemer-lecture.pdf.

[2] Harald Ibach. Physics of Surfaces and Interfaces. Springer-Verlag Berlin Heidelberg (2006), ISBN: 978-3-540-34710-1.

[3] Hans Lüth. Solid Surfaces, Interfaces and Thin Films. Springer-Berlag Berlin Heidelberg (2010), ISBN: 978-3-642-13592-7.

[4] V. Garcia, M. Bibes, L. Bocher, S. Valencia, F. Kronast, A. Crassous, X. Moya, S. Enouz-Vedrenne, A. Gloter, D. Imhoff, C. Deranlot, N. D. Mathur, S. Fusil, K. Bouzehouane und A. Barthélémy. Ferroelectric Control of Spin Polarization. Science 327, 1106 (2010).

[5] C. A. F. Vaz, J. Hoffman, Y. Segal, J. W. Reiner, R. D. Grober, Z. Zhang, C. H. Ahn und F. J. Walker. Origin of the Magnetoelectric Coupling Effect in

$\mathrm{Pb}\left(\mathrm{Zr}_{0.2} \mathrm{Ti}_{\mathbf{0 . 8}}\right) \mathbf{O}_{3} / \mathrm{La}_{0.8} \mathrm{Sr}_{0.2} \mathrm{MnO}_{3}$ Multiferroic Heterostructures. Phys. Rev. Lett. 104, $127202(2010)$.

[6] H. Lu, T. A. George, Y. Wang, I. Ketsman, J. D. Burton, C.-W. Bark, S. Ryu, D. J. Kim, J. Wang, C. Binek, P. A. Dowben, A. Sokolov, C.-B. Eom, E. Y. Tsymbal und A. Gruverman. Electric modulation of magnetization at the $\mathrm{BaTiO}_{3} / \mathrm{La}_{0.67} \mathrm{Sr}_{0.33} \mathrm{MnO}_{3}$ interfaces. Applied Physics Letters 100, 232904 (2012).

[7] A. Ohtomo und H. Y. Hwang. A high-mobility electron gas at the $\mathrm{LaAlO}_{3} / \mathrm{SrTiO}_{3}$ heterointerface. Nature 427, 423 (2004).

[8] Lu Li, C. Richter, J. Mannhart und R. C. Ashoori. Coexistence of magnetic order and two-dimensional superconductivity at $\mathrm{LaAlO}_{3} / \mathrm{SrTiO}_{3}$ interfaces. Nature Physics 7, 762 (2011).

[9] S. Thiel, G. Hammerl, A. Schmehl, C. W. Schneider und J. Mannhart. Tunable QuasiTwo-Dimensional Electron Gases in Oxide Heterostructures. Science 313, 1942 (2006).

[10] Harold Y. Hwang. Atomic Control of the Electronic Structure at Complex Oxide Heterointerfaces. MRS Bulletin 31, 28 (2006). 
[11] Florin Radu und Hartmut Zabel. Exchange Bias Effect of Ferro-/Antiferromagnetic Heterostructures in Magnetic Heterostructures. Springer Berlin Heidelberg (2008), ISBN: 978-3-540-73461-1.

[12] Sebastian Brück, Gisela Schütz, Eberhard Goering, Xiaosong Ji und Kannan M. Krishnan. Uncompensated Moments in the $\mathrm{MnPd} / \mathrm{Fe}$ Exchange Bias System. Phys. Rev. Lett. 101, 126402 (2008).

[13] S. J. May, A. B. Shah, S. G. E. te Velthuis, M. R. Fitzsimmons, J. M. Zuo, X. Zhai, J. N. Eckstein, S. D. Bader und A. Bhattacharya. Magnetically asymmetric interfaces in a $\mathrm{LaMnO}_{3} / \mathrm{SrMnO}_{3}$ superlattice due to structural asymmetries. Phys. Rev. B 77, 174409 (2008).

[14] A. B. Shah, Q. M. Ramasse, S. J. May, Jerald Kavich, J. G. Wen, X. Zhai, J. N. Eckstein, J. Freeland, A. Bhattacharya und J. M. Zuo. Presence and spatial distribution of interfacial electronic states in $\mathrm{LaMnO}_{3} / \mathrm{SrMnO}_{3}$ superlattices. Phys. Rev. B 82, $115112(2010)$.

[15] S. J. May, P. J. Ryan, J. L. Robertson, J.-W. Kim, T. S. Santos, E. Karapetrova, J. L. Zarestky, X. Zhai, S. G. E. te Velthuis, J. N. Eckstein, S. D. Bader und A. Bhattacharya. Enhanced ordering temperatures in antiferromagnetic manganite superlattices. Nature Materials 8, 892 (2009).

[16] V. Moshnyaga und K. Samwer. Ferromagnetic Manganite Films in Handbook of Magnetism and Advanced Magnetic Materials. John Wiley \& Sons Ltd. (2007), ISBN: 978-0-470-02217-7.

[17] Elbio Dagotto. Nanoscale Phase Separation and Colossal Magnetoresistance. Springer Berlin Heidelberg (2003), ISBN: 978-3-662-05244-0.

[18] R. von Helmolt, J. Wecker, B. Holzapfel, L. Schultz und K. Samwer. Giant negative magnetoresistance in perovskitelike $\mathrm{La}_{2 / 3} \mathrm{Ba}_{1 / 3} \mathrm{MnO}_{x}$ ferromagnetic films. Phys. Rev. Lett. 71, 2331 (1993).

[19] S. Jin, T. H. Tiefel, M. McCormack, R. A. Fastnacht, R. Ramesh und L. H. Chen. Thousandfold Change in Resistivity in Magnetoresistive La-Ca-Mn-O Films. Science 264, 413 (1994).

[20] J.-H. Park, E. Vescovo, H.-J. Kim, C. Kwon, R. Ramesh und T. Venkatesan. Nature 392, 794 (1998).

[21] Koichi Momma und Fujio Izumi. VESTA3 for three-dimensional visualization of crystal, volumetric and morphology data. Journal of Applied Crystallography 44, 1272 (2011).

[22] R. Gross. Vorlesungsskript Spinelektronik, Kapitel 4: Der Kolossale Magnetowiderstandseffekt, (2005), URL: http://www.wmi.badw.de/teaching/Lecturenotes/ (besucht am 21.04.2015).

[23] J. M. D. Coey. Magnetism and Magnetic Materials. Cambridge University Press (2010). 
[24] Y. Tokura. Colossal Magnetoresistive Oxides. Taylor \& Francis (2000), ISBN: 978-9056-99231-6.

[25] H. A. Jahn und E. Teller. Stability of Polyatomic Molecules in Degenerate Electronic States. I. Orbital Degeneracy. Proceedings of the Royal Society of London A: Mathematical, Physical and Engineering Sciences 161, 220 (1937).

[26] N. N. Kovaleva, Andrzej M. Ole ś, A. M. Balbashov, A. Maljuk, D. N. Argyriou, G. Khaliullin und B. Keimer. Low-energy Mott-Hubbard excitations in $\mathrm{LaMnO}_{3}$ probed by optical ellipsometry. Phys. Rev. B 81, 235130 (2010).

[27] M. Quijada, J. Černe, J. R. Simpson, H. D. Drew, K. H. Ahn, A. J. Millis, R. Shreekala, R. Ramesh, M. Rajeswari und T. Venkatesan. Optical conductivity of manganites: Crossover from Jahn-Teller small polaron to coherent transport in the ferromagnetic state. Phys. Rev. B 58, 16093 (1998).

[28] Y. Murakami, J. P. Hill, D. Gibbs, M. Blume, I. Koyama, M. Tanaka, H. Kawata, T. Arima, Y. Tokura, K. Hirota und Y. Endoh. Resonant X-Ray Scattering from Orbital Ordering in $\mathrm{LaMnO}_{3}$. Phys. Rev. Lett. 81, 582 (1998).

[29] Anand Bhattacharya und Steven J. May. Magnetic Oxide Heterostructures. Annual Review of Materials Research 44, 65 (2014).

[30] M. Opel. Vorlesungsskript Magnetismus, Kapitel 6: Die Austauschwechselwirkung, (2005), URL: http://www.wmi.badw.de/teaching/Lecturenotes/\%5Cnewline (besucht am 21.04.2015).

[31] P. W. Anderson und H. Hasegawa. Considerations on Double Exchange. Phys. Rev. 100, 675 (1955).

[32] A. J. Millis. Lattice effects in magnetoresistive manganese perovskites. Nature 392 , 147 (1998).

[33] N. Mathur und P. Littlewood. Mesoscopic texture in manganites. Physics Today 56, 25 (2003).

[34] Guo-meng Zhao, K. Conder, H. Keller und K. A. Müller. Giant oxygen isotope shift in the magnetoresistive perovskite $\mathrm{La}_{1-\mathrm{x}} \mathrm{Ca}_{\mathrm{x}} \mathrm{MnO}_{3+\mathrm{y}}$. Nature 381, 676 (1996).

[35] L. Sudheendra, V. Moshnyaga und K. Samwer. Metal-insulator transition and colossal magnetoresistance: relevance of electron-lattice coupling and electronic phase separation. Contemporary Physics 48, 349 (2007).

[36] V. Moshnyaga und K. Samwer. Electron-lattice correlations and phase transitions in CMR manganites. Annalen der Physik 523, 652 (2011).

[37] V. Moshnyaga, A. Belenchuk, S. Hühn, C. Kalkert, M. Jungbauer, O. I. Lebedev, S. Merten, K.-Y. Choi, P. Lemmens, B. Damaschke und K. Samwer. Intrinsic antiferromagnetic coupling underlies colossal magnetoresistance effect: Role of correlated polarons. Physical Review B 89, 024420 (2014). 
[38] J. Hemberger, A. Krimmel, T. Kurz, H.-A. Krug von Nidda, V. Yu. Ivanov, A. A. Mukhin, A. M. Balbashov und A. Loidl. Structural, magnetic, and electrical properties of single-crystalline $\mathrm{La}_{1-x} \mathrm{Sr}_{x} \mathrm{MnO}_{3}(0.4<x<0.85)$. Phys. Rev. B 66, 094410 (2002).

[39] O. Chmaissem, B. Dabrowski, S. Kolesnik, J. Mais, J. D. Jorgensen und S. Short. Structural and magnetic phase diagrams of $\mathrm{La}_{1-x} \mathrm{Sr}_{x} \mathrm{MnO}_{3}$ and $\mathrm{Pr}_{1-y} \mathrm{Sr}_{y} \mathrm{MnO}_{3}$. Phys. Rev. B 67, 094431 (2003).

[40] Takayoshi Takeda und Sōji Ōhara. Magnetic Structure of the Cubic Perovskite Type $\mathrm{SrMnO}_{3}$. J. Phys. Soc. Jpn. 37, 275 (1974).

[41] Despina Louca, T. Egami, E. L. Brosha, H. Röder und A. R. Bishop. Local Jahn-Teller distortion in $\mathrm{La}_{1-x} \mathrm{Sr}_{x} \mathrm{MnO}_{3}$ observed by pulsed neutron diffraction. Phys. Rev. B 56, R8475 (1997).

[42] Jeroen van den Brink und Daniel Khomskii. Double Exchange via Degenerate Orbitals. Phys. Rev. Lett. 82, 1016 (1999).

[43] S. Yunoki, J. Hu, A. L. Malvezzi, A. Moreo, N. Furukawa und E. Dagotto. Phase Separation in Electronic Models for Manganites. Phys. Rev. Lett. 80, 845 (1998).

[44] J. J. Neumeier und J. L. Cohn. Possible signatures of magnetic phase segregation in electron-doped antiferromagnetic $\mathrm{CaMnO}_{3}$. Phys. Rev. B 61, 14319 (2000).

[45] H.Y. Hwang, Y. Iwasa, M. Kawasaki, B. Keimer, N. Nagaosa und Y. Tokura. Emergent phenomena at oxide interfaces. Nature Materials 11, 103 (2012).

[46] James N. Eckstein. Oxide interfaces: Watch out for the lack of oxygen. Nature Materials 6, 473 (2007).

[47] Fabio Miletto Granozio, Gertjan Koster und Guus Rijnders. Functional oxide interfaces. MRS Bulletin 38, 1017 (2013).

[48] N.W. Ashcroft und N.D. Mermin. Solid State Physics. Thomson Learning (1976), ISBN: 978-0030839931.

[49] P. D. C. King, H. I. Wei, Y. F. Nie, M. Uchida, C. Adamo, S. Zhu, X. He, I. Božović, D. G. Schlom und K. M. Shen. Atomic-scale control of competing electronic phases in ultrathin $\mathrm{LaNiO}_{3}$. Nature Nanotechnology 9, 443 (2014).

[50] D. Schumacher, A. Steffen, J. Voigt, J. Schubert, Th. Brückel, H. Ambaye und V. Lauter. Inducing exchange bias in $\mathrm{La}_{0.67} \mathrm{Sr}_{0.33} \mathrm{MnO}_{3-\delta} / \mathrm{SrTiO}_{3}$ thin films by strain and oxygen deficiency. Phys. Rev. B 88, 144427 (2013).

[51] M. P. de Jong, I. Bergenti, V. A. Dediu, M. Fahlman, M. Marsi und C. Taliani. Evidence for $\mathrm{Mn}^{2+}$ ions at surfaces of $\mathrm{La}_{0.7} \mathrm{Sr}_{0.3} \mathrm{MnO}_{3}$ thin films. Phys. Rev. B 71, 014434 (2005).

[52] G. Herranz, F. Sánchez, N. Dix, M. Scigaj und J. Fontcuberta. High mobility conduction at (110) and (111) $\mathrm{LaAlO}_{3} / \mathrm{SrTiO}_{3}$ interfaces. Scientific Reports 2, (2012). 
[53] Ulrich Aschauer, Reto Pfenninger, Sverre M. Selbach, Tor Grande und Nicola A. Spaldin. Strain-controlled oxygen vacancy formation and ordering in $\mathrm{CaMnO}_{3}$. Phys. Rev. B 88, 054111 (2013).

[54] J. Verbeeck, O. I. Lebedev, G. Van Tendeloo und B. Mercey.

$\mathrm{SrTiO}_{3}(100) /\left(\mathrm{LaMnO}_{3}\right)_{m}\left(\mathrm{SrMnO}_{3}\right)_{n}$ layered heterostructures: A combined EELS and TEM study. Phys. Rev. B 66, 184426 (2002).

[55] A. B. Shah, Q. M. Ramasse, S. J. May, Jerald Kavich, J. G. Wen, X. Zhai, J. N. Eckstein, J. Freeland, A. Bhattacharya und J. M. Zuo. Presence and spatial distribution of interfacial electronic states in $\mathrm{LaMnO}_{3}-\mathrm{SrMnO}_{3}$ superlattices. Phys. Rev. B 82, $115112(2010)$.

[56] Hani Dulli, P. A. Dowben, S.-H. Liou und E. W. Plummer. Surface segregation and restructuring of colossal-magnetoresistant manganese perovskites $\mathrm{La}_{0.65} \mathrm{Sr}_{0.35} \mathrm{MnO}_{3}$. Phys. Rev. B 62, R14629 (2000).

[57] Tim T. Fister, Dillon D. Fong, Jeffrey A. Eastman, Peter M. Baldo, Matthew J. Highland, Paul H. Fuoss, Kavaipatti R. Balasubramaniam, Joanna C. Meador und Paul A. Salvador. In situ characterization of strontium surface segregation in epitaxial $\mathrm{La}_{0.7} \mathrm{Sr}_{0.3} \mathrm{MnO}_{3}$ thin films as a function of oxygen partial pressure. Applied Physics Letters 93, 151904 (2008).

[58] S. Turner, O. I. Lebedev, J. Verbeeck, K. Gehrke, V. Moshnyaga und G. Van Tendeloo. Structural phase transition and spontaneous interface reconstruction in $\mathrm{La}_{2 / 3} \mathrm{Ca}_{1 / 3} \mathrm{MnO}_{3} / \mathrm{BaTiO}_{3}$ superlattices. Phys. Rev. B 87, 035418 (2013).

[59] J.D. Jackson. Classical Electrodynamics. John Wiley \& Sons (1998), ISBN: 978-0471309321.

[60] Karsten Gomann, Gunter Borchardt, Michal Schulz, Anissa Gomann, Wolfgang MausFriedrichs, Bernard Lesage, Odile Kaitasov, Susanne Hoffmann-Eifert und Theodor Schneller. Sr diffusion in undoped and La-doped $\mathrm{SrTiO}_{3}$ single crystals under oxidizing conditions. Phys. Chem. Chem. Phys. 7, 2053 (2005).

[61] N. Nakagawa, H. Y. Hwang und D. A. Muller. Why some interfaces cannot be sharp. Nature Materials 5, 204 (2006).

[62] Dieter Wolf. Reconstruction of $\mathrm{NaCl}$ surfaces from a dipolar solution to the Madelung problem. Phys. Rev. Lett. 68, 3315 (1992).

[63] W. A. Harrison, E. A. Kraut, J. R. Waldrop und R. W. Grant. Polar heterojunction interfaces. Phys. Rev. B 18, 4402 (1978).

[64] A. Wander, I. J. Bush und N. M. Harrison. Stability of rocksalt polar surfaces: An ab initio study of $\mathrm{MgO}$ (111) and $\mathrm{NiO}$ (111). Phys. Rev. B 68, 233405 (2003).

[65] D. Cappus, M. Haßel, E. Neuhaus, M. Heber, F. Rohr und H.-J. Freund. Polar surfaces of oxides: reactivity and reconstruction. Surface Science 337, 268 (1995).

[66] R. Hesper, L. H. Tjeng, A. Heeres und G. A. Sawatzky. Photoemission evidence of electronic stabilization of polar surfaces in $\mathrm{K}_{3} \mathrm{C}_{60}$. Phys. Rev. B 62, 16046 (2000). 
[67] M. A. Hossain, J. D. F. Mottershead, D. Fournier, A. Bostwick, J. L. McChesney, E. Rotenberg, R. Liang, W. N. Hardy, G. A. Sawatzky, I. S. Elfimov, D. A. Bonn und A. Damascelli. In situ doping control of the surface of high-temperature superconductors. 4, 527 (2008).

[68] François Bottin, Fabio Finocchi und Claudine Noguera. Facetting and (nx1) reconstructions of $\mathrm{SrTiO}_{3}$ (110) surfaces. Surface Science 574, 65 (2005).

[69] P. R. Willmott, S. A. Pauli, R. Herger, C. M. Schlepütz, D. Martoccia, B. D. Patterson, B. Delley, R. Clarke, D. Kumah, C. Cionca und Y. Yacoby. Structural Basis for the Conducting Interface between $\mathrm{LaAlO}_{3}$ and $\mathrm{SrTiO}_{3}$. Phys. Rev. Lett. 99, 155502 (2007).

[70] A. Annadi, Z. Huang, K. Gopinadhan, X. Renshaw Wang, A. Srivastava, Z. Q. Liu, H. Harsan Ma, T. P. Sarkar, T. Venkatesan und Ariando. Fourfold oscillation in anisotropic magnetoresistance and planar Hall effect at the $\mathrm{LaAlO}_{3} / \mathrm{SrTiO}_{3}$ heterointerfaces: Effect of carrier confinement and electric field on magnetic interactions. Phys. Rev. B 87, 201102 (2013).

[71] Yunzhong Chen, Nini Pryds, Josée E. Kleibeuker, Gertjan Koster, Jirong Sun, Eugen Stamate, Baogen Shen, Guus Rijnders und Søren Linderoth. Metallic and Insulating Interfaces of Amorphous SrTiO3-Based Oxide Heterostructures. Nano Letters 11, 3774 (2011).

[72] M. P. Warusawithana, C. Richter, J. A. Mundy, P. Roy, J. Ludwig, S. Paetel, T. Heeg, A. A. Pawlicki, L. F. Kourkoutis, M. Zheng, M. Lee, B. Mulcahy, W. Zander, Y. Zhu, J. Schubert, J. N. Eckstein, D. A. Muller, C. Stephen Hellberg, J. Mannhart und D. G. Schlom. $\mathrm{LaAlO}_{3}$ stoichiometry is key to electron liquid formation at $\mathrm{LaAlO}_{3} / \mathrm{SrTiO}_{3}$ interfaces. Nature Communications 4, 2351 (2013).

[73] B. R. K. Nanda und S. Satpathy. Polar catastrophe, electron leakage, and magnetic ordering at the $\mathrm{LaMnO}_{3} / \mathrm{SrMnO}_{3}$ interface. Phys. Rev. B 81, 224408 (2010).

[74] Chungwei Lin, Satoshi Okamoto und Andrew J. Millis. Dynamical mean-field study of model double-exchange superlattices. Phys. Rev. B 73, 041104 (2006).

[75] Qinfang Zhang, Shuai Dong, Baolin Wang und Seiji Yunoki. Strain-engineered magnetic order in $\left(\mathrm{LaMnO}_{3}\right)_{\mathrm{n}} /\left(\mathrm{SrMnO}_{3}\right)_{2 \mathrm{n}}$ superlattices. Phys. Rev. B 86, 094403 (2012).

[76] J. Hoffman, I. C. Tung, B. B. Nelson-Cheeseman, M. Liu, J. W. Freeland und A. Bhattacharya. Charge transfer and interfacial magnetism in $\left(\mathrm{LaNiO}_{3}\right)_{\mathrm{n}} /\left(\mathrm{LaMnO}_{3}\right)_{2}$ superlattices. Phys. Rev. B 88, 144411 (2013).

[77] A. Tebano, C. Aruta, S. Sanna, P. G. Medaglia, G. Balestrino, A. A. Sidorenko, R. De Renzi, G. Ghiringhelli, L. Braicovich, V. Bisogni und N. B. Brookes. Evidence of Orbital Reconstruction at Interfaces in Ultrathin $\mathrm{La}_{0.67} \mathrm{Sr}_{0.33} \mathrm{MnO}_{3}$ Films. Phys. Rev. Lett. 100, 137401 (2008).

[78] B. R. K. Nanda und Sashi Satpathy. Effects of strain on orbital ordering and magnetism at perovskite oxide interfaces: $\mathrm{LaMnO}_{3} / \mathrm{SrMnO}_{3}$. Phys. Rev. B 78, 054427 (2008). 
[79] C. Aruta, G. Ghiringhelli, A. Tebano, N. G. Boggio, N. B. Brookes, P. G. Medaglia und G. Balestrino. Strain induced x-ray absorption linear dichroism in $\mathrm{La}_{0.7} \mathrm{Sr}_{0.3} \mathrm{MnO}_{3}$ thin films. Phys. Rev. B 73, 235121 (2006).

[80] Z. Fang, I. V. Solovyev und K. Terakura. Phase Diagram of Tetragonal Manganites. Phys. Rev. Lett. 84, 3169 (2000).

[81] Yoshinori Konishi, Zhong Fang, Makoto Izumi, Takashi Manako, Masahiro Kasai, Hideki Kuwahara, Masashi Kawasaki, Kiyoyuki Terakura und Yoshinori Tokura. OrbitalState-Mediated Phase-Control of Manganites. Journal of the Physical Society of Japan 68, 3790 (1999).

[82] B. Cui, C. Song, F. Li, G. Y. Wang, H. J. Mao, J. J. Peng, F. Zeng und F. Pan. Tuning the entanglement between orbital reconstruction and charge transfer at a film surface. Scientific Reports 4, 4206 (2014).

[83] Marie-Bernadette Lepetit, Bernard Mercey und Charles Simon. Interface Effects in Perovskite Thin Films. Phys. Rev. Lett. 108, 087202 (2012).

[84] M. J. Calderón, L. Brey und F. Guinea. Surface electronic structure and magnetic properties of doped manganites. Phys. Rev. B 60, 6698 (1999).

[85] M. Huijben, L. W. Martin, Y.-H. Chu, M. B. Holcomb, P. Yu, G. Rijnders, D. H. A. Blank und R. Ramesh. Critical thickness and orbital ordering in ultrathin $\mathrm{La}_{0.7} \mathrm{Sr}_{0.3} \mathrm{MnO}_{3}$ films. Phys. Rev. B 78, 094413 (2008).

[86] D. Pesquera, G. Herranz, A. Barla, E. Pellegrin, F. Bondino, E. Magnano, F. Sánchez und J. Fontcuberta. Surface symmetry-breaking and strain effects on orbital occupancy in transition metal perovskite epitaxial films. Nat Comms 3, 1189 (2012).

[87] Eva Benckiser, Maurits W. Haverkort, Sebastian Brück, Eberhard Goering, Sebastian Macke, Alex Frañó, Xiaoping Yang, Ole K. Andersen, Georg Cristiani, HannsUlrich Habermeier, Alexander V. Boris, Ioannis Zegkinoglou, Peter Wochner, HeonJung Kim, Vladimir Hinkov und Bernhard Keimer. Orbital reflectometry of oxide heterostructures. Nat Mater 10, 189 (2011).

[88] James M. Rondinelli, Steven J. May und John W. Freeland. Control of octahedral connectivity in perovskite oxide heterostructures: An emerging route to multifunctional materials discovery. MRS Bulletin 37, 261 (2012).

[89] James M. Rondinelli und Nicola A. Spaldin. Structure and Properties of Functional Oxide Thin Films: Insights From Electronic-Structure Calculations. Advanced Materials 23, 3363 (2011).

[90] T. T. Fister, H. Zhou, Z. Luo, S. S. A. Seo, S. O. Hruszkewycz, D. L. Proffit, J. A. Eastman, P. H. Fuoss, P. M. Baldo, H. N. Lee und D. D. Fong. Octahedral rotations in strained $\mathrm{LaAlO}_{3} / \mathrm{SrTiO}_{3}$ (001) heterostructures. APL Materials 2, 021102 (2014).

[91] Jun He, Albina Borisevich, Sergei V. Kalinin, Stephen J. Pennycook und Sokrates T. Pantelides. Control of Octahedral Tilts and Magnetic Properties of Perovskite Oxide Heterostructures by Substrate Symmetry. Phys. Rev. Lett. 105, 227203 (2010). 
[92] James M. Rondinelli und Nicola A. Spaldin. Substrate coherency driven octahedral rotations in perovskite oxide films. Phys. Rev. B 82, 113402 (2010).

[93] A. Y. Borisevich, H. J. Chang, M. Huijben, M. P. Oxley, S. Okamoto, M. K. Niranjan, J. D. Burton, E. Y. Tsymbal, Y. H. Chu, P. Yu, R. Ramesh, S. V. Kalinin und S. J. Pennycook. Suppression of Octahedral Tilts and Associated Changes in Electronic Properties at Epitaxial Oxide Heterostructure Interfaces. Phys. Rev. Lett. 105, 087204 (2010).

[94] A. Vailionis, H. Boschker, Z. Liao, J. R. A. Smit, G. Rijnders, M. Huijben und G. Koster. Symmetry and lattice mismatch induced strain accommodation near and away from correlated perovskite interfaces. Applied Physics Letters 105, 131906 (2014).

[95] S. J. May, C. R. Smith, J.-W. Kim, E. Karapetrova, A. Bhattacharya und P. J. Ryan. Control of octahedral rotations in $\left(\mathrm{LaNiO}_{3}\right)_{\mathrm{n}} /\left(\mathrm{SrMnO}_{3}\right)_{\mathrm{m}}$ superlattices. Phys. Rev. B 83, 153411 (2011).

[96] K. Gehrke, V. Moshnyaga, K. Samwer, O. I. Lebedev, J. Verbeeck, D. Kirilenko und G. Van Tendeloo. Interface controlled electronic variations in correlated heterostructures. Phys. Rev. B 82, 113101 (2010).

[97] Danny Schwarzbach. Wachstum von dünnen $\mathrm{La}_{0.7} \mathrm{Sr}_{0.3} \mathrm{MnO}_{3}$-Schichten auf verschieden orientierten $\mathrm{SrTiO}_{3}$-Substraten. Bachelorarbeit, Georg-August-Universität Göttingen (2013).

[98] Robert B. Griffiths. Nonanalytic Behavior Above the Critical Point in a Random Ising Ferromagnet. Phys. Rev. Lett. 23, 17 (1969).

[99] Harrison W. A. Electronic Structure and the Properties of Solids. Freeman, San Francisco (1980), ISBN: 978-0-486-66021-9.

[100] Teruo Kanki, Hidekazu Tanaka und Tomoji Kawai. Anomalous strain effect in $\mathrm{La}_{0.8} \mathrm{Ba}_{0.2} \mathrm{MnO}_{3}$ epitaxial thin film: Role of the orbital degree of freedom in stabilizing ferromagnetism. Phys. Rev. B 64, 224418 (2001).

[101] R. Peng, H. C. Xu, M. Xia, J. F. Zhao, X. Xie, D. F. Xu, B. P. Xie und D. L. Feng. Tuning the dead-layer behavior of La0.67Sr0.33MnO3/SrTiO3 via interfacial engineering. Applied Physics Letters 104, 081606 (2014).

[102] J.-S. Lee, D. A. Arena, P. Yu, C. S. Nelson, R. Fan, C. J. Kinane, S. Langridge, M. D. Rossell, R. Ramesh und C.-C. Kao. Hidden Magnetic Configuration in Epitaxial $\mathrm{La}_{1-x} \mathrm{Sr}_{x} \mathrm{MnO}_{3}$ Films. Phys. Rev. Lett. 105, 257204 (2010).

[103] G. Shibata, K. Yoshimatsu, E. Sakai, V. R. Singh, V. K. Verma, K. Ishigami, T. Harano, T. Kadono, Y. Takeda, T. Okane, Y. Saitoh, H. Yamagami, A. Sawa, H. Kumigashira, M. Oshima, T. Koide und A. Fujimori. Thickness-dependent ferromagnetic metal to paramagnetic insulator transition in $\mathrm{La}_{0.6} \mathrm{Sr}_{0.4} \mathrm{MnO}_{3}$ thin films studied by x-ray magnetic circular dichroism. Phys. Rev. B 89, 235123 (2014).

[104] O. Shapoval, S. Hühn, J. Verbeeck, M. Jungbauer, A. Belenchuk und V. Moshnyaga. Interface-controlled magnetism and transport of ultrathin manganite films. Journal of Applied Physics 113, 17C711 (2013). 
[105] H. Boschker, J. Verbeeck, R. Egoavil, S. Bals, G. van Tendeloo, M. Huijben, E. P. Houwman, G. Koster, D. H. A. Blank und G. Rijnders. Preventing the Reconstruction of the Polar Discontinuity at Oxide Heterointerfaces. Advanced Functional Materials 22, 2235 (2012).

[106] M. Minohara, R. Yasuhara, H. Kumigashira und M. Oshima. Termination layer dependence of Schottky barrier height for $\mathrm{La}_{0.6} \mathrm{Sr}_{0.4} \mathrm{MnO}_{3} / \mathrm{Nb}: \mathrm{SrTiO}_{3}$ heterojunctions. Phys. Rev. B 81, 235322 (2010).

[107] Felip Sandiumenge, José Santiso, Lluís Balcells, Zorica Konstantinovic, Jaume Roqueta, Alberto Pomar, Juan Pedro Espinós und Benjamín Martínez. Competing Misfit Relaxation Mechanisms in Epitaxial Correlated Oxides. Phys. Rev. Lett. 110, 107206 (2013).

[108] A. Ohtomo und H. Y. Hwang. Growth mode control of the free carrier density in $\mathrm{SrTiO}_{3}$ films. Journal of Applied Physics 102, 083704 (2007).

[109] E. J. Moon, P. V. Balachandran, B. J. Kirby, D. J. Keavney, R. J. Sichel-Tissot, C. M. Schlepütz, E. Karapetrova, X. M. Cheng, J. M. Rondinelli und S. J. May. Effect of Interfacial Octahedral Behavior in Ultrathin Manganite Films. Nano Letters 14, 2509 (2014).

[110] H. Yamada. Engineered Interface of Magnetic Oxides. Science 305, 646 (2004).

[111] A. Bhattacharya, S. J. May, S. G. E. te Velthuis, M. Warusawithana, X. Zhai, Bin Jiang, J.-M. Zuo, M. R. Fitzsimmons, S. D. Bader und J. N. Eckstein. Metal-Insulator Transition and Its Relation to Magnetic Structure in $\left(\mathrm{LaMnO}_{3}\right)_{2 n} /\left(\mathrm{SrMnO}_{3}\right)_{n}$ Superlattices. Phys. Rev. Lett. 100, 257203 (2008).

[112] H S Kim und H M Christen. Controlling the magnetic properties of LaMnO 3 thin films on SrTiO 3 (100) by deposition in a $\mathrm{O} 2 / \mathrm{Ar}$ gas mixture. Journal of Physics: Condensed Matter 22, 146007 (2010).

[113] C. Aruta, C. Adamo, A. Galdi, P. Orgiani, V. Bisogni, N. B. Brookes, J. C. Cezar, P. Thakur, C. A. Perroni, G. De Filippis, V. Cataudella, D. G. Schlom, L. Maritato und G. Ghiringhelli. Evolution of magnetic phases and orbital occupation in $\left(\mathrm{SrMnO}_{3}\right)_{n} /\left(\mathrm{LaMnO}_{3}\right)_{2 n}$ superlattices. Phys. Rev. B 80, 140405 (2009).

[114] J. F. Mitchell, D. N. Argyriou, C. D. Potter, D. G. Hinks, J. D. Jorgensen und S. D. Bader. Structural phase diagram of $\mathrm{La}_{1-\mathrm{x}} \mathrm{Sr}_{\mathrm{x}} \mathrm{MnO}_{3+\delta}$ : Relationship to magnetic and transport properties. Phys. Rev. B 54, 6172 (1996).

[115] J. A. Alonso, M. J. Martinez-Lope, M. T. Casais, J. L. Macmanus-Driscoll, P. S. I. P. N. de Silva, L. F. Cohen und M. T. Fernandez-Diaz. Non-stoichiometry, structural defects and properties of $\mathrm{LaMnO}_{3+\delta}$ with high $\delta$ values $(0.11 \leq \delta \leq 0.29)$. J. Mater. Chem. 7 , 2139 (1997).

[116] H S Kim und H M Christen. Controlling the magnetic properties of $\mathrm{LaMnO}_{3}$ thin films on $\mathrm{SrTiO}_{3}$ (100) by deposition in a $\mathrm{O}_{2} /$ Ar gas mixture. Journal of Physics: Condensed Matter 22, 146007 (2010). 
[117] Shuai Dong, Rong Yu, Seiji Yunoki, Gonzalo Alvarez, J.-M. Liu und Elbio Dagotto. Magnetism, conductivity, and orbital order in $\left(\mathrm{LaMnO}_{3}\right)_{2 \mathrm{n}} /\left(\mathrm{SrMnO}_{3}\right)_{\mathrm{n}}$ superlattices. Phys. Rev. B 78, 201102 (2008).

[118] Y. S. Hou, H. J. Xiang und X. G. Gong. Intrinsic insulating ferromagnetism in manganese oxide thin films. Phys. Rev. B 89, 064415 (2014).

[119] A. Bhattacharya, S. J. May, S. G. E. te Velthuis, M. Warusawithana, X. Zhai, Bin Jiang, J.-M. Zuo, M. R. Fitzsimmons, S. D. Bader und J. N. Eckstein. Metal-Insulator Transition and Its Relation to Magnetic Structure in $\left(\mathrm{LaMnO}_{3}\right)_{2 n} /\left(\mathrm{SrMnO}_{3}\right)_{n}$ Superlattices. Phys. Rev. Lett. 100, 257203 (2008).

[120] Hiroyuki Yamada, M. Kawasaki, T. Lottermoser, T. Arima und Y. Tokura. $\mathrm{LaMnO}_{3} / \mathrm{SrMnO}_{3}$ interfaces with coupled charge-spin-orbital modulation. Applied Physics Letters 89, 052506 (2006).

[121] P. G. Radaelli, G. Iannone, M. Marezio, H. Y. Hwang, S-W. Cheong, J. D. Jorgensen und D. N. Argyriou. Structural effects on the magnetic and transport properties of perovskite $\mathrm{A}_{1-\mathrm{x}} \mathrm{A}_{\mathrm{x}}^{\prime} \mathrm{MnO}_{3}(x=0.25,0.30)$. Phys. Rev. B 56, 8265 (1997).

[122] J. Rodríguez-Carvajal, M. Hennion, F. Moussa, A. H. Moudden, L. Pinsard und A. Revcolevschi. Neutron-diffraction study of the Jahn-Teller transition in stoichiometric $\mathrm{LaMnO}_{3}$. Phys. Rev. B 57, R3189 (1998).

[123] W. H. Meiklejohn und C. P. Bean. New Magnetic Anisotropy. Phys. Rev. 102, 1413 (1956).

[124] J Nogués und Ivan K Schuller. Exchange bias. J. Magn. Magn. Mater. 192, 203 (1999).

[125] Miguel Kiwi. Exchange bias theory. J. Magn. Magn. Mater. 234, 584 (2001).

[126] A. P. Malozemoff. Random-field model of exchange anisotropy at rough ferromagneticantiferromagnetic interfaces. Phys. Rev. B 35, 3679 (1987).

[127] P. Miltényi, M. Gierlings, J. Keller, B. Beschoten, G. Güntherodt, U. Nowak und K. D. Usadel. Diluted Antiferromagnets in Exchange Bias: Proof of the Domain State Model. Phys. Rev. Lett. 84, 4224 (2000).

[128] K. Westerholt, U. Geiersbach und A. Bergmann. Exchange bias in $\left[\mathrm{Co}_{2} \mathrm{MnGe} / \mathrm{Au}\right]_{\mathrm{n}}$, $\left[\mathrm{Co}_{2} \mathrm{MnGe} / \mathrm{Cr}\right]_{\mathrm{n}}$ and $\left[\mathrm{Co}_{2} \mathrm{MnGe} / \mathrm{Cu}_{2} \mathrm{MnAl}\right]_{\mathrm{n}}$ multilayers. Journal of Magnetism and Magnetic Materials 257, 239 (2003).

[129] V. Moshnyaga, I. Khoroshun, A. Sidorenko, P. Petrenko, A. Weidinger, M. Zeitler, B. Rauschenbach, R. Tidecks und K. Samwer. Preparation of rare-earth manganite-oxide thin films by metalorganic aerosol deposition technique. App. Phys. Lett. 74, 2842 (1999).

[130] I V Khoroshun, E V Karyaev, V T Moshnyaga, G A Kiosse, M A Krachun, V M Zakosarenko und V Yu Davydov. Characteristics of epitaxial $\mathrm{Y}$-Ba-Cu-O thin films grown by aerosol MOCVD technique. Superconductor Science and Technology 3, 493 (1990). 
[131] K. Samwer, A. Belenchuk, O. Shapoval und V. Moshnyaga. "Patent Aerosolerzeugerdüse". DE 102007055936 B4. 30. Dez. 2007.

[132] S. Hühn, M. Jungbauer, M. Michelmann, F. Massel, F. Koeth, C. Ballani und V. Moshnyaga. Modeling of colossal magnetoresistance in $\mathrm{La}_{0.67} \mathrm{Ca}_{0.33} \mathrm{MnO}_{3} / \mathrm{Pr}_{0.67} \mathrm{Ca}_{0.33} \mathrm{MnO}_{3}$ superlattices: Comparison with individual $\left(\mathrm{La}_{1-y} \mathrm{Pr}_{\mathrm{y}}\right)_{0.67} \mathrm{Ca}_{0.33} \mathrm{MnO}_{3}$ films. Journal of Applied Physics 113, $17 \mathrm{D} 701$ (2013).

[133] J. D. Baniecki, M. Ishii, K. Kurihara, K. Yamanaka, T. Yano, K. Shinozaki, T. Imada, K. Nozaki und N. Kin. Photoemission and quantum chemical study of $\mathrm{SrTiO}_{3}(001)$ surfaces and their interaction with $\mathrm{CO}_{2}$. Phys. Rev. B 78, 195415 (2008).

[134] J. M. LeBeau, R. Engel-Herbert, B. Jalan, J. Cagnon, P. Moetakef, S. Stemmer und G. B. Stephenson. Stoichiometry optimization of homoepitaxial oxide thin films using x-ray diffraction. Applied Physics Letters 95, 142905 (2009).

[135] Victor Pfahl. Sauerstoffarme MAD: Struktur und Magnetotransport von Magnetitschichten. Masterarbeit, Georg-August-Universität Göttingen (2014).

[136] P. Laukkanen, J. Sadowski und M. Guina. Semiconductor Research. Springer Series in Materials Science (2012), ISBN: 978-3-642-23350-0.

[137] Guus J. H. M. Rijnders, Gertjan Koster, Dave H. A. Blank und Horst Rogalla. In situ monitoring during pulsed laser deposition of complex oxides using reflection high energy electron diffraction under high oxygen pressure. Applied Physics Letters 70, 1888 (1997).

[138] Darren Dale, Y Suzuki und J D Brock. In situ x-ray reflectivity studies of dynamics and morphology during heteroepitaxial complex oxide thin film growth. Journal of Physics: Condensed Matter 20, 264008 (2008).

[139] J. D. Ferguson, G. Arikan, D. S. Dale, A. R. Woll und J. D. Brock. Measurements of Surface Diffusivity and Coarsening during Pulsed Laser Deposition. Phys. Rev. Lett. 103, 256103 (2009).

[140] H. Fujiwara. Spectroscopic Ellipsometry Principles and Applications. John Wiley \& Sons, Ltd (2007), ISBN: 978-0-470-01608-4.

[141] Hiroyuki Fujiwara, Michio Kondo und Akihisa Matsuda. Real-time spectroscopic ellipsometry studies of the nucleation and grain growth processes in microcrystalline silicon thin films. Phys. Rev. B 63, 115306 (2001).

[142] K. A. Bell, M. Ebert, S. D. Yoo, K. Flock und D. E. Aspnes. Real-time optical characterization of heteroepitaxy by organometallic chemical vapor deposition. Journal of Vacuum Science \& Technology A 18, 1184 (2000).

[143] J.-T. Zettler, T. Wethkamp, M. Zorn, M. Pristovsek, C. Meyne, K. Ploska und W. Richter. Growth oscillations with monolayer periodicity monitored by ellipsometry during metalorganic vapor phase epitaxy of GaAs(001). Applied Physics Letters 67, 3783 (1995). 
[144] D. E. Aspnes, J. P. Harbison, A. A. Studna und L. T. Florez. Optical reflectance and electron diffraction studies of molecular-beam-epitaxy growth transients on GaAs(001). Phys. Rev. Lett. 59, 1687 (1987).

[145] M. Losurdo und K. Hingerl. Ellipsometry at the Nanoscale. Springer-Verlag Berlin Heidelberg (2013), ISBN: 978-3-642-33955-4.

[146] Jeong-Sik Lee, Shigeo Sugou und Yasuaki Masumoto. Mechanism of ellipsometry monolayer oscillations during metalorganic vapor-phase epitaxy. Journal of Applied Physics 88, 196 (2000).

[147] X. D. Zhu, H. B. Lu, Guo-Zhen Yang, Zhi-Yuan Li, Ben-Yuan Gu und Dao-Zhong Zhang. Epitaxial growth of $\mathrm{SrTiO}_{3}$ on $\mathrm{SrTiO}_{3}(001)$ using an oblique-incidence reflectance-difference technique. Phys. Rev. B 57, 2514 (1998).

[148] Y. Y. Fei, X. D. Zhu, L. F. Liu, H. B. Lu, Z. H. Chen und G. Z. Yang. Oscillations in oblique-incidence optical reflection from a growth surface during layer-by-layer epitaxy. Phys. Rev. B 69, 233405 (2004).

[149] X. D. Zhu, Weidong Si, X. X. Xi und Qidu Jiang. Oxidation kinetics in SrTiO3 homoepitaxy on SrTiO3(001). Applied Physics Letters 78, 460 (2001).

[150] Fan Chen, Huibin Lu, Tong Zhao, Kui-juan Jin, Zhenghao Chen und Guozhen Yang. Real-time optical monitoring of the heteroepitaxy of oxides by an oblique-incidence reflectance difference technique. Phys. Rev. B 61, 10404 (2000).

[151] X. D. Zhu, Weidong Si, X. X. Xi, Qi Li, Q. D. Jiang und M. G. Medici. Oxidation kinetics in $\mathrm{La}_{0.67} \mathrm{Ba}_{0.33} \mathrm{MnO}_{3-\delta}$ epitaxy on $\mathrm{SrTiO}_{3}$ (001) during pulsed-laser deposition. Applied Physics Letters 74, 3540 (1999).

[152] David Esteve, Kamil Postava, Philippe Gogol, Gang Niu, Bertrand Vilquin und Philippe Lecoeur. In situ monitoring of $\mathrm{La}_{0.67} \mathrm{Sr}_{0.33} \mathrm{MnO}_{3}$ monolayers grown by pulsed laser deposition. physica status solidi (b) 247, 1956 (2010).

[153] L.D. Landau und E.M. Lifshitz. Electrodynamics of continuous Media. ButterworthHeinemann (1984), ISBN: 978-0750626347.

[154] Optical Society of America. Handbook of Optics. McGraw-Hill (2001), ISBN: 9780070479746.

[155] L. G. Parratt. Surface Studies of Solids by Total Reflection of X-Rays. Phys. Rev. 95, 359 (1954).

[156] G. A. Niklasson, C. G. Granqvist und O. Hunderi. Effective medium models for the optical properties of inhomogeneous materials. Appl. Opt. 20, 26 (1981).

[157] D. E. Aspnes, J. B. Theeten und F. Hottier. Investigation of effective-medium models of microscopic surface roughness by spectroscopic ellipsometry. Phys. Rev. B 20, 3292 (1979).

[158] R. Gross und A. Marx. Festkörperphysik. De Gruyter Oldenbourg (2014), ISBN: 9783110358698. 
[159] D.E. Aspnes. Optical properties of thin films. Thin Solid Films 89, 249 (1982).

[160] D. A. G. Bruggeman. Berechnung verschiedener physikalischer Konstanten von heterogenen Substanzen. I. Dielektrizitätskonstanten und Leitfähigkeiten der Mischkörper aus isotropen Substanzen. Annalen der Physik 416, 636 (1935).

[161] D. E. Aspnes. Bounds on allowed values of the effective dielectric function of twocomponent composites at finite frequencies. Phys. Rev. B 25, 1358 (1982).

[162] H. Fujiwara, Joohyun Koh, P. I. Rovira und R. W. Collins. Assessment of effectivemedium theories in the analysis of nucleation and microscopic surface roughness evolution for semiconductor thin films. Phys. Rev. B 61, 10832 (2000).

[163] Joohyun Koh, Yiwei Lu, C. R. Wronski, Yalei Kuang, R. W. Collins, T. T. Tsong und Y. E. Strausser. Correlation of real time spectroellipsometry and atomic force microscopy measurements of surface roughness on amorphous semiconductor thin films. Applied Physics Letters 69, 1297 (1996).

[164] Bruker AXS GmbH, Karlsruhe. D8 Advancd D8 Discover Röntgen Diffraktometer Handbuch (2001).

[165] S. Macke, S. Brück, P. Audehm, M. Harlander und E. Goering. ReMagX: X-ray Magnetic Reflectivity Tool (2009).

[166] R. G. Munro. Material Properties of a Sintered $\alpha$-SiC. Journal of Physical and Chemical Reference Data 26, 1195 (1997).

[167] Kloehn Ltd. Hardware User's Manual for the VersaPump 6 Syringe Dispenser Module (2002).

[168] S. N. Jasperson und S. E. Schnatterly. An Improved Method for High Reflectivity Ellipsometry Based on a New Polarization Modulation Technique. Review of Scientific Instruments 40, 761 (1969).

[169] CVI Melles-Griot. Datenblatt UV Glan-Thompson prism polarizer (2009).

[170] Hinds-Instruments Inc. PEM-90 Photoelastic Modulator Systems User Manual (1994).

[171] Hinds-Instruments Inc. Det-100, Hinds Instruments (2007).

[172] Keithley Instruments, Inc. Model 2000 Multimeter User's Manual (1994).

[173] Ametek Advanced Measurement Technology, Inc. Model 5210 Dual Phase Lock-inAmplifier Instruction Manual (2002).

[174] R. O. Bell und G. Rupprecht. Elastic Constants of Strontium Titanate. Phys. Rev. 129, 90 (1963).

[175] G. Shirane und Y. Yamada. Lattice-Dynamical Study of the $110^{\circ} \mathrm{K}$ Phase Transition in $\mathrm{SrTiO}_{3}$. Phys. Rev. 177, 858 (1969).

[176] R. Loetzsch, A. Lübcke, I. Uschmann, E. Förster, V. Große, M. Thuerk, T. Koettig, F. Schmidl und P. Seidel. The cubic to tetragonal phase transition in SrTiO3 single 
crystals near its surface under internal and external strains. Applied Physics Letters 96, 071901 (2010).

[177] CrysTec GmbH. Herstellerangabe Gitterparameter $\mathrm{SrTiO}_{3}$ (2014).

[178] Crystal GmbH. Herstellerangabe Gitterparameter $\mathrm{SrTiO}_{3}$ (2014).

[179] SurfaceNet GmbH. Herstellerangabe Gitterparameter $\left(\mathrm{La}_{0.3} \mathrm{Sr}_{0.7}\right)\left(\mathrm{Al}_{0.65} \mathrm{Ta}_{0.35}\right) \mathrm{O}_{3}(2014)$.

[180] Wei Hong, Ho Nyung Lee, Mina Yoon, Hans M. Christen, Douglas H. Lowndes, Zhigang Suo und Zhenyu Zhang. Persistent Step-Flow Growth of Strained Films on Vicinal Substrates. Phys. Rev. Lett. 95, 095501 (2005).

[181] M. Ohring. Materials Science of Thin Films. Deposition and Structure. Academic Press (2001), ISBN: 978-0125249751.

[182] Masashi Kawasaki, Kazuhiro Takahashi, Tatsuro Maeda, Ryuta Tsuchiya, Makoto Shinohara, Osamu Ishiyama, Takuzo Yonezawa, Mamoru Yoshimoto und Hideomi Koinuma. Atomic Control of the SrTiO3 Crystal Surface. Science 266, 1540 (1994).

[183] M. Kawasaki, A. Ohtomo, T. Arakane, K. Takahashi, M. Yoshimoto und H. Koinuma. Atomic control of SrTiO3 surface for perfect epitaxy of perovskite oxides. Applied Surface Science 107, 102 (1996).

[184] Gertjan Koster, Boike L. Kropman, Guus J. H. M. Rijnders, Dave H. A. Blank und Horst Rogalla. Quasi-ideal strontium titanate crystal surfaces through formation of strontium hydroxide. Applied Physics Letters 73, 2920 (1998).

[185] H. L. Liu, K. S. Lu, M. X. Kuo, L. Uba, S. Uba, L. M. Wang und H.-T. Jeng. Magnetooptical properties of $\mathrm{La}_{0.7} \mathrm{Sr}_{0.3} \mathrm{MnO}_{3}$ thin films with perpendicular magnetic anisotropy. Journal of Applied Physics 99, 043908 (2006).

[186] J. B. Wachtman, T. G. Scuderi und G. W. Cleek. Linear Thermal Expansion of Aluminium Oxide and Thorium Oxide from $100 \mathrm{~K}$ to $1100 \mathrm{~K}$. Journal of The American Ceramic Society 45, 319 (1962).

[187] K. van Benthem, C. Elsässer und R. H. French. Bulk electronic structure of $\mathrm{SrTiO}_{3}$ : Experiment and theory. Journal of Applied Physics 90, 6156 (2001).

[188] Rolf H. Muller und Joseph C. Farmer. Macroscopic optical model for the ellipsometry of an underpotential deposit: Lead on copper and silver. Surface Science 135, 521 (1983).

[189] Sergey Bochkanov. ALGLIB (1999-2015), www.alglib.net.

[190] A. Gambardella, P. Graziosi, I. Bergenti, M. Prezioso, D. Pullini, S. Milita, F. Biscarini und V. A. Dediu. Surface Nanostructures in Manganite Films. Scientific Reports 4, 5353 (2014).

[191] Digital Instruments Veeco Metrology Group. Nanoscope IV Controller Manual (2002).

[192] Bruker. Bruker AFM Manual (2013). 
[193] U. Pietsch, V. Holy und T. Baumbach. High-Resolution X-Ray Scattering: From Thin Films to Lateral Nanostructures. Springer (2004), ISBN: 9780387400921.

[194] Gregory S. Rohrer. Structure and Bonding in Crystalline Materials. Cambridge University Press (2001), ISBN: 9780511816116.

[195] B.D. Cullity und S.R. Stock. Elements of X-ray Diffraction. Prentice Hall (2001), ISBN: 9780201610918.

[196] E. Prince. International Tables for Crystallography. International Union of Crystallography (2006).

[197] Eric Fullerton, Ivan Schuller, H. Vanderstraeten und Y. Bruynseraede. Structural refinement of superlattices from x-ray diffraction. Phys. Rev. B 45, 9292 (1992).

[198] Florian Fischgrabe. Zusammenspiel von Ladungs-, Gitter- und magnetischen Ordnungsskalen in hochdotierten LCMO Schichten. Dissertation, Georg-August-Universität Göttingen (2015).

[199] J. Daillant und A. Gibaud. X-ray and Neutron Reflectivity: Principles and Applications. Springer (1999), ISBN: 9783540661955.

[200] E. Beaurepaire, H. Bulou, F. Scheurer und K. Jean-Paul. Magnetism and Synchrotron Radiation: New Trends. Springer (2010), ISBN: 9783642044984.

[201] A. C. Thompson und E. Gullikson. X-Ray Data Booklet. Lawrence Berkeley National Laboratory (2009), ISBN: 9780201610918.

[202] C.T. Chantler, K. Olsen, R.A. Dragoset, J. Chang, A.R. Kishore, S.A. Kotochigova und D.S. Zucker. Detailed Tabulation of Atomic Form Factors, Photoelectric Absorption and Scattering Cross Section, and Mass Attenuation Coefficients for $Z=1$-92 from $E=1-10 \mathrm{eV}$ to $E=0.4-10 \mathrm{MeV}$ (1997), http://www.nist.gov/pml/data/ffast/.

[203] Ricardo Egoavil. STEM investigation of complex oxides at the atomic scale. PhD Thesis, Universität Antwerpen (2015).

[204] Quantum Design. Magnetic Property Measurement System MPMS XL (1996).

[205] M. A. Garcia, E. Fernandez Pinel, J. de la Venta, A. Quesada, V. Bouzas, J. F. Fernández, J. J. Romero, M. S. Martín González und J. L. Costa-Krämer. Sources of experimental errors in the observation of nanoscale magnetism. Journal of Applied Physics 105, 013925 (2009).

[206] Quantum Design. PPMS Hardware Manual (2004).

[207] M. Jungbauer, S. Hühn, J.-O. Krisponeit und V. Moshnyaga. Visible range colossal magnetorefractive effect in $\left(\mathrm{La}_{1-\mathrm{y}} \mathrm{Pr}_{\mathrm{y}}\right)_{2 / 3} \mathrm{Ca}_{1 / 3} \mathrm{MnO}_{3}$ films. New Journal of Physics 16, 063034 (2014).

[208] Markus Jungbauer. Magnetooptische Effekte in dünnen Manganatschichten. Diplomarbeit, Georg-August-Universität Göttingen (2010).

[209] H. J. Lee, J. H. Jung, Y. S. Lee, J. S. Ahn, T. W. Noh, K. H. Kim und S-W. Cheong. Optical properties of a $\mathrm{Nd}_{0.7} \mathrm{Sr}_{0.3} \mathrm{MnO}_{3}$ single crystal. Phys. Rev. B 60, 5251 (1999). 
[210] S. G. Kaplan, M. Quijada, H. D. Drew, D. B. Tanner, G. C. Xiong, R. Ramesh, C. Kwon und T. Venkatesan. Optical Evidence for the Dynamic Jahn-Teller Effect in $N \mathrm{~d}_{0.7} \mathrm{Sr}_{0.3} M n \mathrm{O}_{3}$. Phys. Rev. Lett. 77, 2081 (1996).

[211] P. Orgiani, C. Aruta, R. Ciancio, A. Galdi und L. Maritato. Enhanced transport properties in $\mathrm{La}_{\mathrm{x}} \mathrm{MnO}_{3-\delta}$ thin films epitaxially grown on $\mathrm{SrTiO}_{3}$ substrates: The profound impact of the oxygen content. Applied Physics Letters 95, 013510 (2009).

[212] O. Chmaissem, B. Dabrowski, S. Kolesnik, J. Mais, D. E. Brown, R. Kruk, P. Prior, B. Pyles und J. D. Jorgensen. Relationship between structural parameters and the Néel temperature in $\mathrm{Sr}_{1-x} \mathrm{Ca}_{x} \mathrm{MnO}_{3}(0<x<1)$ and $\mathrm{Sr}_{1-y} \mathrm{Ba}_{y} \mathrm{MnO}_{3}(y<0.2)$. Phys. Rev. B 64, 134412 (2001).

[213] J. H. Jung, K. H. Kim, T. W. Noh, E. J. Choi und Jaejun Yu. Midgap states of $\mathrm{La}_{1-x} \mathrm{Ca}_{x} \mathrm{MnO}_{3}$ : Doping-dependent optical-conductivity studies. Phys. Rev. B 57, R11043 (1998).

[214] A. S. Moskvin, A. A. Makhnev, L. V. Nomerovannaya, N. N. Loshkareva und A. M. Balbashov. Interplay of $p$ - $d$ and $d$-d charge transfer transitions in rare-earth perovskite manganites. Phys. Rev. B 82, 035106 (2010).

[215] K. Tobe, T. Kimura, Y. Okimoto und Y. Tokura. Anisotropic optical spectra in a detwinned $\mathrm{LaMnO}_{3}$ crystal. Phys. Rev. B 64, 184421 (2001).

[216] H. Boschker, M. Huijben, A. Vailionis, J. Verbeeck, S. van Aert, M. Luysberg, S. Bals, G. van Tendeloo, E. P. Houwman, G. Koster, D. H. A. Blank und G. Rijnders. Optimized fabrication of high-quality $\mathrm{La}_{0.67} \mathrm{Sr}_{0.33} \mathrm{MnO}_{3}$ thin films considering all essential characteristics. Journal of Physics D: Applied Physics 44, 205001 (2011).

[217] J. Choi, C. B. Eom, G. Rijnders, H. Rogalla und D. H. A. Blank. Growth mode transition from layer by layer to step flow during the growth of heteroepitaxial $\mathrm{SrRuO}_{3}$ on (001) $\mathrm{SrTiO}_{3}$. Applied Physics Letters 79, 1447 (2001).

[218] Guus Rijnders, Dave H. A. Blank, Junghoon Choi und Chang-Beom Eom. Enhanced surface diffusion through termination conversion during epitaxial $\mathrm{SrRuO}_{3}$ growth. Applied Physics Letters 84, 505 (2004).

[219] S. N. Putilin, E. V. Antipov, O. Chmaissem und M. Marezio. Superconductivity at 94 $K$ in $\mathrm{HgBa}_{2} \mathrm{CuO}_{4+\delta}$. Nature 362, 226 (1993).

[220] C. A-Paz de Araujo, J. D. Cuchiaro, L. D. McMillan, M. C. Scott und J. F. Scott. Fatigue-free ferroelectric capacitors with platinum electrodes. Nature 374, 627 (1995).

[221] Akihiko Kudo, Akira Tanaka, Kazunari Domen, Ken-ichi Maruya, Ken-ichi Aika und Takaharu Onishi. Photocatalytic decomposition of water over $N i O-\mathrm{K}_{4} \mathrm{Nb}_{6} \mathrm{O}_{17}$ catalyst. Journal of Catalysis 111, 67 (1988).

[222] S. N. Ruddlesden und P. Popper. New compounds of the $\mathrm{K}_{2} \mathrm{NIF}_{4}$ type. Acta Crystallographica 10, 538 (1957).

[223] S. N. Ruddlesden und P. Popper. The compound $\mathrm{Sr}_{3} \mathrm{Ti}_{2} \mathrm{O}_{7}$ and its structure. Acta Crystallographica 11, 54 (1958). 
[224] Che-Hui Lee, Nathan D. Orloff, Turan Birol, Ye Zhu, Veronica Goian, Eduard Rocas, Ryan Haislmaier, Eftihia Vlahos, Julia A. Mundy, Lena F. Kourkoutis, Yuefeng Nie, Michael D. Biegalski, Jingshu Zhang, Margitta Bernhagen, Nicole A. Benedek, Yongsam Kim, Joel D. Brock, Reinhard Uecker, X. X. Xi, Venkatraman Gopalan, Dmitry Nuzhnyy, Stanislav Kamba, David A. Muller, Ichiro Takeuchi, James C. Booth, Craig J. Fennie und Darrell G. Schlom. Exploiting dimensionality and defect mitigation to create tunable microwave dielectrics. Nature 502, 532 (2013).

[225] R.J.D. Tilley. An electron microscope study of perovskite-related oxides in the Sr-Ti-O system. Journal of Solid State Chemistry 21, 293 (1977).

[226] W. Tian, X. Q. Pan, J. H. Haeni und D. G. Schlom. Transmission electron microscopy study of $n=1-5 \mathrm{Sr}_{\mathrm{n}+1} \mathrm{Ti}_{\mathrm{n}} \mathrm{O}_{3 \mathrm{n}+1}$ epitaxial thin films. Journal of Materials Research 16, 2013 (2001).

[227] Lei Yan, Hongjun Niu, Craig A. Bridges, Paul A. Marshall, Joke Hadermann, Gustaav van Tendeloo, Paul R. Chalker und Matthew J. Rosseinsky. Unit-Cell-Level Assembly of Metastable Transition-Metal Oxides by Pulsed-Laser Deposition. Angewandte Chemie International Edition 46, 4539 (2007).

[228] J. H. Haeni, C. D. Theis, D. G. Schlom, W. Tian, X. Q. Pan, H. Chang, I. Takeuchi und X.-D. Xiang. Epitaxial growth of the first five members of the $\mathrm{Sr}_{\mathrm{n}+1} \mathrm{Ti}_{\mathrm{n}} \mathrm{O}_{3 \mathrm{n}+1}$ Ruddlesden-Popper homologous series. Applied Physics Letters 78, 3292 (2001).

[229] D.G. Schlom, J.H. Haeni, J. Lettieri, C.D. Theis, W. Tian, J.C. Jiang und X.Q. Pan. Oxide nano-engineering using MBE. Materials Science and Engineering: B 87, 282 (2001).

[230] Robert G. Palgrave, Pavel Borisov, Matthew S. Dyer, Sean R. C. McMitchell, George R. Darling, John B. Claridge, Maria Batuk, Haiyan Tan, He Tian, Jo Verbeeck, Joke Hadermann und Matthew J. Rosseinsky. Artificial Construction of the Layered Ruddlesden-Popper Manganite $\mathrm{La}_{2} \mathrm{Sr}_{2} \mathrm{Mn}_{3} \mathrm{O}_{10}$ by Reflection High Energy Electron Diffraction Monitored Pulsed Laser Deposition. Journal of the American Chemical Society 134, 7700 (2012).

[231] Hidekazu Tanaka und Tomoji Kawai. Artificial construction of $\mathrm{SrO} /(\mathrm{La}, \mathrm{Sr}) \mathrm{MnO}_{3}$ layered perovskite superlattice by laser molecular-beam epitaxy. Applied Physics Letters 76, 3618 (2000).

[232] M. Jungbauer, S. Hühn, R. Egoavil, H. Tan, J. Verbeeck, G. Van Tendeloo und V. Moshnyaga. Atomic layer epitaxy of Ruddlesden-Popper $\mathrm{SrO}\left(\mathrm{SrTiO}_{3}\right)_{n}$ films by means of metalorganic aerosol deposition. Applied Physics Letters 105, 251603 (2014).

[233] Markus Michelmann. Dissertation, Georg-August-Universität Göttingen (2015).

[234] Y. Tokuda, S. Kobayashi, T. Ohnishi, T. Mizoguchi, N. Shibata, Y. Ikuhara und T. Yamamoto. Growth of Ruddlesden-Popper type faults in Sr-excess $\mathrm{SrTiO}_{3}$ homoepitaxial thin films by pulsed laser deposition. Applied Physics Letters 99, 173109 (2011).

[235] K. Szot und W. Speier. Surfaces of reduced and oxidized $\mathrm{SrTiO}_{3}$ from atomic force microscopy. Phys. Rev. B 60, 5909 (1999). 
[236] Y Zhu, C-H Lee, D Schlom und D Muller. Atomic Scale Chemical Mapping in $\mathrm{SrO}\left(\mathrm{SrTiO}_{3}\right)_{6}$ Ruddlesden-Popper Thin Film. Microscopy and Microanalysis 17, 1396 (2011).

[237] J.H. Haeni, C.D. Theis und D.G. Schlom. RHEED Intensity Oscillations for the Stoichiometric Growth of $\mathrm{SrTiO}_{3}$ Thin Films by Reactive Molecular Beam Epitaxy. Journal of Electroceramics 4, 385 (2000).

[238] Sebastian Hühn. Kationen-Ordnung in ferri/ferromagnetischen perowskitischen Dünnfilmen. Dissertation, Georg-August-Universität Göttingen (2015).

[239] Momoji Kubo, Yasunori Oumi, Ryuji Miura, Andras Stirling, Akira Miyamoto, Masashi Kawasaki, Mamoru Yoshimoto und Hideomi Koinuma. Atomic control of layer-bylayer epitaxial growth on $\mathrm{SrTiO}_{3}(001)$ : Molecular-dynamics simulations. Phys. Rev. B 56, 13535 (1997).

[240] R. Takahashi, Y. Matsumoto, T. Ohsawa, M. Lippmaa, M. Kawasaki und H. Koinuma. Growth dynamics of the epitaxial SrO film on $\mathrm{SrTiO}_{3}$ (001). Journal of Crystal Growth 234, 505 (2002).

[241] V. S. Achutharaman, P. A. Kraus, V. A. Vas'ko, C. A. Nordman und A. M. Goldman. Growth of La-Ca-Mn-O films by ozone-assisted molecular beam epitaxy. Applied Physics Letters 67, 1019 (1995).

[242] G. E. Pynchon und E. F. Sieckmann. Refractive Index of Strontium Oxide. Phys. Rev. 143, 595 (1966).

[243] Felix Massel. Wachstum von Manganatschichten mittels Metallorganischer Aerosoldeposition: Atomlagenepitaxie. Masterarbeit, Georg-August-Universität Göttingen (2014).

[244] P. Padhan und W. Prellier. Direct observation of pinned/biased moments in magnetic superlattices. Phys. Rev. B 72, 104416 (2005).

[245] P. Yu, J.-S. Lee, S. Okamoto, M. D. Rossell, M. Huijben, C.-H. Yang, Q. He, J. X. Zhang, S. Y. Yang, M. J. Lee, Q. M. Ramasse, R. Erni, Y.-H. Chu, D. A. Arena, C.-C. Kao, L. W. Martin und R. Ramesh. Interface Ferromagnetism and Orbital Reconstruction in $\mathrm{BiFeO}_{3}-\mathrm{La}_{0.7} \mathrm{Sr}_{0.3} \mathrm{MnO}_{3}$ Heterostructures. Phys. Rev. Lett. 105, 027201 (2010).

[246] M. Huijben, P. Yu, L. W. Martin, H. J. A. Molegraaf, Y.-H. Chu, M. B. Holcomb, N. Balke, G. Rijnders und R. Ramesh. Ultrathin Limit of Exchange Bias Coupling at Oxide Multiferroic/Ferromagnetic Interfaces. Advanced Materials 25, 4739 (2013).

[247] J. Bertinshaw, S. Brück, D. Lott, H. Fritzsche, Y. Khaydukov, O. Soltwedel, T. Keller, E. Goering, P. Audehm, D. L. Cortie, W. D. Hutchison, Q. M. Ramasse, M. Arredondo, R. Maran, V. Nagarajan, F. Klose und C. Ulrich. Element-specific depth profile of magnetism and stoichiometry at the $\mathrm{La}_{0.67} \mathrm{Sr}_{0.33} \mathrm{MnO}_{3} / \mathrm{BiFeO}_{3}$ interface. Phys. Rev. B 90, 041113 (2014).

[248] I. Panagiotopoulos, C. Christides, N. Moutis, M. Pissas und D. Niarchos. Exchange biasing in $\mathrm{La}_{2 / 3} \mathrm{Ca}_{1 / 3} \mathrm{MnO}_{3} / \mathrm{La}_{1 / 3} \mathrm{Ca}_{2 / 3} \mathrm{MnO}_{3}$ multilayers. J. Appl. Phys. 85, 4913 (1999). 
[249] A. L. Kobrinskii, A. M. Goldman, Maria Varela und S. J. Pennycook. Thickness dependence of the exchange bias in epitaxial manganite bilayers. Phys. Rev. B 79, 094405 (2009).

[250] N. Moutis, C. Christides, I. Panagiotopoulos und D. Niarchos. Exchange-coupling properties of $\mathrm{La}_{1-x} \mathrm{Ca}_{x} \mathrm{MnO}_{3}$ ferromagnetic/antiferromagnetic multilayers. Phys. Rev. B 64, 094429 (2001).

[251] S. J. Zhu, J. Yuan, B. Y. Zhu, F. C. Zhang, B. Xu, L. X. Cao, X. G. Qiu, B. R. Zhao und $\mathrm{P} . \mathrm{X}$. Zhang. Exchange bias effect and enhanced magnetoresistance in $\mathrm{La}_{0.67} \mathrm{Sr}_{0.33} \mathrm{MnO}_{3} / \mathrm{SrTiO}_{3}$ superlattices. Appl. Phys. Lett. 90, 112502 (2007).

[252] M. Gibert, P. Zubko, R. Scherwitzl, J. Íñiguez und J.-M. Triscone. Exchange bias in $\mathrm{LaNiO}_{3}-\mathrm{LaMnO}_{3}$ superlattices. Nature Materials 11, 195 (2012).

[253] J. C. Rojas Sánchez, B. Nelson-Cheeseman, M. Granada, E. Arenholz und L. B. Steren. Exchange-bias effect at $\mathrm{La}_{0.75} \mathrm{Sr}_{0.25} \mathrm{MnO}_{3} / \mathrm{LaNiO}_{3}$ interfaces. Phys. Rev. B 85, 094427 (2012).

[254] Shuai Dong, Kunihiko Yamauchi, Seiji Yunoki, Rong Yu, Shuhua Liang, Adriana Moreo, J.-M. Liu, Silvia Picozzi und Elbio Dagotto. Exchange Bias Driven by the Dzyaloshinskii-Moriya Interaction and Ferroelectric Polarization at G-Type Antiferromagnetic Perovskite Interfaces. Phys. Rev. Lett. 103, 127201 (2009).

[255] M. Jungbauer, S. Hühn, M. Michelmann, E. Goering und V. Moshnyaga. Exchange bias in $\mathrm{La}_{0.7} \mathrm{Sr}_{0.3} \mathrm{MnO}_{3} / \mathrm{SrMnO}_{3} / \mathrm{La}_{0.7} \mathrm{Sr}_{0.3} \mathrm{MnO}_{3}$ trilayers. Journal of Applied Physics 113, 17D709 (2013).

[256] Mercy Mathews, Ferry M. Postma, J. Cock Lodder, R. Jansen, Guus Rijnders und Dave H. A. Blank. Step-induced uniaxial magnetic anisotropy of $\mathrm{La}_{0.67} \mathrm{Sr}_{0.33} \mathrm{MnO}_{3}$ thin films. Applied Physics Letters 87, 242507 (2005).

[257] J.-L. Maurice, F. Pailloux, A. Barthélémy, O. Durand, D. Imhoff, R. Lyonnet, A. Rocher und J.-P. Contour. Strain relaxation in the epitaxy of $\mathrm{La}_{2 / 3} \mathrm{Sr}_{1 / 3} \mathrm{MnO}_{3}$ grown by pulsed-laser deposition on $\mathrm{SrTiO}_{3}$ (001). Philosophical Magazine 83, 3201 (2003).

[258] C. Adamo, X. Ke, H. Q. Wang, H. L. Xin, T. Heeg, M. E. Hawley, W. Zander, J. Schubert, P. Schiffer, D. A. Muller, L. Maritato und D. G. Schlom. Effect of biaxial strain on the electrical and magnetic properties of (001) $\mathrm{La}_{0.7} \mathrm{Sr}_{0.3} \mathrm{MnO}_{3}$ thin films. Applied Physics Letters 95, 112504 (2009).

[259] A. Vailionis, H. Boschker, Z. Liao, J. R. A. Smit, G. Rijnders, M. Huijben und G. Koster. Symmetry and lattice mismatch induced strain accommodation near and away from correlated perovskite interfaces. Applied Physics Letters 105, 131906 (2014).

[260] K. Steenbeck und R. Hiergeist. Magnetic anisotropy of ferromagnetic $\mathrm{La}_{0.7}(\mathrm{Sr}, \mathrm{Ca})_{0.3} \mathrm{MnO}_{3}$ epitaxial films. Applied Physics Letters 75, 1778 (1999).

[261] F. Tsui, M. C. Smoak, T. K. Nath und C. B. Eom. Strain-dependent magnetic phase diagram of epitaxial $\mathrm{La}_{0.67} \mathrm{Sr}_{0.33} \mathrm{MnO}_{3}$ thin films. Applied Physics Letters 76, 2421 (2000). 
[262] T. Yu, X. K. Ning, W. Liu, J. N. Feng, X. G. Zhao und Z. D. Zhang. Exchange bias effect in epitaxial $\mathrm{La}_{0.67} \mathrm{Ca}_{0.33} \mathrm{MnO}_{3} / \mathrm{SrMnO}_{3}$ thin film structure. Journal of Applied Physics 116, 083908 (2014).

[263] J. F. Ding, O. I. Lebedev, S. Turner, Y. F. Tian, W. J. Hu, J. W. Seo, C. Panagopoulos, W. Prellier, G. Van Tendeloo und T. Wu. Interfacial spin glass state and exchange bias in manganite bilayers with competing magnetic orders. Phys. Rev. B 87, 054428 (2013).

[264] A. Daoud-Aladine, C. Martin, L. C. Chapon, M. Hervieu, K. S. Knight, M. Brunelli und P. G. Radaelli. Structural phase transition and magnetism in hexagonal $\mathrm{SrMnO}_{3}$ by magnetization measurements and by electron, $x$-ray, and neutron diffraction studies. Phys. Rev. B 75, 104417 (2007).

[265] M. Ali, C. H. Marrows, M. Al-Jawad, B. J. Hickey, A. Misra, U. Nowak und K. D. Usadel. Antiferromagnetic layer thickness dependence of the IrMn/Co exchange-bias system. Phys. Rev. B 68, 214420 (2003).

[266] H. M. Christen und G. Eres. Recent advances in pulsed-laser deposition of complex oxides. Journal of Physics: Condensed Matter 20, 264005 (2008).

[267] Danny Schwarzbach. Magnetismus in $\mathrm{LaMnO}_{3} / \mathrm{SrMnO}_{3}$-Mehrlagensystemen: Einfluss von Grenzflächen- und Verspannungseffekten. Masterarbeit, Georg-August-Universität Göttingen (2015).

[268] P.B. Hirsch. Nucleation and Propagation of Misfit Dislocations in Strained Epitaxial Layer Systems in Polycrystalline Semiconductors II. Springer Berlin Heidelberg (1991), ISBN: 978-3-642-76387-8.

[269] Dominique de Ligny und Pascal Richet. High-temperature heat capacity and thermal expansion of $\mathrm{SrTiO}_{3}$ and $\mathrm{SrZrO}_{3}$ perovskites. Phys. Rev. B 53, 3013 (1996).

[270] F. Tietz. Thermal expansion of SOFC materials. Ionics 5, 129 (1999).

[271] S. Chikazumi und C.D. Graham. Physics of Ferromagnetism. Clarendon Press (1997), ISBN: 978-0-1915-6985-2.

[272] P. Perna, C. Rodrigo, E. Jiménez, F. J. Teran, N. Mikuszeit, L. Méchin, J. Camarero und R. Miranda. Tailoring magnetic anisotropy in epitaxial half metallic $\mathrm{La}_{0.7} \mathrm{Sr}_{0.3} \mathrm{MnO}_{3}$ thin films. Journal of Applied Physics 110, 013919 (2011).

[273] V. Korenivski, R. B. van Dover, Y. Suzuki, E. M. Gyorgy, J. M. Phillips und R. J. Felder. Interlayer exchange coupling in amorphous/crystalline $\mathrm{NiFe}_{2} \mathrm{O}_{4}$ thin-film bilayers. J. Appl. Phys. 79, 5926 (1996).

[274] C. Adamo, X. Ke, P. Schiffer, A. Soukiassian, M. Warusawithana, L. Maritato und D. G. Schlom. Electrical and magnetic properties of $\mathrm{SrMnO}_{3} / \mathrm{LaMnO}_{3}$ superlattices. Applied Physics Letters 92, 112508 (2008).

[275] T. S. Santos. Modulation Doping of Ferromagnetism in Manganite Superlattices (2010), http://www.ncnr.nist.gov/summerschool/ss10/pdf/Talk_Santos.pdf. 
[276] S. J. May, P. J. Ryan, J. L. Robertson, J.-W. Kim, T. S. Santos, E. Karapetrova, J. L. Zarestky, X. Zhai, S. G. E. te Velthuis, J. N. Eckstein, S. D. Bader und A. Bhattacharya. Enhanced ordering temperatures in antiferromagnetic manganite superlattices (Supplementary Information). Nature Materials 8, 892 (2009).

[277] R. D. Shannon. Revised effective ionic radii and systematic studies of interatomic distances in halides and chalcogenides. Acta Crystallographica Section A 32, 751 (1976).

[278] Wojciech Paszkowicz, Jaroslaw Pietosa, Scott M. Woodley, Piotr A. Dluzewski, Miroslaw Kozlowski und Christine Martin. Lattice parameters and orthorhombic distortion of $\mathrm{CaMnO}_{3}$. Powder Diffraction 25, 46 (2010).

[279] Aymeric Sadoc, Bernard Mercey, Charles Simon, Dominique Grebille, Wilfrid Prellier und Marie-Bernadette Lepetit. Large Increase of the Curie Temperature by Orbital Ordering Control. Phys. Rev. Lett. 104, 046804 (2010).

[280] Marius Keunecke. TBA. Masterarbeit, Georg-August-Universität Göttingen (2015). 



\section{Publikationen}

\section{Artikel in wissenschaftlichen Fachzeitschriften}

- Nanoscale Mapping of the Magnetic Properties of (111)-Oriented $\mathrm{La}_{0.67} \mathrm{Sr}_{0.33} \mathrm{MnO}_{3}$. K. J. O'Shea, D. A. MacLaren, D. McGrouther, D. Schwarzbach, M. Jungbauer, S. Hühn, V. Moshnyaga und R. L. Stamps.

Nano Letters 15, 5868 (2015).

- Phase Problem in the B-site ordering of $\mathrm{La}_{2} \mathrm{CoMnO}_{6}$ : Impact on Structure and Magnetism.

R. Egoavil, S. Hühn, M. Jungbauer, N. Gauquelin,

A. Béche, G. van Tendeloo, J. Verbeeck, V. Moshnyaga.

Nanoscale 7, 9835 (2015).

- Atomic Layer Epitaxy of Ruddlesden-Popper $\mathrm{SrO}\left(\mathrm{SrTiO}_{3}\right)_{\mathrm{n}}$ Films by means of Metalorganic Aerosol Deposition.

M. Jungbauer, S. Hühn, R. Egoavil, H. Tan,

J. Verbeeck, G. van Tendeloo, V. Moshnyaga.

Applied Physics Letters 105, 251603 (2014).

- Fabrication of high quality plan-view TEM specimens using the focused ion beam.

K.J. O'Shea, D. McGrouther, C.A. Ferguson,

M. Jungbauer, S. Hühn, V. Moshnyaga, D.A. MacLaren.

Micron 66, 9 (2014).

- Visible range colossal magnetorefractive effect in $\left(\mathrm{La}_{1-y} \mathrm{Pr}_{\mathrm{y}}\right)_{2 / 3} \mathrm{Ca}_{1 / 3} \mathrm{MnO}_{3}$ films.

M. Jungbauer, S. Hühn, J.-O. Krisponeit, V. Moshnyaga.

New Journal of Physics 16, 063034 (2014). 
- Intrinsic antiferromagnetic coupling underlies colossal magnetoresistance effect: Role of correlated polarons.

V. Moshnyaga, A. Belenchuk, S. Hühn, C. Kalkert, M. Jungbauer,

O. I. Lebedev, S. Merten, K.-Y. Choi, P. Lemmens, B. Damaschke, K. Samwer.

Physical Review B 89, 024420 (2014).

- Modeling of colossal magnetoresistance in

$\mathrm{La}_{0.67} \mathrm{Ca}_{0.33} \mathrm{MnO}_{3} / \mathrm{Pr}_{0.67} \mathrm{Ca}_{0.33} \mathrm{MnO}_{3}$ superlattices:

Comparison with individual $\left(\mathrm{La}_{1-\mathrm{y}} \mathrm{Pr}_{\mathrm{y}}\right)_{0.67} \mathrm{Ca}_{0.33} \mathrm{MnO}_{3}$ films.

S. Hühn, M. Jungbauer, M. Michelmann,

F. Massel, F. Koeth, C. Ballani, V. Moshnyaga.

Journal of Applied Physics 113, 17D701 (2013).

- Interface-controlled magnetism and transport of ultrathin manganite films.

O. Shapoval, S. Hühn, J. Verbeeck, M. Jungbauer A. Belenchuk, V. Moshnyaga. Journal of Applied Physics 113, 17C711 8 (2013).

- Exchange bias in $\mathrm{La}_{0.7} \mathrm{Sr}_{0.3} \mathrm{MnO}_{3} / \mathrm{SrMnO}_{3} / \mathrm{La}_{0.7} \mathrm{Sr}_{0.3} \mathrm{MnO}_{3}$ trilayers.

M. Jungbauer, S. Hühn, M. Michelmann, E. Goering, V. Moshnyaga.

Journal of Applied Physics 113, 17D709 (2013).

\section{Beiträge auf Konferenzen}

- Frühjahrstagung der Deutschen Physikalischen Gesellschaft, Dresden, 2009.

- Frühjahrstagung der Deutschen Physikalischen Gesellschaft, Regensburg, 2010.

- Frühjahrstagung der Deutschen Physikalischen Gesellschaft, Dresden, 2011.

- Frühjahrstagung der Deutschen Physikalischen Gesellschaft, Berlin, 2012.

- MMM-Intermag Conference, Chicago, 2013.

- Frühjahrstagung der Deutschen Physikalischen Gesellschaft, Regensburg, 2013. 


\section{Danksagungen}

Diese Arbeit wäre ohne die hilfsbereite Unterstützung vieler Menschen nicht möglich gewesen. Ich möchte hier insbesondere folgenden Personen danken:

- Prof. Dr. Vasily Moshnyaga für die intensive, fast väterliche, Betreuung in meiner Promotionszeit, die sich nicht nur auf wissenschaftliche Aspekte bezog. Vasily hatte immer ein offenes Ohr für meine Probleme und lieferte neue Anregungen für weitere Forschungsvorhaben. Mit ihm ergaben sich immer sehr offene und interessante Diskussionen, für die er sich viel Zeit nahm und sehr genau auf mich einging.

- Prof. Dr. Konrad Samwer für die herzliche Aufnahme in das I.Physikalische Institut, die Unterstützung bei meiner wissenschaftlichen Ausbildung und das spontane Einspringen bei der Unterbrechung der Finanzierung durch die EU.

- Prof. Dr. Christian Jooß, Prof. Dr. Guus Rijnders für ihre Bereitschaft, meine Arbeit als Korreferenten zu begutachten.

- Prof. Dr. Hans Hofsäss, Prof. Dr. Wolfram Kollatschny, Prof. Dr. HansUlrich Krebs, Prof. Dr. Michael Seibt für ihre Zusage zur Teilnahme in der Prüfungskommission meiner Disputation.

- Danny Schwarzbach, Sven Esser und Marius Keunecke für die Herstellung und Untersuchung einiger LMO/SMO-Übergitter, Felix Massel und Fryderyk Lyzwa für die experimentellen Arbeiten an der MAD-Atomlagenepitaxie und der ganzen ManganatGruppe für viele interessante (nicht-)wissenschaftliche Diskussionen.

- Ricardo Egoavil aus der Arbeitsgruppe von Johan Verbeeck sowie Vladimir Roddatis für die Untersuchung der hier vorgestellten Proben mit dem TEM.

- Der feinmechanischen Werkstatt des I. Physikalischen Instituts für die Konstruktion der hier vorgestellten MAD-Anlage, insbesondere Hartmut Eichenberg und dem leider viel zu früh verstorbenen Roberto Rudat.

- Carsten Mahn, Uta Filippich und Katrin Gehrke für die Hilfe bei allen technischen Problemen, die sich zum Teil zu unmöglichen Zeiten ergaben.

- Allen Mitgliedern des I. Physikalischen Instituts für eine freundschaftliche und ungezwungene Arbeitsatmosphäre.

- Meiner Schwester Andrea und Manuel Mchalwat für das Korrekturlesen. 